\title{
Dynamics and Characterization of Composite Quantum Systems
}

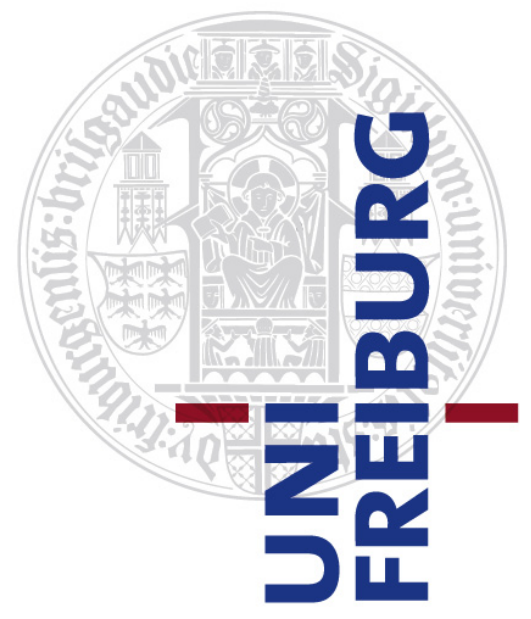

\section{DiSSERTATION}

zur Erlangung des Doktorgrades der

Fakultät für Mathematik und Physik der

Albert-Ludwigs-Universität Freiburg im Breisgau

vorgelegt von

Manuel Gessner

2015 
Dekan: Prof. Dr. Dietmar Kröner Betreuer der Arbeit: Prof. Dr. Andreas Buchleitner

Referent: Prof. Dr. Andreas Buchleitner Koreferent: Prof. Dr. Gerhard Stock

Tag der mündlichen Prüfung: 20.07.2015

Prüfer: Prof. Dr. Andreas Buchleitner Prof. Dr. Hermann Grabert Prof. Dr. Tobias Schätz 



\begin{abstract}
Due to experimental developments over the past decades on quantum optical, and atomic systems, a wealth of composite quantum systems of variable sizes has become accessible under rather well-controlled conditions. Typical systems of tens of trapped ions or thousands of cold neutral atoms are usually too large to fully measure all of their constituents' microscopic quantum properties, but not large enough to be described completely in terms of thermodynamic quantities. This challenging, intermediate regime of controllable quantum few- to many-body systems, is particularly interesting since it combines a variety of different phenomena and applications, ranging from quantum information theory to solid state physics.

The effective characterization of these systems requires flexible and experimentally feasible observables, complemented by efficient theoretical methods and models. In this dissertation we employ concepts from the fields of open quantum systems, quantum information theory, quantum many-body theory and physical chemistry, to construct dynamical approaches for the study of various aspects of correlations, and to describe spectral and dynamical features of complex, interacting quantum systems. Some of the developed theoretical ideas are complemented by experimental realizations with trapped ions or photons.

To facilitate the scalable analysis of bipartite correlation properties in the context of quantum information theory, we introduce a method which allows to detect and estimate discord-type correlations when only one of the two correlated subsystems can be measured. The method makes use of the influence of the correlations on the local subsystem dynamics, which illustrates the fundamental role of initial correlations for the theory of open quantum systems. We present an experimental realization with a single trapped ion, as well as the description of a photonic experiment. Further theoretical studies are presented, including the application to a spin-chain model, which relates the dynamical single-spin signature of the ground-state quantum correlations to a quantum phase transition.

Having established this local detection technique for quantum discord, whose information about the state's correlations is limited, we introduce the correlation rank to assess the degree of total correlations of bipartite quantum states. This allows us to identify strongly correlated states which cannot be generated with local operations. Classically correlated noise processes, however, are able to generate strongly correlated, but separable quantum states. This is confirmed in a trapped-ion experiment, where such noise processes occur naturally, and represent one of the dominant sources of error. We further develop a fully analytical description of the generated ensembleaverage dynamics, allowing us to de derive conditions that ensure the robustness of entanglement in bipartite and multi-particle systems.

Information-theoretic quantifiers of the correlation properties between the constituents no longer represent suitable observables for increasingly complex composite quantum systems. Hence, we develop a multi-configurational mean-field approach in order to understand the dynamical features and, with it, the role of the energy spectrum in the vicinity of the quantum phase transition in a quantum magnet. Specifically, we study a spin-chain model with variable-range interactions in a transverse field, which
\end{abstract}


can be realized in trapped-ion quantum simulators. The obtained semiclassical model allows for an analytical analysis of the excitation spectrum, whose predictions are exact in the limit of very strong or vanishing external magnetic fields. Bifurcations of a series of excited-state energy landscapes below a threshold value of the external magnetic field reflect the quantum phase transition from the paramagnetic phase to the (anti-)ferromagnetic phase in the entire excitation spectrum - and not just in the ground state.

To develop a set of experimentally accessible, suitable observables, able to cope with complex dynamics in quantum optical systems, we develop a general framework based on ideas from nonlinear spectroscopy. Sequences of phase-coherent laser pulses allow us to extract multi-time correlation functions, which may be combined with single-site addressability to achieve spatial resolution. We propose specific schemes to realize the elementary steps with existing trapped-ion technology, and discuss a variety of applications based on second-order and fourth-order signals. The obtained multi-dimensional spectra are particularly suited to extract information about the system's environmental influences, the relevant transport mechanisms, and particleparticle interactions.

The theoretical description of interacting many-body systems becomes particularly hard when the quantum statistics is explicitly taken into account. Generalizing concepts from open-system theory to the case of identical particles, we study the dynamics of a subsystem of interacting bosons. We obtain a hierarchical expansion of the coherent subsystem evolution, which can be truncated by a mean-field ansatz. When applied to a dilute Bose-Einstein condensate, we recover the Gross-Pitaevskii equation. Based on a perturbative second-order expansion in the interaction strength, we establish first steps towards a microscopic derivation of a master-equation description that is able to account for interaction-induced decoherence. 


\section{Zusammenfassung}

Der experimentelle Fortschritt der letzten Jahrzehnte an quantenoptischen und atomaren Systemen ermöglicht heute Experimente unter kontrollierten Bedingungen an einer Vielzahl zusammengesetzter Quantensysteme verschiedener Größen. Typische Systeme, bestehend aus dutzenden gefangenen Ionen oder tausenden kalten, neutralen Atomen, sind typischerweise zu groß, um alle mikroskopischen Eigenschaften ihrer Bestandteile zu messen, während sie noch nicht groß genug sind, um sie vollständig durch thermodynamische Größen zu beschreiben. Dieses herausfordernde Gebiet kontrollierbarer, quantenmechanischer Wenig- bis Vielteilchensysteme ist besonders interessant, da es eine Vielfalt von Phänomenen und Anwendungen kombiniert, die verschiedene Gebiete der Physik, von der Quanteninformationstheorie bis hin zur Festkörperphysik, überspannen.

Die effektive Charakterisierung dieser Systeme bedarf flexibler und experimentell zugänglicher Observablen, ergänzt durch effiziente theoretische Methoden und Modelle. In dieser Dissertation kombinieren wir Konzepte aus den Gebieten der offenen Quantensysteme, der Quanteninformationstheorie, der Quantenvielteilchentheorie und der physikalischen Chemie, um dynamische Zugänge zur Analyse verschiedener Korrelationsaspekte zu konstruieren und, um spektrale und dynamische Eigenschaften komplexer, wechselwirkender Quantensysteme zu beschreiben. Einige der entwickelten theoretischen Ideen werden durch experimentelle Umsetzungen mit gefangenen Ionen und Photonen ergänzt.

Um die skalierbare Analyse bipartiter Korrelationseigenschaften im Kontext der Quanteninformationstheorie zu ermöglichen, führen wir eine Methode ein, die es erlaubt Discord-ähnliche Korrelationen zu detektieren und abzuschätzen, wenn nur eines der beiden korrelierten Untersysteme gemessen werden kann. Die Methode nutzt den Einfluss der Korrelationen auf die Dynamik des Untersystems aus, was wiederum die fundamentale Rolle von Anfangskorrelationen in der Theorie offener Quantensysteme hervorhebt. Wir präsentieren sowohl eine experimentelle Umsetzung dieser theoretischen Idee mit einem einzelnen gefangenen Ion, als auch die Beschreibung eines Experiments mit Photonen. Wir stellen weitere theoretische Studien dazu vor; darunter auch die Anwendung dieser Methode in einer Spinkette, welche die dynamische Signatur eines einzelnen Spins mit einem Quantenphasenübergang und den Quantenkorrelationen des Grundzustands in Verbindung setzt.

Da der Quantendiscord nur wenig Information über die Korrelationen des zugrundeliegenden Zustandes enthält, stellen wir den Korrelationsrang vor, der in der Lage ist, die Gesamtkorrelationen bipartiter Quantenzustände zu erfassen. Dadurch sind wir in der Lage, stark korrelierte Zustände zu identifizieren, die nicht durch lokale Operationen erzeugt werden können. Andererseits ist klassisches Rauschen in der Lage, stark korrelierte, aber separable Quantenzustände zu erzeugen. Dies wird durch ein weiteres Experiment mit gefangenen Ionen bestätigt, wo ein solches Rauschen auf natürliche Weise auftritt und eine der dominanten Fehlerquellen darstellt. Des Weiteren entwickeln wir eine vollständig analytische Beschreibung der zugrundeliegenden Dynamik des Ensemblemittels, welche uns erlaubt, Bedingungen zur Erhaltung von Zwei- und Vielteilchenverschränkung zu bestimmen. 
Informationstheoretische Maße der Korrelationseigenschaften zwischen den Einzelbestandteilen stellen keine geeigneten Observablen mehr dar, sobald die behandelten zusammengesetzten Quantensysteme zunehmend komplex werden. Um die dynamischen Eigenschaften und die damit verbundene Rolle des Energiespektrums in der Umgebung des Quantenphasenübergangs eines Quantenmagneten zu verstehen, entwickeln wir daher eine Molekularfeldnäherung. Das resultierende semiklassische Modell liefert eine Näherung des Anregungsspektrums, welche in den Grenzfällen sehr starker und verschwindender externer Magnetfelder exakte Ergebnisse liefert. Unterhalb eines Grenzwertes des Magnetfeldes entstehen Bifurkationen einer Reihe semiklassischer Energielandschaften, die angeregten Quantenzuständen zugeordnet werden, und daher den Quantenphasenübergang von der paramagnetischen zur (anti-)ferromagnetischen Phase im gesamten Anregungsspektrum, und nicht nur in den Grundzustandseigenschaften, wiederspiegeln.

Um einen Satz geeigneter, experimentell zugänglicher Observablen zu konstruieren, die in der Lage sind, komplexe Dynamik in quantenoptischen Systemen zu erfassen, entwickeln wir einen allgemeinen Ansatz, basierend auf Ideen aus dem Gebiet der nichtlinearen Spektroskopie. Sequenzen phasenkohärenter Laserpulse erlauben es uns Zeit-Mehrpunktskorrelationsfunktionen des Systems zu extrahieren, die wiederum, durch Laser-fokussierte Adressierung einzelner Bestandteile, mit räumlicher Auflösung kombiniert werden können. Wir schlagen spezielle Methoden zur experimentellen Umsetzung der grundlegenden Schritte, basierend auf existierender Technologie gefangener Ionen, vor und diskutieren eine Vielzahl von Anwendungen spezieller Signale zweiter und vierter Ordnung. Die erhaltenen mehrdimensionalen Spektren sind besonders geeignet um Umgebungseinflüsse, relevante Transportprozesse, sowie Wechselwirkungen zu untersuchen.

Die theoretische Beschreibung wechselwirkender Vielteilchensysteme ist besonders schwierig, wenn die Quantenstatistik der Teilchen explizit mitbehandelt wird. Indem wir Konzepte der Theorie offener Quantensysteme auf Systeme ununterscheidbarer Teilchen verallgemeinern, untersuchen wir die Dynamik einer Untergruppe von wechselwirkenden Bosonen. Wir erhalten eine hierarchische Serie von dynamischen Gleichungen zur Beschreibung der kohärenten Zeitentwicklung des Untersystems, welche durch einen Molekularfeldansatz abgebrochen werden kann. Wird dieses Ergebnis auf ein Bose-Einstein Kondensat niedriger Teilchendichte angewandt, bringt es die GrossPitaevskii Gleichung hervor. Durch eine störungstheoretische Entwicklung der Wechselwirkungsstärke zur zweiten Ordnung, können wir erste Schritte zur mikroskopischen Herleitung einer Master-Gleichung unternehmen, die Dekohärenz durch Wechselwirkung beschreiben kann. 

Ich möchte mich bei Andreas Buchleitner, für die Betreuung und Korrektur dieser Arbeit, und die tolle Zeit im 9. Stock des Instituts in Freiburg, herzlich bedanken.

I would like to thank Hartmut Häffner for organizing my stay in Berkeley, and his group, especially Michael Ramm and Thaned (Hong) Pruttivarasin, for a great time, including, but not limited to the lab.

Just like the experiments in Berkeley, many parts of this dissertation are the result of collaborations. In particular, I would like to thank Frank Schlawin for the stimulating joint work on nonlinear spectroscopy, Heinz-Peter Breuer, for continuous advice and collaborations on open quantum systems, and Edoardo Carnio, for his talented work on collective dephasing during his Master's thesis. I would like to thank Elsi-Mari Laine and Jyrki Piilo for hosting me in Turku, as well as Tobias Brandes and Victor Bastidas for the time in Berlin. I would also like to thank Shaul Mukamel for interesting discussions and collaborations. Moreover, my gratitude goes to Christian Roos and his team, in particular, Ben Lanyon, for the collaboration. I also thank Chuan-Feng Li and his group in Hefei.

Moreover, I thank everyone in the 9th floor for the good atmosphere. Special thanks goes to Stefan Fischer and Frank Schlawin with whom I enjoyed uncountable lunch and coffee breaks. I also thank Ugo Marzolino and Mattia Walschaers for frequent discussions. Vielen Dank auch an Gislinde Bühler für viel organisatorische Hilfe.

I thank the German National Academic Foundation (Studienstiftung des deutschen Volkes) for supporting my work on this thesis and providing me with travel funds.

Außerdem bedanke ich mich bei meinen Eltern, meinen Schwestern, und bei Patricia. 



\section{Contents}

1 Introduction 1

2 Background 5

2.1 Elements of quantum information theory . . . . . . . . . . . . 5

2.1.1 Correlations in composite quantum systems: Entanglement and

discord ..................... 7

2.1.2 Role of correlated quantum states in quantum information theory 9

2.2 Controllable quantum systems . . . . . . . . . . . . . . . . . . . 12

2.2 .1 Trapped ions . . . . . . . . . . . . . . . . 12

2.2.1.1 Paul traps . . . . . . . . . . . . . . . 13

2.2.1.2 The Berkeley setup . . . . . . . . . . . . . . 14

2.2.1.3 Motion of trapped ions in linear chains . . . . . . . . 15

2.2.1.4 Electronic levels . . . . . . . . . . . . . . . . 16

2.2.1.5 Quantum state readout and initialization . . . . . . . 18

2.2.1.6 Laser-ion interactions . . . . . . . . . . . . . . . . . 19

2.2.1.7 Experimental quantum computations and quantum sim-

ulations . . . . . . . . . . . . . . . 22

2.2.1.8 Recent experimental developments and challenges . . . 24

2.2.2 Cold gases of neutral atoms in optical lattices . . . . . . . . . . 25

2.2 .3 Photons and (non-)linear optics . . . . . . . . . . . . . 28

2.2 .4 Other systems . . . . . . . . . . . . . . . . . . . . . . 29

2.3 The certification of large-scale quantum devices . . . . . . . . . . . . 31

2.4 Nonlinear spectroscopy . . . . . . . . . . . . . . . . . . . . 33

2.5 Theoretical description of composite quantum systems . . . . . . . . . 37

2.5.1 Semiclassical approximations and mean-field theories . . . . . . . 37

2.5.2 Complex systems, spectral analysis, and random matrix theory . 38

2.5.3 Open quantum systems . . . . . . . . . . . . . . . . . 41

2.5.4 Identical particles . . . . . . . . . . . . . . . . . 46 


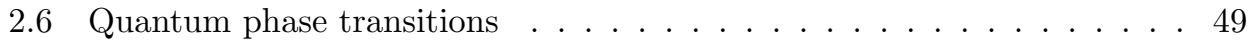

2.7 Scope and structure of this dissertation . . . . . . . . . . . . . 51

3 Local detection of correlations 53

3.1 The local detection protocol . . . . . . . . . . . . . . . 53

3.1.1 Local witness for bipartite quantum discord . . . . . . . . . . . 54

3.1.2 Local bound for the minimum entanglement potential . . . . . . 57

3.1.3 Efficacy of the method . . . . . . . . . . . . . . 60

3.2 Trapped-ion experiment . . . . . . . . . . . . . . . 61

3.2.1 Resonant laser-ion interactions . . . . . . . . . . . 61

3.2.1.1 Carrier transition . . . . . . . . . . . . 62

3.2.1.2 First red sideband transition . . . . . . . . . . . 63

3.2.1.3 First blue sideband transition . . . . . . . . . . . 63

3.2.1.4 Arbitrary order sidebands . . . . . . . . . . . . . 64

3.2.2 The effect of small detunings . . . . . . . . . . . . . . . 66

3.2.3 The local detection protocol for the first blue sideband . . . . . . 68

3.2.3.1 Preparation . . . . . . . . . . . . . 69

3.2.3.2 Implementing the local dephasing operation . . . . . . 69

3.2.3.3 Detection . . . . . . . . . . . . . . . 72

3.2.3.4 Temperature dependence of the local signal . . . . . . . 74

3.2.3.5 Time-averaged local Hilbert-Schmidt distance . . . . . 76

3.2.4 Generalization to arbitrary sidebands . . . . . . . . . . . . 77

3.2.5 Extension of the experimental technique . . . . . . . . . . . 79

3.3 Photonic experiment . . . . . . . . . . . . . . . . 79

3.3.1 The pre-initial state . . . . . . . . . . . . 80

3.3.2 Preparation of the initial state . . . . . . . . . . . . . 82

3.3.3 Local dephasing operation . . . . . . . . . . . . . . . . . 83

3.3.4 Reduced distributions . . . . . . . . . . . . . . . 85

3.3.5 Total trace distance . . . . . . . . . . . . . . . . . . 85

3.3.6 Open-system evolution depending on initial correlations . . . . . 86

3.3.7 Local trace distance . . . . . . . . . . . . . . . . . . . . 87

3.4 Theoretical studies of further examples . . . . . . . . . . . . . 90

3.4.1 Atom-photon correlations during spontaneous decay . . . . . . . 91

3.4.1.1 Atomic evolution with and without local dephasing:

Resolvent method . . . . . . . . . . . . . 91

3.4.1.2 Evolution of atom-field negativity . . . . . . . . . 95

3.4.1.3 Discussion . . . . . . . . . . . . . 96

3.4.2 Many-mode extension of the trapped-ion experiment: A proposal 97

3.4.3 Quantum phase transition in a transverse-field Ising chain . . . . 98

3.4.3.1 Ground-state negativity from the evolution of the single-

spin magnetization . . . . . . . . . . . . 100

3.4.3.2 Local bound for the minimum entanglement potential

of thermal states . . . . . . . . . . . . . . . 102

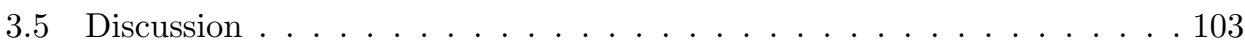


4 From local operations to collective dephasing: Behavior of correlations 107

4.1 Creation of quantum discord by local operations . . . . . . . . . . 107

4.2 Correlation rank: Schmidt decomposition for mixed states . . . . . . . 109

4.3 Trapped-ion experiment . . . . . . . . . . . . . . . . . . 111

4.3.1 Local amplitude damping . . . . . . . . . . . . . . . . . . 111

4.3 .2 Collective dephasing . . . . . . . . . . . . . . . . . 113

4.4 General dynamics of collective dephasing . . . . . . . . . . . . . . 117

4.4 .1 Kraus representation . . . . . . . . . . . . . . 117

4.4.2 Ensemble-average evolution: Interpretation and non-Markovian

effects . . . . . . . . . . . . . . . . . . . . 119

4.4.3 Robustness of bipartite entanglement . . . . . . . . . . . . . 120

4.4.4 Time-invariant states: Multipartite Werner states . . . . . . . . . 121

4.4.5 Robustness of multipartite entanglement . . . . . . . . . . . 122

4.5 Discussion . . . . . . . . . . . . . . . . . . . . . . . 125

5 Quantum phase transition in a family of quantum magnets 127

5.1 Variable-range quantum magnets: From Ising to Lipkin-Meshkov-Glick . 127

5.1 .1 A one-parameter family of models . . . . . . . . . . . 128

5.1 .2 Special case: Nearest-neighbor Ising model . . . . . . . . . . . 129

5.1.3 Special case: Fully-connected Lipkin-Meshkov-Glick model . . 129

5.2 Single-spin signatures of a quantum phase transition . . . . . . . . . 130

5.2.1 Distribution of dephasing-induced excitations . . . . . . . . . 130

5.3 Spectral analysis . . . . . . . . . . . . . . . . . . . . 134

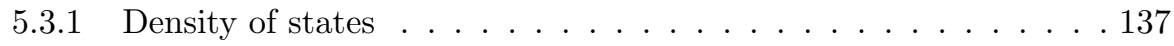

5.3 .2 Level statistics . . . . . . . . . . . . . . . . . . . . 137

5.4 Semiclassical mean-field description . . . . . . . . . . . . . . . . . 139

5.4.1 Semiclassial approximations and quantum phase transitions . . . 140

5.4 .2 Spin-coherent states . . . . . . . . . . . . . . . . . . 142

5.4.3 Multidimensional numerical search for critical points . . . . . . 143

5.4.4 Analytical critical points from a set of single-parameter energy

landscapes . . . . . . . . . . . . . . . . . . . . . . 148

5.4.4.1 Ground state . . . . . . . . . . . . . . . . . . . 148

5.4.4.2 Highest excited state . . . . . . . . . . . . . . . . . 151

5.4.4.3 Generalization for arbitrary states . . . . . . . . . . 151

5.4.5 Performance of the semiclassical ansatz . . . . . . . . . 155

5.4.5.1 Exact results . . . . . . . . . . . . . . . . 156

5.4.5.2 Deviation from the true ground state energy . . . . . . 160

5.4 .6 Scaling of highest and lowest eigenvalues . . . . . . . . . . . . 161

5.4 .7 Distribution of critical fields . . . . . . . . . . . . . . . 166

5.4.7.1 Ground state critical fields . . . . . . . . . . . 166

5.4.7.2 Statistical distribution of excited-state critical fields . . 167

5.5 Discussion . . . . . . . . . . . . . . . . . . . 170

6 Multidimensional nonlinear spectroscopy of controllable quantum systems 173

6.1 Introduction: Nonlinear spectroscopy and controllable quantum systems 173 
6.2 Phase-coherent two-pulse measurements of atomic vapor . . . . . . . . . 174

6.2 .1 Atomic vapor . . . . . . . . . . . . . . . . . . 175

6.2 .2 Light-matter interactions . . . . . . . . . . . . . 176

6.2.3 Fluorescence measurements . . . . . . . . . . . . . . . . . 177

6.2.4 Single quantum coherence . . . . . . . . . . . . . . . . . . 178

6.2 .5 Phase cycling . . . . . . . . . . . . . . . . . . . . . . . 181

6.2.6 Second-order quantum coherence . . . . . . . . . . . . . . . . 182

6.2.7 Higher-order quantum coherence . . . . . . . . . . . . . . . 187

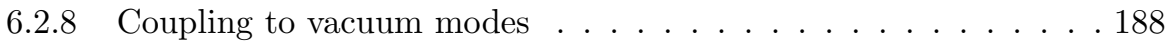

6.2.8.1 Single-photon detection . . . . . . . . . . . . . . 188

6.2.8.2 Two-photon detection . . . . . . . . . . . . . . . . 190

6.2 .9 Interpretation . . . . . . . . . . . . . . . . . . . 191

6.3 Diagrammatic description of nonlinear spectroscopic experiments of con-

trollable quantum systems . . . . . . . . . . . . . . . . . . . . 191

6.3.1 Basic elements for the design of nonlinear measurement schemes 191

6.3.2 Externally induced excitations and de-excitations . . . . . . . . . 192

6.3.3 Time evolution and readout . . . . . . . . . . . . . . . . . . . . 194

6.3.4 Design of a pulse-sequence . . . . . . . . . . . . . . . . . . . . . . 194

6.4 Excitation and readout schemes for trapped ions . . . . . . . . . . 196

6.4.1 Electronic degree of freedom: Dynamics . . . . . . . . . . . 196

6.4.2 Electronic degree of freedom: Excitation and readout . . . . . 197

6.4.3 Vibrational degree of freedom: Dynamics . . . . . . . . . . . 197

6.4.4 Vibrational degree of freedom: Excitation . . . . . . . . . . . . 198

6.4.4.1 Off-resonant stimulated Raman pulses . . . . . . . . . . 199

6.4.4.2 Resonant sideband pulses . . . . . . . . . . . . . . 200

6.4.5 Vibrational degree of freedom: Readout . . . . . . . . . . . 203

6.5 Nonlinear signals and applications for trapped-ion systems . . . . . . . . 204

6.5.1 Two-pulse sequence: Single quantum coherence . . . . . . . . . . . 206

6.5.1.1 Coherent and incoherent phonon transport . . . . . . 206

6.5.1.2 Two-dimensional lineshapes: Distinguishing noise pro-

cesses . . . . . . . . . . . . . . . 210

6.5.1.3 Distribution of couplings and excitations during a quan-

tum phase transition . . . . . . . . . . . . 213

6.5.1.4 Steady-state currents . . . . . . . . . . . . . . . 215

6.5.2 Four-pulse sequence: Double quantum coherence . . . . . . . . 217

6.5.2.1 Detection of anharmonicities and excitation pathways . 218

6.5.2.2 Spatially resolved eigenmode analysis . . . . . . . . 219

6.5.3 Four-pulse sequence: Photon echo . . . . . . . . . . . . . 220

6.6 Discussion . . . . . . . . . . . . . . . . . . . . . . . . . 223

7 Open quantum systems of identical particles 225

7.1 Introduction: Identical particles . . . . . . . . . . . . . . . . . . 225

7.1 .1 Symmetrized states . . . . . . . . . . . . . . . . . 2226

7.1 .2 The single particle subspace . . . . . . . . . . . . . . . . 2227

7.1.3 N-particle operators . . . . . . . . . . . . . . . . . . 229 
7.1.4 The single-particle density operator and partial trace . . . . . . . 230

7.2 General formalism for symmetrized states . . . . . . . . . . . . . . . . . . . . . . . . . . . . . . . . .

7.3 Density matrices and expectation values . . . . . . . . . . . . . . . . . . . . . . . . . . . . . . . . .

7.3.1 N-particle density operator . . . . . . . . . . . . . . 234

7.3.2 Matrix elements and traces in a larger Hilbert space . . . . . . . 235

7.3.3 $M$-particle reduced density operator . . . . . . . . . . . 236

7.3.4 Many-body Hamiltonian . . . . . . . . . . . . . . . . . . . 238

7.3.5 Bosonic product states . . . . . . . . . . . . . . . . 238

7.3.6 Field operator representation . . . . . . . . . . . . . . . 240

7.4 Coherent dynamics of a subgroup of interacting bosons . . . . . . . . . . 242

7.4.1 Time evolution of many-body quantum systems . . . . . . . . . . 243

7.4.2 The Gross-Pitaevskii equation . . . . . . . . . . . . . . . . 245

7.4.3 Hierarchical expansion of the reduced bosonic dynamics . . . . . 246

7.4.3.1 Coherent mean-field evolution . . . . . . . . . . . 252

7.4.4 From the hierarchical expansion to Gross-Pitaevskii . . . . . . . 253

7.5 Second-order master equation for identical particles: Incoherent effects . 254

7.5.1 Single-particle subdynamics of a two-particle bosonic system . . 255

7.5.2 General ansatz: M-particle subdynamics of an N-particle bosonic

system . . . . . . . . . . . . . . . 260

7.5.3 Operator structures within the pure product state approximation 263

7.5.3.1 Terms involving $M+1$ particles . . . . . . . . . . 263

7.5.3.2 Terms involving $M+2$ particles . . . . . . . . . . 265

7.5.3.3 Terms involving $M+3$ particles . . . . . . . . . . 265

7.5.4 General bosonic master equation under the pure product state

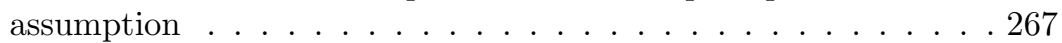

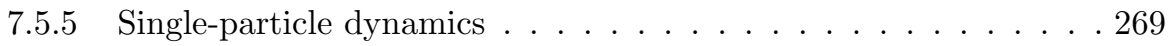

7.5.6 Nonlinear Redfield-type equation . . . . . . . . . . . . . . . . 269

7.5.7 Mean-field approximation . . . . . . . . . . . . . 270

7.5.7.1 Dissipative mean-field master equation for dilute gases 271

7.6 Discussion . . . . . . . . . . . . . . . . . 272

8 Summary and conclusions $\quad 275$

A Derivation of the bosonic master equation (7.151) 279

A.1 First term . . . . . . . . . . . . . . . . . . . . 279

A.2 Second term . . . . . . . . . . . . . . . . . . 284

A.3 Full expression . . . . . . . . . . . . . . . . . . 290

B Representation of double-commutator terms in a larger basis 295

C Transformation properties of interaction-picture operators 299

C.1 Back-transformation to the Schrödinger picture: General properties . . . 299

C.2 Transformation properties of specific operators . . . . . . . . . . 301

C.3 Results for the delta-shaped interaction potential . . . . . . . . . . . 304 
List of Figures

Bibliography 


\section{INTRODUCTION}

While the fundamental laws of quantum mechanics were developed in the last century (Cohen-Tannoudji et al., 1977a; Cohen-Tannoudji et al., 1977b; Sakurai and Napolitano, 1994), recent experimental developments have allowed to probe, and confirm, their validity on the single particle level under well-controlled conditions (Phillips, 1998; Cohen-Tannoudji, 1998; Chu, 1998; Wineland, 2013; Haroche, 2013). The theoretical and experimental characterization of composite quantum systems based on the knowledge of the basic laws is, however, rather challenging due to the rapidly increasing number of degrees of freedom. In the extreme limit of large-scale ensembles of $\sim 10^{23}$ particles, suitable approximations and tools from the well-established fields of solid-state physics or statistical mechanics allow for the efficient description of macroscopic observables (Landau and Lifshitz, 1969; Mahan, 2000). A remaining challenge to both theory and experiment is the intermediate regime of few- to manybody composite quantum systems in the presence of interactions, which shall be the subject of the present dissertation. In recent years, this regime has become available, for example, in experiments on trapped atomic particles (Bloch et al., 2008; Häffner et al., 2008; Schneider et al., 2012; Blatt and Roos, 2012).

Quantum optical experiments, involving, for instance, trapped ions, cold atoms, or photons, represent a flexible testbed for studying quantum systems of variable sizes, ranging from elementary, small-scale systems of few degrees of freedom (Leibfried et al., 2003a; Kok et al., 2007; Häffner et al., 2008), to the many-body dynamics of a BoseEinstein condensate (Pitaevskii and Stringari, 2003; Bloch et al., 2008). As the system size changes, the experimentally accessible observables that characterize the system's properties, as well as the theoretical methods which predict their behavior, need to be adjusted accordingly. Let us first provide a very brief overview of different manifestations of controllable, composite quantum systems, their predominant characteristics, their relevant observables, and the emerging challenges.

As long as one limits to effectively very low-dimensional quantum systems, the full collection of microscopic quantum properties can be measured by quantum state to- 
mography (Paris and Řeháček, 2004) in the experiment, as well as numerically or even analytically handled in theory. Prominent examples are the, by now experimentally well-controlled, elementary building blocks of quantum information theory: Systems of few two-level systems can be encoded, for instance, into the electronic states of trapped ions (Häffner et al., 2008), or the polarization degrees of freedom of individual photons (Kok et al., 2007). In this context, correlation properties are frequently at the center of the theoretical analysis, since they often can identify a boarder between "quantum" and "classical" features, and, in some cases, also play an important role for applications of quantum information theory (Horodecki et al., 2009; Modi et al., 2012).

As the number or the dimension of the constituents of composite quantum systems increases, it is no longer reasonable to measure the entirety of microscopic properties experimentally - feasible full state tomography is limited to systems of roughly no more than ten two-level systems (Häffner et al., 2005a). Thus, the electronic levels of a collection of ten to twenty trapped ions already represent a system that is beyond the reach of standard tools of quantum information theory. Similarly, the system rapidly escapes the easily manageable parameter range if other degrees of freedom, such as the ions' motion in the trap potential, are taken into account. Characterization of the correlation properties in this intermediate, few-particle regime, is significantly complicated by the emerging multipartite nature (Mintert et al., 2005; Gühne and Tóth, 2009), and, experimentally, the preparation of specific quantum states and their efficient isolation from external noise sources becomes more and more challenging (Schindler et al., 2013). When the isolation of the system from its environment is no longer possible, an open-system description that takes decoherence into account is required (Breuer and Petruccione, 2002). In fact, we may also consider the bipartite setting of system and environment as another instance of a composite quantum system.

The larger number of particles also leads to the emergence of new phenomena, such as the onset of collective effects with macroscopically observable signatures; for instance, a measurable magnetization generated by the long-range order of the atom's spins, determined by their mutual interaction. These macroscopic properties may further depend on externally controllable parameters, for example, the strength of an external magnetic field. Tuning these parameters, thus, allows to employ controllable quantum systems for the observation of quantum phase transitions, that is, the macroscopic change of a system's properties induced by the variation of an external parameter (Sondhi et al., 1997; Sachdev, 1999; Vojta, 2003). When a system transitions from one phase into another at zero temperature, its ground state undergoes a non-analytic change. Despite indications that this transition also affects the excitation spectrum (Emary and Brandes, 2003a), the relation between the quantum phase transition and the excited states is not yet well understood in general. Collective effects may further arise due to the interaction of an ensemble of particles with a common environment (Dicke, 1954), which, on the one hand, can lead to enhanced decoherence, but, on the other hand, given a detailed understanding of the resulting dynamics, allows for the efficient control of available parameters, such that coherent evolution can be protected (Palma et al., 1996).

The signature of phase transitions typically becomes more and more pronounced as 
the number of particles increases. Cold, trapped ensembles of neutral atoms represent a platform for composite, controllable quantum systems (Bloch et al., 2008), with yet another parameter regime, characterized by a significantly higher particle number $\left(\sim 10^{3}-10^{6}\right)$ and density than that of trapped-ion systems. Moreover, appropriate design of the potential landscape via optical lattices can render cold-atom systems formally equivalent to solid-state systems (Jaksch et al., 1998). Cold atoms, therefore, allow for the studies of a variety of physical phenomena, including many-particle dynamics from experimentally controlled conditions (Greiner et al., 2002) to truly complex settings (Moore et al., 1995; Raizen, 1999; Oberthaler et al., 1999; Hensinger et al., 2001; Madroñero et al., 2006; Modugno, 2010), and the emergence of a semiclassical limit (Smerzi et al., 1997; Wimberger et al., 2003; Hiller et al., 2009). The high particle density implies that the quantum statistics, induced by the particles' indistinguishability, needs to be taken explicitly into account. Typical experimentally accessible observables that characterize the properties of quantum many-body systems of identical particles are single-particle observables, such as the average momentum. In the presence of interactions their theoretical description is severely complicated by the particles' indistinguishability, and a microscopic theory of the effective dynamics and decoherence (Buchleitner and Kolovsky, 2003; Meinert et al., 2014) of a subset of identical particles is presently not available.

Hence, composite quantum systems, as represented by state-of-the-art quantum optical experiments, span a large range of system sizes and physical phenomena. Their characterization requires the identification of appropriate observables, as well as the development of theoretical models and tools for their efficient description. The systems mentioned above often allow for a surprisingly flexible experimental framework: Despite the large number of degrees of freedom, systems of trapped atomic particles allow for a remarkable level of quantum control, for instance, by providing laser access to individual constituents (Häffner et al., 2008; Weitenberg et al., 2011). Moreover, the interactions between the particles - to some extent - can be controlled externally, and the typical microsecond time scales of the associated evolution allow for the convenient time-resolved measurements of observables, employing nanosecond laser pulses. This rather convenient experimental access to the quantum dynamics stands in contrast to its theoretical description. Interacting composite quantum systems between tens and thousands of particles represent the most challenging parameter regimes: Full diagonalization is no longer plausible, efficient numerical tools are often limited to the treatment of weakly correlated states, and have troubles to predict the dynamical features of systems with more and more complex interactions (Schollwöck, 2005), and, yet, the system is not large enough to be described purely on the level of thermodynamic observables. Therefore, this combination of high-dimensional Hilbert spaces, complex interactions, and high level of experimental access, provides an unprecedented, highly versatile, yet challenging setting.

In the present dissertation we employ methods from fields of rather distinct backgrounds to develop experimentally accessible observables and suitable theoretical approaches that are able to treat a broad range of composite quantum systems of very different sizes and phenomena, as outlined above. The theoretical ideas are furthermore combined with experimental realizations based on trapped ions and photons. In 
particular, chapters 3 and 4 are founded on the fields of quantum information theory and open quantum systems. Specifically, we introduce a dynamical observable for correlation properties of quantum states, when tomographic access to a part of the full system is feasible in chapter 3 , and analyze the generation and protection of correlations under a collective dephasing process in chapter 4 . We move towards more complex systems in chapter 5 , where we develop a semiclassical mean-field approach to describe the excitation spectrum in the context of a quantum phase transition. Chapter 6 deals with the development of a rather general toolbox to experimentally probe multi-time correlation functions of controllable quantum many-body systems based on ideas from nonlinear spectroscopy, a formalism originally developed for physical chemistry. Finally, in chapter 7, combining the open-system perspective with the indistinguishability of quantum particles in many-body systems, we microscopically describe the dynamics of a subsystem of interacting, identical particles, from coherent, mean-field dynamics towards the description of incoherent effects. The following chapter 2 introduces the different basic concepts in further detail, and will also conclude with a more detailed view on the scope and structure of the thesis in section 2.7. 


\section{BACKGROUND}

In this chapter, we provide the background of different conceptual approaches to quantum many-body systems, as they will become relevant in the course of this dissertation. We first introduce basic ideas of quantum information theory, with an emphasis on the role of correlated quantum states. This is followed by an overview over a selection of existing technologies, allowing for high-precision experiments on quantum mechanical systems. Experiments on trapped ions are described in particular detail, as they represent a recurring theme throughout this dissertation. Interesting applications, such as quantum simulations, lead us to the discussion of reliable tools to diagnose increasingly larger quantum systems. In this context, we discuss a very powerful probing technique from a different background: Nonlinear spectroscopy. We further review some commonly employed theoretical methods to describe complex quantum systems, including concepts of random matrix theory, the theory of open quantum systems, and the description of interacting, identical particles. Finally, the phenomenology and description of quantum phase transitions is discussed.

\subsection{Elements of quantum information theory}

The experimental control of objects behaving according to the laws of quantum mechanics has become common practice in many laboratories around the world. Today, there exists a large list of controllable quantum systems, ranging from individual atoms, ions and photons via molecules to nano- or mesoscopic solid-state devices (see also chapter 2.2). Besides the possibility to explore a wide range of interesting physical phenomena, this motivates physicists to study the opportunities provided by quantum mechanics in order to achieve tasks beyond the reach of systems following the laws of classical mechanics.

In classical information theory, an elementary unit of abstract information is encoded into a bit, which can take binary values such as 0 and 1 . Quantum mechanics allows quantum objects, such as atoms, to realize arbitrary superpositions of ground 
and excited state, $|\Psi\rangle=\alpha|0\rangle+\beta|1\rangle$, with complex parameters $\alpha, \beta$ which only have to satisfy the normalization condition $|\alpha|^{2}+|\beta|^{2}=1$. Hence, two-level systems which are used as elementary quantum bits (qubits) in quantum information science can carry significantly more information ${ }^{1}$ than their classical counterparts (Nielsen and Chuang, 2000; Hayashi, 2006). The enormous information content of quantum systems is further manifested when many-particle systems are considered, and a Hilbert space whose dimension grows exponentially with the number of particles is required to describe the many-body quantum state. Quantum theory permits arbitrary coherent superpositions of many-particle states, which includes so-called entangled states. Such states contain correlations which are beyond the reach of classical physics (Bell, 1964), and underline the futility of attempts to grasp certain quantum phenomena with an intuition based on classical physics (Einstein et al., 1935; Bell, 1964; Englert, 2013). Notwithstanding, experimental tests confirmed these predictions of quantum theory (Aspect et al., 1982a; Aspect et al., 1982b), which indicates the novel opportunities that are expected to open up when concepts from classical information theory are extended to the quantum realm.

For instance, the non-classical correlations of quantum states can be harnessed to develop secure key distribution protocols (Ekert, 1991; Acín et al., 2006), in which information is encoded into the quantum states of particle pairs. Each of the parties then receives one of the two strongly correlated particles and performs measurements on it. By sharing the settings of their detectors and part of their measurement data publicly, the two parties can establish a secret key (based on the data kept private) and at the same time confirm that their data is correlated in a way only achievable with undisturbed quantum states, which excludes the influence of a third-party eavesdropper. This protocol, quantum key distribution, is an important example of a field called quantum communication, which strives to make efficient use of the possibilities quantum mechanics offers. Another example is given by quantum teleportation (Bennett et al., 1993): By exchanging a combination of quantum information and classical information, it is possible to transfer the quantum state of one particle to another, possibly in a remote laboratory. Entangled quantum states enable to perform this task deterministically in a single run, without even knowing the teleported quantum state (Bennett et al., 1993). Quantum teleportation has been realized experimentally first using photons (Bouwmeester et al., 1997; Boschi et al., 1998) and later with atoms (Barrett et al., 2004; Riebe et al., 2004).

Besides quantum communication, quantum information theory promises to improve the power of computational algorithms. If it was possible to build computers which work according to the laws of quantum mechanics, the additional resources provided by coherent superpositions of quantum states could be used to achieve tasks which otherwise are believed to be unfeasible (Nielsen and Chuang, 2000; Hayashi, 2006). An important problem, potentially suitable to be treated efficiently with a quantum computer, is given by the factorization of large integer number into its prime factors,

\footnotetext{
${ }^{1}$ The quantum state of a qubit is described by a unit vector in a two-dimensional complex vector space, while a classical bit only contains binary information (Nielsen and Chuang, 2000). Even though a quantum measurement of a qubit only yields a classical bit of information, the full information of the complex coefficients is relevant to describe the quantum evolution of the qubit.
} 
which may be achieved based on Shor's algorithm (Shor, 1994). This algorithms' runtime scales polynomially as a function of the number of classical bits required to represent the integer at question. For classical computers, prime factorization is a very hard task - the runtime of the fastest existing algorithm scales exponentially with the number of bits, which implies that quantum computers have the potential to provide an exponential speed-up over classical computers (Nielsen and Chuang, 2000). The potential implications are immense since the security of standard cryptography methods is based on the assumption that factoring large numbers is computationally intractable.

One prerequisite for the realization of a quantum computer is the ability to control and engineer quantum mechanical systems. Assuming that such level of experimental control was available, one could engineer a quantum system which mimics the dynamics of another interacting many-body system. By measuring the controllable quantum system at hand, one could then infer the properties of the quantum system in question. Without using considerable approximations, predicting the dynamics of large quantum many-body systems is also intractable for classical computers as the dimension of the system, and with it the required computational (classical) memory, grows exponentially with the number of particles in the system. This approach, referred to as quantum simulation (Feynman, 1982), is currently being pursued by experimental groups working on a variety of different platforms (see section 2.2) - the reliability and efficient certification of potential quantum simulations is, however, still debated (see section 2.3).

Entangled states form the basis of many applications of quantum information theory. In the next section, we review the formal definition of quantum entanglement and also introduce the concept of quantum discord, a weaker form of correlations in quantum states which emerges in the context of local measurements of composite quantum systems. Often it turns out difficult to clearly identify the resource which empowers the quantum speedup in the particular protocol. Later, in section 2.1.2, we discuss a selection of examples which rely on entanglement, and others which do not necessarily require entangled states, but instead are enabled by mixed states with nonzero discord.

\subsubsection{Correlations in composite quantum systems: Entanglement and discord}

We now briefly review the definition of separable and entangled states. For further details and pedagogical introductions we refer to the extensive literature on the topic (Nielsen and Chuang, 2000; Mintert et al., 2005; Amico et al., 2008; Horodecki et al., 2009; Tichy, 2011). Formally, quantum states are represented by normalized, positive semi-definite operators (density operators) $\rho$ on a Hilbert space $\mathcal{H}: \rho \geq 1, \operatorname{Tr} \rho=1$ (Cohen-Tannoudji et al., 1977a; Cohen-Tannoudji et al., 1977b), where Tr denotes the trace operation. A composite bipartite system, describing for instance two distinguishable particles or two degrees of freedom, is represented by quantum states on the Hilbert space $\mathcal{H}=\mathcal{H}_{A} \otimes \mathcal{H}_{B}$ formed by the tensor product of the Hilbert spaces $\mathcal{H}_{A}$ and $\mathcal{H}_{B}$ of the individual subsystems. We introduce the notion of correlated quantum states by first defining what is considered a completely uncorrelated state: A product 
state

$$
\rho_{P}=\rho_{A} \otimes \rho_{B}
$$

describes a situation where the two subsystems $A$ and $B$ are statistically completely independent, since probabilities for the measurement outcomes of independent experiments will factorize (Werner, 1989). Quantum states may also exhibit purely classical correlations, which can be seen from the following example: Imagine a device, able to prepare the respective subsystems arbitrarily within the two sets of quantum states $\left\{\rho_{A}^{i}\right\}$ and $\left\{\rho_{B}^{i}\right\}$ (Werner, 1989). If the device is connected to a classical random number generator in a way that with probability $p_{i}$ it prepares system $A$ in state $\rho_{A}^{i}$ and system $B$ in state $\rho_{B}^{i}$, we describe the resulting composite quantum system with the state

$$
\rho_{S}=\sum_{i} p_{i} \rho_{A}^{i} \otimes \rho_{B}^{i}
$$

This state clearly contains some correlations, since the subsystem probabilities do not factorize. However, the example shows that the correlations can be attributed to a classical probability distribution. A state of this form is called separable, and conversely, if a state cannot be represented in this form, it is called entangled. This can be generalized to characterize multiparticle entanglement in a hierarchical order ranging from bipartite to genuine multipartite entangled states (Mintert et al., 2005; Levi and Mintert, 2013); which we will discuss in section 4.4.5.

The definition (2.2) characterizes the most general set of bipartite states whose correlations are of classical nature. A different approach to defining the boarder between classical and quantum states is based on quantum measurements. The measurement uncertainty of two incompatible observables is most prominently determined by nonzero commutators (Heisenberg, 1927). However, quantum mechanics may already predict a finite variance for the measurement of a single observable. For example, if a system is prepared in a pure quantum state $|\Psi\rangle$, an observable $M$ can be measured with zero variance if and only if $|\Psi\rangle$ is an eigenstate of $M$. If the system is described by a mixed state $\rho$, the resulting variance must be attributed to two different origins: One is the lack of knowledge expressed by the statistical mixture in the construction of $\rho$, the other is the remaining intrinsic quantum uncertainty. One may use the commutator of $\rho$ and $M$ to quantify the quantum contribution to the uncertainty (Wigner and Yanase, 1963; Luo, 2003; Girolami et al., 2013) which is consistent with the well-known special case for pure states (Luo, 2003).

This implies certain consequences for correlated quantum states in a bipartite scenario, as considered before. To see this we apply the above reasoning to local measurements on subsystem $A$. It is possible to show that there exists a local observable ${ }^{2}$

\footnotetext{
${ }^{2}$ We assume that the spectrum of $M_{A}$ is non-degenerate, since otherwise a given measurement outcome cannot be uniquely identified with a quantum state, thus, the observable carries limited information.
} 
$M_{A} \otimes \mathbb{I}_{B}$ which commutes with $\rho$ if and only if $\rho$ has the form (Girolami et al., 2013)

$$
\rho=\sum_{i} p_{i}|i\rangle\langle i| \otimes \rho_{B}^{i}
$$

where $\{|i\rangle\}$ represents an orthonormal basis of $\mathcal{H}_{A}$. States of this form are called states of zero discord (Henderson and Vedral, 2001; Ollivier and Zurek, 2001) and the lack of quantum uncertainty motivates to call them classical states ${ }^{3}$ (Modi et al., 2012).

One may also consider a non-selective measurement of $M_{A} \otimes \mathbb{I}_{B}$-a measurement where the outcome was forgotten: ${ }^{4}$ In this case the final state is described by an incoherent mixture of the eigenstates of $M_{A}$,

$$
\rho_{f}=\sum_{i}\left(|i\rangle\langle i| \otimes \mathbb{I}_{B}\right) \rho\left(|i\rangle\langle i| \otimes \mathbb{I}_{B}\right)
$$

An equivalent definition of zero-discord states may be given as follows: There exists a basis $\{|i\rangle\}$ (which can be interpreted as the eigenbasis of an observable), such that $\rho_{f}=\rho$ if and only if $\rho$ is of the form (2.3). This is another indicator for classicality since invariance under measurements is typically not granted in quantum mechanics.

Notice from comparing equations (2.2) and (2.3) that states of zero discord form a subset of separable states: A separable state has zero discord only if the decomposition of the form (2.2) can be expressed in terms of a local orthonormal basis $\{|i\rangle\}$, instead of arbitrary density matrices $\rho_{A}^{i}$.

What does the definition (2.3) tell us about the correlations of nonzero discord states? First of all we note that nonzero discord states are certainly correlated since product states have always zero discord ( $\mathrm{Li}$ and Luo, 2008). Of course the set of nonzero discord states includes the entire set of entangled states, which are quantum correlated - in fact, when restricting to pure states, discord and entanglement are equivalent. Yet, the correlations of separable, nonzero discord states are still of classical origin as they can be characterized by a probability distribution [see discussion preceding equation $(2.2)]$.

In the light of the above discussion, we conclude that states of nonzero discord are correlated quantum states which show quantum properties (quantum noise, measurement disturbance, nonzero commutators) under the influence of local measurements. The nature of their correlations is, however, only quantum when they are also entangled. A more detailed discussion will be given in section 4.1.

\subsubsection{Role of correlated quantum states in quantum information theory}

Much effort is spent to define, characterize, and quantify different notions of correlations in quantum states including quantum entanglement and discord (Horodecki et al.,

\footnotetext{
${ }^{3}$ Note that, due to the asymmetry of this definition, one should always specify which of the two subsystems is being measured.

${ }^{4}$ The post-measurement state after a non-selective measurement is described by a mixture $\rho^{\prime}=$ $\sum_{i} p_{i} \Pi_{i}$ of all measurement projectors $\Pi_{i}$, where the overlap of the initial state $\rho$ with these projectors determines the probability distribution, $p_{i}=\operatorname{Tr}\left\{\Pi_{i} \rho\right\}$.
} 
2009; Modi et al., 2012), usually motivated by referring to their role as a resource for certain quantum information tasks. But which quantity is actually relevant? This question cannot be answered in general. Instead, a clear answer can be given only in few special cases. Even then, it depends very much on the task one aspires to accomplish.

For simplicity, let us first restrict to pure states. In this case, all of the different concepts of correlations collapse into the same notion and the set of zero-discord states coincides with the set of separable states; they even coincide with completely uncorrelated product states. Furthermore, any entangled pure state can violate a Bell inequality (Bell, 1964), which is useful, for instance, to establish certified, secure quantum key distribution ${ }^{5}$ (Ekert, 1991). Pure state entanglement is rather well characterized by now (Mintert et al., 2005; Horodecki et al., 2009), even though this already represents a very intricate task once multipartite states are considered.

The picture becomes considerably more complex when we consider mixed states. Not every mixed entangled state is able to violate a Bell inequality (Popescu, 1994), hence, not all mixed entangled states serve to distribute a certified, secure quantum key. Yet, other tasks, such as quantum teleportation, can be accomplished if only some nonzero entanglement is available (Vidal and Werner, 2002). So clearly, it is not always necessary to ask for the strongest incarnations of correlations in quantum states. This suggests a hierarchical structure of correlated quantum states, with states that violate a Bell inequality on the top. Entangled states form a subset thereof, and an even weaker class of correlated quantum states are those with nonzero discord. The remaining set, the classical (zero discord) states, also includes the completely uncorrelated product states. The set of zero-discord states are actually very sparsethey form a set of Lebesgue measure zero in the full set of quantum states (Ferraro et al., 2010), which demonstrates that quantum features are quite generic to correlated states of composite systems.

Most algorithms for quantum computations employ entangled states at some point (Shor, 1994; Grover, 1997). Some particular implementation schemes even make explicit use of large entangled states (Briegel and Raussendorf, 2001) as their initial resource (Raussendorf and Briegel, 2001). Are there interesting applications of quantum information theory which do not necessarily require entanglement, and if so, is discord useful for certain tasks? This question has been intensively investigated throughout the last decade, since, due to their ubiquity, nonzero discord states are easier to produce than entangled states. The debate was initiated by a quantum algorithm which efficiently determines the trace of large unitary matrices (Knill and Laflamme, 1998), with fixed efficiency independent of the size of the unitary, whereas on classical computers the required resources increase exponentially (Datta et al., 2005). The algorithm only requires one pure qubit, while the rest of the quantum state can be highly mixed, leading to vanishing entanglement in the system (Knill and Laflamme, 1998; Lanyon et al., 2008). However, despite efforts (Datta et al., 2008), it has not been possible

\footnotetext{
${ }^{5}$ Note that the first proposal for a quantum key distribution protocol did not make use of quantum correlations (Bennett and Brassard, 1984). Instead, it uses the destructive nature of the quantum measurement and the no-cloning theorem (Wootters and Zurek, 1982; Dieks, 1982) to rule out the presence of a third party.
} 
to clearly identify the resource for the quantum speed-up in this system, and doubts prevail about the necessity of discord in this context (Dakić et al., 2010).

There exist other examples where the identification of discord as a necessary element is clearer. Consider, for example, a three-partite setup consisting of the two parties $A$ and $B$, as well as a carrier particle $C$. Starting from an initially separable state, $A$ and $B$ can establish quantum entanglement only by exchanging the particle $C$, which, remarkably, is never entangled with either one of the two parties (Cubitt et al., 2003). Nevertheless, there is a quantum cost for this entanglement distribution quantified by a measure of quantum discord (Streltsov et al., 2012; Chuan et al., 2012), which was confirmed in a recent series of experiments (Fedrizzi et al., 2013; Vollmer et al., 2013; Peuntinger et al., 2013).

In a similar scenario, two parties $A$ and $B$ share an initially separable state. A local measurement on $B$ induces a minimal amount of entanglement between the measurement apparatus and the bipartite state of $A$ and $B$, which can be identified by the quantum discord of the state before the measurement (Streltsov et al., 2011b; Piani et al., 2011). Also this entanglement activation protocol has been realized in a photonic experiment (Adesso et al., 2014).

An example from quantum metrology is given when we consider the measurement of an unknown phase-shift induced in one arm of an interferometer. The maximum obtainable information per measurement about the phase, again, is bounded by a measure of the initial state's quantum discord between both arms of the interferometer (Girolami et al., 2014). Essentially, all of these applications make use of the property of nonzero discord states to contain a mixture of states which are non-orthogonal in at least one of the subsystems. Under the influence of local measurements, these non-orthogonal states lead to non-trivial disturbance of the total state which may be harnessed in different ways.

These examples show, that quantum discord can be the figure of merit whenever local measurements and operations play a decisive role. This is often the case in multipartite scenarios, where a large quantum state is shared by many parties while each of them can only manipulate their part of the state - for instance in quantum communication protocols, which explains its relevance for quantum information theory. Another particularly natural setting for such a situation is an open quantum system; that is, a quantum system which is in contact with an inaccessible environment. Thus, the experimentalist can only control the open system with local operations. In this dissertation, we often try to control and measure composite quantum systems by local operations, thus, quantum discord will naturally emerge in various situations. Chapter 3, for instance, is dedicated to a method which detects and estimates the quantum discord in a bipartite setting, when only local access to one of the two parties is available.

As we have seen discussing the previous examples, there exists a remarkable selection of quantum systems which can be controlled with high efficiency. These allow us to develop theoretical ideas in rather abstract terms, considering mostly ideal quantum mechanical systems. In the next section we will review a selection of important experimental platforms for controllable quantum systems. 


\subsection{Controllable quantum systems}

The past century has seen tremendous progress with regard to the understanding and manipulation of particles on the smallest scales. Years after the basic foundations of quantum mechanics had been developed, it was still believed that experiments with individual particles belong to the realm of thought experiments, and can never become an experimental reality (Schrödinger, 1952). When electromagnetic traps for charged particles (Penning, 1936; Paul et al., 1958; Paul, 1990) were first constructed, such experiments became indeed possible, and soon led to the observation of individual electrons (Wineland et al., 1973) and ionized atoms (Neuhauser et al., 1980). This eventually evolved into the nowadays highly successful research field devoted to controlling and manipulating the quantum states of trapped ions, which will be discussed in further detail in section 2.2.1.

Another important development towards the control of quantum mechanical systems in a laboratory was laser cooling (Phillips, 1998; Chu, 1998; Cohen-Tannoudji, 1998), which is not only important for ion trapping, but also renders trapping of neutral atomic ensembles possible. This enabled, for instance, the experimental generation of a Bose-Einstein condensate from ultra-cold atoms (Davis et al., 1995; Anderson et al., 1995). Today, a large number of experimental groups work with cold trapped atoms, often in combination with optical lattices to observe the atom's dynamics in specific potential landscapes. This research field will be discussed in section 2.2.2.

Apart from the quantum states of atomic particles, it is also important to be able to control the quantum state of light. The previous two research fields use light fields to manipulate the quantum states of atoms. The converse approach is followed by cavity quantum electrodynamics experiments, where atoms are sent through highfinesse cavities to probe and manipulate the quantum state of the light mode inside the cavity (Meschede et al., 1985; Benson et al., 1994; Haroche, 2013). Alternatively, the quantum state of individual photons or photon-pairs can be controlled by sending the photons through specifically designed arrays of optical instruments, and analyzed via single-photon detectors. We will briefly discuss such experiments in section 2.2.3.

These examples represent a selection of quantum systems which can be controlled with high precision in today's experiments, and which are most relevant for the present dissertation. A brief overview of further systems can be found in section 2.2.4.

\subsubsection{Trapped ions}

Trapped ions represent one of the most advanced platforms of quantum control at the single particle level (Wineland, 2013). In this section, we summarize some of the essential experimental aspects and introduce some of the key tools which will be needed also for applications later in this dissertation. We will further briefly introduce the experimental setup where part of the research for this dissertation was carried out. 

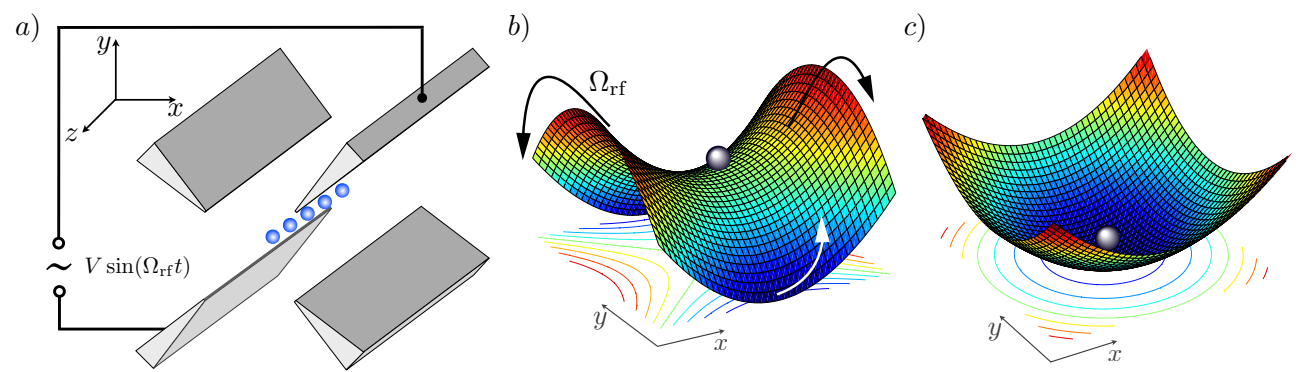

FIGURE 2.1.: a) The radio-frequency electrodes of a linear Paul trap generate a time-dependent quadrupole potential with a saddle point (b), which, upon averaging over the periodic radio-frequency oscillations, can be approximated by a static harmonic potential (c). Subfigures b) and c) are adapted from (Littich, 2011).

\subsubsection{Paul traps}

Due to their positive charge, ions can be trapped using electromagnetic fields. A static field, however, is unable to provide a potential minimum in three dimensions, since, according to Maxwell's equations, the potential has to satisfy the Laplace equation $\Delta \Phi=0$ in the charge-free center of the trap (Jackson, 1999). Thus, a Paul trap uses a combination of static and radio-frequency modulated electric fields to confine charged particles in three dimensions (Paul, 1990). Figure 2.1 a) displays the typical design of a three-dimensional linear Paul trap, where a two-dimensional quadrupole potential generated by the radio-frequency electrodes in the $x$ and $y$ directions (in trapped-ion literature, these directions are referred to as radial directions). The time-dependent potential can be pictured as a saddle point whose edges flop up and down with the radio frequency $\Omega_{\mathrm{rf}}$ (Leibfried et al., 2003a). The time-averaged effective potential can be approximated by a static harmonic potential when the driving frequency is sufficiently strong compared to the frequency of the ion's secular motion (Leibfried et al., 2003a). This approximation is particularly well justified when the ion is close to the center of the trap potential. This is the case when the ion's kinetic energy is low (Leibfried et al., 2003a). Small displacements from the trap center, however, can lead to an additional driving force at the radio frequency called micromotion. This can cause unwanted heating of the ions. Static electric fields are used to shift the ion's equilibrium position striving to compensate this effect. Micromotion becomes more relevant when many ions are placed into the same trap potential since it gets increasingly difficult to maintain all ions close to the trap center.

Along the $z$-axis or the axial direction, two positively charged direct current (dc) endcaps (not shown in the figure) generate a static potential to prevent the ions from escaping into the third dimension. Typical orders of magnitude for the radial trap frequencies are $\nu_{x, y} \approx 2 \pi \times(1-10 \mathrm{MHz})$, while the axial frequencies are usually one order of magnitude smaller, $\nu_{z} \approx 2 \pi \times(0.1-1) \mathrm{MHz}$ (Leibfried et al., 2003a; Schindler et al., 2013). 


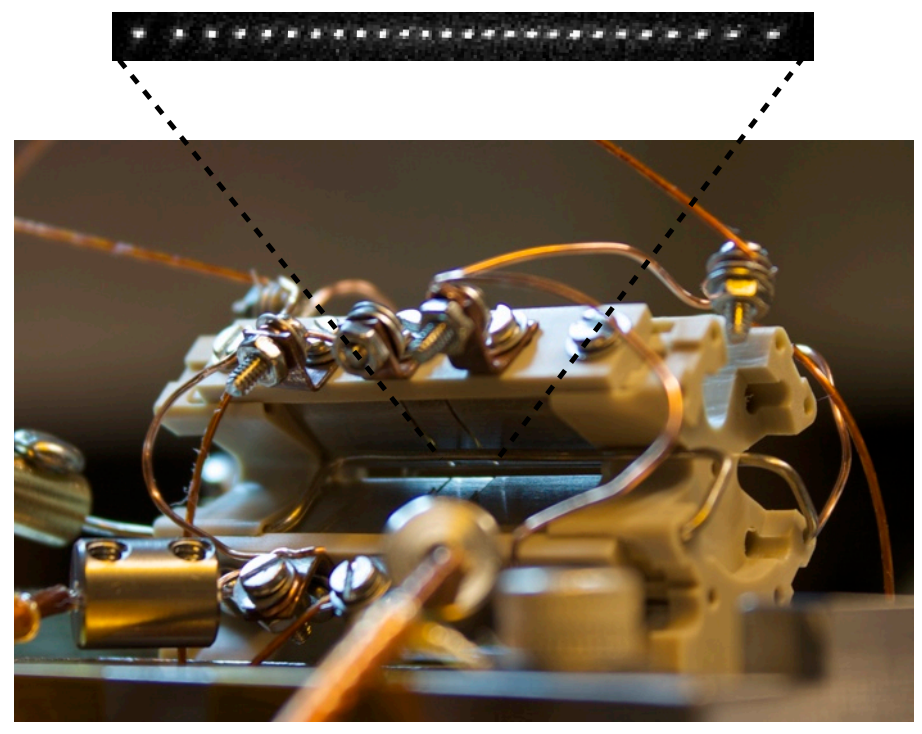

Figure 2.2.: Paul trap used in the experiments carried out at Berkeley, and a fluorescence image of a one-dimensional linear ion chain.

\subsubsection{The Berkeley setup}

Figure 2.2 shows a Paul trap used in the group of Hartmut Häffner at the University of California, Berkeley, where part of the work on this dissertation was done during a ten month stay from September 2012 to June 2013. The trap consists of threeway segmented electrodes of which the center segment is driven with an out-of-phase radio-frequency voltage while the two outer segments are used as dc-endcaps. The out-of-phase drive supplies opposing electrodes with the radio-frequency voltage with an $180^{\circ}$ phase shift. This doubles the radial trap frequency at the same drive amplitude and reduces micromotion along the axial direction, since the potential cancels along the trap axis (Pruttivarasin, 2014; Ramm, 2014). The setup which was used for the experiment discussed in section 3.2, was designed and assembled by Thaned Pruttivarasin and Michael Ramm under the supervision of Hartmut Häffner. In the following we briefly summarize the key elements of the setup. Details can be found in the dissertations (Pruttivarasin, 2014; Ramm, 2014).

To load ions into the trap, an ionization laser pointing at the center of the trap ionizes a beam of thermal atoms emitted from an oven on demand. Interactions with red-detuned lasers Doppler-cool the ions which allows them to fall into the trap potential generated by the electrodes of the Paul trap. The fluorescence light which is scattered in the course of the Doppler cooling process is used to monitor the ions in the trap. Turning off the oven and the photon-ionization laser after the fluorescence light of a certain number of ions is observed allows to steer the number of trapped ions reliably. The Paul trap is placed in an ultra-high-vacuum chamber with a pressure below $5 \times 10^{-9} \mathrm{~Pa}$ to minimize collisions of the ions with background atoms (Ramm, 
2014). The potential generated by such Paul traps can be deep enough to trap atoms at room temperature and, once trapped, the ions can be kept for many hours in the trap; under good conditions the lifetime is only limited by the time the experimental equipment can be continuously supported.

The cooling and quantum state manipulation of ions requires elaborate laser technology which was developed for trapped-ion systems to perform high-precision spectroscopy in the context of atomic frequency standards (Berkeland et al., 1998; Young et al., 1999; Wineland, 2013). Before being sent into the trap, the laser light is guided through acousto-optic modulators (AOM), which generate acoustic standing waves to modulate the incoming laser light and, thus, can be used to fine-tune the laser detuning and may induce a controllable phase shift. The Berkeley group has developed their own python-software to conveniently control the AOM settings to change beam intensities, detunings and phases, and to run frequently used standard pulse sequences and algorithms automatically and on demand (Ramm, 2014). The computer connects to a field-programmable gate array (FPGA), which controls custom-designed direct digital synthesis (DDS) boards to provide the control voltages to the AOMs (Pruttivarasin, 2014). Apart from AOMs the computer also controls the voltages of the trap electrodes and is provided with the photon counts from the photomultiplier tube, which is used to detect the ion's fluorescence. The fluorescence light is guided through the imaging system which at the same time provides laser access to the trap, and eventually is also collected by a CCD (charge coupled device) camera (Pruttivarasin, 2014). The fluorescence of individual ions can be resolved spatially, allowing one to read out the ions' populations independently (Ramm, 2014).

\subsubsection{Motion of trapped ions in linear chains}

When several ions are trapped in the same harmonic potential, the ions are wellseparated due to strong Coulomb repulsions. The full potential consists in the global harmonic trap potential and the Coulomb repulsion between them: ${ }^{6}$

$$
\begin{aligned}
V= & \sum_{i=1}^{N} \frac{1}{2} m\left(\nu_{x}^{2} x_{i}^{2}+\nu_{y}^{2} y_{i}^{2}+\nu_{z}^{2} z_{i}^{2}\right) \\
& +\sum_{\substack{i, j=1 \\
(i>j)}}^{N} \frac{e^{2}}{4 \pi \epsilon_{0}} \frac{1}{\sqrt{\left(x_{i}-x_{j}\right)^{2}+\left(y_{i}-y_{j}\right)^{2}+\left(z_{i}-z_{j}\right)^{2}}},
\end{aligned}
$$

where $N$ is the total number of ions, $m$ is the ion mass, and $\vec{r}_{i}=\left(x_{i}, y_{i}, z_{i}\right)$ the $i$-th ion's position, respectively, $e$ the electron charge and $\epsilon_{0}$ the dielectric constant of the vacuum. Given the ratio of trap frequencies assumed above $\left(\nu_{x, y} \gg \nu_{z}\right)$, their equilibrium positions form a linear chain along the axial $(z)$ direction (James, 1998). The separation of neighboring ions is typically on the order of $10 \mu \mathrm{m}$ (Schindler et al., 2013).

\footnotetext{
${ }^{6}$ Here and in the following, we assume single-ionized ions.
} 
Assuming that the ions remain close to their equilibrium positions, that is, the ions' displacement is much smaller than the inter-ion distance, the Coulomb potential is determined by the ions' equilibrium positions along the axial direction (James, 1998). By expanding the potential around the ions' equilibrium positions to second order, for example in $x_{i}$, we obtain a Hamiltonian for the description in terms of quantized local phonon modes in $x$-direction (and analogously for the $y$-direction) given by

$$
H=\sum_{i=1}^{N} \hbar \omega_{i}^{0} a_{i}^{\dagger} a_{i}+\sum_{\substack{i, j=1 \\(i<j)}}^{N} \hbar t_{i j}\left(a_{i}^{\dagger} a_{j}+a_{j}^{\dagger} a_{i}\right),
$$

where $a_{i}^{\dagger}$ creates a local phonon at site $i$ (Porras and Cirac, 2004a). The average inter-ion distance is given by the length scale $l_{0}^{3}=e^{2} /\left(m \nu_{z}^{2}\right)$, with the axial trap frequency $\nu_{z}$ (James, 1998). The Hamiltonian (2.6) is a valid approximation if the parameter $\beta_{0}:=e^{2} /\left(l_{0}^{3} m \nu_{x}^{2}\right)=\nu_{z}^{2} / \nu_{x}^{2} \ll 1$, assuming that the radial trap frequencies are comparable, $\nu_{x} \approx \nu_{y}$ (Porras and Cirac, 2004a), which is the case for a linear trap architecture as described above. The second-order expansion yields for the local trap frequencies and the coupling matrix (Porras and Cirac, 2004a)

$$
\begin{aligned}
& \omega_{i}^{0} / \nu_{x}=1-\frac{\beta_{0}}{2} \sum_{j \neq i} \frac{1}{\left|u_{i}^{0}-u_{j}^{0}\right|^{3}}, \\
& t_{i j} / \nu_{x}=\frac{\beta_{0}}{2} \frac{1}{\left|u_{i}^{0}-u_{j}^{0}\right|^{3}},
\end{aligned}
$$

where $u_{i}^{0}=z_{i}^{0} / l_{0}$ and $z_{i}^{0}$ denote the ions' equilibrium positions (James, 1998). This leads to a normalized Hamiltonian which is fully determined by the parameters $\nu_{x}$ and $\beta_{0}=\nu_{z}^{2} / \nu_{x}^{2}$.

The large spatial separation between neighboring ions has two important consequences:

(i) The wave functions of individual ions do not overlap. Hence, the ions become distinguishable in space and their quantum statistics does not have to be considered explicitly.

(ii) Focussed lasers are able to address individual ions with very little cross-talk to neighboring ions (Schindler et al., 2013). The Rabi frequency for a next-neighbor ion is typically on the order of $1 \%$ of the Rabi frequency of the addressed ion (Häffner et al., 2008).

The manipulation of ions by means of lasers is extremely important for trapped-ion experiments. Laser-ion interactions are used for laser cooling, as well as for initialization, manipulation and readout of the ions' quantum mechanical states.

\subsubsection{Electronic levels}

There exist two classes of ions which are used for implementations of quantum information tasks. They differ in the physical realization of a long-lived and well-controllable 
a)

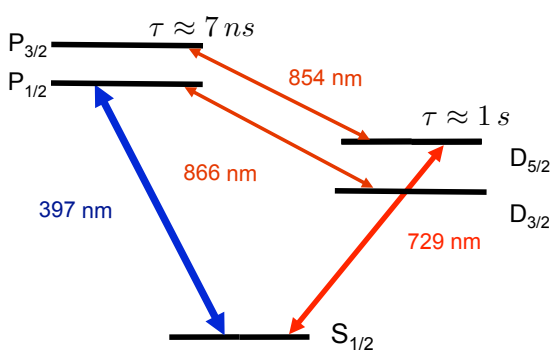

b)

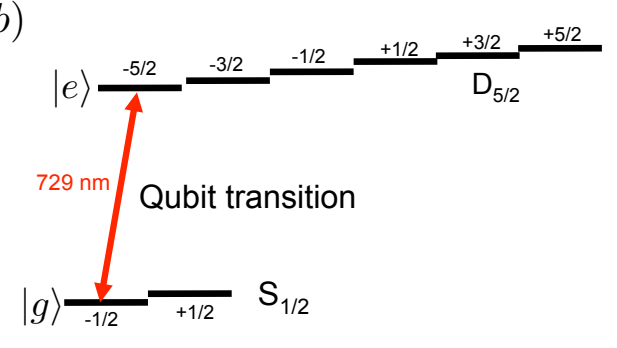

Figure 2.3.: a) Relevant electronic states of ${ }^{40} \mathrm{Ca}^{+}$ions and transition frequencies. Characteristic features are a long-lived dipole-forbidden excited D state, and a short-lived excited $P$ state. b) Under the influence of a magnetic field, the degeneracies are lifted by the Zeeman effect and individual Zeeman levels can be distinguished spectrally. A pair of Zeeman levels is selected from the $S$ and $D$ states as two-level system (qubit).

two-level system (the qubit): One class, represented by ${ }^{40} \mathrm{Ca}^{+}$, uses an optical qubit which can be addressed directly with laser light on a quadrupole transition, whereas the other class, represented by ${ }^{171} \mathrm{Yb}^{+}$, uses a qubit formed by two hyperfine levels separated by a microwave transition, often driven by Raman transitions (Häffner et al., 2008). In this dissertation, we mostly consider systems of ${ }^{40} \mathrm{Ca}^{+}$ions and therefore discuss many of the following experimental considerations in the context of calcium, which was also used in the Berkeley setup.

The relevant electronic transitions of ${ }^{40} \mathrm{Ca}^{+}$ions are displayed in figure $2.3 \mathrm{a}$ ). There are two excited state manifolds (the $\mathrm{P}$ and $\mathrm{D}$ states) which decay into the same ground state (the $\mathrm{S}$ state). The S-P transitions are dipole-allowed and therefore the $\mathrm{P}_{1 / 2}$ state has a rather short lifetime of about $7 \mathrm{~ns}$ (Jin and Church, 1993). In contrast, the S-D transitions are dipole-forbidden and therefore only couple via its quadrupole moment to leading order, resulting in a very long lifetime of about $1.2 \mathrm{~s}$ for the $\mathrm{D}_{5 / 2}$ state (Barton et al., 2000; Kreuter et al., 2005). Under the influence of a magnetic field, the degeneracies of the energy levels are lifted, as shown for the $\mathrm{S}$ and D states in figure $2.3 \mathrm{~b}$ ). One applies an external magnetic field generated by large Helmholtzcoils placed outside the vacuum chamber to lift these degeneracies in a controlled way, and to compensate the earth's field, as well as other stray fields. The applied field is typically of the order of $10^{-4} \mathrm{~T}$. This leads to spectrally well-separated transitions between the Zeeman sublevels, one of which is chosen as the qubit. The dependence of the transition frequencies on the magnetic field remains one of the dominant sources of error in state of the art ion trap experiments since the external magnetic field is subject to fluctuations (Schindler et al., 2013). This induces a dephasing effect which will be discussed in further detail in chapter 4 .

Alternatively, one may encode a qubit into the two Zeeman-splitted ground state levels. These levels can be driven by Raman transitions via the $\mathrm{P}$ state or by microwave fields. This is analogous to the hyperfine-qubit of Yb-type ion species, where the energy splitting is induced by a nonzero nuclear spin. A Raman process involves two laser 
fields, whose relative detuning is resonant with the driven transition. The atom then absorbs one photon from one of the lasers and emits another photon into the light field of the other laser. Effectively, the atom can undergo a transition that is resonant to the energy difference of the two laser fields. Such schemes are especially useful to drive microwave transitions, including motional excitations, with well-focussed optical light fields, as we will make use of in section 6.4.4.

\subsubsection{Quantum state readout and initialization}

The populations of electronic qubit states can be read out with high precision using the electron shelving or quantum jump technique (Leibfried et al., 2003a). This is done by collecting the fluorescence light induced by illumination of the ion with the $397 \mathrm{~nm}$ laser. If the ion is in the $\mathrm{S}_{1 / 2}$ ground state photons will be scattered on the $397 \mathrm{~nm}$ transition with a rate of approximately $\Gamma \approx 2 \pi \times 20 \mathrm{MHz}$, while if the ion is in the excited long-lived $\mathrm{D}_{5 / 2}$ state, no photons will be scattered. The two cases can be distinguished clearly as they produce well-separated histograms of the photon counting distributions. To read out the quantum mechanical population, one has to determine the probabilities by collecting statistics over a significant number of iterations (typically about 100-1000).

The electronic ground state is prepared by optical pumping. This is an incoherent procedure which transfers populations of undesired levels into short-lived excited states of the P-state manifold. These undesired levels consist in the remaining Zeeman sublevels other than the qubit ground state (pumped via the $729 \mathrm{~nm}$ laser to the D-states) and long-lived excited states within the D-state manifold (pumped out using the $866 \mathrm{~nm}$ and $854 \mathrm{~nm}$ lasers). With certain probability, the electron decays into the desired ground state, which is not affected by the optical pumping lasers. After a certain number of cycles, the ion's electronic state can be prepared with fidelities exceeding $99.9 \%$ (Schindler et al., 2013).

Beyond the initialization of the electronic ground state, the motional degree of freedom of the ions is typically cooled down as close as possible to the quantum mechanical ground state. The $\mathrm{S}_{1 / 2}-\mathrm{P}_{1 / 2}$ transition is used for Doppler-cooling (Hänsch and Schawlow, 1975): A red detuned laser at $397 \mathrm{~nm}$ is more likely to be absorbed by atoms moving towards the laser, since in this case the Doppler-shifted laser frequency is close to resonance in those atoms' reference frame. When absorbing the photon, the recoil causes the atoms to slow down. This cooling technique is limited by the gain of momentum when the photon is reemitted. However, since spontaneous emission happens in a random direction while absorptions occurs preferably when the loss of momentum is maximal, the atoms lose kinetic energy on average over many scattering events. To overcome the Doppler limit (Stenholm, 1986), sideband cooling is used (Wineland and Dehmelt, 1975). To describe sideband cooling and to see how the motional state of trapped ions can be controlled coherently, we have to take the quantum mechanical description of laser-ion interactions into account. 


\subsubsection{Laser-ion interactions}

In this section we discuss the laser-induced coupling between the electronic two-level system and the motional degree of freedom of a single trapped ion. For clarity, we restrict to discussing the essential results while a detailed derivation can be found later in this dissertation in section 3.2.1. In the remainder of this dissertation, we neglect the effect of micromotion and approximate the trap potential by a static harmonic potential (see discussion in section 2.2.1.1). We consider a continuous-wave laser illumination of the selected two-level transition, possibly with some detuning $\Delta=\omega-\omega_{L}$, where $\omega$ denotes the atomic resonance frequency and the laser is tuned to $\omega_{L}$. The Hamiltonian describing the system is given by

$$
H(t)=\hbar \omega \sigma_{+} \sigma_{-}+\hbar \nu a^{\dagger} a+\frac{\hbar \Omega}{2}\left(\sigma_{+}+\sigma_{-}\right)\left(e^{i\left(\vec{k} \vec{x}-\omega_{L} t+\varphi\right)}+e^{-i\left(\vec{k} \vec{x}-\omega_{L} t+\varphi\right)}\right),
$$

where $\sigma_{ \pm}=\left(\sigma_{x} \pm i \sigma_{y}\right) / 2$ are the ladder operators of the two-level system, described in terms of the Pauli matrices, $a$ is the annihilation operator for a vibrational mode of frequency $\nu$, and $\Omega$ denotes the Rabi frequency, which depends on the amplitude of the laser light, as well as the effective coupling strength, and $\varphi$ denotes the phase of the laser. Since the ion is moving in a harmonic potential, its position $\vec{x}$ can be expressed in terms of $a$ and $a^{\dagger}$ leading to $\vec{k} \vec{x}=\eta\left(a+a^{\dagger}\right)$, with the Lamb-Dicke parameter

$$
\eta=k \sqrt{\frac{\hbar}{2 m \nu}} \cos \theta,
$$

$\theta$ describing the angle between the direction of laser propagation and the motional axis of the trap, $k$ denotes the modulus of the wave vector of the laser light, and $m$ the ion's mass. To avoid lengthy expressions in this introductory chapter, we assume the Lamb-Dicke limit, $\eta \sqrt{\left\langle\left(a+a^{\dagger}\right)^{2}\right\rangle} \ll 1$, which requires the spread of the ion's motional wavefunction to be always smaller than $1 / k$. This implies that the LambDicke parameter must be small, which means that the energy separation between two consecutive levels of the harmonic oscillator is much larger than the recoil energy provided by the laser light, and therefore the motional state of the ion is unlikely to be changed by photon recoil. A more general treatment beyond the Lamb-Dicke limit will be provided in section 3.2.1.

The Hamiltonian (2.8) is often derived in textbooks for dipole transitions (CohenTannoudji et al., 1992; Scully and Zubairy, 1997). In trapped ions, however, one often encounters quadrupole and stimulated Raman transitions. It can be shown that the same Hamiltonian effectively also applies to the situation of a quadrupole transition or a bichromatic stimulated Raman scheme (Leibfried et al., 2003a).

We first consider the case where the laser is resonant with the atomic transition, $\Delta=0$, which is referred to as the carrier transition. In this case, no coupling between electronic and motional degrees of freedom is induced and the dynamics is described by the Hamiltonian (Häffner et al., 2008)

$$
H^{\mathrm{c}}=\frac{\hbar \Omega}{2}\left(\sigma_{+} e^{i \varphi}+\sigma_{-} e^{-i \varphi}\right)
$$


Thus, the excited state population for an ion, prepared initially in $|g\rangle$, is given by $|\sin (\Omega t / 2)|^{2}$. These driven coherent oscillations can be seen experimentally and are called Rabi oscillations. This can be used to coherently transfer populations from the ground state to the excited state. The entire ground state population is deterministically transferred to the excited state after a time $t=\pi / \Omega$. A pulse of this length is called a $\pi$-pulse, and represents an elementary building block for the quantum state manipulation of trapped ions. Similarly, with a $\pi / 2$-pulse (a pulse half as long as a $\pi$-pulse) one prepares the initial ground-state ion into a coherent superposition of the form $(|g\rangle+|e\rangle) \sqrt{2}$.

Furthermore, controlling the laser phase $\varphi$ allows to map coherences (complex-valued off-diagonal elements of the density matrix) onto the ion's populations, which in turn may be read out efficiently to provide full knowledge of the qubit quantum state (quantum state tomography). Via the two control parameters $\theta=\Omega t$ and $\varphi$, arbitrary unitary rotations of the type

$$
R(\theta, \varphi)=\left(\begin{array}{cc}
\cos \left(\frac{\theta}{2}\right) & -i e^{-i \varphi} \sin \left(\frac{\theta}{2}\right) \\
-i e^{i \varphi} \sin \left(\frac{\theta}{2}\right) & \cos \left(\frac{\theta}{2}\right)
\end{array}\right)
$$

can be applied to the electronic state, employing pulses $R(\theta, \varphi)=e^{-i H^{c} t / \hbar}$ on the carrier transition. Such coherent single-qubit operations are carried out with fidelities of approximately $99.5 \%$ (Schindler et al., 2013).

To analyze the dynamics of coherent superpositions and their decay due to environmental influences, one often employs Ramsey spectroscopy (Ramsey, 1990). In a Ramsey experiment, a coherent superposition of ground and excited state is created with a $\pi / 2$-pulse. This superposition evolves freely, leading the two states to pick up a relative phase difference according to the resonance frequency of their transition. In another $\pi / 2$-pulse, the coherence is mapped back onto the population which is subsequently read out destructively. This measurement scheme reveals the evolution of a coherence and the time scale of its decay. Fourier analysis of the time-resolved data reveals the transition frequency and lifetime via its center frequency and linewidth, respectively.

By scanning the laser around the carrier transition, we expect two additional resonances at $\Delta= \pm \nu$, which in fact can be observed in the spectrum, see figure $2.4 \mathrm{~b}$ ) and are referred to as sidebands. Such laser detunings induce couplings between the electronic and vibrational degrees of freedom. For instance, for $\Delta=-\nu$ we address the first red sideband, and induce a dynamics goverened by the Jaynes-Cummings Hamiltonian (Jaynes and Cummings, 1963; Häffner et al., 2008),

$$
H^{(-1)}=\frac{i \hbar \eta \Omega}{2}\left(\sigma_{+} a e^{i \varphi}-\sigma_{-} a^{\dagger} e^{-i \varphi}\right) .
$$

This effective qubit-mode coupling between pairs of states $|e, n\rangle$ and $|g, n+1\rangle$ is analogous to cavity quantum electrodynamics experiments, where the harmonic oscillator represents the light mode inside a cavity (Haroche and Raimond, 2006). Similarly, when choosing the corresponding blue detuning, $\Delta=\nu$, we drive the first blue side- 
a)
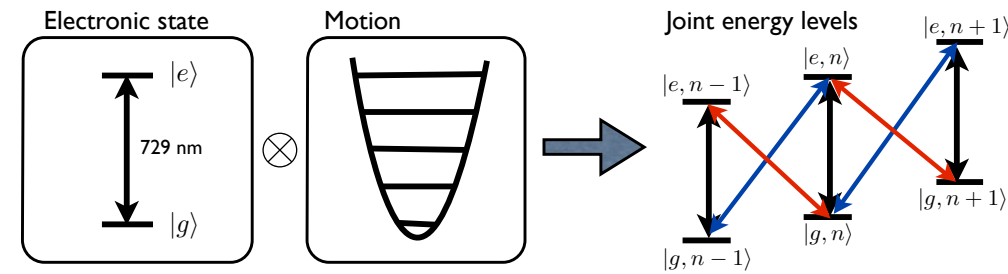

b)

Spectroscopy of resolved sidebands

c)

Carrier Rabi oscillations
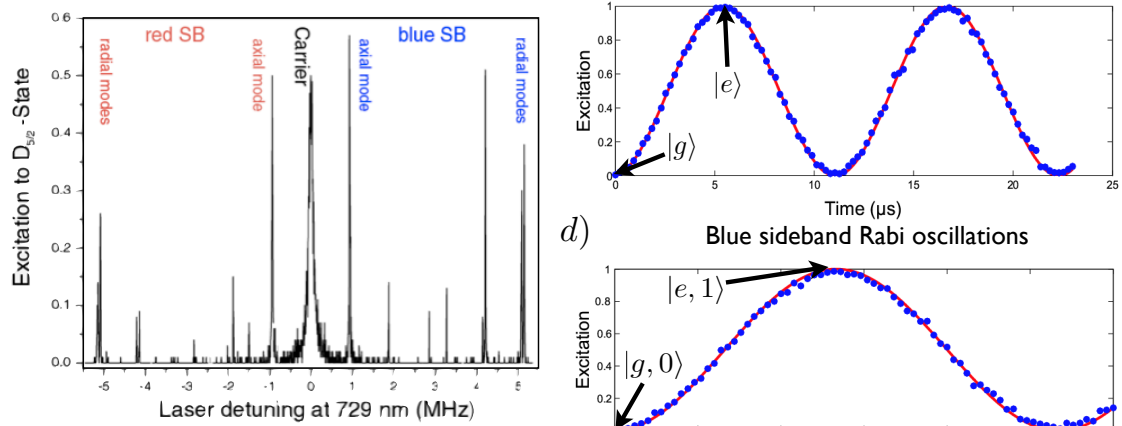

d)

Blue sideband Rabi oscillations

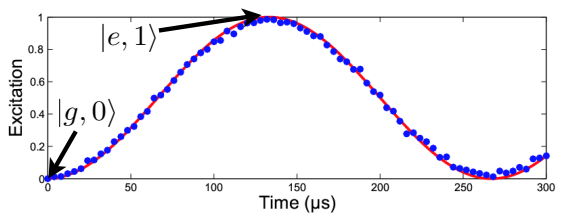

FigURE 2.4.: a) Electronic and motional degrees of freedom of trapped ions can be coupled via laser light, depending on the laser detuning. The black (red / blue) arrows indicate couplings induced by laser light tuned to the carrier (red sideband / blue sideband) frequency. b) The sidebands can be resolved in the spectrum of the $\mathrm{S}_{1 / 2}-\mathrm{D}_{5 / 2}$ (qubit) transition. In a linear trap geometry, the frequencies of axial modes are typically one order of magnitude lower than those of the radial modes. c) Measuring the qubit populations via fluorescence of the $397 \mathrm{~nm}$ transition after driving the qubit transition for different durations, one observes Rabi oscillations. If the laser frequency is resonant with the qubit (carrier Rabi oscillation), motional modes are not coupled. d) By detuning the laser towards one of the sidebands, excitations are coherently exchanged between electronic and motional degree of freedom. Experimental data: Courtesy of H. Häffner (2007).

band, generating dynamics described by (Häffner et al., 2008)

$$
H^{(+1)}=\frac{i \hbar \eta \Omega}{2}\left(\sigma_{+} a^{\dagger} e^{i \varphi}-\sigma_{-} a e^{-i \varphi}\right)
$$

This Hamiltonian coherently exchanges excitations between pairs of states $|g, n\rangle$ and $|e, n+1\rangle$ and is not observed in cavity quantum electrodynamics experiments since such transitions would not conserve the number of total excitations. The transitions induced by the first red (blue) sideband are represented by red (blue) arrows in figure $2.4 \mathrm{a}$ ). The effective Rabi frequencies for first-order sideband Rabi oscillations are proportional to the Lamb-Dicke parameter $\eta$. Since $\eta$ is usually small (depending on the ion species of the order of 0.01-0.1) these transitions are subject to weaker coupling than the carrier transition, leading to slower Rabi oscillations at constant laser intensity, as 
can be seen in figures $2.4 \mathrm{c}$ ) and d). If sufficient laser power is available, sideband transitions can also be driven on a local phonon, which describes a localized vibrational excitation of a single ion. To this end, the pulse must be short enough to encompass the entire spectral range of the contributing eigenmodes, which are driven simultaneously (Harlander et al., 2011; Brown et al., 2011). We will discuss the excitation of local phonons in further detail in section 6.4.4.

From equations (2.12) and (2.13) we see that sideband transitions can be used to transfer excitations between vibrational and electronic degrees of freedom. This is particularly important to cool the ion's motion to the quantum mechanical ground state (Wineland and Dehmelt, 1975; Diedrich et al., 1989). To this end, $729 \mathrm{~nm}$ laser pulses on the (first) red sideband reduce vibrational excitations by transferring excitations from $|g, n\rangle$ to $|e, n-1\rangle$. The electronic population is optically pumped back to the ground state with another laser at $854 \mathrm{~nm}$, such that the ion is able to absorb the next photon on the red sideband.

The combination of ground state cooling with careful elimination of heating and decoherence mechanisms permits remarkably long lifetimes for the quantum states of trapped ions. The lifetime of coherent superpositions of optical electronic excitations is limited mostly by spontaneous decay of the excited state (which occurs on time scales of the order of one second). Encoding the qubit into two sublevels of the ground state can lead to coherence times (time scale of the exponential decay) of 20-30s (Häffner et al., 2005b; Schindler et al., 2013). On the motional degree of freedom, coherence times of about $100 \mathrm{~ms}$ can be achieved (Schindler et al., 2013).

\subsubsection{Experimental quantum computations and quantum simulations}

Given the possibilities for state preparation, readout and coherent manipulation discussed in section 2.2.1, trapped ions are considered a promising candidate for the experimental implementation of theoretical concepts from quantum information science. So far, we have discussed the control of individual ions. Another prerequisite towards a universal quantum computer are entangling gates: operations that generate entangled states of two (or more) qubits (DiVincenzo, 2001). The realization of such gates require the design of effective qubit-qubit interactions. Remember that due to the large separation between the ions, the natural dipole-dipole interaction between neighboring ions is too weak to be considered - in fact it can be completely neglected for the theoretical description of the ions. However, the strong Coulomb repulsion leads to coupled motion of all the ions in the string. This can be used to engineer effective qubit-qubit interactions, where the chain's motional degree of freedom acts as a "quantum bus" (Cirac and Zoller, 1995; Sørensen and Mølmer, 1999). This way, a controlled-NOT gate can be realized experimentally (Monroe et al., 1995; SchmidtKaler et al., 2003; Leibfried et al., 2003b). Together with single-qubit operations (which we have discussed above), this already establishes a simple example of a universal set of gates which, in principle, allows for the realization of arbitrary quantum algorithms (DiVincenzo, 1995; Nielsen and Chuang, 2000).

Even though proof-of-principle experiments with up to 14 entangled qubits have been demonstrated with trapped ions (Monz et al., 2011), a general-purpose large-scale 
universal quantum computer is yet to be achieved. As a more tangible alternative, many groups focus on the controlled engineering of quantum many-body systems, where, currently, the number of constituents ranges between tens of ions in Paul traps (Jurcevic et al., 2014; Richerme et al., 2014) and hundreds of ions in Penning traps (Britton et al., 2012). Since the Hilbert space dimension increases exponentially with the number of particles, the dynamics of large quantum systems cannot be determined efficiently by classical computers. The idea behind quantum simulations is to employ a controllable quantum system, such as a chain of trapped ions, to emulate the dynamics of a computationally intractable quantum system. By measuring the controllable quantum system, one then tries to infer the dynamics of the simulated quantum system (Feynman, 1982; Lloyd, 1996).

Similarly to the realization of quantum gates, by using the motional modes of a chain of trapped ions it is possible to engineer effective, tunable spin-spin interactions across the entire chain. Here, with spin we refer to the electronic qubit, which formally is equivalent to a spin-1/2 system. A combination of off-resonant laser beams on motional sidebands addressing all ions exerts a state-dependent optical dipole force, inducing dynamics described by (Porras and Cirac, 2004b)

$$
H=-\sum_{\substack{i, j=1 \\(i<j)}}^{N} J_{i j} \sigma_{x}^{(i)} \sigma_{x}^{(j)}-B \sum_{i=1}^{N} \sigma_{y}^{(i)}
$$

where $\sigma_{x}^{(i)}$ and $\sigma_{y}^{(i)}$ are Pauli spin operators of the $i$ th spin, and the spin-spin interactions are algebraically decaying,

$$
J_{i j} \approx J_{0} /|i-j|^{\alpha} \text {. }
$$

The range of the tunable exponent $0<\alpha<3$ is limited by the spread of the sideband frequencies and the laser detuning (Jurcevic et al., 2014). The homogeneous, effective magnetic field can be tuned via the Rabi frequency of laser-induced Rabi oscillations on the carrier transition of the spin states. Following this proposal, spin chain models of magnetic systems have been realized experimentally for chains of lengths up to 18 ions (Friedenauer et al., 2008; Kim et al., 2010; Islam et al., 2011; Britton et al., 2012; Schneider et al., 2012; Blatt and Roos, 2012; Jurcevic et al., 2014; Richerme et al., 2014). The Hamiltonian describes a quantum magnet with long-range interactions and shows rich features, such as a quantum phase transition from (anti-)ferromagnetic to paramagnetic phases, as one scans the relative strength of global magnetic field $B$ relative to the spin coupling strength $J_{0}$. In this dissertation, this model will be discussed

(i) in the context of ground-state quantum correlations and their dynamical singlespin signature for the quantum phase transition in section 3.4.3, and

(i) in chapter 5, where we develop a semiclassical approach to further analyze the full spectrum and its role for the quantum phase transition. 
We now turn again to the motional degrees of freedom and summarize some ideas to use these to engineer other quantum systems. In section 2.2.1.3 we have seen that the motion of trapped ions in a linear chain is described in terms of local phonon modes by coupled harmonic oscillators. A standing laser wave (Porras and Cirac, 2004a) modifies the potential landscape by adding a term $V \sum_{i=1}^{N} \cos ^{2}\left(k x_{i}\right)$ to the Hamiltonian (2.6). An expansion in terms of the Lamb-Dicke parameter to fourth order yields a leading additional potential contribution of the form

$$
H_{s w}=U \sum_{i=1}^{N} a_{i}^{\dagger 2} a_{i}^{2},
$$

which completes the system to a Bose-Hubbard Hamiltonian with long-range interactions. This Hamiltonian describes the dynamics of cold trapped atoms on optical lattices; see section 2.2.2 (Jaksch et al., 1998). So far, there have been no experimental realizations of Bose-Einstein condensation with phonons in a trapped-ion system.

In a similar proposal, the axial phonons are studied under the influence of an additional potential induced by a cavity (García-Mata et al., 2007; Pruttivarasin et al., 2011; Benassi et al., 2011). The resulting Hamiltonian was shown to be equivalent to a Frenkel-Kontorova model, which, for instance, describes friction of nanomaterials. The group in Berkeley is currently trying to assemble a linear Paul trap with a cavity along the axial direction with the goal of simulating this model (Pruttivarasin, 2014; Ramm, 2014).

Other proposals for trapped-ion analog quantum simulations involve combinations of electronic and vibrational excitations. By coupling the electronic states via red sideband laser interactions to their local phonon modes, the Jaynes-Cummings-Hubbard model can be simulated (Ivanov et al., 2009; Toyoda et al., 2013). This model conserves quasiparticles composed as superpositions of electronic and vibrational excitations, which formally are analogous to polaritons.

The above examples all represent analog quantum simulations, in which the system follows the engineered dynamics continuously in time (Schneider et al., 2012). A different approach is given by digital quantum simulations, where a specifically designed sequence of quantum gates leads to the desired output. Trapped ions have been used to demonstrate that all basic building blocks to realize a programmable universal digital quantum simulator are available (Lanyon et al., 2011). Moreover, optical pumping can be used to implement different environmental effects to simulate the dynamics of arbitrary open quantum systems described by a Lindblad equation (Barreiro et al., 2011). We will discuss the theoretical description of the dynamics of open quantum systems in section 2.5.3.

\subsubsection{Recent experimental developments and challenges}

The remaining predominant experimental challenge in the field of quantum computation and quantum simulations, particularly relevant for, but not limited to the field of trapped ions, is scalablility: Producing results beyond the reach of classical simulators requires experiments with a significant number of qubits where coherence must be 
upheld for a large number of quantum gates. ${ }^{7}$ Experimentally, this step turns out to be extremely challenging. In a three-dimensional linear trap architecture, as described in the previous sections, it becomes increasingly difficult to avoid decoherence mechanisms which aggravate the fidelity of quantum gates when many ions are sharing the same trap potential (Hughes et al., 1996; Wineland et al., 1998).

One attempt to overcome this issue consists in the fabrication of arrays of segmented micro-traps (Wineland et al., 1998; Cirac and Zoller, 2000; Kielpinski et al., 2002). The quantum gates are then performed in a fixed area, into which only a small number of ions is moved physically from an array of registers (Rowe et al., 2002; Walther et al., 2012). These micro-traps are fabricated in two-dimensional surface architectures on chips, using micro-electromechanical tools and nanotechnology. Surface traps, however, bring new problems as the ions have to be close to the surface for fast gate times and thereby become subject to electric-field noise due to surface impurities caused by atomic adhesion. This generates an additional, strong heating mechanism, which limits coherent operations (Deslauriers et al., 2006). An efficient way to avoid this heating mechanism is to clean the ion trap with energetic ion bombardments (Hite et al., 2012), which effectively take away few atomic layers from the trap surface. This method is now being implemented in several ion-trap laboratories, including by the group at Berkeley (Daniilidis et al., 2014).

Other relevant limitations of current ion traps are

(i) fluctuations of the laser field intensity and beam pointing instabilities. These effects lead to variations of the Rabi frequencies and thereby change the times required to perform a certain gate. When averaged over many repetitions, which is necessary for measuring quantum mechanical expectation values, this leads to an effective dephasing effect (Schindler et al., 2013).

(ii) fluctuations of the laser-qubit detuning caused by (a) laser frequency fluctuation and (b) magnetic field intensity fluctuations due to unstable currents supplying the Helmholtz coils and stray fields. Since the qubit resonance transition depends on the magnetic field strength via the Zeeman effect, this leads to off-resonant excitations and also causes a dephasing effect. This effect has been exploited to generate correlated quantum states in an experiment (Lanyon et al., 2013) described in section 4.3, and a general discussion of the theoretical description and its implications will be provided in section 4.4.

\subsubsection{Cold gases of neutral atoms in optical lattices}

Another versatile controllable atomic quantum system is offered by cold gases of neutral atoms (Bloch et al., 2008). Such gases can be laser-cooled and confined in magnetooptical traps (Raab et al., 1987) followed by a transfer to optical dipole traps (Dalibard and Cohen-Tannoudji, 1985; Chu et al., 1986; Grimm et al., 2000) to achieve temperatures below $1 \mathrm{mK}$. To achieve Bose-Einstein condensation, even lower temperatures

\footnotetext{
${ }^{7}$ Aside from scaling up these experiments, also efficient diagnostic tools need to be developed which are able to cope with large-scale systems. This will be discussed in section 2.3.
} 
must be reached by evaporative cooling (Davis et al., 1995; Anderson et al., 1995). In an optical dipole trap counter-propagating laser beams generate an optical standing wave which provides a periodic trapping potential - an optical lattice. Around its minima, the trap potential can be approximated as harmonic with achievable trap frequencies up to $100 \mathrm{kHz}$ (Bloch et al., 2008). By combining three pairs of orthogonal lasers, cold atoms can be trapped in three-dimensional optical lattice structures.

Under certain conditions, cold atoms on optical lattices are well described by the Bose-Hubbard Hamiltonian (Jaksch et al., 1998),

$$
H=-J \sum_{\langle i, j\rangle} a_{i}^{\dagger} a_{j}+\sum_{i} \epsilon_{i} a_{i}^{\dagger} a_{i}+\frac{U}{2} \sum_{i} a_{i}^{\dagger 2} a_{i}^{2},
$$

where $a_{i}^{\dagger}$ and $a_{i}$ describe creation and annihilation operators for a bosonic particle at site $i$, and the parameters determining the on-site repulsion $U$, the nearest-neighbor tunneling strength $J$, and the local energy offset $\epsilon_{i}$ can be manipulated via parameters of the laser light. The sums extend over all lattice sites and $\langle i, j\rangle$ denote pairs of nearest neighbors. This model predicts the existence of Mott-insulating and superfluid quantum phases, which have been observed experimentally (Greiner et al., 2002), albeit in the presence of an external harmonic confinement.

Originally, the Hubbard model was proposed for fermionic particles to describe electron dynamics in condensed matter, where the potential is generated by a lattice of atomic nuclei (Hubbard, 1963; Kanamori, 1963; Gutzwiller, 1963). Several groups also pursue the goal of simulating the Fermi-Hubbard model to directly mimic the dynamics of solid-state devices, by loading fermionic gases (Greiner et al., 2003) into an optical lattice (Jördens et al., 2008; Wenz et al., 2013). Generating Hubbard models under controlled conditions with tunable parameters opens up a wide range of possibilities for experimental studies of quantum many-body physics (Morsch and Oberthaler, 2006; Bloch et al., 2008). Realizing this with cold atoms is particularly appealing since the spacings of optical lattice sites are three orders of magnitudes larger than those of a real crystal and renders experimental control of the quantum dynamics possible. Moreover, the potential landscape can be adjusted by changing parameters of the lasers to observe, for instance, coherent tunneling dynamics in double-well potentials (Smerzi et al., 1997; Mahmud et al., 2005; Kierig et al., 2008; Hunn et al., 2013).

More complicated potentials can be generated when laser light is reflected from a rough surface. The resulting speckle field can be used to create random disordered potentials (Bouyer, 2010). The interference of multiple scattering events with random potential barriers can lead to surprising effects (Jendrzejewski et al., 2012). For instance, if the disorder is strong enough, the matter-wave describing the quantum state of a Bose-Einstein condensate is expected to localize exponentially in space. This phenomenon is a quite general interference effect known as Anderson localization (Anderson, 1958) which bears important implications for the conductivity of metals in the presence of impurities, and was observed in a wide variety of physical settings (Dalichaouch et al., 1991; Wiersma et al., 1997; Hu et al., 2008) including cold atoms (Roati et al., 2008; Billy et al., 2008; Modugno, 2010).

Alternatively, when the local energy levels $\epsilon_{i}$ are modified via an external potential 

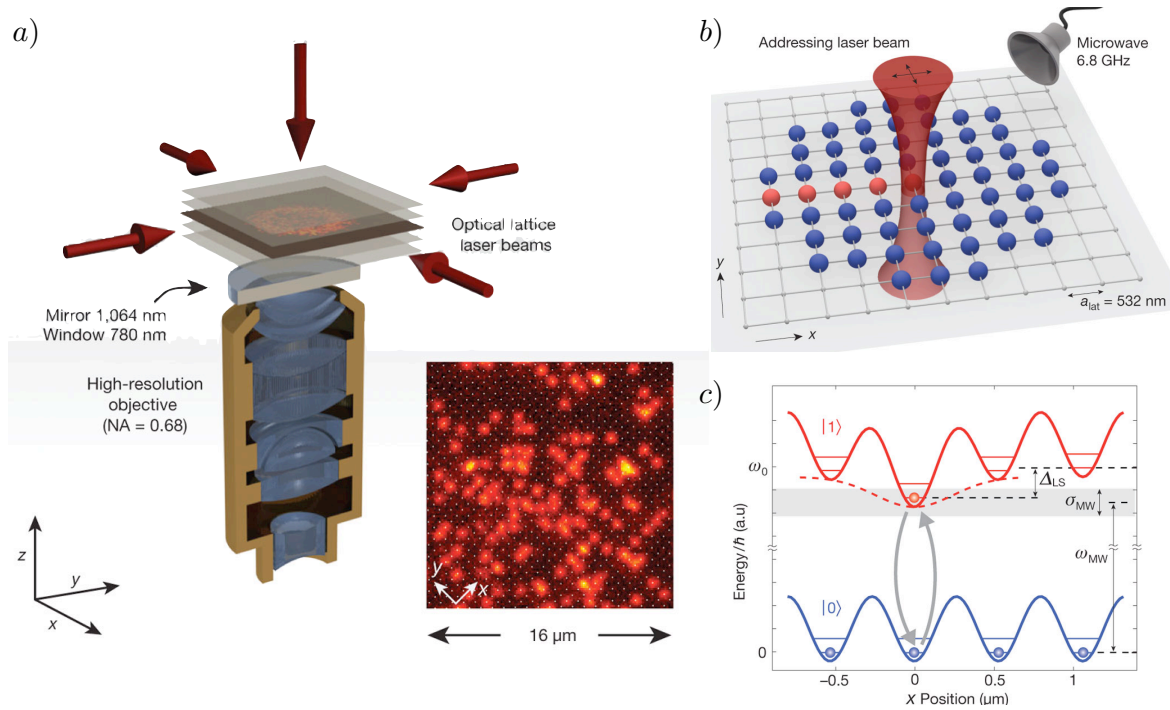

FIGURE 2.5.: a) A high-resolution microscope provides single-site resolved fluorescence images of cold atoms in an optical lattice (Sherson et al., 2010). b) The same imaging system can also be used to send a focussed laser to individual lattice sites (Weitenberg et al., 2011). c) This laser shifts the atom at the selected site into resonance with a global microwave field via an ac Stark effect (Weitenberg et al., 2011). Specifically, in the laser focus, the transition between the atomic hyperfine levels $|0\rangle$ and $|1\rangle$ is shifted by $\Delta_{\mathrm{LS}}$, which renders the an external microwave driving field of frequency $\omega_{\mathrm{MW}}$ and linewidth $\sigma_{\mathrm{MW}}$ resonant with the focussed atom (Weitenberg et al., 2011).

such that they increase linearly with the lattice index, the generated tilted lattice generates the effect of a static driving field on the atoms (Morsch et al., 2001). In this case, rather than uniform motion of the atoms towards the potential minimum, one expects Bloch oscillations (Bloch, 1928; Zener, 1934). Particle-particle interactions can lead to modification (Kolovsky, 2003) and even decoherence of the Bloch oscillations caused by a transition into a quantum chaotic parameter regime (Buchleitner and Kolovsky, 2003). This has also been observed in a recent experiment (Meinert et al., 2014). We avoid discussing the challenges one faces when dealing with the theory of interacting many-body systems at this point (this will be done later in section 2.5.4) however, we already remark here that a microscopic open-system theory for interactioninduced decoherence in systems of indistinguishable particles has not been developed yet.

Cold atoms on tilted optical lattices can also be mapped onto a quantum Ising model (Sachdev et al., 2002), allowing one to study effects of quantum magnetism (Simon et al., 2011; Meinert et al., 2013) with similar models which were discussed in section 2.2.1.7 in the context of trapped-ion quantum simulations. Actual spinspin interactions of the electronic states of cold atoms can be engineered based on the 
exchange interaction which is induced by the quantum statistics of indistinguishable, bosonic particles (Duan et al., 2003; Anderlini et al., 2007).

While neutral atoms generally facilitate the studies of quantum statistics on large ensembles, recently, experiments on Bose-Einstein condensates have been combined with scanning electron microscopes (Würtz et al., 2009) and also optical microscopes using a high-aperture lens (Bakr et al., 2009; Bakr et al., 2010; Sherson et al., 2010), to spatially resolve individual lattice sites (see figure 2.5). The optical microscope has the advantage that it can be used to send a tightly focussed laser beam to induce acStark shifts on individual lattice sites (Weitenberg et al., 2011). In combination with a global microwave field whose frequency is resonant only with the atom in the laser focus, this allows for the manipulation of the electronic quantum state of individual atoms in an optical lattice (Meschede and Rauschenbeutel, 2006; Weitenberg et al., 2011).

\subsubsection{Photons and (non-)linear optics}

In the last two sections we have seen how atomic particles can be trapped and manipulated with electric fields and laser light. Experiments on trapped atomic particles all encompass involved optical setups to control the light fields, which in turn are used as a means to manipulate the quantum state of the atoms. The control of the quantum state of light by means of linear optics provides itself a broad range of possibilities to carry out experiments on individual, well-controlled quantum systems. Single photons can be created from solid-state devices, such as nitrogen-vacancy centers in diamond (Kurtsiefer et al., 2000) or quantum dots (Michler et al., 2000), and detected with single-photon detectors (Eisaman et al., 2011). Many experiments focus on the polarization degree of freedom of photons, since it represents an ideal qubit (Kok et al., 2007), similar to the two-level systems formed by long-lived electronic excitations of trapped ions as discussed above. Furthermore, photons carry a frequency degree of freedom and may also exhibit orbital angular momentum (Allen et al., 1992).

In these experiments, photons are sent through arrays of linear optics devices to manipulate the quantum state of light (Kok et al., 2007). An elementary building block are semi-reflective mirrors, beam splitters, in which part of the incoming light is transmitted while the remaining part is reflected in a fully coherent process (Zeilinger, 1981). The reflectivity of beam-splitters can also depend on the polarization state of light and can therefore be used to prepare photons with specific polarization states. Wave plates rotate the polarization state in the complex plane allowing to switch between linear and circular polarization. Finally a light mode can experience a phase factor when it passes through a material of different diffractive index, called a phase shifter. A combination of these elements allows to generate arbitrary unitary transformations between the incoming and outgoing light modes (Reck et al., 1994).

Such experiments allow for the studies of many-particle dynamics, which are heavily influenced, on the one hand, by the bosonic quantum statistics induced by the indistinguishability of photons (Hong et al., 1987), and, on the other hand, by additional many-body interference phenomena (Tichy, 2011; Ra et al., 2013; Tichy, 2014).

Pairs of entangled photons are created by nonlinear processes which occur in bire- 
fringent crystals $^{8}$ (Kwiat et al., 1995). An incoming pump photon creates a pair of photons with strong frequency correlations. Additionally, under specific conditions, also the polarization of the two photons is described by an entangled quantum state. Nonlinear processes can further be used to induce interactions between polarization and frequency degrees of freedom of the same photon. This effect is used to simulate a controlled environment for the polarization qubit in an experiment described in section 3.3.

Linear optics and photonic qubits are particularly important for quantum communication, since photons - in contrast to atoms - can be sent efficiently from one location to another over long distances (Ursin et al., 2007). Their flexibility renders photonic systems also attractive for the realization of a quantum computer (Knill et al., 2001; Kok et al., 2007). In this context, the main disadvantage over atomic systems is that the desired processes only happen probabilistically, which means that a desired quantum state can only be produced in combination with post-selection. Therefore, observing a certain effect may require a large number of experimental iterations. This is further hampered by imperfect photon sources and detectors, as well as photon losses (Broome et al., 2013).

Experimental efforts to improve the performance of large-scale photonic setups are focussing on integrated photonics, where waveguides and the key building blocks of linear optics are microfabricated onto chips (O'Brien et al., 2009; Peruzzo et al., 2010; Spring et al., 2013; Tillmann et al., 2013; Crespi et al., 2013). So far, however, experiments are carried out on few-particle systems which can be easily treated theoretically on a classical computer. Increasing the numbers of modes and particles such that these experiments become interesting from a complexity point of view (Aaronson and Arkhipov, 2013) remains a major challenge. This resembles the scalability issue which was already pointed out for trapped ions, albeit for different reasons.

\subsubsection{Other systems}

In the previous sections we have discussed the three platforms of trapped ions, cold gases of neutral atoms, and linear optics. There exists a large number of other experimental settings which allow for a similar quality of coherent control of quantum mechanical particles. In this section, we briefly comment on some of them.

Experiments on nuclear magnetic resonance have a long-standing tradition in manipulating large ensembles of spin states with strong coherent pulses (Ernst et al., 1987). Spectrally separated nuclear spins of molecules can be used to identify interacting qubit systems, and have been employed for a proof-of-principle experiment factoring the number 15 with Shor's algorithm (Vandersypen et al., 2001). These molecules are, however, in solution at room temperature, which renders the conservation of quantum properties such as coherence and entanglement unfeasible (Warren, 1997).

In contrast, in a solid-state environment, nitrogen-vacancy centers can provide a platform for coherent quantum experiments in diamond (Jelezko et al., 2004). The challenge here is the difficulty to create nitrogen-vacancy centers with a specific reso-

\footnotetext{
${ }^{8}$ In birefringent crystals, the refractive index depends on the polarization state of light.
} 
nance frequency. Hence, high-precision laser manipulations as with trapped-ions are not possible. The resonance frequency is further influenced by the ambient temperature (Acosta et al., 2010) and can also be sensitive to magnetic fields via the Zeeman effect (Maze et al., 2008). These effects can be used to map out or even change the properties of the complex environments of the nitrogen-vacancy centers with high spatial resolution (Taylor et al., 2008; Kucsko et al., 2013). These systems therefore represent an interesting platform at the interfaces between atomic physics, solid-state physics and soft matter.

Another example is once again defined by atomic systems. When bound electrons are excited to high electronic quantum numbers, the atoms become spatially extended and gain large dipole moments. From a theoretical point of view, such Rydberg atoms (Gallagher, 1994) are an appealing testbed for studies of semiclassics and quantum chaos (Buchleitner et al., 2002; Madroñero et al., 2006), as the electronic quantum motion can be interpreted in terms of classical periodic orbits. Coherent control via external driving fields is able to generate non-dispersive wavepackets, which follow classical trajectories (Buchleitner et al., 2002). Moreover, Rydberg atoms interact strongly with each other via enhanced dipole-dipole interactions, which depend on the inter-atomic distance as $r^{-3}$ (Cohen-Tannoudji et al., 1977b). This enables studies of energy transfer processes (Günter et al., 2013), and the energy shift excerted by an excited atom onto neighboring atoms over large distances (Urban et al., 2009; Gaetan et al., 2009) has potential use for applications in quantum information theory (Lukin et al., 2001; Saffman et al., 2010). Using spatially confined optical dipole traps, optical tweezers, it is possible to trap individual Rydberg atoms in carefully designed optical trap architectures, which allow for the design of arbitrary lattice configurations (Nogrette et al., 2014). A prevailing challenge is the very limited trapping time of Rydberg atoms in such optical tweezers, which do not yet permit deterministic, simultaneous filling of all of the trapping sites.

Rydberg atoms are also used to manipulate and probe the quantum state of a single light mode inside a cavity (Haroche and Raimond, 2006). Strong coupling to a resonant cavity enhances the stimulated emission rate of passing atoms, which is the basic principle of the micromaser (Walther, 1992; Walther et al., 2006). The approach of cavity quantum electrodynamics as incarnated by the micromaser represents a complementary aspect of atom-light interactions, with respect to the field of trapped ions. Here, atoms are used as a means to add or remove individual photonic excitations in the cavity. In an extremely lossless cavity the quantum state of the light mode can be engineered with remarkable precision (Haroche, 2013). Mathematically, this system very much resembles the trapped-ion system, when a sideband transition is addressed. The strong coupling inside the cavity leads to the exchange of excitations between the resonant atomic two-level system and the single light mode, which is described by a quantum harmonic oscillator. The dynamics of such systems was conceived such as to realize the Jaynes-Cummings Hamiltonian (Jaynes and Cummings, 1963), as was already discussed in the context of the first red sideband transition of a trapped ion.

Another mathematically similar realization is given by superconducting qubits built from Josephson junctions (Clarke and Wilhelm, 2008). A prerequisite for quantum information applications is, again, the experimental control of the quantum state 
(Hofheinz et al., 2009). In complete analogy to cavity quantum electrodynamics, one couples artificial atoms of superconducting Josephson junctions to solid-state cavities, in experiments referred to as circuit quantum electrodynamics (Wallraff et al., 2004). Similarly, the field of optomechanics considers the coupling of the quantized motion of matter to light fields, which allows for cooling and control of the quantum state of mesoscopic systems (Meystre, 2013).

We note that a variety of physically very different systems is described by mathematically very similar models. Suitable technologies for quantum information purposes often contain two-level systems (qubits) as the simplest possibility to encode quantum information. The indispensable interaction between such qubits is then mediated via some type of quantum bus, which often is described by a quantum harmonic oscillator.

Finally, closely related to the field of cold atoms in optical lattices, we mention recent experimental steps towards the control of diatomic molecules in optical lattices. These molecules are created from cold atomic gases via a combination of Feshbach resonances and carefully timed Raman pulses (Bergmann et al., 1998) that achieve cooling with optical laser light (Danzl et al., 2008; Ni et al., 2008). Diatomic molecules of two different atomic species can carry a large dipole moment, leading to longrange interactions among the rotational states of the molecules at pinned positions in the optical lattice (Yan et al., 2013). Such polar molecules have the potential to be employed for the studies of interacting many-body systems (Hazzard et al., 2014).

The present overview only presents a subjective selection, and there exist even more interesting experimental platforms for well-controlled quantum experiments, each one with its own characteristic advantages and disadvantages.

\subsection{The certification of large-scale quantum devices}

An obvious reason that comes to mind to motivate the challenge to build large-scale quantum devices is the theoretically proven possibility to computationally outperform computing systems based on classical objects (Feynman, 1982; Nielsen and Chuang, 2000; Aaronson and Arkhipov, 2013). Another motivation may be the possibility of pushing quantum mechanics to larger scales, from which interesting phenomena may emerge. While some even postulate a breakdown of quantum theory at some macroscopic scale (Ghirardi et al., 1986), with interesting phenomena we here mainly refer to novel manifestations of large-scale interacting many-body systems in agreement with quantum theory. One way or the other, the second motivation is certainly sufficient for most physicists to find interest in such endeavors. As we will discuss in the following, the aspect of novel perspectives in the context of computational complexity entails additional challenges.

The small size of current proof-of-principle experiments allows to verify measurement results by comparison with classically computed predictions. When experiments reach the classically intractable realm of parameters, how can the result provided, for instance, by a quantum computer be certified? Conceptually, this certification poses a significant challenge for the field of quantum computations and quantum simulations. Some specific tasks, such as the factorization of prime numbers, solve this issue through 
the simplicity of verifying a result. Given a set of prime numbers, it is a simple task to verify that their product yields the initial number. For quantum simulators, this is more complicated, since their purpose is precisely to solve equations of motion which are not tractable by other means. Similarly, a Boson Sampling device efficiently samples from distributions, generated by the permanents of large, generic complex-valued unitary matrices that map the input on the output state of indistinguishable bosonic particles (Aaronson and Arkhipov, 2013), for instance when transmitted through linear optics setups (Spring et al., 2013; Tillmann et al., 2013; Crespi et al., 2013), or when inscribed into the phonon modes of trapped ions (Shen et al., 2014). The realization of such a device would have far-reaching implications for theoretical computer science (Aaronson and Arkhipov, 2013), but if it exists, how can the functionality of such a device be verified? An important prerequisite to reliably analyze large-scale quantum devices is the development of tools that are able to diagnose systems of increasing size and complexity.

Let us briefly discuss some of the available methods to characterize the prepared quantum states, their dynamics, and the decoherence processes they are exposed to. For medium-sized entangled states of trapped ions it is possible to map out the entire density matrix using quantum state tomography ${ }^{9}$ (Paris and Řeháček, 2004; Häffner et al., 2005a). Already for eight qubits, state tomography requires more than $6 \times 10^{5}$ measurements which corresponds to about ten hours of measurement time (Häffner et al., 2005a). Similarly, by preparing a set of initial states and observing their evolution it is possible to perform quantum process tomography to extract the underlying dynamical map experimentally - at the cost of even higher experimental overhead. In recent years, methods from data compression have been employed to provide tomographic methods with polynomial scaling with the number of qubits, for density matrices with few nonzero elements (Gross et al., 2010; Schwemmer et al., 2014). In general, the number of elements of multi-particle density matrices increases exponentially with the number of particles. Thus, it will neither be possible nor desirable to obtain complete knowledge about the full quantum state in the limit of large-scale quantum systems. Instead, the challenge will consist in identifying the relevant quantities in a given scenario and developing methods to extract these efficiently from complex interacting quantum systems, and to conceive robust statistical descriptions.

A promising direction are spectroscopic techniques, which can map out the excitation spectrum of a quantum simulator (Senko et al., 2014), but still have difficulties to explicitly demonstrate coherent superpositions of many-body quantum states. The spin squeezing parameter as a macroscopic entanglement witnesses (Sørensen et al., 2001) is in this context the only hint at a certain amount of coherent superpositions in the system. To obtain dynamical information on the decay of coherent superpositions, one typically adapts methods of Ramsey spectroscopy to many-body states. Combined with single-site addressability, this technique can be refined to extract spatially resolved correlation functions in thermal equilibrium (Knap et al., 2013; Serbyn et al., 2014). Such interferometric probes of many-body physics entail sequences of

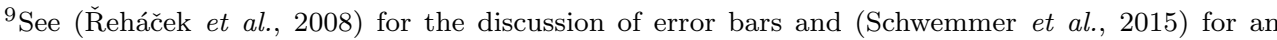
analysis of systematic errors of commonly employed methods for quantum state tomography.
} 
pulses and thereby probe time-correlation functions which contain more information than mere static spectral data (Ernst et al., 1987).

A very general and powerful framework is provided by nonlinear spectroscopy. It is able to describe not only the signal obtained by the above described sequences of strong pulses, but can also account for non-equilibrium initial conditions, provides a perturbative theory of arbitrary order response functions, and allows to include pulse shapes (Mukamel, 1995). We will briefly review the basic ideas and applications of nonlinear spectroscopy in the next section. In chapter 6 , we introduce the application of methods from nonlinear multi-dimensional spectroscopy as a scalable alternative to study specific features of complex quantum optical and atomic systems.

The development of novel experimental tools is just one of two aspects in the context of the reliable and scalable analysis of large many-body quantum systems. These efforts must be complemented by applicable theoretical methods, which eventually can lead to the characterization of the relevant properties, as well as the assessment of the performance of many-body quantum devices. Established methods such as random matrix theory have proven to successfully describe statistical features of sufficiently generic quantum systems. If, however, specific microscopic details are of interest, one must develop an effective description by identifying the relevant degrees of freedom, and treating the less relevant part of the system perturbatively with appropriate approximations. One such approach is represented by the theory of open quantum systems, which describes incoherent influences of an environment on a quantum system of interest efficiently. An overview of different approaches to the theoretical treatment of composite quantum systems will be provided in section 2.5.

\subsection{Nonlinear spectroscopy}

Absorption spectroscopy provides static information about a physical system's spectrum, imparting valuable information about the distribution of energy levels. For a complete dynamical description we additionally need to know the associated eigenvectors. When the initial state can be controlled, the strength of the recorded spectral features can be used to extract information about the shape of the eigenvectors. In truly complex quantum systems, however, the structure of eigenvectors and their overlap with any generic initial state becomes too complicated to be treated explicitly. The size and complex structure of such systems also leads to effectively irreversible dynamics, which is no longer well described by a coherent evolution based on a microscopic Hamiltonian model. In such situations, the information provided by linear absorption spectroscopy is not sufficient to describe the dynamical features of the system. Again, we rather have to sort out the essential, relevant quantities, and identify methods to extract those, depending on the specific question to be answered. In chemical physics, for example, a complete description of the microscopic quantum mechanical properties of molecular aggregates is not considered a realistic and feasible option. Instead, one identifies key features such as the electronic and vibrational resonances, the couplings between them and the nature of resulting transport processes, as well as environmental influences, which lead to decoherence and dissipation. Such information can 
be extracted efficiently with techniques from nonlinear spectroscopy (Mukamel, 1995; Hamm and Zanni, 2011).

The basic idea of nonlinear spectroscopy is to apply a sequence of phase-coherent pulses, with well-defined time delays between them, and scan the signal over many repetitions as a function of these time delays. The resulting multi-dimensional spectra contain all the information of linear absorption spectra, but also are able to identify dynamical information which is inaccessible to linear methods. The basic methods were already developed in the context of nuclear magnetic resonance experiments, which require the excitation and readout of radio-frequency transitions (Ernst et al., 1987). This is done with pulses of well-defined length with specific phase properties, analogous to the manipulation of the ionic qubit, as discussed in section 2.2.1.6. The relative phases between individual pulses in a sequence are an essential tool to control the induced excitations and to monitor the evolution of specific coherent superposition states. In this context, a commonly employed technical term is the excitation pathway, which refers to one of many coherent superpositions that are induced by all of the laser-sample interactions in the course of the full time evolution. Post-processing of the resulting data sets as a function of the accumulated phases allows to extract such excitation pathways, which otherwise remain hidden in the full data comprised of the interference of all contributing transition amplitudes. The extraction of individual pathways is done by a discrete inverse Fourier transform technique called phase cycling (Ernst et al., 1987), which we will explain in further detail in section 6.2.5. The realization of these ideas with optical frequencies is more challenging (Tanimura and Mukamel, 1993), but is common practice in many laboratories today (Cundiff and Mukamel, 2013). In this case, instead of the phases of incoming pulses, one controls their k-vectors, hence, the orientation of the photons' momenta (Mukamel, 1995; Hamm and Zanni, 2011). This allows to extract individual excitation pathways via phase matching.

A typical nonlinear spectroscopic experiment is depicted in figure 2.6. A sequence of weak, perturbative pulses is injected onto the sample from different spatial directions. After the final pulse, the system emits a photon with a well-defined direction, depending on the orientations of the train of incoming pulses and the excitation pathway. By adjusting the orientation of the detector, only the signal which satisfies a certain phase-matching condition, imposed by momentum conservation, is recorded. The pathway depicted in figure $2.6 \mathrm{~b}$ ) is selected by the phase-matching condition $\mathbf{k}_{S}=-\mathbf{k}_{A}+\mathbf{k}_{B}+\mathbf{k}_{C}$. Here, the first pulse creates a coherent superposition of the ground state $|0\rangle$ and an excited state $|1\rangle$. The second pulse transfers the excitation fully to the excited state population. The third pulse brings the system back to a coherent superposition. Finally, after another time delay of duration $t$, the system interacts with a probe pulse, inducing stimulated emission of radiation along $\mathbf{k}_{S}$, which is measured via heterodyne detection. ${ }^{10}$

Typically, the generated, time-dependent signals are Fourier transformed with respect to the time delays $\tau$ and $t$ to obtain a two-dimensional spectrum in the frequency

\footnotetext{
${ }^{10}$ Interference of the sample's signal with the laser field that causes the stimulated emission (Hamm and Zanni, 2011).
} 

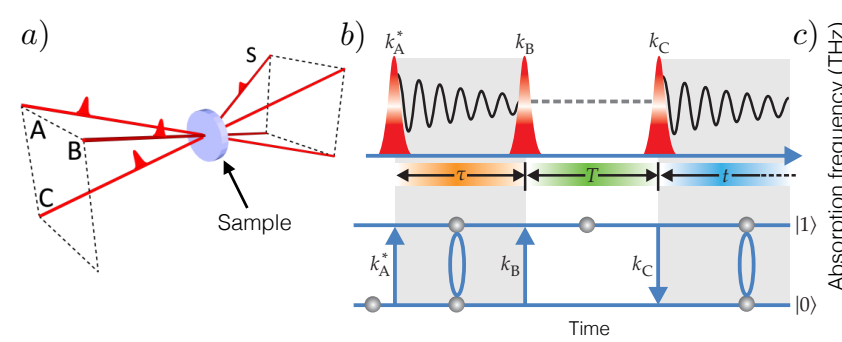

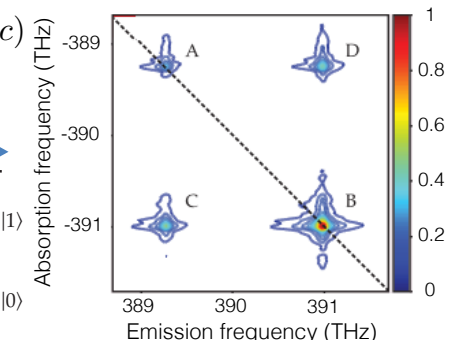

FiguRE 2.6.: Typical experimental setup using nonlinear spectroscopy. Adjustment of the phase-matching conditions (a), allows to select the contribution of individual excitation pathways (b). During the time intervals $\tau$ and $t$, the signal evolves in a coherent superposition, generating an oscillating signal with the transition frequency between the two states. In between, during $T$, the pathway involves an excited state population. The first pulse is applied $180^{\circ}$ out of phase, indicated by the asterisk on the wave vector $\mathbf{k}_{A}$. Part c) shows an experimentally obtained signal resulting from such a pulse sequence applied to an atomic vapor of potassium atoms. The two diagonal peaks (on the dashed line) correspond to the transitions from the ground state to two different excited states, which in linear absorption spectra produce the $D_{1}$ and $D_{2}$ lines. The off-diagonal peaks indicate the correlated nature of these two resonances. The absorption frequency along the $y$-axis is plotted negative by convention to account for the out-of-phase nature of the first pulse. Adapted from (Dai et al., 2012; Cundiff and Mukamel, 2013).

domain. The example in figure 2.6 displays data obtained from atomic potassium vapor. The diagonal peaks correspond to the linear absorption peaks of the $D_{1}$ and $D_{2}$ lines, respectively. The presence of an off-diagonal signal in the two-dimensional spectrum reveals correlations between the two transitions. These are caused, for instance, by the common ground state, and their presence rules out the possibility to attribute the two diagonal resonances to two independent transitions of two different atomic species (Dai et al., 2010). By varying the phase matching condition, other signals can be selected, involving, for instance, coherent superpositions of states beyond the first excited state manifold (Kim et al., 2009), which are able to reveal weak collective resonances induced by particle-particle interactions (Dai et al., 2012). Nonlinear spectroscopy, thus, provides us with a versatile toolbox, which can be adjusted to taylor the adequate experimental observable to quantify a specific target property of the physical system under study.

The strengths of nonlinear spectroscopy become particularly apparent when dealing with systems subject to interaction with challenging environments, fast relaxation time scales, and many-body interactions (Mukamel et al., 2009). In molecular systems, the vibrational excitations can couple to the electronic levels which leads to a broad collection of overlapping resonances (Hamm et al., 1998; Milota et al., 2009). Even in such scenarios, two-dimensional spectra allow to identify structural changes of biologically relevant proteins (Chung et al., 2007), as well as transport processes across 
molecular aggregates (Brixner et al., 2005; Engel et al., 2007). Furthermore, the lineshapes of two-dimensional resonances can be used to distinguish different incoherent processes induced by the interaction of the system with its environment (Tokmakoff, 2000; Siemens et al., 2010). Other scenarios where nonlinear spectroscopy has facilitated the identification of the dynamics and couplings of elementary excitations are encountered in solid state physics, including semiconductors (Zhang et al., 2007) and nitrogen-vacancy centers (Huxter et al., 2013). In semiconductors, two-dimensional spectra have revealed many-body interactions ( $\mathrm{Li}$ et al., 2006) which cannot be described by mean-field theories (Zhang et al., 2007).

Given the information one is able to extract from two-dimensional spectra, these methods are also interesting for artificially engineered, controllable atomic and quantum optical systems, as were discussed in section 2.2. This becomes especially relevant when considering current efforts to increase size and complexity of such experiments, and the entailed necessity to develop powerful, scalable probing tools. Yet, there are considerable differences between synthetic quantum matter such as trapped ions or cold atoms and actual condensed-phase bulk materials or macroscopic ensembles of molecules in solution. Thus, to be able to transfer existing ideas from nonlinear spectroscopy to artificial quantum systems, the available toolbox needs to be extended and adjusted.

Conversely, quantum optical systems can offer significant advantages over bulk materials, regarding, for instance, the possibilities to control a given system. Quantum optical systems often allow for the coherent manipulation of individual quantum particles. The given spatial resolution is able to open up new possibilities to extend standard methods of nonlinear spectroscopy. Adding spatial resolution to phase-coherent nonlinear spectroscopy is a long-standing goal also for bulk materials (Richter et al., 2012). In systems of molecular aggregates, the optical diffraction limit forbids localized excitations that are confined enough to resolve individual interacting chromophores. Current effort is therefore focussed on nano-fields (Vasa et al., 2009) and space-resolved electron emission microscopy (Aeschlimann et al., 2011).

Another advantage, available for, but not limited to, engineered quantum systems, is the possibility to performing experiments on single samples instead of obtaining ensemble-averaged results of a bulk material (see (Hildner et al., 2011) for spectroscopic experiments on single-molecules). The interpretation of multi-dimensional spectra is strongly dependent on the underlying model, which for complex molecular systems is obtained from a combination of physically motivated structures, sometimes based on $\mathrm{X}$-ray data, and parameter adjustment to fit the spectral data that is known from experiments (Schlawin et al., 2013). This is simplified significantly by the austerity of quantum optical systems and facilitates a clean and unambiguous interpretation of multi-dimensional spectra.

Finally, let us also mention the increased interest in concepts from quantum information theory in the context of nonlinear spectroscopy (Schlawin, 2015). For example, entangled photons are being used to enhance time- and frequency resolution, and the signal yield at low intensities (Richter and Mukamel, 2010; Upton et al., 2013). Entangled photons can further be employed in nonlinear spectroscopy to control the population of specific states via many-photon excitation pathways (Schlawin et al., 
2012; Schlawin et al., 2013; Schlawin, 2015).

In section 6, after an introduction to the theoretical treatment of nonlinear response signals, we show how artificial quantum systems can be probed using methods from nonlinear spectroscopy, and how single-site resolution enables us to extend standard methods of nonlinear spectroscopy in this context.

\subsection{Theoretical description of composite quantum systems}

In this section, we briefly review theoretical approaches for the description of composite quantum systems. Specifically, we introduce basic concepts of semiclassical approximations and random matrix theory, review the central ideas behind the theory of open quantum systems, and discuss the theoretical description of interacting systems of identical, indistinguishable particles.

\subsubsection{Semiclassical approximations and mean-field theories}

Semiclassical descriptions of quantum mechanics are, on the one hand, motivated by the question, what is the correspondence between quantum and classical mechanics, and in which limit does classical dynamics emerge (Gutzwiller, 1990)? On the other hand, they have led to the development of efficient methods to describe spectral properties and dynamics of mesoscopic, complex quantum systems (Ozorio de Almeida, 1988; Gutzwiller, 1990; Gutzwiller, 1991; Delande and Buchleitner, 1994; Brack and Bhaduri, 1997; Richter, 2000; Buchleitner et al., 2002).

The starting point of many semiclassical treatments are path integral formulations of the quantum propagator (Feynman, 1948), which expresses the interference of all possible trajectories that connect two configurations $\left(x^{\prime}, t^{\prime}\right)$ and $\left(x^{\prime \prime}, t^{\prime \prime}\right)$, characterized by time and position. The phase factors are of the form $\exp (i R / \hbar)$ with Hamilton's principal function $R$, the time-integral over the Lagrangian. Unless $R$ is stationary, the contributions of different paths to the propagator cancel due to rapidly varying phase factors (Gutzwiller, 1990). According to Hamilton's variational principle, the classical trajectories can be identified as those that lead to a stationary value of $R$, which in turn can be found as the solutions of the Euler-Lagrange equations. Performing a stationary phase approximation of the path integral leads to a semiclassical propagator in form of a sum over the classical trajectories, which interfere with different amplitudes and phases, whereas the corresponding amplitudes increase with the density of trajectories at $x^{\prime \prime}$ (Van Vleck, 1928; Gutzwiller, 1967). Reinserting this semiclassical propagator into the Schrödinger equation shows that this generally ${ }^{11}$ leads to a good approximation to order $\hbar^{2}$, which is why the semiclassical limit is often understood as the limit $\hbar \rightarrow 0$ (Gutzwiller, 1991). In the path integral this translates into the condition that the numerical value of the function $R$ must be much larger than $\hbar$.

For many-body systems, one intuitively expects $R$ to increase with the number $N$ of particles. Indeed, the semiclassical result is often recovered in the limit of $N \rightarrow \infty$

${ }^{11}$ For quadratic potentials, the semiclassical propagator leads to the exact quantum propagator (Kleinert, 2009). 
(Leyvraz and Heiss, 2005; Polkovnikov, 2010; Engl et al., 2014a), which establishes a connection to mean-field theories (Sinatra et al., 2001). The aim of a mean-field ansatz is to reduce the many-body problem, posed by the interaction of a quantum particle with its neighbors, through an effective modification of the single-particle potential (Curie, 1895; Weiss, 1907; Georges et al., 1996; Wen, 2004). In systems of bosons, for instance, this is often realized by treating the quantum fluctuations around an operator's expectation value perturbatively (Bogoliubov, 1947). To lowest order, this corresponds to the expectation value of a suitably chosen product-state ansatz, or equivalently, to the replacement of the field operator by a classical field - further details will be discussed in section 2.5.4 and chapter 7. This, again, motivates the interpretation of a mean-field result as a classical limit to the quantum problem; see, for example (Emary and Brandes, 2003a). In general, however, the correspondence of the thermodynamic limit $N \rightarrow \infty$ and the semiclassical limit $\hbar \rightarrow 0$ is unclear, especially in the case of interacting many-body systems (Engl et al., 2014a; Engl et al., 2014b).

\subsubsection{Complex systems, spectral analysis, and random matrix theory}

In classical systems, complexity is often associated with chaotic dynamics. This can be quantitatively assessed, for instance, in terms of Lyapunov exponents, which express how two trajectories from slightly different initial conditions deviate exponentially as a function of time (Ozorio de Almeida, 1988). The delocalized and dispersive nature of quantum mechanical wave-packets does not allow for a clear definition of such notions in quantum systems. Semiclassical methods can again help to identify appropriate classical limits. In fact, a semiclassical approximation of the Green's function (the Laplace transform of the propagator, which was discussed above), directly links the quantum mechanical energy spectrum and eigenstates to the classical trajectories (Gutzwiller, 1991). For classically chaotic systems, the periodic orbits determine the trace of the Green's function (Gutzwiller, 1970; Gutzwiller, 1971). Spectral analysis takes on a central role in the treatment of complex quantum systems. In the present section 2.5.2 we will provide a brief overview of the central ideas of quantum chaos and random matrix theory.

In a quantum approach to chaotic systems, the distribution of spacings between neighboring energy levels are used as indicators of chaos (Haake, 2001). The symmetries of systems with integrable dynamics in the classical limit tend to generate level clusterings and an abundance of direct level crossings (Berry and Tabor, 1977). On the other hand, quantum systems with chaotic classical counterparts (chaotic quantum systems), display strong interactions between quantum levels which lead to level repulsion and predominantly avoided crossings (Wigner, 1951). These statistics are believed to be universal for generic complex quantum systems, which motivates the description of chaotic quantum systems with ensembles of random matrices ${ }^{12}$ (Bohigas et al., 1984). Random matrix theory follows an approach close in spirit to statistical mechanics and thermodynamics, by describing average properties of ensembles of

\footnotetext{
${ }^{12}$ Conversely, level statistics is also used to characterize quantum systems as chaotic, even if they do
} not allow for a clean identification of a classical counterpart (Haake, 2001). 


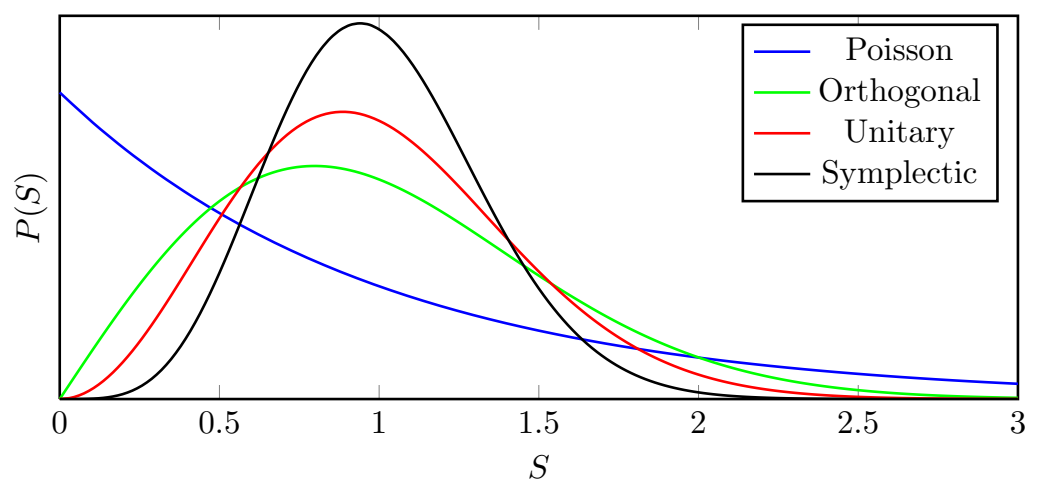

FIGURE 2.7.: Distribution of nearest-neighbor level spacings according to the Poissonian distribution which describes regular systems, and to Wigner-Dyson distributions for the three standard ensembles of random matrix theory, displaying different degrees of level repulsion. Figure taken from (Gessner, 2011).

systems, whereas statistical quantities of individual, generic realizations are typically very well represented by the average value. This allows to describe relevant features of complex quantum systems, often based on analytical results (Mehta, 1991). The predictions made by random matrix theory have been confirmed in a wide range of experiments and numerical studies (Delande and Gay, 1986; Guhr et al., 1998; Gnutzmann et al., 2000; Mirlin, 2000; Haake, 2001; Emary and Brandes, 2003a; Kolovsky and Buchleitner, 2003).

The distribution of the level spacings between the uncorrelated energy eigenvalues of integrable quantum systems is described by a Poisson distribution

$$
P(S) \simeq e^{-S},
$$

which favors small spacings $S$ between neighboring energy levels. Standard random matrix theory employs three Gaussian ensembles of matrices to model complex quantum systems. Depending on the system's symmetries, different degrees of level repulsion are found in the resulting nearest-neighbor spacings, which are described by Wigner-Dyson distributions (Wigner, 1959),

$$
P(S) \simeq S^{k} e^{-S^{2}} .
$$

The degree of level repulsion, described by $k$, depends on the invariance under canonical transformations of the underlying random matrix ensemble. For quantum systems with no symmetries, the most general class of transformations which preserve Hermiticity and leave the eigenvalues invariant is given by the unitary group. The corresponding Gaussian unitary ensemble has $k=2$. When time-reversal symmetry applies, that is, when the Hamiltonian has only real entries, the canonical transformations are constrained to the orthogonal group, leading to $k=1$ (Gaussian orthogonal ensemble). A third class of time-reversal symmetric systems can emerge for half-integer spins. As 
the name suggests, the corresponding Gaussian symplectic ensemble is invariant under transformations of the symplectic group, and has quartic level repulsion, $k=4$, see figure 2.7.

These Gaussian ensembles can be generalized to circular ensembles of unitary matrices, allowing for applications in the context of scattering theory (Dyson, 1962). Average expressions in terms of integrals over the unitary, orthogonal or symplectic groups based on Haar measure integrals can often be evaluated analytically (Mello, 1990; Collins, 2003; Collins and Sniady, 2006; Matsumoto, 2012). With such powerful tools, dynamic expectation values and their variances can be analyzed analytically, which confirms the success of the ensemble-approach to complex systems (Mello and Seligman, 1980; Mehta, 1991; Brouwer and Beenakker, 1996; Beenakker, 1997; Guhr et al., 1998; Haake, 2001; Jalabert and Pastawski, 2001; Gorin and Seligman, 2002; Gorin et al., 2006; Hastings, 2009; Gessner, 2011; Gessner and Breuer, 2013a).

For further details on the well-established field of quantum chaos and random matrix theory, we refer to the extensive literature and textbooks on the topic (Mehta, 1991; Guhr et al., 1998; Haake, 2001). In the context of this dissertation, we employ spectral analysis as a top-down approach to the description of statistical quantities entailing macroscopic observables, which require knowledge of the full quantum system, such as the entire energy spectrum. These global properties of the system directly influence the microscopic dynamics of its local constituents. Conversely, certain situations may require the precise knowledge of the microscopic properties of a small part of the system (local properties). Yet, microscopic knowledge about the full system can in many cases not be achieved, which requires a complementary bottom-up approach, aiming to approximatively describe the effective dynamics of a small, controllable subsystem, and develop methods to probe global quantities of the total system on a local level. The dynamics of small systems in contact with a large number of degrees of freedom is in many cases efficiently described by the theory of open quantum systems (Davies, 1976; Grabert, 1982; Weiss, 1993; Breuer and Petruccione, 2002; Gardiner and Zoller, 2004).

Before turning to the discussion of open quantum systems, we briefly remark on the connection of spectral properties to numerical methods for the simulation of quantum many-body systems. Efficient methods based on matrix product states (Fannes et al., 1992) and density-matrix renormalization group algorithms (White, 1992; Schollwöck, 2005) and its variants are extremely successful in the description of ground-state properties and of the low-energy sector of the excitation spectrum (Verstraete et al., 2008). The capacities of these methods, however, are limited when dealing with a large number of excited states, especially when these are highly entangled (Schuch et al., 2008). Such situations are commonly encountered when the spectrum shows signatures of quantum chaos, which suggests the conjecture that efficient numerical methods based on matrix product states or density-matrix renormalization group are limited to integrable quantum systems (Prosen and Žnidarič, 2007; Venzl et al., 2009). 


\subsubsection{Open quantum systems}

The theory of open quantum systems describes the dynamics of small quantum systems that are coupled to a typically inaccessible and large environment. This situation describes the unavoidable influence of noise in all quantum experiments, but can also be interpreted as the restriction to few, controllable degrees of freedom of a large, interacting many-body quantum system, effectively yielding a small, lower-dimensional subsystem which can be treated and controlled efficiently, both experimentally and theoretically. The goal of the open-system approach is to derive an efficient, approximative description of the dynamics which contains the incoherent and dissipative effects induced by the influence of the environment (Davies, 1976; Grabert, 1982; Weiss, 1993; Breuer and Petruccione, 2002; Gardiner and Zoller, 2004).

Formally, an open quantum system is described by a bipartite tensor structure $\mathcal{H}=\mathcal{H}_{S} \otimes \mathcal{H}_{E}$, comprised of the Hilbert spaces of system and environment, $\mathcal{H}_{S}$ and $\mathcal{H}_{E}$, respectively. We have encountered such structures already when discussing quantum correlations, and the interpretation in terms of system and environment is solely one of the many physical manifestations for this mathematical structure. Due to the inability to control or access the environment, we are constrained to the measurement of local operators on the system which have the form $A \otimes \mathbb{I}$, where $\mathbb{I}$ denotes the identity operator on the environment. The expectation values of operators of this kind are completely determined by the reduced density operator of the system $\rho_{S}$, which is obtained by tracing over the degrees of freedom of the environment. In abstract terms, if the total density operator $\rho=\sum_{k} A_{k} \otimes B_{k}$ is given by a completely general decomposition in terms of arbitrary operators $A_{k}$ and $B_{k}$ of system and environment, respectively, the reduced density operator of the system is written as

$$
\rho_{S}=\operatorname{Tr}_{E} \rho=\sum_{k} A_{k} \operatorname{Tr} B_{k}
$$

where $\operatorname{Tr}_{E}$ denotes the partial trace over the environment and $\operatorname{Tr}$ is the trace of an operator. The reduced density operator is the object of central interest to the theory of open quantum systems, since it contains all the information available over the accessible part of the full system. The principal task is, thus, to describe the time evolution of this operator, given certain initial conditions.

There exist different approaches to deriving a master equation for the dynamics of the reduced density operator. In a more formally oriented ansatz, one introduces a set of conditions that any physically meaningful map should satisfy to define a set of quantum operations. Any quantum operation $\Phi$ must be (Nielsen and Chuang, 2000)

- convex linear: For any probablity distribution $p_{i}$, the following must hold

$$
\Phi\left(\sum_{i} p_{i} \rho_{i}\right)=\sum_{i} p_{i} \Phi\left(\rho_{i}\right) .
$$

- completely positive: This requires not only that $\Phi(\rho)$ represents a positive operator, but that this remains true when we attach arbitrary environments to the 


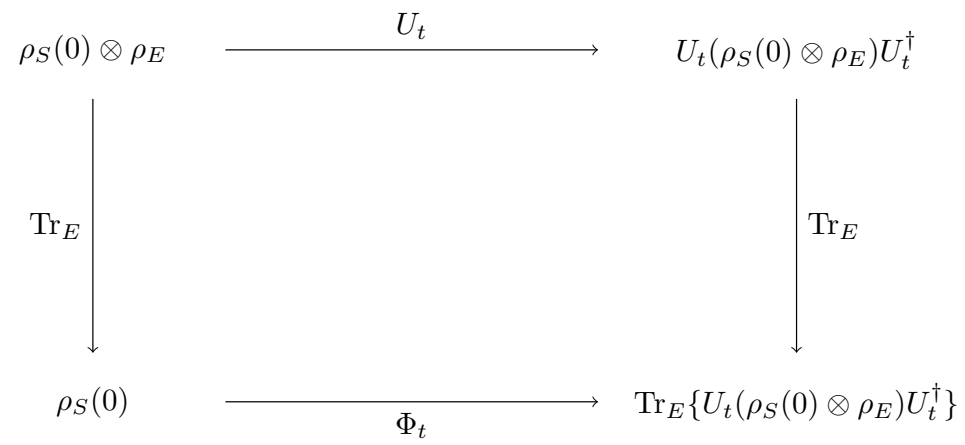

FIGURE 2.8.: Diagram defining the dynamical map $\Phi_{t}$ for initial states without correlations between system and environment. Adapted from (Breuer and Petruccione, 2002).

system, which are not affected by the operation. Thus, $(\Phi \otimes \mathbb{I}) \rho$ must yield a positive operator, whereas $\mathbb{I}$ denotes the identity operator on an arbitrary ancilla system, of arbitrary dimension $d$, and described by the Hilbert space $\mathbb{C}^{d}$. The reason for this requirement is that for some states with system-environment entanglement, a local positive operation may produce a global non-positive operator (Peres, 1996).

- trace non-increasing: The density operator at the outcome of the quantum operation must be normalizable to a positive semi-definite operator of trace one, $\operatorname{Tr} \Phi(\rho) \leq \operatorname{Tr} \rho$.

Quantum operations include actual manipulations of the quantum state, as discussed in the context of trapped ions, but also the measurement process and time evolution. They are often referred to as completely positive maps and allow for a Kraus representation (Kraus, 1983; Nielsen and Chuang, 2000; Bengtsson and Życzkowski, 2006)

$$
\Phi(\rho)=\sum_{i} K_{i} \rho K_{i}^{\dagger}
$$

where the Kraus operators satisfy

$$
\sum_{i} K_{i}^{\dagger} K_{i} \leq \mathbb{I}
$$

The time evolution of the open system is also described in terms of a completely positive map. It contains the combination of the unitary time evolution operator $U_{t}$, describing a solution of the Schrödinger or of the von Neumann equation for the combined system including the environment, and the partial trace operation. To be able to map within states of the open system only, one must assume an uncorrelated initial state of the form $\rho_{S}(0) \otimes \rho_{E}$ (Lindblad, 1996). In this case

$$
\rho_{S}(t)=\Phi_{t} \rho_{S}(0)=\operatorname{Tr}_{E}\left\{U_{t}\left(\rho_{S}(0) \otimes \rho_{E}\right) U_{t}^{\dagger}\right\}
$$


describes a completely positive map for arbitrary fixed states of the environment $\rho_{E}$, see figure 2.8. A formal statement about the dynamical map $\Phi_{t}$ is made by the Lindblad theorem, if the one-parameter family of maps $\Phi_{t}$ form a semigroup, that is, if $\Phi_{t+s}=$ $\Phi_{t} \circ \Phi_{s}$ and $\Phi_{0}=\mathbb{I}$. In this case the bounded superoperator $\mathcal{L}$ is the generator of this group, $\Phi_{t}=e^{\mathcal{L} t}$, if and only if it has the form (Lindblad, 1976; Gorini et al., 1976)

$$
\mathcal{L} \rho_{S}(t)=-\frac{i}{\hbar}\left[H_{S}, \rho_{S}(t)\right]+\sum_{i} \gamma_{i}\left(L_{i} \rho_{S}(t) L_{i}^{\dagger}-\frac{1}{2}\left\{\rho_{S}(t), L_{i}^{\dagger} L_{i}\right\}\right) .
$$

The system Hamiltonian $H_{S}$ may not coincide with the bare Hamiltonian of the system, as it usually contains environment-induced energy shifts (Cohen-Tannoudji et al., 1992). This generalizes the von Neumann equation by including incoherent effects on the dynamics induced by the environment, quantified by the decay rates $\gamma_{i}$. The semigroup assumption is a rather strong requirement since it demands that the evolution during each arbitrary time slice is described by the same dynamical map. Nevertheless, the Lindblad master equation has wide applications and has been very successful in describing the dynamics of open systems, for instance in quantum optics (CohenTannoudji et al., 1992; Gardiner and Zoller, 2004), despite the fact that actual physical operators are often unbounded and the Lindblad theorem formally does not apply (Breuer and Petruccione, 2002). The formal statement of the Lindblad theorem does not specify the specific form of the Lindblad operators $L_{i}$, which are often assumed phenomenologically. For a proof of the theorem, further discussions, applications and rigorous mathematical background we refer to the original literature (Lindblad, 1976; Gorini et al., 1976), review articles (Spohn, 1980; Breuer, 2012) and standard textbooks (Davies, 1976; Alicki and Lendi, 1987; Breuer and Petruccione, 2002; Gardiner and Zoller, 2004).

Alternatively, a master equation can be derived microscopically, starting from a complete description of system and environment. In the weak-coupling limit, the starting point of such a derivation is a second-order expansion of the von Neumann equation in the interaction picture, leading to an integro-differential equation for $\rho_{S}$. Central element of a microscopic derivation of a quantum master equation is the BornMarkov approximation. This contains the approximation to coarse-grained time-scales: Excitations in the environment are assumed to happen on unresolved, faster time scales than the system dynamics (Born approximation). Formally this is done by truncating the second-order expansion with a factorized total state containing a timeindependent environmental reduced state. Additionally, the time-dependence of $\rho_{S}$ on previous times is removed in the integrand, which corresponds to a truncation in second order of the system-environment interaction strength (Markov approximation). At this point one obtains a Redfield equation (Redfield, 1957), which does not lead to semigroup dynamics. Finally, the upper integration limit is shifted to infinity and a secular approximation or rotating wave approximation removes rapidly oscillating terms to produce a master equation with Lindblad structure (Breuer and Petruccione, 2002).

A master equation in Lindblad form describes a completely memoryless (Markovian) time evolution, where time-autocorrelation functions of the environment decay expo- 
nentially. In many practical situations, mostly when considering environments of finite extent or with well-defined spectral structures, the semigroup property does not apply (Lambropoulos et al., 2000; Breuer and Petruccione, 2002). A natural generalization of the Lindblad form is found by allowing for time-dependent Lindblad operators and decay rates,

$$
\mathcal{K}(t) \rho_{S}(t)=-\frac{i}{\hbar}\left[H_{S}(t), \rho_{S}(t)\right]+\sum_{i} \gamma_{i}(t)\left(L_{i}(t) \rho_{S}(t) L_{i}^{\dagger}(t)-\frac{1}{2}\left\{\rho_{S}(t), L_{i}^{\dagger}(t) L_{i}(t)\right\}\right)
$$

which describes the general form of a generator of time-local master equations (Breuer, 2012)

$$
\frac{\partial}{\partial t} \rho_{S}(t)=\mathcal{K}(t) \rho_{S}(t)
$$

As a less stringent requirement than the semigroup property, the divisibility of the dynamical map demands that for any $t_{2} \geq t_{1} \geq 0$, there exists a completely positive and trace preserving map $\Phi\left(t_{2}, t_{1}\right)$ such that

$$
\Phi\left(t_{2}, 0\right)=\Phi\left(t_{2}, t_{1}\right) \circ \Phi\left(t_{1}, 0\right)
$$

Divisibility can be directly linked to the decay functions $\gamma_{i}(t)$ in the time-local master equation (2.26). In fact, the dynamics induced by (2.26) is divisible if and only if the $\gamma_{i}(t)$ are positive functions (Breuer, 2012). Yet, many physical situations are not even described by divisible time evolutions. This implies that the time evolution depends on previous times which may be interpreted as a quantum memory effect (non-Markovianity). Recently, a variety of approaches have been proposed to formally define and quantify such memory effects in terms of quantum information theoretic measures (Breuer et al., 2009; Rivas et al., 2010; Breuer, 2012).

An elementary tool in this context is the trace distance

$$
\left\|\rho_{1}-\rho_{2}\right\|=\frac{1}{2}\left|\rho_{1}-\rho_{2}\right|
$$

where the trace norm is defined as $|A|=\operatorname{Tr} \sqrt{A^{\dagger} A}$. The prefactor is a mathematical convenience and restricts the range of the trace distance to real numbers between 0 and 1 . The trace distance constitutes a formal mathematical distance, that is, it satisfies the triangle inequality and $\left\|\rho_{1}-\rho_{2}\right\| \geq 0$, whereas equality is reached if and only if $\rho_{1}=\rho_{2}$. Furthermore, it has appealing properties for quantum information applications. For instance, the trace distance allows for an intuitive interpretation in terms of the distinguishability of quantum states (Helstrom, 1967; Nielsen and Chuang, 2000; Hayashi, 2006): The probability to successfully identify which of two quantum states was randomly (with equal probability) prepared, based on a single measurement, is given by $p=\left(\left\|\rho_{1}-\rho_{2}\right\|+1\right) / 2$. Under arbitrary positive operations, the trace distance between two states can only decrease (Ruskai, 1994), which expresses the loss 
of distinguishability in the course of noisy quantum processes. Thus, in mathematical terms, the trace distance is a contraction under positive operations.

One approach to quantum non-Markovianity is based on the ability of certain time evolutions to increase the trace distance temporarily. This increase of distinguishability is interpreted as a flow of information from the environment to the system - a quantum manifestation of memory effects induced by a non-Markovian time evolution (Breuer et al., 2009). This definition is in general not equivalent to the divisibility condition (2.28) (Breuer, 2012). Inspired by entanglement measures (Vidal and Werner, 2002), one may also employ the trace norm to quantify the amount to which complete positivity is violated by $\Phi\left(t_{2}, t_{1}\right)$ to define a notion of quantum non-Markovianity which is equivalent to divisibility (Rivas et al., 2010). A situation where divisibility and increasing trace distance coincide is given when the sum in equation (2.26) contains only a single decay term.

Non-Markovian time evolutions are characterized by a coupling of the system to a structured reservoir with pronounced resonances (Caldeira and Leggett, 1983; Riseborough et al., 1985; Haake and Reibold, 1985). Spontaneous emission rates, for example, are determined by the density of modes at the atomic transition frequency (Cohen-Tannoudji et al., 1992). In structured environments, such as cavities (Walther et al., 2006; Haroche and Raimond, 2006) or photonic crystals (Lambropoulos et al., 2000), this factor can increase significantly in comparison to free space vacuum modes (Purcell, 1946; Kleppner, 1981), leading to strongly enhanced spontaneous emission. The nearly resonant exchange of excitations between the atom and some of the electromagnetic field modes can no longer be described in terms of the traditional opensystem ansatz, where the environment is considered a memoryless heat bath, and non-Markovian extensions are required. Extrapolating this example even further, one reaches a limit where the master-equation treatment of the atom-light interaction is no longer appropriate: In the extreme case of an (almost) resonant lossless single-mode cavity, which one may still formally consider as a simple environment, the cavity mode can conveniently be treated as a part of the system (Jaynes and Cummings, 1963); see also section 3.2 .

As mentioned before, the standard approach to open systems furthermore implies the absence of initial correlations between the system and the environment. This condition can be realized to a good approximation in experiments where the initial state of the system can be prepared with little uncertainty, that is, in a pure quantum state. In many of the settings discussed in section 2.2 , the initial states are well controlled for systems of few particles. ${ }^{13}$ However, there exist physically relevant situations where disregarding the initial correlations is not appropriate, in particular in realistic scenarios where system and environment have been in contact long before the experiment is conducted, and therefore have settled in a correlated thermal equilibrium state. On the one hand, a measurement of a continuous system variable, for instance, of a particle's position, does not fully remove the system-environment correlations of the

${ }^{13}$ Even for such controllable quantum systems this is not always the case: For example, laser cooling the vibrational state of a string of trapped ions still poses a considerable experimental challenge (Eschner et al., 2003; Segal and Wunderlich, 2014), and instead of the pure ground state, it is more realistic to assume an initial condition described by a low-temperature thermal state. 
thermal state, and, thus, the subsequent evolution cannot be described properly when these correlations are ignored (Hakim and Ambegaokar, 1985; Smith and Caldeira, 1987; Grabert et al., 1988; Gorini et al., 1989; Hu et al., 1992). On the other hand, the theoretical description of the system's autocorrelation functions also requires a treatment which includes correlated initial states (Sassetti and Weiss, 1990; Breuer et al., 2001) - even for discrete variables such as spins, and the effect of these correlations can still be visible in the system's long-time behavior (Leggett et al., 1987; Smith and Caldeira, 1990; Orth et al., 2013). We may encounter both these situations also in the context of controllable quantum systems. In quantum optical experiments, control over the initial state is often precise enough to avoid unwanted effects arising from initially correlated states. Yet, such systems may still serve to study the formal consequences of initial system-environment correlations - both experimentally and theoretically. When the initial state is not a product state, the time evolution can no longer be given in terms of completely positive operators (Pechukas, 1994; Alicki, 1995; Pechukas, 1995) that are defined independently of the correlations in the initial state (Lindblad, 1996). For further details on this topic we refer to the extensive original literature, as listed in the course of this paragraph; see also (Gessner, 2011).

In any time evolution with factorizing initial conditions, the trace distance is bounded from above by its value at the initial time. Non-Markovianity may temporarily lead to increased distinguishability, but any two quantum states can never become more distinguishable than at the initial time. This is different when the initial state contains system-environment correlations: Any temporary increase of the trace distance of an arbitrary pair of states above its initial value constitutes a witness for initial systemenvironment correlations (Laine et al., 2010), which has been observed in photonic proof-of-principle experiments (Li et al., 2011; Smirne et al., 2011). This allows one to extract information about a large, composite system by accessing only a small, open subsystem. In the same spirit, in chapter 3, we review and extend a method to detect quantum correlations in a bipartite setting with only local access to one of the two subsystems, which is applicable in the context of an open quantum system.

\subsubsection{Identical particles}

Another aspect of complexity is added by the quantum statistics of identical particles (Cohen-Tannoudji et al., 1977b). In the context of linear optics experiments, we have already pointed out that the symmetrization of quantum states for the description of bosonic particles can be a computationally hard task. For both, fermions and bosons, one usually introduces creation and annihilation operators which satisfy canonical commutation and anti-commutation relations, to ensure the appropriate quantum statistics when many-body quantum states are constructed from them (Mahan, 2000). We restrict the treatment of the following section to bosons, but the considerations can be easily adapted to fermions.

Consider the creation operator $a_{i}^{\dagger}$ of a particle in the state $a_{i}^{\dagger}|0\rangle=\left|\varphi_{i}\right\rangle$, where $|0\rangle$ is the vacuum state, which contains no particles. The $\left\{\left|\varphi_{i}\right\rangle\right\}$ form the eigenbasis of a single-particle observable. The creation operator and its adjoint counterpart, the 
annihilation operator $a_{i}$, satisfy the canonical commutation relations

$$
\left[a_{i}, a_{j}^{\dagger}\right]=\delta_{i j}, \quad\left[a_{i}^{\dagger}, a_{j}^{\dagger}\right]=0, \quad\left[a_{i}, a_{j}\right]=0, \quad \forall i, j .
$$

Arbitrary many-particle states can be constructed from the vacuum using the creation operator, and we can express operators in terms of $a_{i}$ and $a_{i}^{\dagger}$, such as arbitrary manybody Hamiltonians of the form

$$
H=\sum_{i j} H_{i j}^{(1)} a_{i}^{\dagger} a_{j}+\frac{1}{2} \sum_{i j k l} H_{i j ; k l}^{(2)} a_{i}^{\dagger} a_{j}^{\dagger} a_{k} a_{l},
$$

which may contain a single-particle energy $H^{(1)}$ and a two-particle interaction term $H^{(2)}$.

To theoretically describe experiments on many-body quantum systems, we can either propagate an initial quantum state according to the Schrödinger equation, or determine the Heisenberg-picture evolution of an observable. Since any operator can be constructed from the elementary creation and annihilation operators, it would suffice to solve the Heisenberg equations of motion of

$$
\frac{\partial}{\partial t} a_{i}(t)=\frac{i}{\hbar}\left[H, a_{i}(t)\right]
$$

with the formal solution $a_{i}(t)=e^{i H t} a_{i}(0) e^{-i H t}, a_{i}(0)=a_{i}$, and $H$ is given by $(2.31)$. Whenever the two-particle interaction term $H^{(2)}$ is nonzero, solving equation (2.32) constitutes a full many-body problem, and it is usually not possible to provide an explicit solution without additional approximations (Mahan, 2000).

Different perturbation theoretical methods have been developed to cope with this problem, for instance, using Green's functions in solid state systems, which express the expectation value of such operators in the Heisenberg picture at different times (Mahan, 2000; Wen, 2004; Kamenev, 2005). This involves a separation of the Hamiltonian into a soluble part, which can be treated exactly, and a perturbation. The expectation values of many-body observables can be predicted using perturbation theory. Many important physical quantities, however, are already fully determined on the basis of single-particle expectation values, such as the particle density or momentum distribution of Bose-Einstein condensates (Bogoliubov, 1967a; Bogoliubov, 1967b; Pitaevskii and Stringari, 2003). For the description of such quantities, the unwieldy task of handling the full Hilbert space of a many-body quantum system may be avoided if appropriate methods for the effective description of few-particle observables can be developed.

A prominent example of such an effective description in the case of dilute BoseEinstein condensates is given by the Gross-Pitaevskii equation. In this introductory chapter, we restrict to a brief overview without providing any derivations. Further details will be presented in chapter 7 . In a representation in terms of field-operators $\hat{\Psi}(t, \mathbf{x})$, equation (2.32) takes on the form (Bogoliubov, 1967a)

$$
i \hbar \frac{\partial}{\partial t} \hat{\Psi}(t, \mathbf{x})=\left(-\frac{\hbar^{2}}{2 m} \nabla_{\mathbf{x}}^{2}+V_{0}(\mathbf{x})+\int d \mathbf{x}^{\prime} V\left(\mathbf{x}, \mathbf{x}^{\prime}\right) \hat{\Psi}^{\dagger}\left(t, \mathbf{x}^{\prime}\right) \hat{\Psi}\left(t, \mathbf{x}^{\prime}\right)\right) \hat{\Psi}(t, \mathbf{x})
$$


Based on the Bogoliubov approximation (Bogoliubov, 1947; Pitaevskii and Stringari, 2003), which consists in the description of the macroscopic occupation of a singleparticle quantum state by a classical field $\Psi_{0}(t, \mathbf{x})$, whereas quantum corrections are considered to be weak, one obtains a closed expression for the time evolution of the classical field (Gross, 1961; Pitaevskii, 1961; Pitaevskii and Stringari, 2003),

$$
i \hbar \frac{\partial}{\partial t} \Psi_{0}(t, \mathbf{x})=\left(-\frac{\hbar^{2}}{2 m} \nabla_{\mathbf{x}}^{2}+V_{0}(\mathbf{x})+g\left|\Psi_{0}(t, \mathbf{x})\right|^{2}\right) \Psi_{0}(t, \mathbf{x})
$$

when the interactions are assumed to be short-range, $V\left(\mathbf{x}, \mathbf{x}^{\prime}\right)=g \delta\left(\mathbf{x}-\mathbf{x}^{\prime}\right)$. From equation (2.34), we see that the dynamics becomes nonlinear, since the mean-field, induced by the ensemble of particles, which are all described by the same single-particle state, creates an effective potential for each of these particles. The Gross-Pitaevskii equation has shown to provide an excellent description of the dynamics of dilute BoseEinstein condensates (Pitaevskii and Stringari, 2003), but, by construction, it cannot include dissipative effects akin to those described by the influence of an environment, as in the theory of open quantum systems.

Trying to describe only single-particle observables, or, equivalently, the evolution of single-particle quantum states, instead of the entire many-body system is close in spirit to the open-system approach to quantum systems. Formally, this corresponds to tracing over the entire Hilbert space apart from the single-particle subspace. Due to quantum statistics, the definitions of the single-particle subspace and partial trace operation must be adjusted, since in a many-body system all of the single particles are identical and cannot be distinguished. The first-quantized description of a wellseparated tensor structure between system and environment is only applicable if either the particles in system and environment become distinguishable by some degree of freedom, for example via fixed lattice site positions in mean-field master equations as the Hartree equation, or by phenomenological approaches which involve an artificial symmetrization of the decoherence operators, as for example in the context of the Boltzmann equation (Spohn, 1980; Breuer and Petruccione, 2002; Röpke, 2013).

A microscopic expression for the reduced dynamics of a subsystem of identical particles is given by the Bogoliubov-Born-Green-Kirkwood-Young (BBGKY) hierarchy, which was originally derived for classical observables but can be generalized to quantum systems (Bogoliubov, 1967a). In chapter 7, we will provide a derivation of this hierarchy based on an appropriate description of a subsystem of identical particles in terms of creation and annihilation operators. We obtain this hierarchy from the von Neumann equation for the full many-body quantum state by tracing over the degrees of freedom of all but few particles. A pure product state assumption then truncates this hierarchy to first order, which reproduces the Gross-Pitaevskii equation for the single-particle mean-field.

Experiments on Bose-Einstein condensates, where Bloch oscillations of the singleparticle momentum distribution in a periodic lattice potential are measured (Morsch et al., 2001), show that these coherent oscillations decay due to interactions between the particles (Buchleitner and Kolovsky, 2003; Meinert et al., 2014). This raises the question whether an open quantum system description including incoherent effects 
can be derived microscopically for a subsystem of identical particles. In chapter 7 we take on first steps towards a microscopic derivation of a second-order perturbative expansion of interacting bosonic particles.

\subsection{Quantum phase transitions}

The importance of developing an understanding of the microscopic description of complex systems is underlined by its observable macroscopic signatures, for instance in form of phase transitions. When an external control parameter is varied, the system's behavior at large scales can change qualitatively due to changes in its microscopic structure. Classical, thermally induced phase transitions can be observed in every day life. When a system's temperature increases over a given critical value, thermal fluctuations can destroy macroscopic long-range order and produce a disordered phase. Such phase transitions can be described by methods of classical statistical mechanics (Landau and Lifshitz, 1969; Ma, 1973; Wilson, 1975). Temperature, however, is not the only external parameter that can induce a phase transition (Gilmore and Feng, 1978; Feng et al., 1981). Consider for example a material with strong internal spinspin couplings. The energy of such a ferromagnetic system is minimized by a highly ordered spin configuration where each spin's orientation depends on the alignment of its neighbors, which in turn leads to a macroscopic magnetic moment. However, once a sufficiently strong external field is applied, the spin configuration is determined by the strength and direction of the external field, which dominates over the internal spin-spin coupling. This behavior characterizes a paramagnetic system. Hence, one can observe a macroscopic change of the material's behavior, from ferromagnet to paramagnet, caused by an external control parameter, the magnetic field. Such phase transitions become especially interesting at very low temperatures, where all thermal fluctuations are frozen out, and only quantum fluctuations prevail (Hertz, 1976; Suzuki, 1976).

Phase transitions which are generated by changes of an external control parameter at zero temperature, such as the above example of a magnetic material, are called quantum phase transitions (Sondhi et al., 1997; Sachdev, 1999; Vojta, 2003). The formal analogy to classical phase transitions becomes apparent by realizing that $d$-dimensional quantum systems can be mapped onto $d+1$-dimensional classical systems, where the additional dimension is determined by the inverse temperature $\beta$ of the quantum system (Suzuki, 1976; Hertz, 1976; Sondhi et al., 1997; Sachdev, 1999; Vojta, 2003; Batrouni and Scalettar, 2011). This can be seen by interpreting the temperaturedependent exponent $-\beta H$, which occurs in the partition function, in the spirit of Feynman's path integral formulation (Feynman, 1948), as a propagator in the imaginary time $t=-i \beta$. A formal analogy can be established between this imaginary-time propagator of the $d$-dimensional quantum system and a product of classical transfer matrices of a $d+1$-dimensional classical system (Sondhi et al., 1997). To this end, one discretizes the imaginary time interval into $N=\hbar \beta / \delta \tau$ equidistant steps of (real) length $\delta \tau$. In a chain of spins, a sequence of transfer matrices is used to efficiently determine the partition function by connecting each spin via a transfer matrix to its nearest neighbor, thereby constructing an expression for the entire one-dimensional 
chain spin by spin. Here, the imaginary-time propagator connects the entire system to another copy of itself, one discrete step later in imaginary time, effectively yielding a step-by-step construction of a $d+1$-dimensional system (Vojta, 2003). The extent of the additional dimension is limited by $\beta$, and the dimension becomes only truly infinite when the temperature approaches zero.

The above mapping can be worked out explicitly, and for the example of the one-dimensional quantum Ising model at zero temperature, one obtains the twodimensional classical Ising model; see, for example (Batrouni and Scalettar, 2011). By studying the classical partition function, one finds that some of the system parameters of the quantum system take the role of temperature in the classical system. This enables one to apply the theory of classical phase transitions and to identify the critical temperature of the classical system with critical parameter values of the quantum system (Sondhi et al., 1997). Also the scaling behavior expressed by critical exponents can be mapped to the quantum system (Vojta, 2003). This does not imply that every aspect of a quantum phase transition can be described in terms of classical statistical mechanics by employing this quantum-classical mapping. Issues may arise, for instance, when trying to describe dynamical properties, or the correlation length of the coherence of the quantum mechanical wave function (Sachdev, 1999).

Quantum phase transitions can be observed in experiments, even though the point of absolute zero temperature cannot be reached experimentally. The reason is that at low, finite temperatures, the dominant contribution to the thermal distribution of energy eigenstates still stems from the ground state, and residual excited state populations tend to wash out otherwise sharp transitions. Observations of quantum phase transitions have been reported in various condensed-matter bulk materials (Vojta, 2003). Recent progress in quantum optics, as summarized in section 2.2, allows to study models exhibiting quantum phase transitions under well-controllable conditions and on less demanding time-scales. One example is the transition from a ferro- or anti-ferromagnet to a paramagnet, induced by an external field, which can be observed in systems described by the spin-chain model (2.14), for instance a chain of trapped ions with specifically designed interactions (Islam et al., 2011). The Bose-Hubbard model (2.17), which we discussed in the context of cold atoms in an optical lattice, predicts a quantum phase transition from a superfluid phase, where the atoms are delocalized over all lattice sites, to a Mott-insulating phase, where a constant number of atoms is fixed on each lattice site (Jaksch et al., 1998). This quantum phase transition has been observed in cold-atom experiments (Greiner et al., 2002). Note, however, that the transition from a cold gas to a Bose-Einstein condensate is not a quantum, but rather a classical phase transition, since it occurs at a finite temperature and therefore is thermally induced. As a final example of a quantum phase transition, we mention the 3D Anderson transition from metallic to insulating, localized phases, with increasing strength of the disorder (Anderson, 1958; Evers and Mirlin, 2008).

The fact that a quantum phase transition occurs at zero temperature motivates studies to conveniently restrict the analysis to the ground state. Indeed, non-analyticities of the ground state energy currently serve as the commonly accepted definition of a quantum phase transition (Sachdev, 1999). Moreover, correlation properties of the ground state often show clear signatures of the quantum phase transition, for instance, 
in terms of correlation functions (Sachdev, 1999), or of quantum entanglement (Osterloh et al., 2002; Osborne and Nielsen, 2002; Vidal et al., 2003; Wu et al., 2004). This can be understood intuitively by studying the system's spectrum: When the external parameter is changed, the different states of the system react differently until at some point an excited level turns into the new ground state. The new ground state then may exhibit completely different properties than the previous one. In finite systems the above described transition of the ground state is typically described by an avoided crossing of the two lowest-lying energy levels, whereas the energy gap at the critical point shrinks with increasing system size, and the phase transition becomes sharper (Sachdev, 1999). Nonetheless, the emergence of (avoided) level crossings is certainly not limited to the ground state. Often, a quantum phase transition implies a qualitative change of the entire level statistics at the critical point (Wu et al., 1990; Heiss, 1994; Mirlin, 2000; Emary and Brandes, 2003b); see also (Kolovsky and Buchleitner, 2003). Recently, non-analytic behavior of properties of the excited states (Cejnar et al., 2006; Caprio et al., 2008; Cejnar and Stránský, 2008), and of quasi-energy states in driven systems (Bastidas et al., 2014), have also been identified in the context of quantum phase transitions.

In chapter 5 we study the (anti-)ferromagnet to paramagnet quantum phase transition in variable-range quantum magnets, taking into account both ground-state quantum correlations as well as the excitation spectrum. A brief introduction to the semiclassical intuition associated with quantum phase transitions will further be provided in section 5.4.1.

\subsection{Scope and structure of this dissertation}

In this chapter we discussed selected topics of state of the art theoretical and experimental quantum physics, focussing, in particular, on the description of controllable quantum optical systems, on their applications in quantum information theory, and on their time evolution. For an efficient characterization of composite quantum systems of variable sizes and levels of complexity, we develop a versatile set of tools in the present dissertation. This consists in the definition of instructive, and, at the same time, experimentally accessible observables, as well as suitable theoretical methods to describe spectral features and dynamics.

Chapters 3 and 4 mostly focus on the characterization of correlation properties of quantum systems of which - at least - a part is experimentally well under control. Specifically, in chapter 3 we refine and extend a method that allows to detect bipartite discord-type correlations between two quantum systems, when access is restricted to only one of them. Exploiting the interaction between the two subsystems, this allows to detect correlations between a system and an environment with only local operations on the system. The "environment" can be either a truly inaccessible part of the full system, or a part of the system that is too large to be fully characterized. We further present experimental realizations of this method with trapped ions and photons, and a series of theoretical studies to explore the limits of its applicability. We end the chapter by studying the example of a spin-chain model, where we select out a single 
spin as the accessible subsystem, whereas the remaining spins act as environment. The interplay of ground-state correlations and single-spin dynamics in the context of the quantum phase transition shifts the focus from quantum information concepts to more complex multipartite systems.

Before focussing on the in-depth analysis of the spin-chain model, we study the effect of local operations and classical noise processes on correlation properties in bipartite and multipartite systems in chapter 4 . We discuss the creation and robustness of correlations in another experiment with a chain of trapped ions, where stochastic noise processes are naturally present due to fluctuating control fields - one of the dominant error sources of current experiments. We provide an analytical description of the corresponding ensemble-averaged dynamics and discuss the implications of the generated dynamics in the context of entanglement preservation.

Chapter 5 , then, is dedicated to a detailed analysis of the quantum phase transition in a family of quantum magnets with interactions of variable range. Developing a multi-configurational mean-field ansatz, we analytically reproduce key features of the excitation spectrum. This semiclassical mean-field ansatz provides us with the exact quantum spectrum in certain limits of the external field strength. Moreover, an intuitive semiclassical interpretation provides an instructive picture of the quantum phase transition, which relates to the entire excitation spectrum, whereas such phenomena are usually analyzed solely based on ground-state properties.

A general framework for the construction of observables that characterize the spectral and dynamical features of controllable quantum systems will be introduced in chapter 6 , based on ideas from nonlinear spectroscopy. Single-site addressability, available in controllable quantum systems, can add spatial resolution to the experimentally recorded multi-time correlation functions. We discuss implementations with systems of trapped ions in great detail, and elaborate on a variety of applications.

Finally, in chapter 7 we describe the dynamics of a subset of indistinguishable, interacting quantum particles. We microscopically derive a general coherent mean-field equation, which reproduces the Gross-Pitaevskii equation in the special case of a dilute Bose-Einstein condensate. Furthermore, first steps towards a master-equation description of interaction-induced decoherence of single-particle observables are presented. 


\section{LOCAL DETECTION OF CORRELATIONS IN COMPOSITE QUANTUM SYSTEMS}

We discuss a method for the detection of quantum discord between two subsystems, when access is limited to only one of them. The method is based on local operations on the accessible subsystem and makes use of the interaction to reveal the bipartite correlations via the measurable, local subsystem dynamics. We further present experimental realizations with a trapped ion and with single photons, as well as additional theoretical examples.

\subsection{The local detection protocol}

In the present section we will review a method which was originally developed in the author's Diploma thesis (Gessner, 2011), and published in (Gessner and Breuer, 2011). During the work on the present dissertation, this method was refined and extended (Gessner and Breuer, 2013b; Gessner et al., 2014a; Gessner et al., 2014b). Here we present it from an updated perspective and restrict to presenting only those details and derivations which have not been presented elsewhere.

In the previous chapter, we discussed the central role of correlated quantum states in many applications of quantum information theory, as well as for the dynamics of open quantum systems. The detection of correlations can pose a difficult task, especially when considering multi-particle systems, since standard methods usually require access to all subsystems (Mintert et al., 2005; Horodecki et al., 2009; Gühne and Tóth, 2009; Auccaise et al., 2011; Silva et al., 2013; Walter et al., 2013). It was discussed in the previous chapter that a complete characterization of the correlation properties of large ensembles does not represent a scalable approach. Also in most quantum 


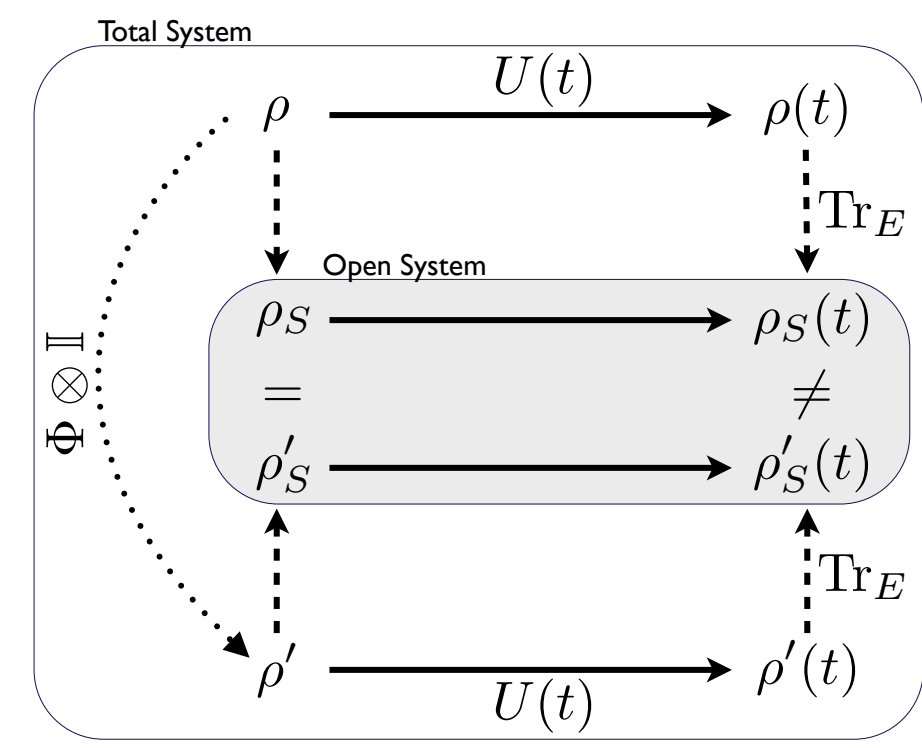

FIgURE 3.1.: The local detection protocol, as outlined in section 3.1.1, is based on a local dephasing operation $\Phi$ which removes the discord-type correlations from the initial state $\rho$. If the subsystem dynamics is affected by this operation, the correlations of the initial state are revealed to observables of the subsystem. Adapted from (Gessner et al., 2014a).

communication setups, each party typically has only local access to his share of the total quantum state.

Our strategy consists in detecting correlated quantum states in a possibly multipartite setting by accessing only a small subsystem. This has the advantage that operations are always limited to the small, low-dimensional, controllable Hilbert space of the accessible subsystem, regardless of the total size of the system. Since we will not even assume knowledge over the remaining subsystems, this scenario is applicable in an open quantum system context, where correlations may emerge between the a wellcontrolled system and its inaccessible environment. As we discussed in the previous chapter, such system-environment correlations can have significant influence on the dynamics of the reduced subsystem. Factorizing initial conditions are indeed central to standard attempts to describe the dynamics of open quantum systems, for both the microscopic and the formal approach. Here, we harness the impact of correlations on the reduced dynamics to reveal the bipartite correlations to the subsystem.

\subsubsection{Local witness for bipartite quantum discord}

To develop the local detection protocol, we assume a high degree of control over the quantum system described by the Hilbert space $\mathcal{H}_{S}$, which is coupled to an inaccessible second system, described by $\mathcal{H}_{E}$. Here the subscripts $S$ and $E$ are inspired from the 
separation of the total system in system and environment, which can be an artificial decomposition of a many-body quantum system and does not have to describe an actual open system coupled to a heat bath. We further assume no knowledge about the second system, the interaction between the two systems and the properties of a given initial quantum state $\rho$ of the composite system $\mathcal{H}_{S} \otimes \mathcal{H}_{E}$. The goal is to detect and possibly quantify the bipartite correlations contained in the quantum state $\rho$. Since the total state $\rho$ is only partially accessible, the complete obtainable information at a single moment in time is contained in the corresponding reduced density matrix for the accessible subsystem, given, after diagonalization, by its spectral decomposition

$$
\rho_{S}=\operatorname{Tr}_{E} \rho=\sum_{i} p_{i}|i\rangle\langle i|,
$$

with the local eigenbasis $|i\rangle$. Unless we encounter a pure state $\rho_{S}=|\varphi\rangle\langle\varphi|$, in which case we could conclude that the total state is a product state, this does not immediately allow us to draw conclusions about the correlations of the total state $\rho$. This is due to the fact that the statistical mixture of $\rho_{S}$ can have two different causes. On the one hand, the observer's ignorance about the reduced state can be a result of the quantum correlations of the total state: A pure maximally entangled total state leads to maximally mixed reduced states in both subsystems. On the other hand, if the total state is already mixed, then the reduced state will be mixed, even in the absence of quantum correlations. To distinguish these two cases we need to take on further steps.

The general strategy will be to reveal the correlations via their effect on the local dynamics. Let $U(t)$ be the unitary time evolution operator describing the coherent evolution of the total system. Note that we assume unitary dynamics for convenience, and the local detection protocol does not rely on this assumption (Gessner and Breuer, $2013 \mathrm{~b})$. The subsystem state after time $t$ will be given by

$$
\rho_{S}(t)=\operatorname{Tr}_{E}\left\{U(t) \rho U^{\dagger}(t)\right\}
$$

This evolution contains the influence of the correlations which are possibly present in the initial state $\rho$. To detect these correlations, we will compare this evolution with the subsystem evolution after removing correlations from the total state with the help of a carefully designed local operation.

To be specific, we define a local dephasing operation to produce a classically correlated reference state where all discord-type correlations are removed. The action of the local dephasing operation on an arbitrary operator $X$ is defined in terms of the eigenbasis $\{|i\rangle\}$ of $\rho_{S}$ as (Gessner and Breuer, 2011)

$$
\Phi(X)=\sum_{i}|i\rangle\langle i|X| i\rangle\langle i| .
$$

It is important to notice that this constitutes a local operation only on the Hilbert space $\mathcal{H}_{S}$, and does not affect the second subsystem at all. If we apply this operation locally to the total bipartite initial state $\rho$, we obtain

$$
\rho^{\prime}=(\Phi \otimes \mathbb{I}) \rho=\sum_{i}(|i\rangle\langle i| \otimes \mathbb{I}) \rho(|i\rangle\langle i| \otimes \mathbb{I}) .
$$


This operation corresponds to a local, non-selective measurement of $\rho_{S}$ and induces complete dephasing in the local basis $\{|i\rangle\}$. Later in this chapter, we will demonstrate possibilities to realize this method with trapped ions and photonic polarization qubits. Recall from section 2.1.1 that the correlations assessed by the quantum discord can indeed be defined on the basis of this dephasing operation. In fact, the state $\rho$ is classical (zero discord) if and only if

$$
\rho=\rho^{\prime} .
$$

Otherwise it contains quantum discord. This terminology is motivated by the fact that $\{|i\rangle\}$ can be interpreted as the eigenbasis of an observable. In the case of a classical state, one may perform non-selective measurements on this local observable without disturbing the total quantum state, and the quantum uncertainty for this observable vanishes (Girolami et al., 2013). Furthermore, uncorrelated product states are always classical, whereas all entangled states contain nonzero discord. For further details see section 2.1.1.

Thus, we see that the local dephasing operation has no effect on the initial quantum state if there are no discord-type correlations present in $\rho$. Next, we study the effect of the local dephasing on the reduced density matrices of both subsystems. Since the dephasing, by construction, is a local operation on $\mathcal{H}_{S}$, the reduced state of the inaccessible subsystem will not be affected. Moreover, the dephasing is carried out in the eigenbasis of $\rho_{S}$, which means that $\rho_{S}$ will also be invariant under such an operation. This implies that both reduced states of system and environment (or of the accessible and inaccessible subsystems) are not at all affected by the local dephasing. Finally, we note that the state $\rho^{\prime}$ is always a state of zero discord, as it can be readily cast into the form of equation (2.3). Formal proofs of these statements can be found in references (Gessner, 2011; Gessner and Breuer, 2013b).

These properties allow for a straight-forward interpretation of the local dephasing operation: This locally implementable operation removes all discord-type correlations, while the local subsystem distributions are unaffected. We can therefore interpret the state $\rho^{\prime}$ as the incoherent counterpart to $\rho$ (Gessner and Breuer, 2013b). For example in the case of a pure state $\rho=|\Psi\rangle\langle\Psi|$, the local eigenbasis $\{|i\rangle\}$ corresponds to the local Schmidt basis of $|\Psi\rangle$, and the state $\rho^{\prime}$ is the incoherent mixture (with same weights) of the states which are coherently superposed to produce the entangled state $|\Psi\rangle$ (Gessner and Breuer, 2013b). Yet, in order to directly check whether or not the total state changed under the influence of the local dephasing, we would still require access to both subsystems.

To reveal the possible change of the total state to the subsystem, we make use of the latter's time evolution. After preparing the reference state $\rho^{\prime}$ by locally dephasing the initial state $\rho$, we again observe the local time evolution in the accessible subsystem, given by

$$
\rho_{S}^{\prime}(t)=\operatorname{Tr}_{E}\left\{U(t) \rho^{\prime} U^{\dagger}(t)\right\} .
$$

As discussed before, at $t=0$ we will find that, in the local subsystem, we start out in the exact same reduced state as without the dephasing, $\rho_{S}(0)=\rho_{S}^{\prime}(0)$. Furthermore, 
the unitary time evolution operator is the same, since we did not change the environment or the couplings between the two subsystems. One detail, however, did change: The discord-type correlations with the environment have been removed completely by the dephasing. Thus, if we find that the local time evolution changes due to the dephasing-induced removal of the quantum discord, we have found a witness for the initial quantum discord in the state $\rho$ (Gessner and Breuer, 2011; Gessner and Breuer, 2013b).

At the same time, this demonstrates the influence of initial system-environment correlations on the open-system dynamics. It becomes obvious that the reduced dynamics can no longer be defined in terms of a dynamical map which is independent of the environmental state, since the same initial state $\rho_{S}(0)$ is mapped onto two different final states. The fact that any difference between $\rho_{S}(t)$ and $\rho_{S}^{\prime}(t)$ demonstrates the presence of quantum discord in the initial state $\rho$ also implies that any approximate description of the resulting dynamics in terms of factorized initial conditions would be inappropriate (Laine et al., 2010; Gessner and Breuer, 2011; Gessner and Breuer, $2013 \mathrm{~b})$. The steps of the local detection protocol are summarized in the diagram in figure 3.1 .

\subsubsection{Local bound for the minimum entanglement potential}

The question now arises, can we go beyond mere detection and also quantify correlations using this protocol? In fact, the difference between two quantum states can be quantified in terms of distance measures, such as the trace distance. Let us start by discussing the quantification of quantum discord in terms of trace-distance measures.

Considering the results of the previous section, an evident attempt to quantify the quantum discord of the state $\rho$ is based on the disturbance (Luo, 2008) which is induced by the local dephasing operation (3.4), for instance, via the trace distance (Gessner et al., 2014a)

$$
D(\rho)=\left\|\rho-\rho^{\prime}\right\| .
$$

Due to the contractivity property of the trace distance under positive operations (Ruskai, 1994), such as the partial trace and unitary time evolution, a lower bound for this quantity is given by the local trace distance, which can be measured in the accessible subsystem (Gessner and Breuer, 2013b),

$$
\begin{aligned}
d(t)=\left\|\rho_{S}(t)-\rho_{S}^{\prime}(t)\right\| & =\left\|\operatorname{Tr}_{E}\left\{U(t)\left(\rho-\rho^{\prime}\right) U^{\dagger}(t)\right\}\right\| \\
& \leq\left\|U(t)\left(\rho-\rho^{\prime}\right) U^{\dagger}(t)\right\| \\
& =\left\|\rho-\rho^{\prime}\right\|, \quad \forall t \geq 0
\end{aligned}
$$

Since this holds for arbitrary $t \geq 0$, the best strategy to provide a tight bound to the global trace distance (3.7) consists in monitoring the local time evolution for long times and taking the maximum value (Gessner et al., 2014a)

$$
d_{\max }=\max _{t}\left\|\rho_{S}(t)-\rho_{S}^{\prime}(t)\right\| \leq\left\|\rho-\rho^{\prime}\right\|
$$


From an information-theoretic perspective, geometric measures of discord-type correlations should determine the minimal distance to the set of all dephased or classical states (Horodecki et al., 2009; Modi et al., 2012). A priori, it is unclear whether the dephasing-induced disturbance (3.7) minimizes this distance. Indeed, when compared to measures involving a minimization, one finds that, for certain mixed states, equation (3.7), overestimates the amount of correlations in $\rho$ (Girolami et al., 2011; Campbell et al., 2011). Instead of the fixed dephasing in the eigenbasis, one could therefore consider to extend the dephasing process to arbitrary basis sets, defined by a set of orthogonal projection operators $\Pi=\left\{\Pi_{1}, \Pi_{2}, \ldots,\right\}$ as

$$
\Phi_{\Pi}(\rho)=\sum_{i}\left(\Pi_{i} \otimes \mathbb{I}\right) \rho\left(\Pi_{i} \otimes \mathbb{I}\right) .
$$

Minimizing the distance over all projections,

$$
D_{\min }(\rho)=\min _{\Pi}\left\|\rho-\Phi_{\Pi}(\rho)\right\|,
$$

yields an information-theoretically sound measure for quantum discord, the minimal dephasing disturbance. When the accessible subsystem is a qubit, $\mathcal{H}_{S}=\mathbb{C}^{2}$, this measure coincides with the minimum entanglement potential (Nakano et al., 2013), which measures the maximal amount of entanglement which can be activated from discord in a measurement (Streltsov et al., 2011b; Piani et al., 2011; Adesso et al., 2014); see also section 2.1.2. When $\rho$ describes a pure state, this measure reduces to the negativity (Vidal and Werner, 2002),

$$
\mathcal{N}(\rho)=\frac{\left\|\rho^{\Gamma}\right\|-1}{2},
$$

where $\rho^{\Gamma}$ denotes the partial transpose of $\rho$ in an arbitrary basis of one of the two subsystems (Piani and Adesso, 2012). This result is expected since in the case of pure states, we know that the only type of correlation is entanglement. Furthermore, the trace distance and the negativity are both linear in the Schmidt coefficients $\lambda_{i}$. These are defined via a representation of the total state $\rho=|\Psi\rangle\langle\Psi|$ in terms of arbitrary bases of $\mathcal{H}_{S}$ and $\mathcal{H}_{E}$, followed by a singular value decomposition, which leads to (Schmidt, 1907)

$$
|\Psi\rangle=\sum_{i} \lambda_{i}\left|\varphi_{i}\right\rangle \otimes\left|\chi_{i}\right\rangle
$$

with the Schmidt basis $\left|\varphi_{i}\right\rangle \otimes\left|\chi_{i}\right\rangle$ of $\mathcal{H}_{S} \otimes \mathcal{H}_{E}$.

The question remains, which is the basis that minimizes the distance (3.11) in the above situation? The local eigenbasis, considered for dephasing in equation (3.4), here, is given by the local Schmidt basis $\left|\varphi_{i}\right\rangle$. In the case of $\mathcal{H}_{S}=\mathbb{C}^{2}$, the dephasing disturbance (3.7) in this particular basis $\Pi_{0}=\left\{\left|\varphi_{0}\right\rangle\left\langle\varphi_{0}|,| \varphi_{1}\right\rangle\left\langle\varphi_{1}\right|\right\}$ is given by

$$
\begin{aligned}
D(\rho) & =\left\|\rho-\Phi_{\Pi_{0}}(\rho)\right\| \\
& =\| \lambda_{1} \lambda_{0}\left|\varphi_{1}\right\rangle\left\langle\varphi_{0}|\otimes| \chi_{1}\right\rangle\left\langle\chi_{0}\left|+\lambda_{1} \lambda_{0}\right| \varphi_{0}\right\rangle\left\langle\varphi_{1}|\otimes| \chi_{0}\right\rangle\left\langle\chi_{1}\right| \| \\
& =\frac{1}{2} \operatorname{Tr} \sqrt{C^{\dagger} C},
\end{aligned}
$$


where the $2 \times 2$ matrix of operators on $\mathcal{H}_{E}$ can be represented in the local Schmidt basis as

$$
C=\lambda_{0} \lambda_{1}\left(\begin{array}{cc}
0 & \left|\chi_{1}\right\rangle\left\langle\chi_{0}\right| \\
\left|\chi_{0}\right\rangle\left\langle\chi_{1}\right| & 0
\end{array}\right) .
$$

Since $\lambda_{0,1} \geq 0$, the trace distance yields

$$
D(\rho)=\lambda_{0} \lambda_{1}=\mathcal{N}(\rho) .
$$

Employing the same decomposition, the negativity yields (for arbitrary dimensions of $\left.\mathcal{H}_{S}\right)$

$$
\mathcal{N}(|\Psi\rangle\langle\Psi|)=\frac{1}{2} \sum_{\substack{i, j \\ i \neq j}} \lambda_{i} \lambda_{j}
$$

We find that the disturbance on a pure state induced by dephasing in its local qubit eigenbasis is quantified by the negativity. Since the minimal disturbance must also yield the negativity, we have shown that the local eigenbasis minimizes the expression (3.11) (Gessner et al., 2014b).

In general, however, we do not know whether the total state is pure or mixed. The goal is therefore to find a local lower bound for the minimal disturbance. This expression can indeed by estimated by dephasing in many different bases instead of only the local eigenbasis (Gessner et al., 2014b). Based on a particular selection of projection operators $\Pi$, we introduce the local distance

$$
d_{\Pi}(t)=\left\|\rho_{S}(t)-\rho_{S}^{\Pi}(t)\right\|,
$$

where

$$
\rho_{S}^{\Pi}(t)=\operatorname{Tr}_{E}\left\{U(t) \Phi_{\Pi}(\rho) U^{\dagger}(t)\right\}
$$

describes the local time evolution after dephasing. Since the contractivity property which leads to the inequality (3.8) is independent of the dephasing basis, we can extend the statement to obtain a lower bound for the minimal dephasing disturbance (Gessner et al., 2014b)

$$
d_{\min }(\rho)=\max _{t} \min _{\Pi} d_{\Pi}(t) \leq D_{\min }(\rho)
$$

This quantity is accessible when control is restricted to the subsystem since both the dephasing operations and the reduced time evolution are processes which affect the local system. Note, however, that unlike to what we observed when dephasing was implemented in the local eigenbasis, here, we may find a nonzero difference between $\rho_{S}(0)$ and $\rho_{S}^{\Pi}(0)$.

The general procedure then consists in dephasing the initial state systematically in different bases. For each dephasing process, one observes the subsequent local time evolution. At each time $t$, the minimum value of all local distances (there exists one for each dephasing basis) is kept. Out of all the minimum values (one for each recorded time-step), one keeps the largest to obtain the tightest possible bound. 


\subsubsection{Efficacy of the method}

The local detection method is - in principle - always applicable, regardless of the environment to which the locally accessible system couples. Whether or not the presence of initial correlations is revealed, depends on the dynamics of system and environment. In the extreme case of no interaction between the two subsystems it is impossible to map the global correlations to the local subsystem dynamics (Gessner and Breuer, 2013 b).

The distance measures introduced in the previous section can be evaluated easily when, instead of the trace distance, the Hilbert-Schmidt distance, based on the Hilbert-Schmidt norm $\|X\|_{2}^{2}=\operatorname{Tr} X^{\dagger} X$, is used, even when the minimization procedure is included (Dakić et al., 2010). It further allows for analytic determination of the unitary expectation value and its variance (Gessner and Breuer, 2013b) using Haar measure integration techniques (Gessner and Breuer, 2013a). The resulting average local distance is given by (Gessner and Breuer, 2011)

$$
\int d \mu(U)\left\|\operatorname{Tr}_{E}\left\{U\left(\rho-\rho^{\prime}\right) U^{\dagger}\right\}\right\|_{2}^{2}=\frac{d_{S}^{2} d_{E}-d_{E}}{d_{S}^{2} d_{E}^{2}-1}\left\|\rho-\rho^{\prime}\right\|_{2}^{2},
$$

where $d \mu$ denotes the Haar measure on the unitary group, and $d_{S}$ and $d_{E}$ are the dimensions of system and environment, respectively. Together with the corresponding variance, which can be found in (Gessner and Breuer, 2013b), this result can be used to assess the success probability of the local detection method. The unitary average value (3.21) shows that generic dynamical systems will reveal the quantum discord (right-hand side) to the open-system dynamics (left-hand side); see also (Gessner, 2011).

In many cases we can also establish a direct connection between the time average over the local Hilbert-Schmidt distance and the dephasing disturbance as quantified via the Hilbert-Schmidt distance (Gessner et al., 2014a). The Hilbert-Schmidt distance is, however, not contractive under positive maps, which can cause unphysical increases of these quantities under trivial operations (Ozawa, 2000; Piani, 2012). In this thesis, we will mostly employ the trace distance, which does not suffer from these limitations (Paula et al., 2013).

The result (3.21) further shows that the local signal of these correlations shrinks as the dimension of the environment increases when the dynamical evolution is sufficiently generic to be described by the random-matrix average. For such complex systemenvironment interactions, this suggests the necessity of a finite-dimensional Hilbert space in order to be able to successfully reveal initial correlations using the present method. In the following sections, we present a series of theoretical and experimental applications of the local detection method to study its efficacy in different scenarios. These include examples of infinite-dimensional, memoryless environments where the correlations are revealed, and others where they cannot be detected. 


\subsection{Trapped-ion experiment}

In this section we describe a trapped-ion experiment where the local detection protocol was implemented to detect discord-type correlations between the electronic qubit and the motional degree of freedom of the same trapped ion, while restricting to local operations on the electronic qubit (Gessner et al., 2014a).

\subsubsection{Resonant laser-ion interactions}

Before describing the actual experiment regarding the detection of discord-type correlations, we derive a general theoretical description of the quantum dynamics induced by laser light on an electronic two-level transition as a function of the laser detuning, restricting the treatment to a single oscillator mode (Leibfried et al., 2003a; Meekhof et al., 1996; Gardiner et al., 1997). The derived expressions are then applied to the local detection scenario in section 3.2.3. Our initial point in this section is the Hamiltonian (2.8), however, here we do not make use of the Lamb-Dicke limit (Roghani et al., 2011).

We start by transforming to a moving reference frame with the unitary operator $U(t)=e^{-i \omega_{L} \sigma_{+} \sigma_{-} t}$. Subsequently, we apply the rotating wave approximation, that is, we ignore rapidly oscillating terms corresponding to sums of optical frequencies over terms whose slow oscillation is determined by differences of optical frequencies; see, for example (Cohen-Tannoudji et al., 1992). This yields the time-independent Hamiltonian (Blockley et al., 1992; Vogel and Filho, 1995)

$$
H=-\hbar \Delta \sigma_{+} \sigma_{-}+\hbar \nu a^{\dagger} a+\frac{\hbar \Omega}{2}\left(\sigma_{+} e^{i \eta\left(a+a^{\dagger}\right)} e^{i \varphi}+\sigma_{-} e^{-i \eta\left(a+a^{\dagger}\right)} e^{-i \varphi}\right) .
$$

In the following, we consider different choices for the detuning $\Delta$ between the qubit transition and the laser frequency. To find the most relevant terms we transform to the interaction picture with respect to $H_{0}=-\hbar \Delta \sigma_{+} \sigma_{-}+\hbar \nu a^{\dagger} a$. This transformation is effectively implemented via the replacements $a \rightarrow a e^{-i \nu t}, a^{\dagger} \rightarrow a^{\dagger} e^{i \nu t}, \sigma_{ \pm} \rightarrow \sigma_{ \pm} e^{\mp i \Delta t}$, which yield

$$
H_{I}(t)=\frac{\hbar \Omega}{2}\left(\sigma_{+} e^{i \eta\left(a e^{-i \nu t}+a^{\dagger} e^{i \nu t}\right)} e^{-i \Delta t} e^{i \varphi}+\sigma_{-} e^{-i \eta\left(a e^{-i \nu t}+a^{\dagger} e^{i \nu t}\right)} e^{i \Delta t} e^{-i \varphi}\right)
$$

Using the Campbell-Baker-Hausdorff relation (Mandel and Wolf, 1995), we obtain

$$
e^{ \pm i \eta\left(a^{\dagger} e^{i \nu t}+a e^{-i \nu t}\right)}=e^{-\eta^{2} / 2} \sum_{k, l=0}^{\infty} \frac{\left( \pm i \eta a^{\dagger}\right)^{k}( \pm i \eta a)^{l}}{k ! l !} e^{i(k-l) \nu t} .
$$

For additional resonance conditions $\Delta=m \nu, m \in \mathbb{Z}$, some of the terms do not oscillate at all. These will make the most relevant contribution to the dynamics as the oscillating terms can be ignored after a second application of the rotating wave approximation. This works best for a large ratio $\nu / \Omega$. Then, the rapid oscillations of frequencies $\nu$ and higher average to zero within the relevant time scale given by $1 / \Omega$. 


\subsubsection{Carrier transition}

For $\Delta=0$ we address the carrier transition since the laser is resonant with the atomic transition. The relevant terms are found by the condition $k=l$. Neglecting the counter-rotating terms we obtain

$$
H_{I}^{(0)}=\frac{\hbar \Omega}{2} e^{-\eta^{2} / 2} \sum_{k=0}^{\infty} \frac{\left(-\eta^{2}\right)^{k}\left(a^{\dagger}\right)^{k} a^{k}}{k !^{2}}\left(\sigma_{+} e^{i \varphi}+\sigma_{-} e^{-i \varphi}\right)
$$

This decomposes into a family of effective $2 \times 2$-Hamiltonians $H_{n}^{(0)}=\hbar \Omega_{n}^{(0)} / 2\left(\sigma_{+} e^{i \varphi}+\right.$ $\left.\sigma_{-} e^{-i \varphi}\right)$ with

$$
\Omega_{n}^{(0)}=\Omega e^{-\eta^{2} / 2} \sum_{k=0}^{n} \frac{\left(-\eta^{2}\right)^{k} n !}{(n-k) ! k !^{2}} .
$$

When $\eta \ll 1$, this frequency reduces to $\Omega$, thereby reproducing the approximate expression given in equation (2.10) in this special case. Multiple application of the creation and annihilation operators on a Fock state $|n\rangle$ with $n$ vibrational excitations yields

$$
\begin{aligned}
\left(a^{\dagger}\right)^{k}|n\rangle & =\sqrt{\frac{(n+k) !}{n !}}|n+k\rangle, \\
a^{k}|n\rangle & =\sqrt{\frac{n !}{(n-k) !}}|n-k\rangle,
\end{aligned}
$$

which leads to

$$
\left(a^{\dagger}\right)^{k} a^{k}|n\rangle=\frac{n !}{(n-k) !}|n\rangle
$$

For an arbitrary state of the form $|\Psi(t)\rangle=\sum_{n=0}^{\infty}\left(g_{n}(t)|g, n\rangle+e_{n}(t)|e, n\rangle\right)$, where $|g\rangle$ and $|e\rangle$ denote ground and excited state of the two-level system, respectively, we solve Schrödinger's equation to obtain the following time evolution

$$
\begin{aligned}
& g_{n}(t)=g_{n}(0) \cos \left(\frac{\Omega_{n}^{(0)} t}{2}\right)-i e^{-i \varphi} e_{n}(0) \sin \left(\frac{\Omega_{n}^{(0)} t}{2}\right), \\
& e_{n}(t)=e_{n}(0) \cos \left(\frac{\Omega_{n}^{(0)} t}{2}\right)-i e^{i \varphi} g_{n}(0) \sin \left(\frac{\Omega_{n}^{(0)} t}{2}\right) .
\end{aligned}
$$

Beyond coherent operations as discussed in section 2.2.1.6, Rabi oscillations on the carrier transition are also used to estimate the ion's temperature. For a thermal distribution, $\rho(0)=|g\rangle\left\langle g\left|\otimes \sum_{n} p_{n}\right| n\right\rangle\langle n|$, we get

$$
p_{e}(t)=\left\langle e\left|\rho_{S}(t)\right| e\right\rangle=\sum_{n} p_{n}\left|\sin \left(\frac{\Omega_{n}^{(0)} t}{2}\right)\right|^{2}
$$


where the Boltzmann-distributed populations of excited vibrational state can be expressed in terms of the average occupation number $\bar{n}$ as $p_{n}=\bar{n}^{n} /(\bar{n}+1)^{n+1}$ (Leibfried et al., 2003a). This relation is often used to estimate the temperature of thermal excitations by fitting the experimentally measured Rabi-flop to the predicition (3.30) with the free parameter $\bar{n}$. This method has to be taken with some precaution since other dephasing effects (see section 2.2.1.8) may also lead to a decay of the Rabi oscillations which is hard to distinguish from increased temperatures.

\subsubsection{First red sideband transition}

If we choose a red detuning corresponding to the trap frequency, $\Delta=-\nu$, we drive the first red sideband transition. The time-independent terms in equations (3.23) and (3.24) are those that satisfy the condition $l=k+1$ in the sum corresponding to $e^{i k x}$ and $k=l+1$ for $e^{-i k x}$. The interaction Hamiltonian assumes a form close to the on-resonance Jaynes-Cummings Hamiltonian,

$$
H_{I}^{(-1)}=i \eta \frac{\hbar \Omega}{2} e^{-\eta^{2} / 2}\left(\sum_{k=0}^{\infty} \frac{\left(-\eta^{2}\right)^{k}\left(a^{\dagger}\right)^{k} a^{k}}{k !(k+1) !} \sigma_{+} a e^{i \varphi}-\sigma_{-} a^{\dagger} e^{-i \varphi} \sum_{k=0}^{\infty} \frac{\left(-\eta^{2}\right)^{k}\left(a^{\dagger}\right)^{k} a^{k}}{k !(k+1) !}\right) .
$$

Here the state space splits up in families of pairs $\{|e, n\rangle,|g, n+1\rangle\}$ coupled via the Hamiltonians

$$
H_{n}^{(-1)}=\frac{i \hbar \Omega_{n}^{(1)}}{2 \sqrt{n+1}}\left(\sigma_{+} a e^{i \varphi}-\sigma_{-} a^{\dagger} e^{-i \varphi}\right)
$$

and effective Rabi frequencies

$$
\Omega_{n}^{(1)}=\eta \sqrt{n+1} \Omega e^{-\eta^{2} / 2} \sum_{k=0}^{n} \frac{\left(-\eta^{2}\right)^{k} n !}{k !(k+1) !(n-k) !} .
$$

As already discussed in section 2.2, the induced Jaynes-Cummings dynamics describes a variety of different physical systems, including cavity quantum electrodynamics, where light modes inside a cavity take on the role of the phonons.

\subsubsection{First blue sideband transition}

The first blue sideband is addressed for $\Delta=\nu$. Relevant terms for the $e^{i k x}$ term are identified by $k=l+1$, and by $l=k+1$ for the $e^{-i k x}$ term. We obtain the anti Jaynes-Cummings interaction,

$$
H_{I}^{(+1)}=i \eta \frac{\hbar \Omega}{2} e^{-\eta^{2} / 2}\left(\sigma_{+} a^{\dagger} e^{i \varphi} \sum_{k=0}^{\infty} \frac{\left(-\eta^{2}\right)^{k}\left(a^{\dagger}\right)^{k} a^{k}}{k !(k+1) !}-\sum_{k=0}^{\infty} \frac{\left(-\eta^{2}\right)^{k}\left(a^{\dagger}\right)^{k} a^{k}}{k !(k+1) !} \sigma_{-} a e^{-i \varphi}\right) .
$$


This Hamiltonian couples the pairs $\{|e, n+1\rangle,|g, n\rangle\}$ with the $2 \times 2$ Hamiltonians

$$
H_{n}^{(+1)}=\frac{i \hbar \Omega_{n}^{(1)}}{2 \sqrt{n+1}}\left(\sigma_{+} a^{\dagger} e^{i \varphi}-\sigma_{-} a e^{-i \varphi}\right) .
$$

This interaction cannot be found in cavity quantum electrodynamics experiments, since it would correspond to the creation of a photon, while at the same time the atom gets excited. Since in an ion trap this interaction is induced via an external laser, which can add or dissipate arbitrary amounts of energy into or out of the system, such processes can now occur. In fact, they form the basis of sideband cooling techniques, where vibrational excitations, phonons, are removed.

\subsubsection{Arbitrary order sidebands}

The previous considerations can be generalized to arbitrary order with $\Delta= \pm m \nu$, $m \in \mathbb{N}$ (Cahill and Glauber, 1969; Wineland and Itano, 1979; Leibfried et al., 2003a). We speak of the $m$ th red (blue) sideband whenever the sign is negative (positive). Let us first consider the mth blue sideband. Equations. (3.23) and (3.24) yield the conditions $k=l+m$, for $e^{i k x}$, and $l=k+m$, for $e^{-i k x}$, respectively. The Hamiltonian yields

$$
\begin{aligned}
H_{I}^{(+m)}=\frac{\hbar \Omega}{2} e^{-\eta^{2} / 2} & \left(\sigma_{+}\left(i \eta a^{\dagger}\right)^{m} e^{i \varphi} \sum_{k=0}^{\infty} \frac{\left(-\eta^{2}\right)^{k}\left(a^{\dagger}\right)^{k} a^{k}}{k !(k+m) !}\right. \\
& \left.+\sum_{k=0}^{\infty} \frac{\left(-\eta^{2}\right)^{k}\left(a^{\dagger}\right)^{k} a^{k}}{k !(k+m) !} \sigma_{-}(-i \eta a)^{m} e^{-i \varphi}\right),
\end{aligned}
$$

and splits up in $2 \times 2$ blocks of

$$
H_{n}^{(+m)}=\sqrt{\frac{n !}{(n+m) !}} \frac{\hbar \Omega_{n}^{(m)}}{2}\left(i^{m} \sigma_{+}\left(a^{\dagger}\right)^{m} e^{i \varphi}+(-i)^{m} \sigma_{-} a^{m} e^{-i \varphi}\right),
$$

coupling $\{|g, n\rangle,|e, n+m\rangle\}$, with

$$
\Omega_{n}^{(m)}=\eta^{m} \sqrt{\frac{(n+m) !}{n !}} \Omega e^{-\eta^{2} / 2} \sum_{k=0}^{n} \frac{\left(-\eta^{2}\right)^{k} n !}{k !(k+m) !(n-k) !} .
$$

It is possible to express this term as a function of the generalized Legendre polynomials (Morse and Feshbach, 1953),

$$
L_{n}^{(\alpha)}(x)=\sum_{k=0}^{n}(-1)^{k}\left(\begin{array}{c}
n+\alpha \\
n-k
\end{array}\right) \frac{x^{k}}{k !}
$$

as

$$
\Omega_{n}^{(m)}=\eta^{m} \sqrt{\frac{n !}{(n+m) !}} \Omega e^{-\eta^{2} / 2} L_{n}^{(m)}\left(\eta^{2}\right)
$$


The evolution of a general state,

$$
|\Psi(t)\rangle=\sum_{n=0}^{\infty}\left[g_{n}(t)|g, n\rangle+e_{n+m}(t)|e, n+m\rangle\right]
$$

is given by

$$
\begin{aligned}
g_{n}(t) & =g_{n}(0) \cos \left(\frac{\Omega_{n}^{(m)} t}{2}\right)+(-i)^{m+1} e^{-i \varphi} e_{n+m}(0) \sin \left(\frac{\Omega_{n}^{(m)} t}{2}\right), \\
e_{n+m}(t) & =e_{n+m}(0) \cos \left(\frac{\Omega_{n}^{(m)} t}{2}\right)+i^{m-1} e^{i \varphi} g_{n}(0) \sin \left(\frac{\Omega_{n}^{(m)} t}{2}\right) .
\end{aligned}
$$

By analogous methods the Hamiltonian for the $m$ th red sideband yields

$$
\begin{aligned}
H_{I}^{(-m)}=\frac{\hbar \Omega}{2} e^{-\eta^{2} / 2} & \left(\sum_{k=0}^{\infty} \frac{\left(-\eta^{2}\right)^{k}\left(a^{\dagger}\right)^{k} a^{k}}{k !(k+m) !} \sigma_{+}(i \eta a)^{m} e^{i \varphi}\right. \\
& \left.+\sigma_{-}\left(-i \eta a^{\dagger}\right)^{m} e^{-i \varphi} \sum_{k=0}^{\infty} \frac{\left(-\eta^{2}\right)^{k}\left(a^{\dagger}\right)^{k} a^{k}}{k !(k+m) !}\right),
\end{aligned}
$$

again splitting up in $2 \times 2$ blocks,

$$
H_{n}^{(-m)}=\sqrt{\frac{n !}{(n+m) !}} \frac{\hbar \Omega_{n}^{(m)}}{2}\left(i^{m} \sigma_{+} a^{m} e^{i \varphi}+(-i)^{m} \sigma_{-}\left(a^{\dagger}\right)^{m} e^{-i \varphi}\right),
$$

coupling $\{|g, n+m\rangle,|e, n\rangle\}$. This evolves a general state,

$$
|\Psi(t)\rangle=\sum_{n=0}^{\infty}\left[g_{n+m}(t)|g, n+m\rangle+e_{n}(t)|e, n\rangle\right],
$$

according to

$$
\begin{aligned}
g_{n+m}(t) & =g_{n+m}(0) \cos \left(\frac{\Omega_{n}^{(m)} t}{2}\right)+(-i)^{m+1} e^{-i \varphi} e_{n}(0) \sin \left(\frac{\Omega_{n}^{(m)} t}{2}\right), \\
e_{n}(t) & =e_{n}(0) \cos \left(\frac{\Omega_{n}^{(m)} t}{2}\right)+i^{m-1} e^{i \varphi} g_{n+m}(0) \sin \left(\frac{\Omega_{n}^{(m)} t}{2}\right) .
\end{aligned}
$$

While, so far, we assumed perfect resonance between laser and sideband frequency, one experimentally always has to account for detunings, with drifts of the trap frequency as dominant source. In the following section, we generalize the analytical description to include a small detuning from the sideband transition. 


\subsubsection{The effect of small detunings}

To be able to drop the idealized assumption that the sideband transition is addressed resonantly, we include the influence of an additional detuning $\delta \ll \Delta$. The rotating wave approximation is still valid in this situation. However, a nonzero $\delta$ introduces additional time-dependent terms into the Hamiltonian, as can be seen by replacing $\Delta$ with $\Delta+\delta$ in equation (3.23). For red and blue sidebands, we obtain the timedependent Hamiltonians

$$
H_{n}^{(-m)}(t)=\sqrt{\frac{n !}{(n+m) !}} \frac{\hbar \Omega_{n}^{(m)}}{2}\left(i^{m} \sigma_{+} a^{m} e^{i \varphi} e^{-i \delta t}+(-i)^{m} \sigma_{-}\left(a^{\dagger}\right)^{m} e^{-i \varphi} e^{i \delta t}\right),
$$

and

$$
H_{n}^{(+m)}(t)=\sqrt{\frac{n !}{(n+m) !}} \frac{\hbar \Omega_{n}^{(m)}}{2}\left(i^{m} \sigma_{+}\left(a^{\dagger}\right)^{m} e^{i \varphi} e^{-i \delta t}+(-i)^{m} \sigma_{-} a^{m} e^{-i \varphi} e^{i \delta t}\right),
$$

respectively. The Schrödinger equation leads for the red sidebands to the set of coupled differential equations

$$
\begin{aligned}
\dot{g}_{n+m}(t) & =(-i)^{m+1} e^{-i \varphi} e^{i \delta t} \frac{\Omega_{n}^{(m)}}{2} e_{n}(t), \\
\dot{e}_{n}(t) & =i^{m-1} e^{i \varphi} e^{-i \delta t} \frac{\Omega_{n}^{(m)}}{2} g_{n+m}(t) .
\end{aligned}
$$

These can be solved by an ansatz of the form (Scully and Zubairy, 1997)

$$
\begin{aligned}
g_{n+m}(t) & =\left(a_{1} e^{i \widetilde{\Omega}_{n}^{(m)} t / 2}+a_{2} e^{-i \widetilde{\Omega}_{n}^{(m)} t / 2}\right) e^{i \delta t / 2}, \\
e_{n}(t) & =\left(b_{1} e^{i \widetilde{\Omega}_{n}^{(m)} t / 2}+b_{2} e^{-i \widetilde{\Omega}_{n}^{(m)} t / 2}\right) e^{-i \delta t / 2} .
\end{aligned}
$$

The solution can be written in terms of the initial conditions as

$$
\begin{aligned}
g_{n+m}(t)= & \left(g_{n+m}(0)\left[\cos \left(\frac{\widetilde{\Omega}_{n}^{(m)} t}{2}\right)-\frac{i \delta}{\widetilde{\Omega}_{n}^{(m)}} \sin \left(\frac{\widetilde{\Omega}_{n}^{(m)} t}{2}\right)\right]\right. \\
& \left.+(-i)^{m+1} e^{-i \varphi} \frac{\Omega_{n}^{(m)}}{\widetilde{\Omega}_{n}^{(m)}} e_{n}(0) \sin \left(\frac{\widetilde{\Omega}_{n}^{(m)} t}{2}\right)\right) e^{i \delta t / 2}, \\
e_{n}(t)= & \left(e_{n}(0)\left[\cos \left(\frac{\widetilde{\Omega}_{n}^{(m)} t}{2}\right)+\frac{i \delta}{\widetilde{\Omega}_{n}^{(m)}} \sin \left(\frac{\widetilde{\Omega}_{n}^{(m)} t}{2}\right)\right]\right. \\
& \left.-i^{m+1} e^{i \varphi} \frac{\Omega_{n}^{(m)}}{\widetilde{\Omega}_{n}^{(m)}} g_{n+m}(0) \sin \left(\frac{\widetilde{\Omega}_{n}^{(m)} t}{2}\right)\right) e^{-i \delta t / 2},
\end{aligned}
$$

where

$$
\widetilde{\Omega}_{n}^{(m)}=\sqrt{\left(\Omega_{n}^{(m)}\right)^{2}+\delta^{2}}
$$


For the blue sidebands we obtain

$$
\begin{aligned}
\dot{g}_{n}(t) & =(-i)^{m+1} e^{-i \varphi} e^{i \delta t} \frac{\Omega_{n}^{(m)}}{2} e_{n+m}(t), \\
\dot{e}_{n+m}(t) & =i^{m-1} e^{i \varphi} e^{-i \delta t} \frac{\Omega_{n}^{(m)}}{2} g_{n}(t),
\end{aligned}
$$

with the solution

$$
\begin{aligned}
g_{n}(t)= & g_{n}(0)\left[\cos \left(\frac{\widetilde{\Omega}_{n}^{(m)} t}{2}\right)-\frac{i \delta}{\widetilde{\Omega}_{n}^{(m)}} \sin \left(\frac{\widetilde{\Omega}_{n}^{(m)} t}{2}\right)\right] \\
& \left.+(-i)^{m+1} e^{-i \varphi} \frac{\Omega_{n}^{(m)}}{\widetilde{\Omega}_{n}^{(m)}} e_{n+m}(0) \sin \left(\frac{\widetilde{\Omega}_{n}^{(m)} t}{2}\right)\right) e^{i \delta t / 2}, \\
e_{n+m}(t)= & \left(e_{n+m}(0)\left[\cos \left(\frac{\widetilde{\Omega}_{n}^{(m)} t}{2}\right)+\frac{i \delta}{\widetilde{\Omega}_{n}^{(m)}} \sin \left(\frac{\widetilde{\Omega}_{n}^{(m)} t}{2}\right)\right]\right. \\
& \left.-i^{m+1} e^{i \varphi} \frac{\Omega_{n}^{(m)}}{\widetilde{\Omega}_{n}^{(m)}} g_{n}(0) \sin \left(\frac{\widetilde{\Omega}_{n}^{(m)} t}{2}\right)\right) e^{-i \delta t / 2} .
\end{aligned}
$$

We can use this to explicitly write out the elements of the unitary time evolution operator,

$$
U^{( \pm m)}\left(t_{1}, t_{0}\right)=\mathcal{T} \exp \left(-\frac{i}{\hbar} \int_{t_{0}}^{t_{1}} H^{( \pm m)}(\tau) d \tau\right)
$$

where $H^{( \pm m)}=\bigoplus_{n=0}^{\infty} H_{n}^{( \pm m)}$, and $\mathcal{T}$ denotes the time-ordering operator. According to equations (3.51) and (3.54), and using $U\left(t_{1}, t_{0}\right)=U\left(t_{1}, 0\right) U^{\dagger}\left(t_{0}, 0\right)$, we obtain

$$
\begin{aligned}
u_{g g}^{m n}\left(t_{1}, t_{0}\right) & :=\left\langle g, n\left|U^{(+m)}\left(t_{1}, t_{0}\right)\right| g, n\right\rangle=\left\langle g, n+m\left|U^{(-m)}\left(t_{1}, t_{0}\right)\right| g, n+m\right\rangle \\
& =\left\langle e, n+m\left|U^{(+m)}\left(t_{1}, t_{0}\right)\right| e, n+m\right\rangle^{*}=\left\langle e, n\left|U^{(-m)}\left(t_{1}, t_{0}\right)\right| e, n\right\rangle^{*} \\
& =\left[\cos \left(\frac{\widetilde{\Omega}_{n}^{(m)}\left(t_{1}-t_{0}\right)}{2}\right)-\frac{i \delta}{\widetilde{\Omega}_{n}^{(m)}} \sin \left(\frac{\widetilde{\Omega}_{n}^{(m)}\left(t_{1}-t_{0}\right)}{2}\right)\right] e^{i \delta\left(t_{1}-t_{0}\right) / 2},
\end{aligned}
$$

and

$$
\begin{aligned}
u_{e g}^{m n}\left(t_{1}, t_{0}\right) & :=\left\langle e, n+m\left|U^{(+m)}\left(t_{1}, t_{0}\right)\right| g, n\right\rangle=\left\langle e, n\left|U^{(-m)}\left(t_{1}, t_{0}\right)\right| g, n+m\right\rangle \\
& =\left\langle g, n\left|U^{(+m)}\left(t_{1}, t_{0}\right)\right| e, n+m\right\rangle^{*}=\left\langle g, n+m\left|U^{(-m)}\left(t_{1}, t_{0}\right)\right| e, n\right\rangle^{*} \\
& =-i^{m+1} e^{i \varphi} \frac{\Omega_{n}^{(m)}}{\widetilde{\Omega}_{n}^{(m)}} \sin \left(\frac{\widetilde{\Omega}_{n}^{(m)}\left(t_{1}-t_{0}\right)}{2}\right) e^{-i \delta\left(t_{1}+t_{0}\right) / 2} .
\end{aligned}
$$




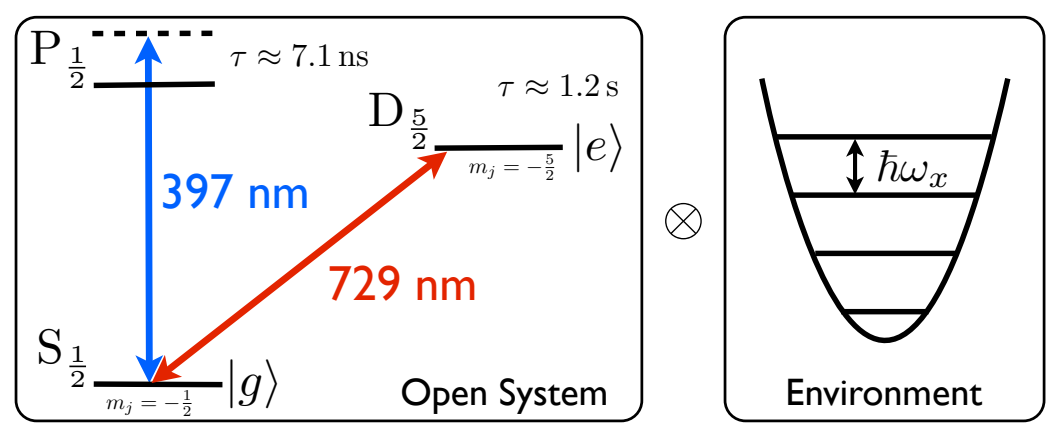

FIGURE 3.2.: The electronic qubit transition can be driven coherently via the quadrupole transition at $729 \mathrm{~nm}$. Incoherent readout and dephasing operations are carried out via the $397 \mathrm{~nm}$ laser. When the detuning of the qubit laser corresponds to multiples of the trap frequency $\omega_{x}$, the qubit is coupled via the laser to the harmonic ion motion. Compare also to figure 2.3. Adapted from (Gessner et al., 2014a).

\subsubsection{The local detection protocol for the first blue sideband}

Having established these exact theoretical tools for the complete description of the dynamics of an atomic two-level system interacting with a harmonic oscillator mode, we can apply this to analyze the local detection protocol in the context of a trapped-ion experiment. We consider the electronic qubit of a single trapped ion as the accessible quantum system, which is coupled via laser-induced interactions to the a single mode of its own motion in the harmonic trap potential. This motional mode takes on the role of an environment. In this case it is to some extent possible to control the environment-nevertheless, coherent control over the ion's motion is technically much more demanding and by far not as well-established as for the electronic qubit. For a complete measurement of the quantum state of qubit and motion, correlated operations on both degrees of freedom would be necessary. The present experiment makes use only of local operations on the qubit degree of freedom and is therefore in principle - applicable independently of the environment to which the qubit couples. We shift the discussion about whether or not the scheme - in practice - would successfully reveal the presence of correlations for arbitrary environments to the end of this chapter.

The present experiment was performed in the quantum information group at Berkeley, and the experimental setup was already described in the introduction. Further details can be found in references (Ramm et al., 2013; Ramm, 2014; Pruttivarasin, 2014). The most important electronic levels of ${ }^{40} \mathrm{Ca}^{+}$are displayed in figure 3.2 .

After a combination of Doppler and sideband cooling, the ion is prepared in a lowtemperature thermal state of motion, while its electronic degree of freedom is initialized in the ground state by optical pumping. The prepared state,

$$
\rho_{0}=\sum_{n=0}^{\infty} p_{n}|g\rangle\langle g|\otimes| n\rangle\langle n|
$$


is an uncorrelated product state, characterized by the average thermal occupation number $\bar{n}$ via the probability distribution $p_{n}=\bar{n}^{n} /(\bar{n}+1)^{n+1}$; see, for example, (Leibfried et al., 2003a), section II.C.4. To create a correlated state of open system (qubit) and environment (motion), we drive the first blue sideband for a time $t_{0}$. In section 3.2.4, we present a general theoretical treatment of arbitrary red or blue sidebands. The sideband resonance is chosen to match the trap frequency along the $x$ direction, that is, we select the motional frequency $\nu=\omega_{x}$. The experimental trap frequencies of the linear Paul trap are $\left(\omega_{x}, \omega_{y}, \omega_{z}\right)=2 \pi \times(2.8,2.6,0.2) \mathrm{MHz}$.

\subsubsection{Preparation}

The initial state $\rho_{0}$ evolves under the influence of the first blue sideband,

$$
\begin{aligned}
\rho\left(t_{0}\right)= & U^{(+1)}\left(t_{0}, 0\right) \rho_{0} U^{(+1)}\left(t_{0}, 0\right)^{\dagger} \\
= & \sum_{n=0}^{\infty} p_{n}\left[\left|u_{g g}^{1 n}\left(t_{0}, 0\right)\right|^{2}|g, n\rangle\left\langle g, n\left|+u_{e g}^{1 n}\left(t_{0}, 0\right)^{*} u_{g g}^{1 n}\left(t_{0}, 0\right)\right| g, n\right\rangle\langle e, n+1|\right. \\
& \left.\quad+u_{e g}^{1 n}\left(t_{0}, 0\right) u_{g g}^{1 n}\left(t_{0}, 0\right)^{*}|e, n+1\rangle\left\langle g,\left.n|+| u_{e g}^{1 n}\left(t_{0}, 0\right)\right|^{2} \mid e, n+1\right\rangle\langle e, n+1|\right],
\end{aligned}
$$

which describes a correlated quantum state. This first time evolution therefore represents the preparation of the correlated initial state. The next step is the detection of these correlations by local means. Of the total correlated state, we can only access the reduced density matrix of the qubit, which now is described by

$$
\rho_{S}\left(t_{0}\right)=\operatorname{Tr}_{E} \rho\left(t_{0}\right)=\sum_{n=0}^{\infty} p_{n}\left[\left|u_{g g}^{1 n}\left(t_{0}, 0\right)\right|^{2}|g\rangle\left\langle\left. g|+| u_{e g}^{1 n}\left(t_{0}, 0\right)\right|^{2} \mid e\right\rangle\langle e|\right] .
$$

Figure 3.3 shows the key steps of the local detection protocol, tomography data, and the time evolution of $\left\langle e\left|\rho_{S}\left(t_{0}\right)\right| e\right\rangle$ for different initial temperatures of the motional state. For the time evolution, an average with slightly fluctuating parameters was performed to account for magnetic field noise and instabilities of the trap frequency. Typically, the detuning was assumed to fluctuate according to a Gaussian distribution with both center and variance of the order of $2 \pi \times 1 \mathrm{kHz}$ (Gessner et al., 2014a).

To reveal the correlations to the local dynamics, we implement the local dephasing operation (3.4) in the eigenbasis of the qubit. This local eigenbasis is always given by $|g\rangle$ and $|e\rangle$, as can be seen from equation (3.60) and which is confirmed experimentally by state tomography shown in figure $3.3 \mathrm{~b}$ ).

\subsubsection{Implementing the local dephasing operation}

The key step of the local detection protocol consists in the removal all coherences from the total quantum state by a local dephasing operation. In the present setting, coherences in the local eigenbasis can be removed in a controlled way by applying a far-detuned laser on the $397 \mathrm{~nm}$ transition, see figure 3.2. Such a laser will induce an AC-Stark shift (Cohen-Tannoudji et al., 1992) on the ground state $|g\rangle$, while the 
a)

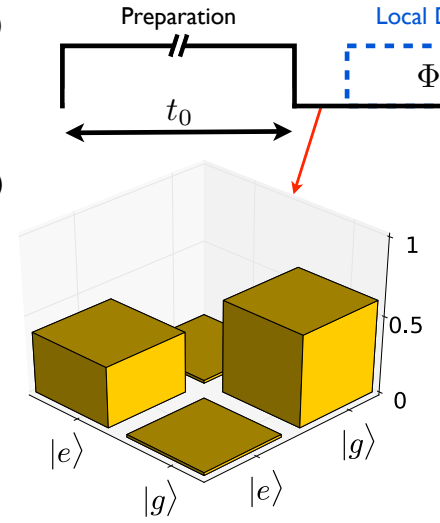

State tomography without dephasing

d)

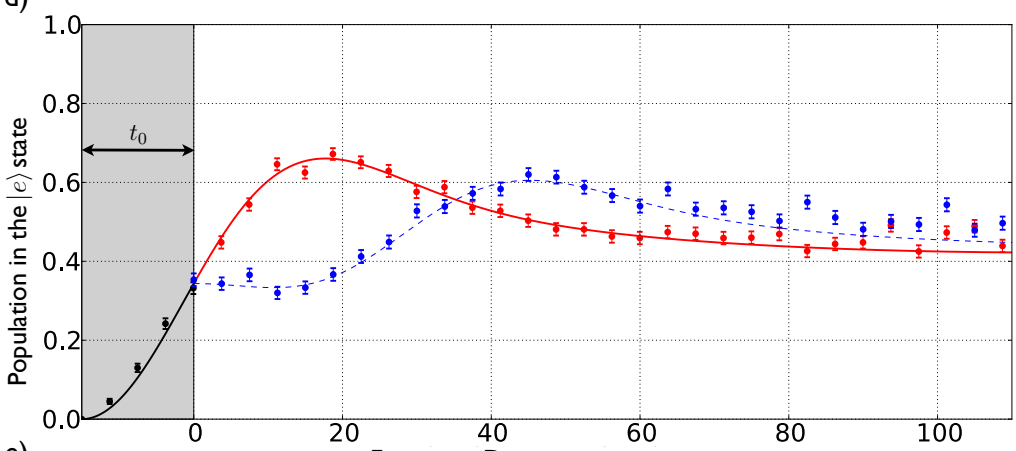

e)

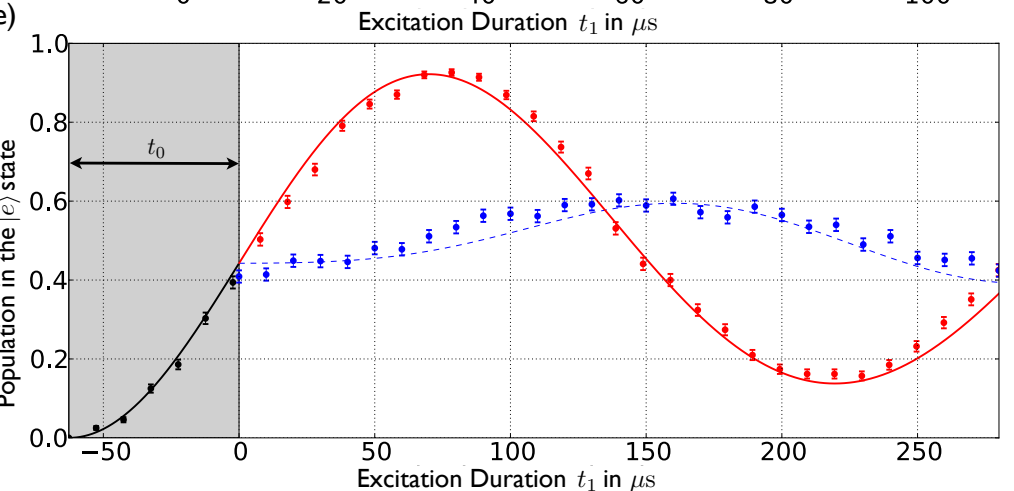

FigURE 3.3.: a) The experimental sequence consists in the comparison of the time evolution with and without dephasing. b-c) Full state tomography of the qubit system confirms that dephasing in the local eigenbasis does not affect the local initial state. d-e) The subsequent time evolution is dramatically influenced by the removal of initial discord-type correlations by local dephasing, which is shown for different environmental thermal distributions: Parameters are $\bar{n}=5.9$ in d) and $\bar{n}=0.2$ in e). The error bars describe the statistical error $\sigma_{p}=\sqrt{p(1-p) / N}$ for $N=1000$ measurements per timestep. Adapted from (Gessner et al., 2014a). 
excited state $|e\rangle$ is not affected. If the detuning is sufficiently large, the population of the qubit ground state will not be affected, which is important, since we only need to remove certain coherences. In this limit, one can describe the dynamics of the relevant electronic states with the laser mode via an effective Hamiltonian (Schneider et al., 1997; Gerry and Knight, 2005; Foot, 2007)

$$
H_{\mathrm{eff}}=\hbar \frac{\Omega_{f}^{2}}{4 \Delta_{f}}\left(|f\rangle\langle f|+(|f\rangle\langle f|-| g\rangle\langle g|) b^{\dagger} b\right),
$$

where $b$ is the annihilation operator of the laser light mode and $|f\rangle=\left|P_{1 / 2}\right\rangle$ describes the short-lived excited $\mathrm{P}_{1 / 2}$ state, which is addressed by the $397 \mathrm{~nm}$ laser light. We see that this Hamiltonian does not induce population transfer, but instead induces an energy shift. Thus, during the application of this off-resonant laser, the ground state will accumulate a phase shift with respect to the unperturbed excited state. This phase shift oscillates with frequency $\Omega_{f}^{2} / 4 \Delta_{f}$, and can be precisely manipulated via the pulse duration. This oscillation of the relative phase was also experimentally confirmed via Ramsey experiments (Ramm, 2014).

By averaging the subsequent time evolution over a series of phase shifts whose average value is zero, we effectively remove all coherences and with it the quantum discord of the total state, while only manipulating the local state of the qubit. To ensure that indeed the ground-state population is not affected by accidental photon scattering events from the $397 \mathrm{~nm}$ laser (Ozeri et al., 2005; Uys et al., 2010), we estimate the number of scattered photons (Gessner et al., 2014a). The scattering rate is given by $\Gamma p_{f}$, where the $\mathrm{P}_{1 / 2}$-state population is obtained from the steady-state solution of the optical Bloch equations (Cohen-Tannoudji et al., 1992),

$$
p_{f}=\frac{s / 2}{1+s+\left(2 \Delta_{f} / \Gamma\right)^{2}}
$$

with the saturation parameter $s=2 \Omega_{f}^{2} / \Gamma^{2}$ and the decay rate $\Gamma=1 / \tau$ of the $\mathrm{P}_{1 / 2}$ state, which has a lifetime of $\tau \approx 7.1 \mathrm{~ns}$. All achievable phase shifts are reached within the oscillation period

$$
T=\frac{8 \pi \Delta_{f}}{\Omega_{f}^{2}}
$$

which therefore determines the longest duration of all required dephasing pulses. We further write $s=I / I_{\text {sat }}$ with (Foot, 2007)

$$
I_{\mathrm{sat}}=\frac{\pi}{3} \frac{h c}{\lambda^{3} \tau} .
$$

Inserting $\lambda=397 \mathrm{~nm}$ leads to $I_{\text {sat }}=46.8 \mathrm{~mW} / \mathrm{cm}^{2}$, and we find $s \approx 255$, which is consistent with independent measurements of the laser beam size in the focus and the intensities of the lasers entering the trap. For the experimental parameters $T=25 \mu \mathrm{s}$, $\Delta=2 \pi \times 400 \mathrm{GHz}$, we obtain an estimation of $3.5 \times 10^{-4}$ scattering events during the longest dephasing pulse. 
Hence, this dephasing method indeed does not affect the populations and describes the local dephasing operation,

$$
\begin{aligned}
\rho^{\prime}\left(t_{0}\right) & =(\Phi \otimes \mathbb{I}) \rho\left(t_{0}\right) \\
& =\sum_{i \in\{e, g\}}(|i\rangle\langle i| \otimes \mathbb{I}) \rho\left(t_{0}\right)(|i\rangle\langle i| \otimes \mathbb{I}) \\
& =\sum_{n=0}^{\infty} p_{n}\left[\left|u_{g g}^{1 n}\left(t_{0}, 0\right)\right|^{2}|g, n\rangle\left\langle g,\left.n|+| u_{e g}^{1 n}\left(t_{0}, 0\right)\right|^{2} \mid e, n+1\right\rangle\langle e, n+1|\right] .
\end{aligned}
$$

This produces the reference state $\rho^{\prime}\left(t_{0}\right)$ which differs from the original state $\rho\left(t_{0}\right)$ only by lacking quantum discord. The trace-distance dephasing disturbance (3.7) is easily evaluated and yields

$$
D\left(\rho\left(t_{0}\right)\right)=\sum_{n=0}^{\infty} p_{n}\left|u_{e g}^{1 n}\left(t_{0}, 0\right) u_{g g}^{1 n}\left(t_{0}, 0\right)\right| .
$$

\subsubsection{Detection}

Finally, the correlations (3.66) are detected by monitoring the subsequent time evolution in the local subsystem after dephasing. At $t_{0}$ there is no difference between the local states with and without dephasing, as confirmed by the state tomography in figure $3.3 \mathrm{c}$ ). The local time evolution of the excited state population

$$
\begin{aligned}
& \left\langle e\left|\rho_{S}^{\prime}\left(t_{1}+t_{0}\right)\right| e\right\rangle \\
= & \left\langle e\left|\operatorname{Tr}_{E}\left\{U^{(+1)}\left(t_{1}+t_{0}, t_{0}\right) \rho^{\prime}\left(t_{0}\right) U^{(+1)}\left(t_{1}+t_{0}, t_{0}\right)^{\dagger}\right\}\right| e\right\rangle \\
= & \sum_{n=0}^{\infty} p_{n}\left[\left|u_{g g}^{1 n}\left(t_{0}, 0\right)\right|^{2}\left|u_{e g}^{1 n}\left(t_{1}+t_{0}, t_{0}\right)\right|^{2}+\left|u_{e g}^{1 n}\left(t_{0}, 0\right)\right|^{2}\left|u_{g g}^{1 n}\left(t_{1}+t_{0}, t_{0}\right)\right|^{2}\right],
\end{aligned}
$$

can differ significantly from the evolution of the unperturbed initial state. This difference

$$
\begin{aligned}
d_{e}\left(t_{0}, t_{1}\right) & =\left\langle e\left|\rho_{S}\left(t_{1}+t_{0}\right)-\rho_{S}^{\prime}\left(t_{1}+t_{0}\right)\right| e\right\rangle \\
& =\sum_{n=0}^{\infty} p_{n}\left[u_{e g}^{1 n}\left(t_{0}, 0\right)^{*} u_{g g}^{1 n}\left(t_{0}, 0\right) u_{e g}^{1 n}\left(t_{1}+t_{0}, t_{0}\right) u_{g g}^{1 n}\left(t_{1}+t_{0}, t_{0}\right)+\text { c.c. }\right]
\end{aligned}
$$

directly determines the local trace distance

$$
d\left(t_{0}, t_{1}\right)=\left\|\rho_{S}\left(t_{1}+t_{0}\right)-\rho_{S}^{\prime}\left(t_{1}+t_{0}\right)\right\|=\left|d_{e}\left(t_{0}, t_{1}\right)\right|,
$$

since the coherences in the qubit system are always zero. Any nonzero value of $d\left(t_{0}, t_{1}\right)$ establishes a witness of quantum discord in the initial state $\rho\left(t_{0}\right)$, and provides a lower bound to equation (3.66). Figure 3.4 compares the measured local trace distance evolution with the theoretical prediction obtained from the parameters of the measured, 

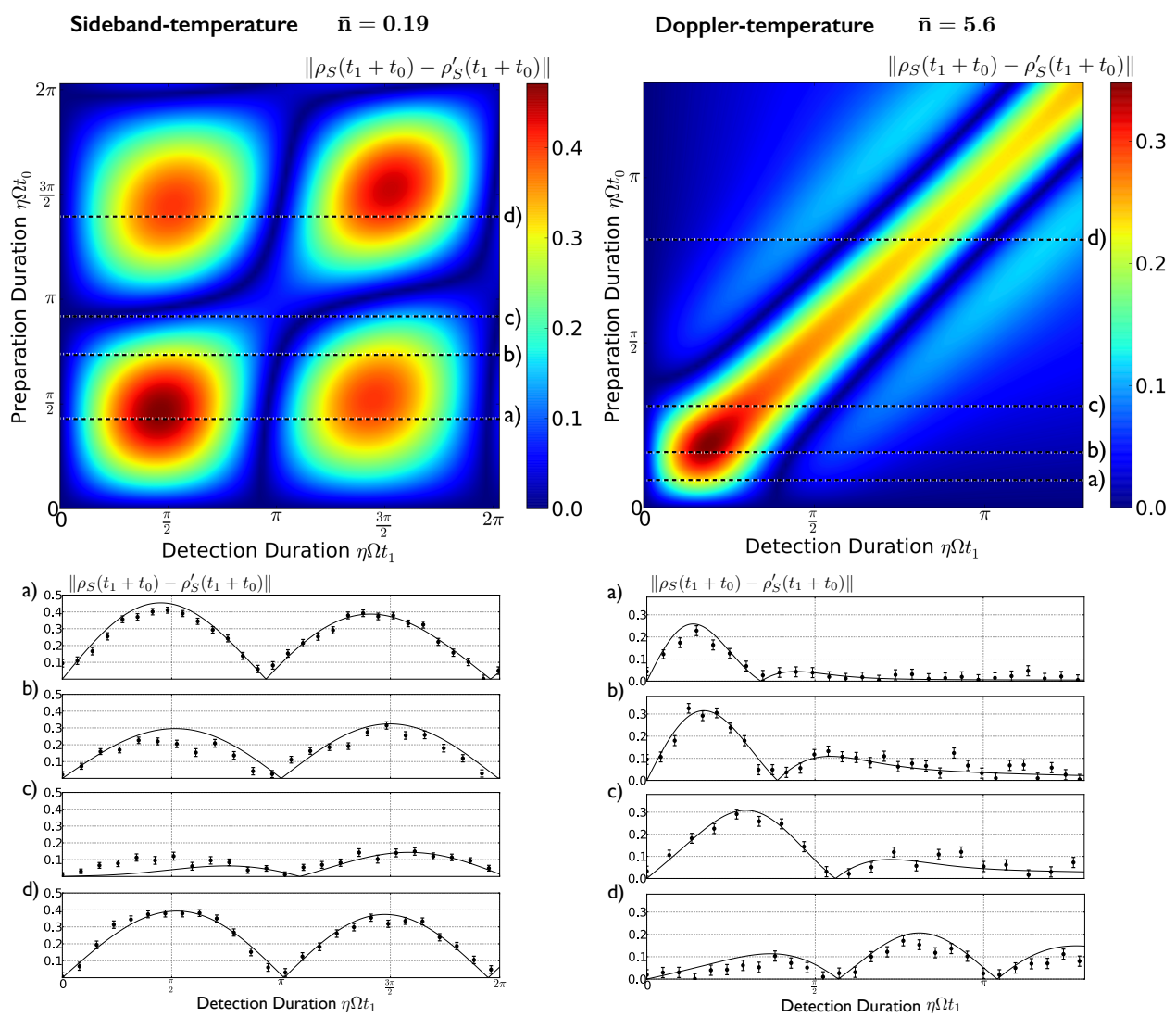

FIGURE 3.4.: The local trace distance is theoretically predicted [equation (3.69)] for the parameters obtained from independent measurements of the unperturbed Rabi oscillations. After different preparation times, we employ the local detection protocol and monitor the local trace distance experimentally, for two different initial environmental temperature states. The error bars label statistical errors over 1000 experimental repetitions per timestep. Adapted from (Gessner et al., 2014a).

unperturbed Rabi oscillation. The parameters are $\Omega \approx 2 \pi \times 100 \mathrm{kHz}$ and $\eta=0.04$. We find strong signatures of the correlations in the local dynamics, even for the highertemperature environment, which was prepared only by Doppler cooling. When additionally sideband cooling is implemented, the deviations almost reach the maximum value of $1 / 2$.

To find the closest lower bound on the dephasing disturbance of the total states for each initial state, characterized by $t_{0}$, we maximize the local trace distance over all measured times $t_{1}$. The result can be seen in figure 3.5. The local witness almost reaches the actual value of the global correlations for the low-temperature environment. In the case of higher temperature, the contribution of many different frequencies to 

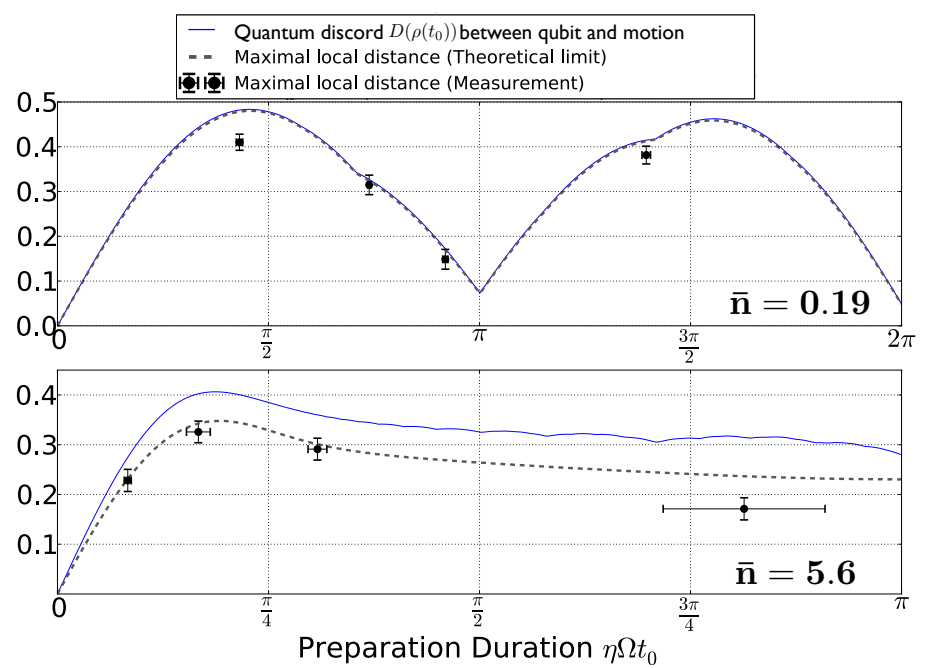

FIgURE 3.5.: The maximum local trace distance provides a lower bound for the dephasing disturbance (3.7). While this bound is almost tight for the low-temperature initial state, the contributions of many different Rabi frequencies limit the reachable maximum value at finite temperatures; see also section 3.2.3.4 for further discussions. Adapted from (Gessner et al., 2014a).

the time evolution does not lead to a full revival. The dashed line shows the theoretically computed upper limit for the maximum local distance within an experimentally motivated, observable time window, which shows that our measurements provide the experimentally closest possible lower bound.

\subsubsection{Temperature dependence of the local signal}

In the experiment, the local signature of the correlations was studied for two different environmental temperatures. The case of a sideband-cooled environment is special in a sense that we almost couple to a pure Fock state, which allows to adjust the detection duration $t_{1}$ such that the local bound is tight (Gessner et al., 2014a). In general, this is not possible, since each environmental state couples the system with a different Rabi frequency, which can be seen from the dependence of $\Omega_{n}^{(m)}$ on $n$ in equations (3.33) and (3.40). For the first blue sideband, this dependence is approximately given by $\Omega_{n}^{(1)} \approx \eta \sqrt{n+1} \Omega$, when $\eta \ll 1$. Does this mean that the local signature of the initial correlations vanishes in the high-temperature limit? In fact, numerical analysis of the local signal (3.69) suggests that the locally accessible lower bound converges to a finite value in the present scenario when $\bar{n} \rightarrow \infty$, as we will show in the following.

To estimate the temperature-dependence of the local signal we consider $\delta=0$, and, for convenience we fix the preparation and detection times to $t_{0}=t_{1}=\eta \Omega \pi / 2$. After inserting these parameters, together with equations (3.56) and (3.57) with $m=1$ into 


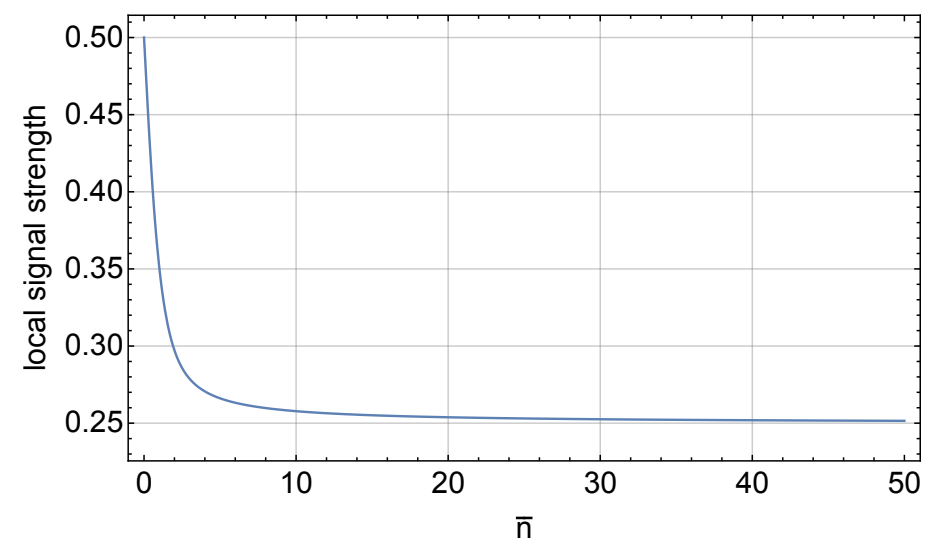

FiguRE 3.6.: The local signal strength, as quantified by the trace distance (3.70), is plotted for the special case where preparation time and detection time are both chosen as $t_{0}=t_{1}=\eta \Omega \pi / 2$. While for $\bar{n}=0$ this leads to the maximum value of $1 / 2$, the signal decays as $\bar{n}$ increases, which was also experimentally observed in figures 3.3 and 3.5 for $\bar{n} \approx 5.6$. For larger values of $\bar{n}$, the signal does not decay to zero and instead reaches an asymptotic value of $1 / 4$. The sum in equation (3.70) was evaluated numerically over 10000 environmental states.

the expression (3.69) for the local signal, we obtain

$$
\left.d\left(t_{0}, t_{1}\right)\right|_{t_{0}=t_{1}=\eta \Omega \pi / 2}=\frac{1}{2} \sum_{n=0}^{\infty} \frac{\bar{n}^{n}}{(\bar{n}+1)^{n+1}} \sin ^{2}\left(\frac{\pi \sqrt{n+1}}{2}\right) .
$$

One can indeed readily verify that the above choice of $t_{0}$ and $t_{1}$, which corresponds to the $\pi / 2$-time of the ground-state Rabi oscillation on the first sideband, leads to the maximum local signal of $d=1 / 2$ when $\bar{n}=0$; see also the supplementary material of (Gessner et al., 2014a) for further details. To observe the dependence on temperature, we plot equation (3.70) as a function of $\bar{n}$ in figure 3.6. We find that after an initial reduction of the local signal, the recordable local signal approaches the finite value $1 / 4$ in the limit $\bar{n} \rightarrow \infty$, and does not decay to zero as one might have expected. Note that it might even be possible to achieve a stronger signal by changing the parameters $t_{0}$ and $t_{1}$. In the limit $\bar{n} \rightarrow \infty$, an increasing number of environmental states become relevant for the dynamics of the electronic two-level system. While this result confirms the efficacy of the method also in the limit of large effective environmental dimensions, extrapolation of these results to even higher values of $\bar{n}$ would require a careful analysis of the validity of previously made assumptions, such as the time-averaged, effectively static potential, and the controlled resonant interaction of the ion with a laser.

Note that, in the light of the discussion of section 3.1.3, this result also implies that the present Jaynes-Cummings-type interaction between system and environment is not generic in the sense that it is not well described by the Haar-measure average over all unitary evolutions, since in that case we expect the signal to approach zero in the high-dimensional limit; see also (Gessner, 2011). 


\subsubsection{Time-averaged local Hilbert-Schmidt distance}

In the previous sections, we have discussed the local detection of trace-distance based quantum discord based on the contraction property. When instead the Hilbert-Schmidt distance is employed, an interesting relation between the total distance and the timeaveraged local distance can be derived. Nevertheless, we must recall the caveat mentioned already in section 3.1.3, which implies that the absolute information content of the Hilbert-Schmidt distance is limited when employed as a formal informationtheoretic measure of discord-type correlations (Piani, 2012).

The dephasing disturbance, expressed by the Hilbert-Schmidt distance reads

$$
\begin{aligned}
D_{H S}\left(\rho\left(t_{0}\right)\right) & =\left\|\rho\left(t_{0}\right)-\rho^{\prime}\left(t_{0}\right)\right\|_{2}^{2} \\
& =2 \sum_{n=0}^{\infty} p_{n}^{2}\left|u_{e g}^{1 n}\left(t_{0}, 0\right)\right|^{2}\left|u_{g g}^{1 n}\left(t_{0}, 0\right)\right|^{2} .
\end{aligned}
$$

As mentioned in section 3.1.3, employing methods from random matrix theory, one can show that the unitary Haar-measure average of the local Hilbert-Schmidt distance reproduces the total Hilbert-Schmidt distance except for a dimension-dependent prefactor (Gessner and Breuer, 2011; Gessner and Breuer, 2013a; Gessner and Breuer, 2013b), see equation (3.21). Assuming an ergodicity-type connection between the average over all unitary time evolution operators and the time average for a given Hamiltonian, one can expect to draw similar conclusions from the time average (Gessner and Breuer, 2013b).

The local Hilbert-Schmidt distance is given by

$$
\left\|\rho_{S}\left(t_{1}+t_{0}\right)-\rho_{S}^{\prime}\left(t_{1}+t_{0}\right)\right\|_{2}^{2}=2\left|d_{e}\left(t_{0}, t_{1}\right)\right|^{2} .
$$

For on-resonance sideband interactions $(\delta=0)$, this yields

$$
\begin{aligned}
& \left\|\rho_{S}\left(t_{0}+t_{1}\right)-\rho_{S}^{\prime}\left(t_{0}+t_{1}\right)\right\|_{2}^{2} \\
= & \frac{1}{2} \sum_{n, m} p_{n} p_{m} \sin \left(\Omega_{n}^{(1)} t_{0}\right) \sin \left(\Omega_{n}^{(1)} t_{1}\right) \sin \left(\Omega_{m}^{(1)} t_{0}\right) \sin \left(\Omega_{m}^{(1)} t_{1}\right) .
\end{aligned}
$$

The time average over $t_{1}$ can be evaluated analytically, using

$$
\begin{aligned}
& \left\langle\sin \left(\Omega_{n}^{(1)} t_{1}\right) \sin \left(\Omega_{m}^{(1)} t_{1}\right)\right\rangle_{t_{1}} \\
= & \frac{1}{2} \lim _{T \rightarrow \infty} \frac{1}{T} \int_{0}^{T} d t_{1}\left[\cos \left(\left(\Omega_{n}^{(1)}-\Omega_{m}^{(1)}\right) t_{1}\right)-\cos \left(\left(\Omega_{n}^{(1)}+\Omega_{m}^{(1)}\right) t_{1}\right)\right] \\
= & \frac{1}{2} \delta_{\Omega_{n}^{(1)} \Omega_{m}^{(1)} .}
\end{aligned}
$$

For relevant frequencies of low-temperature thermal states, $\Omega_{n}^{(1)}$ and $\Omega_{m}^{(1)}$ coincide only 


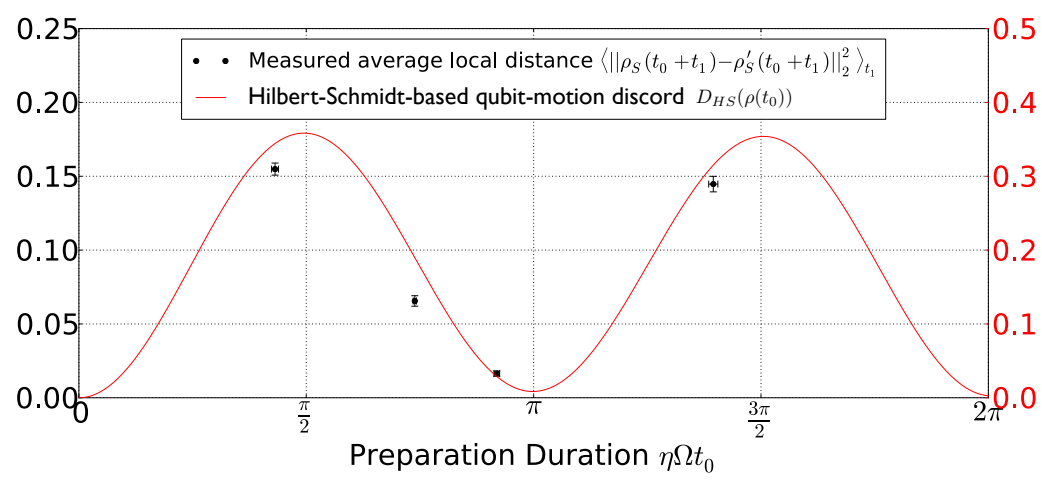

FiguRE 3.7.: The measurement data is evaluated in terms of the Hilbert-Schmidt distance instead of the trace distance, which shows that the local time average (3.75) is proportional to the Hilbert-Schmidt distance (3.71) of the total states. Adapted from (Gessner et al., 2014a).

if $n=m$. We finally obtain

$$
\begin{aligned}
\left\langle\left\|\rho_{S}\left(t_{0}+t_{1}\right)-\rho_{S}^{\prime}\left(t_{0}+t_{1}\right)\right\|_{2}^{2}\right\rangle_{t_{1}} & =\sum_{n} p_{n}^{2} \cos ^{2}\left(\frac{\Omega_{n}^{(1)} t_{0}}{2}\right) \sin ^{2}\left(\frac{\Omega_{n}^{(1)} t_{0}}{2}\right) \\
& =\frac{1}{2}\left\|\rho\left(t_{0}\right)-\rho^{\prime}\left(t_{0}\right)\right\|_{2}^{2} \\
& =\frac{1}{2} D_{H S}\left(\rho\left(t_{0}\right)\right) .
\end{aligned}
$$

As anticipated, the time-average of the local Hilbert-Schmidt distance over the detection time $t_{1}$ is directly proportional to $D_{H S}\left(\rho\left(t_{0}\right)\right)$. The corresponding experimental data is shown in figure 3.7 .

\subsubsection{Generalization to arbitrary sidebands}

The theoretical treatment from the previous sections can be extended to the dynamics on arbitrary red and blue sidebands. Starting from the uncorrelated, thermal state (3.58), a pulse on the $m$ th red sideband produces the correlated state

$$
\begin{aligned}
& \rho\left(t_{0}\right)=U^{(-m)}\left(t_{0}, 0\right) \rho_{0} U^{(-m)}\left(t_{0}, 0\right)^{\dagger} \\
&=\sum_{n=0}^{\infty} p_{n+m} {\left[\left|u_{g g}^{m n}\left(t_{0}, 0\right)\right|^{2}|g, n+m\rangle\langle g, n+m|\right.} \\
&+u_{e g}^{m n}\left(t_{0}, 0\right)^{*} u_{g g}^{m n}\left(t_{0}, 0\right)|g, n+m\rangle\langle e, n| \\
&+u_{e g}^{m n}\left(t_{0}, 0\right) u_{g g}^{m n}\left(t_{0}, 0\right)^{*}|e, n\rangle\langle g, n+m| \\
&\left.+\left|u_{e g}^{m n}\left(t_{0}, 0\right)\right|^{2}|e, n\rangle\langle e, n|\right] .
\end{aligned}
$$


Note that, if the initial state is prepared with low temperature, there will be almost no signal from the red sideband pulse, since the first $m$ populations are skipped in the sum. This expresses the simple fact that there are no resonant red sideband states to couple to, when the ion is already prepared in $|g, 0\rangle$. For blue sideband transitions, we can easily generalize (3.59) to arbitrary $m$ beyond 1 . Both cases of red and blue sidebands can be combined to a single expression as

$$
\begin{aligned}
\rho\left(t_{0}\right)= & U^{(s)}\left(t_{0}, 0\right) \rho(0) U^{(s)}\left(t_{0}, 0\right)^{\dagger} \\
=\sum_{n=0}^{\infty} p_{n+x}[ & \left|u_{g g}^{m n}\left(t_{0}, 0\right)\right|^{2}|g, n+x\rangle\langle g, n+x| \\
& +u_{e g}^{m n}\left(t_{0}, 0\right)^{*} u_{g g}^{m n}\left(t_{0}, 0\right)|g, n+x\rangle\langle e, n+x+s| \\
& +u_{e g}^{m n}\left(t_{0}, 0\right) u_{g g}^{m n}\left(t_{0}, 0\right)^{*}|e, n+x+s\rangle\langle g, n+x| \\
& \left.+\left|u_{e g}^{m n}\left(t_{0}, 0\right)\right|^{2}|e, n+x+s\rangle\langle e, n+x+s|\right],
\end{aligned}
$$

with $m=|s|$ and $x=\max \{0,-s\}$. The corresponding reduced state of the two-level system is for $s \neq 0$ given by

$$
\rho_{S}\left(t_{0}\right)=\operatorname{Tr}_{E} \rho\left(t_{0}\right)=\sum_{n=0}^{\infty} p_{n+x}\left[\left|u_{g g}^{m n}\left(t_{0}, 0\right)\right|^{2}|g\rangle\left\langle\left. g|+| u_{e g}^{m n}\left(t_{0}, 0\right)\right|^{2} \mid e\right\rangle\langle e|\right] .
$$

This state is always diagonal in the computational basis $|g\rangle$ and $|e\rangle$. The reference state after dephasing yields

$$
\begin{aligned}
\rho^{\prime}\left(t_{0}\right)= & (\Phi \otimes \mathbb{I}) \rho\left(t_{0}\right) \\
= & \sum_{n=0}^{\infty} p_{n+x}\left[\left|u_{g g}^{m n}\left(t_{0}, 0\right)\right|^{2}|g, n+x\rangle\langle g, n+x|\right. \\
& \left.\quad+\left|u_{e g}^{m n}\left(t_{0}, 0\right)\right|^{2}|e, n+x+s\rangle\langle e, n+x+s|\right] .
\end{aligned}
$$

This leads to a trace distance of

$$
D\left(\rho\left(t_{0}\right)\right)=\left\|\rho\left(t_{0}\right)-\rho^{\prime}\left(t_{0}\right)\right\|=\sum_{n=0}^{\infty} p_{n+x}\left|u_{e g}^{m n}\left(t_{0}, 0\right) u_{g g}^{m n}\left(t_{0}, 0\right)\right|,
$$

and a squared Hilbert-Schmidt distance of

$$
D_{H S}\left(\rho\left(t_{0}\right)\right)=\left\|\rho\left(t_{0}\right)-\rho^{\prime}\left(t_{0}\right)\right\|_{2}^{2}=2 \sum_{n=0}^{\infty} p_{n+x}^{2}\left|u_{e g}^{m n}\left(t_{0}, 0\right)\right|^{2}\left|u_{g g}^{m n}\left(t_{0}, 0\right)\right|^{2} .
$$

Analogous to the considerations in the previous section, we find for the generalized expression for the excited state population after a subsequent time evolution

$$
\begin{aligned}
& \left\langle e\left|\rho_{S}^{\prime}\left(t_{1}+t_{0}\right)\right| e\right\rangle \\
= & \left\langle e\left|\operatorname{Tr}_{E}\left\{U^{(s)}\left(t_{1}+t_{0}, t_{0}\right) \rho^{\prime}\left(t_{0}\right) U^{(s)}\left(t_{1}+t_{0}, t_{0}\right)^{\dagger}\right\}\right| e\right\rangle \\
= & \sum_{n=0}^{\infty} p_{n+x}\left[\left|u_{g g}^{m n}\left(t_{0}, 0\right)\right|^{2}\left|u_{e g}^{m n}\left(t_{1}+t_{0}, t_{0}\right)\right|^{2}+\left|u_{e g}^{m n}\left(t_{0}, 0\right)\right|^{2}\left|u_{g g}^{m n}\left(t_{1}+t_{0}, t_{0}\right)\right|^{2}\right] .
\end{aligned}
$$


The local trace- and Hilbert-Schmidt distances can then be evaluated using equations (3.69) and (3.72), based on the resulting difference in the excited state population, which yields

$$
\begin{aligned}
d_{e}\left(t_{0}, t_{1}\right) & =\left\langle e\left|\rho_{S}\left(t_{1}+t_{0}\right)-\rho_{S}^{\prime}\left(t_{1}+t_{0}\right)\right| e\right\rangle \\
& =\sum_{n=0}^{\infty} p_{n+x}\left[u_{e g}^{m n}\left(t_{0}, 0\right)^{*} u_{g g}^{m n}\left(t_{0}, 0\right) u_{e g}^{m n}\left(t_{1}+t_{0}, t_{0}\right) u_{g g}^{m n}\left(t_{1}+t_{0}, t_{0}\right)+\text { c.c. }\right]
\end{aligned}
$$

\subsubsection{Extension of the experimental technique}

The experimental techniques, developed in the course of the described trapped-ion experiment, enable to dephase in the computational basis of the qubit. In section 3.1.2, we have seen that, in order to avoid overestimation of the discord-type correlations for all classes of mixed states, one should consider dephasing over a large set of basis sets. Dephasing in different bases is also important to increase the flexibility of the method to ensure applicability in situations where the local eigenbasis is not the computational basis. In this section we briefly discuss how the AC-Stark shift method can be easily extended to achieve this.

To induce dephasing in a different basis, a combination of the AC-Stark shift dephasing procedure with single-qubit unitary rotations is sufficient. Let us assume that dephasing $\Phi_{\Pi_{A}}$ can be implemented in the basis $\Pi_{A}=\{|1\rangle\langle 1|| 2\rangle,\langle 2|, \ldots\}$ (recall equation (3.10) for the definition of the notation). To achieve dephasing in another basis $\Pi_{B}=\left\{\left|\varphi_{1}\right\rangle\left\langle\varphi_{1}|,| \varphi_{2}\right\rangle\left\langle\varphi_{2}\right|, \ldots\right\}$, we consider the unitary operation,

$$
U=\sum_{i}\left|\varphi_{i}\right\rangle\langle i|
$$

which rotates one basis into the other. As discussed in section 2.2.1, on trapped-ion qubits such operations are typically implemented with fidelities of 0.995 (Schindler et al., 2013). The desired dephasing operation $\Phi_{\Pi_{B}}$ is then achieved as a combination of unitary rotations and the implementable dephasing $\Phi_{\Pi_{A}}$ as (Gessner et al., 2014a)

$$
\begin{aligned}
U \Phi_{\Pi_{A}}\left(U^{\dagger} X U\right) U^{\dagger} & =\sum_{i j k l m}\left|\varphi_{i}\right\rangle\langle i \mid k\rangle\langle k \mid l\rangle\left\langle\varphi_{l}|X| \varphi_{m}\right\rangle\langle m \mid k\rangle\langle k \mid j\rangle\left\langle\varphi_{j}\right| \\
& =\sum_{i}\left|\varphi_{i}\right\rangle\left\langle\varphi_{i}|X| \varphi_{i}\right\rangle\left\langle\varphi_{i}\right|=\Phi_{\Pi_{B}}(X)
\end{aligned}
$$

\subsection{Photonic experiment}

After having discussed the experimental realization of the local detection protocol with a single trapped ion, we now turn to an implementation with photons, which are sent through linear and nonlinear optical elements. This section describes an experiment which was carried out in the Key Laboratory of Quantum Information in Hefei, China (Tang et al., 2013). Since photons are often used as traveling carriers of quantum information (Duan et al., 2001), which can be sent over long distances, it is important to 
characterize the correlations encoded into their quantum states under experimentally realistic constraints. When photons are sent through noisy environments, decoherence effects can be detrimental to the capacity of the quantum channel. A turbulent atmosphere can cause decay of orbital angular momentum entanglement (Paterson, 2005), but also the typically more robust polarization qubits are subject to decoherence in optical fibers.

Decoherence is a manifestation of interaction with another degree of freedom, which produces correlated states that, on the level of the reduced system, appear as incoherent mixtures. As we have seen in the previous sections, these correlations can influence the time evolution of the reduced system and thereby be revealed. In the present experiment, we consider a photon in a birefringent fiber, which induces interactions between the polarization qubit and the photon's frequency degree of freedom. Formally, this resembles the coupling of a two-level system to a harmonic oscillator which we discussed in the previous section-however, here, the environment is composed of a continuum of modes, instead of a single mode as the harmonic motion of the trapped ion.

For the experiment we apply the local detection method in a typical two-party quantum communication scenario. We suppose that Alice prepares a photon in a possibly correlated state of qubit and motion and sends it to Bob. Being only able to access the qubit degree of freedom, Bob then tries to identify and estimate these correlations making use of interactions between both degrees of freedom, which, in the spirit of open-system theory, we consider system (polarization qubit) and environment (continuum of frequency modes).

\subsubsection{The pre-initial state}

On Alice's side, the single photons are created by a quantum dot, where spectral diffusion $^{1}$ (Sallen et al., 2010) generates a mixed frequency state, given by a sum over closely spaced frequencies (Tang et al., 2013),

$$
\rho_{E}=\sum_{\omega} \Delta \omega G(\omega)|\omega\rangle\langle\omega|
$$

with a Lorentzian line shape

$$
G(\omega)=\frac{1}{\pi} \frac{\delta \omega}{\delta \omega^{2}+\left(\omega-\omega_{0}\right)^{2}},
$$

and measured parameters of $\omega_{0}=2 \pi c /(914 \mathrm{~nm})$, and $1 / \delta \omega=9.703 \pm 0.124 \mathrm{ps,}$ with $c$ denoting the speed of light.

To ensure proper normalization of $\rho_{E}$, we have introduced a description by a discrete sum of frequency states $|\omega\rangle$, which form a complete orthonormal set, that is, $\left\langle\omega \mid \omega^{\prime}\right\rangle=$ $\delta_{\omega, \omega^{\prime}}$ and $\sum_{\omega}|\omega\rangle\langle\omega|=\mathbb{I}$. Positivity and normalization of $\rho_{E}$ are warranted since $\Delta \omega G(\omega) \geq 0$ and $\sum_{\omega} \Delta \omega G(\omega)=1$. After determining the relevant quantities, such as the trace distance, we will perform the continuum limit $\Delta \omega \rightarrow 0$.

\footnotetext{
${ }^{1}$ Random jumps of a narrow resonance caused by environmental fluctuations.
} 


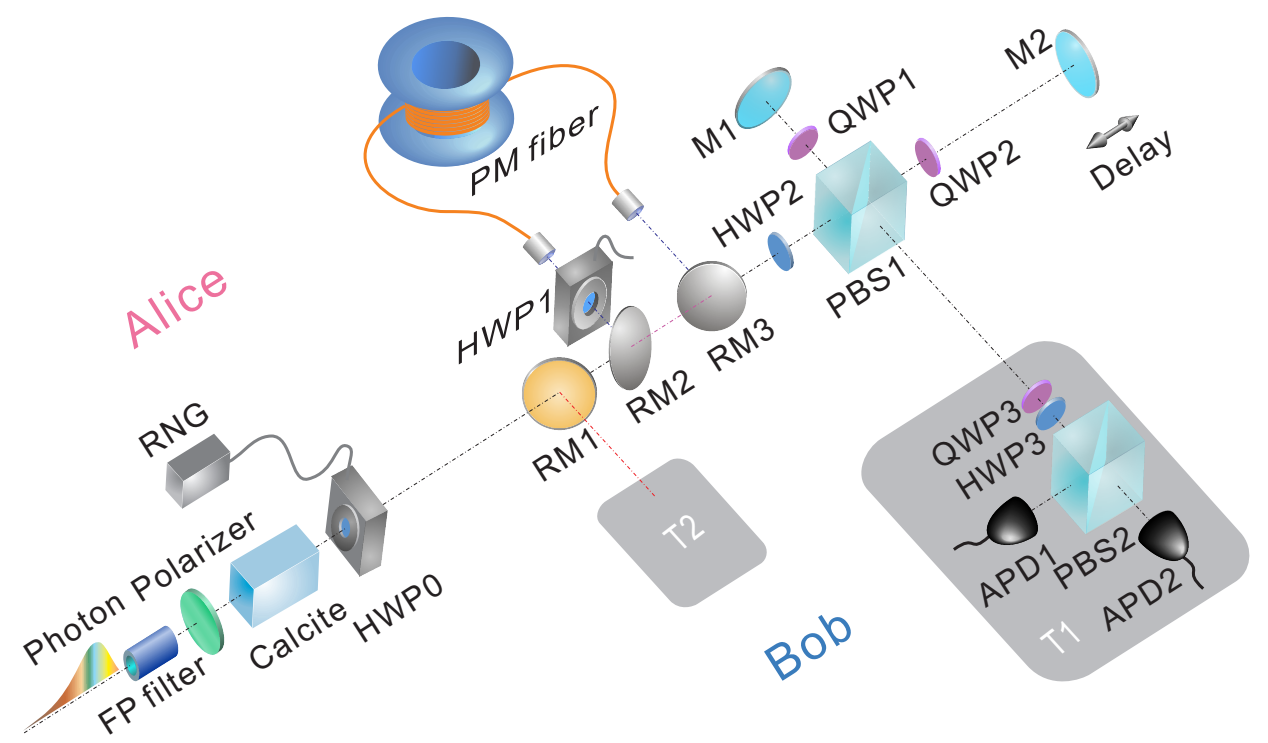

FIGURE 3.8.: Alice prepares a polarization-frequency correlated single photon state using a combination of polarizer and calcite crystal. A half-wave plate (HWP0) whose orientation is driven by a random number generator hides Alice's choice for the local eigenbasis before the state is sent to Bob whose task it is to detect and estimate the correlations with local operations on the qubit degree of freedom. Removable mirrors (RM1, RM2 and RM3) are employed to realize the different steps of the local detection protocol. First, qubit tomography (T2) is performed to obtain the local eigenbasis. The dephasing is performed using a half-wave plate (HWP1) followed by a long polarization-maintaining fiber. Original and dephased state are submitted to a unitary time evolution generated by a Michelson interferometer, which consists in another half-wave plate (HWP2), a polarizing beam splitter (PBS1), and two interferometer arms comprised of mirrors (M1 and M2) and quarter-wave plates (QWP1 and QWP2). The second mirror M2 is movable to vary the phase shift. Finally, another qubit tomography reveals whether the removal of discord-type correlations by local dephasing had an observable effect on the subsequent qubit dynamics, which then serves as a witness and lower bound for the correlations in the initial state. The tomography sections (T1 and T2) consist of quarter-wave plate (QWP3) and half-wave plate (HWP3), a polarizing beam splitter (PBS2) and two avalanche single photon detectors (APD1 and APD2). Adapted from (Tang et al., 2013).

The total pre-initial state describes an uncorrelated product state, $\rho_{p i}=\rho_{0} \otimes \rho_{E}$, where the state of the polarization qubit is represented in the basis $\{|H\rangle,|V\rangle\}$ as

$$
\rho_{0}=\left(\begin{array}{cc}
\alpha & \beta e^{i \varphi} \\
\beta e^{-i \varphi} & 1-\alpha
\end{array}\right) .
$$

The states $|H\rangle$ and $|V\rangle$ represent horizontal and vertical polarization eigenstates, re- 
spectively. The parameters $\beta$ and $\alpha$ describe the qubit quantum state. While the following theoretical treatment is applicable for arbitrary values of $\alpha, \beta \in \mathbb{R}$ (as long as $\rho_{0}$ describes a valid quantum state), for the ease of notation, we will restrict to the case of $\alpha=1 / 2$, which describes the experiment as well. This equal superposition between $|H\rangle$ and $|V\rangle$ is realized by sending the qubit through a $45^{\circ}$ polarization plate.

\subsubsection{Preparation of the initial state}

In order to generate polarization-frequency correlations, Alice sends the pre-initial state,

$$
\begin{aligned}
\rho_{p i}=\sum_{\omega} \Delta \omega G(\omega)( & \frac{1}{2}|H, \omega\rangle\left\langle H, \omega\left|+\beta e^{i \varphi}\right| H, \omega\right\rangle\langle V, \omega| \\
& \left.+\beta e^{-i \varphi}|V, \omega\rangle\left\langle H, \omega\left|+\frac{1}{2}\right| V, \omega\right\rangle\langle V, \omega|\right),
\end{aligned}
$$

through a birefringent calcite crystal of length $L$. The induced interaction between polarization and frequency degrees of freedom is described by

$$
U_{\text {cal }}(t):\left\{\begin{array}{l}
|H, \omega\rangle \rightarrow|H, \omega\rangle \\
|V, \omega\rangle \rightarrow e^{-i \omega t}|V, \omega\rangle
\end{array}\right.
$$

where the time spent inside the crystal is given by $t=\Delta n_{\text {cal }} L / c$. The birefringence $\Delta n_{\text {cal }}$ describes the difference between the refraction indices for the two polarization states and can be measured from the shift of the single-photon spectra with and without the calcite crystal in a Michelson interferometer (Tang et al., 2013). The experimental parameters are $\Delta n_{\text {cal }}=0.179 \pm 0.001$ and $L=35.92 \mathrm{~mm}$. This evolution results in the generation of the correlated initial state

$$
\begin{aligned}
\rho_{S E}= & U_{\text {cal }}(t) \rho_{p i} U_{\text {cal }}^{\dagger}(t) \\
=\sum_{\omega} \Delta \omega G(\omega)( & \frac{1}{2}|H, \omega\rangle\left\langle H, \omega\left|+\beta e^{i(\omega t+\varphi)}\right| H, \omega\right\rangle\langle V, \omega| \\
& \left.\quad+\beta e^{-i(\omega t+\varphi)}|V, \omega\rangle\left\langle H, \omega\left|+\frac{1}{2}\right| V, \omega\right\rangle\langle V, \omega|\right) .
\end{aligned}
$$

The reduced state for the qubit is obtained by tracing over the frequency degrees of freedom,

$$
\rho_{S}=\left(\begin{array}{cc}
1 / 2 & \beta C(t) e^{i \Psi(t)} \\
\beta C(t) e^{-i \Psi(t)} & 1 / 2
\end{array}\right)
$$

with

$$
C(t)=\sum_{\omega} \Delta \omega G(\omega) e^{i\left(\omega-\omega_{0}\right) t}
$$


and $\Psi(t)=\omega_{0} t+\varphi$. The initial phase $\varphi$ can be adjusted, such that it cancels the time-dependent phase for any fixed time $t$, that is, $\Psi(t) \equiv 0$, which implies the choice $\varphi=-\omega_{0} t$. The function $C(t)$ is real valued since $G(\omega)$ is symmetric about $\omega=\omega_{0}$.

Before this state is sent to Bob, Alice hides the choice of her local eigenbasis by performing an additional random local unitary basis rotation of the qubit (HWP0 in figure 3.8). This rotation does not affect the correlation properties of the quantum state. Formally, this rotation can be taken into account by replacing the polarization eigenbasis $|H\rangle$ and $|V\rangle$ with the rotated eigenvectors. We can therefore neglect this additional local rotation in the theoretical treatment.

\subsubsection{Local dephasing operation}

The first step on Bob's side consists in obtaining the local eigenbasis of $\rho_{S}$. To this end the removable mirror RM1 is placed to guide the photon into the tomography section T2, see figure 3.8). Diagonalization of (3.92) yields the eigenvalues,

$$
\begin{aligned}
& \lambda_{0}=1 / 2+\beta C(t), \\
& \lambda_{1}=1 / 2-\beta C(t),
\end{aligned}
$$

and eigenvectors,

$$
\begin{aligned}
& |0\rangle=\frac{1}{\sqrt{2}}\left(\begin{array}{c}
1 \\
1
\end{array}\right)=\frac{1}{\sqrt{2}}(|H\rangle+|V\rangle), \\
& |1\rangle=\frac{1}{\sqrt{2}}\left(\begin{array}{c}
1 \\
-1
\end{array}\right)=\frac{1}{\sqrt{2}}(|H\rangle-|V\rangle) .
\end{aligned}
$$

Dephasing in the local eigenbasis is described by

$$
\Phi(X)=|0\rangle\langle 0|X| 0\rangle\langle 0|+| 1\rangle\langle 1|X| 1\rangle\langle 1| .
$$

This produces the classically correlated reference state (with $\varphi=-\omega_{0} t$, see above, in equation (3.91)),

$$
\begin{aligned}
\rho_{S E}^{\prime}=(\Phi \otimes \mathbb{I}) \rho_{S E} & \\
=\frac{1}{2} \sum_{\omega} \Delta \omega G(\omega) & {[|H, \omega\rangle\langle H, \omega|+| V, \omega\rangle\langle V, \omega|} \\
& +\beta\left(e^{i\left(\omega-\omega_{0}\right) t}+e^{-i\left(\omega-\omega_{0}\right) t}\right)|H, \omega\rangle\langle V, \omega| \\
& \left.+\beta\left(e^{-i\left(\omega-\omega_{0}\right) t}+e^{i\left(\omega-\omega_{0}\right) t}\right)|V, \omega\rangle\langle H, \omega|\right] .
\end{aligned}
$$

To realize the local dephasing operation on the polarization qubit, the photon is sent through a very long polarization-maintaining fiber. Considering the setup described in figure 3.8, this is achieved by removing the mirror RM1, and placing the mirrors RM2 and RM3. Before entering the fiber, the photon passes a computer-controlled half-wave plate (HWP1) which rotates the eigenstates $|0\rangle$ and $|1\rangle$ onto the principal axes of the fiber. The fiber also shows a small birefringence of $\Delta n_{\mathrm{pmf}}=3 \times 10^{-4}$, which 
we exploit to generate a dephasing effect after long interaction times. The interaction in the fiber is described by

$$
U_{\mathrm{pmt}}:\left\{\begin{array}{ll}
|0, \omega\rangle & \longrightarrow|0, \omega\rangle \\
|1, \omega\rangle & \longrightarrow e^{-i \omega s}|1, \omega\rangle
\end{array},\right.
$$

where $s=\Delta n_{\mathrm{pmf}} l / c \gg 1 / \delta \omega$ describes the time the photon spends inside the fiber. We first rewrite the effect of this dynamics in terms of $|H\rangle$ and $|V\rangle$,

$$
U_{\mathrm{pmt}}:\left\{\begin{array}{ll}
|H, \omega\rangle & \longrightarrow a|H, \omega\rangle+b|V, \omega\rangle \\
|V, \omega\rangle & \longrightarrow b|H, \omega\rangle+a|V, \omega\rangle
\end{array},\right.
$$

where we introduced

$$
a=\frac{1}{2}\left(1+e^{-i \omega s}\right), \quad b=\frac{1}{2}\left(1-e^{-i \omega s}\right) .
$$

This dynamics evolves the initial state (3.91), again with the choice $\varphi=-\omega_{0} t$, into the state

$$
\begin{aligned}
\rho_{S E}^{\prime \prime}=U_{\mathrm{pmt}} \rho_{S E} U_{\mathrm{pmt}}^{\dagger} & \\
=\sum_{\omega} \Delta \omega G(\omega)[ & \frac{1}{2}|H, \omega\rangle\left\langle H, \omega\left|+\frac{1}{2}\right| V, \omega\right\rangle\langle V, \omega| \\
& +\beta\left(e^{i\left(\omega-\omega_{0}\right) t} a b^{*}+e^{-i\left(\omega-\omega_{0}\right) t} a^{*} b\right)|H, \omega\rangle\langle H, \omega| \\
& +\beta\left(e^{i\left(\omega-\omega_{0}\right) t} a^{*} b+e^{-i\left(\omega-\omega_{0}\right) t} a b^{*}\right)|V, \omega\rangle\langle V, \omega| \\
& +\beta\left(e^{i\left(\omega-\omega_{0}\right) t}|a|^{2}+e^{-i\left(\omega-\omega_{0}\right) t}|b|^{2}\right)|H, \omega\rangle\langle V, \omega| \\
& \left.+\beta\left(e^{i\left(\omega-\omega_{0}\right) t}|b|^{2}+e^{-i\left(\omega-\omega_{0}\right) t}|a|^{2}\right)|V, \omega\rangle\langle H, \omega|\right] .
\end{aligned}
$$

To realize that this state indeed describes the desired dephased state $\rho_{S E}^{\prime}$, equation (3.97), to a good approximation, it is important to recall that the time spent inside the fiber is much larger than the inverse spectral width of the frequency state, $\delta \omega \cdot s \gg 1$. Hence, the rapid oscillations of the function

$$
|a|^{2}=\frac{1}{2}(1+\cos \omega s) \text {. }
$$

cannot be resolved since the uncertainty for the time $s$ in the fiber exceeds the period of this oscillation. We must therefore replace $|a|^{2}$ by its mean value, obtained by averaging over one period

$$
|a|^{2} \rightarrow\left\langle|a|^{2}\right\rangle=\frac{1}{2}
$$

Similarly,

$$
|b|^{2} \rightarrow\left\langle|b|^{2}\right\rangle=\frac{1}{2}, \quad a b^{*} \rightarrow\left\langle a b^{*}\right\rangle=0 .
$$

Inserting this into equation (3.101), we see that indeed $\rho_{S E}^{\prime \prime}$ reduces to $\rho_{S E}^{\prime}$, as given by equation (3.97). 


\subsubsection{Reduced distributions}

Before we proceed, we confirm that the reduced states of $\rho_{S E}$ and $\rho_{S E}^{\prime}$ coincide, which should be the case by construction of the local dephasing operation. To this end, we take the difference between the two total states

$$
\Delta \rho_{S E}=\rho_{S E}-\rho_{S E}^{\prime}=\sum_{\omega} \Delta \omega G(\omega) f(\omega)[|H, \omega\rangle\langle V, \omega|-| V, \omega\rangle\langle H, \omega|]
$$

where we introduced the imaginary-valued function

$$
f(\omega)=\frac{\beta}{2}\left(e^{i\left(\omega-\omega_{0}\right) t}-e^{-i\left(\omega-\omega_{0}\right) t}\right)=i \beta \sin \left[\left(\omega-\omega_{0}\right) t\right]
$$

The equivalence of the environmental states is readily confirmed due to the orthogonality of $|H\rangle$ and $|V\rangle$,

$$
\rho_{E}-\rho_{E}^{\prime}=\operatorname{Tr}_{S}\left\{\rho_{S E}-\rho_{S E}^{\prime}\right\}=0
$$

To confirm this also for the qubit states, we trace over the frequency degrees of freedom, obtaining

$$
\rho_{S}-\rho_{S}^{\prime}=\operatorname{Tr}_{E}\left\{\rho_{S E}-\rho_{S E}^{\prime}\right\}=\sum_{\omega} \Delta \omega G(\omega) f(\omega)[|H\rangle\langle V|-| V\rangle\langle H|]
$$

We now perform the continuum limit $\Delta \omega \rightarrow 0$, leading to

$$
\sum_{\omega} \Delta \omega G(\omega) f(\omega) \longrightarrow \int d \omega G(\omega) f(\omega)=0
$$

The integral vanishes since $G(\omega)$ is a symmetric about $\omega=\omega_{0}$, while $f(\omega)$ is antisymmetric. To summarize, we have confirmed that the reduced distributions of original and dephased states coincide for both the environmental degree of freedom and the qubit, $\rho_{S}-\rho_{S}^{\prime}=0$.

\subsubsection{Total trace distance}

To obtain the trace distance between the states $\rho_{S E}$ and $\rho_{S E}^{\prime}$, we diagonalize the operator $\Delta \rho_{S E}$, equation (3.105), which yields the eigenvalues

$$
\mu_{ \pm}(\omega)= \pm i \Delta \omega G(\omega) f(\omega)
$$

and eigenstates

$$
\left|\mu_{ \pm}(\omega)\right\rangle=\frac{1}{\sqrt{2}}(|H\rangle \pm i|V\rangle) \otimes|\omega\rangle
$$


The trace distance of $\rho_{S E}$ and $\rho_{S E}^{\prime}$ corresponds to the trace norm of $\Delta \rho_{S E}$

$$
\begin{aligned}
D\left(\rho_{S E}\right) & =\left\|\rho_{S E}-\rho_{S E}^{\prime}\right\| \\
& =\frac{1}{2} \operatorname{Tr} \sqrt{\Delta \rho_{S E}^{\dagger} \Delta \rho_{S E}} \\
& =\frac{1}{2} \sum_{\omega}\left(\left|\mu_{-}(\omega)\right|+\left|\mu_{+}(\omega)\right|\right) \\
& =\sum_{\omega}|\Delta \omega G(\omega) f(\omega)| \\
& =\frac{\beta}{2} \sum_{\omega} \Delta \omega G(\omega)\left|e^{i\left(\omega-\omega_{0}\right) t}-e^{-i\left(\omega-\omega_{0}\right) t}\right| .
\end{aligned}
$$

We finally perform the continuum limit to obtain the trace-distance dephasing disturbance (3.7),

$$
D\left(\rho_{S E}\right)=\frac{\beta}{2} \int d \omega G(\omega)\left|e^{i\left(\omega-\omega_{0}\right) t}-e^{-i\left(\omega-\omega_{0}\right) t}\right|,
$$

which quantifies the quantum discord in $\rho_{S E}$.

\subsubsection{Open-system evolution depending on initial correlations}

By now we have established a reference state, which was produced by local dephasing of the original, unknown state $\rho_{S E}$. We know that the two total states differ if the initial state contained discord-type correlations, but since we cannot measure the full state, we try to map the influence of the correlations onto the local, observable dynamics of the qubit. To this end, both, the original state $\rho_{S E}$ and the dephased state $\rho_{S E}^{\prime}$ are subjected to the same time evolution, which is observed locally on the qubit degree of freedom.

This dynamics is generated by a Michelson delay setup, which consists of a halfwave plate (HWP2) with an adjustable angle $\eta / 2$, followed by a polarizing beam splitter (PBS1), see figure 3.8. The beam splitter then sends the two polarization components $|\eta\rangle$ and $\left|\eta_{\perp}\right\rangle$, into different arms of an interferometer. The two polarization states accumulate a relative phase shift which depends on the difference of the two interferometer path lengths. This is described by

$$
U(\eta, \tau):\left\{\begin{array}{ll}
|\eta, \omega\rangle & \longrightarrow|\eta, \omega\rangle \\
\left|\eta_{\perp}, \omega\right\rangle & \longrightarrow e^{-i \omega \tau}\left|\eta_{\perp}, \omega\right\rangle
\end{array},\right.
$$

where $\tau=2 x / c$ correponds to the coordinate of the delayable mirror (M2). The half-wave plate HWP2 generates the basis vectors

$$
|\eta\rangle=\left(\begin{array}{c}
\cos \eta \\
\sin \eta
\end{array}\right), \quad\left|\eta_{\perp}\right\rangle=\left(\begin{array}{c}
-\sin \eta \\
\cos \eta
\end{array}\right) .
$$




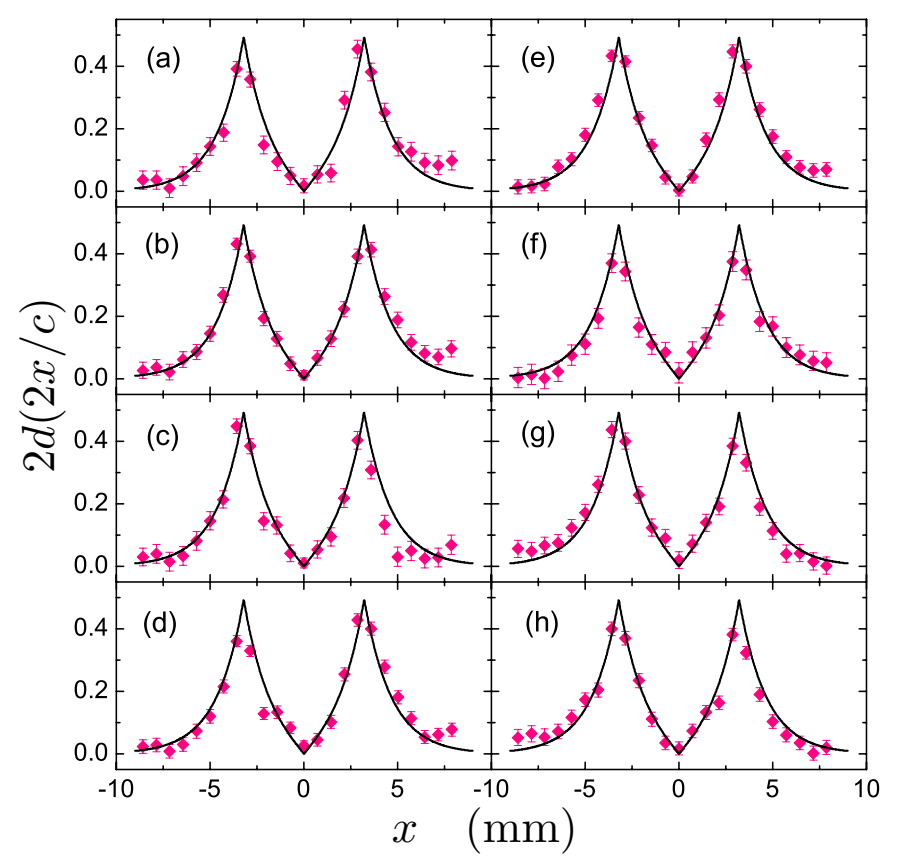

FIGURE 3.9.: The trace distance evolution of the qubit reveals the initial correlations to the local subsystem. The time variable $\tau=2 x / c$ is determined by the difference $x$ between the path lengths of the interferometer's arms, which is adjusted via the position of the mirror M2, see figure 3.8. The plots (a)-(h) show the evolution for different orientations of the half-wave plate HWP2, specified by the angles $\eta=n \pi / 16$ with $n=0, \ldots, 7$. The data was obtained for an initial state (3.91) whose correlation properties (3.113) are determined via the length of the calcite crystal leading to interaction time $t=\Delta n_{\text {cal }} L / c$ with $L=35.92 \mathrm{~mm}$. The black lines represent the theoretical prediction (3.128), which is independent of $\eta$, and therefore makes identical predictions for all subfigures $(a)-(h)$, as confirmed by the experimental data. Adapted from (Tang et al., 2013).

Using

$$
|H\rangle=\cos \eta|\eta\rangle-\sin \eta\left|\eta_{\perp}\right\rangle, \quad|V\rangle=\sin \eta|\eta\rangle+\cos \eta\left|\eta_{\perp}\right\rangle
$$

we express the interferometer dynamics in terms of the basis $|H\rangle$ and $|V\rangle$,

$$
U(\eta, \tau): \begin{cases}|H, \omega\rangle & \longrightarrow \cos \eta|\eta, \omega\rangle-\sin \eta e^{-i \omega \tau}\left|\eta_{\perp}, \omega\right\rangle \\ |V, \omega\rangle & \longrightarrow \sin \eta|\eta, \omega\rangle+\cos \eta e^{-i \omega \tau}\left|\eta_{\perp}, \omega\right\rangle\end{cases}
$$

\subsubsection{Local trace distance}

To determine the evolution of the local trace distance as a function of the correlations we study the evolution of the difference operator $\Delta \rho_{S E}=\rho_{S E}-\rho_{S E}^{\prime}$; using 
equations (3.105) and (3.117):

$$
\begin{aligned}
& U(\eta, \tau)\left(\rho_{S E}-\rho_{S E}^{\prime}\right) U^{\dagger}(\eta, \tau) \\
= & \sum_{\omega} \Delta \omega G(\omega) f(\omega)\left[\sin \eta \cos \eta|\eta, \omega\rangle\left\langle\eta, \omega\left|+\cos ^{2} \eta e^{i \omega \tau}\right| \eta, \omega\right\rangle\left\langle\eta_{\perp}, \omega\right|\right. \\
& \left.\quad-\sin ^{2} \eta e^{-i \omega \tau}\left|\eta_{\perp}, \omega\right\rangle\left\langle\eta, \omega|-\sin \eta \cos \eta| \eta_{\perp}, \omega\right\rangle\left\langle\eta_{\perp}, \omega\right|-\text { H.c. }\right] \\
= & \sum_{\omega} \Delta \omega G(\omega) f(\omega)\left[e^{i \omega \tau}|\eta, \omega\rangle\left\langle\eta_{\perp}, \omega\left|-e^{-i \omega \tau}\right| \eta_{\perp}, \omega\right\rangle\langle\eta, \omega|\right] .
\end{aligned}
$$

The qubit dynamics is given after the partial trace over the frequency space

$$
\begin{aligned}
\rho_{S}(\eta, \tau)-\rho_{S}^{\prime}(\eta, \tau) & =\operatorname{Tr}_{E}\left\{U(\eta, \tau)\left(\rho_{S E}-\rho_{S E}^{\prime}\right) U^{\dagger}(\eta, \tau)\right\} \\
& =\sum_{\omega} \Delta \omega G(\omega) f(\omega)\left[e^{i \omega \tau}|\eta\rangle\left\langle\eta_{\perp}\left|-e^{-i \omega \tau}\right| \eta_{\perp}\right\rangle\langle\eta|\right] .
\end{aligned}
$$

We represent this operator in the basis $\left\{|\eta\rangle,\left|\eta_{\perp}\right\rangle\right\}$ as

$$
\rho_{S}(\eta, \tau)-\rho_{S}^{\prime}(\eta, \tau)=\left(\begin{array}{cc}
0 & z \\
z^{*} & 0
\end{array}\right),
$$

where

$$
z=\sum_{\omega} \Delta \omega G(\omega) f(\omega) e^{i \omega \tau} .
$$

Since this result does not depend on $\eta$, we omit the argument $\eta$ in the following. The trace distance yields

$$
\begin{aligned}
d(\tau) & =\left\|\rho_{S}(\tau)-\rho_{S}^{\prime}(\tau)\right\| \\
& =|z| \\
& =\left|\sum_{\omega} \Delta \omega G(\omega) f(\omega) e^{i \omega \tau}\right| \\
& =\frac{\beta}{2}\left|\sum_{\omega} \Delta \omega G(\omega)\left(e^{i\left(\omega-\omega_{0}\right) t}-e^{-i\left(\omega-\omega_{0}\right) t}\right) e^{i \omega \tau}\right| .
\end{aligned}
$$

In the continuum limit we obtain,

$$
d(\tau)=\frac{\beta}{2}\left|\int d \omega G(\omega)\left(e^{i\left(\omega-\omega_{0}\right) t}-e^{-i\left(\omega-\omega_{0}\right) t}\right) e^{i \omega \tau}\right| .
$$

Comparison with equation (3.113) confirms that the general contractivity property, as expressed via the inequality

$$
\left\|\operatorname{Tr}_{E}\left\{U(\eta, \tau)\left(\rho_{S E}-\rho_{S E}^{\prime}\right) U^{\dagger}(\eta, \tau)\right\}\right\| \leq\left\|\rho_{S E}-\rho_{S E}^{\prime}\right\|,
$$




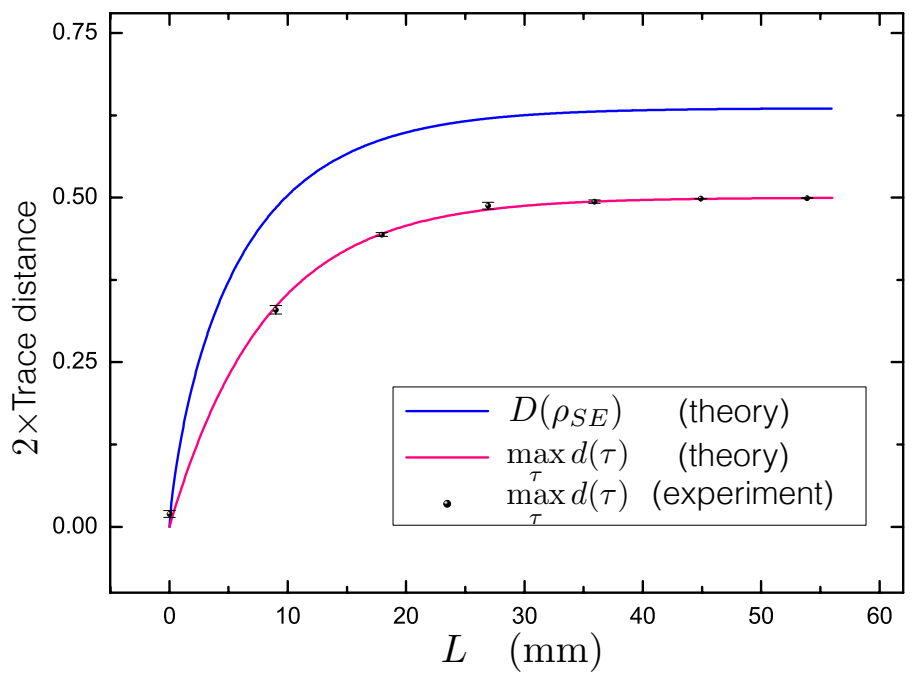

FiguRE 3.10.: For each initial state (3.91), the maximum value of the local trace distance (3.129) bounds the total trace distance (3.113) from below. The dynamics does not reveal the full correlations to the local system, but the theoretical upper limit is experimentally reached. These functions hardly depend on $\delta \omega$ in equation (3.87) when the propagation time is rescaled accordingly, and as long as (3.125) is valid. Adapted from (Tang et al., 2013).

is satisfied here, as a consequence of the triangle inequality.

We can further analytically simplify the local trace distance (3.123), since due to a sufficiently sharp, optical frequency range, $\omega_{0} \gg \delta \omega>0$, the support of the function $G(\omega)$ restricts to a good approximation to the positive domain,

$$
\int_{-\infty}^{\infty} G(\omega) \simeq \int_{0}^{\infty} G(\omega)=1
$$

The Fourier transform is given by

$$
\int_{0}^{\infty} G(\omega) e^{ \pm i \omega t} d \omega=e^{ \pm i \omega_{0} t} C(t)
$$

with

$$
C(t)=e^{-\delta \omega|t|} .
$$

Using this in the expression for the local trace distance (3.123), we obtain

$$
d(\tau)=\frac{\beta}{2}\left|e^{-\delta \omega|t+\tau|}-e^{-\delta \omega|t-\tau|}\right| .
$$

Figure 3.9 shows the local trace distance, for $\beta=1 / 2$ in (3.88), as a function of $x=c \tau / 2$ for different angles of the half-wave plate HWP2. As becomes evident from 
equation (3.117), this angle determines the parts of the polarization state that are sent into the short and long interferometer arms after passing the beam splitter PBS1. The maximum value represents the closest lower bound to the total trace distance. Using equation (3.128), the maximum local distance is found as

$$
\max _{\tau} d(\tau)=\frac{\beta}{2}\left(1-e^{-2 \delta \omega t}\right) .
$$

This procedure is repeated for different lengths of the calcite crystal, which varies the correlations in the initial state. The nature of the polarization-frequency interaction, induced by the birefringence of the calcite crystal, converts the initial coherence of the polarization state gradually into entanglement with the frequency modes, by delocalizing the initial populations over both degrees of freedom. The longer the photon spends inside the crystal, the stronger are the correlations at the end of the evolution, as illustrated by the blue line in figure 3.10. The maximum values of (3.129), obtained via local monitoring of the polarization state, are compared to the theoretically determined total trace distance and the predicted theoretical bound of the local distance in figure 3.10. Even though the local bound does not reach the actual value of the total trace distance, it provides a clear signature of the influence of the initial polarization-frequency correlations on the local dynamics of the qubit, even when the environmental state represents a continuum of infinite-dimensional modes.

The induced, effective open-system dynamics of the polarization degree of freedom is described by a monotonous decay of coherences. This is an irreversible decoherence process whose time scale is determined by $\delta \omega$ in the spectral distribution of the environmental state (3.87), and such an environment is therefore considered Markovian ${ }^{2}$ (Liu et al., 2011). Numerical studies of the total trace distance (3.113) and of the observable local distance (3.129) (as plotted in figure 3.10) confirm that the observed results do not depend on $\delta \omega$, as long as the environmental frequency range can be assumed positive, as in equation (3.125). Since changing the width $\delta \omega$ of the spectral distribution also changes the time scale of the exponential decay of coherences, the propagation time in the calcite crystal must be rescaled accordingly.

In conclusion, the present all-optical experiment demonstrates the applicability of the local detection method in the presence of a memoryless environment, generated by a continuum of modes with a Lorentzian spectral density. Upon appropriate rescaling of the time scale, the result is independent of the spectral width. In the next section, we will theoretically explore the limitations of the method, by studying the example of spontaneous emission.

\subsection{Theoretical studies of further examples}

So far, in this chapter, we have introduced a method to detect discord-type correlations in a bipartite scenario with local operations, and presented experimental realizations

\footnotetext{
${ }^{2} \mathrm{~A}$ non-Markovian environment can be generated in this scenario by inducing additional peaks and structure in the environmental spectral distribution, as was realized in the experiment reported in (Liu et al., 2011).
} 
with photons and ions. Recently, another experimental realization with polarization qubits was reported, where correlations are created with two spatial propagation modes (Cialdi et al., 2014). In this section we discuss the application of the method in different scenarios theoretically. Some applications were already discussed in (Gessner, 2011; Gessner and Breuer, 2011; Gessner and Breuer, 2013a; Gessner and Breuer, 2013b), mostly in the context of random, generic systems, which we do not review here.

\subsubsection{Atom-photon correlations during spontaneous decay}

In the course of the preceding sections, we have often tried to test the limits of the efficacy of the local detection method, for example by studying the high-temperature limit in the trapped-ion environment in section 3.2, and a broad frequency distribution of the photonic environment in section 3.3. So far, numerical studies always confirmed that the method successfully reveals the system-environment correlations to the local dynamics.

In the trapped-ion experiment, the electronic qubit exchanges excitations with a rather simple model environment, described by only a single frequency mode. The frequency degree of freedom of a single photon contains a continuum of modes. Here, the resulting irreversible dynamics is described by a pure decoherence process, where no excitations are transferred between the system and the environment (Liu et al., 2011). In this section, we combine the infinite-dimensional Hilbert-space, represented by a continuum of modes, with the excitation-exchanging coupling. To this end, we apply the local detection protocol to the extreme case of the spontaneous, irreversible emission process into an infinite environment. Our goal is to study the influence of the atom-field entanglement which builds up in the transient state, when an initial atomic excitation decays spontaneously into the free-space modes of the electromagnetic field. In this case we do not expect any back-action of the correlations on the system dynamics. Indeed, we will find that in this case, employing the local dephasing at any time during the decay process of an excited atom will not produce any effect on the atomic dynamics.

Given the difficulty to detect a spontaneously emitted photon in a random spatial direction (Volz et al., 2006), the local detection method, in principle, represents a convenient alternative to other entanglement detection methods ${ }^{3}$ since it only demands access to the controllable open system, which here is described by the atom. The fact that no effect on the local dynamics can be observed when the entanglement is destroyed by local dephasing indicates that the effect of the correlations on this particular irreversible dynamics are negligible.

\subsubsection{Atomic evolution with and without local dephasing: Resolvent method}

Following standard treatments of spontaneous emission (Weisskopf and Wigner, 1930; Cohen-Tannoudji et al., 1992), we assume a unitary evolution $U(t)=e^{-i H t / \hbar}$ which

\footnotetext{
${ }^{3}$ Note that in (Volz et al., 2006), the entanglement between the atomic state and the emitted photon's polarization degree of freedom is investigated, instead of the atom-field entanglement which we discuss here.
} 
transfers excitations between the atomic states $|e\rangle$ and $|g\rangle$ and the modes $|\mathbf{k}\rangle$ of the electromagnetic field. The Hamiltonian is given by $H=H_{0}+V$ with

$$
H_{0}=E_{e}|e\rangle\left\langle e\left|+E_{g}\right| g\right\rangle\langle g|+\sum_{\mathbf{k}} \hbar \omega_{\mathbf{k}} a_{\mathbf{k}}^{\dagger} a_{\mathbf{k}}
$$

and

$$
V=\sum_{\mathbf{k}}\left(g_{\mathbf{k}} a_{\mathbf{k}}^{\dagger}|g\rangle\left\langle e\left|+g_{\mathbf{k}}^{*} a_{\mathbf{k}}\right| e\right\rangle\langle g|\right) .
$$

The combined states $\{|g, \mathbf{k}\rangle,|e, \mathbf{k}\rangle\}$ form a basis of the free Hamiltonian, $H_{0}|i, \mathbf{k}\rangle=$ $\left(E_{i}+\hbar \omega_{\mathbf{k}}\right)|i, \mathbf{k}\rangle, i \in\{e, g\}$ and $\omega_{e g}=E_{e}-E_{g}$. We start with the dynamics

$$
\begin{aligned}
U(t)|e, 0\rangle & =u_{00}(t)|e, 0\rangle+\sum_{\mathbf{k}} u_{\mathbf{k} 0}(t)|g, \mathbf{k}\rangle, \\
U(t)|g, \mathbf{k}\rangle & =u_{0 \mathbf{k}}(t)|e, 0\rangle+\sum_{\mathbf{k}^{\prime}} u_{\mathbf{k}^{\prime} \mathbf{k}}(t)\left|g, \mathbf{k}^{\prime}\right\rangle .
\end{aligned}
$$

Irreversibility is later introduced when the transition amplitudes are determined and by performing the continuum limit of the sum over all modes. We use the resolvent method (Cohen-Tannoudji et al., 1992) to determine the matrix elements nonperturbatively

$$
\begin{aligned}
u_{00}(t) & =\langle e, 0|U(t)| e, 0\rangle, \\
u_{\mathbf{k} 0}(t) & =\langle g, \mathbf{k}|U(t)| e, 0\rangle, \\
u_{0 \mathbf{k}}(t) & =\langle e, 0|U(t)| g, \mathbf{k}\rangle, \\
u_{\mathbf{k k}^{\prime}}(t) & =\left\langle g, \mathbf{k}|U(t)| g, \mathbf{k}^{\prime}\right\rangle .
\end{aligned}
$$

Unitarity requires $u_{0 \mathbf{k}}(t)=u_{\mathbf{k} 0}^{*}(-t)$ for time-independent Hamiltonians.

The initial state $|e, 0\rangle$ evolves after time $t_{0}$ into

$$
\left|\Psi\left(t_{0}\right)\right\rangle=u_{00}\left(t_{0}\right)|e, 0\rangle+\sum_{\mathbf{k}} u_{\mathbf{k} 0}\left(t_{0}\right)|g, \mathbf{k}\rangle .
$$

Local dephasing in the local eigenbasis $\{|e\rangle,|g\rangle\}$ erases all except the off-diagonal matrix elements and generates the classically correlated reference state,

$$
\rho^{\prime}\left(t_{0}\right)=(\Phi \otimes \mathbb{I})\left|\Psi\left(t_{0}\right)\right\rangle\left\langle\Psi\left(t_{0}\right)\right|,
$$

from the initially entangled state $\rho\left(t_{0}\right)=\left|\Psi\left(t_{0}\right)\right\rangle\left\langle\Psi\left(t_{0}\right)\right|$. When dephasing is implemented after time $t_{0}$, the difference between the two states is given by

$$
\rho\left(t_{0}\right)-\rho^{\prime}\left(t_{0}\right)=\sum_{\mathbf{k}}\left(u_{00}\left(t_{0}\right) u_{\mathbf{k} 0}^{*}\left(t_{0}\right)|e, 0\rangle\left\langle g, \mathbf{k}\left|+u_{00}^{*}\left(t_{0}\right) u_{\mathbf{k} 0}\left(t_{0}\right)\right| g, \mathbf{k}\right\rangle\langle e, 0|\right) .
$$


Further evolution from $t_{0}$ to $t$ yields, after tracing out the electromagnetic field modes,

$$
\begin{aligned}
\rho_{S}\left(t, t_{0}\right)-\rho_{S}^{\prime}\left(t, t_{0}\right) & =\operatorname{Tr}_{E}\left\{U\left(t-t_{0}\right)\left(\rho\left(t_{0}\right)-\rho^{\prime}\left(t_{0}\right)\right) U^{\dagger}\left(t-t_{0}\right)\right\} \\
& =2 \Re\left[\sum_{\mathbf{k}} u_{00}\left(t_{0}\right) u_{\mathbf{k} 0}^{*}\left(t_{0}\right) u_{00}\left(t-t_{0}\right) u_{0 \mathbf{k}}^{*}\left(t-t_{0}\right)\right] \sigma_{z},
\end{aligned}
$$

where $\Re$ denotes the real part of a complex number.

The unitary time evolution operator is expressed in terms of the resolvent $G(z)=$ $1 /(z-H)$ as (Cohen-Tannoudji et al., 1992),

$$
U(t)=\lim _{\eta \rightarrow 0^{+}} \frac{1}{2 \pi i} \int_{-\infty}^{\infty} d E e^{-i E t / \hbar}[G(E-i \eta)-G(E+i \eta)] .
$$

Projecting this matrix onto the subspace spanned by $|e, 0\rangle$ and assuming that the radiative level shift $\Delta_{e}$ and the decay constant $\Gamma$ are energy-independent, we obtain (see, for instance, (Cohen-Tannoudji et al., 1992), III.C.1)

$$
u_{00}(t)=e^{-i \widetilde{E}_{e} t / \hbar-\Gamma|t| / 2}
$$

where $\widetilde{E}_{e}=E_{e}+\hbar \Delta_{e}$. If the above assumptions are satisfied, this expression is exact. In order to obtain $u_{\mathbf{k} 0}(t)$, we use equation (C.29), III.C.2 in (Cohen-Tannoudji et al., 1992):

$$
\langle g, \mathbf{k}|G(E \pm i \eta)| e, 0\rangle \simeq \frac{g_{\mathbf{k}}}{\left(E \pm i \eta-\widetilde{E}_{g}-\hbar \omega_{\mathbf{k}}\right)\left(E-\widetilde{E}_{e} \pm i \hbar(\Gamma / 2)\right)}
$$

where, again, the radiative level shift $\Delta_{g}$ yields $\widetilde{E}_{g}=E_{g}+\hbar \Delta_{g}$. Equation (3.138) can be integrated using a contour integral if we choose the integration path appropriately. This requires the numerator $e^{-i z t / \hbar}$ to tend towards zero for $|z| \rightarrow \infty$. Inserting $z=|z|(\cos \phi+i \sin \phi)$ yields

$$
\left|e^{-i z t / \hbar}\right|=\left|e^{-i|z|(\cos \phi+i \sin \phi) / \hbar}\right|=\left|e^{-i t|z| \cos \phi / \hbar} e^{|z| t \sin \phi / \hbar}\right| \leq e^{|z| t \sin \phi / \hbar} .
$$

Hence, for $t>0$ we must close the contour below the $\mathrm{x}$-axis, with $\sin \phi<0$ for $\phi \in(\pi, 2 \pi)$. Since the only poles of $G(z)$ are on the x-axis, it follows from Cauchy's integral theorem that the contribution of $G(E-i \eta)$ is zero. Conversely, for $t<0$ the contour must be closed above the x-axis, $\sin \phi>0$ for $\phi \in(0, \pi)$ and in this case the term $G(E+i \eta)$ does not contribute. We start with $t>0$ :

$$
\begin{aligned}
u_{\mathbf{k} 0}(t) & =\lim _{\eta \rightarrow 0^{+}} \frac{1}{2 \pi i} \int_{\infty}^{-\infty} d E e^{-i E t / \hbar}\langle g, \mathbf{k}|G(E+i \eta)| e, 0\rangle \\
& \simeq \lim _{\eta \rightarrow 0^{+}} \frac{1}{2 \pi i} \int_{\infty}^{-\infty} d E e^{-i E t / \hbar} \frac{g_{\mathbf{k}}}{\left(E+i \eta-\widetilde{E}_{g}-\hbar \omega_{\mathbf{k}}\right)\left(E-\widetilde{E}_{e}+i \hbar(\Gamma / 2)\right)} .
\end{aligned}
$$


Substituting $E$ by a complex number $z$ and integrating in the complex plane over a contour from $R \in \mathbb{R}$ to $-R$, which is closed below the real axis as described before we obtain by the residue theorem

$$
\oint_{C} f(z) d z=\int_{R}^{-R} f(z) d z+\int_{\operatorname{arc}} f(z) d z=2 \pi i\left(\operatorname{Res}\left(f, z_{0}\right)+\operatorname{Res}\left(f, z_{1}\right)\right),
$$

where

$$
f(z)=e^{-i z t / \hbar} \frac{g_{\mathbf{k}}}{\left(z-z_{0}\right)\left(z-z_{1}\right)},
$$

has two complex poles of first order at $z_{0}=\widetilde{E}_{g}+\hbar \omega_{\mathbf{k}}-i \eta$ and $z_{1}=\widetilde{E}_{e}-i \hbar(\Gamma / 2)$. The residues are given by

$$
\begin{aligned}
\operatorname{Res}\left(f, z_{0}\right) & =\frac{e^{-i z_{0} t / \hbar}}{z_{0}-z_{1}}, \\
\operatorname{Res}\left(f, z_{1}\right) & =\frac{e^{-i z_{1} t / \hbar}}{z_{1}-z_{0}} .
\end{aligned}
$$

After the limits $R \rightarrow \infty$ and $\eta \rightarrow 0^{+}$we finally obtain

$$
u_{\mathbf{k} 0}(t)=g_{\mathbf{k}} \frac{e^{-i\left(\widetilde{E}_{g} / \hbar+\omega_{\mathbf{k}}\right) t}-e^{-i \widetilde{E}_{e} t / \hbar-\Gamma t / 2}}{\hbar \omega_{\mathbf{k}}-\left(\widetilde{E}_{e}-\widetilde{E}_{g}\right)+i \hbar \Gamma / 2} .
$$

Analogously, for $t<0$ we get

$$
\begin{aligned}
u_{\mathbf{k} 0}(t) & \simeq \lim _{\eta \rightarrow 0^{+}} \frac{1}{2 \pi i} \int_{-\infty}^{\infty} d E e^{-i E t / \hbar} \frac{g_{\mathbf{k}}}{\left(E-i \eta-\widetilde{E}_{g}-\hbar \omega_{\mathbf{k}}\right)\left(E-\widetilde{E}_{e}-i \hbar(\Gamma / 2)\right)} \\
& =g_{\mathbf{k}} \frac{e^{-i\left(\widetilde{E}_{g} / \hbar+\omega_{\mathbf{k}}\right) t}-e^{-i \widetilde{E}_{e} t / \hbar+\Gamma t / 2}}{\hbar \omega_{\mathbf{k}}-\left(\widetilde{E}_{e}-\widetilde{E}_{g}\right)-i \hbar \Gamma / 2} .
\end{aligned}
$$

Using the unitarity condition for time-independent Hamiltonians we get $(t>0)$

$$
u_{0 \mathbf{k}}(t)=u_{\mathbf{k} 0}^{*}(-t) \simeq g_{\mathbf{k}}^{*} \frac{e^{-i\left(\widetilde{E}_{g} / \hbar+\omega_{\mathbf{k}}\right) t}-e^{-i \widetilde{E}_{e} t / \hbar-\Gamma t / 2}}{\hbar \omega_{\mathbf{k}}-\left(\widetilde{E}_{e}-\widetilde{E}_{g}\right)+i \hbar \Gamma / 2} .
$$

We now use this to determine the difference in the open-system evolution of the atomic electronic levels, after the entanglement with the electromagnetic field has been removed and replaced by classical correlations. To this end, we insert the derived expressions into equation (3.137), which yields

$$
\begin{aligned}
& \rho_{S}\left(t, t_{0}\right)-\rho_{S}^{\prime}\left(t, t_{0}\right) \\
& \simeq \frac{2}{\hbar} \Re \sum_{\mathbf{k}}\left|g_{\mathbf{k}}\right|^{2} e^{-\Gamma t}\left(\frac{e^{-i\left(\widetilde{\omega}_{e g}-\omega_{\mathbf{k}}\right) t+\Gamma t / 2}-e^{-i\left(\widetilde{\omega}_{e g}-\omega_{\mathbf{k}}\right)\left(t-t_{0}\right)+\Gamma\left(t-t_{0}\right) / 2}}{\left(\omega_{\mathbf{k}}-\widetilde{\omega}_{e g}-i \Gamma / 2\right)^{2}}\right. \\
&\left.+\frac{-e^{-i\left(\widetilde{\omega}_{e g}-\omega_{\mathbf{k}}\right) t_{0}+\Gamma t_{0} / 2}+1}{\left(\omega_{\mathbf{k}}-\widetilde{\omega}_{e g}-i \Gamma / 2\right)^{2}}\right) \sigma_{z},
\end{aligned}
$$


with the radiation-shifted atomic resonance $\hbar \widetilde{\omega}_{e g}=\widetilde{E}_{e}-\widetilde{E}_{g}$. We now evaluate the sum in the continuum limit (Weisskopf and Wigner, 1930),

$$
\begin{aligned}
& \rho_{S}\left(t, t_{0}\right)-\rho_{S}^{\prime}\left(t, t_{0}\right) \\
& \simeq \frac{2\left|\mu_{e g}\right|^{2}}{3(2 \pi)^{2} \hbar \epsilon_{0} c^{3}} \frac{2}{\hbar} \Re \int_{0}^{\infty} d \omega_{\mathbf{k}} \omega_{\mathbf{k}}^{3} e^{-\Gamma t}\left(\frac{e^{-i\left(\widetilde{\omega}_{e g}-\omega_{\mathbf{k}}\right) t+\Gamma t / 2}-e^{-i\left(\widetilde{\omega}_{e g}-\omega_{\mathbf{k}}\right)\left(t-t_{0}\right)+\Gamma\left(t-t_{0}\right) / 2}}{\left(\omega_{\mathbf{k}}-\widetilde{\omega}_{e g}-i \Gamma / 2\right)^{2}}\right.\left.+\frac{-e^{-i\left(\widetilde{\omega}_{e g}-\omega_{\mathbf{k}}\right) t_{0}+\Gamma t_{0} / 2}+1}{\left(\omega_{\mathbf{k}}-\widetilde{\omega}_{e g}-i \Gamma / 2\right)^{2}}\right) \sigma_{z} \\
& \approx \frac{\left|\mu_{e g}\right|^{2} \widetilde{\omega}_{e g}^{3}}{3 \pi^{2} \hbar^{2} \epsilon_{0} c^{3}} e^{-\Gamma t} \Re \int_{-\infty}^{\infty} d \omega_{\mathbf{k}}\left(\frac{e^{-i\left(\widetilde{\omega}_{e g}-\omega_{\mathbf{k}}\right) t+\Gamma t / 2}-e^{-i\left(\widetilde{\omega}_{e g}-\omega_{\mathbf{k}}\right)\left(t-t_{0}\right)+\Gamma\left(t-t_{0}\right) / 2}}{\left(\omega_{\mathbf{k}}-\widetilde{\omega}_{e g}-i \Gamma / 2\right)^{2}}\right. \\
&\left.\quad+\frac{-e^{-i\left(\widetilde{\omega}_{e g}-\omega_{\mathbf{k}}\right) t_{0}+\Gamma t_{0} / 2}+1}{\left(\omega_{\mathbf{k}}-\widetilde{\omega}_{e g}-i \Gamma / 2\right)^{2}}\right) \sigma_{z},
\end{aligned}
$$

where $\mu_{e g}$ denotes the dipole moment of the atomic transition, and $\epsilon_{0}$ is the dielectric constant of the vacuum. Due to a narrow resonance of the transition amplitudes at $\omega_{\mathbf{k}}=\widetilde{\omega}_{e g}$, we approximate the $\omega_{\mathbf{k}}^{3}$-term in the integral with the term $\widetilde{\omega}_{e g}^{3}$. We evaluate the remaining integral with the same contour integral methods as before. Closing the contour above the $\mathrm{x}$-axis, we find a second order pole at $z_{0}=\widetilde{\omega}_{e g}+i \Gamma / 2$ whereas the residue for functions of the type $f(z)=e^{i z t} /\left(z-z_{0}\right)^{2}$ is given by

$$
\operatorname{Res}\left(f, z_{0}\right)=i t e^{i z_{0} t} .
$$

Finally, this leads to a vanishing difference in the evolution of the atom:

$$
\rho_{S}\left(t, t_{0}\right)-\rho_{S}^{\prime}\left(t, t_{0}\right) \simeq \frac{2\left|\mu_{e g}\right|^{2}}{3(2 \pi)^{2} \hbar \epsilon_{0} c^{3}} \frac{2}{\hbar} e^{-\Gamma t} \Re\left\{i t-i\left(t-t_{0}\right)-i t_{0}\right\} \sigma_{z}=0,
$$

independently of the observation time $t$ and the dephasing time $t_{0}$.

\subsubsection{Evolution of atom-field negativity}

Before concluding that the atom-field entanglement cannot be detected in the atomic evolution based on the local detection method, we have to demonstrate that, within the same approximations, the atom-field entanglement is finite. Based on (3.16) we can use the trace-distance dephasing disturbance (3.7) to determine the atom-field negativity of the transient state $\left|\Psi\left(t_{0}\right)\right\rangle$, as given in equation (3.134). To this end, we determine the trace norm of the difference operator $M\left(t_{0}\right)=\rho\left(t_{0}\right)-\rho^{\prime}\left(t_{0}\right)$, which is given in equation (3.136). We obtain

$$
M^{\dagger}\left(t_{0}\right) M\left(t_{0}\right)=\left|u_{00}\left(t_{0}\right)\right|^{2}(|e\rangle\langle e|+| g\rangle\langle g|) \otimes N\left(t_{0}\right)\left(|0\rangle\left\langle 0|+| \Psi_{E}\left(t_{0}\right)\right\rangle\left\langle\Psi_{E}\left(t_{0}\right)\right|\right),
$$

with the pure state

$$
\left|\Psi_{E}\left(t_{0}\right)\right\rangle=\frac{1}{\sqrt{N\left(t_{0}\right)}} \sum_{\mathbf{k}} u_{\mathbf{k} 0}\left(t_{0}\right)|\mathbf{k}\rangle,
$$


and the normalization constant $N\left(t_{0}\right)=\sum_{\mathbf{k}}\left|u_{\mathbf{k} 0}\left(t_{0}\right)\right|^{2}$. We express $N\left(t_{0}\right)$ using equations (3.146) and employ the same approximations as before:

$$
\begin{aligned}
N\left(t_{0}\right) & =\frac{1}{\hbar^{2}} \sum_{\mathbf{k}}\left|g_{\mathbf{k}}\right|^{2} \frac{1-2 \cos \left[\left(\omega_{\mathbf{k}}-\widetilde{\omega}_{e g}\right) t_{0}\right] e^{-\Gamma t_{0} / 2}+e^{-\Gamma t_{0}}}{\left(\omega_{\mathbf{k}}-\widetilde{\omega}_{e g}\right)^{2}+\Gamma^{2} / 4} \\
& \simeq \frac{1}{\hbar^{2}} \frac{\left|\mu_{e g}\right|^{2}}{6 \pi^{2} \epsilon_{0} \hbar c^{3}} \int_{0}^{\infty} d \omega_{\mathbf{k}} \omega_{\mathbf{k}}^{3} \frac{1-2 \cos \left[\left(\omega_{\mathbf{k}}-\widetilde{\omega}_{e g}\right) t_{0}\right] e^{-\Gamma t_{0} / 2}+e^{-\Gamma t_{0}}}{\left(\omega_{\mathbf{k}}-\widetilde{\omega}_{e g}\right)^{2}+\Gamma^{2} / 4} \\
& \approx \frac{1}{\hbar^{2}} \frac{\left|\mu_{e g}\right|^{2} \widetilde{\omega}_{e g}^{3}}{6 \pi^{2} \epsilon_{0} \hbar c^{3}} \int_{0}^{\infty} d \omega_{\mathbf{k}} \frac{1-2 \cos \left[\left(\omega_{\mathbf{k}}-\widetilde{\omega}_{e g}\right) t_{0}\right] e^{-\Gamma t_{0} / 2}+e^{-\Gamma t_{0}}}{\left(\omega_{\mathbf{k}}-\widetilde{\omega}_{e g}\right)^{2}+\Gamma^{2} / 4} \\
& =\frac{1}{\hbar^{2}} \frac{\left|\mu_{e g}\right|^{2} \widetilde{\omega}_{e g}^{3}}{6 \pi^{2} \epsilon_{0} \hbar c^{3}} \frac{2 \pi}{\Gamma}\left(1-e^{-\Gamma t_{0}}\right) .
\end{aligned}
$$

Using (3.139), we obtain the atom-field negativity via the trace distance from (3.153),

$$
\begin{aligned}
\mathcal{N}\left(\left|\Psi\left(t_{0}\right)\right\rangle\left\langle\Psi\left(t_{0}\right)\right|\right) & =\frac{1}{2} \operatorname{Tr} \sqrt{M^{\dagger}\left(t_{0}\right) M\left(t_{0}\right)} \\
& =2\left|u_{00}\left(t_{0}\right)\right| \sqrt{N\left(t_{0}\right)} \\
& =2 \sqrt{\frac{\left|\mu_{e g}\right|^{2} \widetilde{\omega}_{e g}^{3}}{3 \pi \epsilon_{0} \hbar^{3} c^{3} \Gamma} e^{-\Gamma t_{0}}\left(1-e^{-\Gamma t_{0}}\right) .}
\end{aligned}
$$

The temporal behavior described by (3.156) matches the physical intuition: At initial times $t_{0} \sim 0$, the atom is described by the excited state $|e\rangle$, whereas after long waiting times $t_{0} \rightarrow \infty$, the atom has decayed with high probability into the ground state $|g\rangle$. In both cases, the system is completely decoupled from the environment, and, hence, no atom-field entanglement is present in those limits. However, at transient times $t_{0}$, we find finite amount of entanglement, as quantified by the trace distance of the total atom-field states with and without quantum correlations, which coincides with the negativity. Since the local state difference (3.152) vanishes also for transient values of $t_{0}$, we find that the local detection method is not able to reveal these dynamically in the present context.

\subsubsection{Discussion}

As indicated in the beginning of this section, the atomic evolution is not influenced by removing the entanglement between the radiation field and the atom during the spontaneous decay process. This can be attributed to the complete lack of structure in the environmental frequency modes, and a system-environment coupling which induces exchange of excitations. The resulting irreversible exponential decay of the atomic population has the unique property that it can be interrupted at arbitrary moments, and the subsequent dynamics always has the exact same shape. Slight modulations thereof, for example, by higher-order corrections (Cohen-Tannoudji et al., 1992) or coupling the atom to a lossy cavity (Puri and Agarwal, 1986), are expected to change this behavior (Breuer and Petruccione, 2002). 


\subsubsection{Many-mode extension of the trapped-ion experiment: A proposal}

The example of an infinite-size continuum of free-space modes shows that there exists a limit in which the bipartite correlations no longer significantly influence the local subsystem dynamics. In contrast, the trapped-ion experiment showed strong signatures of the correlations on the qubit dynamics when the ion couples to a single mode where only few discrete energy levels are occupied. The question is, what happens in between, when the open system couples to a finite-size mesoscopic environment? At which size can we neglect the influence of correlations on the reduced dynamics and how long is it possible to locally detect these correlations dynamically?

In this section we discuss possible extensions of the trapped-ion experiment, where the number of harmonic modes can be tuned to probe the transition from small model environments with low effective dimensions to an almost irreversible and memoryless decay into a continuum of modes. To this end, instead of a single ion, we consider a full chain of $N$ ions. We now find $3 N$ motional modes, whereas depending on the specific geometric arrangement of the laser system one can restrict to the $N$ modes in just one spatial dimension. How can we entangle a single qubit with several motional modes at the same time? Driving one of the sidebands resonantly would still only couple the ion to the addressed single eigenmode, and the theoretical treatment would be equivalent to the one presented in section 3.2.

A possibility to generate interactions with many modes at the same time are fast pulses on local sidebands. This corresponds to a sideband pulse which is fast enough such that its Fourier bandwidth contains all eigenmodes (Brown et al., 2011; Harlander et al., 2011), which is possible if sufficient laser power is available. These local sideband pulses are essentially local, spin-dependent kicks, which displace the ion in the laser focus on time scales which are too fast for the rest of the chain to follow the movement. The created local vibrational excitations are non-equilibrium wave-packets of the phonon system (Haze et al., 2012; Toyoda et al., 2013) and can be used to study energy transport within the ion chain (Ramm et al., 2014; Ramm, 2014); see also section 6.4.

An interaction of a single electronic qubit with an ensemble of modes can be generated based on the following scheme:

- Apply a spin-dependent kick on the selected ion.

- Let the system evolve freely for time $t$.

- Apply another spin-dependent kick, $180^{\circ}$ out of phase to the first one.

To illustrate the mechanism of this sequence, let us assume that the ion was prepared in a product state of $|\varphi\rangle=|0\rangle$ and $\rho_{\mathrm{m}}=|0\rangle\langle 0|$, which denote the respective ground states of qubit and motion. The first spin-dependent kick promotes the ion in a superposition state (Monroe et al., 1996)

$$
|\Psi\rangle=\frac{1}{\sqrt{2}}\left(\left|0,0_{1}\right\rangle+\left|1, \alpha_{1}\right\rangle\right)
$$


where $\left|\alpha_{1}\right\rangle$ denotes a coherent state on the Fock space describing the motion of the addressed ion. Such an excitation is not an eigenstate of the full coupled ion chain, and will therefore evolve during $t$, which constitutes the next step. During the free evolution, the populations of the electronic qubit do not evolve. Finally, the phonon evolution is mapped back onto the electronic degree of freedom with a second displacement. The $180^{\circ}$ out-of-phase shift of the second kick ensures that at $t=0$, the excitation is mapped right back to the initial state. This combination of $\pi / 2$-pulses (creating equal superpositions of ground and excited states) and free evolution basically constitutes a Ramsey scheme (Ramsey, 1990) on the local phonons.

The advantage of this scheme is that it can be implemented with arbitrary ion numbers $N$. Furthermore the ratio of the trap frequencies in axial and radial directions controls how densely the motional frequencies along the observable direction are packed, which allows to tune the spectral density of the phonon bath. When the interion distance reduces, it becomes experimentally more challenging to focus the laser only onto a single ion. This technical problem may be circumvented using the fact that the outer ions usually show significantly larger spacings than those in the center when the chain becomes large, which facilitates the local addressing, see also the inset in figure 2.2. A study of a related scheme (De Chiara et al., 2008) demonstrated that the trace-distance information backflow from the phonons to the qubit - a quantifier of non-Markovian effects - peaks at the structural phase transition (Borrelli et al., 2013), which occurs when the axial trap frequency exceeds a threshold value and the ions change their arrangement from a linear to a zig-zag structure (Birkl et al., 1992; Waki et al., 1992).

Using the above Ramsey scheme, the qubit can be coupled to a tunable bath of phonons. To explore the influence of correlations on the qubit dynamics, several possibilities emerge. The most natural choice would be to submit correlated states to the Ramsey dynamics, and to check for a different resulting qubit evolution when additionally the local dephasing operation is implemented before the first spin-dependent kick. Correlated states of spin and motion may be generated by the same spin-dependent displacement operations or other sideband pulses. Alternatively, one may interrupt the Ramsey scheme to implement a dephasing operation after the first spin-dependent kick.

\subsubsection{Quantum phase transition in a transverse-field Ising chain}

Finally, we study another scenario of a many-body interacting system. We now move from an interacting multi-phonon environment to a system of interacting spins which describes a quantum magnet and shows interesting phenomena, such as a quantum phase transition (Sachdev, 1999). A detailed study of this model will be provided in section 5 .

Quantum phase transitions are essentially crossings or avoided crossings of energy levels, as a function of an external control parameter, which involve the ground state. After such a crossing, a new state has lower energy than the ground state in the previous quantum phase, which renders it the new ground state. Quite generically, this new ground state exhibits different properties than the original ground state, includ- 
ing, for example its entanglement properties. This makes the ground-state quantum correlations perceptive to the quantum phase transition, as confirmed in a series of case studies (Osterloh et al., 2002; Osborne and Nielsen, 2002; Vidal et al., 2003; Wu et al., 2004).

Detecting quantum correlations in a large crystal can, however, not be achieved on the basis of conventional detection schemes - which require access to each individual spin. For this reason, a direct experimental verification of diverging entanglement quantifiers at the critical point of a phase transitions has not yet been achieved. Such an experiment would directly demonstrate the existence of a striking link between concepts from quantum information and solid state physics. In controlled quantum systems, Hamiltonians that mimic the dynamics of solid-state systems can be designed, and individual access is given to individual constituents; recall section 2.2 for a brief overview. Thus, in this last study, we apply the local detection protocol to detect the quantum correlations in the ground state of large spin chains, by considering only one of the spins as the accessible subsystem and treating the remaining spins as environment (Gessner et al., 2014b).

To be precise, we study a family of spin chain models with variable-range interactions, given by

$$
H=-\sum_{\substack{i, j=1 \\(i<j)}}^{N} \frac{J_{0}}{|i-j|^{\alpha}} \sigma_{x}^{(i)} \sigma_{x}^{(j)}-B \sum_{i=1}^{N} \sigma_{y}^{(i)}
$$

As discussed in sections 2.2.1.7 and 2.2.2, this Hamiltonian can be simulated efficiently with a chain of trapped ions, and cold atoms on tilted optical lattices. The interaction range is determined by the continuous parameter $\alpha$, and in trapped-ion experiments this parameter can be tuned within the values $0<\alpha<3$ (Kim et al., 2010), whereas typically, these experiments work around $\alpha \approx 1$. A detailed study of this model and the associated quantum phase transition will be provided in chapter 5 . In this section, we restrict to the application and interpretation of the local detection protocol, for both zero- and nonzero temperature states.

The Hamiltonian (3.158) shows a quantum phase transition from (anti-)ferromagnet to paramagnet when the external magnetic field $B$ exceeds the strength of the spinspin interactions, which are determined by $J_{0}$. The sign of $J_{0}$ determines whether the system behaves as a ferromagnet $\left(J_{0}>0\right)$ or anti-ferromagnet $\left(J_{0}<0\right)$ when $B=0$. While both cases are scrutinized in chapter 5 , in this section we restrict to the ferromagnetic case $J_{0}>0$. The theoretical treatment, however, is easily transferred to the anti-ferromagnet. In the ferromagnetic phase, for example at $B=0$, the ground state $^{4}$ is described by superposition states of Greenberger-Horne-Zeilinger ( $G H Z$ ) type (Greenberger et al., 1989; Dür et al., 2000; Štelmachovič and Bužek, 2004),

$$
\left|\mathrm{GHZ}_{\varphi}\right\rangle=\frac{1}{\sqrt{2}}\left(|\uparrow \uparrow \ldots\rangle+e^{i \varphi}|\downarrow \ldots\rangle\right)
$$

\footnotetext{
${ }^{4}$ See also (Heisenberg, 1928; Bethe, 1931) for pioneering work on quantum magnetism.
} 
In such a configuration, each spin is entangled with the rest of the chain. ${ }^{5}$ The groundstate entanglement reduces when $B$ increases since the spins start to align along the $y$ direction of the external magnetic field. In the extreme case of $B \gg J_{0}$, the ground state forms a completely uncorrelated product state $\left|\uparrow_{y}\right|^{\otimes N}$. Hence, we expect the bipartite ground-state entanglement between a randomly selected spin and the rest of the chain to decay monotonously as the external magnetic field is scanned from zero to large fields, where the transition from entangled to separable becomes sharper with increasing system size.

\subsubsection{Ground-state negativity from the evolution of the single-spin magnetization}

Let us first consider the case of the pure ground state $\left|\Psi_{0}\right\rangle$ of $H$, as given in (3.158). We apply the local detection protocol to the state $\left|\Psi_{0}\right\rangle$, by considering the left-most spin the accessible subsystem, whereas the remaining spins are considered as part of an inaccessible reservoir. In this case, the initial state $\left|\Psi_{0}\right\rangle$ does not evolve in time, since it is the ground state, hence, an eigenstate of $H$. Dephasing in the local eigenbasis, however, populates a combination of excited states, which can lead to time evolution. The signatures of this time evolution on the single spin are then employed to detect and estimate the ground-state entanglement.

The $Z_{2}$ symmetry of the Hamiltonian $H$ (invariance under a $180^{\circ}$ rotation around the $y$-axis) requires that the reduced, single-spin density matrices are diagonal in the eigenbasis of $\sigma_{y}$, for all eigenstates. For further details we refer to chapter 5 . Therefore, the local dephasing is always implemented by projecting non-selectively in the eigenbasis of $\sigma_{y}$ of the left-most spin. The resulting dephasing operation yields the reference state $(\Phi \otimes \mathbb{I})\left|\Psi_{0}\right\rangle\left\langle\Psi_{0}\right|$, which, in general, is no longer an eigenstate of the system. Recall from section 3.1.2 that the dephasing in the eigenbasis minimizes the trace distance over all possible dephasing bases in the case of a pure state. Since the local spin is described by a two-level system, we obtain the negativity (3.12) as a figure of merit for the quantum correlations as [see equations (3.7) and (3.16)]

$$
\left.D\left(\left|\Psi_{0}\right\rangle\left\langle\Psi_{0}\right|\right)=\| \Psi_{0}\right\rangle\left\langle\Psi_{0}|-(\Phi \otimes \mathbb{I})| \Psi_{0}\right\rangle\left\langle\Psi_{0}\right| \|=\mathcal{N}\left(\left|\Psi_{0}\right\rangle\left\langle\Psi_{0}\right|\right) .
$$

To obtain a locally observable lower bound on this quantity, we monitor the time evolution of the dephased state on the single-spin level

$$
\rho_{S}(t)=\operatorname{Tr}_{E}\left\{U(t)(\Phi \otimes \mathbb{I})\left|\Psi_{0}\right\rangle\left\langle\Psi_{0}\right| U^{\dagger}(t)\right\},
$$

with $U(t)=e^{-i H t / \hbar}$. Any deviation from $\rho_{S}(0)$ indicates the presence of entanglement in $\left|\Psi_{0}\right\rangle$ and the trace distance

$$
d(t)=\left\|\rho_{S}(t)-\rho_{S}(0)\right\|
$$

\footnotetext{
${ }^{5}$ Multipartite entanglement will only be introduced in the next chapter. We already remark here that the GHZ-states contain global multipartite entanglement, but when one or more particles are traced out, no entanglement remains in the subsystem (Dür et al., 2000).
} 

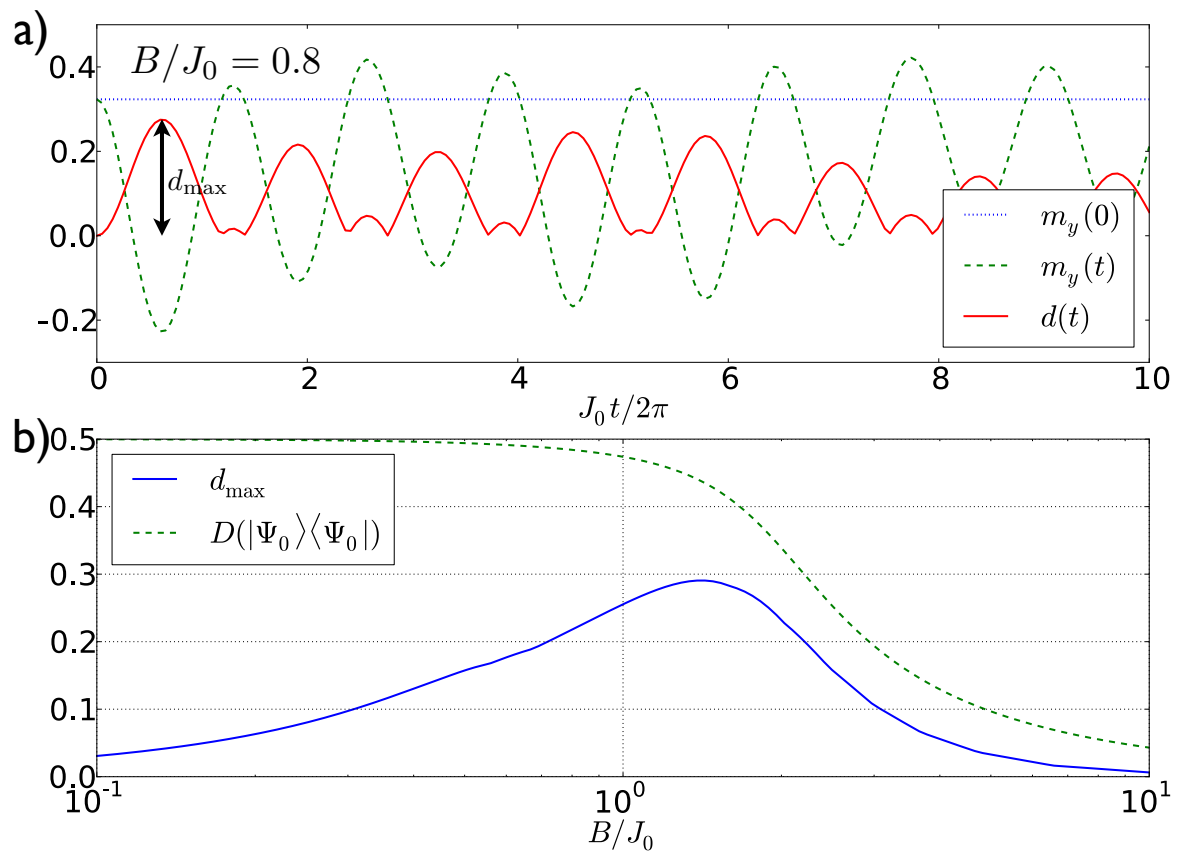

FIGURE 3.11.: Monitoring the dephasing-induced time evolution (3.163) of the local single-spin magnetization along the $y$-axis is sufficient to provide a lower bound for the total ground state negativity (3.160). The maximal local trace distance [maximized over $t$, see equation (3.164)] shows a peak close to the critical point of the quantum phase transition, of which predecessors can be observed in this small system of $N=7$ spins. Observation of the time evolution over a longer interval can further improve the lower bound. The total trace distance $D\left(\left|\Psi_{0}\right\rangle\left\langle\Psi_{0}\right|\right)$ is equal to the ground-state negativity, see equation (3.160). Adapted from (Gessner et al., 2014b).

provides a lower bound on the negativity $\mathcal{N}\left(\left|\Psi_{0}\right\rangle\left\langle\Psi_{0}\right|\right)$ at all times $t$. Since the local state $\rho_{S}(t)$ must remain diagonal in the eigenbasis of $\sigma_{y}$, the trace distance (3.162) is fully determined by the magnetization $m_{y}(t)=\operatorname{Tr}\left\{\rho_{S}(t) \sigma_{y}\right\}$ of the observable spin along the $y$ direction as

$$
d(t)=\frac{1}{2}\left|m_{y}(t)-m_{y}(0)\right|
$$

The maximum local distance

$$
d_{\max }=\max _{t} d(t) \leq \mathcal{N}\left(\left|\Psi_{0}\right\rangle\left\langle\Psi_{0}\right|\right)
$$

is plotted in figure 3.11. We find that for very small values of $B$, despite large amounts of entanglement in the ground state (as quantified by $D\left(\left|\Psi_{0}\right\rangle\left\langle\Psi_{0}\right|\right.$ ) and plotted by the green dashed line in figure $3.11 \mathrm{~b}$ ), the dynamics does not reveal those correlations to 


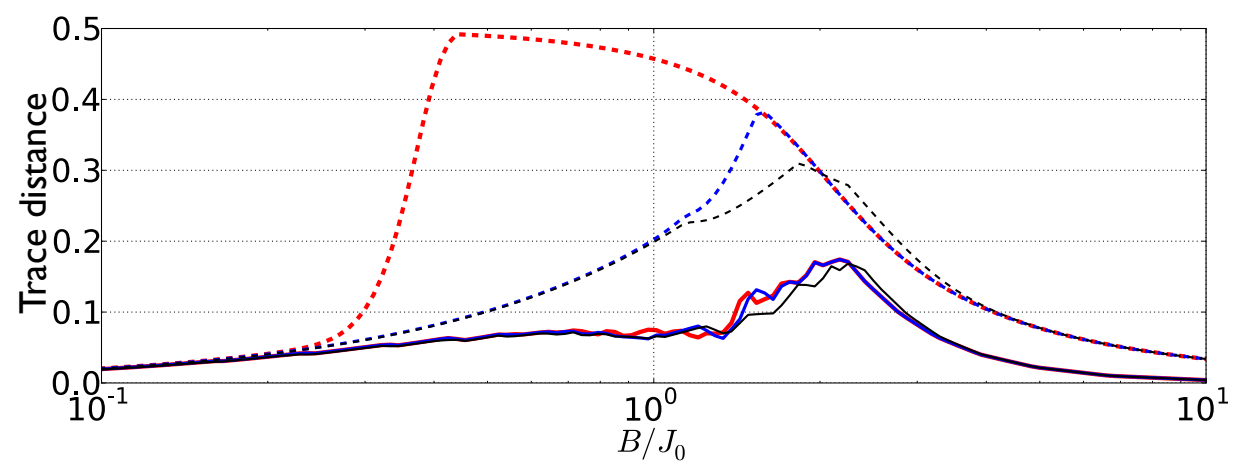

FIGURE 3.12.: For low, finite temperatures, the minimum dephasing disturbance $D_{\text {min }}$ (dashed lines), defined in equation (3.11), vanishes when the excitation gap between the almost-degenerate ground states is smaller than the thermal energy $k T$ (see figure 3.13). The parameters are $N=7$ spins with $k T=10^{-5}$ (thick, red), $k T=0.1$ (medium, blue), and $k T=1$ (black, thin line). The local signature $d_{\min }$ (corresponding continuous lines), defined in equation (3.20), is, however, only weakly affected by finite temperatures. The simulations consider a set of 20 uniformly distributed pairs of qubit basis states for the different dephasing bases that occur in the definition (3.11) of the minimum dephasing disturbance. Adapted from (Gessner et al., 2014b).

the local spin state. When we approach the quantum critical point around $B \simeq J_{0}$, a strong, visible signal emerges and almost reaches the upper bound which is given by the negativity of the total state. As the external field is further increased, the total correlations decrease, and consequently the local signal disappears. The distinct peak identifies the critical point of the quantum phase transition. An explanation for this behavior can be given after realizing that the dephasing operation connects the ground-state properties to the excitation spectrum, since these are revealed via the dephasing-induced dynamics. Indeed, the excitation spectrum undergoes a significant structural change around the critical field of the quantum phase transition, which induces complex dynamics which is more likely to reveal correlation properties to the local system, as we will discuss in further detail in chapter 5 .

Typical parameters are $J_{0} \approx 2 \pi \times 500 \mathrm{~Hz}$ and coherence times are usually around $3 \mathrm{~ms}$. In the simulations, we therefore restrict the observable time window to a range of $5 \mathrm{~ms}$. The simulations are implemented with a python code employing the qutip package (Johansson et al., 2013).

\subsubsection{Local bound for the minimum entanglement potential of thermal states}

For finite-sized ferromagnetic systems, there exists a small energy gap $\Delta$ between the ground state and the first excited state of $H$. This gap vanishes in the thermodynamic limit, $N \rightarrow \infty$, where the ground state becomes two-fold degenerate. Despite very low 
temperatures, it is therefore more realistic to consider the thermal state

$$
\rho_{\beta}=\frac{e^{-\beta H}}{\operatorname{Tr} e^{-\beta H}}
$$

instead of the pure ground state, with $\beta=1 / k T$, where $k$ denotes the Boltzmann constant and $T$ the temperature. Even in the zero-temperature limit, the degeneracy produces an incoherent mixture of the two ground states instead of a pure state. This has the effect that the entanglement, and even the discord-type correlations vanish: The mixture of the two ground states

$$
\left.\rho_{\infty}\right|_{B=0}=\frac{1}{2}\left(\left|\mathrm{GHZ}_{\varphi}\right\rangle\left\langle\mathrm{GHZ}_{\varphi}|+| \mathrm{GHZ}_{\varphi+\pi}\right\rangle\left\langle\mathrm{GHZ}_{\varphi+\pi}\right|\right)
$$

describes a state of zero discord since the opposing phase factors lead to complete cancellation of the coherences when both states are mixed. The minimum dephasing disturbance $D_{\min }$, as introduced in equation (3.11), therefore yields zero for the lowtemperature thermal ground state (3.166) when $B=0$, and increases to a nonzero value when the energy gap between the ground states exceeds the thermal excitation energy $k T$, and recovers the negativity of the energetically lower-lying state, as can be seen from the dashed lines in figure 3.12 and the inset in figure 3.13. From these figures we can also observe how the sharpness of this transition increases with decreasing $T$. A lower bound for $D_{\min }$ can be obtained by dephasing in a large ensemble of basis states, and monitoring the subsequent time evolutions, which yields the optimal local witness $d_{\text {min }}$, as defined in equation (3.20). While the total correlations are strongly affected by the mixing process, the local dynamical signature remains largely unaffected, and still shows a maximum in the vicinity of the quantum phase transition.

In the present study, we have established a dynamical witness for the critical field of the quantum phase transition based on entanglement. It is based on measurements of dephasing-induced dynamics of the the single-spin magnetization. This dynamics is entirely determined by the excitation spectrum which is populated by the local dephasing operation. The fact that we observe a strong peak at the phase transition point indicates a connection of the quantum phase transition to the excitation spectrum, which we will study in further detail in chapter 5 .

\subsection{Discussion}

We have seen in this chapter that the coupling of a small controllable subsystem to additional, inaccessible degrees of freedom can be harnessed to reveal global properties, such as bipartite correlations, via the local subsystem dynamics. The basic mechanism which enables the local detection protocol presented in this chapter is the strong influence of correlations on the subsequent time evolution of an open quantum system. The method can always be applied when full coherent control is available over a part of the full system. Whether or not the method successfully reveals the presence of correlations through the local dynamics depends on the time evolution of the full system. 


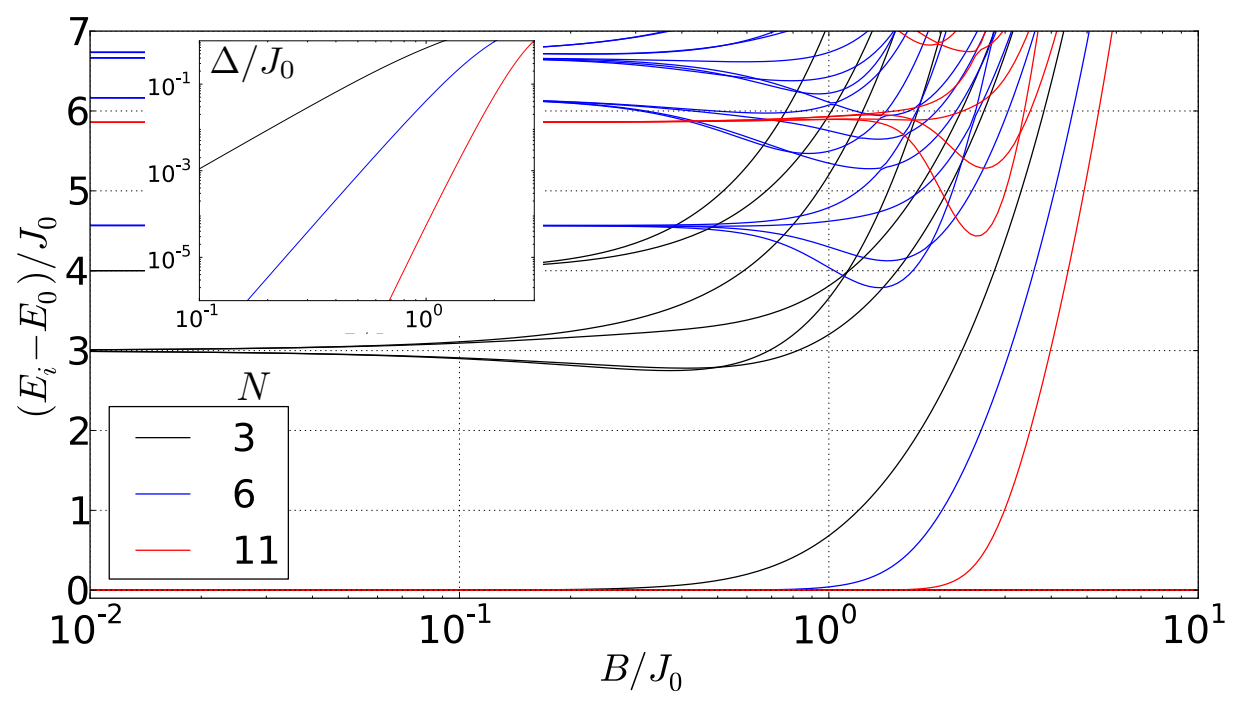

FIGURE 3.13.: Lower part of the excitation spectrum of the ferromagnetic spin chain (3.158) at $\alpha=1$ for different system sizes. The gap $\Delta$ between the ground- and first excited state (inset) determines at which temperature the low-temperature thermal state (3.165) turns from a pure ground state to an incoherent mixture of both ground states. Unlike the pure ground state, which contains GHZ-type entanglement (3.159), the incoherent mixture (3.166) contains neither entanglement nor discord-type correlations. In the thermodynamic limit the two ground states are degenerate for values of $B$ below the critical field. A detailed discussion of the spectrum is provided in chapter 5 .

The examples studied in this chapter have explored the limitations of the method for different couplings, environments, and physical settings. Scenarios where the detection was successful included the dissipative coupling to a single-mode harmonic oscillator, which was studied in the trapped-ion experiment. Numerical simulations also suggest an observable local signature of correlations in a high-temperature limit. Moreover, the photonic experiment demonstrated a clearly observable signature of initial correlations in the presence of coupling to a continuum of modes that lead to irreversible, memoryless dephasing of a qubit. The important difference to the trapped-ion case is that, here, the coupling does not induce exchange of excitations between system and environment. A completely memoryless, dissipative environment was studied theoretically using the example of spontaneous emission of an atomic excitation into the free-field modes. In this case, there is no influence of the atom-field entanglement on the atomic subdynamics. Taken together, these findings suggest that the efficacy of the method does not rely on non-Markovian memory effects or structured environments, as long as the reduced dynamics is described by pure dephasing (no excitation exchange). For dissipative environments (including excitation exchange), a structured environment, leading to back-action on the system, seems to be a necessary requirement for a local 
signature of initial correlations based on the present method.

The boarder where the influence of initial correlations on the reduced dynamics vanishes due to dispersion into many modes of a high-dimensional system may be explored thoroughly with a proposed generalization of the trapped-ion experiment. Based on the intuition developed in this chapter, we expect the local signal to decrease when the environment becomes sufficiently large and spectrally broad, to be effectively memoryless. In this case, when we find irreversible dissipation, free of revivals, we expect little influence of the initial correlations on the open-system dynamics. Similarly, theoretical studies in the context of a lossy cavity, described by coupling to a continuum of modes of variable spectral width, may also directly test the dependence of the method on non-Markovian effects in the presence of dissipative couplings.

The separation into system and environment can formally be carried over to fewbody or many-body systems, where local control of some of the constituents is possible, while complete control over the state of the full system is not available. To verify the presence of discord-type correlations, which are necessary for carrying out specific quantum information protocols, one may employ the local detection method, given that interactions between the constituents are present. To this end, one treats the accessible subsystem as the "open system" and the remaining constituents of the interacting system as "environment". The method is expected to be useful when the total system becomes too large to employ tomographic reconstruction methods or to measure complicated entanglement witnesses, which require measurements on all parties. In this case, one may restrict to controlling a low-dimensional subsystem, which is more feasible. In the case of excitation-exchanging interactions, however, we must consider the limitation of the method in the extreme case when the environmental part becomes effectively memoryless, as discussed above. The discussion of the example of the spin-chain model furthermore raises the intriguing question about the connection between ground-state quantum correlations, the excitation spectrum and the quantum phase transition, which will be analyzed in chapter 5 . 



\section{FROM LOCAL OPERATIONS TO COLLECTIVE DEPHASING: BEHAVIOR OF CORRELATED QUANTUM STATES}

To analyze the correlation properties of quantum states with nonzero discord, we investigate the possibilities to create quantum discord with local operations. We introduce the correlation rank as a complementary notion for correlations in quantum states. Furthermore we study an experimentally relevant, classically correlated dephasing processes and its effect on different notions of correlations in quantum states.

\subsection{Creation of quantum discord by local operations}

In the previous chapter, the detection of quantum discord was discussed on the basis of local operations. In the present chapter, we take on the converse point of view and analyze the possibility to use local operation to create discord (Gessner et al., 2012). As we will see, this possibility indeed exists, which affirms that quantum discord is not a measure for correlations. ${ }^{1}$ Correlations are required to be non-increasing under local operations (Bennett et al., 1996a): The impossibility to increase under local operations (and classical communications) is one of the fundamental requirements for appropriate measures of quantum entanglement (Werner, 1989; Bennett et al., 1996b; Vedral et al., 1997). Also total correlations - which express how far a given quantum state differs from a completely uncorrelated product state, without distinguishing between the quantum or classical nature of these correlations - cannot be created by

\footnotetext{
${ }^{1}$ In the original publication, it was introduced as a "measure for the quantumness of correlations" (Ollivier and Zurek, 2001).
} 
local operations. If a local operation $\Phi \otimes \mathbb{I}$ is applied to a product state (which has zero total correlations), the resulting state

$$
(\Phi \otimes \mathbb{I}) \rho_{A} \otimes \rho_{B}=\Phi\left(\rho_{A}\right) \otimes \rho_{B},
$$

is still an uncorrelated product state. How can we picture the creation of nonzero quantum discord from a classical state by local operations? Consider the following example of a classically correlated, zero-discord state of two qubits (Dakić et al., 2010),

$$
\rho_{c}=\frac{1}{2}(|00\rangle\langle 00|+| 11\rangle\langle 11|) .
$$

The local orthogonal states $|0\rangle$ and $|1\rangle$ can be mapped onto new states

$$
|0\rangle \rightarrow\left|\varphi_{0}\right\rangle, \quad|1\rangle \rightarrow\left|\varphi_{1}\right\rangle .
$$

with a completely positive and trace-preserving local operation in one of the systems. The resulting state

$$
\rho_{q}=\frac{1}{2}\left(\left|\varphi_{0} 0\right\rangle\left\langle\varphi_{0} 0|+| \varphi_{1} 1\right\rangle\left\langle\varphi_{1} 1\right|\right) .
$$

has nonzero discord as soon as the states $\left|\varphi_{0}\right\rangle$ and $\left|\varphi_{1}\right\rangle$ are non-orthogonal, $\left\langle\varphi_{0} \mid \varphi_{1}\right\rangle \neq 0$. In this example, the quantum discord assesses the non-orthogonality of local states in an incoherent mixture. This non-orthogonality can lead to effects, such as noncommutativity with certain observables and disturbance by measurements, which are interpreted as quantum phenomena.

Furthermore, it is crucial to note that a key prerequisite for such a local creation of discord is the presence of classical correlations in the initial state $\rho_{c}$, as becomes apparent by reconsidering the case of a product state (4.1). It is therefore not correct to assume that a local operation can create discord "from nothing". Instead, a more appropriate interpretation is the conversion of classical correlations into discord by manipulating the local orthogonality of the basis states. The final state is then still separable and cannot be more strongly correlated than the initial state. Indeed, recalling section 2.1.1, we know that nonzero quantum discord only reflects the absence of a local observable that commutes with the total state (Girolami et al., 2013), which, in principle, does not quantify the state's correlation properties. However, since such an observable can always be found for uncorrelated states (see section 2.1.1), nonzero discord also implies nonzero correlations.

The above scenario demonstrates that the mere presence of quantum discord does not imply the presence of correlations which are necessarily stronger than those of other classical (zero-discord) states. Since the maximum number of local orthogonal basis states is bounded by the dimension of the local subsystem, the nonzero discord states which can be created from classically correlated states by local operations must be severely limited, even when we exclude the entire set of entangled states, which of course have nonzero discord but, by definition, cannot be reached with local operations. In order to put these two statements into a more quantitative picture, and to complement the information provided by the quantum discord, we introduce the correlation rank (Gessner et al., 2012). 


\subsection{Correlation rank: Schmidt decomposition for mixed states}

To introduce the correlation rank, we employ a decomposition of the total, possibly mixed density matrix, close in spirit to the Schmidt decomposition of a pure entangled state. We consider a bipartite Hilbert space $\mathcal{H}=\mathcal{H}_{A} \otimes \mathcal{H}_{B}$ where the two subsystems are described by their respective Hilbert spaces of dimensions $d_{A}$ and $d_{B}$. We express an arbitrary bipartite quantum state $\rho$, that is, a normalized, positive, and bounded operator on $\mathcal{H}$, in terms of arbitrary operator bases $\left\{A_{i}\right\}$ and $\left\{B_{i}\right\}$ on the individual respective Hilbert spaces (Dakić et al., 2010),

$$
\rho=\sum_{i=1}^{d_{A}^{2}} \sum_{j=1}^{d_{B}^{2}} r_{i j} A_{i} \otimes B_{j} .
$$

A singular value decomposition of the real-valued correlation matrix $R=\left(r_{i j}\right)$ yields a diagonal representation

$$
\rho=\sum_{i=1}^{L} c_{i} S_{i} \otimes F_{i}
$$

with $S_{i}=\sum_{j} u_{j i} A_{j}, F_{i}=\sum_{j} v_{j i} B_{j}$ and orthogonal matrices $U=\left(u_{i j}\right)$ and $V=\left(v_{i j}\right)$ which transform the correlation matrix into diagonal form,

$$
R=U \operatorname{diag}\left(c_{1}, c_{2}, \ldots, c_{L}, 0, \ldots\right) V^{T},
$$

and the $c_{i}$ denote the nonzero singular values of $R$. The number $L$ denotes the correlation rank of the quantum state $\rho$. Before we proceed, we remark that the diagonal form (4.6) allows for a simple check for nonzero discord, which nicely generalizes the above intuition about local non-orthogonality to non-separable states: The state $\rho$ has zero quantum discord with respect to subsystem $\mathcal{H}_{A}$ if and only if $\left[S_{i}, S_{j}\right]=0$ for all $i$ and $j$ (Dakic et al., 2010). Analogously, the commutativity of the $F_{j}$ determine the quantum discord with respect to measurements on subsystem $\mathcal{H}_{B}$. The $S_{i}$ and $F_{i}$, however, can in general not be interpreted as quantum states since, for example, they may exhibit negative eigenvalues.

The correlation rank quantifies the total correlations by precisely counting the number of uncorrelated product operators that are necessary to decompose a given quantum state. Let us consider a pure state $\rho=|\Psi\rangle\langle\Psi|$ with Schmidt decomposition $|\Psi\rangle=\sum_{i=1}^{S} \lambda_{i}\left|\varphi_{i}\right\rangle \otimes\left|\chi_{i}\right\rangle$ (Schmidt, 1907) to investigate how the two concepts are related. The Schmidt number $S$ denotes the number of nonzero Schmidt coefficients $\lambda_{i}$ and quantifies the entanglement of $|\Psi\rangle$ (Barnett and Phoenix, 1991; Ekert and Knight, 1995). We write

$$
\rho=|\Psi\rangle\left\langle\Psi\left|=\sum_{i=1}^{S} \sum_{j=1}^{S} \lambda_{i} \lambda_{j}\right| \varphi_{i}\right\rangle\left\langle\varphi_{j}|\otimes| \chi_{i}\right\rangle\left\langle\chi_{j}\right| .
$$

Combining the two indices $i$ and $j$ into a single index $k=(i, j)=1, \ldots, S^{2}$ allows us to cast this decomposition into the form (4.6) with local operator bases $A_{k}=\left|\varphi_{i}\right\rangle\left\langle\varphi_{j}\right|$ 
and $B_{k}=\left|\chi_{i}\right\rangle\left\langle\chi_{j}\right|$ and coefficients $c_{k}=\lambda_{i} \lambda_{j}$. This decomposition cannot be further reduced, and we find $L=S^{2}$, demonstrating that the correlation rank represents a direct generalization of the Schmidt decomposition to mixed states. It is important to emphasize here that $L$ is not an entanglement measure, but, rather quantifies total correlations. As we have confirmed with the previous example, the total correlations of pure states are equivalent to their entanglement.

We first establish that the correlation rank cannot increase under local operations. To this end, we employ the diagonal representation (4.6),

$$
\begin{aligned}
(\Phi \otimes \mathbb{I}) \rho & =\sum_{i=1}^{L} c_{i} \Phi\left(S_{i}\right) \otimes F_{i} \\
& =\sum_{i=1}^{L} \sum_{j=1}^{d_{A}^{2}} c_{i} d_{i j} A_{j} \otimes F_{i}
\end{aligned}
$$

where we decomposed each of the $\Phi\left(S_{i}\right)$ in terms of the local operator basis $A_{j}$ using the coefficient matrix $D=\left(d_{i j}\right)$. The resulting correlation rank $L^{\prime}$ is given by the rank of the matrix $M=\left(c_{i} d_{i j}\right)$, which yields

$$
L^{\prime}=\operatorname{rk} M \leq \min \{L, \operatorname{rk} D\} \leq L,
$$

and can never exceed the initial correlation rank (Gessner et al., 2012). This can be generalized straight-forwardly to show that this still holds for bilocal operations of the form $\Phi_{A} \otimes \Phi_{B}$ (Lanyon et al., 2013).

This provides a strict constraint on the nonzero discord states which can be created from zero discord states by local operations. Consider a general classical state, that is, a state of zero discord with respect to both subsystems,

$$
\rho_{c c}=\sum_{i=1}^{d_{A}} \sum_{j=1}^{d_{B}} p_{i j} \Pi_{i}^{A} \otimes \Pi_{j}^{B} .
$$

The number of projectors $\Pi_{i}^{A}=\left|i_{A}\right\rangle\left\langle i_{A}\right|$ and $\Pi_{j}^{B}=\left|j_{B}\right\rangle\left\langle j_{B}\right|$ onto orthogonal pure states $\left|i_{A}\right\rangle$ and $\left|j_{B}\right\rangle$ is fundamentally limited by the dimensions of the respective local Hilbert spaces $\mathcal{H}_{A}$ and $\mathcal{H}_{B}$. Therefore, the correlation rank of classical states is bounded from above by the minimal subsystem dimension $d_{\min }=\min \left\{d_{A}, d_{B}\right\}$ (Dakić et al., 2010). Since local operations cannot increase the correlation rank, the same must hold for all nonzero discord states that can be created from classical states with local operations (Gessner et al., 2012).

From this we draw the conclusion that any state with $L>d_{\min }$ contains correlations that are beyond the reach of any classical state in the same Hilbert space, which separates the nonzero discord states into the classes above and below this threshold. This information about the total correlations, then, can be complemented by measures of quantum discord, which determine the quantum nature of these states with respect to local measurements. Note that even some (not maximally) entangled states may have $L \leq d_{\text {min }}$ in Hilbert space dimensions larger than two (Gessner et al., 2012). 
Appropriate local transformations of the local bases $\left|i_{A}\right\rangle$ and $\left|j_{B}\right\rangle$ allow to convert the state (4.11) into arbitrary states of the form (Gessner et al., 2012)

$$
\rho=\sum_{i=1}^{d_{\text {min }}} p_{i} \rho_{i}^{A} \otimes \rho_{i}^{B},
$$

with local density matrices $\rho_{i}^{A}$ and $\rho_{i}^{B}$. Note that the number of product states that are mixed in (4.12) is upper bounded by the minimum dimension of the two subsystems $d_{\text {min }}$. At the same time, states of the form (4.12) form the most general class of states that can be created from classical states with local operations and it can be shown that these states form a set of Lebesgue measure zero in the total set of quantum states (Gessner et al., 2012). One can, however, design an example of a mixed separable state in a bipartite Hilbert space with unequal subsystem dimensions $d_{B}>d_{A}>2$, which has $L=d_{\text {min }}$ but cannot be represented as a convex linear combination of less than $d_{\text {min }}+1$ product states (Zhao, 2012). This example shows that not all separable states whose correlation rank is compatible with classical states can be created from a classical state with local operations, as one may have suspected intuitively.

The correlation rank has also been employed (Giorgi, 2013) to argue against the universality of claims which identify quantum discord as a resource in the remote state preparation protocol (Dakić et al., 2012). Moreover, the information which can be transmitted from one party to another in a tomographic multiple-copy teleportation scenario with mixed states is determined by the correlation rank (Wang et al., 2013).

\subsection{Trapped-ion experiment}

Theoretical studies showed that even local Markovian noise processes can suffice to create states of nonzero discord from classically correlated initial states ( $\mathrm{Hu}$ et al., 2011; Ciccarello and Giovannetti, 2012; Streltsov et al., 2011a), and an experiment with two trapped ions, carried out at the Institute for Quantum Optics and Quantum Information in Innsbruck, measured this effect (Lanyon et al., 2013). The experiment also confirmed that the correlation rank was at all times bounded by the initial value during the local noise process.

\subsubsection{Local amplitude damping}

In the experiment, the classical two-qubit state

$$
\rho_{1}=\frac{1}{2}(|++\rangle\langle++|+|--\rangle\langle--|),
$$

with $| \pm\rangle=(|0\rangle \pm|1\rangle) / \sqrt{2}$ was exposed to a local amplitude damping channel on the second qubit, described by $\mathcal{E}_{a d}(\rho)=E_{0} \rho E_{0}^{\dagger}+E_{1} \rho E_{1}^{\dagger}$, with $E_{0}=|0\rangle\langle 0|+\sqrt{1-p}| 1\rangle\langle 1|$ and $E_{1}=\sqrt{p}|0\rangle\langle 1|$. This channel describes one of the elementary loss processes of quantum computers due to energy dissipation (Nielsen and Chuang, 2000). Figure 4.1 displays the experimental result of full state tomography of the two-qubit density 

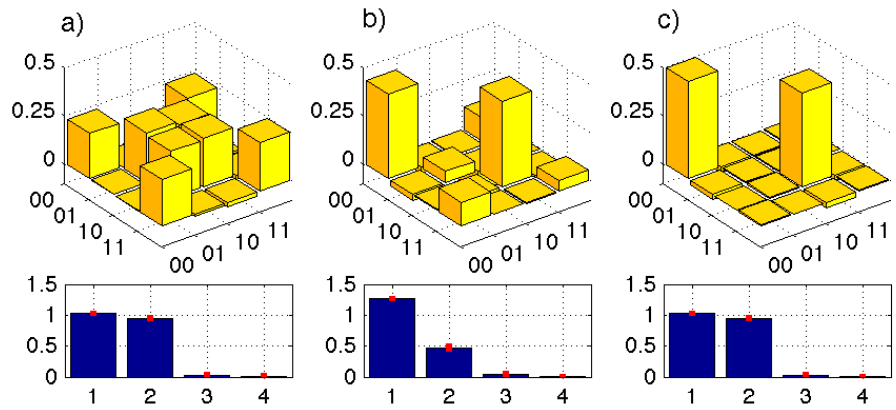

FIGURE 4.1.: Real values of the elements of the full two-qubit density matrix under the influence of a local amplitude damping channel [see text below equation (4.13)] for $p=0$ (a), $p=0.79$ (b) and $p=1$ (c). The initial state $\rho_{1}$, defined in (4.13), has zero discord and correlation rank $L=2$. While the discord can be shown to be nonzero in (b), the singular values of the correlation matrix (blue bars) never indicate a correlation rank above the initial value of $L=2$. Figure taken from (Lanyon et al., 2013).

matrices for different values of $0 \leq p \leq 1$, as well as the singular values of the associated correlation matrices. While the quantum discord in the reconstructed quantum states can be shown to increase for intermediate values of $p$, the correlation rank is never found to exceed the initial value $L=2$, within the experimental error bars (Lanyon et al., 2013).

Since the qubit resonance frequencies are controlled by the magnitude of the external magnetic field, an additional dephasing effect is induced by fluctuations of the latter; see section 2.2.1. This results in a correlated dephasing process since the field is, to a good approximation, spatially homogeneous within the trap center, and, thus, the fluctuations affect all ions equally. Under such separable operations, $\sum_{i} p_{i} \Phi_{i}^{A} \otimes \Phi_{i}^{B}$, the correlation rank is no longer bounded and can increase arbitrarily, as can be seen by starting from equation (4.6), and employing the decompositions $\Phi_{i}^{A}\left(S_{j}\right)=\sum_{k=1}^{d_{A}^{2}} d_{j k}^{i} A_{k}$ and $\Phi_{i}^{B}\left(F_{j}\right)=\sum_{l=1}^{d_{B}^{2}} e_{j l}^{i} B_{l}$ to describe the final state as (Lanyon et al., 2013)

$$
\rho^{\prime}=\sum_{i=1}^{P} p_{i}\left(\Phi_{i}^{A} \otimes \Phi_{i}^{B}\right) \rho=\sum_{i=1}^{P} \sum_{j=1}^{L} \sum_{k=1}^{d_{A}^{2}} \sum_{l=1}^{d_{B}^{2}} p_{i} c_{j} d_{j k}^{i} e_{j l}^{i} A_{k} \otimes B_{l} .
$$

The rank of the correlation matrix $f_{k l}=\sum_{i=1}^{P} \sum_{j=1}^{L} p_{i} c_{j} d_{j k}^{i} e_{j l}^{i}$ can now become as large as $\min \left\{P \cdot L, d_{A}^{2}, d_{B}^{2}\right\}$.

Thus, separable processes, such as collective dephasing are able to synthesize separable quantum states with strong total correlations. The dephasing effect is quite interesting as a process on its own. By ensemble-averaging over classically fluctuating external parameters, an effective, incoherent atom-atom interaction is induced, allowing for the generation of correlations even when the atoms are not coupled by other mechanisms. We will first discuss the asymptotics for the special case of two qubits 
and its implications for the created correlations to describe the trapped-ion experiment reported in (Lanyon et al., 2013). In section 4.4, a general description of the dynamics towards the asymptotic state, for arbitrary numbers of spins, will be provided and applied to study the decay and robustness of multipartite entanglement.

\subsubsection{Collective dephasing}

As described in section 2.2.1, the transition frequency of the electronic trapped-ion qubits are determined by the strength of the external magnetic field $B$ due to the Zeeman effect. The fluctuations of this external field, which in turn are caused by instabilities of the currents of the generating coils, lead to fluctuations of the qubit frequencies. This effect is strongly correlated, since all ions in the trap experience the same magnetic field due to the relatively small inter-ion distances. Usually, the induced effect on the qubit dynamics is small on relevant time scales of trapped-ion experiments. By intentionally exposing the ions to such fluctuations for long times, full dephasing towards some asymptotic state can be achieved (Lanyon et al., 2013).

To describe this effect, we derive the corresponding Hamiltonian and the resulting ensemble-averaged dynamics induced by the fluctuations. The direction $\mathbf{n}$ of the external magnetic field $\mathbf{B}=B \mathbf{n}$ determines the eigenbasis of the electronic two-level system

$$
H_{\omega}=\frac{\hbar \omega}{2} \mathbf{n} \cdot \boldsymbol{\sigma}
$$

and the transition frequency is proportional to the magnitude of the field $B$,

$$
\omega=\mu B
$$

where the proportionality factor $\mu$ can be interpreted as a magnetic moment. The resulting time evolution describes a rotation of the qubit,

$$
U_{\omega}(t)=e^{-i H_{\omega} t / \hbar}=e^{-i \omega t \mathbf{n} \cdot \boldsymbol{\sigma} / 2},
$$

where the angle of rotation $\theta=\omega t$ is determined by the duration of the evolution. In this section we restrict to the case of two ions, subject to the same fluctuating external field. To estimate expectation values, experiments have to be repeated many times. The appropriate description for the associated resulting quantum state is the ensemble average

$$
\rho(t)=\mathcal{E}_{t, 0}^{\mathbf{n}}[\rho(0)]=\int d \omega p(\omega) U_{\omega}(t) \otimes U_{\omega}(t) \rho(0) U_{\omega}^{\dagger}(t) \otimes U_{\omega}^{\dagger}(t)
$$

which is governed by the frequency distribution $p(\omega)$. In writing (4.18), we assume that the fluctuations occur on time scales that are much longer than the typical time scale of the atomic evolution, which allows us to model the dynamics of a single experimental run by a time-independent Hamiltonian, characterized by a constant magnetic field. From one experimental repetition to another, the magnetic field may have changed. 
Since the time scale of the atomic evolution, and with it the typical duration of a single experimental run, is determined by the strength of the magnetic field, the above condition is met when the fluctuation amplitude of $B$ is much weaker than its mean value.

When this condition is satisfied we can also investigate the asymptotic limit of this evolution, which is reached when the system evolves for a time $\tau$, which is long enough, such that the fluctuations of $\omega$ distribute the resulting phases $e^{-i \omega \tau}$ uniformly over the unit circle, but not too long to conflict with the assumption of a time-independent Hamiltonian. Formally, the asymptotic state is given by

$$
\rho_{s}=\lim _{t \rightarrow \infty} \rho(t)
$$

whereas we will show in section 4.4 that for experimentally realistic distributions $p(\omega)$, this state is independent of the exact form of $p(\omega)$. Hence, we restrict the analysis in this section to

$$
\rho_{s}=\mathcal{E}^{\mathbf{n}}[\rho(0)]=\frac{1}{2 \pi} \int_{0}^{2 \pi} d \theta \mathcal{R}_{\mathbf{n}}(\theta) \otimes \mathcal{R}_{\mathbf{n}}(\theta) \rho(0) \mathcal{R}_{\mathbf{n}}^{\dagger}(\theta) \otimes \mathcal{R}_{\mathbf{n}}^{\dagger}(\theta),
$$

with $\mathcal{R}_{\mathbf{n}}(\theta)=e^{-i \theta \mathbf{n} \cdot \boldsymbol{\sigma} / 2}$. As we will show in section 4.4 , this map can be written in Kraus form as (Lanyon et al., 2013)

$$
\mathcal{E}^{\mathbf{n}}[\rho(0)]=\sum_{i=0}^{2} \Theta_{i} \rho(0) \Theta_{i}
$$

with Kraus operators

$$
\begin{aligned}
& \Theta_{0}=\Lambda_{+} \otimes \Lambda_{+}, \\
& \Theta_{1}=\Lambda_{+} \otimes \Lambda_{-}+\Lambda_{-} \otimes \Lambda_{+}, \\
& \Theta_{2}=\Lambda_{-} \otimes \Lambda_{-},
\end{aligned}
$$

and the orthogonal projectors

$$
\Lambda_{ \pm}=(\mathbb{I} \pm \mathbf{n} \cdot \boldsymbol{\sigma}) / 2 .
$$

To analyze the evolution and correlation properties of arbitrary two-qubit states, it is most convenient to represent those in the Fano form (Fano, 1983; Bengtsson and Życzkowski, 2006)

$$
\rho=\frac{1}{4}\left(\mathbb{I} \otimes \mathbb{I}+\sum_{i=1}^{3} r_{i}^{A} \sigma_{i} \otimes \mathbb{I}+\sum_{i=1}^{3} r_{i}^{B} \mathbb{I} \otimes \sigma_{i}+\sum_{i=1}^{3} \sum_{j=1}^{3} \beta_{i j} \sigma_{i} \otimes \sigma_{j}\right) .
$$

The two reduced Bloch vectors $\mathbf{r}^{A}, \mathbf{r}^{B}$, and the matrix $\beta$ can be chosen real. The correlation matrix is given by

$$
R=\frac{1}{4}\left(\begin{array}{cc}
1 & \left(\mathbf{r}^{B}\right)^{T} \\
\mathbf{r}^{A} & \beta
\end{array}\right)
$$




\begin{tabular}{l|ccc} 
& $\mathbf{n}=\mathbf{w}$ & $\mathbf{n} \cdot \mathbf{w}=0$ & $|\mathbf{n} \cdot \mathbf{w}|>0$ \\
\hline $\mathbf{n}=\mathbf{v}$ & 2 & 1 & 2 \\
$\mathbf{n} \cdot \mathbf{v}=0$ & 1 & 3 & 3 \\
$|\mathbf{n} \cdot \mathbf{v}|>0$ & 2 & 3 & 4
\end{tabular}

FIGURE 4.2.: The correlation rank $L$ after collective dephasing into the asymptotic state can be controlled via the orientation $\mathbf{n}$ of the external magnetic field. The initial classically correlated state with $L=2$ and maximally mixed reduced density matrices is characterized by the two vectors $\mathbf{v}$ and $\mathbf{w}$. Appropriate choice of $\mathbf{n}$ can lead to a strongly correlated state of maximal correlation rank, $L=4$.

with the $1 \times 3$ matrix $\mathbf{r}^{T}=\left(r_{1}, r_{2}, r_{3}\right)$. This representation allows for direct determination of the rank $L$ of the correlation matrix $R$ by (Meyer, 1973)

$$
L=1+\operatorname{rk}\left(\beta-\mathbf{r}^{A} \otimes \mathbf{r}^{B}\right),
$$

where the elements of $\mathbf{r}^{A} \otimes \mathbf{r}^{B}$ are defined as $\left(\mathbf{r}^{A} \otimes \mathbf{r}^{B}\right)_{i j}=r_{i}^{A} r_{j}^{B}$. The matrix $\beta$ can always be decomposed into singular values $d_{k}$ as $\beta_{i j}=\sum_{k=1}^{3} v_{i k} d_{k} w_{j k}$, with orthogonal matrices $V$ and $W$. We rewrite the state $\rho$ as

$$
\rho=\frac{1}{4}\left(\mathbb{I} \otimes \mathbb{I}+\mathbf{r}^{A} \cdot \boldsymbol{\sigma} \otimes \mathbb{I}+\mathbb{I} \otimes \mathbf{r}^{B} \cdot \boldsymbol{\sigma}+\sum_{k=1}^{3} d_{k} \mathbf{v}_{k} \cdot \boldsymbol{\sigma} \otimes \mathbf{w}_{k} \cdot \boldsymbol{\sigma}\right),
$$

where we have introduced two sets of orthonormal vectors $\left(\mathbf{v}_{k}\right)_{i}=v_{i k}$ and $\left(\mathbf{w}_{k}\right)_{j}=$ $w_{j k}$.

Using this representation, the correlation rank of the asymptotic state $\rho_{s}$ can be deduced from geometric relations between the vectors $\left\{\mathbf{v}_{k}, \mathbf{w}_{k}\right\}$ and the direction $\mathbf{n}$ of the external magnetic field. For details we refer to (Lanyon et al., 2013). In the experiment, two classes of initial states were considered:

(A) uncorrelated states $(L=1)$,

(B) classically correlated, zero-discord states $(L=2)$ with maximally mixed reduced density matrices.

One can show that the states of class (A), that is, states with $L=1$, coincide with the set of product states and that, in this case, $\beta=\mathbf{r}^{A} \otimes \mathbf{r}^{B}$ (Lanyon et al., 2013). Based on elementary geometric considerations, this allows to conclude that application of the collective dephasing operation yields a state of $L=3$ if and only if $\mathbf{n}$ coincides neither with $\mathbf{r}^{A}$ nor $\mathbf{r}^{B}$; otherwise the asymptotic state remains at $L=1$ (Lanyon et al., 2013). An explicit expression for the two-qubit density matrix after collective dephasing of a product state under arbitrary magnetic field orientations can be found in (Carnio, 2014). Moreover, two consecutive applications of the collective dephasing operation can transform arbitrary initial states into states with $L=4$. This requires that the direction of the magnetic field differs for the two dephasing processes, since otherwise the map is idempotent (Carnio, 2014). 
a)
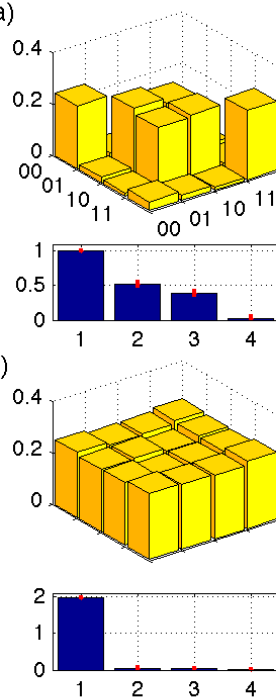

b)
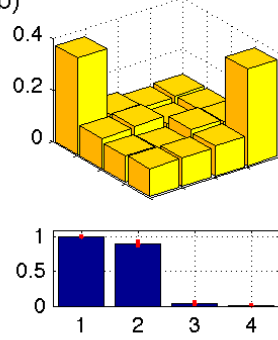

e)

0.4
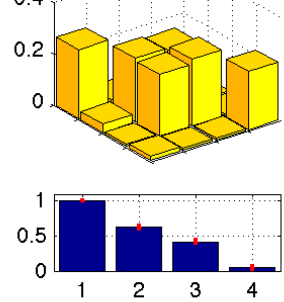

c)
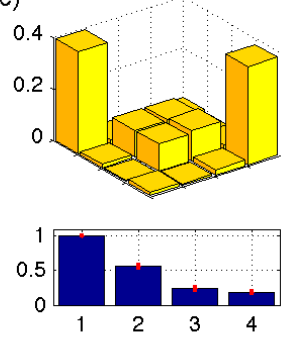

f)
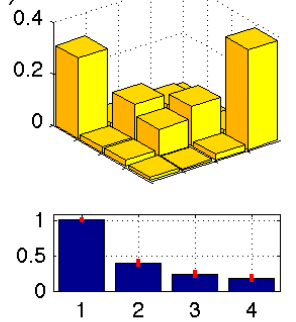

FiguRE 4.3.: Real values of the elements of the full two-qubit density matrix under the influence of collective dephasing. The plots show $\mathcal{E}^{\mathbf{z}}\left(\rho_{1}\right)$ with $\rho_{1}$ defined in equation (4.13) and measured in the left-most tomography (a) of figure 4.1; the map $\mathcal{E}^{\mathbf{n}}$ is defined in equation (4.21). The other subplots show $\rho_{2}=$ $\mathcal{R}_{\mathbf{y}}(\pi / 8)^{\otimes 2} \rho_{1} \mathcal{R}_{\mathbf{y}}^{\dagger}(\pi / 8)^{\otimes 2}(\mathrm{~b}) ; \mathcal{E}^{\mathbf{n}}\left(\rho_{2}\right)$ (c); $|\Psi\rangle=|++\rangle$ (d); $\mathcal{E}^{\mathbf{z}}(|\Psi\rangle\langle\Psi|)$ (e); $\mathcal{E}^{\mathbf{z}}\left(\mathcal{R}_{\mathbf{y}}(\pi / 2)^{\otimes 2} \mathcal{E}^{\mathbf{z}}(|\Psi\rangle\langle\Psi|) \mathcal{R}_{\mathbf{y}}^{\dagger}(\pi / 2)^{\otimes 2}\right)(\mathrm{f})$; with $\mathbf{y} \cdot \boldsymbol{\sigma}=\sigma_{y}$ and $\mathbf{z} \cdot \boldsymbol{\sigma}=\sigma_{z}$. The number $L$ of nonzero singular values (blue bars) after collective dephasing agrees with the predictions made from figure 4.2 within uncertainties. Figure taken from (Lanyon et al., 2013).

All states of class (B) have zero discord and can be characterized by $\beta=d \mathbf{v} \otimes \mathbf{w}$. The geometric interpretation yields the possible values of the asymptotic correlation rank, which are summarized in Table 4.2. (Lanyon et al., 2013). We see that the full spectrum of correlation ranks $1 \leq L \leq 4$ for a two-qubit system can be reached after a single collective dephasing operation, including the maximally correlated states with $L=4$. This is confirmed in the experimental data of figure 4.3, which displays different initial and final states under the influence of collective dephasing after long times.

To summarize, we have seen that local operations are able to transform classical correlations into correlations with quantum nature with respect to local measurements as quantified by quantum discord. The correlation rank is able to assess the amount of total correlations and cannot increase under local operations. Classically correlated noise processes, however, can produce strongly correlated states. In trapped-ion experiments such noise processes are naturally present due to fluctuations of the magnetic field. Manipulation of the orientation of this field, or equivalently, rotations of the qubit state before dephasing, allow to control the correlation properties of the asymptotic state. In the next section, we generalize the present analysis of the steady-state 
of two qubits to a full description of the general multipartite dephasing process of $N$ qubits, including its transient time evolution.

\subsection{General dynamics of collective dephasing}

The collective dephasing process, discussed in the trapped-ion experiment of the previous section, represents one of the remaining dominant error sources for experimental quantum computations. To correct or avoid the errors induced thereby, it is of primordial importance to fully understand and characterize the associated dynamics in the general setting of $N$ qubits. Due to the necessity to repeat experiments with few atoms many times to obtain statistically significant measurements of populations, this represents a natural embedding of an ensemble-averaged effective dynamics. From a theoretical point of view, this constitutes an intuitive alternative to the conventional system-environment scenario for the description of noise and decoherence.

The present section summarizes a selection of results on the dynamics of the multipartite collective dephasing process, which were obtained in collaboration with Edoardo Carnio in the course of his Master's thesis. We refer to the thesis (Carnio, 2014) and the corresponding publication (Carnio et al., 2015) for most of the details and further discussions. The treatment presented here generalizes the considerations of the previous section by describing

- a system of $N$ qubits,

- the transient time evolution as a function of the intensity fluctuations.

Finally, aside from Zeeman shifted qubit resonances, a dynamics of the form (4.15) describes the general interaction of a dipole with an external field, and applies to paramagnets as well as electric dipoles.

\subsubsection{Kraus representation}

The first goal in this section is to derive an expression for the dynamics of $N$ qubits under the influence of collective dephasing. The task is, thus, to evaluate the ensemble average

$$
\rho(t)=\int d \omega p(\omega) U_{\omega}(t)^{\otimes N} \rho(0) U_{\omega}^{\dagger}(t)^{\otimes N}
$$

where $\rho(t)$ and $\rho(0)$ denote states of $N$ qubits, and the unitary dynamics during each realization is given by equation (4.17) with the same frequency for each atom. Again, this expression implies a separation of time scales, as discussed below equation (4.18).

Using the pairs of orthogonal projectors $\Lambda_{ \pm}$, which were defined in equation (4.23), allows us to rewrite the time evolution operator as

$$
U_{\omega}(t)^{\otimes N}=\left(e^{-i \omega t / 2} \Lambda_{+}+e^{i \omega t / 2} \Lambda_{-}\right)^{\otimes N}
$$


To expand this we introduce the permutation operator $V_{\sigma}$, which mediates the permutation $\sigma \in S_{N}$ on the $N$-partite tensor space,

$$
V_{\sigma}=\sum_{i_{1} \ldots i_{N}}\left|i_{\sigma(1)} \ldots i_{\sigma(N)}\right\rangle\left\langle i_{1} \ldots i_{N}\right|
$$

where $S_{N}$ denotes the symmetric group of $N$ elements. We obtain (Carnio et al., 2015),

$$
\begin{aligned}
U_{\omega}(t)^{\otimes N} & =\sum_{j=0}^{N} \frac{e^{i \omega t(j-N / 2)}}{j !(N-j) !} \sum_{\sigma \in S_{N}} V_{\sigma}\left[\Lambda_{-}^{\otimes j} \otimes \Lambda_{+}^{\otimes N-j}\right] V_{\sigma}^{\dagger} \\
& =\sum_{j=0}^{N} e^{i \omega t(j-N / 2)} \Theta_{j},
\end{aligned}
$$

where we introduced the operators

$$
\Theta_{j}=\frac{1}{j !(N-j) !} \sum_{\sigma \in S_{N}} V_{\sigma}\left[\Lambda_{-}^{\otimes j} \otimes \Lambda_{+}^{\otimes N-j}\right] V_{\sigma}^{\dagger} .
$$

Inserting equation (4.31) into the ensemble average (4.28) yields the time evolution in shape of a Kraus form (Bengtsson and Życzkowski, 2006)

$$
\rho(t)=\sum_{i, j=0}^{N} M_{i j}(t) \Theta_{i} \rho(0) \Theta_{j},
$$

which is characterized by

$$
M_{j k}(t)=\int d \omega p(\omega) e^{i \omega(j-k) t}=\varphi[(j-k) t] .
$$

In the last step we expressed the matrix elements $M_{j k}(t)$ via the characteristic function

$$
\varphi(t)=\int d \omega p(\omega) e^{i \omega t}
$$

of the probability distribution $p(\omega)$. The $(N+1) \times(N+1)$ matrix $M(t)=\left(M_{i j}(t)\right)$ is Hermitian and semi-positive definite for all $t$, due to Bochner's theorem (Rudin, 1990). At each time $t$, we can therefore diagonalize this matrix using a unitary matrix $V(t)=\left(v_{i j}(t)\right)$ with $M_{i j}(t)=\sum_{k} v_{i k}(t) \lambda_{k}(t) v_{j k}^{*}(t)$, to obtain the canonical Kraus form (Bengtsson and Życzkowski, 2006)

$$
\begin{aligned}
\rho(t)=\mathcal{E}_{t, 0}^{\mathbf{n}}[\rho(0)] & =\sum_{k=0}^{N} \lambda_{k}(t)\left(\sum_{i=0}^{N} v_{i k}(t) \Theta_{i}\right) \rho(0)\left(\sum_{j=0}^{N} v_{j k}^{*}(t) \Theta_{j}\right) \\
& =\sum_{i=0}^{N} A_{i}(t) \rho(0) A_{i}^{\dagger}(t),
\end{aligned}
$$


with the canonical Kraus operators

$$
A_{i}(t)=\sqrt{\lambda_{i}(t)} \sum_{j=0}^{N} v_{j i}(t) \Theta_{j} .
$$

Notice that the $A_{i}$ are composed of the permutationally invariant $\Theta_{i}$, as defined in equation (4.32), which implies that they act upon all the atoms simultaneously and cannot be decomposed into local, single-atom operators. Thus, the coupling to a common, classical, fluctuating field mediates an incoherent atom-atom interaction.

While for the details we refer to (Carnio et al., 2015), let us remark here that one can use the orthogonality of the projectors $\Lambda_{ \pm}$to show that

$$
\sum_{i} A_{i}^{\dagger}(t) A_{i}(t)=\sum_{i} \Theta_{i}^{\dagger} \Theta_{i}=\mathbb{I}
$$

which ensures that the map $\mathcal{E}_{t, 0}^{\mathbf{n}}$ in equation (4.36) is completely positive and tracepreserving for all $t$.

Let us now consider the implications of this description for the asymptotic state, which is reached in the limit $t \rightarrow \infty$. Note that in a common scenario, we will find that the characteristic function $\varphi(t)$ vanishes for long times. Exceptions are cases where, for example, only few discrete frequencies play a role in the frequency spectrum, which happens if $p(\omega)$ is described by a discrete sum of delta functions. Instead, we assume that the frequency distribution has finite, continuous support and typically can be described by Lorentzian or Gaussian distributions, for which $\lim _{t \rightarrow \infty} \varphi(t)=0$ is satisfied. In this case we find $\lim _{t \rightarrow \infty} M_{i j}(t)=\delta_{i j}$ which leads to the asymptotic form of the Kraus operators $\lim _{t \rightarrow \infty} A_{i}(t)=\Theta_{i}$, and

$$
\mathcal{E}^{\mathbf{n}}[\rho(0)]=\sum_{i=0}^{N} \Theta_{i} \rho(0) \Theta_{i},
$$

independently of the specific form of $p(\omega)$. This behavior is intuitively expected if we recall the discussion of the asymptotic state preceding equation (4.19): If the waiting time $\tau$ is chosen long enough, any shape of continuously distributed fluctuations of $\omega$ will lead to the same, uniformly distributed phases $e^{-i \omega \tau}$. The general expression (4.39) reduces to equation (4.21) in the special case of $N=2$.

\subsubsection{Ensemble-average evolution: Interpretation and non-Markovian effects}

We have derived an explicit form to describe the dynamics (4.36) and asymptotics (4.39) of the collective dephasing process of atomic two-level systems in an external classical field. The Kraus representation describes this dynamics in a canonical form, which is commonly applied to describe the dynamics of open quantum systems (Breuer and Petruccione, 2002), for example in terms of noisy quantum channels (Nielsen and Chuang, 2000; Bengtsson and Życzkowski, 2006). Unlike typical microscopic derivations of theoretical models describing incoherent effects induced by an environment, we 
started from the unitary evolution of a closed system. The resulting Kraus representation shows that the ensemble-average over fluctuating system parameters leads to an equivalent description, where the fluctuation distribution $p(\omega)$ takes on the role of the spectral density of a conventional open-system approach. Given the necessity to repeat experiments many times to measure an expectation value, our derivation represents an experimentally very intuitive approach to quantum noise. While, here, we restrict to the formal solution of the dynamics, in (Carnio, 2014) it is further shown that this problem can be cast equivalently into the form of a master equation, including effective incoherent effects described by Lindblad operators as in the standard treatment of open quantum systems; see also (Benatti et al., 2012; Rossi et al., 2014).

Let us briefly discuss the transient time evolution towards the asymptotic state, whereas for the details we refer to (Carnio, 2014). Whether this process will be irreversible or display revivals is determined by the spectral probability distribution $p(\omega)$. To formally quantify the induced memory effects we can observe the evolution of $\rho(t)$ towards the steady-state $\rho_{s}$. A suitable auto-correlation function is defined on the basis of the trace distance $D(t)=\left\|\rho(t)-\rho_{s}\right\|$. It is indeed found to be monotonically decaying for Lorentzian and Gaussian spectral distributions $p(\omega)$, whereas the distance reaches zero and revives in a damped oscillatory fashion for a uniform box distribution (Carnio et al., 2015), similarly to what one intuitively expects from couplings to structured reservoirs (Lambropoulos et al., 2000; Walther et al., 2006; Haroche and Raimond, 2006). In this sense, the finite support of the box distribution represents an environment with pronounced structure, while in the other distributions the full frequency spectrum contributes. From an information-theoretic point of view, the distance $D(t)$ represents the auto-correlation function of just one of many state-dependent time evolutions. For a state-independent analysis of the underlying dynamical map, one would try to identify the pair of initial states which would maximize the increase of the trace distance (Breuer et al., 2009).

\subsubsection{Robustness of bipartite entanglement}

For a complete analysis of the bipartite case, we refer to (Carnio, 2014), where the influence of collective dephasing on arbitrary initial states is discussed in terms of correlation rank and the concurrence, which measures the entanglement of arbitrary mixed states (Wootters, 1998; Mintert et al., 2005). These results generalize the discussions of the previous section in the context of the trapped-ion experiment. Bipartite states can be represented conveniently in a tetrahedron picture (Horodecki and Horodecki, 1996), in which the entanglement, as well as the time evolution under collective dephasing have clear geometric interpretations. In particular, for arbitrary initial entangled states, a magnetic field direction can be identified, which preserves the entanglement at all times (Carnio et al., 2015). There also exists an entire family of entangled states whose entanglement remains time-invariant under arbitrary magnetic field directions - even if the states do evolve in time (Carnio et al., 2015). Under the influence of strongly structured spectral distributions $p(\omega)$, entanglement can also increase temporarily, which shows that the dynamics, as defined in equation (4.28), can lose its separable character for intermediate time intervals. 
Furthermore, applications are discussed, such as the noise-assisted generation of bipartite Werner states (see next section for a definition), which always have correlation rank $L=4$, by exposing a classical state of $L=2$ to collective dephasing with appropriate field direction. In a three-partite setting, the resulting Werner state can then be used as a separable carrier for entanglement distribution (Chuan et al., 2012); see section 2.1.2.

\subsubsection{Time-invariant states: Multipartite Werner states}

We identify a set of time-invariant states, characterized by the condition

$$
\rho(t)=\mathcal{E}_{t, 0}^{\mathbf{n}}[\rho(0)]=\rho(0), \quad \forall t,
$$

which we require to hold for all orientations $\mathbf{n}$ of the external field. Using the permutation invariance of the operators $\Theta_{j}$, one finds that all permutations $s \in S_{N}$, which in operator form can be written as $V_{\sigma}$ in equation (4.30), are invariant under the time evolution (4.33) (Carnio et al., 2015). Here, $\left\{\left|0_{k}\right\rangle,\left|1_{k}\right\rangle\right\}$ represents a basis of the $k$ th qubit. The set of time-invariant states under collective dephasing is given by all linear combinations

$$
\rho_{W}=\sum_{\sigma \in S_{N}} c_{\sigma} V_{\sigma}
$$

This set of states is known as the multipartite Werner states (Eggeling and Werner, 2001), with the defining property that they are invariant under local unitary transformations $U^{\otimes N}$ (Weyl, 1950; Werner, 1989; Vollbrecht and Werner, 2001). The timeinvariance under collective dephasing applies to arbitrary directions of the external field, which, given the invariance property of Werner states, is quite intuitive since the rotation of the field can be compensated by such local unitary transformations. The set of multipartite Werner states (4.41) identifies an $(N !-1)$-parameter family of states which span a decoherence-free subspace (Lidar et al., 1998) under collective dephasing. We consider any subspace that is completely insusceptible to the incoherent dynamics a decoherence-free subspace, implying that this subspace may contain mixed states.

This relates directly to a pure dephasing model, obtained from a microscopic description of non-interacting qubits, which are coupled, without exchange of excitations, to a common bath of harmonic oscillators (Palma et al., 1996). This model is exactly solvable and shows that collective interactions can lead to strongly enhanced decoherence or complete suppression of decoherence (Palma et al., 1996; Fischer and Breuer, 2013). Decoherence can be enhanced by a factor of $N^{2}$ compared to the decoherence rates of individual qubits, in analogy to the collective decay of excited states, called superradiance, which can occur when the coupling is dissipative (Dicke, 1954). On the other hand, decoherence in the pure dephasing model is completely suppressed for coherent superpositions of quantum states whose quantum numbers sum up to the same value. This is indeed the case for all multipartite Werner states, since each term of the permutations, as defined in equation (4.30), satisfies $\sum_{k} i_{k}=\sum_{k} i_{\sigma(k)}$. 
This identifies the Werner states as a remarkable family of states characterized by resilience to collective decoherence. Decoherence-free subspaces are considered useful in the context of quantum memories, where a given superposition of states must be conserved for long times (Lidar et al., 1998; Bacon et al., 1999). In a dynamical scenario, which is required, for example, to describe quantum computations, the decoherence-free subspaces are, however, fragile to perturbations and therefore do not represent an experimentally feasible alternative to error correction methods (Bacon et al., 1999).

Consequently, the identification of the time-invariant set of states is not sufficient. A more promising alternative, which still needs further investigation, might be given by the phenomenon of time-invariant entanglement outside of decoherence-free subspaces, which was briefly mentioned in section 4.4.3; see also (Carnio et al., 2015). We complement this result with the description of the decay of multipartite entanglement properties under collective dephasing, as a function of the external field's orientation.

\subsubsection{Robustness of multipartite entanglement}

Multipartite entanglement can be characterized in a hierarchical way, assessing the number of parties which are entangled with each other (Levi and Mintert, 2013). The definition of separability, given by equation (2.2), can be straight-forwardly extended to a multipartite setting by defining a completely separable multi-particle state as a state which can be written as a convex linear combination of product states (Linden and Popescu, 1998; Mintert et al., 2005), that is

$$
\rho_{s}=\sum_{i} p_{i} \rho_{1}^{i} \otimes \cdots \otimes \rho_{N}^{i}
$$

Otherwise the state is considered entangled. To express the number of parties which are described by a non-separable superposition, the above definition is refined. A $k$-separable state is a state of an $N$-partite quantum system which can be written as

$$
\rho_{k}=\sum_{i} p_{i} \rho_{A_{1}}^{i} \otimes \cdots \otimes \rho_{A_{k}}^{i}
$$

where $A_{1}, \ldots, A_{k}$ represents an arbitrary division of the total $N$-partite Hilbert space. If the state is $N$-separable, it is completely separable across any division of the Hilbert space. Conversely, states with $k=1$ are fully $N$-partite entangled, which, in an $N$ partite system is commonly referred to as genuine multipartite entangled or genuine multi-particle entangled.

Efficient separability criteria can be derived in form of inequalities, which avoid the optimization over complicated convex-roof constructions ${ }^{2}$ (Gühne and Seevinck,

\footnotetext{
${ }^{2}$ One typically tries to characterize the entanglement of mixed states in terms of the pure states of which it is decomposed. Since there exist many equivalent decompositions of a pure state into mixed states, one minimizes the entanglement over all possible convex decompositions (Uhlmann, 1998). This commonly referred to as a convex-roof.
} 
2010; Huber et al., 2010). When expressed in an arbitrary basis, the elements of any $k$-separable density matrix must satisfy the inequality (Gao et al., 2013)

$$
\sum_{0 \leq i<j \leq N-1}\left|\rho_{2^{i}+1,2^{j}+1}\right| \leq \sum_{0 \leq i<j \leq N-1} \sqrt{\rho_{1,1} \rho_{2^{i}+2^{j}+1,2^{i}+2^{j}+1}}+\frac{N-k}{2} \sum_{i=0}^{N-1} \rho_{2^{i}+1,2^{i}+1} .
$$

Solving for the largest integer $k$ saturating this bound, we define $k_{\text {eff }} \geq k$. For $k_{\text {eff }}<2$, the state contains genuine multi-particle entanglement, and the state may be separable only if $k_{\text {eff }} \geq N$ (Gao et al., 2014).

We study the evolution of an initially multipartite entangled $W$-state (Dür et al., 2000),

$$
|W\rangle=\frac{1}{\sqrt{N}}(|10 \ldots 0\rangle+|01 \ldots 0\rangle+\cdots+|0 \ldots 01\rangle),
$$

under collective dephasing. Again, the time evolution of $k_{\text {eff }}$ can be non-monotonic, depending on the spectral distribution $p(\omega)$ (Carnio, 2014). Similarly to the results discussed in the bipartite setting, we can control the entanglement properties of the asymptotic state by changing the external magnetic field direction. The $z$ direction represents a natural axis for the state (4.45), since it defines the eigenbasis of each individual qubit state, and if the magnetic field is chosen in this direction, the multipartite entanglement is perfectly preserved (Carnio et al., 2015). It is further possible to numerically extract a finite angle $\theta_{\mathrm{NPE}}$ which defines the allowed deviation from the $z$ axis to maintain multipartite entanglement in the asymptotic state. We find that this angle is well described, as a function of $N$, by (Carnio et al., 2015)

$$
\theta_{\mathrm{NPE}}=\arctan \left(\frac{1}{\sqrt{N(N-1)}}\right) \text {. }
$$

Analogously, the critical angle which defines the range of directions around the $z$ axis that certainly lead to preservation of at least bipartite entanglement follows the relation

$$
\theta_{\mathrm{E}}=\arctan \left(\frac{1}{\sqrt{N}}\right)
$$

General symmetry of the dephasing evolution under $\mathbf{n} \rightarrow-\mathbf{n}$, and rotational symmetry around $z$, which also holds for the initial state (4.45), allow to characterize the resulting $k_{\text {eff }}$ using only the polar angle $\theta$ to the $z$ axis, see figure 4.4 . In general, the $k_{\text {eff }}$ show a non-monotonic distribution over the sphere of directions in $\mathbb{R}^{3}$.

Finally, we mention a special class of $W$ states, equipped with the phase relations

$$
|\widetilde{W}\rangle=\frac{1}{\sqrt{N}} \sum_{i=1}^{N} e^{i 2 \pi k / N}\left|1_{k}\right\rangle
$$



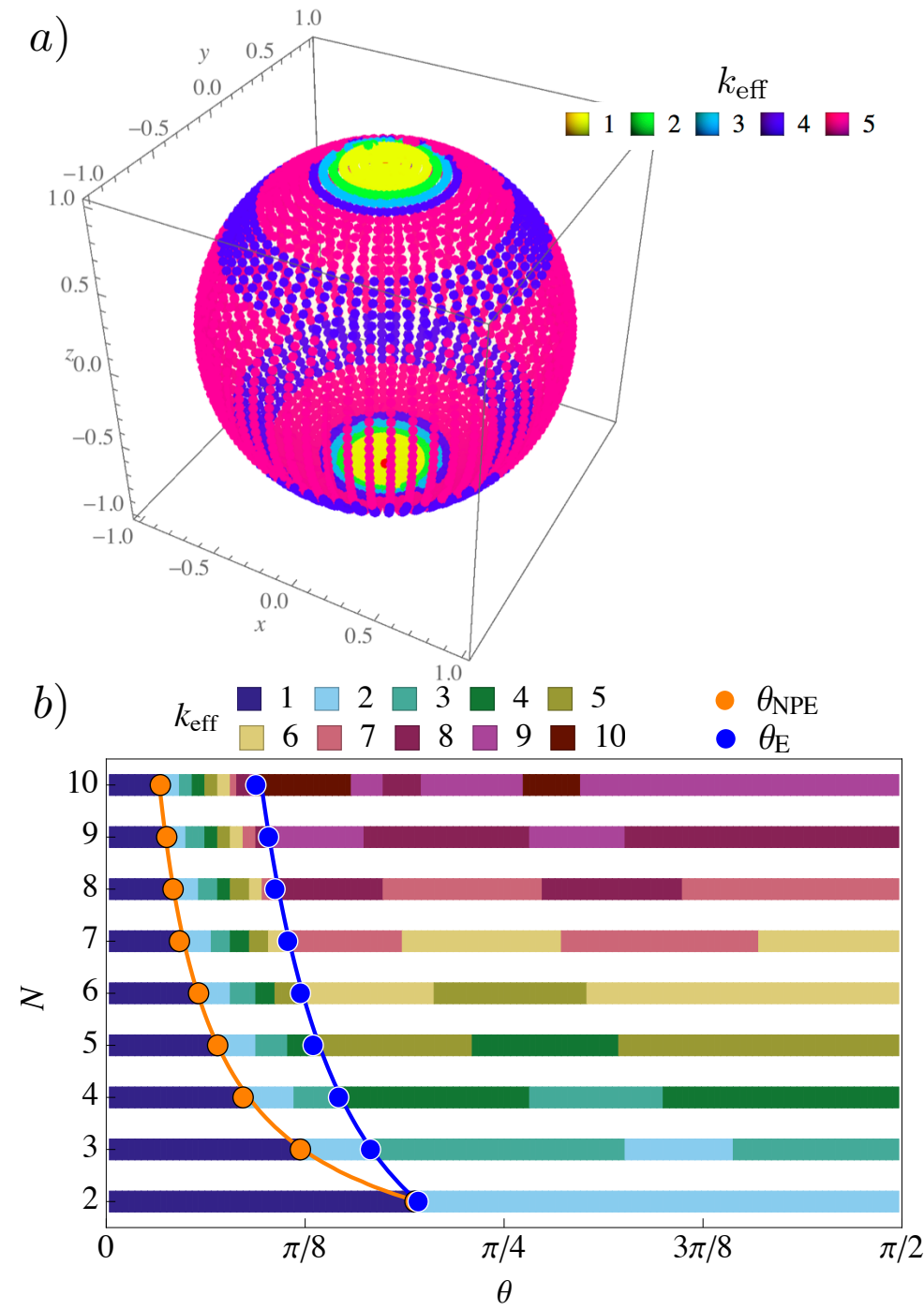

FIGURE 4.4.: Decoherence and robustness of multipartite entanglement of a $|W\rangle$-state (4.45), subject to collective dephasing (4.39). The integer $k_{\text {eff }}$, defined in (4.44), is an upper bound to the $k$-separability class of the asymptotic state. The resulting distribution is rotationally symmetric around the $z$ axis, and mirror symmetric around the $x-y$ plane, which is shown in a) for $N=5$. Thus, the distribution of $k_{\text {eff }}$ can be characterized solely based on the polar angle angle $\theta$ of $\mathbf{n}$ with respect to the $z$ axis, which is shown for $N=2 \ldots 10$ in b). The solid lines represent the angles (4.46) and (4.47), which provide lower bounds for the allowed deviation from $z$, such that multipartite and bipartite entanglement, respectively, can be preserved, as a function of $N$. Adapted from (Carnio, 2014; Carnio et al., 2015). 
where the $\left|1_{k}\right\rangle$ denote states where all qubits are in the ground state, except for one excited state at the $k$ th position, in an arbitrary, but fixed ordering. These states share the remarkable property that, despite themselves not being time-invariant, their multipartite entanglement is completely preserved at all times under the collective dephasing dynamics. This effect is not yet well understood, and the question remains whether further states can be identified that share this property, We expect this entanglement-preservation to be potentially more robust than decoherence-free subspaces, since it does not require complete time-evolution invariance. For further discussions and details we refer to (Carnio, 2014; Carnio et al., 2015).

\subsection{Discussion}

In the previous chapter, we had detected quantum discord with the help of local operations, which was based on a local dephasing operation that destroyed quantum discord. Conversely, in the present section, we discussed the possibility to create discord with local operations. Introducing the correlation rank, we were able to derive strict boundaries for the set of discordant states which can be created with local operations. Classically correlated dephasing processes, which occur naturally in trapped-ion experiments, can, however, generate separable states with nonzero discord, whose correlations are not subject to these bounds.

We further developed an analytical description of a general, multipartite collective dephasing process, which describes an important noise process in an intuitive ensemble-average approach to noisy quantum systems. The resulting dynamics describes effective, noise-induced atom-atom interactions which can generate classically correlated states and ensure the preservation of entanglement. Most interestingly, the dynamics allows to identify states with time-invariant entanglement even beyond decoherence-free subspaces, that is, the states do evolve in time, but their entanglement remains constant. These states have not yet been systematically explored in a multipartite scenario, and the measure of the set of states exhibiting this feature is so far unclear. 



\section{QUANTUM PHASE TRANSITION IN A FAMILY OF QUANTUM MAGNETS}

We analyze the role of the excitation spectrum for the quantum phase transition in the spin-chain model, describing a quantum magnet with variable-range interactions. Developing a variational mean-field ansatz we reproduce the semiclassical backbone of the quantum spectrum with simple analytical tools. The semiclassical result is exact in certain limits, and further provides an intuitive interpretation of the second-order quantum phase transition, which affects the entire excitation spectrum.

\subsection{Variable-range quantum magnets: From Ising to Lipkin-Meshkov- Glick}

Recalling section 2.6, we had introduced quantum phase transitions as changes of a material's macroscopic character (phase) that are induced by changes of an external parameter at zero temperature, and not, as "classical" phase transitions by thermal fluctuations at finite temperatures. We had also introduced the example of a ferromagnetic material that behaves paramagnetically, as soon as the external field exceeds a certain threshold value. Since, by definition, these transitions occur at zero temperature, the quantum mechanical ground state plays an important role in their description.

In section 3.4.3, we saw that single-spin dynamics can be made susceptible to the ground-state quantum correlations characteristic of a quantum phase transition. To link ground-state properties to the locally observable dynamics, we have to take the excitation spectrum into account. In this chapter, we revisit this model and describe its features by focussing on the complete energy spectrum rather than only on the 
ground state. Let us first recall the model.

\subsubsection{A one-parameter family of models}

We consider a family of spin chain models described by the Hamiltonian

$$
H=-\sum_{\substack{i, j=1 \\ i<j)}}^{N} J_{i j} \sigma_{x}^{(i)} \sigma_{x}^{(j)}-B \sum_{i=1}^{N} \sigma_{y}^{(i)},
$$

where $\sigma_{x}^{(i)}$ and $\sigma_{y}^{(i)}$ are Pauli spin operators of the $i$ th spin, and the spin-spin interactions are algebraically decaying,

$$
J_{i j}=\frac{J_{0}}{|i-j|^{\alpha}} .
$$

The model is continuously parametrized by $\alpha \in[0, \infty)$, which determines the range of the spin-spin coupling. Roughly speaking, and disregarding the term proportional to $B$, for $J_{0}>0$, the energy minimizes when two neighboring spins align in parallel. In this case, the model describes a ferromagnet. Conversely, for $J_{0}<0$, neighboring spins favor opposite orientations, and the model describes an anti-ferromagnet.

The properties of the system are determined by the competition between the two terms that enter the Hamiltonian (5.1). On the one hand, the strength of the internal spin-spin coupling (depending on the spins' relative orientation along the $x$ direction) is determined via the exchange interaction by the term $J_{0}$ (Cohen-Tannoudji et al., 1977b). On the other hand, a paramagnetic contribution to the total energy is generated by an external, transverse (in $y$ direction) magnetic field with strength $B$. The relative strength of these two interaction terms determines the system's microscopic ordering, which can become observable on macroscopic scales. When $B / J_{0} \ll 1$, the spins aim to minimize their potential energy with respect to their neighbors, whereas, for $B / J_{0} \gg 1$, the spins follow the external magnetic field's orientation. Consequently, for increasing $B / J_{0}$, the system undergoes a quantum phase transition from an (anti-)ferromagnet to a paramagnet.

As was already mentioned in section 3.4.3, the Hamiltonian exhibits a $Z_{2}$ symmetry, which reflects the invariance under a $180^{\circ}$ rotation around the $y$ axis,

$$
Z_{2}=\bigotimes_{j=1}^{N} e^{i \pi \sigma_{y}^{(j)} / 2}=\bigotimes_{j=1}^{N} i \sigma_{y}^{(j)} .
$$

This rotational invariance of the Hamiltonian is expressed by

$$
\left[H, Z_{2}\right]=0,
$$

and the rotation effectively leads to the substitution of all of the local spin operators as follows:

$$
Z_{2} \sigma_{x}^{(i)} Z_{2}^{\dagger}=-\sigma_{x}^{(i)}, \quad Z_{2} \sigma_{y}^{(i)} Z_{2}^{\dagger}=\sigma_{y}^{(i)}, \quad Z_{2} \sigma_{z}^{(i)} Z_{2}^{\dagger}=-\sigma_{z}^{(i)} .
$$


Furthermore, the system is invariant under a mirror operation with respect to the center of the chain, described by replacing the indices by

$$
i \longrightarrow N-i+1 \text {. }
$$

While for such systems only few results are known for arbitrary, intermediate values of $\alpha$ (Dyson, 1969; Cannas and Tamarit, 1996; Koffel et al., 2012), the model reduces to very well known models in the special cases $\alpha=0$ and $\alpha \rightarrow \infty$.

\subsubsection{Special case: Nearest-neighbor Ising model}

For $\alpha \rightarrow \infty$, the spin-spin interactions are limited to nearest neighbors. In this case, we recover the well-known one-dimensional quantum Ising model (Ising, 1925),

$$
H_{\mathrm{I}}=-J_{0} \sum_{i=1}^{N-1} \sigma_{x}^{(i)} \sigma_{x}^{(i+1)}-B \sum_{i=1}^{N} \sigma_{y}^{(i)}
$$

There exists an exact solution for this model, which can be obtained by employing a Jordan-Wigner transform, leading to a system of noninteracting fermions (Jordan and Wigner, 1928). The quantum phase transition from the symmetry-broken (anti-)ferromagnetic phase to the symmetric paramagnetic phase occurs at the critical field $B=\left|J_{0}\right|$ (Sachdev, 1999). A semiclassical intuition for the different symmetries of the two phases will be provided in section 5.4.4.

\subsubsection{Special case: Fully-connected Lipkin-Meshkov-Glick model}

For $\alpha=0$, all spins are coupled to each other with the same strength - the interaction range is infinite. In this case, one can combine all spins into one large spin: We introduce

$$
\mathbf{S}=\frac{1}{2} \sum_{i=1}^{N} \boldsymbol{\sigma}^{(i)}
$$

with $\boldsymbol{\sigma}^{(i)}=\left(\sigma_{x}^{(i)}, \sigma_{y}^{(i)}, \sigma_{z}^{(i)}\right)$, to rewrite the Hamiltonian as ${ }^{1}$

$$
H_{\mathrm{LMG}}=-2 J_{0} S_{x}^{2}-2 B S_{y}+\frac{J_{0}}{2} N \mathbb{I} .
$$

In this limit we recover the Lipkin-Meshkov-Glick model (Lipkin et al., 1965; Meshkov et al., 1965; Glick et al., 1965). In addition to the $Z_{2}$ symmetry of the general model, the total angular momentum $\mathbf{S}^{2}$ becomes a conserved quantity, since it commutes with all of the components of $\mathbf{S}$. Exact analytical solutions exist for special cases (Lipkin et al., 1965), and can, in principle, generally be obtained algebraically (Pan and Draayer, 1999; Links et al., 2003) using a "Bethe ansatz" (Bethe, 1931). This is,

\footnotetext{
${ }^{1}$ The final term proportional to the identity is caused by restricting to $i<j$ in the first term in (5.1).
} 
however, a rather complicated task in practice, and in many cases becomes computationally more expensive than numerically exact diagonalization (Ribeiro et al., 2007). The model further allows for an efficient perturbative treatment for large $N$. In this limit, the total angular momentum $\mathbf{S}$ becomes larger and, eventually, is effectively described by a classical Hamiltonian (Leyvraz and Heiss, 2005). Semiclassical meanfield approximations are derived most efficiently by employing spin coherent states (Ribeiro et al., 2007), which we will introduce in section 5.4.2. In fact, this ansatz is exact in the thermodynamic limit $N \rightarrow \infty$ (Ribeiro et al., 2008). A suitable method to gain an expansion of quantum corrections in orders of $1 / N$ is the "Holstein-Primakoff mapping" onto a system of bosons (Holstein and Primakoff, 1940). The semiclassical solution based on spin coherent states constitutes the zeroth order of this expansion (Dusuel and Vidal, 2005).

\subsection{Single-spin signatures of a quantum phase transition}

Let us start by briefly recalling the results of section 3.4.3. We considered a partition of the spin chain into the left-most spin, which was considered an easily controllable local subsystem, and the rest of the chain. The bipartite correlations across this split are expected to indicate the quantum phase transition. To detect these correlations with reasonable experimental overhead, we implement the local detection protocol. By preparing the system in the ground state and locally dephasing the left-most spin, the incoherent counterpart to the ground state is created. However, this locally dephased state may still contain coherences in the energy eigenbasis, which allow the state to evolve in time, and the time evolution serves as a witness for the correlations of the ground state.

The dynamics of the locally dephased state represents the key feature which maps signatures of the correlations, and with it of the quantum phase transition, to the locally controllable subsystem. This evolution, in turn, is determined by the distribution of excited states that are populated when the local dephasing operation is applied to the ground state.

In this section, we study the effect of the dephasing on the ground state, to better understand the mechanism behind the successful local detection of ground-state quantum correlations via the coupled spin dynamics.

\subsubsection{Distribution of dephasing-induced excitations}

The distribution of excitations which are induced by locally dephasing the pure ground state $\left|\Psi_{0}\right\rangle$ in the eigenbasis of the accessible spin may give us a first hint on why the single-spin dynamics is much richer in some parts of the parameter range than in others. As noted in section 3.4.3, due to the $Z_{2}$ symmetry of the Hamiltonian, the single-spin state is always diagonal in the eigenbasis $\left\{\left|\uparrow_{y}\right\rangle,\left|\downarrow_{y}\right\rangle\right\}$ of $\sigma_{y}$. Thus, the locally dephased state ground state is given by

$$
\rho_{\Phi}=(\Phi \otimes \mathbb{I})\left|\Psi_{0}\right\rangle\left\langle\Psi_{0}\left|=\sum_{\varphi \in\left\{\uparrow_{y}, \downarrow_{y}\right\}}(|\varphi\rangle\langle\varphi| \otimes \mathbb{I})\right| \Psi_{0}\right\rangle\left\langle\Psi_{0}\right|(|\varphi\rangle\langle\varphi| \otimes \mathbb{I}) .
$$



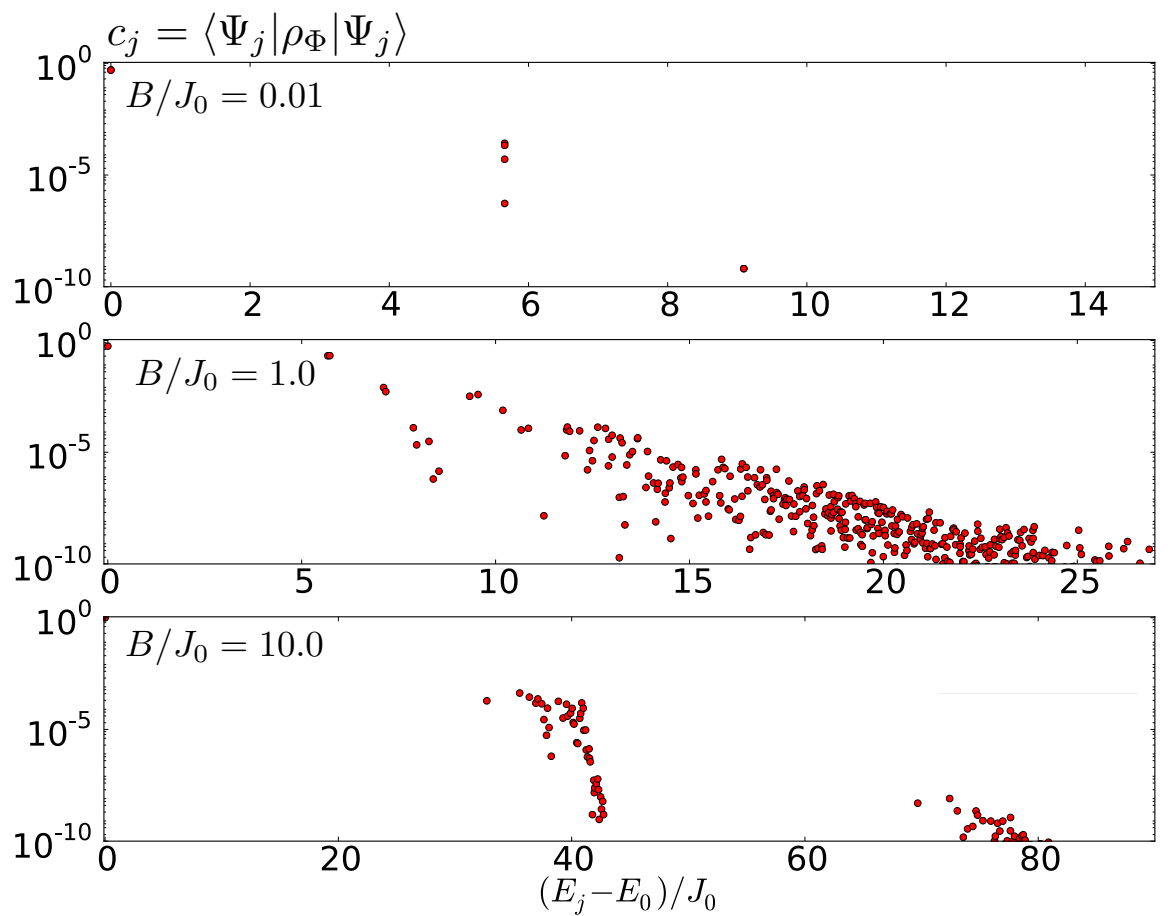

FIgURE 5.1.: Dephasing-induced excitations of the ground state for $\alpha=1$ and $N=10$. The coefficients $c_{j}$, equation (5.11), which quantify the overlap of the locally dephased ground state (5.10) with the energy eigenstates, demonstrate the broad excitation spectrum in the vicinity of the phase transition (center), while hardly any (top) or regularly spaced (bottom) excitations are populated far away from the phase transition.

To analyze the excitation spectrum of $\rho_{\Phi}$, we introduce the overlap

$$
c_{j}=\left\langle\Psi_{j}\left|\rho_{\Phi}\right| \Psi_{j}\right\rangle,
$$

with the eigenstates $\left|\Psi_{j}\right\rangle$ of $H$. The distribution of dephasing-induced excitations is shown in figure 5.1 for a ferromagnetic system, $J_{0}>0$. We find that for very weak external fields $B$, the excitations hardly extend beyond the ground state manifold, while for very strong external fields, the band structure of paramagnets can be recognized. These equally-spaced bands are determined by the number of spins which align against the direction of the external field. The energy of the bands increases linearly with $B$, leading to energy bands around the values of $E / J_{0}=-N B,-(N-2) B,-(N-4) B, \ldots$, as we will discuss later in equation (5.64). This means that beyond the populated bands at $\left(E-E_{0}\right) / J_{0}=40,80$ there exist energy bands at $\left(E-E_{0}\right) / J_{0}=20,60$ which are not populated. This is explained by the symmetry of the ground state and the dephasing operation, as we will see in the following. 
Most interestingly, in the intermediate range, when $B \simeq J_{0}$, the excitations are broadly distributed over the entire energy spectrum. This is also nicely seen in figure 5.2, where the dephasing-induced population $c_{j}$ of excited states is plotted as a function of the index $j$, which labels the excited states according to their corresponding energy eigenvalues. Naturally, such a broad population distribution leads to the most complex dynamics, which is responsible for the local signature of the quantum phase transition, observed in section 3.4.3.

Now let us come back to the reason for the omission of some excited states in the dephasing-induced excitation spectrum. Being an eigenstate of $H$, the ground state can be defined to have definite parity under the $Z_{2}$ rotation, $Z_{2}\left|\Psi_{0}\right\rangle= \pm\left|\Psi_{0}\right\rangle$. The operation $\widetilde{\Phi}=(\Phi \otimes \mathbb{I})$ as defined in equation (5.10) does not break the $Z_{2}$ symmetry, since it operates in the $y$ basis:

$$
\widetilde{\Phi}\left(Z_{2} \rho Z_{2}^{\dagger}\right)=Z_{2} \widetilde{\Phi}(\rho) Z_{2}^{\dagger}
$$

This means that only half of the excitation spectrum can be reached by the local dephasing operation, since we are limited to stay within the parity subspace of the ground state.

The dephasing-induced population of excited states is an important ingredient to understand the strong local signal close to the quantum phase transition, shown in figures 3.11 and 3.12. The complex dynamics which we expect from the broad spectral distribution of excitations in the dephased state can be considered more likely to reveal global properties to the local subsystem. For weak external fields we see only few populated excited states, which nevertheless does not exclude the possibility that there might be non-trivial time evolution of the dephased state.

Another important point to consider in this context is the partial trace operation. How do we know that tracing over the remaining spins does not hide all the interesting dynamics of the total dephased state from the local dynamics of the accessible spin? In order to study the full dynamics of the dephased state, we consider the global time-autocorrelation function

$$
C(t)=\frac{1}{\mathcal{P}\left(\rho_{\Phi}\right)} \operatorname{Tr}\left\{\rho_{\Phi} U(t) \rho_{\Phi} U^{\dagger}(t)\right\}
$$

with $U(t)=e^{-i H t / \hbar}$. We have renormalized this function with the purity $\mathcal{P}\left(\rho_{\Phi}\right)=$ $\operatorname{Tr} \rho_{\Phi}^{2}$ to ensure that $C(0)=1$. This function is directly related to the coherences of $\rho_{\Phi}$ in the energy eigenbasis, which govern the time evolution. This can be seen from the following decomposition:

$$
C(t)=\frac{1}{\mathcal{P}\left(\rho_{\Phi}\right)} \sum_{i j}\left|\left\langle\Psi_{j}\left|\rho_{\Phi}\right| \Psi_{i}\right\rangle\right|^{2} e^{-i\left(E_{i}-E_{j}\right) t / \hbar} .
$$

In figure 5.3, we display the maximum deviation from the initial state given by the minimum value of $C(t)$. This confirms that, indeed, for very weak and very strong $B$, almost no dynamics is observable on the global level, and the fact that we do not observe dynamics on the local level is not caused by locally monitoring only a small 


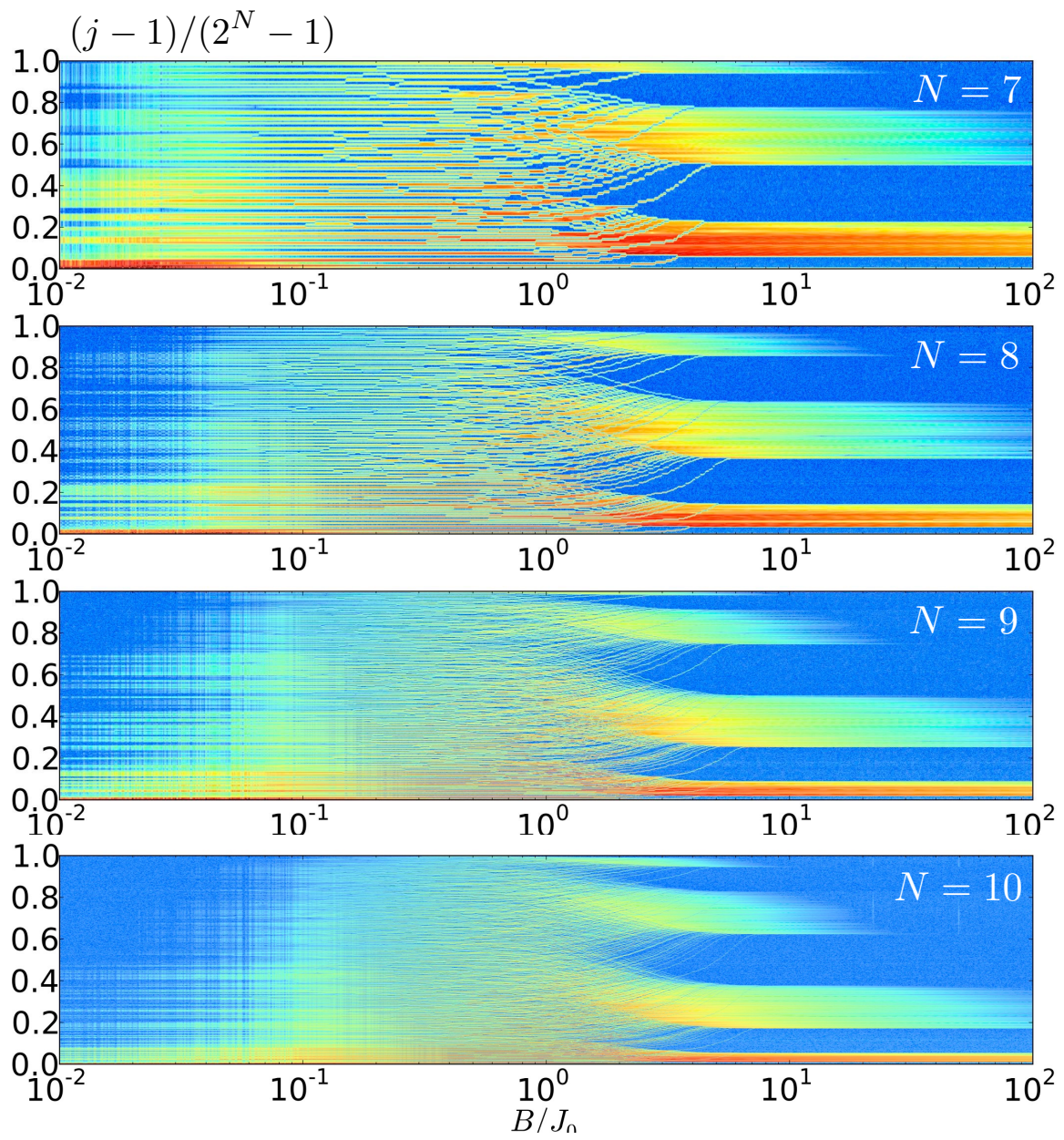

FIGURE 5.2.: Dephasing-induced excited-state populations $c_{j}$, equation (5.11), in the dephased state $\rho_{\Phi}$, equation (5.10), for $\alpha=1$. The $y$-axes show the (renormalized) index of the excited state, which is why the paramagnetic energy bands seem broader than they are when plotted against the energy. Again, we see mostly groundstate contributions when $B$ is small. Most prominently, the broad distribution around $B \simeq J_{0}$ confirms the broad distribution of excited-state populations close to the quantum phase transition. The plot further shows the rapid convergence with increasing $N$, due to the exponentially increasing Hilbert space dimension. For values of $B / J_{0} \gtrsim 10$, we observe how excited states are skipped due to the $Z_{2}$ symmetry of the dephasing operation; see discussion around equation (5.12). The color code is logarithmically scaled and normalized to 100 steps between the respective minimum and maximum values of $c_{j}$, increasing from blue to red. 


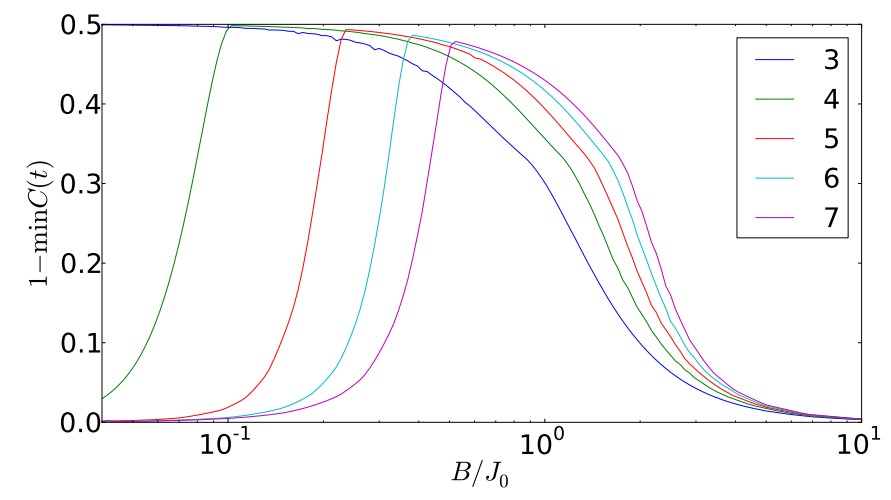

FiguRE 5.3.: The global time-autocorrelation function demonstrates that even on the global level, no dynamics can be observed when $B$ is very large or very small. This is plotted for various values of $N$ (see legend) with $J_{0}>0$ and $\alpha=1$. The strongest deviation from the initial state is found around $B \sim J_{0}$, which agrees with the findings of the local single-spin dynamics, shown in figure 3.11.

part of the full system. The most pronounced time evolution of the dephased ground state is observed at values of $B \simeq J_{0}$. In order to find out what causes such rich dynamics, we will study the full spectrum of $H$ in the next section.

\subsection{Spectral analysis}

The dephasing-induced populations seen, for example, in figure 5.1, already indicate a significant qualitative change of the excitation spectrum as the external field is scanned. This intuition is confirmed already for small system sizes, when we plot the full spectrum in figure 5.4. The red and black plotted spectral lines separate odd and even parity subspaces of the $Z_{2}$ operation, respectively. For weak external fields $B$, pairs of eigenstates, comprised of states from both parity subspaces, approach each other energetically. For example, in the thermodynamic limit $(N \rightarrow \infty)$, the ground state becomes two-fold degenerate below $B \lesssim J_{0}$. In fact, the magnetic field above which the ground-state degeneracy is suddenly lifted, defines the critical point of the quantum phase transition. Already in finite-size systems, we observe at intermediate $B$ that the levels within each subspace start to bend, inducing avoided crossings due to nonzero couplings between the eigenstates. The two subspaces, however, evolve independently of each other and the lack of interaction causes frequent direct crossings between two levels from different subspaces. When $B$ increases even further, the two subspaces occupy completely disjunct spaces of the energy spectrum. The paramagnetic bands alternate from one subspace to the other as the energy is increased.

Our initial motivation to dig into the spectral features of this spin chain model was to explain the success of the local detection method in revealing the ground-state correlations to the single-spin dynamics. The effects observed in section 3.4.3 are 

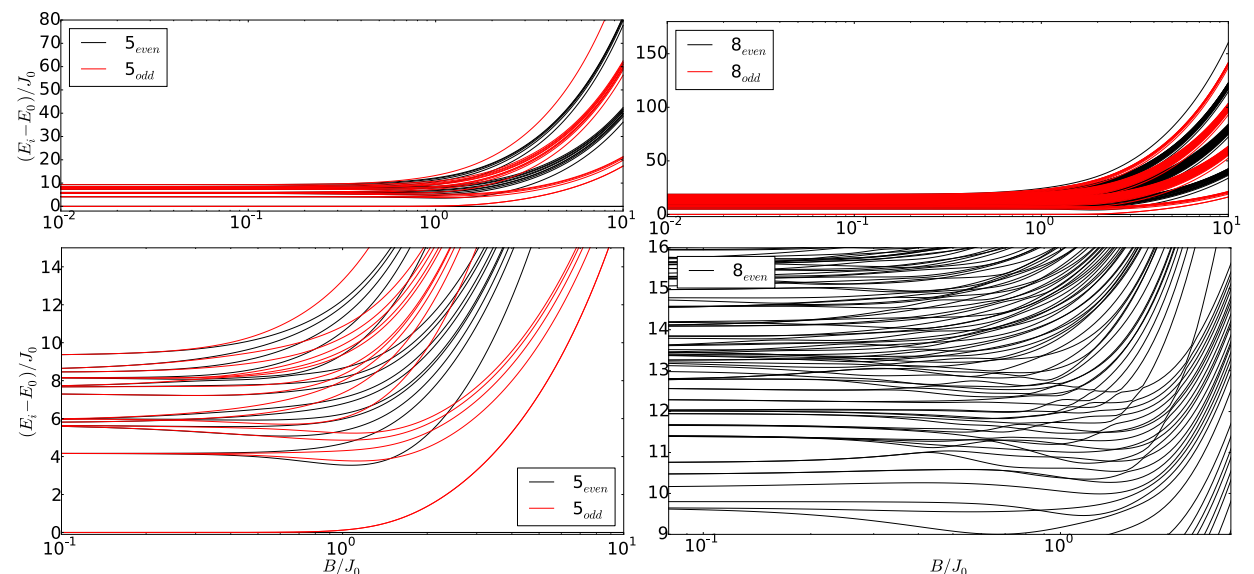

FiguRE 5.4.: Spectra of the spin chain Hamiltonian (5.1) for $\alpha=1, J_{0}>0$ and $N=5$ (left) and $N=8$ (right), respectively. Energy eigenvalues are plotted in red (black) when the corresponding eigenstate belongs to the odd (even) parity subspace with respect to the $Z_{2}$ operation, as defined in equation (5.3). We observe the onset of two-fold degeneracy (which occurs in the limit $N \rightarrow \infty$ ) comprising both subspaces for $B \ll J_{0}$, broadly distributed spectra around $B \simeq J_{0}$, with frequent avoided crossings within each subspace, and paramagnetic energy bands at $B \gg J_{0}$, which have definite parity.

well explained by the interplay of dephasing-induced excitations and complex level structures in the vicinity of the quantum phase transition. The characteristic spectral features that accompany this quantum phase transition are intriguing, independently of their relevance for the local detection method. For arbitrary $\alpha$, the system is poorly understood since previous studies mostly focussed on the limiting cases of the Ising and Lipkin-Meshkov-Glick models. We thus dedicate the rest of this chapter to the development of a general understanding of the spectral features of the spin chain Hamiltonian as a function of the parameters $J_{0}$ and $\alpha$ while the external field $B$ is being varied. After a brief, qualitative analysis of the level statistics in the next two subsections, we develop a semiclassical ansatz to describe features of the excitation spectrum, which we relate to the quantum phase transition in section 5.4.

In order to extract statistical information about the level dynamics, we run statistical analysis of the nearest-neighbor spacings. This may allow us to identify chaotic structures in the quantum spectrum; recall section 2.5.2. We remark, however, that in this section, we restrict to elementary analysis of the spectral features, whose goal is to establish a qualitative understanding without necessarily providing a stringent random-matrix analysis which would be able to extract quantitative statements. The results of the semiclassical mean-field analysis, presented in section 5.4, are independent of the spectral analysis in the present section. 

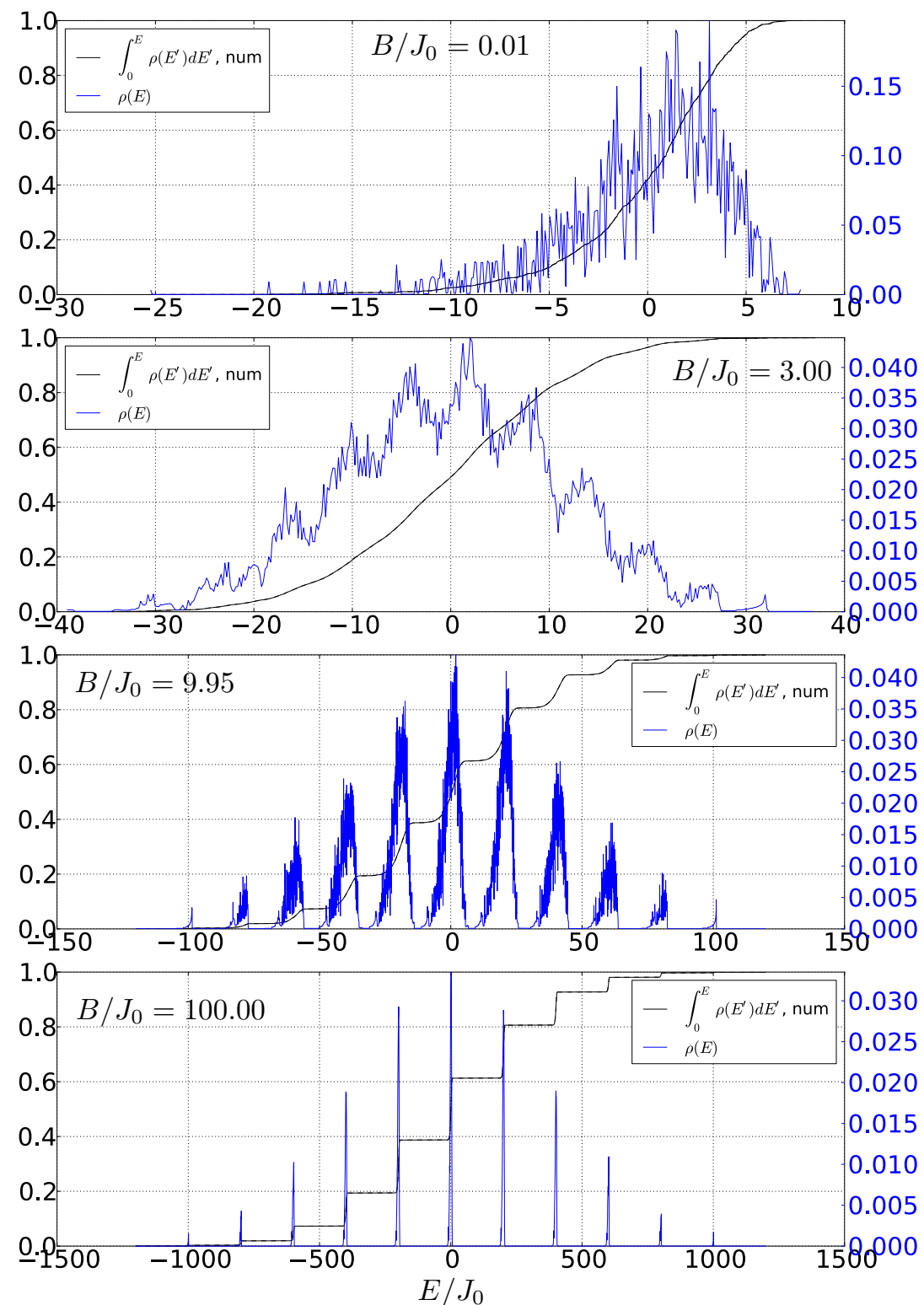

Figure 5.5.: Density of states $\rho(E)$, equation (5.17), and its integral, the counting function (5.15), for a spin chain (5.1) with parameters $N=12, \alpha=1, J_{0}>0$. The numerically obtained density of states shows a transition from a broadly distributed, asymmetric ferromagnetic spectrum at $B=0$ (see also figure 5.13 later in this chapter) to a paramagnetic spectrum, characterized by strongly degenerate, equally-spaced energy bands at $B \gg J_{0}$. 


\subsubsection{Density of states}

We begin with an analysis of the staircase or counting function

$$
N(E)=\sum_{i=0}^{2^{N}-1} \theta\left(E-E_{i}\right),
$$

where $\theta$ denotes the Heaviside function. The counting function is related to the density of states $\rho(E)$ as

$$
N(E)=\int_{0}^{E} \rho\left(E^{\prime}\right) d E^{\prime},
$$

and the density of states is given by

$$
\rho(E)=\sum_{i=0}^{2^{N}-1} \delta\left(E-E_{i}\right) .
$$

Both quantities can be obtained numerically from the generated spectra. Figure 5.5 shows the normalized density of states, as well as the corresponding counting function for different values of $B$ in a ferromagnetic chain of $N=12$ spins at $\alpha=1$. At $B=0$, the spectrum is strongly influenced by $\alpha$. The case $\alpha=1$ leads to an extremely broadly distributed ferromagnetic spectrum. We will see later in this chapter, that this is no longer the case when $\alpha$ becomes very large or very small, when many degeneracies emerge. The influence of $\alpha$ diminishes with increasing $B$. In the opposite limit, when $B \gg J_{0}$, the counting function shows a regular staircase with consecutive steps of equal spacings. The height of the steps are, however, not equal, since the center bands of the paramagnetic system are more strongly degenerate than the outer values, which can already be anticipated from the two top plots in figure 5.4. In the intermediate regime, for moderate values of $B$, a smooth interpolation between the two cases can be observed.

\subsubsection{Level statistics}

Trying to identify signatures of quantum chaos, we finally turn to an analysis of the level statistics. To obtain significant statistics, we collect the nearest neighbor spacings over different intervals of $B$. At each fixed value of $B$, the nearest-neighbor spacings $s_{i}=E_{i+1}-E_{i}$ are renormalized as $s_{i} / \bar{s}$ with the average value $\bar{s}=2^{-N} \sum_{i=0}^{2^{N}-1} s_{i}$. This simple form of spectral unfolding ensures that the statistics of systems with different energy scales can be compared (Haake, 2001). We emphasize again that the purpose of the present section is only to provide a brief qualitative impression of the level dynamics. For a more thorough analysis, the unfolding needs to be performed on the basis of a local mean value of the level spacings, whereas the range would have to be tested through the robustness of the resulting level statistics (Haake, 2001). Moreover, in the present section we only distinguish between the two parity subspaces, 


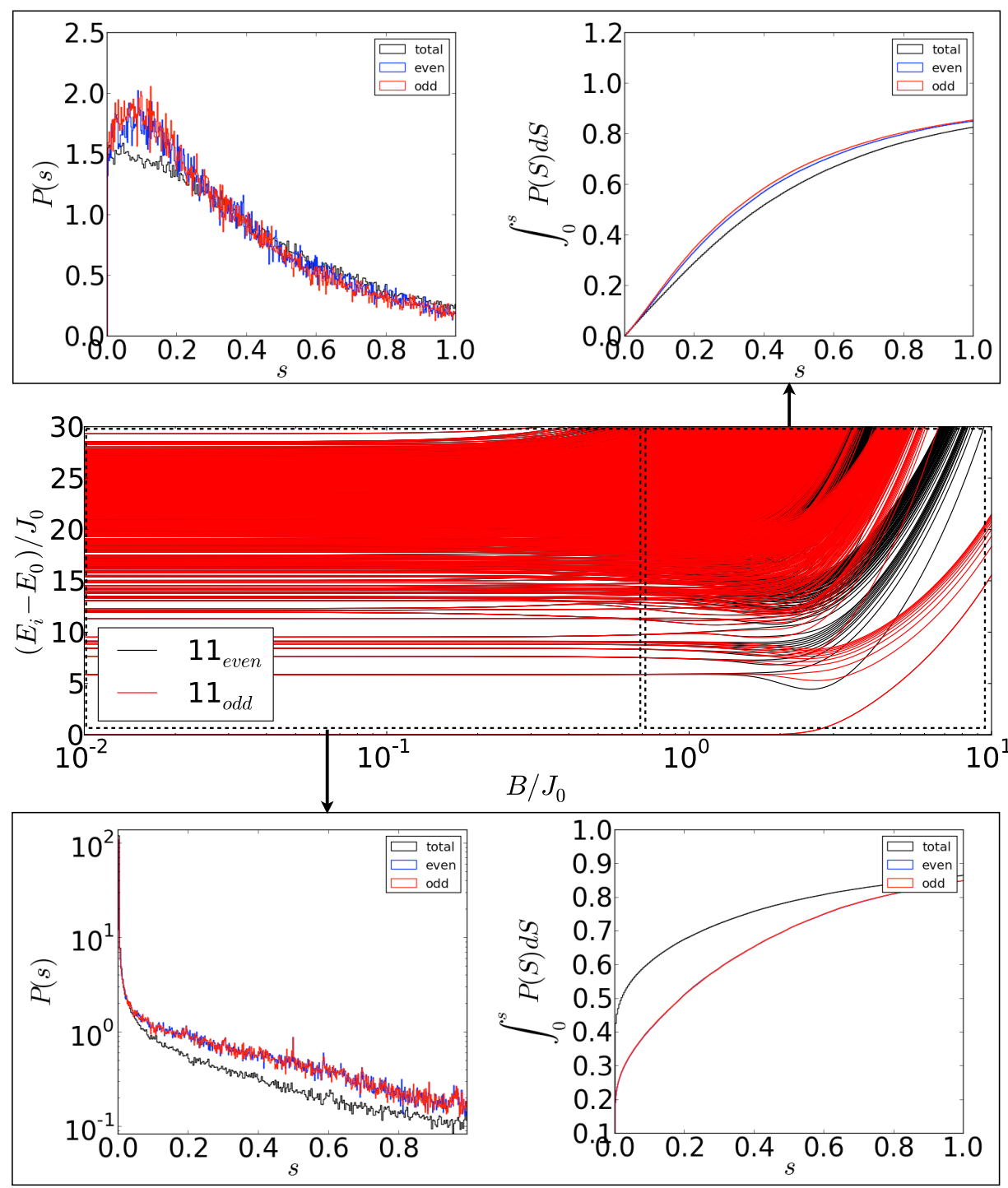

FigURE 5.6.: Spectrum of a $N=11, J_{0}>0$, and $\alpha=1$ spin chain, described by (5.1). The statistical analysis of the nearest-neighbor level spacing distributions $P(s)$ shows a strong cusp in the vicinity of $s=0$, followed by an exponential decay for $s>0$, for $0.01 \leq B / J_{0} \leq 0.7$ (bottom left). In the regime $0.7 \leq B / J_{0} \leq 10$, level repulsion becomes more prominent. However, the level statistics (top left) does not match a Wigner-Dyson distribution (2.19); compare to predictions from random matrix theory in figure 2.7. The integrated level spacing distributions (top and bottom right) confirm this observation. 
whereas an unambiguous identification of chaotic signatures requires that all known symmetries are removed. Apart from the $Z_{2}$ symmetry, the model discussed here is mirror symmetric with respect to the center spin, see section 5.1.1.

Figure 5.6 provides qualitative intuition of the level statistics using the example of $N=11, \alpha=1$ and $J_{0}>0$. Over the range $0.01 \leq B / J_{0} \leq 0.7$ we see exponential decay of the nearest-neighbor probability distribution $P(s)$. This is expected for uncorrelated level statistics, which lead to a Poissonian distribution $P(s)$ and indicate regular dynamics; see equation (2.18). However, we observe a strong cusp for very small values of $s$, which is not predicted by the Poissonian distribution. This cusp persists even when we separate the two parity subspaces and indicates the tendency to form degenerate energy levels in the ferromagnetic spectrum. A possible explanation for this cusp could be the system's mirror-symmetry, which leads to further degeneracies.

When we move on to the intermediate range $0.7 \leq B / J_{0} \leq 10$, we find clear signatures of level repulsion in the individual parity subspaces. Nevertheless, the distribution does not match a Wigner-Dyson distribution (2.19), as apparent, for example, from the nonzero value of $P(s)$ at $s=0$. Again, the remaining mirror symmetry certainly leads to degenerate levels, but it remains unclear whether the spectrum would follow a clean Wigner-Dyson distribution if this symmetry was removed. The figure also shows the integrated level spacing distributions, which often allow for a more precise comparison to Wigner-Dyson and Poissonian distributions. Yet, the present case constitutes an example of a mixed level statistics, in which neither completely regular nor to totally chaotic structures can be identified. We are limited by the exponentially increasing Hilbert space dimension, which complicates exact numerical diagonalization. $^{2}$ Conversely, for small systems, the limited number of levels at a single value of $B$ is not sufficient to obtain significant statistical data. To circumvent this, we mix the statistics of many systems over a range of values for $B$, which complicates the extraction of clean signatures of either regular or chaotic structures.

The spectral analysis provided in this section has provided a qualitative understanding of the essential features of the quantum spectrum throughout different values of the external field parameter $B$. In the next section, we describe features of the excitation spectrum by developing a semiclassically motivated, variational ansatz.

\subsection{Semiclassical mean-field description}

We now turn to the main part of the present chapter. We begin by reviewing semiclassical approximations for spin systems, and their implications for quantum phase transitions.

\footnotetext{
${ }^{2}$ Exact numerical diagonalization (Johansson et al., 2013) of the full Hamiltonian (5.1) for $N=12$ spins, described by a complex-valued $4096 \times 4096$-matrix, requires about $1 \mathrm{~GB}$ of RAM, whereas each additional spin increases the memory requirements by at least a factor of two.
} 


\subsubsection{Semiclassial approximations and quantum phase transitions}

In section 2.5.1, we had already mentioned different approaches to derive semiclassical limits of quantum mechanics. One class of such approaches is represented by mean-field theories. By discarding the quantum fluctuations, the quantum mechanical Hamilton operator is transformed into a classical Hamiltonian. The magnitude of these fluctuations typically decreases as the number of particles increases, and the classical model is recovered in the thermodynamic limit, $N \rightarrow \infty$; see for example (Raghavan et al., 1999; Pitaevskii and Stringari, 2003; Wen, 2004; Leyvraz and Heiss, 2005; Shchesnovich and Konotop, 2007; Caprio et al., 2008).

This is realized effectively by replacing operators with complex numbers, which in turn correspond to the operator's expectation value, when, for example, the trial state is chosen as a coherent state (Zhang et al., 1990; Gnutzmann and Kuś, 1998; Caprio et al., 2008). Thus, in many cases, a mean-field approximation can be formally understood as a variational ansatz, where the classical Hamiltonian corresponds to the energy expectation value of the Hamilton operator, and suitably chosen variational parameters of the trial function emerge as the coordinates of the corresponding classical system (Botet et al., 1982; Wen, 2004; Dusuel and Vidal, 2005). To achieve this also in the context of spin systems one defines formal analogues of coherent states based on the spin algebra, which we will review in the next section. A variational ansatz in terms of such "spin-coherent states" can then be interpreted as a semiclassical limit of the spin model (Balakrishnan and Bishop, 1985; Schliemann and Mertens, 1998; Ribeiro et al., 2007; Ribeiro et al., 2008). Trial functions for such variational meanfield approaches are mostly product states, in which all particles are described by the same single-particle state. Such an ansatz completely neglects the correlations between the particles. Yet, mean-field approaches have successfully provided valuable insight into various systems, ranging from magnetic systems (Curie, 1895; Weiss, 1907) to dilute Bose-Einstein condensates (Bogoliubov, 1947).

Semiclassical descriptions become especially relevant in the context of phase transitions, which describe the discontinuous change of a system's properties in the thermodynamic limit - when mean-field approaches typically yield good results. Characteristic features of a quantum phase transition (recall section 2.6) are

(i) non-analytic behavior of the ground state energy, and

(ii) a closing energy gap between the ground state and the first excited state.

Both of these phenomena occur when the external control parameter reaches its critical value. Using the Feynman-Hellmann theorem ${ }^{3}$ (Feynman, 1939), feature (i) can be linked to the discontinuous behavior of an order parameter at the critical value (Caprio et al., 2008). The order parameter, in turn, reflects the spontaneous breaking of symmetry, when the critical field is crossed - in analogy to the well-known classical theory of thermal phase transitions (Landau and Lifshitz, 1969).

\footnotetext{
${ }^{3}$ The Feynman-Hellmann theorem determines the change of a Hamiltonian's energy eigenvalue as a function of a parameter of the Hamiltonian (Feynman, 1939).
} 
All of these features are intuitively illustrated at the hand of the example of a double-well potential with tunable barrier-we will encounter such structures later in this chapter; an illustration can be found in figure $5.10 \mathrm{a}$ ). In fact, semiclassical methods, as described above, often map the quantum model undergoing the quantum phase transition onto such a problem (Leyvraz and Heiss, 2005; Caprio et al., 2008). The barrier then emerges exactly when the control parameter drops below its critical value. Above this value, there is no barrier, and the minimum is always found at the center of the potential. This implies that in this case, the ground state is symmetric under reflections with respect to the potential's symmetry axis. Below the critical value, when the barrier appears, one finds two degenerate potential minima, with their associated ground states being localized in the respective left and right well. Hence, below the critical value, the symmetry of the two ground states is broken. The nonzero displacement (the ground-state expectation value) from the potential center reflects this observation and can be interpreted as an order parameter.

In the above example the ground state becomes two-fold degenerate in the symmetric phase, thus, as anticipated in (ii), the energy gap closes. However, the double-well potential also entails consequences for the excited states, which are, again, most efficiently described by semiclassical methods. When the barrier appears, the fixed point at the potential center becomes unstable. Hence, the energy at the top of the barrier separates the phase space into three regions and a separatrix emerges (Ozorio de Almeida, 1988). For energies below the separatrix the motion is constrained to either one of the two wells, which corresponds to the symmetry-broken phase. Above this energy threshold, the trajectories explore both sides of the barrier and the symmetry is restored, similarly to the case where no barrier was present. Close to the separatrix energy, the periods of the classical orbits diverge, which, from semiclassical correspondence, leads to a diverging density of states of the associated quantum system; see, for example, (Tennyson et al., 1986; Leyvraz and Heiss, 2005). This is a direct extension of (ii) to higher excited states, and is therefore labelled an excited-state quantum phase transition (Caprio et al., 2008; Bastidas et al., 2014).

The singularity of the ground state at a given value of the external control parameter can be considered a boundary between phases, which can be distinguished via the ground-state expectation value of a suitably chosen order parameter. Thus, the ground state exhibits qualitatively different properties, with macroscopic signatures, depending on the value of the order parameter. In the case of excited-state quantum phase transitions, the properties that characterize the "phase" of the respective excited state can only be assessed via dynamical quantities, as becomes apparent from the above semiclassical illustration (Caprio et al., 2008; Engelhardt et al., 2015).

In this dissertation, we will analyze features of the quantum phase transition that manifest in the excitation spectrum by developing a variational product-state ansatz for the spin chain Hamiltonian (5.1). First, we generalize the commonly employed mean-field ansatz of coherent spin states to a multi-variable product state. This leads to an high-dimensional energy landscape which we can optimize numerically. Second, by introducing physically motivated constraints, we reduce the problem to a series of one-dimensional optimization problems (each one characterized by a specific spin configuration), which can be treated analytically. This multi-configurational mean- 
field ansatz is able to reproduce key features of the quantum model, including the second-order quantum phase transition.

\subsubsection{Spin-coherent states}

Coherent states are well known in quantum optics for the description of laser light (Glauber, 1963; Klauder, 1963; Sudarshan, 1963). They form an overcomplete basis of states of the quantum harmonic oscillator, describing, for instance, a single light mode. Their expectation values of momentum and position follow the classical equations of motion, ${ }^{4}$ and the quantum uncertainty which defines the spread of the state in phase space remains constantly at the lowest possible value, saturating Heisenberg's uncertainty relation (Heisenberg, 1927). Hence, coherent states behave very classically. Formally, they can be created by a coherent displacement of the vacuum - or in the language of the harmonic oscillator, the state of zero excitations. For further details, see (Mandel and Wolf, 1995; Scully and Zubairy, 1997; Schleich, 2001).

In close analogy, such states can be defined for spin-1/2 systems (Radcliffe, 1971; Arecchi et al., 1972). To this end, we first consider total angular momentum eigenstates of a collection of $N$ spin-1/2 particles. The eigenvalues of $\mathbf{S}^{2}$ are given by $l(l+1)$ with $-N / 2 \leq l \leq N / 2$, and for each $l$ the $2 l+1$ sublevels are characterized by the eigenvalue $m=-l,-l+1, \ldots, l-1, l$ via the relation $S_{z}|l, m\rangle=m|l, m\rangle$. The states $|l, m\rangle$ are denoted Dicke states (Dicke, 1954), and for $l=N / 2$ they contain $N / 2+m$ particles in the excited state while the remaining $N / 2-m$ particles are in the ground state. These states were first introduced to describe the influence on the photon emission rate of a collection of two-level atoms subject to coupling to a common electromagnetic field, which can lead to strongly enhanced radiation. We have already briefly commented on this effect, called superradiance (Dicke, 1954), when we discussed collective dephasing processes in section 4.4.4; note also the direct correspondence of the Dicke states and the projectors that occur in the spectral decomposition (4.31).

All Dicke states can be generated by multiple applications of the raising operator $S_{+}$to the respective ground state $|l,-l\rangle$, which has the lowest possible value of $m$, and we define $S_{ \pm}=S_{x} \pm i S_{y}$ (Mandel and Wolf, 1995). We further note that the spectrum of the Dicke states resembles the equidistant spectrum of the quantum harmonic oscillator. In the spirit of this analogy, we can now formally introduce an atomic displacement operator (Arecchi et al., 1972)

$$
D(z)=\exp \left(\zeta S_{+}-\zeta^{*} S_{-}\right),
$$

where $z=\tan |\zeta| e^{i \arg \zeta}$, to define a spin coherent state

$$
|l, z\rangle=D(z)|l,-l\rangle,
$$

as a displaced ground state. These states are the analog of the well-known coherent states of the boson field, ${ }^{5}$ and sometimes (for reasons that will become clear soon) they

\footnotetext{
${ }^{4}$ This is a result of Ehrenfest's theorem for the special case of a quadratic potential (Ehrenfest, 1927).

${ }^{5}$ Note, however, that spin coherent states are not eigenstates of $S_{-}$, but instead of a much less intuitive linear combination of $S_{-}$and $S_{z}$ (Arecchi et al., 1972).
} 
are referred to as Bloch states or atomic coherent states (Arecchi et al., 1972; Mandel and Wolf, 1995). In the special case $l=N / 2$, these states reduce to the rather simple expression

$$
|N / 2, z\rangle=\bigotimes_{i=1}^{N} \frac{1}{\sqrt{1+|z|^{2}}}\left(|\downarrow\rangle^{(i)}+z|\uparrow\rangle^{(i)}\right)
$$

As they form a pure product of identical single-spin states, these states can be represented conveniently via the Bloch vector of the single-spin states (Bloch, 1946). The parameter $z$ can be identified with the Bloch vector coordinates via $z=\cot (\theta / 2) e^{-i \phi}$, which yields the expectation values

$$
\begin{aligned}
& \left\langle N / 2, z\left|S_{x}\right| N / 2, z\right\rangle=\frac{N}{2} \sin \theta \cos \phi, \\
& \left\langle N / 2, z\left|S_{y}\right| N / 2, z\right\rangle=\frac{N}{2} \sin \theta \sin \phi, \\
& \left\langle N / 2, z\left|S_{z}\right| N / 2, z\right\rangle=\frac{N}{2} \cos \theta,
\end{aligned}
$$

and, thus, we rewrite

$$
|N / 2, z\rangle=|\theta, \phi\rangle=\bigotimes_{i=1}^{N}\left[\sin \left(\frac{\theta}{2}\right)|\downarrow\rangle^{(i)}+\cos \left(\frac{\theta}{2}\right) e^{-i \phi}|\uparrow\rangle^{(i)}\right] .
$$

We see that this decomposition is completely general on the level of a single spin $(N=$ 1 ), hence, any pure state of a spin- $1 / 2$ particle can be written as a spin coherent state. The Bloch vector representation can be generalized to arbitrary $l$, where $l$ generally enters as the length of the Bloch vector (Mandel and Wolf, 1995). Spin coherent states further form an overcomplete basis, and represent wavepackets of minimum uncertainty with respect to the components of the rotated total angular momentum, where the angles of rotation correspond to the angles characterizing the Bloch vector (Arecchi et al., 1972).

\subsubsection{Multidimensional numerical search for critical points}

Considering the spin-spin interactions in the variable-range quantum magnet (5.1), we do not necessarily expect that the symmetry of the spin-coherent states (5.24) in their standard construction applies, that is, it is unlikely that all spins align along the same direction and can be characterized by a single Bloch vector. Instead, we will formulate a variational ansatz which allows to tune the orientation of individual spins independently. Furthermore, we see from the Hamiltonian (5.1) that the energy expectation value does only depend on the Bloch vector's $x$ and $y$ components, but not on the $z$ component. Hence, to analyze the energy landscape of the variable-range quantum magnet semiclassically, we suggest a product state of individual states of the form

$$
|\Psi(\phi)\rangle=\bigotimes_{i=1}^{N}\left|\phi_{i}\right\rangle
$$


where $\boldsymbol{\phi}=\left(\phi_{1}, \ldots, \phi_{N}\right)$ are the local angles characterizing the single spin states

$$
\left|\phi_{i}\right\rangle=\frac{1}{\sqrt{2}}\left(|\downarrow\rangle^{(i)}-i e^{i \phi_{i}}|\uparrow\rangle^{(i)}\right) .
$$

The states $\left|\phi_{i}\right\rangle$ can be interpreted as spin coherent states for $N=1$ and take the usual form, equation (5.24), upon replacing $\theta \rightarrow \pi / 2$ and $\phi \rightarrow \pi / 2-\phi_{i}$. Using equations (5.21) to (5.23), the expectation values of the Pauli matrices yield the Bloch vector coordinates ${ }^{6}$

$$
\begin{aligned}
\left\langle\phi_{i}\left|\sigma_{x}\right| \phi_{i}\right\rangle & =\sin \phi_{i}, \\
\left\langle\phi_{i}\left|\sigma_{y}\right| \phi_{i}\right\rangle & =\cos \phi_{i}, \\
\left\langle\phi_{i}\left|\sigma_{z}\right| \phi_{i}\right\rangle & =0 .
\end{aligned}
$$

The ansatz was chosen such that it parameterizes the $x y$ plane without adding unnecessary variables in the $z$ direction. Using this ansatz and (5.1), the energy expectation value per site yields

$$
E(\phi)=\frac{1}{N}\langle\Psi(\phi)|H| \Psi(\phi)\rangle=-\frac{J_{0}}{N} \sum_{\substack{i, j=1 \\(i<j)}}^{N} \frac{\sin \phi_{i} \sin \phi_{j}}{|i-j|^{\alpha}}-\frac{B}{N} \sum_{i=1}^{N} \cos \phi_{i},
$$

which represents an $N$-dimensional semiclassical energy landscape. We find that the minimum of $E(\phi)$ reproduces the ground state energy of the true quantum spectrum reasonably well over a broad range of parameters, except for $B \sim\left|J_{0}\right|$, see the circles in figure 5.7. This approach can be understood as an application of the variational principle with (5.25) as trial state (Ritz, 1909; Cohen-Tannoudji et al., 1977b). The agreement with the quantum ground state energy is somewhat unexpected for $B /\left|J_{0}\right| \ll 1$, since the trial state is a product state, whereas we know that the true ground state must be a GHZ-type $\left(J_{0}>0\right)$ or W-type $\left(J_{0}<0\right)$ multipartite entangled state (Štelmachovič and Bužek, 2004). A possible reason for this could be the symmetry of the system, which allows to approximate the description of the subsystems by assuming statistical independence between them (de Finetti, 1937; Fannes et al., 1988; Renner, 2007).

Next, we use this ansatz to analyze the semiclassical energy values beyond the ground state, by classifying extremal points of the energy manifold. We first define a set $\mathcal{C}$ of critical points, characterized by vectors $\phi^{0}$ of local angles, where the gradient of $E$ vanishes:

$$
\left.\frac{\partial E(\phi)}{\partial \phi_{i}}\right|_{\phi=\phi^{0}}=0, \quad \forall i=1, \ldots, N \quad \forall \phi^{0} \in \mathcal{C} .
$$

The condition $\partial E(\phi) / \partial \phi_{i}=0$ leads to

$$
\tan \phi_{i}^{0}=\frac{J_{0}}{B} \sum_{\substack{j=1 \\(j \neq i)}}^{N} \frac{\sin \phi_{j}^{0}}{|i-j|^{\alpha}}
$$

\footnotetext{
${ }^{6}$ The factor $1 / 2$ in comparison to equations (5.21) to (5.23) stems from the definition (5.8).
} 

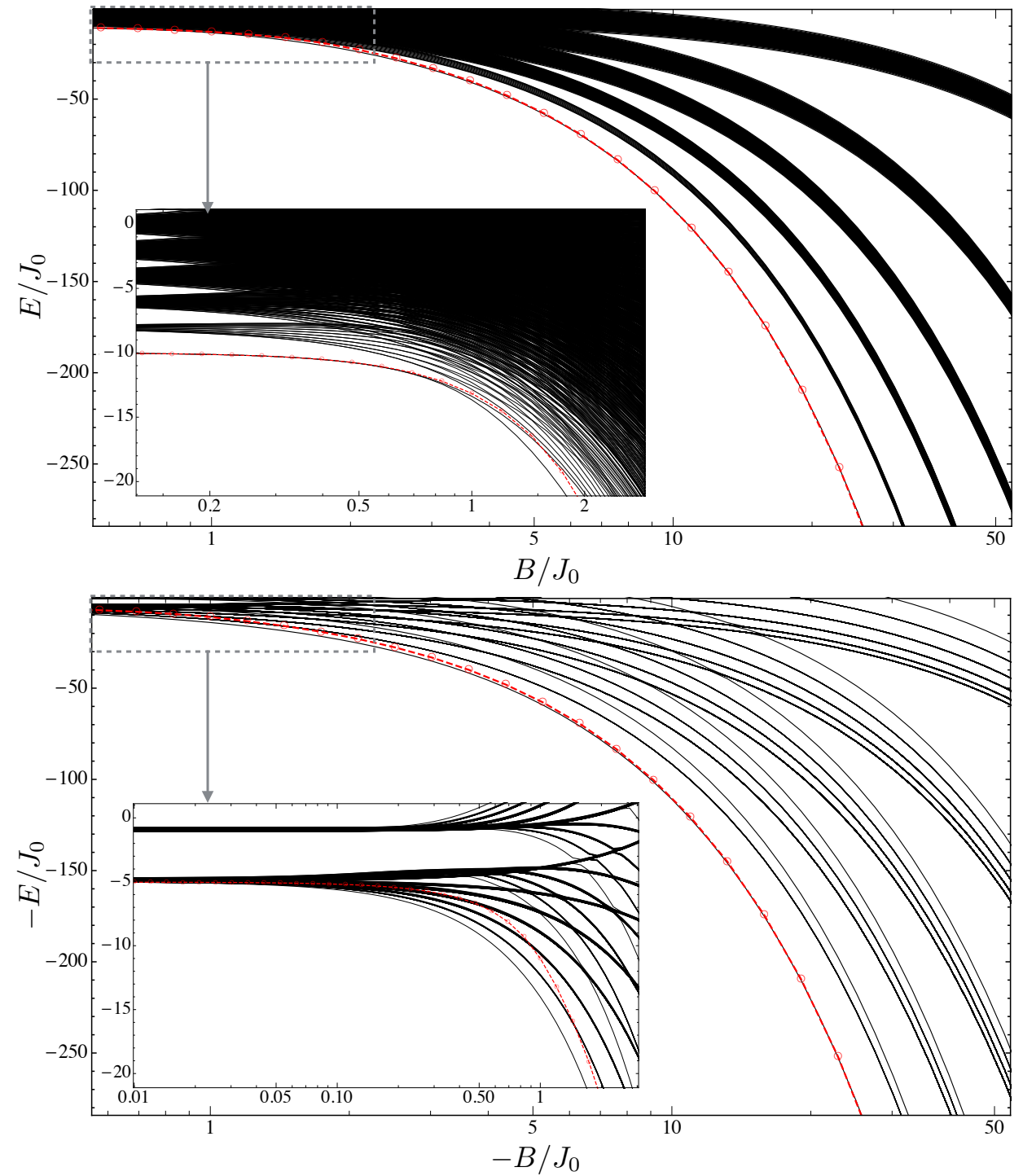

FiguRE 5.7.: Lower part of the exact quantum spectrum (black lines) of (5.1) for $N=11$, $J_{0}>0, \alpha=100$ (top), and for $J_{0}<0, \alpha=0.01$ (bottom). The ground state energy is very well approximated by the minimum of the semiclassical energy manifold $E(\phi)$, defined in (5.30), (red circles and dashed line), for $B /\left|J_{0}\right| \gg 1$ and $B /\left|J_{0}\right| \ll 1$. Deviations emerge in the vicinity of the critical magnetic field, when $B \sim\left|J_{0}\right|$, especially for small $\alpha$ (see insets). 
which must hold for all $i=1, \ldots, N$. The diagonal elements of the Hessian matrix then yield

$$
h_{i i}=\frac{\partial^{2} E(\phi)}{\partial \phi_{i}^{2}}=\frac{J_{0}}{N} \sin \phi_{i} \sum_{\substack{j=1 \\(j \neq i)}}^{N} \frac{\sin \phi_{j}}{|i-j|^{\alpha}}+\frac{B}{N} \cos \phi_{i},
$$

which, by equation (5.32), at any critical point $\phi^{0}$ reduces to

$$
\left.h_{i i}\right|_{\phi=\phi^{0}}=\frac{B}{N} \frac{1}{\cos \phi_{i}^{0}} .
$$

The Hessian matrix $h=\left(h_{i j}\right)$ is completed by its off-diagonal entries $(i \neq j)$,

$$
h_{i j}=\frac{\partial^{2} E(\phi)}{\partial \phi_{i} \partial \phi_{j}}=-\frac{J_{0}}{N} \frac{\cos \phi_{i} \cos \phi_{j}}{|i-j|^{\alpha}} .
$$

Critical points which satisfy $\left.\operatorname{det} h\right|_{\phi=\phi^{0}}=0$ are called degenerate critical points. They correspond, for instance, to plateaus of $E(\phi)$. Non-degenerate critical points can be classified by the dimension of the positive-definite subspace of the Hessian matrix, which is defined as the index $\lambda$ of the critical point (Milnor, 1963). Local minima of $E(\phi)$ are characterized by a positive definite Hessian matrix, hence, they have $\lambda=N$, while maxima satisfy $\lambda=0$. For values $0<\lambda<N$ we find different classes of saddle points, which can be visualized as crossings of two lines of constant energy $E$. Those saddle points are classified by their index $\lambda$ : In a local environment around the critical point, there are $\lambda$ directions in which the potential shows a local minimum, whereas it exhibits local maxima with respect to the the remaining $N-\lambda$ directions.

The critical points and their indices are determined numerically, ${ }^{7}$ and the corresponding critical values, $E\left(\phi^{0}\right)$, are plotted for $N=11, J_{0}>0, \alpha=100$ in figure 5.8. We find the paramagnetic bands $\left(B /\left|J_{0}\right| \gg 1\right)$ are perfectly labeled by the indices $\lambda$. The semiclassical energy spectrum also reproduces the values of the strongly degenerate energy levels deep in the (anti-)ferromagnetic regime $\left(B /\left|J_{0}\right| \ll 1\right)$. However, there a labelling of the energy levels in terms of $\lambda$ is no longer possible.

In the regime where the two competing interaction strengths $J_{0}$ and $B$ are of equal order of magnitude, which corresponds to the parameter range where we expect the quantum phase transition in the thermodynamic limit, we find a very broadly distributed quantum spectrum, while the semiclassical critical points only reproduce certain central aspects thereof. Moreover, the semiclassical spectrum describes energy levels that transition continuously from a paramagnetic band to a ferromagnetic bundle, as can be seen, for example by following the dots of equal $\lambda$ in figure (5.8). In the quantum spectrum the occurrence of avoided crossing can prevent such a direct connection; see figure 5.9. Nevertheless, despite the rather simple ansatz, the essential features are well reproduced by the semiclassical spectrum.

\footnotetext{
${ }^{7}$ For $\alpha \rightarrow \infty, h$ becomes a tridiagonal matrix, which allows for efficient calculation of its determinant in terms of a recursion formula (Muir, 1960).
} 

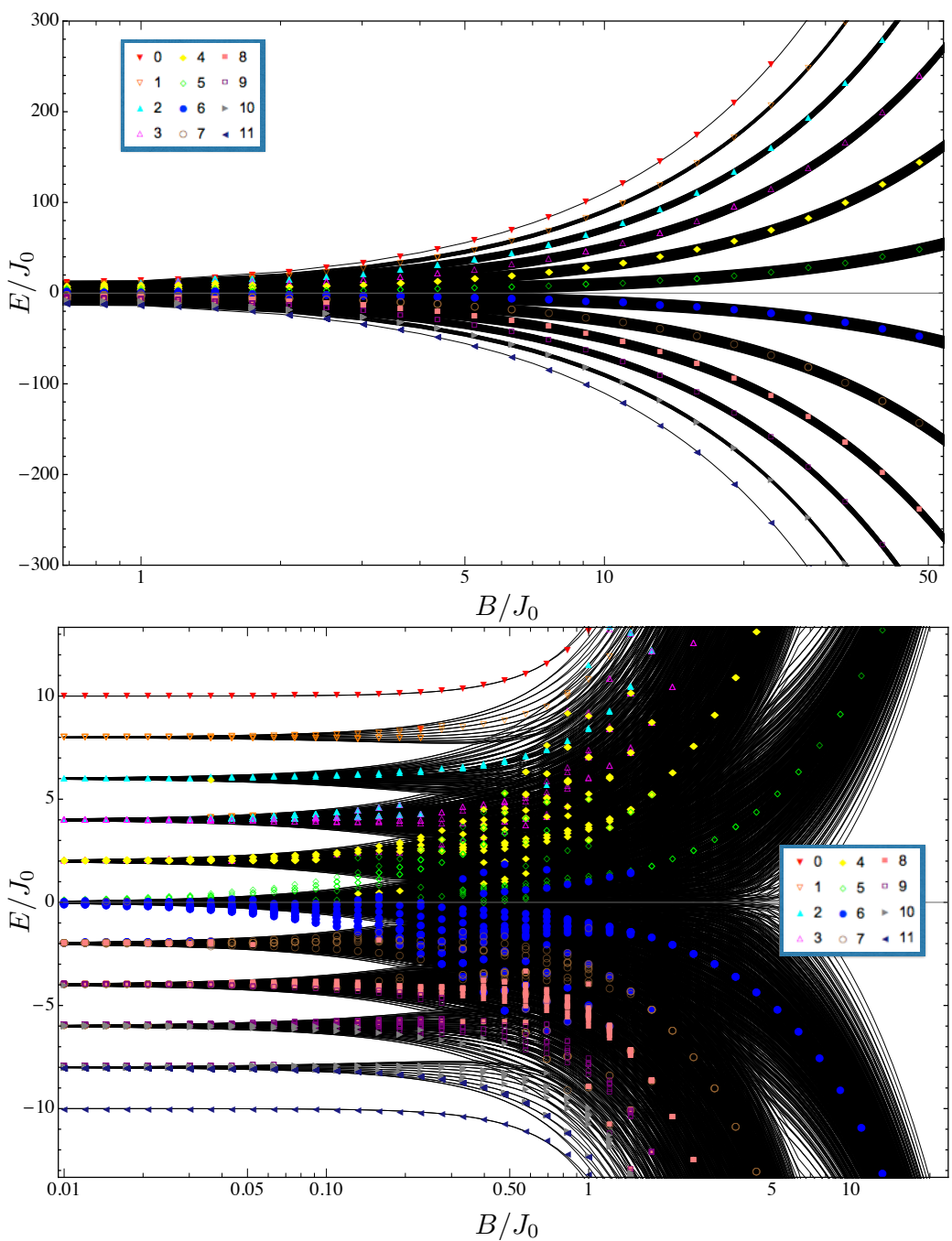

FIGURE 5.8.: Quantum spectrum (black lines) and saddle points of the semiclassical energy manifold $E(\phi)$ for a ferromagnetic system $\left(J_{0}>0\right)$ of $N=11$ spins with short-range interactions defined by $\alpha=100$; see equations (5.1) and (5.30). The saddle points are marked according to their index $\lambda$, represented by symbols in the box insets, which determine the number of positive eigenvalues of the Hessian matrix at the critical point. Thus, $\lambda$ ranges from $\lambda=0$ (maxima) to $\lambda=N=11$ (minima), and in between labels the saddle points, which were obtained numerically. The quantum spectrum is well represented, and in the paramagnetic regime, $B / J_{0} \gtrsim 1, \lambda$ labels the energy bands. This is no longer true for $B / J_{0} \lesssim 1$, where also some deviations can be observed at $\lambda=5$ and $\lambda=6$ around $E / J_{0} \approx 0$ (lower panel). 


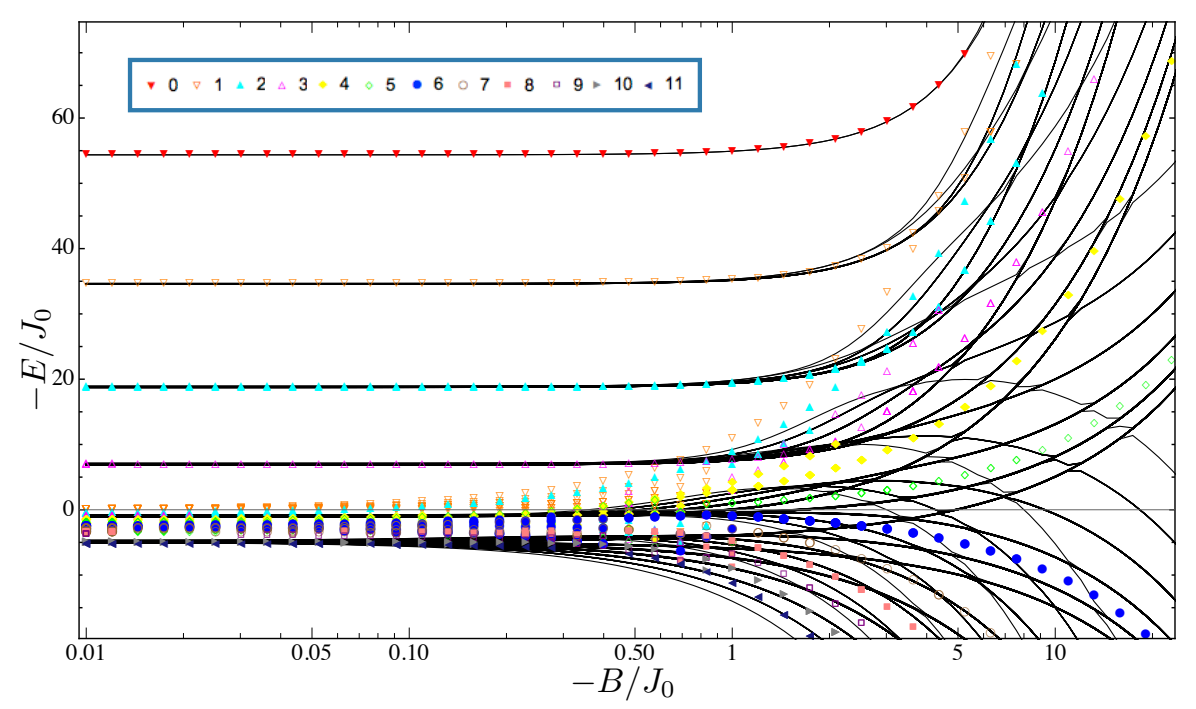

FigURE 5.9.: The saddle-point search over the multidimensional semiclassical energy landscape (5.30) fails to describe the quantum mechanical energy levels (black lines) of very long-range spin chain models when $B \ll\left|J_{0}\right|$. The plot compares the critical points, labeled by their index $\lambda$ (see legend) to the spectrum of (5.1) for $N=11, \alpha=0.01, J_{0}<0$. This shortcoming can be compensated by a simplified ansatz, which puts physically motivated constraints on the hitherto independent spin variables $\phi_{i}$ (see section 5.4.4 and figure 5.11)

One may have expected an adequate description of the vicinity of the ground state in the limit of large $N$, for small $\alpha$, when we recover the Lipkin-Meshkov-Glick model with a well-known semiclassical approximation (Botet et al., 1982; Leyvraz and Heiss, 2005; Ribeiro et al., 2007). Counterintuitively, the semiclassical spectrum reflects the quantum spectrum particularly well when $\alpha \gg 1$, which includes the nearest-neighbor Ising model. However, we are not able to reproduce the (anti-)ferromagnetic part of the quantum spectrum, when $\alpha \ll 1$. In the next section, we remedy this problem by introducing an even simpler ansatz, which allows for an analytical optimization.

\subsubsection{Analytical critical points from a set of single-parameter energy landscapes}

In the previous section, we allowed the spins in the chain to independently adjust the orientation of their local pure state. In this section, we impose physically motivated constraints on the relative orientation of the spins across the chain, which reduces the dimensionality of the energy manifold.

\subsubsection{Ground state}

We first focus on the ferromagnetic case, $J_{0}>0$, to construct a physically motivated trial function for the ground state. Since the ferromagnetic interaction favors 

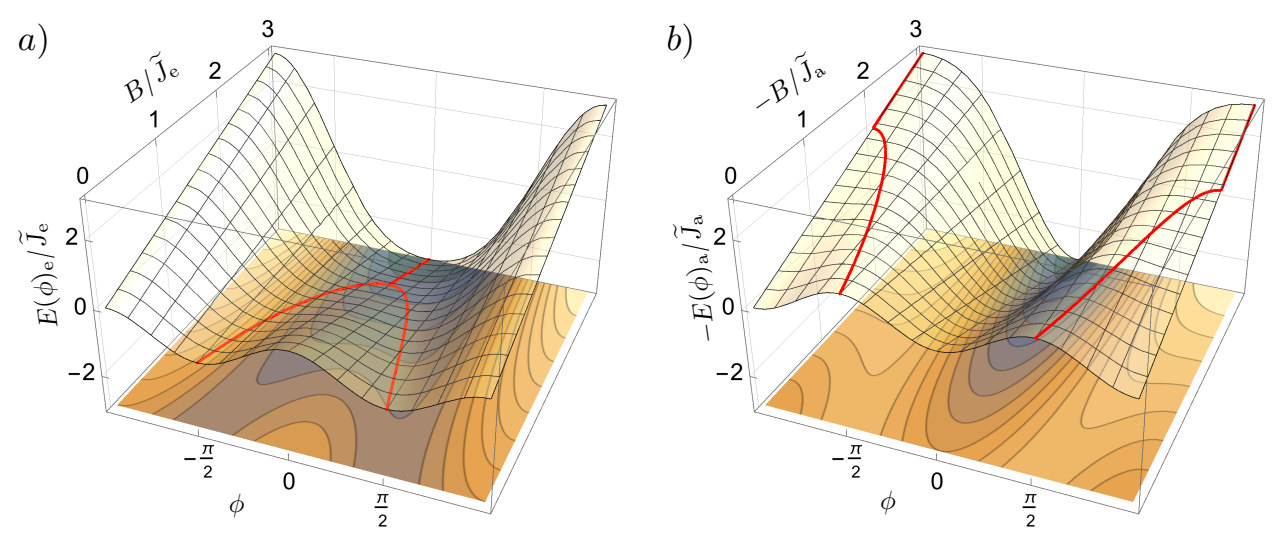

FiguRE 5.10.: a) The semiclassical energy manifold $E_{\mathrm{e}}(\phi)$, equation (5.37), obtained from the equal mean-field ansatz shows a bifurcation when the magnetic field $B$ reaches its critical value $B_{\mathrm{e}}^{c}=2 \widetilde{J}_{\mathrm{e}}$, where $\widetilde{J}_{\mathrm{e}}>0$; see equation (5.42). Then, the minimum energy changes from quadratic to linear dependence on $B$. This provides a good approximation for the ground state energy of the ferromagnet. b) By maximizing $E_{\mathrm{a}}(\phi)$, equation (5.48), for the alternating mean-field, we estimate the energy of the highest excited state. Analogously, we observe a second order transition at $B_{\mathrm{a}}^{c}=-2 \widetilde{J}_{\mathrm{a}}$, with $\widetilde{J}_{\mathrm{a}}<0$; see equation (5.50). The coordinates $\phi=\pi$ and $\phi=-\pi$ are equivalent.

neighboring spins to align along the same direction, we impose the equal mean-field constraint

$$
\phi_{1}=\phi_{2}=\cdots=\phi_{N} \equiv \phi \in[-\pi, \pi] .
$$

This corresponds to a conventional spin coherent state, as defined in equation (5.24), which is constrained to the $x y$ plane. In this case the energy manifold (5.30) reduces to the one-dimensional function ${ }^{8}$

$$
E_{\mathrm{e}}(\phi)=-\widetilde{J}_{\mathrm{e}} \sin ^{2} \phi-B \cos \phi,
$$

with

$$
\widetilde{J}_{\mathrm{e}}=\frac{J_{0}}{N} \sum_{\substack{i, j=1 \\(i<j)}}^{N} \frac{1}{|i-j|^{\alpha}} .
$$

As in the previous section, $E_{\mathrm{e}}(\phi)$ is rescaled by $N$ and thus denotes the energy per site. We note that for ferromagnetic interactions, $J_{0}>0$, we obviously obtain $\widetilde{J}_{\mathrm{e}}>0$. Critical points are easily identified by the condition

$$
\frac{d E_{\mathrm{e}}(\phi)}{d \phi}=B \sin \phi-2 \widetilde{J}_{\mathrm{e}} \sin \phi \cos \phi \stackrel{!}{=} 0,
$$

\footnotetext{
${ }^{8}$ The subscript e stands for equal (mean-field).
} 
which always has at least the three solutions

$$
\phi^{(1)}=-\pi, \quad \phi^{(2)}=0, \quad \phi^{(3)}=\pi .
$$

If the condition $B \leq 2 \widetilde{J}_{\mathrm{e}}$ is met, we find two additional solutions

$$
\phi^{(4,5)}= \pm \arccos \frac{B}{2 \widetilde{J}_{\mathrm{e}}} .
$$

Thus, we find a bifurcation at a critical magnetic field of

$$
B_{\mathrm{e}}^{c}=2 \widetilde{J}_{\mathrm{e}}
$$

The corresponding critical values are

$$
E_{\mathrm{e}}\left(\phi^{(1)}\right)=E_{\mathrm{e}}\left(\phi^{(3)}\right)=B, \quad E_{\mathrm{e}}\left(\phi^{(2)}\right)=-B
$$

and

$$
E_{\mathrm{e}}\left(\phi^{(4)}\right)=E_{\mathrm{e}}\left(\phi^{(5)}\right)=-\widetilde{J}_{\mathrm{e}}-\frac{B^{2}}{4 \widetilde{J}_{\mathrm{e}}}
$$

Since we are trying to reproduce the ground state energy, we look for the global minimum. We find

$$
E_{\mathrm{e}}^{\min }(B)= \begin{cases}-\widetilde{J}_{\mathrm{e}}-B^{2} /\left(4 \widetilde{J}_{\mathrm{e}}\right), & B \leq B_{\mathrm{e}}^{c} \\ -B, & B>B_{\mathrm{e}}^{c}\end{cases}
$$

The energy landscapes and their minima are shown in figure 5.10 a) for different $B$.

The transition from quadratic to linear dependence on $B$ implies a jump in the second derivative of the ground state energy at the bifurcation point, which identifies the second-order quantum phase transition. The two degenerate minima nicely illustrate the spontaneous breaking of symmetry, which happens in the semiclassical picture when the system relaxes into one of the two degenerate solutions $\phi^{(4,5)}$ in the ferromagnetic phase (Wen, 2004). The symmetry of the paramagnetic phase is also represented in the semiclassical picture since the absolute minimum is always found at the center, $\phi^{(2)}=0$.

For ferromagnetic systems, $J_{0}>0$, the analytical ground state energy $E_{\min }$ reproduces the numerical minimum of the multi-variable optimization of the previous section. However, if we maximize the function $E(\phi)$, we obtain $E_{\max }=B$, which matches the true spectrum for $B \gg J_{0}$, but does not describe the behavior for $B \ll J_{0}$. This is quite intuitive, since the energy of the ferromagnetic interaction term is maximal when neighboring spins are oriented in opposite directions, which cannot be achieved by the equal mean-field ansatz. 


\subsubsection{Highest excited state}

Thus we ask, is it possible to modify the equal mean-field ansatz (5.36) to reproduce other features of the spectrum? To recover the highest excited state deep inside the ferromagnetic regime, we impose the alternating mean-field condition

$$
\phi_{i}=(-1)^{i} \phi, \quad i=1, \ldots, N .
$$

We expect this arrangement of spins to be the energetically least favorable for ferromagnetic interaction. Furthermore, one can see that this ansatz does not change the contribution of the paramagnetic term in (5.30), since the cosine is symmetric. Intuitively, this can be attributed to the transverse orientation of the external field: The field is oriented along the $y$ direction, while the natural spin-spin interaction is determined on the basis of the spin's orientation along $x$. The resulting one-dimensional energy manifold has the same form as equation (5.37), where $\widetilde{J}_{\mathrm{e}}$ is replaced by ${ }^{9}$

$$
\widetilde{J}_{\mathrm{a}}=\frac{J_{0}}{N} \sum_{\substack{i, j=1 \\(i<j)}}^{N} \frac{(-1)^{i+j}}{|i-j|^{\alpha}} .
$$

We analyze this expression later in section 5.4.7. For now it is sufficient to know that $\widetilde{J}_{\mathrm{a}}<0$ when $J_{0}>0$, and, thus, the maxima of

$$
E_{\mathrm{a}}(\phi)=-\widetilde{J}_{\mathrm{a}} \sin ^{2} \phi-B \cos \phi
$$

are given by

$$
E_{\mathrm{a}}^{\max }(B)= \begin{cases}-\widetilde{J}_{\mathrm{a}}-B^{2} /\left(4 \widetilde{J}_{\mathrm{a}}\right), & B \leq B_{\mathrm{a}}^{c} \\ B, & B>B_{\mathrm{a}}^{c}\end{cases}
$$

Strikingly, we again can observe a second-order transition-this time at the critical magnetic field strength

$$
B_{\mathrm{a}}^{c}=-2 \widetilde{J}_{\mathrm{a}}
$$

see figure $5.10 \mathrm{~b}$ ). We will see soon that this semiclassical description represents an adequate approximation of the highest excited energy eigenvalue. Before doing so, we generalize this ansatz even further.

\subsubsection{Generalization for arbitrary states}

In the preceding two sections, we have recovered the energy of the ground state and of the highest excited state of a ferromagnetic spin chain from a semiclassical approach of spin coherent states. Can we further modify this ansatz to reproduce also intermediate energy levels? We develop such a general ansatz, by considering first on the two limiting

\footnotetext{
${ }^{9}$ The subscript a stands for alternating (mean-field).
} 
cases: the perfect paramagnet and ferromagnet. For example, when $B \ll J_{0}$, we have already seen that by flipping half of the spins against the mean field $\phi$,

$$
\phi_{i}=-\phi,
$$

we can transform the ansatz for the ground state to an ansatz for the highest excited state. If none of the spins are flipped [equation (5.36)], the ferromagnetic ground state energy is extracted by minimizing the resulting energy manifold, whereas if we flip every second spin [equation (5.46)], we obtain the highest excited state by maximizing.

To extract the values of intermediate energy levels, we start from the equal meanfield ansatz and flip a subset of $k \leq N / 2$ spins $(k \in \mathbb{N})$. We then proceed as before and study the resulting one-dimensional energy manifold, obtained from inserting the ansatz into equation (5.30). By flipping more than half of the spins we do not generate new energy manifolds since the replacement of $\phi \rightarrow-\phi$ would yield an equivalent manifold with less than half the spins flipped. The number and position of the spin flips govern the resulting effective spin-spin coupling energy

$$
\widetilde{J}_{\mu}=\frac{J_{0}}{N} \sum_{\substack{i, j=1 \\(i<j)}}^{N} \frac{\operatorname{sgn}\left(\phi_{i}^{\mu} / \phi\right) \operatorname{sgn}\left(\phi_{j}^{\mu} / \phi\right)}{|i-j|^{\alpha}},
$$

where the configuration is defined by the vector $\phi^{\mu}=\left(\phi_{1}^{\mu}, \ldots, \phi_{N}^{\mu}\right)$ with $\phi_{i}^{\mu}= \pm \phi$. For finite values of $\alpha$, the distance-dependent denominator can trigger a strong dependence of $\widetilde{J}_{\epsilon}$ on, not only the number of flips, but also their relative orientation.

These local spin flips are able to modify the energy deep inside the ferromagnetic regime. However, they have no influence on the potential energy generated by the transverse magnetic field. Thus, we now turn our attention to the paramagnetic regime. For $B \gg J_{0}$, the ground state will be a product state where all spins align along the direction of the external magnetic field. Conversely, if all spins are shifted $180^{\circ}$ with respect to the external field direction, the energy is maximal. The intermediate levels are determined by the number of spins which are shifted. In the semiclassical model, such local spin shifts are described by

$$
\phi_{i}=\phi+\pi .
$$

Indeed, due to $\cos (\phi+\pi)=-\cos (\phi)$, we see that the paramagnetic energy changes, depending on the number of shifted spins. If $s$ spins experience a shift, we obtain

$$
\frac{B}{N} \sum_{i=1}^{N} \cos \phi_{i}=B \frac{N-2 s}{N} \cos \phi .
$$

In general, given a configuration $\phi^{\mu}$, with all elements satisfying either $\phi_{i}^{\mu}= \pm \phi$ or $\phi_{i}^{\mu}= \pm \phi+\pi$, we define

$$
\widetilde{B}_{\mu}=B \frac{N-2 s^{\mu}}{N}
$$


where

$$
s^{\mu}=\#\left\{i=1, \ldots, N \text { which satisfy }\left.\phi_{i}^{\mu}\right|_{\phi=0} \neq 0\right\}
$$

denotes the number of shifted spins. This means, by adjusting the number of shifted spins, we can scan through all of the $N+1$ paramagnetic bands. We consider at most $s_{\max }^{\mu}=N / 2, s_{\max }^{\mu} \in \mathbb{N}$ shifted spins, since we obtain the negative-energy part of the paramagnetic spectrum by minimizing the semiclassical energy manifold, and the positive sector by maximizing. This finally yields the values

$$
\widetilde{B}_{\mu}=B, B \frac{N-2}{N}, B \frac{N-4}{N}, \ldots, \widetilde{B}_{\min },
$$

with $\widetilde{B}_{\min }=0$ if $N$ is even and $\widetilde{B}_{\min }=B / N$ if $N$ is odd.

How do such spin flips modify the ferromagnetic interaction? Since $\sin (\phi+\pi)=$ $-\sin (\phi)$, a shift will affect the spin-spin interaction energy. This effect, however, can be compensated by combining the spin shift with a spin flip,

$$
\phi_{i}=-\phi+\pi .
$$

We modify equation (5.52) to take possible shifts into account:

$$
\widetilde{J}_{\mu}=\frac{J_{0}}{N} \sum_{\substack{i, j=1 \\(i<j)}}^{N} \frac{\epsilon_{i}^{\mu} \epsilon_{j}^{\mu}}{|i-j|^{\alpha}},
$$

with

$$
\epsilon_{i}^{\mu}=\left\{\begin{array}{ll}
+1, & \phi_{i}^{\mu}=\phi \quad \text { or } \quad \phi_{i}^{\mu}=-\phi+\pi \\
-1, & \phi_{i}^{\mu}=-\phi \quad \text { or } \quad \phi_{i}^{\mu}=\phi+\pi
\end{array} .\right.
$$

Hence, combinations of the two operations, shifts and flips, allow us to independently tune the effective spin-spin coupling $\widetilde{J}_{\mu}$ and the effective magnetic field $\widetilde{B}_{\mu}$. This means that for every combination of possible values for $\widetilde{J}_{\mu}$ and $\widetilde{B}_{\mu}$, we can design a one-parameter spin configuration $\phi^{\mu}$, which would produce this scenario.

In conclusion, appropriate modifications of the equal mean-field ansatz can generate a family of single-parameter energy manifolds of the type

$$
E_{\mu}(\phi)=-\widetilde{J}_{\mu} \sin ^{2} \phi-\widetilde{B}_{\mu} \cos \phi .
$$

While the $\widetilde{B}_{\mu}$ are strictly positive, the $\widetilde{J}_{\mu}$ can either be positive or negative. These two cases effectively reduce to the expressions which were already discussed for the ground state and the highest excited state (see figure 5.10). If $\widetilde{J}_{\mu}>0$, we analytically minimize the energy $E_{\mu}(\phi)$, yielding a semiclassical energy level

$$
E_{\mu}^{\min }(B)=\left\{\begin{array}{ll}
-\widetilde{J}_{\mu}-\widetilde{B}_{\mu}^{2} /\left(4 \widetilde{J}_{\mu}\right), & \widetilde{B}_{\mu} \leq 2 \widetilde{J}_{\mu} \\
-\widetilde{B}_{\mu}, & \widetilde{B}_{\mu}>2 \widetilde{J}_{\mu}
\end{array},\right.
$$



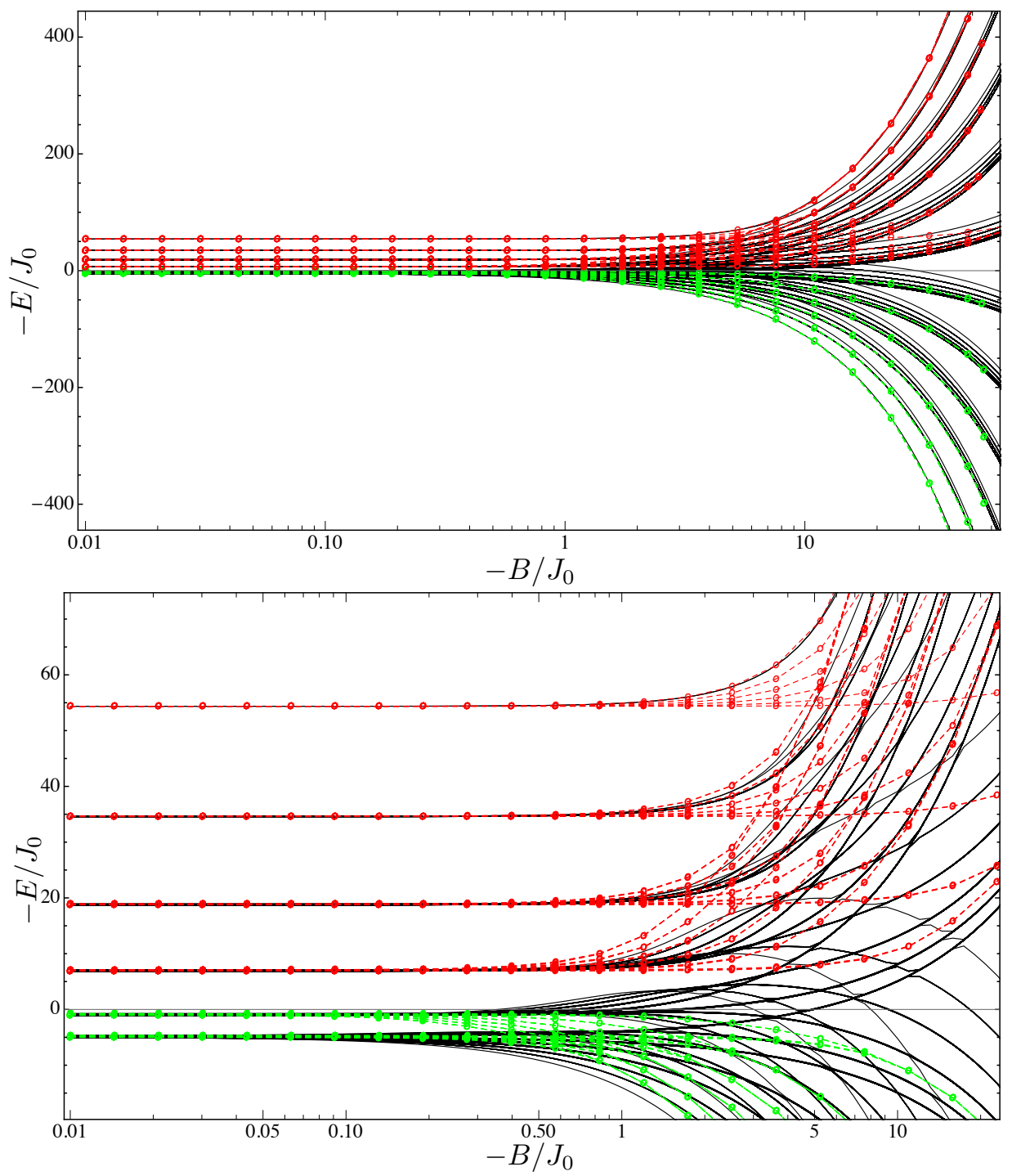

FIGURE 5.11.: Quantum spectrum (black lines) of (5.1) and maxima (minima) of the onedimensional semiclassical energy manifolds $E_{\mu}(\phi)$, given in (5.61), [red (green) dots and dashed lines] for an anti-ferromagnetic system $\left(J_{0}<0\right)$ of $N=11$ spins with $\alpha=0.01$. For $B \gg\left|J_{0}\right|$ and $B \ll\left|J_{0}\right|$, the quantum spectrum is well reproduced by the semiclassical approximation. Despite significantly simplifying the multi-dimensional ansatz, the level scheme is much better represented (compare to figure 5.9), in particular in the anti-ferromagnetic parameter regime. Still, deviations are observed around the critical field $B \sim\left|J_{0}\right|$. 
where $\widetilde{B}_{\mu}$ depends on $B$ via equation (5.55). We further find semiclassical energy levels for $\widetilde{J}_{\mu}<0$ by analytically maximizing the energy manifold $E_{\mu}(\phi)$, with the result

$$
E_{\mu}^{\max }(B)= \begin{cases}-\widetilde{J}_{\mu}-\widetilde{B}_{\mu}^{2} /\left(4 \widetilde{J}_{\mu}\right), & \widetilde{B}_{\mu} \leq-2 \widetilde{J}_{\mu} \\ \widetilde{B}_{\mu}, & \widetilde{B}_{\mu}>-2 \widetilde{J}_{\mu}\end{cases}
$$

The results of the two preceding sections, that is, ground state and highest excited state for $J_{0}>0$, are recovered using the equal mean-field ansatz $\phi_{i}^{\mathrm{e}}=\phi$ or the alternating mean-field ansatz $\phi_{i}^{\mathrm{a}}=(-1)^{i} \phi, i=1, \ldots, N$, respectively.

We emphasize that the generalized approach can also be applied to anti-ferromagnetic interactions, $J_{0}<0$. In this case, we will find that the alternating mean-field ansatz actually yields a positive value for $\widetilde{J}_{\mathrm{a}}$, which will minimize the energy, while the equal mean-field is energetically the least favorable and generates the highest excited state.

By imposing physically motivated constraints on the spin orientation, we have reduced the high-dimensional optimization problem of section 5.4.3 to a simple, onedimensional problem, which can be treated analytically. Remarkably, the simplified ansatz also significantly improves the performance of the semiclassical approximation. The resulting semiclassical spectrum reproduces all of the paramagnetic bands, as well as all of the energy levels in the (anti-)ferromagnetic regime, independently of the parameter $\alpha$. This is displayed for in Figs. 5.11 and 5.12. There are essentially two quantum features, which are not reflected by this simple family of energy levels:

(i) The finite width of energy bands. These are generated in the paramagnetic regime by residual influence of the (anti-)ferromagnetic interaction term, when $B$ is not sufficiently large compared to $J_{0}$. The semiclassical energy does not take this into account, since the maximal and minimal energy do no longer depend on $J_{0}$ once the external field exceeds the value $B^{c}$. Similarly, when the energy levels are mostly determined by the spin-spin coupling $J_{0}$, nonzero values of $B$ lead to a broadening of the lines.

(ii) Avoided crossings. We see that, by construction, we can generate a semiclassical energy line which continuously moves from any given (anti-)ferromagnetic coupling energy to any given paramagnetic band with the same sign. In the actual quantum spectrum, such lines are often bent by strong couplings to other states.

Hence, we can interpret the semiclassical energy levels as the structured backbone of the full quantum spectrum. In the next sections we try to quantify the performance of this approximation, and show that in some cases we actually recover exact quantum mechanical results. Moreover, (almost) each of the parametrized semiclassical energy levels can be assigned a critical magnetic field $B^{c}$, at which the dependence on $B$ changes from quadratic to linear, implying a jump of the second derivative. The distribution of these critical fields will be discussed in section 5.4.7.

\subsubsection{Performance of the semiclassical ansatz}

We now proceed towards a quantitative comparison between the true quantum spectrum and the semiclassical prediction. First, we focus on special cases where we can 

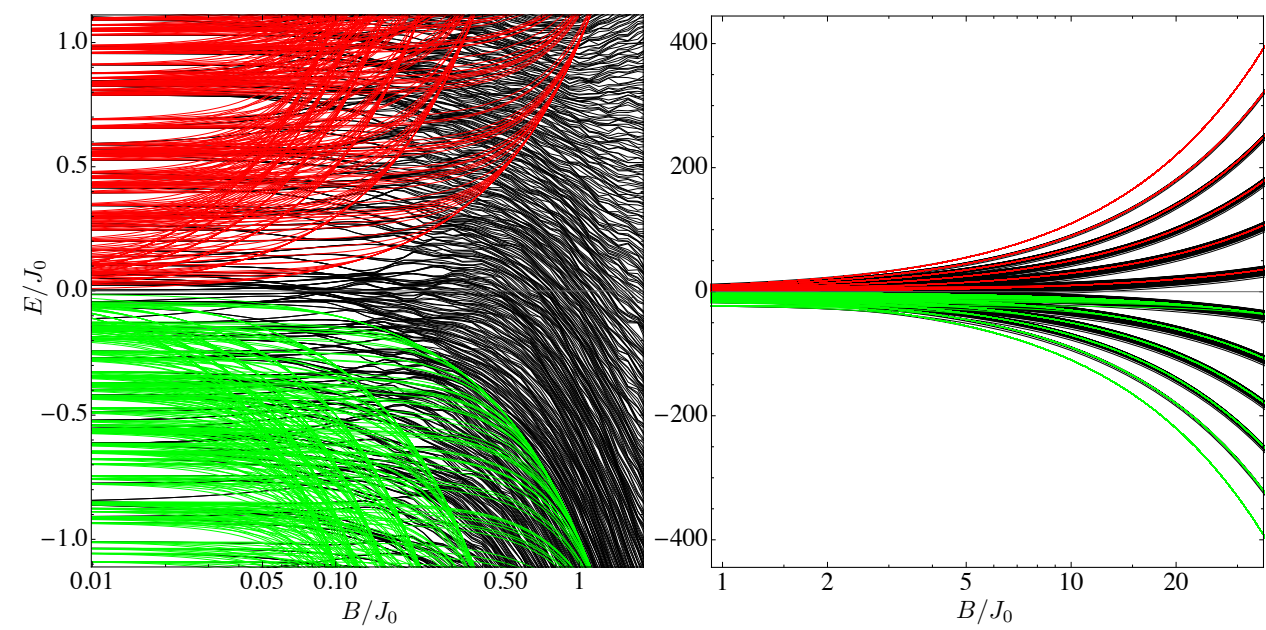

FIGURE 5.12.: The semiclassical energy levels (green and red lines) coincide with the exact quantum spectrum (black lines) for $B \ll J_{0}$ and $B \gg J_{0}$. This is plotted here for a ferromagnetic system $\left(J_{0}>0\right)$ of $N=11$ spins with intermediate-range interactions, $\alpha=1$, which leads to a broadly distributed ferromagnetic spectrum at $B=0$. Around the critical field $B \sim\left|J_{0}\right|$, competing interactions lead to an abundance of avoided crossings which cannot be captured by the semiclassical approach.

establish analytically that the semiclassical spectrum is exact. Then, in the subsequent section, we analyze the deviation to the exact ground state energy as a function of the system parameters.

\subsubsection{Exact results}

From Figs. 5.11 and 5.12 we observe the striking agreement of the semiclassical prediction with the quantum spectrum in the limiting cases $B \gg\left|J_{0}\right|$ and $B \ll\left|J_{0}\right|$. It is crucial to recall equations (5.62) and (5.63) to realize that the sets of admissible values for $\widetilde{J}_{\mu}$ and $\widetilde{B}_{\mu}$ precisely determine the entire semiclassical spectrum in the two extreme cases, when $J_{0}=0$ and $B=0$, respectively.

For example, for $J_{0}=0$, the (semiclassically predicted) paramagnetic energy levels ${ }^{10}$ are given by the $N+1$ possible values of

$$
\mathrm{E}_{\mu}= \pm N \widetilde{B}_{\mu}=-N B,-B(N-2), \ldots,+B(N-2),+N B
$$

where the positive (negative) solutions corresponds to $N E_{\mu}^{\max }\left(N E_{\mu}^{\min }\right)$, and the possible values for $\widetilde{B}_{\mu}$ were given in equation (5.57). It is a straight-forward task to

\footnotetext{
${ }^{10}$ We introduce the sans-serif letter to distinguish the total energy $\mathrm{E}$ from the energy per site $E=\mathrm{E} / \mathrm{N}$.
} 
determine the exact quantum spectrum of the paramagnetic Hamiltonian

$$
H_{\mathrm{p}}=\left.H\right|_{J_{0}=0}=-B \sum_{i=1}^{N} \sigma_{i}^{(y)} .
$$

This Hamiltonian immediately reminds us of the spin coherent states, as introduced in section 5.4.2, and is equivalent to the Hamiltonian of non-interacting two-level atoms, as discussed in sections 4.3.2 and 4.4: We have $H_{\mathrm{p}}=-2 B S_{y}$ [see equation (5.8)], where the spectra of all three components of the total angular momentum coincide. Thus, the spectrum of $S_{y}$ is also given by $-N / 2,-N / 2+1, \ldots, N / 2-1, N / 2$, where the different energy levels can be labeled by the number of spins which are oriented against the direction of the external field. This implies that the spectrum of $H_{\mathrm{p}}$ is exactly given by equation (5.64). Hence, independently of $\alpha$ and $N$, the semiclassical energy levels always recover the exact quantum spectrum in the limit $B \gg\left|J_{0}\right|$.

In the opposite limit, when $B=0$, we see from equations (5.62) and (5.63) that the total energy is entirely determined by the set of possible values for $\widetilde{J}_{\mu}$ :

$$
\mathrm{E}_{\mu}=-N \widetilde{J}_{\mu}
$$

Can we predict the distribution of the $\widetilde{J}_{\mu}$ ? Let us first consider $\alpha=0$. In this case we find the following set of effective spin-spin couplings from (5.59):

$$
\begin{aligned}
\left.\widetilde{J}_{\mu}\right|_{\alpha=0} & =\frac{J_{0}}{2 N} \sum_{\substack{i, j=1 \\
i \neq j}}^{N} \epsilon_{i}^{\mu} \epsilon_{j}^{\mu}=\frac{J_{0}}{2 N}\left[\left(\sum_{i=1}^{N} \epsilon_{i}^{\mu}\right)^{2}-\sum_{i=1}^{N}\left(\epsilon_{i}^{\mu}\right)^{2}\right] \\
& =\frac{J_{0}}{2 N}\left[\left(N-2 l^{\mu}\right)^{2}-N\right], \quad l^{\mu}=0, \ldots, N
\end{aligned}
$$

Here, $l^{\mu}$ denotes the number of negative $\epsilon_{i}^{\mu}$ in a given configuration $\phi^{\mu}$, which can be any number between 0 and $N$. There are however only $\lceil(N+1) / 2\rceil$ different solutions, which reflects a two-fold degeneracy for each solution. Here $\lceil x\rceil$ denotes the smallest integer $\geq x$. Quantum mechanically, this case corresponds to the Lipkin-MeshkovGlick model (Lipkin et al., 1965; Meshkov et al., 1965; Glick et al., 1965) without external field; see equation (5.9),

$$
H_{\mathrm{LMG}_{0}}=\left.H\right|_{B=0, \alpha=0}=-2 J_{0} S_{x}^{2}+\frac{J_{0}}{2} N \mathbb{I} .
$$

This Hamiltonian's spectrum is directly related to the paramagnetic spectrum, which was described below equation (5.65). Inserting this result immediately yields the energy values $-2 J_{0}(N / 2-k)^{2}+J_{0} N / 2$, for $k=0, \ldots, N$. These coincide exactly with the semiclassical results provided by equations (5.66) and (5.67). We also recognize the same two-fold degeneracy caused by the invariance of the squared expression to changes of the sign of the argument. 
Second, in the limit $\alpha \rightarrow \infty$, the $\widetilde{J}_{\mu}$ yield the following equally spaced sequence:

$$
\lim _{\alpha \rightarrow \infty} \widetilde{J}_{\mu}=\frac{J_{0}}{N} \sum_{i=1}^{N-1} \epsilon_{i}^{\mu} \epsilon_{i+1}^{\mu}=J_{0} \frac{N-1-2 k^{\mu}}{N}, \quad k^{\mu}=0, \ldots, N-1 .
$$

Here $k^{\mu}$ labels the number of nearest-neighbor pairs which have opposite orientations and thus produce $\epsilon_{i}^{\mu} \epsilon_{i+1}^{\mu}=-1$. The corresponding quantum mechanical Hamiltonian is given by the Ising model (Ising, 1925) in absence of an external field,

$$
H_{\mathrm{m}}^{0}=\left.\lim _{\alpha \rightarrow \infty} H\right|_{B=0}=-J_{0} \sum_{i=1}^{N-1} \sigma_{x}^{(i)} \sigma_{x}^{(i+1)} .
$$

This is a special case of the transverse-field Ising model (5.7), as well as of the anisotropic $X Y$-model (Lieb et al., 1961). Both can be solved generally by using a Jordan-Wigner transformation (Jordan and Wigner, 1928; Lieb et al., 1961). For the present special case, however, we can immediately diagonalize the Hamiltonian by introducing the states

$$
\left|\phi^{\mu}\right\rangle=\left|\epsilon_{1}^{\mu}, \ldots, \epsilon_{N}^{\mu}\right\rangle,
$$

where $\left|\epsilon_{i}^{\mu}\right\rangle$ denote eigenstates of $\sigma_{x}^{(i)}$ :

$$
\sigma_{x}^{(i)}\left|\epsilon_{i}^{\mu}\right\rangle=\epsilon_{i}^{\mu}\left|\epsilon_{i}^{\mu}\right\rangle
$$

with $\epsilon_{i}^{\mu}= \pm 1$. Hence, the energy eigenvalue equation becomes

$$
\begin{aligned}
H_{\mathrm{m}}^{0}\left|\phi^{\mu}\right\rangle & =-J_{0} \sum_{i=1}^{N-1} \sigma_{x}^{(i)} \sigma_{x}^{(i+1)}\left|\epsilon_{1}^{\mu}, \ldots, \epsilon_{N}^{\mu}\right\rangle \\
& =-J_{0} \sum_{i=1}^{N-1} \epsilon_{i}^{\mu} \epsilon_{i+1}^{\mu}\left|\epsilon_{1}^{\mu}, \ldots, \epsilon_{N}^{\mu}\right\rangle \\
& =-\lim _{\alpha \rightarrow \infty} \widetilde{J}_{\mu} N\left|\phi^{\mu}\right\rangle \\
& =\mathrm{E}_{\mu}\left|\phi^{\mu}\right\rangle .
\end{aligned}
$$

In the last step we have used equation (5.66) to show that the semiclassical spectrum exactly reproduces the quantum spectrum also in this case. In fact, in the quantum model, the number $k^{\mu}$ characterizes the number of domain walls; see, for example (Sachdev, 1999). In a ferromagnetic system, a domain refers to a collection of adjacent spins with the same orientation, and domain walls occur when two domains with different orientations meet, which connects to our interpretation of the semiclassical configurations. Each domain wall increases the energy by $2 J_{0}$ and there can be between 0 and $N-1$ domain walls, which labels all energy levels. For anti-ferromagnets, the same concept can be applied with the inverse argument; that is, a domain is formed by a collection of perfectly alternating spins, and excitations (domain walls) 


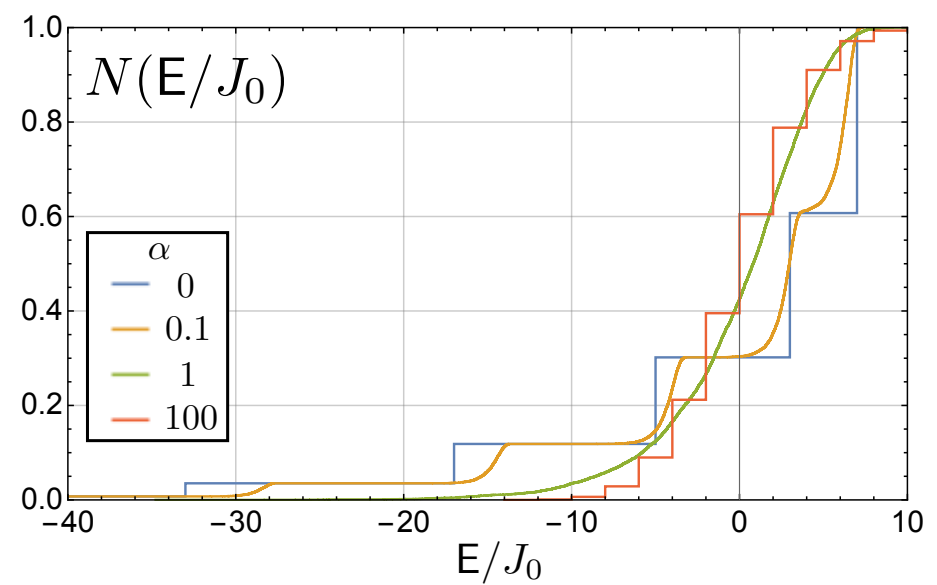

FIGURE 5.13.: Normalized counting function (5.15), the integrated density of states, for a ferromagnetic system $\left(J_{0}>0\right)$ without external field $(B=0)$, as introduced in (5.74), for various values of $\alpha$, and $N=15$. For $\alpha=0$, we obtain quadratic spacings between neighboring energy bands, while, for $\alpha \rightarrow \infty$, the spectrum becomes harmonic (see text). For intermediate values of $\alpha \sim 1$, the energy levels are broadly distributed, and all degeneracies are lifted. Compare also figure 5.17.

are introduced when neighboring spins have the same orientation. This implies that the transition from ferromagnet to anti-ferromagnet does not affect the spectrum of the nearest-neighbor Ising model, even though the eigenstates are ordered in reverse. From equation (5.69) we can see that the spectrum is symmetric around zero and consists of $N$ values. This is similar to the paramagnetic case, equation (5.64), where we had $N+1$ values, symmetric around zero. The resulting symmetry of the spectrum over the entire span of magnetic fields can be see nicely in the quantum spectrum displayed in figure 5.8.

The states $\left|\phi^{\mu}\right\rangle$ span an orthonormal basis. In fact, they also represent eigenstates of the magnetic Hamiltonian in the most general case for arbitrary couplings $J_{i j}$,

$$
H_{\mathrm{m}}^{0}=\left.H\right|_{B=0}=-\sum_{\substack{i, j=1 \\(i<j)}}^{N} J_{i j} \sigma_{x}^{(i)} \sigma_{x}^{(j)} .
$$

The eigenvalues are given by

$$
H_{\mathrm{m}}^{0}\left|\phi^{\mu}\right\rangle=-\sum_{\substack{i, j=1 \\ i<j)}}^{N} J_{i j} \epsilon_{i}^{\mu} \epsilon_{i}^{\mu}\left|\phi^{\mu}\right\rangle=\mathrm{E}_{\mu}\left|\phi^{\mu}\right\rangle .
$$

In the case of algebraically decaying couplings $J_{i j}=J_{0}|i-j|^{-\alpha}$, these correspond to the semiclassical energy values, as determined by the most general form of $\widetilde{J}_{\mu}$, 

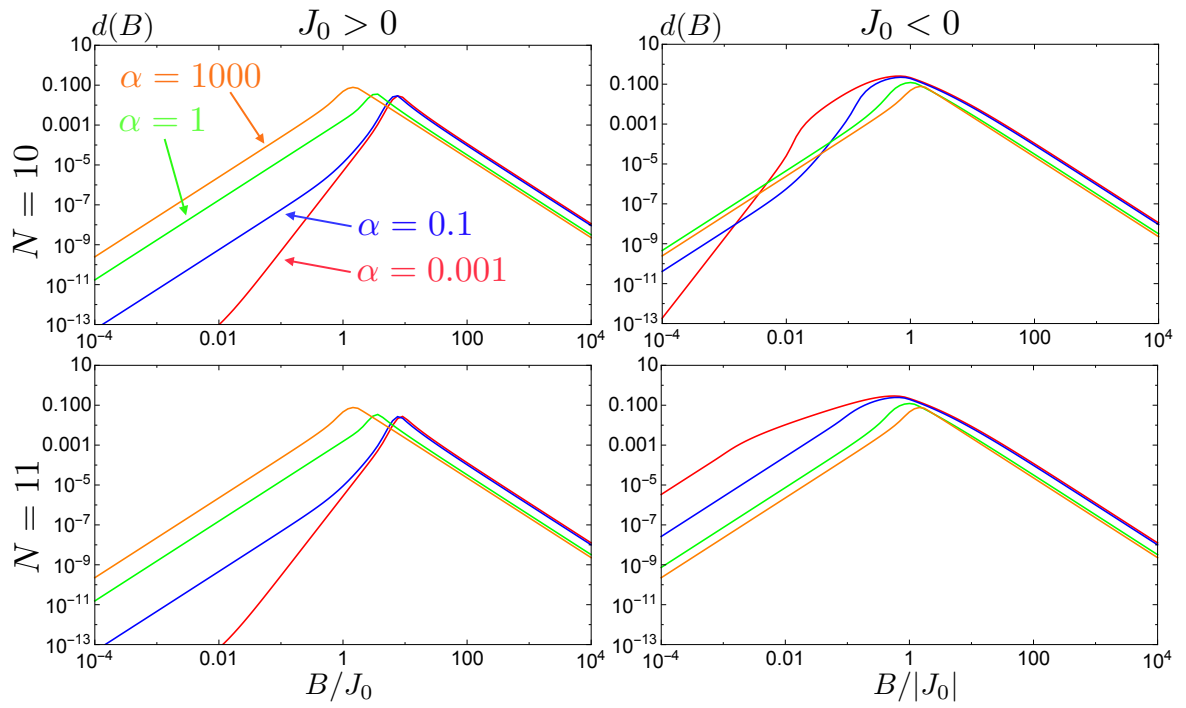

FiguRE 5.14.: Relative deviation $d(B)$, equation (5.76), between the semiclassical and numerically exact ground state energies, for $N=10$ and $N=11$ spins of both ferroand anti-ferromagnetic interactions of (5.1). The colors represent different interaction ranges, characterized by $\alpha=0.001$ (long-range; red), 0.1 (blue), 1.0 (green), and 1000 (short-range; orange).

for all values of $\alpha$, as given in equation (5.59). Notice that each eigenvalue is at least two-fold degenerate, due to the invariance under the inversion of the signs of all $\epsilon_{i}^{\mu}$. This reflects the Hamiltonian's symmetry under the operation $\sigma_{x} \rightarrow-\sigma_{x}$, which originates from the $Z_{2}$ symmetry of the full Hamiltonian $H$, see equation (5.4). Moreover, the chain is symmetric under a reflection with respect to its center, $i \rightarrow$ $N+1-i$. These two symmetries reduce the maximal number of different eigenvalues. For intermediate values of $\alpha \sim 1$, the resulting spectrum rarely contains degeneracies within the remaining levels, and its interpretation is not as easy as in the two special cases discussed above. The counting function ${ }^{11}$ is shown in figure 5.13 , for different values of $\alpha$. We can recognize the quadratic spacing between neighboring energy levels for $\alpha=0$, as well as the harmonic, equidistant spectrum for $\alpha \gg 1$. The smooth density of states at $\alpha=1$ was already observed in figure 5.5.

To summarize, we have analytically demonstrated that the semiclassical energy levels exactly reproduce the quantum mechanical spectrum in the limits $B \rightarrow \infty$ and $B=0$, for all values of $\alpha$.

\subsubsection{Deviation from the true ground state energy}

In order to quantify the approximation of the quantum spectrum, which is provided by the semiclassical energies for arbitrary intermediate values of $B$, we determine the

\footnotetext{
${ }^{11}$ In figure 5.13, this function is renormalized by dividing by the total number of states, $2^{N}$.
} 
deviation from the exact ground state energy. The latter can be determined exactly using numerical diagonalization, for moderate sizes of $N$. To compare the exact ground state energy $E_{0}(B)$ to the corresponding semiclassical value $E^{\min }(B)$, we introduce the relative deviation

$$
d(B)=\frac{E^{\min }(B)-E_{0}(B)}{\left|E_{0}(B)\right|} .
$$

Considering the ground state, our semiclassical approach can be interpreted as an instance of the Ritz variational principle, of which we know that the approximate ground state energy always represents an upper bound to the true value (Ritz, 1909; Cohen-Tannoudji et al., 1977b), rendering $d(B)$ a positive quantity.

Figure 5.14 shows the relative deviation for various values of $\alpha$ and different lengths of the spin chain. The largest deviation, as expected, is observed close to $B \simeq J_{0}$. For long-range ferromagnetic interactions, the peak moves to larger $B$ as we increase $N$, while for short-range ferromagnetic or arbitrary anti-ferromagnetic interactions, the position of the maximum deviation remains largely unaffected by $N$. This is in agreement with the position of the critical field that induces the quantum phase transition, as will be discussed in further detail in the next sections. Thus, the semiclassical approach works better the further we are away from the quantum phase transition, which confirms the intuition provided, for example, by figure 5.12; see also (Mayer et al., 2014) for a similar result for the Bose-Hubbard model. In fact, we know (recall section 5.4.5.1 above) that, if we increase or decrease $B$ far enough, the semiclassical approximation will become exact. Furthermore, the deviation, even at the critical field, is always small compared to the absolute ground state energy, and approaches zero quadratically as we proceed towards the exact solution of the paramagnetic bands. This is independent of $\alpha$, since the spin-spin coupling loses influence when $\left|J_{0}\right|$ becomes weak compared to $B$. In the opposite limit, the exact solution is approached quadratically, except for very long-range interactions (red lines), which tend towards the exact solution even faster, approximately following a quartic dependence on $B$. This may be due to the well-defined semiclassical limit of the infinite-range Lipkin-Meshkov-Glick model. In the anti-ferromagnetic case, we find strong dependence of $d(B)$ on $N$ when the interaction is long-range (red and blue lines). For odd $N$ we observe a quadratic approach to the exact solution, whereas we find that the deviation increases with the length of the interaction range, in contrast to what was observed in the ferromagnetic case. For even $N$, the long-range systems show a dramatic change in behavior when $B$ drops below a certain threshold value. Beyond this value, the deviation of longrange systems falls below that of short-range systems, and even recovers the quartic approach towards zero in the limit of very long range. Note also that the deviation from the ground state energy of the system corresponds to the deviation from the highest excited state of the same system when the sign of $J_{0}$ is inverted.

\subsubsection{Scaling of highest and lowest eigenvalues}

In section 5.4.5.1, we saw that the spectra of (5.1) at $B=0$ are determined by the $\widetilde{J}_{\mu}$, equation (5.59). The latter further determine the critical fields of all of the excited 

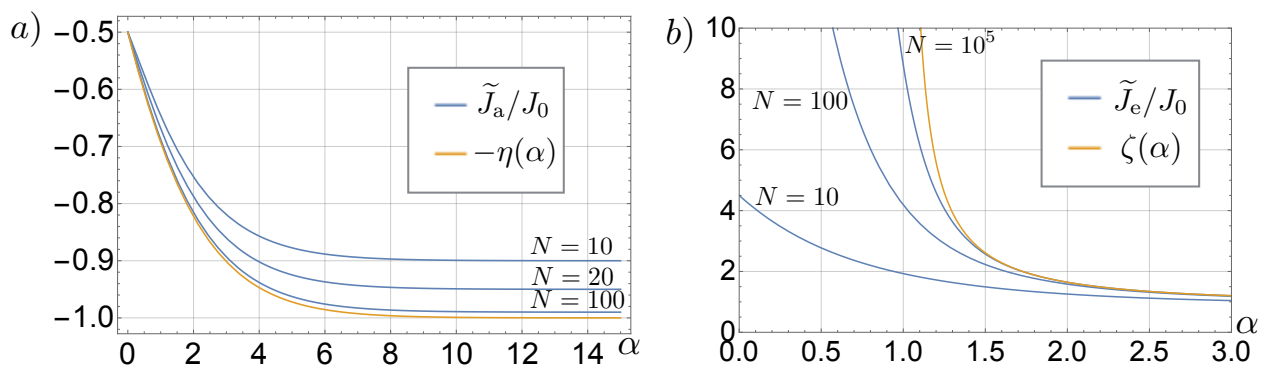

FIGURE 5.15.: a) For the alternating mean-field ansatz (5.46), describing the anti-ferromagnetic ground state, the effective coupling $\widetilde{J}_{\mathrm{a}}$, equation (5.78), converges to Dirichlet's eta function $\eta(\alpha)$. b) For the equal mean-field ansatz (5.36), which describes the ground state of a ferromagnetic system, the effective spin-spin coupling strength $\widetilde{J}_{\mathrm{e}}$, equation (5.83), converges to Riemann's zeta function $\zeta(\alpha)$ for $\alpha>1$ in the thermodynamic limit.

states via the condition $\widetilde{B}_{\mu}=2\left|\widetilde{J}_{\mu}\right|$, where the $\widetilde{B}_{\mu}$ always display a very regular distribution, see equation (5.57). All values of $\widetilde{J}_{\mu}$ can be bounded by those that lead to the ground state and the highest excited state. These, in turn, are given by the two special cases of the equal and alternating mean-fields, $\widetilde{J}_{\mathrm{e}}$ and $\widetilde{J}_{\mathrm{a}}$, respectively, as defined in equations (5.38) and (5.47). In this section, we analyze these upper and lower bounds of the (anti-)ferromagnetic spectrum and their behavior in the thermodynamic limit.

Let us start with $\widetilde{J}_{\mathrm{a}}$, whose sign is always opposite to the sign of $J_{0}$. We take a moment to analyze the expression (5.47), which turns out to be a mathematically interesting construction. First, we regroup the terms as a function of $|i-j|$,

$$
\frac{N}{J_{0}} \widetilde{J}_{\mathrm{a}}=\underbrace{-\sum_{i=1}^{N-1} \frac{1}{1^{\alpha}}}_{j=i+1} \underbrace{\sum_{i=1}^{N-2} \frac{1}{2^{\alpha}}}_{j=i+2} \underbrace{\sum_{i=1}^{N-3} \frac{1}{3^{\alpha}}}_{j=i+3}+\ldots,
$$

which leads to

$$
\widetilde{J}_{\mathrm{a}}=\frac{J_{0}}{N} \sum_{k=1}^{N}(-1)^{k} \frac{(N-k)}{k^{\alpha}} .
$$

From Leibnitz' alternating series test (Knopp, 1956) follows that this sum is convergent in the thermodynamic limit $(N \rightarrow \infty)$ for all values of $\alpha \geq 0$. We can further rearrange the terms to obtain

$$
\widetilde{J}_{\mathrm{a}}=J_{0} \sum_{k=1}^{N} \frac{(-1)^{k}}{k^{\alpha}}+\frac{J_{0}}{N} \sum_{k=1}^{N} \frac{(-1)^{k+1}}{k^{\alpha-1}} .
$$

For $\alpha>0$, the thermodynamic limit identifies the first term with Dirichlet's eta function, $\eta(x)$, which is defined as the analytic continuation of $\sum_{n=1}^{\infty}(-1)^{k+1} k^{-x}$ (Edwards, 1974; Titchmarsh, 1986), and sometimes called the alternating zeta function. 
We find numerically that ${ }^{12}$

$$
\frac{J_{0}}{N} \sum_{k=1}^{N} \frac{(-1)^{k+1}}{k^{\alpha-1}}=\mathcal{O}\left(N^{-\alpha}\right),
$$

which allows us to neglect the second term in the thermodynamic limit when $\alpha>0$. Thus, we obtain

$$
\lim _{N \rightarrow \infty} \widetilde{J}_{\mathrm{a}}=-J_{0} \eta(\alpha) .
$$

For $\alpha=0$, the sum (5.78) can be evaluated explicitly,

$$
\left.\widetilde{J}_{\mathrm{a}}\right|_{\alpha=0}=J_{0} \frac{1-(-1)^{N}}{4 N}-J_{0} \frac{1}{2} \stackrel{N \rightarrow \infty}{\longrightarrow}-J_{0} \frac{1}{2} .
$$

Since $\eta(0)=1 / 2$ (Titchmarsh, 1986), we can extend the relation (5.81) to include $\alpha=0$. The convergence of $\widetilde{J}_{\mathrm{a}} / J_{0}$ towards $-\eta(\alpha)$ is displayed in figure 5.15 a) as a function of $\alpha$ for different $N$.

After discussing the alternating mean-field ansatz, we now turn to the opposite limit, and analyze the effective coupling constant $\widetilde{J}_{\text {e }}$, equation $(5.38)$, which corresponds to the equal mean-field ansatz. This quantity has the same sign as $J_{0}$. An analogous rearrangement of terms as before leads to

$$
\begin{aligned}
\widetilde{J}_{\mathrm{e}} & =\frac{J_{0}}{N} \sum_{\substack{i, j=1 \\
(i<j)}}^{N} \frac{1}{|i-j|^{\alpha}} \\
& =\frac{J_{0}}{N} \sum_{k=1}^{N} \frac{N-k}{k^{\alpha}} \\
& =J_{0} \sum_{k=1}^{N} \frac{1}{k^{\alpha}}+\frac{J_{0}}{N} \sum_{k=1}^{N} \frac{1}{k^{\alpha-1}} .
\end{aligned}
$$

For $\alpha>1$, the first term converges in the thermodynamic limit, $N \rightarrow \infty$, and can be identified as Riemann's zeta function, $\zeta(\alpha)$, which is defined as the analytic continuation of the series $\sum_{k=1}^{\infty} k^{-\alpha}$ (Riemann, 1859; Edwards, 1974; Titchmarsh, 1986). Using the Euler-Maclaurin formula (Knopp, 1956), one can show for $\alpha<2$, that $\sum_{k=1}^{N} k^{1-\alpha} \sim \mathcal{O}\left(N^{2-\alpha}\right)$ (Graham et al., 1989; Cannas and Tamarit, 1996). Thus, for the second term we obtain for $2>\alpha>1$ :

$$
\frac{J_{0}}{N} \sum_{k=1}^{N} \frac{1}{k^{\alpha-1}} \sim \mathcal{O}\left(N^{1-\alpha}\right) \stackrel{N \rightarrow \infty}{\longrightarrow} 0 .
$$

\footnotetext{
${ }^{12}$ The asymptotic scaling $\sim N^{-\alpha}$ seems to coincide with the actual growth rate of the left-hand-side of equation (5.80), and is not just an upper bound as the $\mathcal{O}$ notation suggests. Note that, for small $\alpha$, this expression is still of the order of 1 for very large values of $N$, and approaches zero very slowly as $N$ increases. A formal proof may be achieved using the Euler-Maclaurin mapping of the sum to an integral expression (Knopp, 1956).
} 

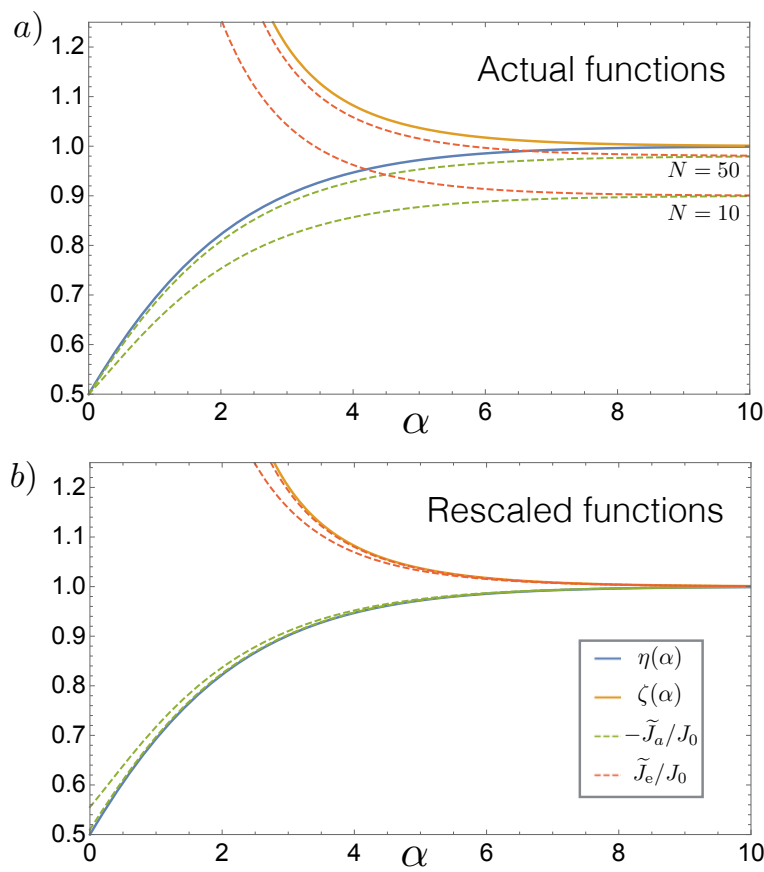

FigURE 5.16.: a) In the thermodynamic limit $(N \rightarrow \infty)$, the effective spin-spin coupling $\widetilde{J}_{\mathrm{a}}\left[\widetilde{J}_{\mathrm{e}}\right]$, defined in (5.78) [(5.83)], which reproduces the ground state energy if $J_{0}<0$ $\left[J_{0}>0\right]$, converges to $\eta(\alpha)[\zeta(\alpha)]$. The functions $\eta(\alpha)$ and $\zeta(\alpha)$ coincide for $\alpha \rightarrow \infty$, that is, in the limit of nearest-neighbor interactions. The figure further displays the finite-size effects $(N=10$ and $N=50$, dashed lines) on the effective couplings $\widetilde{J}_{\mathrm{a}}$ and $\widetilde{J}_{\mathrm{e}}$, respectively. b) These effects can be taken into account by rescaling these functions with the correction term $c_{N}=1-1 / N$. All rescaled effective spin-spin couplings $\widetilde{J}_{\mathrm{a}} /\left(J_{0} c_{N}\right)$ and $\widetilde{J}_{\mathrm{e}} /\left(J_{0} c_{N}\right)$ collapse onto the curve of the thermodynamic limit, except for small deviations at very small values of $\alpha$; see equations (5.88) and (5.89).

This term also vanishes for all values of $\alpha>2$, since in this case the sum converges to $\zeta(\alpha-1)$. While for $\alpha=1$, the sum diverges logarithmically, $\sum_{k=1}^{N} 1 / k \sim \mathcal{O}(\ln N)$, we can conclude for $\alpha>1$ that

$$
\lim _{N \rightarrow \infty} \widetilde{J}_{\mathrm{e}}=J_{0} \zeta(\alpha), \quad(\alpha>1)
$$

This can be observed graphically in figure $5.15 \mathrm{~b}$ ).

Let us briefly discuss the finite size effects in the case when this expression converges $(\alpha>1)$. The convergence in both cases, $\widetilde{J}_{\mathrm{e}}$ and $\widetilde{J}_{\mathrm{a}}$ is represented in a unified picture in figure 5.16. The discrepancy between finite- $N$ terms and the thermodynamic limit 


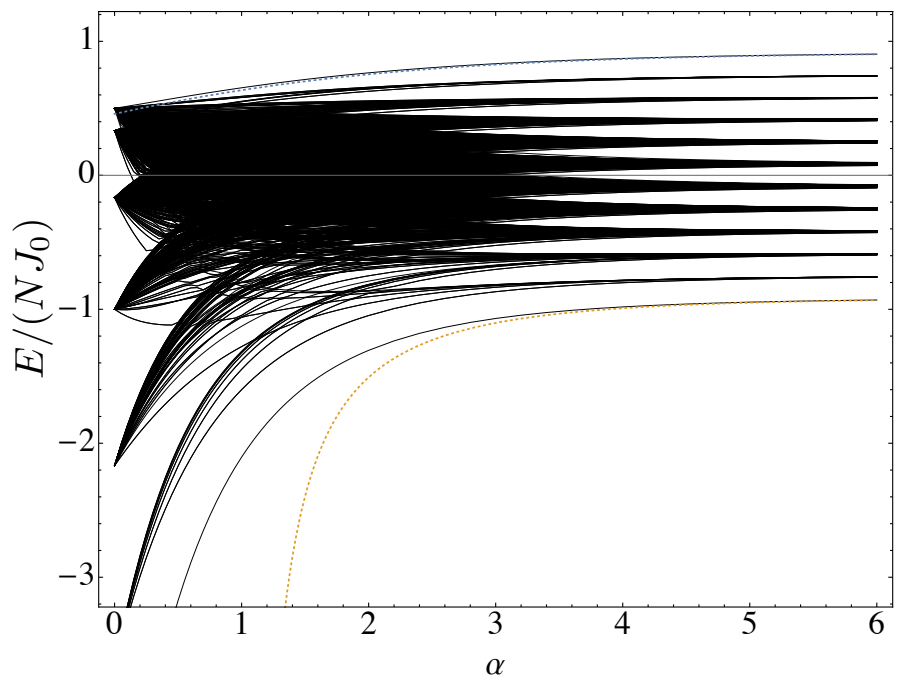

FiguRE 5.17.: The ferromagnetic $\left(J_{0}>0\right)$ spectrum of $(5.74)$ for $N=12$ as a function of $\alpha$ (black lines). In the thermodynamic limit, highest and lowest eigenvalues are given by the Riemann $\zeta$ and Dirichlet $\eta$ functions, respectively, which generally serve as upper and lower bounds of the spectrum. In the thermodynamic, limit the spectrum becomes unbounded from below (above, when $J_{0}<0$ ) for $\alpha \leq 1$. The figure shows the rescaled functions $-(1-1 / N) \zeta(\alpha)$ and $(1-1 / N) \eta(\alpha)$ (yellow and blue dotted lines), introduced in equations (5.88) and (5.89).

can be compensated by a scaling factor as

$$
\begin{aligned}
& \widetilde{J}_{\mathrm{e}} \simeq\left(1-\frac{1}{N}\right) J_{0} \zeta(\alpha), \quad(\alpha>1) \\
& \widetilde{J}_{\mathrm{a}} \simeq\left(1-\frac{1}{N}\right) J_{0} \eta(\alpha),
\end{aligned}
$$

which expresses the rate of convergence towards the thermodynamic limit in leading order of $N$. With this factor, we observe universal behavior already for moderate values of $N$; see figure 5.16. Moreover, since $\zeta(\alpha)$ and $\eta(\alpha)$ constitute upper and lower bounds on the spectra at $B=0$, for $N \rightarrow \infty$, we can predict the energy spread of the spectrum for quantum magnetic systems of finite $N$ with great accuracy using the above finite-size corrections; see figure 5.17. Specifically, for $J_{0}<0$ we have $-\eta(\alpha) \leq E_{k} / J_{0} \leq \zeta(\alpha)$, while for $J_{0}>0$, we have $-\zeta(\alpha) \leq E_{k} / J_{0} \leq \eta(\alpha)$, where $E_{k}=\mathrm{E}_{k} / N$ represents any arbitrary renormalized energy eigenvalue of $H_{\mathrm{m}}^{0}$.

Now let us come back to the case of $\widetilde{J}_{\mathrm{e}}$ when $\alpha \leq 1$. Using generalized Harmonic numbers $\mathrm{H}_{n}^{(m)}$ (Conway and Guy, 1998), $\widetilde{J}_{\mathrm{e}}$ can immediately be written as $\widetilde{J}_{\mathrm{e}}=J_{0}\left(\mathrm{H}_{N}^{(\alpha)}+\mathrm{H}_{N}^{(\alpha-1)} / N\right)$ for arbitrary $\alpha$. The thermodynamic limit of $\widetilde{J}_{\mathrm{e}}$, however, 
does not exist for $\alpha \leq 1$. For example, for $\alpha=1$ we obtain

$$
\left.\widetilde{J}_{\mathrm{e}}\right|_{\alpha=1}=\frac{J_{0}}{2}(N-1) \text {. }
$$

Hence, the energy per spin of (anti-)ferromagnetic systems is unbounded from below (above) whenever the interaction range decays too slowly as a function of distance, as specified by the condition $\alpha \leq 1$, see also figure 5.17. For this reason, one typically introduces a renormalized coupling constant $J_{0} \rightarrow J_{0} / N$ for long-range spin chain models (Botet et al., 1982; Ribeiro et al., 2007).

\subsubsection{Distribution of critical fields}

The semiclassical treatment generates a family of energy levels which approximate the quantum spectrum. As mentioned before, almost each of these levels can be assigned a critical magnetic field $B^{c}$, where a change from quadratic to linear dependence occurs and the second derivative jumps. To be precise, when $N$ is even, there always exists a family of levels which do not depend on $B$, and consequently do not have a critical magnetic field. The configurations $\phi^{\mu}$ which generate these levels satisfy $s^{\mu}=N / 2$, and therefore $\widetilde{B}_{\mu}=0$. These configurations are important to obtain the full spectrum in the limiting case $B \gg\left|J_{0}\right|$, but they cannot generate a nontrivial energy landscape of the type (5.61) as a function of $B$. The analogous situation (for $B \ll\left|J_{0}\right|$ ) arises when configurations with $\widetilde{J}_{\mu}=0$ exist. These rare configurations are excluded from the following analysis of critical fields. All other levels do show non-analytic behavior in the second derivative at some point.

\subsubsection{Ground state critical fields}

Let us first focus on the critical field associated with the ground state, since, in a conventional approach (Sachdev, 1999), this is the decisive quantity to pin-point the quantum phase transition in the thermodynamic limit. However, we have to be careful since our semiclassical mean-field ansatz is not guaranteed to deliver exact results in thermodynamic limit for all values of $\alpha$.

The bifurcation point of the semiclassical ground state is determined by $\widetilde{J}_{\mathrm{e}}$ when $J_{0}>0$, and by $\widetilde{J}_{\mathrm{a}}$ for $J_{0}<0$. To connect these with the quantum phase transition, we have to consider the thermodynamic limit, which was done in the detailed analysis of the previous section. Let us start with $J_{0}<0$, in which case the thermodynamic limit is always well defined. Recall from section 5.4.4.1 that the bifurcation point is defined by the condition $B_{\mathrm{a}}^{c}=2 \widetilde{J}_{\mathrm{a}}$. In the thermodynamic limit, we obtain, using equation (5.81),

$$
\lim _{N \rightarrow \infty} B_{\mathrm{a}}^{c}=-2 J_{0} \eta(\alpha) .
$$

For nearest-neighbor interactions $(\alpha \rightarrow \infty)$ the above expression yields the limit ${ }^{13}$ $\lim _{\alpha \rightarrow \infty} \lim _{N \rightarrow \infty} B_{\mathrm{a}}^{c}=-2 J_{0}$, which does not agree with the critical field of the quan-

\footnotetext{
${ }^{13}$ From equations (5.78) and (5.83), we see that the limits for $\alpha$ and $N$ commute for both $J_{0}>0$ and $J_{0}<0$.
} 
tum phase transition in the Ising model, which is found at $B=-J_{0}$. However, the semiclassically predicted critical point yields the correct order of magnitude and differs from the exact result only by a factor of 2 . In the opposite limit, we have seen that, in order to maintain a bounded spectrum in the thermodynamic limit, one must renormalize the spin-spin coupling constant by dividing it by $N$. Using these renormalized constants, the quantum phase transition is found at zero field, $B=0$ (Vidal et al., 2004). This is consistent with the semiclassical result $\lim _{\alpha \rightarrow 0} \lim _{N \rightarrow \infty} B_{\mathrm{a}}^{c}=-J_{0}$, which, in renormalized units approaches zero as $1 / N$.

In the ferromagnetic case, $J_{0}>0$, we obtain the bifurcation at

$$
\lim _{N \rightarrow \infty} B_{\mathrm{e}}^{c}=-2 J_{0} \zeta(\alpha)
$$

when $\alpha>1$. This converges towards the same critical field for the nearest-neighbor Ising model, $\lim _{\alpha \rightarrow \infty} \lim _{N \rightarrow \infty} B_{\mathrm{e}}^{c}=2 J_{0}$, which also deviates from the exact result by a factor of 2 . The agreement of nearest-neighbor ferro- and anti-ferromagnetic ground-state behavior is expected since both $\eta(\alpha)$ and $\zeta(\alpha)$ converge to 1 for $\alpha \rightarrow \infty$, albeit from different sides, see figure 5.16 a). To study the quantum phase transition in the opposite limit, we need to take the growth rate with $N$ into account, since the corresponding expressions do not converge in our units. For $\alpha=0$, we find that $\lim _{\alpha \rightarrow 0} B_{\mathrm{e}}^{c}=(3 N+1) J_{0}$. Translating back from rescaled units into our units, the non-analytic behavior of the ground state energy is found at $B=N J_{0}$ (Botet et al., 1982; Botet and Jullien, 1983), which again matches the order of magnitude, but does not exactly coincide with the semiclassical result.

\subsubsection{Statistical distribution of excited-state critical fields}

What can we say about the distribution of critical fields of intermediate levels? For each configuration $\phi^{\mu}$, according to equations (5.62) and (5.63) we find a critical field at

$$
\widetilde{B}_{\mu}^{c}=2\left|\widetilde{J}_{\mu}\right|
$$

from which we obtain, inserting equation (5.55) for $s^{\mu}<N / 2$ (as mentioned before, we exclude those families of states for which either $\widetilde{B}_{\mu}=0$ or $\widetilde{J}_{\mu}=0$ ):

$$
B_{\mu}^{c}=\frac{N}{N-2 s^{\mu}} 2\left|\widetilde{J}_{\mu}\right| .
$$

We can derive an upper bound for this quantity, by using $\left|\widetilde{J}_{\mu}\right| \leq\left|\widetilde{J}_{\mathrm{e}}\right|$,

$$
B_{\mu}^{c} \leq\left\{\begin{array}{ll}
N\left|\widetilde{J}_{\mathrm{e}}\right|, & N \text { even } \\
2 N\left|\widetilde{J}_{\mathrm{e}}\right|, & N \text { odd }
\end{array} .\right.
$$

This bound is indeed tight, since it is reached for the configuration $\mu_{0}$ that satisfies $\widetilde{J}_{\mu_{0}}=\widetilde{J}_{\mathrm{e}}$ and $s^{\mu_{0}}=\lfloor N / 2\rfloor$, where $\lfloor x\rfloor$ denotes the largest integer $\leq x$.

We conclude that the critical fields of intermediate levels are unbounded in the thermodynamic limit. The distribution of critical fields is determined by the distribution 
of the $\widetilde{J}_{\mu}$, and the $\widetilde{B}_{\mu}$. As a matter of fact, this precisely connects the spectra of the limiting cases of $B$, since the spectrum for $B=0$ is entirely determined by the $\widetilde{J}_{\mu}$, whereas for $B \rightarrow \infty$, it is entirely determined by $\widetilde{B}_{\mu}$. This was already pointed out in section 5.4.5.1, where it was also noted that, for $\alpha=\infty$ and $\alpha=0$, the $\widetilde{J}_{\mu}$ show very regular distributions, see figure 5.13 . Since the $\widetilde{B}_{\mu}$ merely label paramagnetic energy levels at $B \rightarrow \infty$, the resulting spectrum for $J_{0}=0$ is always harmonic. Furthermore, in the limits $B=0$ and $J_{0}=0$, the semiclassical prediction for the spectrum was shown to be exact. Since there exists a semiclassical energy level for each pair of $\widetilde{J}_{\mu}$ and $\widetilde{B}_{\mu}$ of the same sign, the critical fields associated with these exited-state levels will in general not fall onto the same spot. The ground state only represents one of the many realizations. Instead, we will encounter a broad distribution of critical fields generated by the entire semiclassical energy spectrum, which we analyze in the following.

Recall from section 5.4.4.3 that some of the critical fields correspond to minima of semiclassical energy landscapes, while others correspond to maxima. For the statistical analysis of the critical fields, we will treat those two cases separately. We consider those critical fields which stem from a maximum (these are always found at positive energies) as unstable critical points, while those which belong to a minimum are considered semiclassically stable (at negative energies); see also figure 5.10. Actually, the replacement $J_{0} \rightarrow-J_{0}$ leads to an inversion of the signs of all of the eigenvalues, since the paramagnetic part is always symmetric with respect to zero. Thus, the stable critical fields of the ferromagnetic system correspond to the unstable critical fields of the same system with anti-ferromagnetic couplings, and vice-versa. For the special cases of $\alpha=\infty$ and $\alpha=0$, we can make use of the analytic results, equations (5.67) and (5.69), derived in section 5.4.5.1, to efficiently generate statistical data of large many-body spin chain models.

Figure 5.18 shows the histograms of stable critical points on logarithmically spaced bins. Strikingly, this spacing allows us to identify clear maxima. In the case $\alpha=0$, we obtain a maximum at $B=\left|J_{0}\right|$ when $J_{0}<0$. This reflects the ground state bifurcation point as discussed before and agrees with the critical field in the thermodynamic limit in rescaled units. A clear power-law decay can be identified when $B$ is increased beyond the position of the maximum. Then, the distribution decays as $1 / B$.

In the case $J_{0}>0$, we have seen that the position of the ground state bifurcation point tended towards infinity with a slope of $3 N+1$, while we expect from the critical field of the quantum phase transition to do the same with a slope of $N$. The maximum of the corresponding histogram distribution reproduces exactly this behavior. This maximum is approached from both sides algebraically. Below the critical point, the maximum is approached as $\sqrt{B}$, whereas above the maximum the distribution, again, decays as $1 / B$.

Finally, for $\alpha=\infty$ the distribution does no longer depend on the sign of $J_{0}$. The maximum is always found at $B=2\left|J_{0}\right|$, which agrees with the ground state bifurcation point, but differs from the actual critical point by a factor of 2 . Here, the distribution proceeds towards the maximum as $B$ from below, and as $1 / B$ from above. Hence, in all cases we observe the $1 / B$-type decay above the maximum. This universality is 


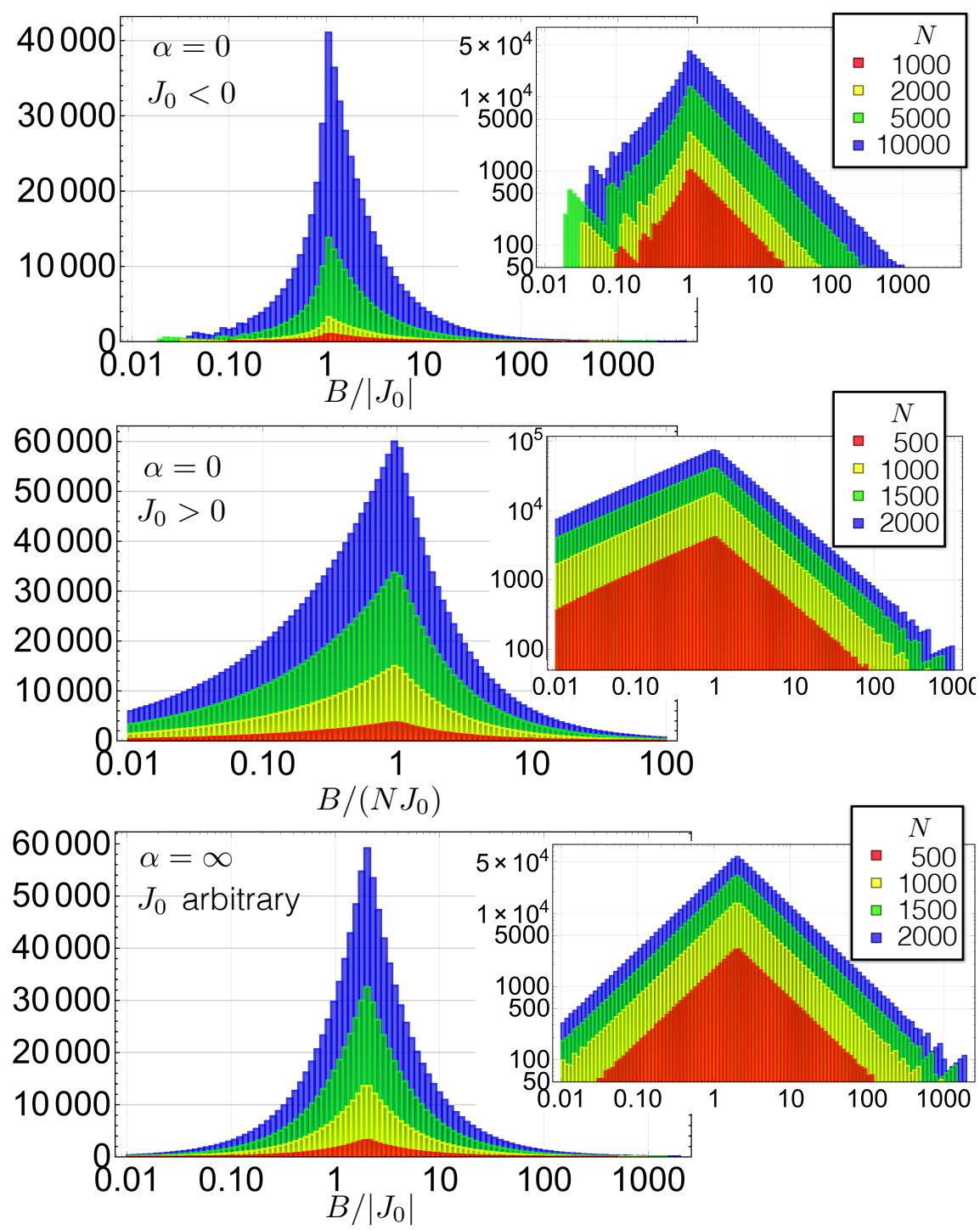

FiguRE 5.18.: Histograms of stable critical fields of semiclassical energy levels for spin chains (5.1) of variable sizes. For $\alpha=0$ and $J_{0}<0$, the maximum is found at $B=\left|J_{0}\right|$, whereas for $J_{0}>0$, the global maximum if found at $B=N J_{0}$. For $\alpha=\infty$, the distribution does no longer depend on the sign of $J_{0}$, and always yields a maximum at $B=2\left|J_{0}\right|$. The logarithmically scaled insets show that the maxima are in most cases approached following a power-law dependence (see text). 
caused by the vanishing influence of $J_{0}$ and $\alpha$ in the limit of large $B$.

It is now most interesting to extract similar information from the histograms of quantum magnets with intermediate interaction ranges, $0<\alpha<\infty$. This is, however, computationally much more demanding despite the explicit formula provided by equation (5.75), since in the most general case, especially for $\alpha \simeq 1$, the eigenvalues are broadly distributed (see also figure 5.17) and have to be figured out by probing exponentially many configurations $\phi^{\mu}$. At this point we are not able to provide conclusive and statistically relevant data for intermediate values of $\alpha$.

\subsection{Discussion}

In this chapter, we have explored the excitation spectrum of one-dimensional spin chain models over the full range of tunable interaction ranges. Based on a semiclassical approach, we were able to reproduce large parts of the quantum mechanical excitation spectrum. The semiclassical approach further identifies an energy landscape with each excited level. These landscapes depend on the external field parameter, and when a threshold value is surpassed, a bifurcation from a single extremal value at zero, to two degenerate extremal points occurs, which reflects the transition from a symmetric paramagnetic phase to the symmetry-broken (anti-)ferromagnetic phase in each of the excited states levels. This shows that the quantum phase transition affects the entire excitation spectrum just as much as it affects the ground state. The results of this chapter, thus, imply a significant influence of the quantum phase transition on the system's dynamical features, which are not accounted for by (purely static) properties of the ground state.

The semiclassical approach is of course limited in its possibilities and unable to generate the full quantum spectrum at all points in the phase transition. In particular, at intermediate values of $B$, the abundance of avoided level crossings cannot be expected to be seen in the semiclassical ansatz. The spectra far away from the phase transition, however, are exactly reproduced semiclassically. To identify information about the phase transition, we have analyzed the distribution of the excited state bifurcation points, which essentially consists in a combination of the two extremal spectra far away from the phase transition. The resulting histograms were found to be able to identify the critical point of the quantum phase transition in some cases. Deviations were seen in the case of the nearest-neighbor Ising model, where the discrepancy consists in a factor of 2. In the opposite, infinite-range limit, the maximum is even found exactly at the critical point. In this limit, semiclassical approaches have been known to work well (Botet et al., 1982; Dusuel and Vidal, 2004; Leyvraz and Heiss, 2005; Dusuel and Vidal, 2005; Ribeiro et al., 2007), in contrast to the Ising model. The intuitive reason for this discrepancy is simply the number of interacting partners for each of the spins: While in the Ising mode, each spin only interacts with its two neighbors, in the Lipkin-Meshkov-Glick model, each spin is in contact with all other spins, which improves the performance of mean-field methods (Georges et al., 1996). Thus, we expect that extensions to two- or three-dimensional lattice arrangements could give rise to promising future applications of the present method. 
The developed ansatz can be applied to arbitrary interaction ranges and can, in principle, also predict the statistics of intermediate-range spin chains. The semiclassical ansatz allows to avoid diagonalization of a large Hilbert space at the cost of scanning through combinations of spin configurations. Exploiting symmetries reduces the number of configurations to values below the dimension of the Hilbert space, but the remaining exponential growth still limits the size of tractable systems significantly. 



\section{MULtidimensionaL NONLINEAR SPECTROSCOPY OF CONTROLLABLE QUANTUM SYSTEMS}

Employing concepts from nonlinear spectroscopy, we develop a general method to probe multi-time correlation functions of interacting many-body quantum dynamics. Singlesite addressability of controllable quantum systems further provides spatial resolution which allows us to extend standard methods of multidimensional spectroscopy. This provides us with a scalable framework for the targeted construction of a probing scheme to extract information about specific aspects of a complex, interacting many-body quantum system.

The results of the present section have been obtained in collaboration with Frank Schlawin; a related presentation can be found in (Schlawin, 2015).

\subsection{Introduction: Nonlinear spectroscopy and controllable quantum systems}

In the previous chapters we have discussed various examples of controllable quantum systems. This includes, for example, the dynamics of trapped ions, involving the laserinduced interaction between different degrees of freedom or the dynamics of long-range spin-spin couplings in competition with an external field. We have also analyzed environmental influences and incoherent dynamics induced by fluctuating external fields. In chapter 3, our strategy for the detection of quantum correlations consisted in analyzing only a small subsystem, which allowed us to resort to methods which provide full information about the subsystem, such as state tomography. To analyze the dy- 
namics of large-scale quantum systems, standard methods from quantum information theory must be complemented with scalable tools, which are able to identify and extract relevant and robust information. That is, in complex systems we are interested in average values and higher moments (or equivalently multi-point correlation functions), rather than in the details of the distributions that generate these moments; recall also section 2.5.2.

After successfully demonstrating high-level experimental control over the elementary constituents of quantum optical systems, experiments are being pushed towards larger and larger system sizes. This is necessary to bring experimental quantum computations and quantum simulations to a computationally relevant level of complexity. On the other hand, one must ensure that the high level of coherent control can be maintained in the course of the expansion towards larger system sizes.

The goal of the present chapter is to complement the theoretical methods of chapters 4 and 5 with experimental methods, able to extract spectral and dynamical information from possibly complex many-body quantum systems. To this end, we refine methods from nonlinear spectroscopy, which have already proven to be successful in probing higher-order correlation functions in much more complex situations. Their straight-forward application to quantum optical systems is, however, not possible. For example, typical trapped-ion experiments contain only tens or hundreds of ions, with a rather large separation between them due to the Coulomb repulsion. This leads to a much smaller optical density and reduces the photon yield for absorption spectroscopic methods. ${ }^{1}$ Conversely, this difference between bulk materials and artificial quantum systems offers new opportunities to extend standard methods of nonlinear spectroscopy.

Before we develop our formalism for controllable quantum systems in section 6.3, we introduce the general principles and the theoretical description of nonlinear spectroscopic measurements employing the example of atomic vapor. In this context, we already discuss the measurement of fluorescence signals in combination with phasecycling, and introduce a diagrammatic description which simplifies the theoretical treatment significantly. These ingredients will be essential for the development of multidimensional probing techniques for controllable quantum systems later in this chapter.

\subsection{Phase-coherent two-pulse measurements of atomic vapor}

To develop an intuition for the principles of nonlinear spectroscopy, we describe a phase-coherent two-pulse experiment carried out on atomic vapor. Similar experiments have been reported in references (Fourkas et al., 1989; Tekavec et al., 2006; Tekavec et al., 2007; Dai et al., 2010; Dai et al., 2012), and are currently also being realized in the group of Frank Stienkemeier at the University of Freiburg. We first discuss how the lowest-order single quantum coherence signals can be extracted by changing the phases of the incoming pulses. We extend the theoretical treatment to higher-order

\footnotetext{
${ }^{1}$ This is why one usually employs the electron shelving method to probe the electronic state of the ions; see section 2.2.1.5.
} 
terms, allowing for several interactions of the light field with an ensemble of atoms. In the course of this section, we will gradually introduce the conventional diagrammatic representation of excitation pathways in terms of Feynman ladder diagrams, which is commonly employed to treat nonlinear signals (Mukamel, 1995). Its convenience will become apparent during the following explicit calculations, and in the following sections, we will make exclusive use the diagrammatic approach.

\subsubsection{Atomic vapor}

The Hamiltonian describing the relevant electronic states of a single atom, labelled by the index $n$, is given as $^{2}$

$$
\hat{H}_{0}^{(n)}=\sum_{i} \epsilon_{i}|i\rangle_{n}\left\langle\left. i\right|_{n}\right.
$$

In the presence of atom-atom interactions, we add terms of the form

$$
\hat{V}=\sum_{i>j} \sum_{m>n} v_{i j}^{n m}\left(|i\rangle_{n}\left\langle\left. j\right|_{m}+\mid j\right\rangle_{m}\left\langle\left. i\right|_{n}\right)\right.
$$

such that the total atomic Hamiltonian reads

$$
\hat{H}_{0}=\sum_{n} \sum_{i} \epsilon_{i}|i\rangle_{n}\left\langle\left. i\right|_{n}+\sum_{i>j} \sum_{m>n} v_{i j}^{n m}\left(|i\rangle_{n}\left\langle\left. j\right|_{m}+\mid j\right\rangle_{m}\left\langle\left. i\right|_{n}\right) .\right.\right.
$$

The coupling constants $v_{i j}^{n m}$ express the strength of the interaction between two atoms $n$ and $m$, when they are occupying the states $|i\rangle_{n}$ and $|j\rangle_{m}$, respectively. In an atomic vapor, such interactions are typically given by dipole-dipole interactions. The dipoledipole interaction between atoms in the $P$ state, scales with the inter-atom distance $r$ as $r^{-3}$, and for atoms in an $S$ state (which is the case for the ground state of hydrogenlike atoms, such as alkali metals), we have induced-dipole interactions, which depend on the distance as $r^{-6}$ (Cohen-Tannoudji et al., 1977b). Except for very large densities, such interactions are very weak in an atomic vapor and hard to detect from the width of spectral lines (Dai et al., 2012).

The atomic Hamiltonian can be written in terms of well-defined excited-state manifolds of the collective state of all $N$ atoms,

$$
\hat{H}_{0}=\epsilon_{g}|g\rangle\left\langle g\left|+\sum_{i} \epsilon_{i}^{(1)}\right| e_{i}^{(1)}\right\rangle\left\langle e_{i}^{(1)}\left|+\sum_{i} \epsilon_{i}^{(2)}\right| e_{i}^{(2)}\right\rangle\left\langle e_{i}^{(2)}\right|+\ldots
$$

where $\left|e_{i}^{(n)}\right\rangle$ denote the $n$-fold excited states ( $n$ out of $N$ atoms are excited). If there are no interactions between the atoms, the first excited state manifold is $N$-fold degenerate, that is, $\epsilon_{i}^{(1)}=\epsilon^{(1)} \forall i$-it is comprised of all states, where one atom is excited while all others remain in the ground state. Weak atom-atom interactions lift these

\footnotetext{
${ }^{2}$ Throughout this section, we denote operators with a hat to avoid ambiguities when we replace the classical electromagnetic field modes by quantized operators.
} 
degeneracies and produce a band of resonances whose width depends on the strength of the interaction. Writing out the eigenstates of the full Hamiltonian in terms of different excitation manifolds is close in spirit to the theoretical treatment of molecular systems. To describe, for instance, the nonlinear spectra generated by the excitons (bounded electron-hole paris) in molecular aggregates, one frequently employs a similar decomposition of the Hamiltonian (Schröter et al., 2015). The different energy bands - or in a the language of chemical physics, the exciton manifolds - are coupled via single-photon absorption and emission processes. We will, at some points, make use of this language for the description and interpretation of nonlinear spectroscopic signals of quantum optical systems. For example, if the system is initially in the ground state, we need to absorb two photons to populate the second exciton manifold. This is important for the perturbative treatment of nonlinear signals which we will develop in the next section.

\subsubsection{Light-matter interactions}

To spectroscopically analyze the system, we have to probe it with laser pulses. We therefore start from the description of the system in the presence of atom-light interactions, given by the Hamiltonian

$$
\hat{H}=\hat{H}_{0}+\hat{H}_{\text {int }}^{S}(t)
$$

where

$$
\hat{H}_{\mathrm{int}}^{S}(t)=-\hat{\mu}^{S} E(t)
$$

represents the light-matter coupling via the dipole operator

$$
\hat{\mu}^{S}=\sum_{i} \mu_{i g}^{(0)}\left|e_{i}^{(1)}\right\rangle\left\langle g\left|+\sum_{n} \sum_{i j} \mu_{i j}^{(n)}\right| e_{i}^{(n+1)}\right\rangle\left\langle e_{j}^{(n)}\right|+\text { H.c. },
$$

of the atomic ensemble. We have introduce dipole moments $\mu_{i g}^{(0)}$, which couple the ground state to the first excited state manifold, and similarly, couplings induced between higher-order energy bands described by $\mu_{i j}^{(n)}$. The superscript $S$ denotes the Schrödinger picture. We treat the electromagnetic field as a classical field, which is justified for typical laser pulses. We will provide a quantum treatment of the vacuum modes in a later section, when we discuss the emission process of the sample after a pulse sequence. For the perturbative expansion in the number of light-matter interactions, we transform the quantum state of the atomic ensemble to the interaction picture using

$$
|\Psi(t)\rangle=e^{i \hat{H}_{0} t / \hbar}\left|\Psi^{S}(t)\right\rangle,
$$

which leads to a time evolution described by

$$
i \hbar \frac{\partial}{\partial t}|\Psi(t)\rangle=\hat{H}_{\mathrm{int}}(t)|\Psi(t)\rangle
$$


with $\hat{H}_{\text {int }}(t)=e^{i \hat{H}_{0} t / \hbar} \hat{H}_{\text {int }}^{S}(t) e^{-i \hat{H}_{0} t / \hbar}$. We assume that the laser frequency, determining $E(t)$, is close to the transition frequency between excited state manifolds (see below). In the rotating wave approximation (see, for example, section 3.2.1), the interaction Hamiltonian reads

$$
\hat{H}_{\text {int }}(t)=-\hat{\mu}^{\dagger}(t) E^{+}(t)-\hat{\mu}(t) E^{-}(t)
$$

where we have introduced the positive-frequency component of the interaction-picture dipole operator

$$
\hat{\mu}^{\dagger}(t)=\sum_{i} \mu_{i g}^{(0)} e^{i \omega_{i g} t}\left|e_{i}^{(1)}\right\rangle\left\langle g\left|+\sum_{n} \sum_{i j} \mu_{i j}^{(n)} e^{i \omega_{i j}^{(n)} t}\right| e_{i}^{(n+1)}\right\rangle\left\langle e_{j}^{(n)}\right|,
$$

with $\omega_{i g}=\left(\epsilon_{i}^{(1)}-\epsilon_{g}\right) / \hbar$ and $\omega_{i j}^{(n)}=\left(\epsilon_{i}^{(n+1)}-\epsilon_{j}^{(n)}\right) / \hbar$. The electromagnetic field is decomposed into individual pulses $E(t)=E_{1}(t)+E_{2}(t)+\ldots$ of the form

$$
\begin{aligned}
E_{j}(t) & =A_{j}\left(t-t_{j}\right) \cos \left[\omega_{L}\left(t-t_{j}\right)+\Phi_{j}\right] \\
& =\frac{1}{2} A_{j}\left(t-t_{j}\right) e^{i\left[\omega_{L}\left(t-t_{j}\right)+\Phi_{j}\right]}+\frac{1}{2} A_{j}\left(t-t_{j}\right) e^{-i\left[\omega_{L}\left(t-t_{j}\right)+\Phi_{j}\right]} \\
& =E_{j}^{-}(t)+E_{j}^{+}(t),
\end{aligned}
$$

where $\omega_{L}$ denotes the laser frequency and $A_{j}$ describes the time-dependent amplitude, that is, the temporal envelope of the $j$-th pulse. The phase $\Phi_{j}$ of the laser pulse will play a crucial role for the phase cycling, to be introduced in section 6.2.5. For the above rotating-wave-approximation, we assume $\omega_{L} \approx \omega_{i g} \approx \omega_{j k}^{(n)}$, for all single excited states labeled by $i$ and higher-order excited states labeled by $j, k$ and $n$.

\subsubsection{Fluorescence measurements}

There are several possibilities to collect the signal at the end of the pulse sequence. Bulk materials are usually measured by heterodyne detection of the stimulated emission light, or by collecting the light emitted via spontaneous decay at a random time after the last interaction. This is possible for atomic vapor (Dai et al., 2010; Dai et al., 2012), but when we consider extending these methods to cold atomic ensembles of roughly only hundreds of atoms, the number of scattered photons will be too low to implement such methods. Furthermore, we will also develop methods to probe, for instance, the dynamics of phonons in an ion trap. These excitations dissipate slowly into the environment instead of decaying radiatively. Hence different methods need to be developed for probing artificial quantum systems. A promising possibility is to consider the measurement of fluorescence light, which is induced externally, and its intensity is proportional to the population of the excited states (Tekavec et al., 2006; Tekavec et al., 2007; Lott et al., 2011; Hildner et al., 2011). In the context of controllable atomic systems with long lifetimes, considered in the next section, this technique brings the additional advantage that the time-delay between the final pulse of the sequence and the collection of the signal can be fully controlled and, as we will see later, its variation can yield further information about the decay of excitations in the system. 


\subsubsection{Single quantum coherence}

We describe the spectroscopic experiment in the weak-coupling limit by a perturbative expansion in the light-matter interaction. Since we consider a two-pulse measurement scheme, we have to extend the expansion at least to second order contributions in the interaction Hamiltonian, with $E(t)=E_{1}(t)+E_{2}(t)$. In this section, we restrict to a fully coherent perturbation expansion, which means that we consider the time evolution of a pure state governed by the Schrödinger equation. This simplifies the theoretical treatment significantly, and, furthermore, is well justified for the description of electronic excitations in atomic vapor. Incoherent effects can be analyzed efficiently with methods from nonlinear spectroscopy, and their theoretical description will be taken into account in later sections. A perturbative expansion of the quantum state in the number of light-matter interactions yields

$$
|\Psi\rangle=\left|\Psi^{(0)}\right\rangle+\left|\Psi^{(1)}\right\rangle+\left|\Psi^{(2)}\right\rangle+\ldots,
$$

where the superscript denotes the number of interaction events, which can be absorption or emission processes of the atomic ensemble. We measure fluorescence light immediately after the final interaction. Thus, the final signal is given, on the one hand, by the overlap of the final state with the excited states, and, on the other hand, by their respective coupling to the nearest manifold of energetically lower states (as expressed by the dipole moments):

$$
S=\sum_{n} \sum_{k j}\left|\mu_{k j}^{(n-1)}\right|^{2}\left\langle e_{k}^{(n)} \mid \Psi\right\rangle\left\langle\Psi \mid e_{k}^{(n)}\right\rangle .
$$

Here we only consider a single decay process for each excited state, while for higherexcited states, there exists the possibility that the fluorescence yield is enhanced by cascaded emissions. When a higher excited state decays into a lower excited state, the subsequent decay of that lower excited state occurs - on average - after that state's lifetime has passed, and is therefore emitted later. Thus, the above approximation is not justified if two conditions are met: First the time scale over which the fluorescence light is recorded is much longer than the lifetime, and, second, the emitted photons are recorded with high efficiency (that is: if the decay of an excited state leads to the emission of multiple photons, the photon count will only be affected if also multiple photons are registered). For the theoretical treatment presented in the following, we assume that the above approximation is valid, and we will discuss other scenarios qualitatively at the end of this section.

We assume that initially, the system is prepared in its ground state $\left|\Psi^{(0)}\right\rangle=|g\rangle$, which does not contribute to the fluorescence light. Inserting equation (6.13) and only retaining contributions up to second order, we obtain

$$
\begin{aligned}
S & =\sum_{n} \sum_{k j}\left|\mu_{k j}^{(n-1)}\right|^{2}\left\langle e_{k}^{(n)} \mid \Psi^{(1)}\right\rangle\left\langle\Psi^{(1)} \mid e_{k}^{(n)}\right\rangle \\
& =\sum_{k}\left|\mu_{k g}^{(0)}\right|^{2}\left\langle e_{k}^{(1)} \mid \Psi^{(1)}\right\rangle\left\langle\Psi^{(1)} \mid e_{k}^{(1)}\right\rangle,
\end{aligned}
$$


where with a single interaction, only the first excited-state manifold $(n=1)$ can be reached from the ground state. It is thus sufficient to determine the first order terms

$$
\left|\Psi^{(1)}(t)\right\rangle=-\frac{i}{\hbar} \int_{t_{0}}^{t} d \tau \hat{H}_{\mathrm{int}}(\tau)\left|\Psi\left(t_{0}\right)\right\rangle .
$$

The above mentioned assumption, that long before the interaction, the system was prepared in its ground state, is now expressed formally by replacing $t_{0} \rightarrow-\infty$ and $\left|\Psi\left(t_{0}\right)\right\rangle=|g\rangle$. Moreover, we assume that the measurement is performed long after the final light-matter interaction, thus, we perform the limit $t \rightarrow \infty$. Since, from equation (6.11), we have $\hat{\mu}(t)|g\rangle=0$, a single interaction with the field $E(t)=E_{1}(t)+$ $E_{2}(t)$ is determined by the respective positive frequency parts,

$$
\begin{aligned}
\left|\Psi^{(1)}\right\rangle & =\frac{i}{\hbar} \int_{-\infty}^{\infty} d \tau \hat{\mu}^{\dagger}(\tau) E_{1}^{+}(\tau)|g\rangle+\frac{i}{\hbar} \int_{-\infty}^{\infty} d \tau \hat{\mu}^{\dagger}(\tau) E_{2}^{+}(\tau)|g\rangle \\
& =\left|\Psi_{1}^{(1)}\left(t_{1}\right)\right\rangle+\left|\Psi_{2}^{(1)}\left(t_{2}\right)\right\rangle
\end{aligned}
$$

with

$$
\begin{aligned}
\left|\Psi_{j}^{(1)}\left(t_{j}\right)\right\rangle & =\frac{i}{\hbar} \int_{-\infty}^{\infty} d \tau \hat{\mu}^{\dagger}(\tau) E_{j}^{+}(\tau)|g\rangle \\
& =\frac{i}{\hbar} \sum_{i} \int_{-\infty}^{\infty} d \tau \mu_{i g}^{(0)} e^{i \omega_{i g} \tau} E_{j}^{+}(\tau)\left|e_{i}^{(1)}\right\rangle\langle g \mid g\rangle \\
& =\frac{i}{\hbar} \sum_{i} \mu_{i g}^{(0)} \tilde{E}_{j}^{+}\left(\omega_{i g}\right)\left|e_{i}^{(1)}\right\rangle .
\end{aligned}
$$

In the last step, we define the time integral, with equation (6.12), as

$$
\begin{aligned}
\tilde{E}_{j}^{+}\left(\omega_{i g}\right) & =\frac{1}{2} \int_{-\infty}^{\infty} d \tau A_{j}\left(\tau-t_{j}\right) e^{i \omega_{i g} \tau} e^{-i\left[\omega_{L}\left(\tau-t_{j}\right)+\Phi_{j}\right]} \\
& =\frac{1}{2} \int_{-\infty}^{\infty} d \tau^{\prime} A_{j}\left(\tau^{\prime}\right) e^{i\left(\omega_{i g}-\omega_{L}\right) \tau^{\prime}} e^{i \omega_{i g} t_{j}} e^{-i \Phi_{j}} \\
& =\alpha_{j}\left(\omega_{i g}\right) e^{i \omega_{i g} t_{j}} e^{-i \Phi_{j}}
\end{aligned}
$$

which contains the spectral amplitude $\alpha_{j}(\omega)$, which, formally represents the Fourier transform of the temporal envelope $A_{j}(t)$. Inserting this back into equation (6.18), we obtain

$$
\left|\Psi_{j}^{(1)}\left(t_{j}\right)\right\rangle=\frac{i}{\hbar} \sum_{i} \mu_{i g}^{(0)} \alpha_{j}\left(\omega_{i g}\right) e^{i \omega_{i g} t_{j}} e^{-i \Phi_{j}}\left|e_{i}^{(1)}\right\rangle .
$$

Thus, if we expand a phase-coherent two-pulse sequence to second order in the lightmatter interaction (one excitation induced by each pulse), we obtain an excited state 


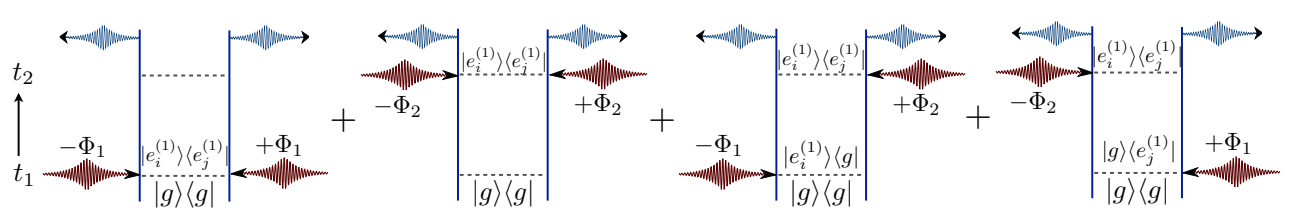

FiguRE 6.1.: Diagrammatic representation of the contributions to the total signal in a simple two-pulse measurement scheme. Excitations (red arrows) apply to the 'bra'and 'ket' side of the density matrix and induce characteristic phase shifts $\Phi_{j}$, which label different excitation pathways. The fluorescence readout of excitedstate populations is depicted by two blue arrows. The first two terms describe populations which are created by a single pulse, and show no time evolution between the two pulses. These contributions also do not depend on the laser phase. This is different for the coherences (third and fourth term), which can be selected based on their phase dependence.

population that leads to the fluorescence signal, given by (6.15), with (6.17) and (6.20),

$$
\begin{aligned}
S= & \sum_{k}\left|\mu_{k g}^{(0)}\right|^{2}\left|\left\langle e_{k}^{(1)} \mid \Psi_{1}^{(1)}\left(t_{1}\right)\right\rangle\right|^{2}+\sum_{k}\left|\mu_{k g}^{(0)}\right|^{2}\left|\left\langle e_{k}^{(1)} \mid \Psi_{2}^{(1)}\left(t_{2}\right)\right\rangle\right|^{2} \\
& +\sum_{k}\left|\mu_{k g}^{(0)}\right|^{2}\left\langle e_{k}^{(1)} \mid \Psi_{1}^{(1)}\left(t_{1}\right)\right\rangle\left\langle\Psi_{2}^{(1)}\left(t_{2}\right) \mid e_{k}^{(1)}\right\rangle \\
& +\sum_{k}\left|\mu_{k g}^{(0)}\right|^{2}\left\langle e_{k}^{(1)} \mid \Psi_{2}^{(1)}\left(t_{2}\right)\right\rangle\left\langle\Psi_{1}^{(1)}\left(t_{1}\right) \mid e_{k}^{(1)}\right\rangle .
\end{aligned}
$$

At this point, we introduce a diagrammatic representation of the resulting four terms, by writing out the initial pure state as a projector and introducing time as the upwards direction, see figure 6.1. The first two contributions,

$$
\sum_{k}\left|\mu_{k g}^{(0)}\right|^{2}\left|\left\langle e_{k}^{(1)} \mid \Psi_{j}^{(1)}\left(t_{j}\right)\right\rangle\right|^{2}=\frac{1}{\hbar^{2}} \sum_{i}\left|\mu_{i g}^{(0)}\right|^{4}\left|\alpha_{j}\left(\omega_{i g}\right)\right|^{2},
$$

describe processes in which the ensemble only interacts with one of the two pulses and an excited-state population is created by either the first or the second pulse. This does not lead to any time evolution between the pulses. This is different for the two interference terms,

$$
\sum_{k}\left|\mu_{k g}^{(0)}\right|^{2}\left\langle e_{k}^{(1)} \mid \Psi_{2}^{(1)}\left(t_{2}\right)\right\rangle\left\langle\Psi_{1}^{(1)}\left(t_{1}\right) \mid e_{k}^{(1)}\right\rangle=\sum_{i}\left|\mu_{i g}^{(0)}\right|^{4} \alpha_{1}\left(\omega_{i g}\right) \alpha_{2}\left(\omega_{i g}\right) e^{i \omega_{i g} t_{21}} e^{-i \Phi_{21}}
$$

which evolve in a coherent superposition of ground- and excited states. Unlike the populations, these terms evolve as a function of the time delay $t_{21}=t_{2}-t_{1}$ between the pulses. A Fourier transform of the corresponding signal would yield the energy spectrum of the first excited states which are coupled to the ground state via $\hat{\mu}$. Had we included incoherent processes, we would be able to observe the decay of this coherent 
superposition as a function of the delay time. The corresponding terms define the single quantum coherence signal and they are identified by the characteristic phase shift $\Phi_{21}=$ $\Phi_{2}-\Phi_{1}$. In the present setting, these coherences represent the only relevant excitation pathway. In higher-order signals, different pathways may be able to reveal different information about the dynamical and spectral features of the system, and it becomes increasingly important to selectively extract only specific contributions, characterized by their phase-dependence. As we already indicated in the introductory section 2.4 , in bulk material, this can be done by adjusting phase-matching conditions. The analogue of this method in the current setting is phase cycling, which was developed already in the context of two-dimensional nuclear magnetic resonance experiments (Ernst et al., 1987).

\subsubsection{Phase cycling}

Phase cycling is a post-processing method which allows to extract only those contributions with a specific phase dependence from a convoluted signal with many different contributions. It relies on the ability to control the phases $\Phi_{i}$ in a sequence of pulses. As we have seen in the previous section, each interaction process with a light field, described by (6.12), adds another phase factor $\pm \Phi_{j}$ to the quantum state. Let us assume the total signal contains contributions with phase signatures $k \Phi_{12}$, with $k=0, \pm 1, \pm 2, \ldots, \pm k_{\max }$ and $k_{\max }$ is determined by type and strength of the interaction. The total signal can be decomposed into terms labeled by their phase shifts

$$
S\left(\Phi_{12}\right)=\sum_{k=-k_{\max }}^{k_{\max }} S_{k} e^{i k \Phi_{12}},
$$

and the goal is to extract the contribution $S_{k}$. This can be achieved by an inverse discrete Fourier transform (Ernst et al., 1987)

$$
S_{k}=\frac{1}{2 k_{\max }+1} \sum_{j=0}^{2 k_{\max }} S\left(\delta \Phi_{j}\right) e^{-i k \delta \Phi_{j}},
$$

with $\delta \Phi_{j}=2 \pi j /\left(2 k_{\max }+1\right)$. Experimentally, this requires repeating the measurement while scanning the phase shifts over the set of $\delta \Phi_{j}$. In a perturbative treatment, typical small values of $k_{\max } \leq 2$ allow to extract the relevant terms already with few repetitions of the experiment. Since the development for nuclear magnetic resonance (Ernst et al., 1987), phase cycling has been implemented with optical signals (Tian et al., 2003) and enables for example phase-coherent experiments with single molecules (Hildner et al., 2011). In the example of the atomic vapor, phase-cycling is implemented by a slow acousto-optical modulation of the laser pulses, which is extracted with a lock-in amplifier (Tekavec et al., 2006).

In higher-order pulse sequences, more complicated dependences on the phase difference between each consecutive pair of pulses may occur. The above method can be extended easily to a multidimensional inverse Fourier analysis, and optimized phasecycling schemes have been developed to reduce the number of experimental repetitions (Ernst et al., 1987). 


\subsubsection{Second-order quantum coherence}

From equation (6.21) we see that in the above second-order perturbative treatment, the only contributions to the fluorescence signal are the induced populations of the first excited state manifold. If we further employ phase-cycling to extract the signature $\Phi_{12}$ from the total signal, there cannot be any contribution of higher excited statesirrespective of the intensities of the pulses. In order to study the dynamics of higherexcited states in the above two-pulse scheme we must therefore extend the perturbation theory to higher orders, and adjust the phase-cycling condition.

Thus, we now allow for two interactions with each pulse, leading to a total of four light-matter interactions. Due to the orthogonality of the excited state manifolds, there can be no contribution from the third order since this would involve asymmetric populations in the left and right arm of the ladder diagrams. The next leading order is therefore the fourth order. We assume the initial state to be the ground state again, and expand equation (6.14) to fourth order:

$$
\begin{aligned}
S=\sum_{n} \sum_{k j}\left|\mu_{k j}^{(n-1)}\right|^{2} & \left(\left\langle e_{k}^{(n)} \mid \Psi^{(2)}\right\rangle\left\langle\Psi^{(2)} \mid e_{k}^{(n)}\right\rangle\right. \\
& \left.+\left\langle e_{k}^{(n)} \mid \Psi^{(1)}\right\rangle\left\langle\Psi^{(3)} \mid e_{k}^{(n)}\right\rangle+\left\langle e_{k}^{(n)} \mid \Psi^{(3)}\right\rangle\left\langle\Psi^{(1)} \mid e_{k}^{(n)}\right\rangle\right) .
\end{aligned}
$$

The first-order contributions were already determined in equation (6.20). The secondorder contributions can also generate second-excited states $(n=2)$, and therefore the above sum can no longer be restricted to terms with $n=1$. Under the same assumptions as before, the second-order terms yield, with (6.10), and recalling that $\hat{\mu}|g\rangle=0$

$$
\begin{aligned}
\left|\Psi^{(2)}\right\rangle & =-\frac{1}{\hbar^{2}} \int_{-\infty}^{\infty} d \tau \int_{-\infty}^{\tau} d \tau^{\prime} \hat{H}_{\mathrm{int}}(\tau) \hat{H}_{\mathrm{int}}\left(\tau^{\prime}\right)|g\rangle \\
& =-\frac{1}{\hbar^{2}} \sum_{i, j=1,2} \int_{-\infty}^{\infty} d \tau \int_{-\infty}^{\tau} d \tau^{\prime}\left[\hat{\mu}^{\dagger}(\tau) E_{i}^{+}(\tau) \hat{\mu}^{\dagger}\left(\tau^{\prime}\right) E_{j}^{+}\left(\tau^{\prime}\right)\right. \\
& =\sum_{i, j=1,2}\left|\Psi_{i j}^{(2)}\left(t_{i}, t_{j}\right)\right\rangle,
\end{aligned}
$$

where, with (6.11),

$$
\begin{aligned}
& \left|\Psi_{i j}^{(2)}\left(t_{i}, t_{j}\right)\right\rangle \\
= & -\frac{1}{\hbar^{2}} \sum_{a b c} \int_{-\infty}^{\infty} d \tau \int_{-\infty}^{\tau} d \tau^{\prime} \mu_{c b}^{(1)} e^{i \omega_{c b}^{(1)} \tau} E_{i}^{+}(\tau) \mu_{a g}^{(0)} e^{i \omega_{a g} \tau^{\prime}} E_{j}^{+}\left(\tau^{\prime}\right)\left|e_{c}^{(2)}\right\rangle\left\langle e_{b}^{(1)} \mid e_{a}^{(1)}\right\rangle \\
& -\frac{1}{\hbar^{2}} \sum_{a b} \int_{-\infty}^{\infty} d \tau \int_{-\infty}^{\tau} d \tau^{\prime} \mu_{b g}^{(0) *} e^{-i \omega_{b g} \tau} E_{i}^{-}(\tau) \mu_{a g}^{(0)} e^{i \omega_{a g} \tau^{\prime}} E_{j}^{+}\left(\tau^{\prime}\right)|g\rangle\left\langle e_{b}^{(1)} \mid e_{a}^{(1)}\right\rangle
\end{aligned}
$$




$$
\begin{aligned}
= & -\frac{1}{\hbar^{2}} \sum_{a c} \int_{-\infty}^{\infty} d \tau \int_{-\infty}^{\tau} d \tau^{\prime} \mu_{c a}^{(1)} e^{i \omega_{c a}^{(1)} \tau} E_{i}^{+}(\tau) \mu_{a g}^{(0)} e^{i \omega_{a g} \tau^{\prime}} E_{j}^{+}\left(\tau^{\prime}\right)\left|e_{c}^{(2)}\right\rangle \\
& -\frac{1}{\hbar^{2}} \sum_{a} \int_{-\infty}^{\infty} d \tau \int_{-\infty}^{\tau} d \tau^{\prime}\left|\mu_{a g}^{(0)}\right|^{2} e^{-i \omega_{a g}\left(\tau-\tau^{\prime}\right)} E_{i}^{-}(\tau) E_{j}^{+}\left(\tau^{\prime}\right)|g\rangle
\end{aligned}
$$

The dependence of these terms on the times $t_{1}$ and $t_{2}$ at which the two pulses are applied will become apparent later. The last term in (6.28), which describes an excitation to a first excited state and a stimulated emission back to the ground state, does not contribute to the fluorescence light, and we will therefore omit it for future calculations. For the other term, which describes a two-photon excitation to a doubleexcited state, we employ the approximation $\omega_{c b}^{(1)} \approx \omega_{a g} \equiv \omega_{0} \forall c, b, a$. This means that we neglect inter-atom interactions, which would lead to broad bands instead of degenerate excited state manifolds. We first consider contributions in which both absorbed photons origin from the same pulse, that is $i=j$. The integral in the only contributing term of (6.28), then, can be written as

$$
\left|\Psi_{j j}^{(2)}\left(t_{j}\right)\right\rangle=-\frac{1}{\hbar^{2}} \sum_{a c} \mu_{c a}^{(1)} \mu_{a g}^{(0)} \int_{-\infty}^{\infty} d \tau \int_{-\infty}^{\tau} d \tau^{\prime} e^{i \omega_{0} \tau} E_{j}^{+}(\tau) e^{i \omega_{0} \tau^{\prime}} E_{j}^{+}\left(\tau^{\prime}\right)\left|e_{c}^{(2)}\right\rangle .
$$

For simplicity, the equality sign in this, and henceforth in similar terms, reflects equality up to terms that can lead to a nonzero contribution to the fluorescence light (equality modulo terms that are proportional to the ground state, and therefore are irrelevant for the fluorescence signal). For functions satisfying $f\left(\tau, \tau^{\prime}\right)=f\left(\tau^{\prime}, \tau\right)$ the following holds:

$$
\int_{-\infty}^{\infty} d \tau \int_{-\infty}^{\tau} d \tau^{\prime} f\left(\tau, \tau^{\prime}\right)=\frac{1}{2} \int_{-\infty}^{\infty} d \tau \int_{-\infty}^{\infty} d \tau^{\prime} f\left(\tau, \tau^{\prime}\right)
$$

This follows from

$$
\iint_{\mathbb{R}^{2}} d \tau d \tau^{\prime}=\iint_{\tau>\tau^{\prime}} d \tau d \tau^{\prime}+\iint_{\tau \leq \tau^{\prime}} d \tau d \tau^{\prime}=\int_{-\infty}^{\infty} d \tau \int_{-\infty}^{\tau} d \tau^{\prime}+\int_{-\infty}^{\infty} d \tau^{\prime} \int_{-\infty}^{\tau^{\prime}} d \tau
$$

together with the symmetry of $f$. We use equation (6.30) to simplify the integral (6.29)

$$
\begin{aligned}
\left|\Psi_{j j}^{(2)}\left(t_{j}\right)\right\rangle & =-\frac{1}{2 \hbar^{2}} \sum_{a c} \mu_{c a}^{(1)} \mu_{a g}^{(0)} \int_{-\infty}^{\infty} d \tau \int_{-\infty}^{\infty} d \tau^{\prime} e^{i \omega_{0} \tau} E_{j}^{+}(\tau) e^{i \omega_{0} \tau^{\prime}} E_{j}^{+}\left(\tau^{\prime}\right)\left|e_{c}^{(2)}\right\rangle \\
& =-\frac{1}{2 \hbar^{2}} \sum_{a c} \mu_{c a}^{(1)} \mu_{a g}^{(0)} \tilde{E}_{j}^{+}\left(\omega_{0}\right) \tilde{E}_{j}^{+}\left(\omega_{0}\right)\left|e_{c}^{(2)}\right\rangle
\end{aligned}
$$

Using equation (6.19) yields

$$
\left|\Psi_{j j}^{(2)}\left(t_{j}\right)\right\rangle=-\frac{1}{2 \hbar^{2}} \sum_{a c} \mu_{c a}^{(1)} \mu_{a g}^{(0)} \alpha_{j}^{2}\left(\omega_{0}\right) e^{2 i \omega_{0} t_{j}} e^{-2 i \Phi_{j}}\left|e_{c}^{(2)}\right\rangle .
$$


In the presence of weak atom-atom interactions, the above integration trick can still be considered a good approximation (assuming that the resulting width of the excitedstate bands is still small compared to their center value), and we obtain

$$
\left|\Psi_{j j}^{(2)}\left(t_{j}\right)\right\rangle \simeq-\frac{1}{2 \hbar^{2}} \sum_{a c} \mu_{c a}^{(1)} \mu_{a g}^{(0)} \alpha_{j}\left(\omega_{c a}^{(1)}\right) \alpha_{j}\left(\omega_{a g}\right) e^{i\left(\omega_{c a}^{(1)}+\omega_{a g}\right) t_{j}} e^{-2 i \Phi_{j}}\left|e_{c}^{(2)}\right\rangle
$$

Alternatively, the impulsive limit allows us to include atom-atom interactions without approximations. In the impulsive limit, we assume that the finite pulse duration can be neglected compared to the time scale of the interacting system dynamics, transforming equation (6.12) into

$$
E_{j}(t)=A_{j} \delta\left(t-t_{j}\right) \cos \left[\omega_{L}\left(t-t_{j}\right)+\Phi_{j}\right]
$$

Using this in equation (6.28), and omitting again the ground-state contribution, we obtain,

$$
\begin{aligned}
\left|\Psi_{j j}^{(2)}\left(t_{j}\right)\right\rangle & =-\frac{1}{\hbar^{2}} \sum_{a c} \int_{-\infty}^{\infty} d \tau \int_{-\infty}^{\tau} d \tau^{\prime} \mu_{c a}^{(1)} e^{i \omega_{c a}^{(1)} \tau} E_{j}^{+}(\tau) \mu_{a g}^{(0)} e^{i \omega_{a g} \tau^{\prime}} E_{j}^{+}\left(\tau^{\prime}\right)\left|e_{c}^{(2)}\right\rangle \\
& =-\frac{1}{\hbar^{2}} \sum_{a c} A_{j}^{2} \mu_{c a}^{(1)} \mu_{a g}^{(0)} e^{i\left(\omega_{c a}^{(1)}+\omega_{a g}\right) t_{j}} e^{-2 i \Phi_{j}}\left|e_{c}^{(2)}\right\rangle
\end{aligned}
$$

Note that in general, excited-state contributions created from a sequence of two pulses carry a phase shift $\Phi_{i}+\Phi_{j}$ :

$$
\left|\Psi_{i j}^{(2)}\left(t_{i}, t_{j}\right)\right\rangle \sim e^{-i\left(\Phi_{i}+\Phi_{j}\right)}
$$

If $i \neq j$, this means that both pulses excite the system once. Such excitations can only lead to a measurable population when they occur in combination with the Hermitian conjugate event, in which case the pathway is described by the creation of a population by each of the pulses. Since these pathways do not involve coherences, they do not provide information about the spectrum or the dissipative dynamics. They further do not carry a total phase signature, and, hence, will be discarded by phase-cycling. Instead, the evolution of double-excited states can be observed from the pathways involving events with $i=j$. To measure the second-order quantum coherence ${ }^{3}$ signal, one selects the terms with phase signature $2 \Phi_{21}=2 \Phi_{2}-2 \Phi_{1}$ from the total signal (6.26). The only relevant contribution from second-order perturbation expressions is therefore, using the expression obtained from the impulsive limit, and recalling the

\footnotetext{
${ }^{3}$ We reserve the term double quantum coherence for a particular four-pulse signal, which will be discussed later in this chapter.
} 
a)

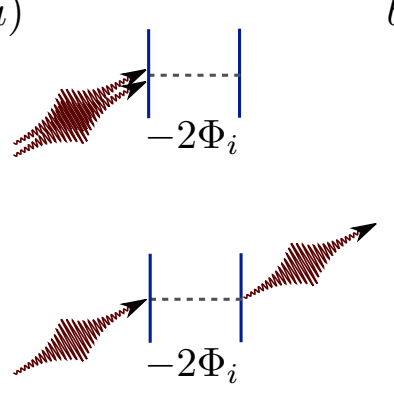

b)

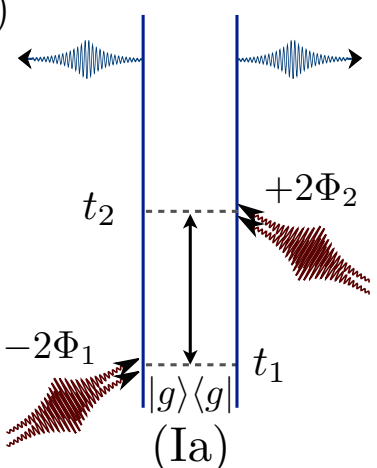

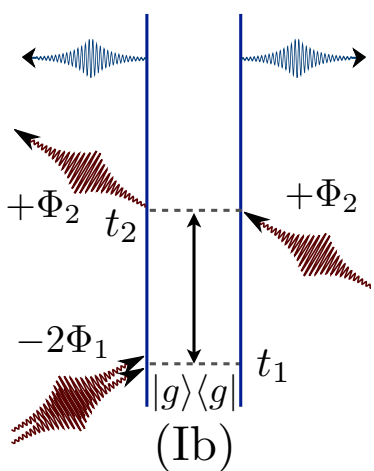

FIGURE 6.2.: Diagrammatic representation of contributions to the second-order quantum coherence signal. Two elementary processes involving two photons from the same laser pulse have to be considered (a). Feynman ladder diagrams represent a useful formalism to visualize the relevant perturbative expressions. For the second-order quantum coherence signal, one selects the phase signature $2 \Phi_{21}$ (see text). Two pathways with opposite signs contribute (b). In the current considerations, we assume that fluorescence happens immediately after the second pulse.

definitions for $\omega_{i g}$ and $\omega_{i j}^{(n)}$ below (6.11),

$$
\begin{aligned}
& \sum_{n} \sum_{k j}\left|\mu_{k j}^{(n-1)}\right|^{2}\left\langle e_{k}^{(n)} \mid \Psi_{22}^{(2)}\left(t_{2}\right)\right\rangle\left\langle\Psi_{11}^{(2)}\left(t_{1}\right) \mid e_{k}^{(n)}\right\rangle \\
= & \frac{1}{\hbar^{4}} \sum_{a b c j}\left|\mu_{c j}^{(1)}\right|^{2} A_{1}^{2} A_{2}^{2} \mu_{c a}^{(1)} \mu_{a g}^{(0)} \mu_{c b}^{(1) *} \mu_{b g}^{(0) *} e^{i\left(\omega_{c a}^{(1)}+\omega_{a g}\right) t_{2}-i\left(\omega_{c b}^{(1)}+\omega_{b g}\right) t_{1}} e^{-2 i \Phi_{21}} \\
= & \frac{1}{\hbar^{4}} \sum_{a b c j}\left|\mu_{c j}^{(1)}\right|^{2} A_{1}^{2} A_{2}^{2} \mu_{c a}^{(1)} \mu_{a g}^{(0)} \mu_{c b}^{(1) *} \mu_{b g}^{(0) *} e^{i\left(\epsilon_{c}^{(2)}-\epsilon_{g}\right) t_{2}-i\left(\epsilon_{c}^{(2)}-\epsilon_{g}\right) t_{1}} e^{-2 i \Phi_{21}} \\
= & \frac{1}{\hbar^{4}} \sum_{c} A_{1}^{2} A_{2}^{2}\left(\sum_{j}\left|\mu_{c j}^{(1)}\right|^{2}\right)\left(\sum_{a b} \mu_{c a}^{(1)} \mu_{a g}^{(0)} \mu_{c b}^{(1) *} \mu_{b g}^{(0) *}\right) e^{i\left(\epsilon_{c}^{(2)}-\epsilon_{g}\right) t_{21}} e^{-2 i \Phi_{21}} .
\end{aligned}
$$

These terms describe the fluorescence signal of double-excited states and can be represented by the Feynman diagram (Ia) in figure 6.2.

To determine the remaining two contributions to the total signal (6.26), we turn to the third order terms,

$$
\left|\Psi^{(3)}\right\rangle=\left(\frac{-i}{\hbar}\right)^{3} \int_{-\infty}^{\infty} d \tau \int_{-\infty}^{\tau} d \tau^{\prime} \int_{-\infty}^{\tau^{\prime}} d \tau^{\prime \prime} \hat{H}_{\mathrm{int}}(\tau) \hat{H}_{\mathrm{int}}\left(\tau^{\prime}\right) \hat{H}_{\mathrm{int}}\left(\tau^{\prime \prime}\right)|g\rangle .
$$

Here, excitation pathways leading to an excited state are comprised of the sequences $E_{i}^{+}(\tau) E_{j}^{+}\left(\tau^{\prime}\right) E_{k}^{+}\left(\tau^{\prime \prime}\right), E_{i}^{+}(\tau) E_{j}^{-}\left(\tau^{\prime}\right) E_{k}^{+}\left(\tau^{\prime \prime}\right)$, and $E_{i}^{-}(\tau) E_{j}^{+}\left(\tau^{\prime}\right) E_{k}^{+}\left(\tau^{\prime \prime}\right)$. The first terms create triple-excited states $\left|e_{m}^{(3)}\right\rangle$. For the fluorescence detection, these terms are later 
combined with first order terms, and, since the resulting coherence is not diagonal in the basis of excited states, this pathway will not contribute to a measurable population in the total signal (6.26). We can further restrict the number of contributions by already taking into account the phase dependence $2 \Phi_{21}$, which is selected from the total signal. The first order terms will be first excited states $\left|e_{n}^{(1)}\right\rangle$ with phase signature $-\Phi_{i}$. Thus, a third-order term will only contribute if it carries the phase signature $2 \Phi_{j}-\Phi_{i}$. This implies that the two excitations ( $E^{+}$terms) must stem from the same pulse $j$ and the de-excitation must be induced by the same pulse $i$ which creates the first excited state in the corresponding first-order term. We thus only need to consider the term

$$
\begin{aligned}
\left|\Psi_{i j}^{(3)}\left(t_{i}, t_{j}\right)\right\rangle=-\left(\frac{-i}{\hbar}\right)^{3} & \sum_{a b c} \mu_{b c}^{(1) *} \mu_{b a}^{(1)} \mu_{a g}^{(0)} \int_{-\infty}^{\infty} d \tau \int_{-\infty}^{\tau} d \tau^{\prime} \int_{-\infty}^{\tau^{\prime}} d \tau^{\prime \prime} \\
& \times E_{i}^{-}(\tau) E_{j}^{+}\left(\tau^{\prime}\right) E_{j}^{+}\left(\tau^{\prime \prime}\right) e^{-i \omega_{b c}^{(1)} \tau} e^{i \omega_{b a}^{(1)} \tau^{\prime}} e^{i \omega_{a g} \tau^{\prime \prime}}\left|e_{c}^{(1)}\right\rangle .
\end{aligned}
$$

In the impulsive limit we obtain

$$
\left|\Psi_{i j}^{(3)}\left(t_{i}, t_{j}\right)\right\rangle=-\left(\frac{-i}{\hbar}\right)^{3} \sum_{a b c} \mu_{b c}^{(1) *} \mu_{b a}^{(1)} \mu_{a g}^{(0)} A_{i} A_{j}^{2} e^{-i \omega_{b c}^{(1)} t_{i}} e^{i \omega_{b a}^{(1)} t_{j}} e^{i \omega_{a g} t_{j}} e^{-i\left(2 \Phi_{j}-\Phi_{i}\right)}\left|e_{c}^{(1)}\right\rangle .
$$

The contribution to the fluorescence light is given by, see equation (6.26),

$$
\begin{aligned}
& \sum_{k}\left|\mu_{k g}^{(0)}\right|^{2}\left\langle e_{k}^{(1)} \mid \Psi_{12}^{(3)}\left(t_{1}, t_{2}\right)\right\rangle\left\langle\Psi_{1}^{(1)}\left(t_{1}\right) \mid e_{k}^{(1)}\right\rangle \\
= & \frac{-i^{4}}{\hbar^{4}} \sum_{a b c}\left|\mu_{b g}^{(0)}\right|^{2} \mu_{b c}^{(1) *} \mu_{c g}^{(0) *} \mu_{b a}^{(1)} \mu_{a g}^{(0)} A_{1}^{2} A_{2}^{2} e^{-i \omega_{b c}^{(1)} t_{1}} e^{i \omega_{b a}^{(1)} t_{2}} e^{i \omega_{a g} t_{2}} e^{-i \omega_{c g} t_{1}} e^{-i 2 \Phi_{21}} \\
= & -\frac{1}{\hbar^{4}} \sum_{b} A_{1}^{2} A_{2}^{2}\left|\mu_{b g}^{(0)}\right|^{2}\left(\sum_{a c} \mu_{b c}^{(1) *} \mu_{c g}^{(0) *} \mu_{b a}^{(1)} \mu_{a g}^{(0)}\right) e^{i\left(\epsilon_{b}^{(2)}-\epsilon_{g}\right) t_{21}} e^{-i 2 \Phi_{21}}
\end{aligned}
$$

where we have used Eqs. (6.20) and (6.41). These terms correspond to the Feynman ladder diagram $(\mathrm{Ib})$ in figure 6.2. Note how the third-order interaction on the 'ket' side and the first order on the 'bra' side of the density matrix can be intuitively read off the diagram. Furthermore, since this pathway also involves a de-excitation, the total pathway occurs with a different sign than the one described by diagram (Ia) and equation (6.38). Moreover, by comparing the two contributions (6.38) and (6.42), we find that both terms coincide when $\left|\mu_{k g}^{(0)}\right|^{2}=\sum_{j}\left|\mu_{k j}^{(1)}\right|^{2}$. This condition is physically satisfied when the decay probability from the second to the first excited-state manifold is equal to the decay probability from the first excited-state manifold into the ground state. This is indeed the case for weakly interacting atomic gases, where the $n$-th excited-state manifold is just characterized by $n$ excited atoms, and, due to the opposing signs of (6.38) and (6.42), the two contributions effectively cancel each other. However, recall that (6.42) is obtained from the fluorescence of first excited states, 

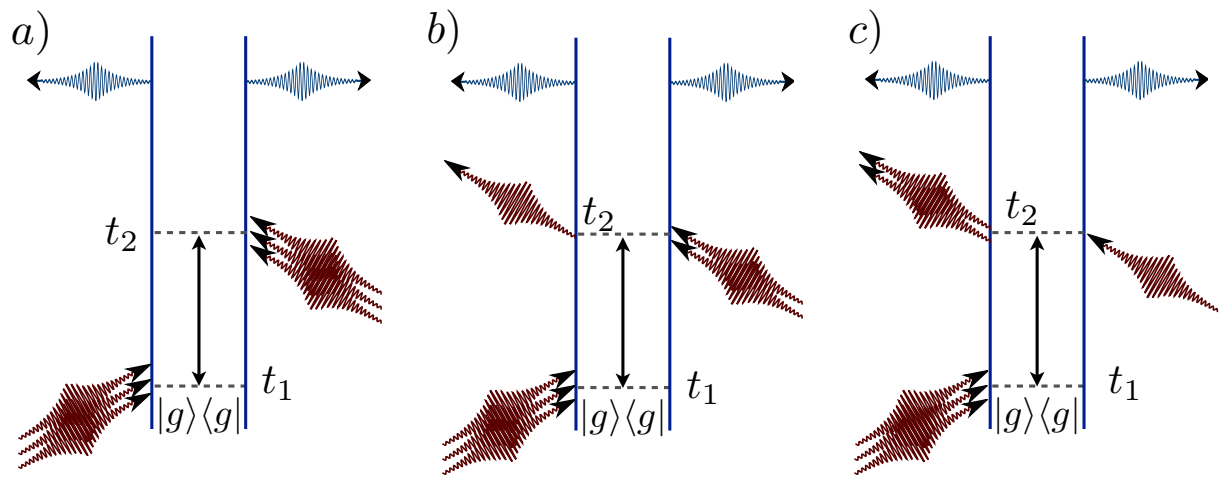

FIGURE 6.3.: Diagrammatic representation of contributions to the third-order quantum coherence signal. Three pathways have to be considered, each one corresponding to the fluorescence light of triple- (a), double- (b), and single (c) excited states, respectively. Note that, according to the third diagram rule, the diagram (b) contributes with a different sign than $(a)$ and $(c)$ to the total signal.

while (6.38) originates from second-excited states. In writing equation (6.26), we assumed that the same number of photons is collected from the decay of all excited states. When working with short-lived excited states (typical dipole-allowed transitions yield lifetimes on the order of few nanoseconds), it is more realistic to assume that higher excited states (of the full atomic ensemble) emit more photons during the detection period, and thus produce a stronger contribution to the fluorescence signal. In fact, these second-order quantum coherences can be measured (Bruder, 2014), and allow to extract the spectrum and the decoherence of the second excited-state manifold.

\subsubsection{Higher-order quantum coherence}

The above methods can be extended to higher orders in perturbation theory and selecting the phase signatures $m \Phi_{21}$. It is convenient to use the diagrammatic theory to determine the relevant pathways and their signs. These are depicted in figure 6.3 for $m=3$. In general, the following diagram rules can generate the entire set of contributing pathways for arbitrary pulse sequences and phase signatures efficiently:

- Left and right side of the ladder diagram describe the interactions of 'ket' and 'bra' side of the density matrix, respectively.

- Excitations (de-excitations) are described by arrows pointing towards (away from) the density matrix. They imprint the phase shift $-\Phi(+\Phi)$ on the 'ket' side of the density matrix, and $+\Phi(-\Phi)$ on the 'bra' side.

- Each de-excitation adds a factor -1 to the overall sign of the diagram.

- To contribute to the fluorescence signal, the diagram must end in a population of an excited state. 
Finally, the total signal is generated by summing up all relevant diagrams, taking into account their relative signs. This can lead to destructive interference and cancellation of pathways. In the context of controllable quantum systems, we will provide a systematic introduction to the diagrammatic representation of pathways, see section 6.3.1. For further background on the diagrammatic method and the theoretical treatment of nonlinear response functions, we refer to the extensive literature on the topic, see for instance references (Mukamel, 1995; Hamm and Zanni, 2011; Brańczyk et al., 2014; Schlawin, 2015).

\subsubsection{Coupling to vacuum modes}

Fluorescence readout, which was assumed throughout the theoretical treatment of this chapter, is particularly useful to probe the populations of long-lived excited states and will be our method of choice to study controllable quantum systems. For short-lived excited states, another possibility is provided by collecting spontaneously emitted photons. In this section, we briefly discuss how the theoretical treatment of the preceding section can be modified to describe the signal obtained by collecting spontaneously emitted photons, instead of externally induced fluorescence light. One major difference between the two scenarios is that fluorescence light involves a macroscopic number of scattered photons whereas spontaneous emission from an ensemble of atoms allows to distinguish between single photons and coincident two-photon processes. To treat emission into the vacuum, we have to replace the classical electromagnetic field $E$ with a quantized operator $\hat{E}$. The Hilbert space, thus, now also contains the field modes, and the Hamilton operator reads (Cohen-Tannoudji et al., 1992; Loudon, 1973)

$$
\hat{H}=\hat{H}_{0}+\hat{H}_{F}+\hat{H}_{\mathrm{int}},
$$

where $\hat{H}_{F}$ denotes the Hamiltonian of the quantized electromagnetic field. We work in the interaction picture with respect to $\hat{H}_{0}+\hat{H}_{F}$.

\subsubsection{Single-photon detection}

We first determine the intensity, as measured, for example, by a photomultiplier tube from independent single-photon events. Assuming that each emitted photon is detected (otherwise we add a factor $\gamma<1$ to rescale), we measure the expectation value of the quantized intensity operator (Loudon, 1973)

$$
S=\int d t \operatorname{Tr}\left\{\hat{E}^{\dagger}(t) \hat{E}(t) \rho(t)\right\}
$$

Here, $\rho(t)$ denotes the density operator of the atomic ensemble and the electromagnetic field, which is determined perturbatively. The interaction-picture operator $\hat{E}(t)$ contains the destruction operators of a continuum of quantized electromagnetic field modes (Loudon, 1973). This expression can be interpreted as the population of the reduced statistical operator describing the single-particle subspace in second quantization. We will encounter similar expressions frequently in chapter 7. Here, we do not 
describe the excitation pathway that leads to the population of certain excited states, which was already done in previous sections. Instead, we are only concerned with the decay of excited states accompanied by photon emission into the vacuum modes. Moreover, we also do not care about the time it takes to emit the photon, hence, our integrated expression reflects the total detected intensity over an infinite time interval. To describe the single-photon signal obtained by decay of a second-excited state into a first-excited state, we assume the initial state to be

$$
\rho_{0}=\left|e_{i}^{(2)}\right\rangle\left\langle e_{j}^{(2)}|\otimes| 0\right\rangle\langle 0|,
$$

where $|0\rangle$ describes the vacuum state of the electromagnetic field. Since, eventually, only populations of at least one photon will yield a signal, the most relevant contribution of a perturbative expansion in terms of the light-matter interactions, expressed by Dyson's series (Sakurai and Napolitano, 1994; Mukamel, 1995), will be the secondorder term:

$$
S \simeq-\frac{1}{\hbar^{2}} \int d t \int_{0}^{t} d \tau \int_{0}^{\tau} d \tau^{\prime} \operatorname{Tr}\left\{\hat{E}^{\dagger}(t) \hat{E}(t)\left[\hat{H}_{\mathrm{int}}(\tau),\left[\hat{H}_{\mathrm{int}}\left(\tau^{\prime}\right), \rho_{0}\right]\right]\right\}
$$

The only contributing terms are those where the atom is de-excited and the field mode gains one photon:

$$
\begin{array}{r}
S \simeq \frac{1}{\hbar^{2}} \int d t \int_{0}^{t} d \tau \int_{0}^{\tau} d \tau^{\prime} \operatorname{Tr}\left\{\hat { E } ^ { \dagger } ( t ) \hat { E } ( t ) \left(\hat{\mu}(\tau) \hat{E}^{\dagger}(\tau) \rho_{0} \hat{\mu}^{\dagger}\left(\tau^{\prime}\right) \hat{E}\left(\tau^{\prime}\right)\right.\right. \\
\left.\left.+\hat{\mu}\left(\tau^{\prime}\right) \hat{E}^{\dagger}\left(\tau^{\prime}\right) \rho_{0} \hat{\mu}^{\dagger}(\tau) \hat{E}(\tau)\right)\right\} .
\end{array}
$$

Inserting the initial state yields terms of the form

$$
\begin{aligned}
\operatorname{Tr}\left\{\hat{E}^{\dagger}(t) \hat{E}(t) \hat{E}^{\dagger}(\tau)|0\rangle\langle 0| \hat{E}\left(\tau^{\prime}\right)\right\} & =\left\langle 0\left|\hat{E}\left(\tau^{\prime}\right) \hat{E}^{\dagger}(t) \hat{E}(t) \hat{E}^{\dagger}(\tau)\right| 0\right\rangle \\
& =\left(\frac{\hbar \omega_{0}}{2 \epsilon_{0} c^{3} V}\right)^{2} \delta\left(t-\tau^{\prime}\right) \delta(t-\tau),
\end{aligned}
$$

to describe the contribution of the light field to the expectation value. In the last step we have used the commutation relations

$$
\left[\hat{E}\left(t_{1}\right), \hat{E}^{\dagger}\left(t_{2}\right)\right]=\frac{\hbar \omega_{0}}{2 \epsilon_{0} c^{3} V} \delta\left(t_{2}-t_{1}\right)
$$

with the quantization volume $V$. Employing the narrow-bandwidth approximation (Loudon, 1973), we assume that the frequencies of emitted photons are close to some central resonance frequency $\omega_{0}$, which determines the energy gap between consecutive excited state manifolds. For the atomic expectation value we obtain

$$
\begin{aligned}
\operatorname{Tr}\left\{\hat{\mu}(\tau)\left|e_{i}^{(2)}\right\rangle\left\langle e_{j}^{(2)}\right| \hat{\mu}^{\dagger}\left(\tau^{\prime}\right)\right\} & =\left\langle e_{j}^{(2)}\left|\hat{\mu}^{\dagger}\left(\tau^{\prime}\right) \hat{\mu}(\tau)\right| e_{i}^{(2)}\right\rangle \\
& =\sum_{k} \mu_{j k}^{(1)} \mu_{i k}^{(1) *} e^{i \omega_{j k}^{(1)} \tau^{\prime}} e^{-i \omega_{i k}^{(1)} \tau} .
\end{aligned}
$$


Inserting this yields

$$
\begin{aligned}
S & \simeq \frac{2}{\hbar^{2}}\left(\frac{\hbar \omega_{0}}{2 \epsilon_{0} c^{3} V}\right)^{2}\left(\sum_{k} \mu_{j k}^{(1)} \mu_{i k}^{(1) *}\right) \int d t e^{i\left(\epsilon_{j}^{(2)}-\epsilon_{i}^{(2)}\right) t} \\
& =4 \pi\left(\frac{\omega_{0}}{2 \epsilon_{0} c^{3} V}\right)^{2}\left(\sum_{k} \mu_{j k}^{(1)} \mu_{i k}^{(1) *}\right) \delta\left(\epsilon_{j}^{(2)}-\epsilon_{i}^{(2)}\right) .
\end{aligned}
$$

As expected, we will only obtain a nonzero signal when $i=j$, that is, when the final state describes a population rather than a coherence. The intensity is further determined by the relevant dipole couplings. Similarly, for the single-photon signal from single-excited states, that is, $\rho_{0}=\left|e_{i}^{(1)}\right\rangle\left\langle e_{j}^{(1)}|\otimes| 0\right\rangle\langle 0|$, we obtain

$$
S \simeq 4 \pi\left(\frac{\omega_{0}}{2 \epsilon_{0} c^{3} V}\right)^{2} \mu_{i g}^{(0)} \mu_{j g}^{(0) *} \delta\left(\epsilon_{j}^{(1)}-\epsilon_{i}^{(1)}\right) .
$$

\subsubsection{Two-photon detection}

For the expectation value of coincident two-photon detection events, we determine (Loudon, 1973)

$$
S=\int d t \operatorname{Tr}\left\{\hat{E}^{\dagger}(t) \hat{E}^{\dagger}(t) \hat{E}(t) \hat{E}(t) \rho(t)\right\}
$$

This expression extends the definition of the intensity operator considered before and represents the populations of the reduced statistical operator describing expectation values of the two-particle subspace. Here, the leading order terms are given by the fourth order perturbation in $\hat{H}_{\text {int }}$. Extending the above analysis to fourth order yields expectation values of the form

$$
\begin{aligned}
& \left\langle 0\left|\hat{E}\left(\tau_{i}\right) \hat{E}\left(\tau_{j}\right) \hat{E}^{\dagger}(t) \hat{E}^{\dagger}(t) \hat{E}(t) \hat{E}(t) \hat{E}^{\dagger}\left(\tau_{k}\right) \hat{E}^{\dagger}\left(\tau_{l}\right)\right| 0\right\rangle \\
= & 4\left(\frac{\hbar \omega_{0}}{2 \epsilon_{0} c^{3} V}\right)^{4} \delta\left(\tau_{i}-t\right) \delta\left(\tau_{j}-t\right) \delta\left(\tau_{k}-t\right) \delta\left(\tau_{l}-t\right),
\end{aligned}
$$

and

$$
\begin{aligned}
& \left\langle e_{\beta}^{(2)}\left|\hat{\mu}^{\dagger}\left(\tau_{i}\right) \hat{\mu}^{\dagger}\left(\tau_{j}\right) \hat{\mu}\left(\tau_{k}\right) \hat{\mu}\left(\tau_{l}\right)\right| e_{\alpha}^{(2)}\right\rangle \\
= & \sum_{m n} \mu_{\beta m}^{(1)} \mu_{m g}^{(0)} \mu_{n g}^{(0) *} \mu_{\alpha n}^{(1) *} e^{i \omega_{\beta m}^{(1)} \tau_{i}} e^{i \omega_{m g} \tau_{j}} e^{-i \omega_{n g} \tau_{k}} e^{-i \omega_{\alpha n}^{(1)} \tau_{l}}
\end{aligned}
$$

We obtain

$$
\begin{aligned}
S & \simeq \frac{4}{\hbar^{4}}\left(\frac{\hbar \omega_{0}}{2 \epsilon_{0} c^{3} V}\right)^{4}\left(\sum_{m n} \mu_{\beta m}^{(1)} \mu_{m g}^{(0)} \mu_{n g}^{(0) *} \mu_{\alpha n}^{(1) *}\right) \int d t e^{i\left(\epsilon_{\beta}^{(2)}-\epsilon_{g}\right) t} e^{-i\left(\epsilon_{\alpha}^{(2)}-\epsilon_{g}\right) t} \\
& =8 \pi\left(\frac{\omega_{0}}{2 \epsilon_{0} c^{3} V}\right)^{4}\left(\sum_{m n} \mu_{\beta m}^{(1)} \mu_{m g}^{(0)} \mu_{n g}^{(0) *} \mu_{\alpha n}^{(1) *}\right) \delta\left(\epsilon_{\beta}^{(2)}-\epsilon_{\alpha}^{(2)}\right) .
\end{aligned}
$$




\subsubsection{Interpretation}

In this section, we discussed higher-order quantum coherence signals using the example of atomic vapor. The described nonlinear measurement protocols are able to reveal the energies of specific excited state manifolds beyond single excited states with a twopulse measurement scheme. It further allows to monitor the time evolution of these coherences, and their environment-induced decay.

If resolution permits, one could infer about the strength of atom-atom interactions and collective effects from the width of an excitation band. However, at low densities such shifts are typically weak and may be hard to detect. The present two-pulse method can be extended to a four-pulse sequence which is able to reveal such collective resonances using the double quantum coherence pulse sequence (Dai et al., 2012). This scheme is similar to the second-order quantum coherence scheme discussed above, however, with the crucial difference that the time delays between each of the four interactions can be scanned. Separating the third and fourth interaction allows to design a measurement scheme which only contributes if degeneracies are lifted, for instance by collective resonances. We will discuss a fluorescence-based analogue of this method in the context of controllable quantum systems.

\subsection{Diagrammatic description of nonlinear spectroscopic experiments of controllable quantum systems}

After discussing a simple nonlinear spectroscopic measurement scheme for atomic vapor, we now turn towards our principal goal, which is the development of efficient and scalable probing methods to assess spectral and dynamical features of controllable, many-body quantum optical systems. Atomic vapor represents a quantum optical sample system which is comprised of a macroscopic number of constituents. Experiments with cold atoms on optical lattices and especially with trapped ions are often carried out on samples which are not optically dense enough to detect absorption spectra or direct photon scattering events. The standard methods of nonlinear spectroscopy must therefore be adjusted. We have already seen that fluorescence readout, which is routinely implemented, for instance, with trapped ions, can provide a promising method to measure the population at the end of a pulse sequence. Furthermore, acousto-optical modulators allow to control and change the phases of incoming excitation pulses, which allows to perform phase cycling. Furthermore, in many cases, single-site addressability may be exploited to add spatial resolution to the measured time-correlation functions. This feature is usually not available in bulk materials, and may enable us to extract further information about the system at hand.

\subsubsection{Basic elements for the design of nonlinear measurement schemes}

We start by formally introducing the basic building blocks, which are required to design an arbitrary pulse sequence to perform a nonlinear spectroscopic experiment. The basic principle of such experiments is illustrated in figure 6.4. The system is probed with a sequence of phase-coherent pulses, and the signal is collected as a function of the 


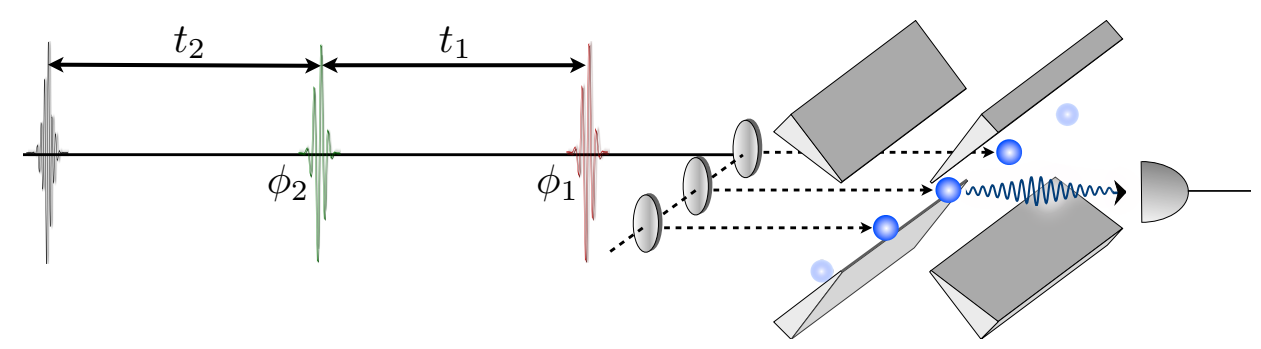

FIGURE 6.4.: Experimental scheme for a two-pulse nonlinear measurement protocol. Two pulses with phases $\Phi_{1}$ and $\Phi_{2}$ are applied to a controllable quantum system. The pulses can be applied to the collection of all atoms in the sample, or by employing strongly focussed laser beams, they can be targeted onto specific local sites. After the pulse sequence, the fluorescence light is collected. The experiment is repeated many times while the time delays $t_{1}$ and $t_{2}$ are scanned. Figure taken from (Schlawin et al., 2014).

time delays between these pulses. The phases are then used to extract the contribution of individual pathways via phase-cycling. The figure further suggests the possibility to induce localized excitations using tightly focussed laser beams, which is not essential but can open up additional possibilities. One may picture such an experiment as an interference experiment in which each pulse creates or probes excitations in the system, close in spirit to wave packet interferometry (Fourkas et al., 1989; Scherer et al., 1991).

The essential building blocks of nonlinear spectroscopic experiments on controllable quantum systems are (Gessner et al., 2014c; Schlawin et al., 2014),

- interactions of the system with a well-controlled external field,

- the time evolution of the system,

- readout schemes,

- phase cycling.

\subsubsection{Externally induced excitations and de-excitations}

We describe the interaction of the system with an external field in the impulsive limit, which means that we assume the duration of external pulses to be much shorter than typical time scales of the system dynamics. This allows us to avoid treating the electromagnetic fields explicitly as in the previous section, and we can effectively describe the action of these pulses in terms of creation and annihilation operators of the system only. This is realized by a transition operator which, in general, can be written as

$$
V_{i}(\phi)=\alpha \mathbb{I}+\beta e^{i \phi} A_{i}^{\dagger}+\gamma e^{-i \phi} A_{i}
$$




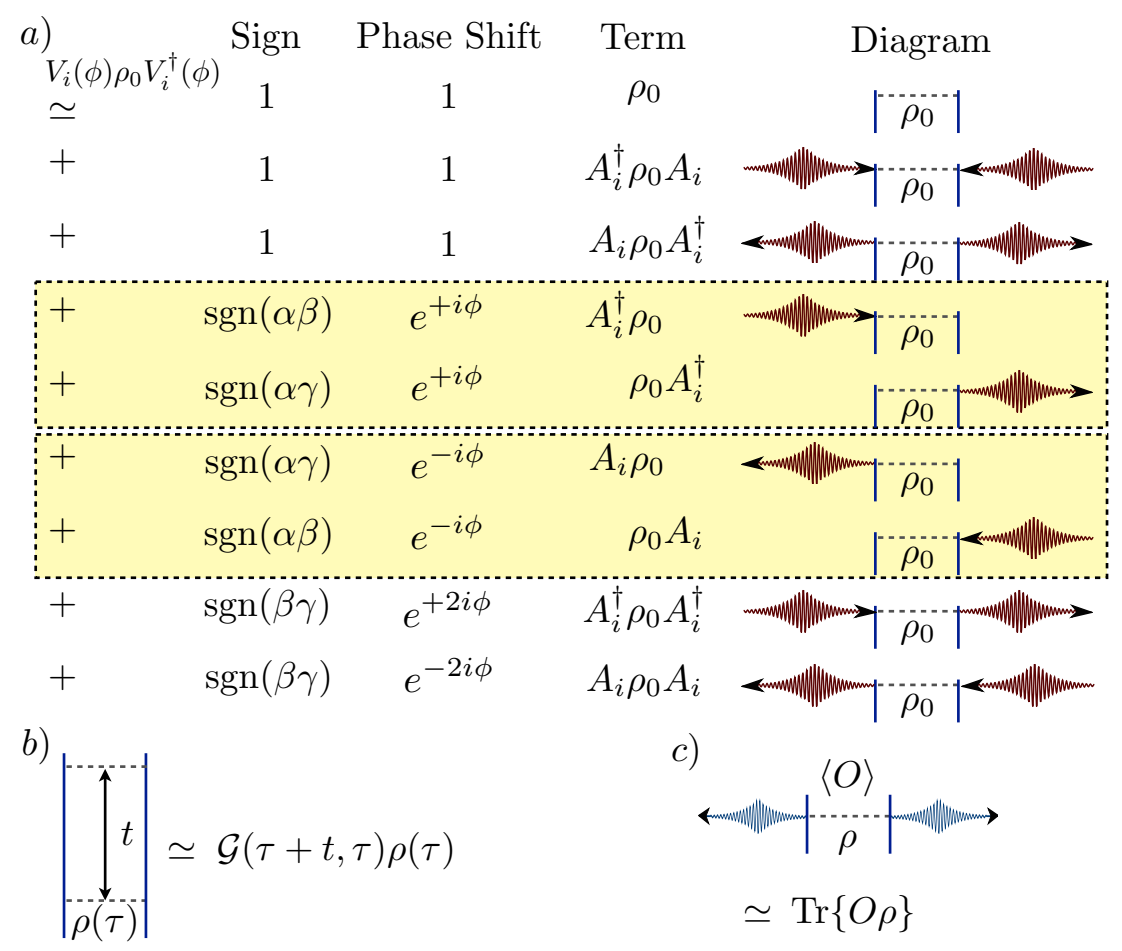

FiguRE 6.5.: a) Elementary excitation and de-excitation processes in a single interaction event. Red arrows pointing towards and away from the 'bra' and 'ket' sides of the quantum state represent excitations and de-excitations, respectively. The different terms contribute with different signs and can be grouped in terms of the phase shift, which is used for phase-cycling. b) Time-evolution without external interactions is represented in the upwards direction. c) Readout of populations is represented by two blue arrows. Adapted from (Schlawin et al., 2014).

where $A_{i}$ is the annihilation operator of an arbitrary mode of the system, labeled by $i$. This mode can be any excitation in the system which can be addressed by external control, for example, localized or collective vibrational excitations, or excitations of electronic states. Higher-order excitations are also possible, for instance, when $A_{i}$ represents a displacement operator. For now, we treat $A_{i}$ as an abstract, unspecified operator whereas examples and possible experimental implementations will be discussed later in great detail. The phase $\phi$ is a property of the pulse that induces this transition. During interaction events, this phase shift is imprinted onto the quantum state of matter, which is crucial for phase cycling. The real-valued parameters $\alpha, \beta$ and $\gamma$ depend on the properties of the applied pulses. For example, weak perturbative pulses are characterized by $\beta \sim \gamma \ll 1$, while strong pulses with well-defined duration are described by $\beta=\gamma$ with $\alpha^{2}+\beta^{2}=1$.

To develop a diagrammatic representation of the elementary excitation and de- 
excitation events, we group the terms that describe the possible transitions by their net phase shift. Consider the system to be described by the density matrix $\rho_{0}$ before the interaction event. The induced transitions are described by the superoperator

$$
\begin{aligned}
\mathcal{V}_{i}(\phi) \rho_{0}= & V_{i}(\phi) \rho_{0} V_{i}^{\dagger}(\phi) \\
= & \alpha^{2} \rho_{0}+\beta^{2} A_{i}^{\dagger} \rho_{0} A_{i}+\gamma^{2} A_{i} \rho_{0} A_{i}^{\dagger}+\alpha e^{-i \phi}\left(\beta \rho_{0} A_{i}+\gamma A_{i} \rho_{0}\right) \\
& +\alpha e^{i \phi}\left(\gamma \rho_{0} A_{i}^{\dagger}+\beta A_{i}^{\dagger} \rho_{0}\right)+e^{-2 i \phi} \gamma \beta A_{i} \rho_{0} A_{i}+e^{2 i \phi} \beta \gamma A_{i}^{\dagger} \rho_{0} A_{i}^{\dagger} .
\end{aligned}
$$

These contributions are depicted in figure 6.5 a). Excitations are represented by red arrows pointing towards the 'ket' or 'bra' side of the density matrix, while de-excitations are denoted by arrows pointing away from the quantum state. The figure also lists the corresponding signs, which must be considered when various diagrams add up to yield the total signal. Finally, the different phase shifts allow to distinguish groups of interaction events via phase-cycling. Note that for transitions of the form (6.57), the maximal phase shift is $\pm 2 \phi$. For perturbative pulses, the terms with the largest phase shift can also be neglected since $\beta \sim \gamma \ll 1$ and $\alpha \sim 1$. This reduces the required overhead for phase cycling significantly, since distinguishing terms with phase shift 0 or $\pm \phi$ is possible by repeating the experiment with no more than three sets of phases, as was shown in equation (6.25).

\subsubsection{Time evolution and readout}

The time evolution of the system without interactions with the external probing fields is governed by the system Hamiltonian, and possibly, by dissipative processes caused by couplings of the system to its environment. In general, we describe the time evolution from $\tau_{a}$ to $\tau_{b}$ by a propagator (Mukamel, 1995)

$$
\mathcal{G}\left(\tau_{b}, \tau_{a}\right)=\mathcal{T} \exp \left[\int_{\tau_{a}}^{\tau_{b}} d t \mathcal{L}(t)\right],
$$

with the Liouvillian $\mathcal{L}(t)$. In the diagrammatic representation, the time axis evolves upwards, see figure $6.5 \mathrm{~b}$ ).

Finally, to collect the signal a readout mechanism must be identified. In general, we describe this by the measurement of the expectation value of an arbitrary operator at the end of the pulse sequence, which is realizable with significant flexibility in quantum optical systems. Diagrammatically, this is represented by two outgoing blue arrows at the end of the sequence, see figure $6.5 \mathrm{c}$ ).

\subsubsection{Design of a pulse-sequence}

To probe an $n$-point time-correlation function of the system, a pulse sequence is designed by specifying a series of $n$ transition operators $\mathcal{V}_{i_{1}}, \ldots, \mathcal{V}_{i_{n}}$ and a readout observable $O_{j}$. The appropriate pulses are then applied with tunable time delays $t_{1}, \ldots, t_{n}$ 


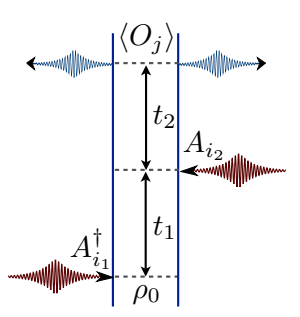

$L R$

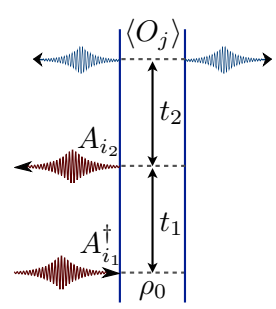

$L l$

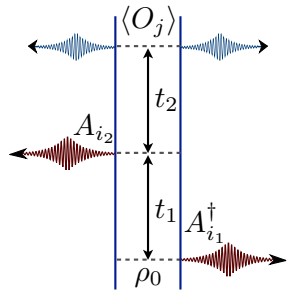

$r l$

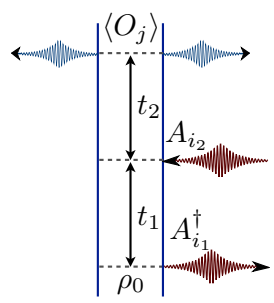

$r R$

FIGURE 6.6.: The four diagrams which comprise the single quantum coherence signal. To label the diagrams, excitations on the 'ket' ('bra') side are described by capital letters $L(R)$, while de-excitations are indicated by the respective small letters $l(r)$. Adapted from (Schlawin et al., 2014).

in between them. The initial density matrix $\rho_{0}=\rho\left(\tau_{0}\right)$ hence evolves into

$$
\rho_{i_{1}, \ldots, i_{n}}^{(n)}\left(t_{1}, \ldots, t_{n}\right)=\prod_{k=1}^{n}\left[\mathcal{G}\left(\tau_{k}, \tau_{k-1}\right) \mathcal{V}_{i_{k}}\left(\phi_{k}\right)\right] \rho\left(\tau_{0}\right),
$$

where $t_{i}=\tau_{i}-\tau_{i-1}$. This evolution is probed by measuring the observable $O_{j}$, leading to the signal

$$
S_{i_{1}, \ldots, i_{n} ; j}^{(n)}\left(t_{1}, \ldots, t_{n}\right)=\operatorname{Tr}\left\{O_{j} \rho_{i_{1}, \ldots, i_{n}}^{(n)}\left(t_{1}, \ldots, t_{n}\right)\right\} .
$$

Depending on the choice of excitation and readout pulses, this $n$-point time-correlation function of the system can reveal specific properties of the system, such as internal couplings and transport processes, as well as environmental effects on the dynamics. We will discuss a series of examples at the end of this chapter.

Let us consider the example of a two-pulse measurement scheme. We extend the measurement scheme which was discussed in section 6.2 .4 by allowing for another time evolution between the last pulse and the readout. The general single quantum coherence signal is obtained by applying the two pulses with phases $\phi_{1}$ and $\phi_{2}$, respectively, and extracting only those terms which carry the phase shift $\phi_{1}-\phi_{2}$ via phase cycling; see section 6.2.5 (Gessner et al., 2014c). The corresponding interaction events create coherent superpositions and are highlighted in yellow in figure 6.5. Assuming an observable $O_{j}$ which measures populations of those modes which are created by the excitation operators $A_{i_{k}}^{\dagger}$, the four diagrams depicted in figure 6.6 contribute to the signal.

If the initial state $\rho_{0}=|0\rangle\langle 0|$ contains no excitations and if the ground state does not contribute to the signal, $\left\langle 0\left|O_{j}\right| 0\right\rangle=0$, one can restrict to the left-most diagram, denoted $L R$. This can be seen easily using the diagrammatic representation: The two diagrams $r l$ and $r R$ involve a de-excitation in the first pulse, which causes them to drop out when the initial state cannot be further de-excited. Moreover, the diagram $\mathrm{Ll}$ is first excited and subsequently de-excited on the 'ket' side, while the 'bra' side 
does not experience interactions, such that the diagram ends in a population of the ground state, which does not contribute either.

\subsection{Excitation and readout schemes for trapped ions}

After introducing the formal principles and constituents of nonlinear measurement protocols in the previous section (Gessner et al., 2014c), we now turn to the discussion of the actual physical implementation of these ideas (Schlawin et al., 2014). In this section we develop specific methods to realize transitions of the form (6.57) with current experimental trapped-ion technology. We also discuss possibilities to read out excitations and address both the electronic and vibrational degrees of freedom, equipping us with a flexible set of tools to probe the dynamics of ion crystals using nonlinear spectroscopy. Trapped-ion experiments were discussed in detail in section 2.2.1. Even though nonlinear measurement protocols can yield valuable information on the eigenmode dynamics, in this secion, we make explicit use of the local addressing of individual ions, which allows us to study non-equilibrium transport processes. Different methods allow for the addressing of individual sites in an ion chain. We will mostly consider tightly focussed lasers (Schindler et al., 2013), which may be redirected by a controllable acousto-optic deflector. Other possibilities include spatially dependent qubit resonance frequencies induced by the Zeeman effect in a magnetic field gradient (Mintert and Wunderlich, 2001; Johanning et al., 2009; Ospelkaus et al., 2011), or by resorting to different ion species which can be distinguished spectrally (Schneider et al., 2012).

\subsubsection{Electronic degree of freedom: Dynamics}

Let us briefly recall different models which describe the dynamics of electronic excitations in a Coulomb crystal under appropriate conditions, as was already discussed in section 2.2.1.7. The large inter-ion separation on the order of $\sim 5 \mu \mathrm{m}$ leads to negligibly small dipole-dipole couplings between the ions. Effective spin-spin interactions can, however, be mediated using lasers that couple the electronic states to the common modes of motion. This allows to induce interactions of the form

$$
H_{\mathrm{MS}}=\frac{\hbar \Omega}{2} \sigma_{x}^{(1)} \otimes \sigma_{x}^{(2)}
$$

by appropriate driving of sideband transitions (Sørensen and Mølmer, 1999). Such interactions can realize entangling gates which form a basic building block of quantum algorithms. This can be pushed further to generate spin-spin interactions throughout the entire chain, described by the long-range spin-chain model which was subject of chapter 5 (Porras and Cirac, 2004b),

$$
H_{\mathrm{sc}}=-\sum_{\substack{i, j=1 \\(i<j)}}^{N} \frac{J_{0}}{|i-j|^{\alpha}} \sigma_{x}^{(i)} \sigma_{x}^{(j)}-B \sum_{i=1}^{N} \sigma_{y}^{(i)} .
$$


For further possibilities, details and background on quantum simulations with trapped ions we refer to references (Schneider et al., 2012; Blatt and Roos, 2012).

\subsubsection{Electronic degree of freedom: Excitation and readout}

The electronic states of trapped ions are routinely controlled with highest precision to realize methods from quantum information and quantum computations (Leibfried et al., 2003a; Häffner et al., 2008; Schindler et al., 2013). As was shown in equation (2.11), application of a pulse of duration $t$ and phase $\varphi$ on the carrier transition induces a transition described by the unitary operator

$$
U_{\varphi}^{c}(t)=\cos \left(\frac{\Omega t}{2}\right) \mathbb{I}-i \sin \left(\frac{\Omega t}{2}\right)\left(e^{i \varphi} \sigma_{+}+e^{-i \varphi} \sigma_{-}\right) .
$$

A $\pi / 2$-pulse is characterized by $\Omega t=\pi / 2$, which leads to $\cos (\Omega t / 2)=\sin (\Omega t / 2)=$ $1 / \sqrt{2}$. Assuming that this pulse is applied with a spatially focussed laser onto the ion at site $i$, we can cast this transition into the form of equation (6.57) by identifying

$$
\phi=\varphi-\pi / 2, \quad A_{i}=\sigma_{-}^{(i)}, \quad \alpha=\beta=-\gamma=1 / \sqrt{2}
$$

In the development of the general formalism, we have assumed the impulsive limit, which requires that the interactions with the external control field happen on faster time scales than those of the relevant system dynamics. With sufficient laser intensity, carrier pulses can be applied within less than one microsecond, while typical time scales of the trapped-ion dynamics are tens to hundreds of microseconds, depending on the experimental parameters (Häffner et al., 2008; Schneider et al., 2012; Blatt and Roos, 2012). If these do not permit to apply such strong pulses with well-defined durations this does, however, not limit the possibilities to realize nonlinear spectroscopy experiments: Recall that by phase-cycling we essentially post-select those events in which the desired sequence of excitations occurs. For pulse schemes as the single quantum coherence, the yield of processes involving these events is maximized by applying $\pi / 2$ pulses. In order to ensure that the induced excitations are applied on fast time-scales, one can employ shorter pulses at the cost of reduced usable signal yield. This also manifests in the fact that the final signal does not depend on the actual values of the coefficients $\alpha, \beta$ and $\gamma$, assuming they are nonzero.

Readout of electronic observables is typically implemented using the electron shelving method (Leibfried et al., 2003a), which provides detection efficiencies close to unity, and corresponds to measurement of the observable $O_{j}=\sigma_{z}^{(j)}$; see also section 2.2.1.5. Combination of this fluorescence-based readout scheme with unitary operations of the form (6.64) allow for the measurement of arbitrary spin observables.

\subsubsection{Vibrational degree of freedom: Dynamics}

The motional degrees of freedom of linear chains of trapped ions were discussed in section 2.2.1.3. We describe the common motion in terms of local phonons, using 
equation (2.6) (James, 1998), and include the effect of nonlinear corrections (2.16) yielding the Hamiltonian (Porras and Cirac, 2004a)

$$
H_{\mathrm{ph}}=\sum_{i=1}^{N} \hbar \omega_{i}^{0} a_{i}^{\dagger} a_{i}+\sum_{\substack{i, j=1 \\(i<j)}}^{N} \hbar t_{i j}\left(a_{i}^{\dagger} a_{j}+a_{j}^{\dagger} a_{i}\right)+U \sum_{i=1}^{N} a_{i}^{\dagger 2} a_{i}^{2} .
$$

Microscopic expressions for the local frequencies $\omega_{i}^{0}$ and inter-site couplings $t_{i j}$ were given in equation (2.7). The nonlinear term $U$ may be attributed to higher-order terms of the trap potential, which become especially relevant when the ratio of axial and radial trap frequencies $\beta_{0}=\left(\nu_{z} / \nu_{x}\right)^{2}$ is no longer small. When $\beta_{0} \ll 1$, one may induce nonlinear correction terms under well-controlled conditions using a standing laser wave or a cavity; see section 2.2.1.3.

Throughout the remainder of this section, we will show various theoretical simulations of the dynamics induced by the Hamiltonian $H_{\mathrm{ph}}$, with $\beta_{0}=0.1$, for different $N$ and $U$, employing a python code based on the qutip package (Johansson et al., 2013).

Similarly to our description of the Hamiltonian of atomic vapor, and close in spirit to quantum chemistry, we label different excitation manifolds by the number of excited local phonons. In particular, we introduce the single excited states

$$
\left|e_{i}\right\rangle=\sum_{j=1}^{N} c_{i j} a_{j}^{\dagger}|0\rangle
$$

and the double excited states

$$
\left|f_{i}\right\rangle=\sum_{j, k=1}^{N} d_{i j k} a_{j}^{\dagger} a_{k}^{\dagger}|0\rangle
$$

These are formed by coherent superpositions of states containing one or two local phonons, respectively, and involve one or two applications of the creation operator on the ground state $|0\rangle$, which contains no phonons.

\subsubsection{Vibrational degree of freedom: Excitation}

Laser pulses can be used to coherently drive sideband transitions, which couple the electronic degree of freedom to a resonant common mode of motion. This allows for the controlled excitation of global phonons in the system. Moreover, individual resolution of ions, and intense, tightly focussed laser pulses allow for the creation of local nonstationary vibrational excitations in the system (Harlander et al., 2011; Brown et al., 2011), which open up new possibilities for studies of energy transport through an ion chain (Haze et al., 2012; Ramm et al., 2014).

In order to excite a local phonon, a sideband pulse must be realized faster than the time scale of phonon hopping. We have seen in Eqs. (2.12) and (2.13), or (3.33), that the time scale of first-order sideband transitions is given by $\eta \Omega$, where $\Omega$ is determined by the laser intensity. Furthermore, the time scale of the local phonon dynamics 

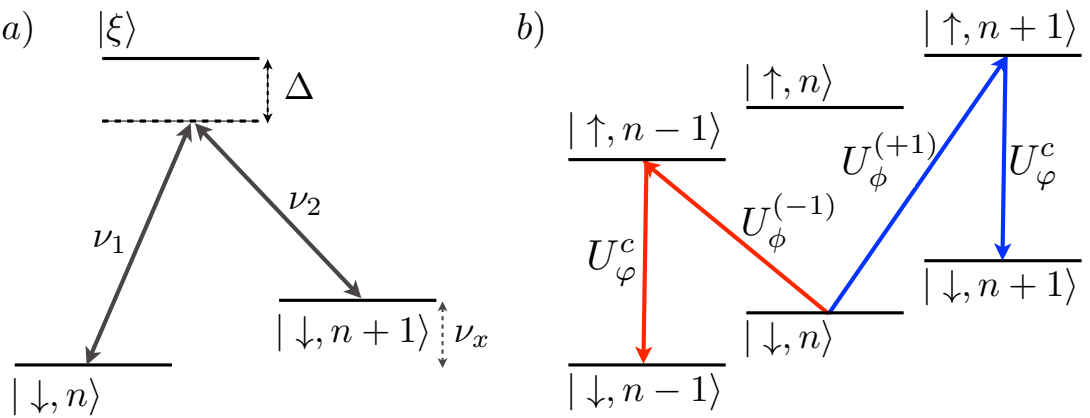

Figure 6.7.: a) A coherent displacement operation can be induced using a bichromatic, offresonant, stimulated Raman scheme. b) Combinations of sideband and carrier pulses allow to selectively create or destroy phonons. Adapted from (Schlawin et al., 2014).

is determined by the parameter $\beta_{0}$, see equation (2.7). Thus the creation of local phonons requires $\eta \Omega \gg \beta_{0} \nu_{x}$. The parameter $\beta_{0}$ can be adjusted via the ratio of axial and radial trap frequencies. This can even be realized adiabatically to change the time- and energy scales within the same experiment (Haze et al., 2012).

The following methods to excite phonons are rather general, and do not depend on whether one considers a global beam, which excites common excitations of all ions, or whether the above conditions are satisfied and a single ion is addressed with fast pulses to create local vibrational excitations. We first discuss a method based on stimulated Raman pulses, before we proceed to consider combinations of sideband pulses to excite phonons.

\subsubsection{Off-resonant stimulated Raman pulses}

Experiments with hyperfine qubits routinely employ Raman pulses to manipulate the qubit state. Such pulses are similar to those employed to probe molecular systems (Hamm and Zanni, 2011) and can also be applied to induce motional excitations. To this end, consider a combination of two lasers off-resonantly addressing a short-lived excited state $|\xi\rangle$, such that their frequency difference matches the chosen vibrational transition, for instance the local phonon frequency of the ion in the laser focus,

$$
\nu_{1}-\nu_{2}=\nu_{x},
$$

as depicted in figure 6.7 a). The detuning $\Delta$ is chosen sufficiently large, such that the population of the state $|\xi\rangle$ can be neglected and this level can be adiabatically eliminated (Heinzen and Wineland, 1990; Leibfried et al., 2003a). This leads to an effective coupling between the two vibrational levels $|n\rangle$ and $|n+1\rangle$ given by (Leibfried et al., 2003a)

$$
(\hbar / 2) \Omega_{\mathrm{eff}}=-\hbar \frac{\Omega_{n, \xi} \Omega_{n+1, \xi}}{\Delta} e^{i \phi},
$$


where $\Omega_{n, \xi}$ and $\Omega_{n+1, \xi}$ denote the couplings of the ground states (we assume the electronic degree of freedom to be in the ground state $|\downarrow\rangle)$ to the excited state $|\xi\rangle$ and the phase shift $\phi$ can be controlled through the phases of the two individual laser pulses. This process generates a displacement operation on the addressed phonon mode (Monroe et al., 1996). If, for instance, the local phonon mode at site $j$ is addressed, this is described by

$$
D_{j}\left(\epsilon e^{i \phi}\right)=e^{\epsilon e^{i \phi} a_{j}^{\dagger}-\epsilon e^{-i \phi} a_{j}} .
$$

The magnitude $\epsilon=\eta \Omega_{\text {eff }} t$ of the displacement is controlled by the intensity and duration of the pulse. In the limit of weak, perturbative pulses, $\epsilon \ll 1$, we can expand this operator to linear order, leading to

$$
D_{j}\left(\epsilon e^{i \phi}\right)=\mathbb{I}+\epsilon e^{i \phi} a_{j}^{\dagger}-\epsilon e^{-i \phi} a_{j}+\mathcal{O}\left(\epsilon^{2}\right) .
$$

This reduces to the general transition operator (6.57) with the parameters $\alpha \approx 1$ and $\beta=-\gamma=\epsilon \ll 1$ (Schlawin et al., 2014).

\subsubsection{Resonant sideband pulses}

Instead of the off-resonant Raman scheme one may also employ pulses which involve the electronic degree of freedom. These can also be induced using a bichromatic Raman transition, when the qubit is encoded in a radiofrequency transition. Alternatively, optical qubits can be manipulated directly using visible (or near-visible) laser frequencies; see section 2.2.1. In this section, we discuss the controlled, selective excitation or de-excitation of vibrations based on sideband pulses.

We consider the following protocol to create a vibrational excitation (Schlawin et al., 2014):

(i) Before the first pulse, the addressed ion is assumed to be in the electronic ground state, that is, we start in the state $|\downarrow, n\rangle$. The first pulse is applied on the first blue sideband with duration $t_{1}$ and phase $\phi$ which, according to equation (3.42) with $m=1$, generates the superposition

$$
U_{\phi}^{(+1)}\left(t_{1}\right)|\downarrow, n\rangle=\cos \left(\frac{\Omega_{n}^{(1)} t_{1}}{2}\right)|\downarrow, n\rangle+e^{i \phi} \sin \left(\frac{\Omega_{n}^{(1)} t_{1}}{2}\right)|\uparrow, n+1\rangle .
$$

(ii) Next, we apply a pulse on the carrier transition of duration $t_{2}$ and phase $\varphi$, as described by equation (6.64). The combination of the two pulses generates the 
state

$$
\begin{aligned}
U_{\varphi}^{c}\left(t_{2}\right) U_{\phi}^{(+1)}\left(t_{1}\right)|\downarrow, n\rangle= & \cos \left(\frac{\Omega_{n}^{(1)} t_{1}}{2}\right) \cos \left(\frac{\Omega t_{2}}{2}\right)|\downarrow, n\rangle \\
& +e^{i \phi} \sin \left(\frac{\Omega_{n}^{(1)} t_{1}}{2}\right) \cos \left(\frac{\Omega t_{2}}{2}\right)|\uparrow, n+1\rangle \\
& -i e^{i \varphi} \cos \left(\frac{\Omega_{n}^{(1)} t_{1}}{2}\right) \sin \left(\frac{\Omega t_{2}}{2}\right)|\uparrow, n\rangle \\
& -i e^{-i \varphi} e^{i \phi} \sin \left(\frac{\Omega_{n}^{(1)} t_{1}}{2}\right) \sin \left(\frac{\Omega t_{2}}{2}\right)|\downarrow, n+1\rangle .
\end{aligned}
$$

This induced transition is depicted by blue arrows in figure $6.7 \mathrm{~b}$ ).

This pulse combination creates a phonon, but the ion's electronic state now contains a contribution of the excited electronic state $|\uparrow\rangle$. Thus, another pulse of the same form may de-excite a phonon, instead of inducing another excitation. As a consequence, without further modification this sequence effectively produces a combination of excitation and de-excitation operators, as in the Raman scheme, equation (6.72).

To selectively excite or de-excite a phonon in a multi-pulse sequence based on the above pulse combination, the population of $|\uparrow\rangle$ must become invisible for subsequent pulses on the carrier and sideband transition. We now discuss two alternatives to achieve this, at the hand of the specific example of the ${ }^{40} \mathrm{Ca}^{+}$level scheme, which was introduced in section 2.2.1.4. Both alternatives involve coherent transitions to other long-lived electronic levels. ${ }^{4}$ Recall from figure $2.3 \mathrm{~b}$ ) that the $|\uparrow\rangle$-state is arbitrarily chosen as one of the six Zeeman sublevels of the $D_{5 / 2}$-state manifold, while the $|\downarrow\rangle$ state is one of the two $\mathrm{S}_{1 / 2}$ levels.

The first alternative is to address a different electronic transition, that is, one encodes the $|\uparrow\rangle$-state into another electronic level for each of the excitations that are induced during the nonlinear measurement protocol. This way, given that there are six different states in the excited $\mathrm{D}_{5 / 2}$-state manifold, sequences of up to six pulses can be realized. This is no restriction since the most convenient nonlinear measurements, to be discussed in section 6.5, only require up to four pulses. One must, however, be careful to leave some population in the ground state after each of the pulses, and avoid accidental resonances between the different carrier and sideband transitions, for example by applying a large magnetic field.

The second alternative allows to use the same electronic transition for all of the excitations in the measurement protocol, but requires additional pulses to hide the $|\uparrow\rangle$-state population from subsequent excitations. These pulses coherently transfer the electronic $|\uparrow\rangle$-state population to another electronic state which is not coupled by

\footnotetext{
${ }^{4}$ Note that in (Schlawin et al., 2014), a method based on incoherent optical pumping of the $|\uparrow\rangle$ state to the $|\downarrow\rangle$-state was suggested. This process, however, is not suitable since it destroys the coherence of the motional degree of freedom, which is required to realize the phase cycling protocol.
} 
the employed electronic transitions of steps (i) and (ii). For each pulse of this form, the $|\uparrow\rangle$-state population must be hidden in a different level. Hence, this recipe can be applied to an $n$-pulse sequence if at least $n$ long-lived levels are available which do not respond to the carrier $|\downarrow\rangle \rightarrow|\uparrow\rangle$ transition and the corresponding sideband transition. In the $\mathrm{D}_{5 / 2}$-state manifold, figure $2.3 \mathrm{~b}$ ), we identify four such levels: For the first three pulses, the population can be hidden in the $+1 / 2,+3 / 2,+5 / 2$ states (by going through the $\mathrm{S}_{+1 / 2}$ ground state), while for the final pulse, it is sufficient to transfer the population to the $S_{+1 / 2}$ ground state. Moreover, if coherent laser access is provided to the $\mathrm{D}_{3 / 2}$-state manifold, four additional long-lived electronic levels are available.

After discarding the excited-state population using either one of the two alternatives, the state is effectively described by

$$
\begin{aligned}
\left|\Psi_{f}\right\rangle \simeq|\downarrow\rangle \otimes & \left(\cos \left(\frac{\Omega_{n}^{(1)} t_{1}}{2}\right) \cos \left(\frac{\Omega t_{2}}{2}\right)|n\rangle\right. \\
& \left.-i e^{i(\phi-\varphi)} \sin \left(\frac{\Omega_{n}^{(1)} t_{1}}{2}\right) \sin \left(\frac{\Omega t_{2}}{2}\right)|n+1\rangle\right) .
\end{aligned}
$$

Hence, the desired transition can be identified in a phase cycling scheme by the signature $\phi-\varphi$. This further allows to keep either one of the two phases fixed, and scan the other for phase cycling.

We did not specify the pulse durations $t_{1}$ and $t_{2}$, considered in the above pulse scheme. This, again, is not essential, since, after phase-cycling, the signal does not depend on these parameters. For the same reason we also did not explicitly account for the loss of electronic population by hiding the $|\uparrow\rangle$-state population from subsequent pulses. To maximize the usable signal yield, which in the above case corresponds to the contributions of the phase-dependent coherences, one would employ $\pi / 2$-pulses on the sideband and on the carrier. The slowest of the three above processes is the sideband pulse, step (i), since $\Omega_{n}^{(1)} \approx \eta \sqrt{n+1} \Omega$, see equation (3.33), where the Lamb-Dicke factor $\eta$, defined in equation (2.9), is typically of the order of $1 \%$, and $\Omega$ determines the time scale of the carrier transition, step (ii). If one chooses to transfer the excited state population to another long-lived state (the second alternative discussed above), the required pulses would also be realized on the same time scale as the carrier transition. Thus, in order to apply the above sequence of pulses on a local sideband, we need to comply with the condition $2 \eta \Omega / \pi \gg \beta_{0} \nu_{x}$.

The combination of the three processes can then be approximately described by an instantaneous transition of the form

$$
V_{+}=\alpha \mathbb{I}+\beta e^{i \phi} A^{\dagger},
$$

which has the shape of equation (6.57) with $\gamma=0$, allowing to selectively create an excitation, without leaving the possibility to de-excite the system with the same pulse. Such transitions are not available for the studies of molecular or solid-state systems and demonstrate the high degree of flexibility which is provided by quantum optical 
experiments on artificial, synthetic systems. The operator $A^{\dagger}$ can either describe the excitation of a local phonon $a_{j}^{\dagger}$, but may also induce global collective excitations when the above pulse sequence is applied on slow time scales involving sidebands of the common motional eigenmodes, such as the center of mass mode $A_{\mathrm{cm}}^{\dagger}=a_{1}^{\dagger}+\cdots+a_{N}^{\dagger}$. In order to selectively annihilate a vibrational excitation, the sequence can be modified by replacing the blue sideband pulse, step (i), with a pulse on the red sideband, which induces a transition from $|\downarrow, n\rangle$ to $|\uparrow, n-1\rangle$, and removes one phonon. This would yield the effective transition operator

$$
V_{-}=\alpha \mathbb{I}+\gamma e^{-i \phi} A
$$

which selectively de-excites the system. The generated transitions allow to choose between the excitation or de-excitation mechanisms which correspond to those depicted in the yellow boxes in figure 6.5. The opportunity to suppress one of the two processes generates novel possibilities to extend standard methods of nonlinear spectroscopy, which is a unique feature of controllable quantum systems (Gessner et al., 2014c; Schlawin et al., 2014).

Combinations of resonant sideband pulses also allow for the generation of a controlled displacement on the selected motional mode. To this end, one drives both sidebands with equal intensity $\eta \Omega$, and opposite phases $\phi_{r}=-\phi_{b}=\phi+\pi / 2$. The scenario resembles the one employed to generate the Mølmer-Sørensen interaction, with the crucial difference that here the sidebands are addressed resonantly, while for the generation of entangling gates one drives them off-resonantly (Sørensen and Mølmer, 1999). In complete analogy to the Raman scheme discussed in the previous section, this results in a displacement operation $D\left(\epsilon e^{i \phi}\right)$, with $\epsilon=\eta \Omega t / 2$ (Hayes et al., 2012).

\subsubsection{Vibrational degree of freedom: Readout}

The vibrational populations can be read out by mapping them onto the electronic degrees of freedom, which in turn are easily measured. The relative intensity of red and blue sidebands allow to directly infer the motional temperature, if the vibrations are in a thermal state of motion. This will hardly be the case in coherent nonlinear spectroscopy experiments considered in this section, which aims to probe non-equilibrium distributions. In many cases, however, we consider phonon populations close to the ground state. To measure the population of a low- $n$ phonon Fock state $|n\rangle$, we apply the following scheme (Gessner et al., 2014c; Schlawin et al., 2014); see also (Heinzen and Wineland, 1990):

Since the red sideband dissapears when $n=0$, there is no more notion of a $\pi$-pulse in this limit. To define a related pulse for small but nonzero $n$, consider the length of a $\pi$-pulse on the blue sideband for a ground-state ion, which is given by $t=\pi / \Omega_{0}^{(1)}$. A pulse on the red sideband with this duration is described by

$$
U^{(-1)}(\pi)|\downarrow, n\rangle=\cos \left(\frac{\Omega_{n-1}^{(1)}}{\Omega_{0}^{(1)}} \frac{\pi}{2}\right)|\downarrow, n\rangle+\sin \left(\frac{\Omega_{n-1}^{(1)}}{\Omega_{0}^{(1)}} \frac{\pi}{2}\right)|\uparrow, n-1\rangle,
$$


where we discard the phase which is irrelevant for the present readout method, see equation (3.46). The ratio of Rabi frequencies is given by

$$
\frac{\Omega_{n-1}^{(1)}}{\Omega_{0}^{(1)}}=\sqrt{n}+\mathcal{O}\left(\eta^{2}\right)
$$

Therefore, if a ground-state ion in an arbitrary motional state,

$$
\rho=\sum_{n, m=0}^{\infty} \rho_{n m}|\downarrow, n\rangle\langle\downarrow, m|,
$$

is driven with the above pulse, its electronic excited-state population will be given by

$$
\left\langle\uparrow\left|\operatorname{Tr}_{M}\left\{U^{(-1)}(\pi) \rho U^{(-1)}(\pi)^{\dagger}\right\}\right| \uparrow\right\rangle=\sum_{n=0}^{\infty} \rho_{n n} \sin ^{2}\left(\frac{\sqrt{n} \pi}{2}\right),
$$

where $\operatorname{Tr}_{M}$ denotes the partial trace over the motional degrees of freedom. This quantity is, again, easily measured by electron shelving. Assuming that the individual steps are carried out sufficiently fast, the above scheme effectively realizes a measurement of the motional observable

$$
O=\sum_{n=0}^{\infty} \sin ^{2}\left(\frac{\sqrt{n} \pi}{2}\right)|n\rangle\langle n|,
$$

which distinguishes well between low- $n$ Fock states (Heinzen and Wineland, 1990). This scheme can also be applied on a local sideband transition to read out the phonon population at a specific site within the ion chain (Gessner et al., 2014c). Yet, this is only one of many possibilities to measure the vibrational quantum states of a trapped ion. If time scales permit, appropriate pulse sequences allow for the readout of arbitrary motional observables (Meekhof et al., 1996; Gardiner et al., 1997).

\subsection{Nonlinear signals and applications for trapped-ion systems}

We have so far developed a general theoretical framework with a convenient diagrammatic representation in section 6.3 , and specific methods to realize flexible excitation and readout schemes of electronic and vibrational degrees of freedom of trapped ions in section 6.4. Before proceeding to discuss nonlinear signals and their interpretation, we briefly summarize the main differences between nonlinear spectroscopy of artificial, controllable quantum systems and bulk material, which was discussed in section 2.4 (Gessner et al., 2014c).

- The optical density of bulk material allows for the selection of pathways by phase matching, which involves adjusting the spatial orientation of the incoming pulses and the detector. This is only possible because a significant amount of light is scattered when the material is being excited by external pulses. There is no 
light directly scattered when we apply sideband pulses to a trapped ion, and hardly any scattering for Raman pulses. This is caused by choosing long-lived, meta-stable excited levels to encode the two-level system, which, hence, do not decay within the relevant time scales by emitting a detectable photon. Moreover, if we study the dynamics of phonons, a radiative decay does not happen on any time scale. To circumvent this, we measure the relevant populations using well-established fluorescence techniques. To allow for pathway selection, we resort to the phase cycling method, which constitutes an adequate alternative for phase matching, rendering our experimental approach close to multidimensional spectroscopy of nuclear spins (Ernst et al., 1987).

- Using externally induced fluorescence to read out the populations provides us with an additional time interval, which we can tune at will, whereas in bulk material induced emission happens immediately after the final pulse, or spontaneous decay occurs after an uncontrollable time delay.

- Controllable quantum systems provide flexibility for the design of readout and excitation schemes, by employing well-controlled pulses. These tools are not available in bulk material. Making efficient use of these possibilities allows us to distinguish diagrams which otherwise cannot be distinguished, or to read out different observables other than plain excited-state populations, which may contain more information.

- Similarly, single-site addressability represents another unique feature of controllable quantum systems, which we may harness to extend standard methods from nonlinear spectroscopy.

- The time scales of the atomic evolution in quantum optical systems are usually on the order of microseconds, which is experimentally much less challenging to handle than the femtosecond processes of electronic excitations, for instance in molecular systems.

- Quantum optical systems can be well-isolated from their environment, which suppresses natural decay processes, and allows for the controlled design of coherent interactions and quantum simulations. The corresponding coherence times are often on the orders of milliseconds (Harlander et al., 2011; Brown et al., 2011; Schindler et al., 2013; Ramm et al., 2014), while, for example, for photosynthetic complexes one faces coherence decay on the order of picoseconds (Brixner et al., 2005; Engel et al., 2007).

- Experiments with large ensembles in the bulk yield a directly measureable signal, reflecting the ensemble average. In quantum optical experiments with few atoms, expectation values are obtained by many repetitions of the same measurement. Assuming that the shot-to-shot fluctuations are weak - recall, however, section 4.3.2 - repeated single-system measurements are equivalent to direct measurements of the ensemble average. Such repeated measurements are also encountered in single-molecule experiments (Hildner et al., 2011). 
In the remainder of this chapter, we make use of the above advantages to design nonlinear measurement protocols and to theoretically study their application in controllable quantum systems. This provides us with non-equilibrium multi-time correlation functions of interacting many-body quantum systems. We will make use of single-site addressability to study spatially resolved dynamics, allowing us to probe, for instance, the role of coherence during phonon transport across an ion chain. The necessity of developing scalable methods to study long-range coherences is important, not only for the validation of quantum simulators and the identification of error sources for quantum computers (Gessner et al., 2014c), but can also help to understand quantum critical phenomena in the simulated models (Kim et al., 2010).

To be able to explain the visible features of the resulting two-dimensional spectra, and to identify each of the resonances, we mostly restrict to the theoretical study of relatively small systems. Notwithstanding, the methods developed in this section are - in principle - applicable to large systems, where the intuition gained from studying small sample systems can be applied. The pulse sequences always contain the same number of steps, that is, excitation and readout pulses, independently of the size of the system. The number of scanned time steps, however, depends on the desired frequency resolution, which implies that when the number of energy levels, and their density increases, the number of time steps must be increased accordingly to maintain the same ratio of resolution and mean level spacing.

\subsubsection{Two-pulse sequence: Single quantum coherence}

The single quantum coherence signal is a second-order, two-pulse signal with the phase signature $\phi_{1}-\phi_{2}$ (Gessner et al., 2014c) and was already discussed in the context of atomic vapor in section 6.2.4. It was introduced in terms of the formalism for controllable quantum systems in section 6.3.4, and the relevant diagrams are depicted in figure 6.6. To see how this signal can be interpreted, we consider the example of phonon transport.

\subsubsection{Coherent and incoherent phonon transport}

We now combine the general formalism of section 6.3 with the excitation and readout schemes of section 6.4. We study the phonon dynamics in a chain of trapped ions. For simplicity, we consider the special case where an ion chain was cooled to the motional ground state before applying the single quantum coherence pulse sequence. To probe phonon transport, we employ a local excitation and readout scheme, depicted in figure 6.8 for the example of a five ion chain. The outer ion (with index 1) is excited with two local phonon pulses, creating the transition operators

$$
V_{1}\left(\phi_{i}\right) \simeq \mathbb{I}+\epsilon e^{i \phi_{i}} a_{1}^{\dagger}-\epsilon e^{-i \phi_{i}} a_{1}, \quad i=1,2,
$$

which corresponds to the linear expansion of a weak, coherent displacement, as discussed in section 6.4.4. At the end of the sequence, the population of the center ion (index 3) is measured employing the motional observable $O_{3}$, which corresponds to a local readout of the observable developed in equation (6.82). The time delays between 

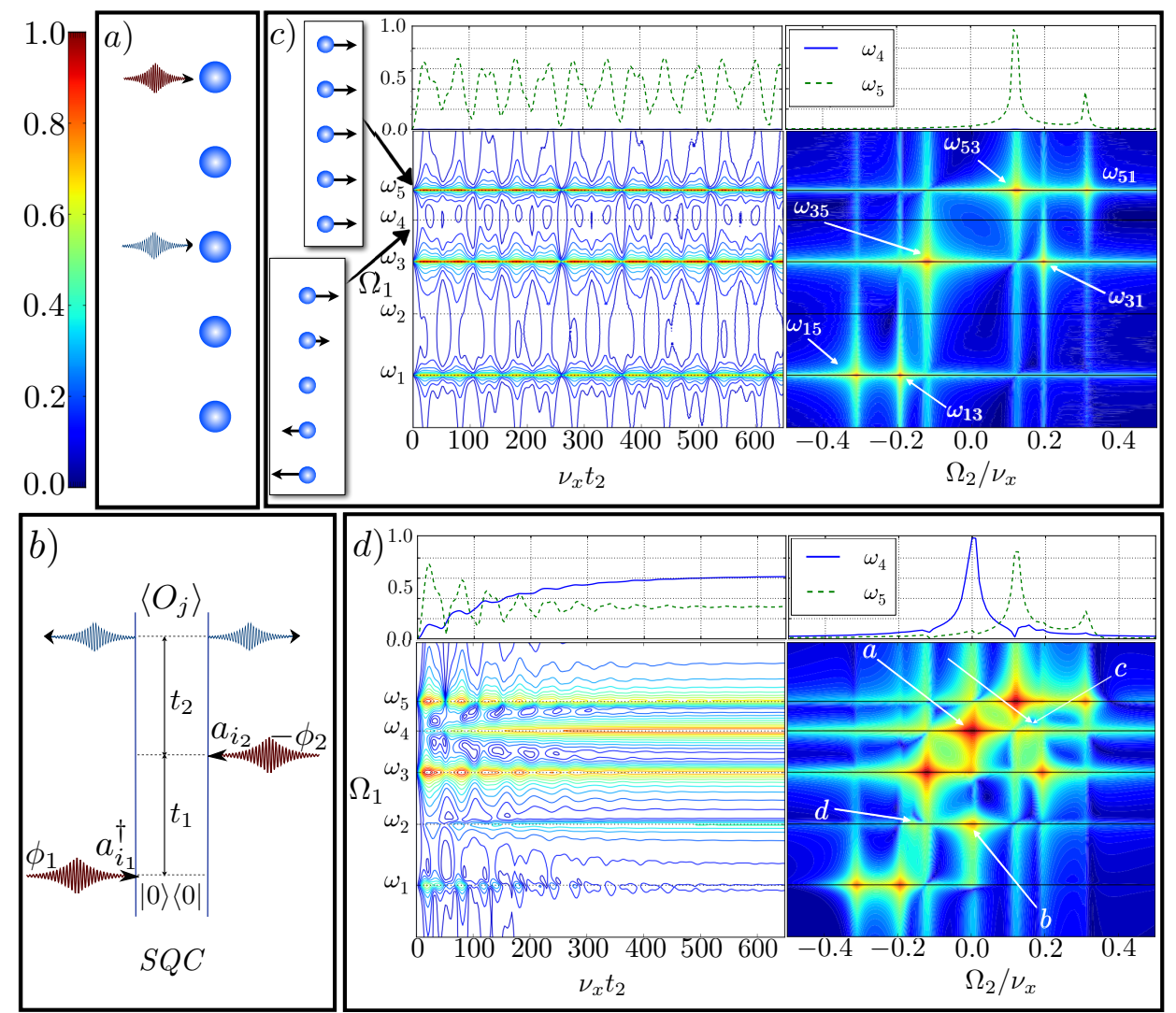

FIGURE 6.8.: a) A string of five ions is excited at one end with a phase-coherent two-pulse scheme followed by readout of the phonon populations at the center. b) The signal (6.85) is described by the contributing single quantum coherence diagram. c) Fully coherent phonon transport does not transfer populations between eigenmodes of the chain. The left panel shows the signal (6.87) while in the right panel, both time intervals have been Fourier transformed, as described by (6.90). The latter reveals all contributing eigenmode frequencies and their couplings. d) When incoherent local noise is added to the system dynamics, populations from the unobservable breathing mode are incoherently transferred to the center-offmass mode, which can be measured at the center ion. Adapted from (Gessner et al., 2014c).

the two pulses and before readout are scanned. The induced excitations evolve according to the dynamics generated by $H_{\mathrm{ph}}$ as defined in equation (6.66), with $U=0$; see also section 2.2.1.3. This Hamiltonian is time-independent and therefore the propagators correspond to unitary time evolution operators which can be expressed, as a 
function of the time delays $t_{1}$ and $t_{2}$, as

$$
\mathcal{G}\left(t_{i}\right) \rho=e^{-i H_{\mathrm{ph}} t_{i} / \hbar} \rho e^{i H_{\mathrm{ph}} t_{i} / \hbar}, \quad i=1,2 .
$$

The total signal is then described, according to equation (6.61), as (recall, for example, from equation (6.58) that the calligraphic letters denote superoperators)

$$
S_{1,1 ; 3}^{(2)}\left(t_{1}, t_{2}\right)=\operatorname{Tr}\left\{O_{3} \mathcal{G}\left(t_{2}\right) \mathcal{V}_{1}\left(\phi_{2}\right) \mathcal{G}\left(t_{1}\right) \mathcal{V}_{1}\left(\phi_{1}\right)|0\rangle\langle 0|\right\}
$$

From the total signal we extract only those terms with phase signature $\phi_{1}-\phi_{2}$ which, in this case, are described by the diagram depicted in figure $6.8 \mathrm{~b}$ ). We can describe the corresponding signal by retaining only the contributing terms from the total transition operators (6.57) as (Gessner et al., 2014c)

$$
S_{1,1 ; 3}^{(\mathrm{SQC})}\left(t_{1}, t_{2}\right)=\operatorname{Tr}\left\{O_{3} \mathcal{G}\left(t_{2}\right)\left[\mathcal{G}\left(t_{1}\right)\left[a_{1}^{\dagger}|0\rangle\langle 0|\right] a_{1}\right]\right\} .
$$

In general, nonlinear measurement protocols are uniquely defined via their characteristic phase signature, which is extracted by phase cycling - provided that excitation and readout methods are well defined. The most instructive way to represent a given signal is by providing the contributing diagrams, which are readily translated into explicit formulas, such as equation (6.86). To extract the relevant, contributing frequencies, it is most instructive to Fourier transform with respect to one or both time delays. The signal

$$
S_{1,1 ; 3}^{\mathrm{SQC})}\left(\Omega_{1}, t_{2}\right)=\int_{0}^{\infty} d t_{1} e^{i \Omega_{1} t_{1}} S_{1,1 ; 3}^{(\mathrm{SQC})}\left(t_{1}, t_{2}\right)
$$

is displayed in the left panel of figure $6.8 \mathrm{c}$ ). On the $\Omega_{1}$ axis, we observe three resonances corresponding to the eigenmodes which are excited with the two excitation pulses, and read out at the end. These are caused by the coherence $a_{1}^{\dagger}|0\rangle\left\langle 0|=| 1_{1}\right\rangle\langle 0|$, which is created by the first pulse, and whose contribution is selected by phase cycling. Here $\left|n_{i}\right\rangle$ denotes the $n$-th Fock state of the vibrationas at ion $i$. When expressed in terms of a decomposition of eigenmodes (6.67), characterized by $H_{\mathrm{ph}}\left|e_{i}\right\rangle=\hbar \omega_{i}\left|e_{i}\right\rangle$,

$$
a_{1}^{\dagger}|0\rangle\left\langle 0\left|=\sum_{j} c_{j 1}^{*}\right| e_{j}\right\rangle\langle 0|,
$$

the coherence evolves as

$$
\sum_{j} c_{j 1}^{*} \mathcal{G}\left(t_{1}\right)\left|e_{j}\right\rangle\left\langle 0\left|=\sum_{j} c_{j 1}^{*} e^{-i \omega_{j} t}\right| e_{j}\right\rangle\langle 0|,
$$

and, thus, reveals the contributing eigenfrequencies in $\left|1_{1}\right\rangle$. We order the frequencies such that $\omega_{1} \leq \omega_{2} \leq \ldots$ Even though all eigenmodes contribute to the decomposition of $\left|1_{1}\right\rangle$, not all of the five eigenmodes of can be observed in the two-dimensional spectrum in figure $6.8 \mathrm{c}$ ). This is due to the local readout at the center of the chain. The center ion does not participate in the motion of, for instance, the breathing mode 
with frequency $\omega_{4}$, which is characterized by a displacement of all ions proportional to their distance to the center (James, 1998), and therefore cannot be read out at the center of the chain - in contrast to the center-of-mass mode with $\omega_{5}$, where all ions are displaced equally, see insets in figure $6.8 \mathrm{c}$ ).

While during $t_{1}$, we have observed the time evolution of a coherence between single excited states and the ground state, during $t_{2}$, we observe the coherence between two single excited states. The local readout reflects the coherent hopping of local phonons between the sites of the chain, and can be observed on the $t_{2}$ axis. Another Fourier transform,

$$
S_{1,1 ; 3}^{(\mathrm{SQC})}\left(\Omega_{1}, \Omega_{2}\right)=\int_{0}^{\infty} d t_{1} \int_{0}^{\infty} d t_{2} e^{i \Omega_{1} t_{1}} e^{i \Omega_{2} t_{2}} S_{1,1 ; 3}^{(\mathrm{SQC})}\left(t_{1}, t_{2}\right)
$$

reveals the energy differences $\omega_{i j}=\omega_{i}-\omega_{j}$ of single excited states along $\Omega_{2}$, which are directly related to the couplings between the ions and their corresponding tunneling rates. To see that the frequencies $\omega_{i j}$ indeed determine the periods of phonon tunneling, consider the transition probability from ion $a$ to ion $b$ (Gessner et al., 2014c)

$$
p_{a b}(t)=\left|\left\langle 1_{b}\left|e^{-i H_{\mathrm{ph}} t / \hbar}\right| 1_{a}\right\rangle\right|^{2}=\sum_{i j}\left\langle 1_{b} \mid e_{i}\right\rangle\left\langle e_{i} \mid 1_{a}\right\rangle\left\langle 1_{a} \mid e_{j}\right\rangle\left\langle e_{j} \mid 1_{b}\right\rangle e^{-i \omega_{i j} t},
$$

where, henceforth, we indicate a Fourier-transformed signal by replacing the time variable $t_{i}$ with the corresponding frequency variable $\Omega_{i}$.

Aside from the naturally present incoherent couplings between neighboring sites, we can artificially induce incoherent processes in an ion trap which induce couplings between different eigenmodes. For this theoretical study, we do not consider natural dephasing processes, because they are usually correlated and do not lead to redistribution of the eigenmode populations. To achieve the latter, we rather assume that the natural dephasing process is negligible, and instead, a simulated environment, induced by focussed lasers, creates the dissipative dynamics described by the Lindblad master equation (2.25) with $L_{i}=a_{i}^{\dagger} a_{i}$ and equal rates $\gamma_{i}=\gamma$, leading to the time evolution

$$
\mathcal{L} \rho(t)=-\frac{i}{\hbar}\left[H_{\mathrm{ph}}, \rho(t)\right]+\gamma \sum_{i=1}^{N}\left(a_{i}^{\dagger} a_{i} \rho(t) a_{i}^{\dagger} a_{i}-\frac{1}{2}\left\{a_{i}^{\dagger} a_{i} a_{i}^{\dagger} a_{i}, \rho(t)\right\}\right) .
$$

Such dynamics can be induced via appropriately designed laser interaction (Poyatos et al., 1996; Myatt et al., 2000; Barreiro et al., 2011), combined with local addressing. The resulting two-dimensional spectrum can be observed in figure $6.8 \mathrm{~d}$ ). The induced coupling between the center-of-mass mode to the breathing mode now leads to an observable signal at $\omega_{4}$, which emerges after some time on the $t_{2}$ axis has passed. In the double Fourier-transformed plot, the incoherent nature of this coupling process manifests via a peak at $\left(\Omega_{1}, \Omega_{2}\right)=\left(\omega_{4}, 0\right)$, labelled peak $a$ in the figure. The same phenomenon can be observed for the mode $\omega_{2}$ (peak $b$ ). Thus, the local dephasing process, ${ }^{5}$ described by equation (6.92), incoherently distributes the initial population

\footnotetext{
${ }^{5}$ This dynamics is not directly related to the controlled dephasing process in chapter 3 .
} 
of the breathing mode to other modes, which can be read out at the center ion. There are, however, also coherent hopping processes which involve the breathing mode, which without dephasing could not be observed. The slow oscillatory modulation at transient times, and the resulting side-peaks at $\left(\omega_{4}, \omega_{42}\right)$ and $\left(\omega_{2}, \omega_{24}\right)$, peaks $c$ and $d$, reveal these in the single quantum coherence signal in the presence of dephasing. These slow oscillations and resulting peaks are best seen in the frequency slices on the top of the figure, detailing the contributions of $\omega_{4}$ and $\omega_{5}$.

The analysis of the single quantum coherence signal can thus be used to identify the coherent or incoherent nature of couplings in the system. It further allows to monitor the decay of coherent superpositions, and to assess different transport processes. The treatment above is easily extended to a more general scenario, and for the interpretation of the signal one can follow the intuition provided by the above example (Gessner et al., 2014c; Schlawin et al., 2014).

\subsubsection{Two-dimensional lineshapes: Distinguishing noise processes}

As already mentioned in section 2.4, the lineshapes of two-dimensional resonances disclose information about the system's interaction with an environment, which cannot be read off the linewidth of linear absorption spectra. Identifying such decoherence processes is particularly relevant for quantum computations, which mostly rely on purely coherent dynamics. Let us therefore consider the electronic degrees of freedom (recall sections 6.4.1 and 6.4.2), subject to the phonon-mediated effective interaction which is induced to generate the Mølmer-Sørensen entangling gate between two spins (Sørensen and Mølmer, 1999),

$$
H_{\mathrm{MS}}=\frac{\hbar \Omega}{2} \sigma_{x}^{(1)} \otimes \sigma_{x}^{(2)} .
$$

If this interaction is applied with a duration $t_{g}=\pi /(2 \Omega)$, it converts the two-qubit ground state $|00\rangle$ into the maximally entangled Bell state

$$
U_{\mathrm{MS}}|00\rangle=e^{-i H_{\mathrm{MS}} t_{g} / \hbar}|00\rangle=\frac{1}{\sqrt{2}}(|00\rangle-i|11\rangle) .
$$

We probe the induced dynamics using the single quantum coherence signal, by applying the excitation pulses

$$
V_{j}(\phi)=\alpha \mathbb{I}+\beta\left(e^{i \phi} \sigma_{+}^{(j)}-e^{-i \phi} \sigma_{-}^{(j)}\right),
$$

which can be generated by resonant carrier pulses with $\alpha^{2}+\beta^{2}=1$ and a welldefined phase, as shown in equation (6.64). We assume that these pulses are applied locally to ion $j$. Similarly, local readout of the electronic populations is described by measurement of the expectation value of $O_{j}=\sigma_{z}^{(j)}$.

After optical pumping, the two ions are initialized in their electronic ground state $|00\rangle$. In the following, all excitations and the readout are carried out on the same spin, as described by the signal $S_{1,1 ; 1}^{(\mathrm{SQC})}\left(\Omega_{1}, \Omega_{2}\right)$. Following the interpretation of the phonon example from before, we can predict four peaks at $\left(\Omega_{1}, \Omega_{2}\right)= \pm(0, \Omega)$ and 
$\pm(\Omega, \Omega)$ (Gessner et al., 2014c). In the phonon example, we studied the evolution of local excitations by decomposing them into eigenstates of the total system. Also in the present case, it is most instructive to interpret the resulting signals in terms of Bell-states $\left\{\left|\Psi^{ \pm}\right\rangle,\left|\Phi^{ \pm}\right\rangle\right\}$, which are eigenstates of $H_{\mathrm{MS}}$ with eigenvalues $\pm \hbar \Omega / 2$. The first excitation creates the state $|10\rangle=1 / \sqrt{2}\left(\left|\Psi^{+}\right\rangle-\left|\Psi^{-}\right\rangle\right)$on the 'ket' side, while on the 'bra' side the initial state $|00\rangle=1 / \sqrt{2}\left(\left|\Phi^{+}\right\rangle+\left|\Phi^{-}\right\rangle\right)$remains unperturbed. For unitary evolution, each eigenstate acquires a phase-shift of the form $e^{ \pm i \Omega t_{1} / 2}$ during the interval $t_{1}$, such that the Fourier transform yields the three frequencies $\{-\Omega, 0, \Omega\}$, depending on the pairing of eigenstates in a coherence.

Let us first focus on the two time-independent coherences $\left|\Psi^{ \pm}\right\rangle\left\langle\Phi^{ \pm}\right|$, which must lead to resonances along the $\Omega_{1}=0$ axis. The second interaction on the 'bra' side creates a superposition $\left|\Psi^{ \pm}\right\rangle\left(\left\langle\Psi^{ \pm}\right|-\left\langle\Psi^{\mp}\right|\right)$. The Bell states are maximally mixed in each of the single spin Hilbert spaces, and, hence, no signal will be observable by local readout of populations of the form $\left|\Psi^{ \pm}\right\rangle\left\langle\Psi^{ \pm}\right|$. Thus, the only signal of this pathway stems from the coherences $\left|\Psi^{ \pm}\right\rangle\left\langle\Psi^{\mp}\right|$ which oscillate with respective frequencies $\pm \Omega$ during $t_{2}$. Hence, these pathways generate two peaks in $S_{1,1 ; 1}^{(\mathrm{SQC})}\left(\Omega_{1}, \Omega_{2}\right)$, at $\left(\Omega_{1}, \Omega_{2}\right)= \pm(0, \Omega)$.

We next consider the evolution of the coherences $\left|\Psi^{ \pm}\right\rangle\left\langle\Phi^{\mp}\right|$, which evolve during $t_{1}$, with frequencies $\pm \Omega$ : The second interaction creates the superposition $\left|\Psi^{ \pm}\right\rangle\left(\left\langle\Psi^{\mp}\right|-\right.$ $\left.\left\langle\Psi^{ \pm}\right|\right)$, where, again, only the terms $\left|\Psi^{ \pm}\right\rangle\left\langle\Psi^{\mp}\right|$ lead to a signal. These oscillate during $t_{2}$ with frequencies $\pm \Omega$, inducing two additional peaks at $\pm(\Omega, \Omega)$. Without dissipation, all four peaks are delta peaks, yet the coupling to an environment can broaden them and gives rise to distinct two-dimensional lineshapes.

Figure 6.9 a) shows $\left|S_{1,1 ; 1}^{(\mathrm{SQC})}\left(\Omega_{1}, \Omega_{2}\right)\right|$ including the effect of a local noise process, described by the two Lindblad operators $L_{\mathrm{ld}}^{(j)}=\sqrt{\gamma} \sigma_{z}^{(j)}, j=1,2$, while figure $6.9 \mathrm{~b}$ ) depicts the influence of correlated dephasing described by $L_{\mathrm{cd}}=\sqrt{\gamma} \sigma_{z}^{(1)} \otimes \sigma_{z}^{(2)}$. The correlated dephasing process is analogous to the ensemble-averaged dynamics discussed in section 4.4. However, here it is described in terms of an effective Lindblad master equation. Local dephasing broadens the resonances equally along both frequency axes, whereas correlated dephasing only affects the width along $\Omega_{1}$, which is best seen in the close-up figures $\mathrm{c}$ ) and $\mathrm{d}$ ), which zoom in on the peak on $\left(\Omega_{1}, \Omega_{2}\right)=(\Omega, \Omega)$. This is due to the fact that the coherences $\left|\Psi^{ \pm}\right\rangle\left\langle\Psi^{\mp}\right|$ are protected by a decoherence-free subspace during $t_{2}$ (Lidar et al., 1998); see also section 4.4.4.

For local dephasing, the peak width along $\Omega_{1}$, quantified by the full-width at halfmaximum (FWHM), allows to estimate the error probability of the corresponding gate $U_{\text {MS }}$, since both quantities depend linearly on the dephasing strength $\gamma$, to a good approximation for perturbative noise processes $\gamma<0.1 \Omega$, which is shown in figure 6.9 e) (Gessner et al., 2014c). We quantify the error probability $1-\mathcal{F}$, using the fidelity

$$
\mathcal{F}=\sqrt{\left\langle 00\left|U_{\mathrm{MS}}^{\dagger} \rho_{\gamma} U_{\mathrm{MS}}\right| 00\right\rangle}
$$

which expresses the overlap between the target state $U_{\mathrm{MS}}|00\rangle$ and the state

$$
\rho_{\gamma}=\mathcal{G}\left(\frac{\pi}{2 \Omega}\right)|00\rangle\langle 00|
$$



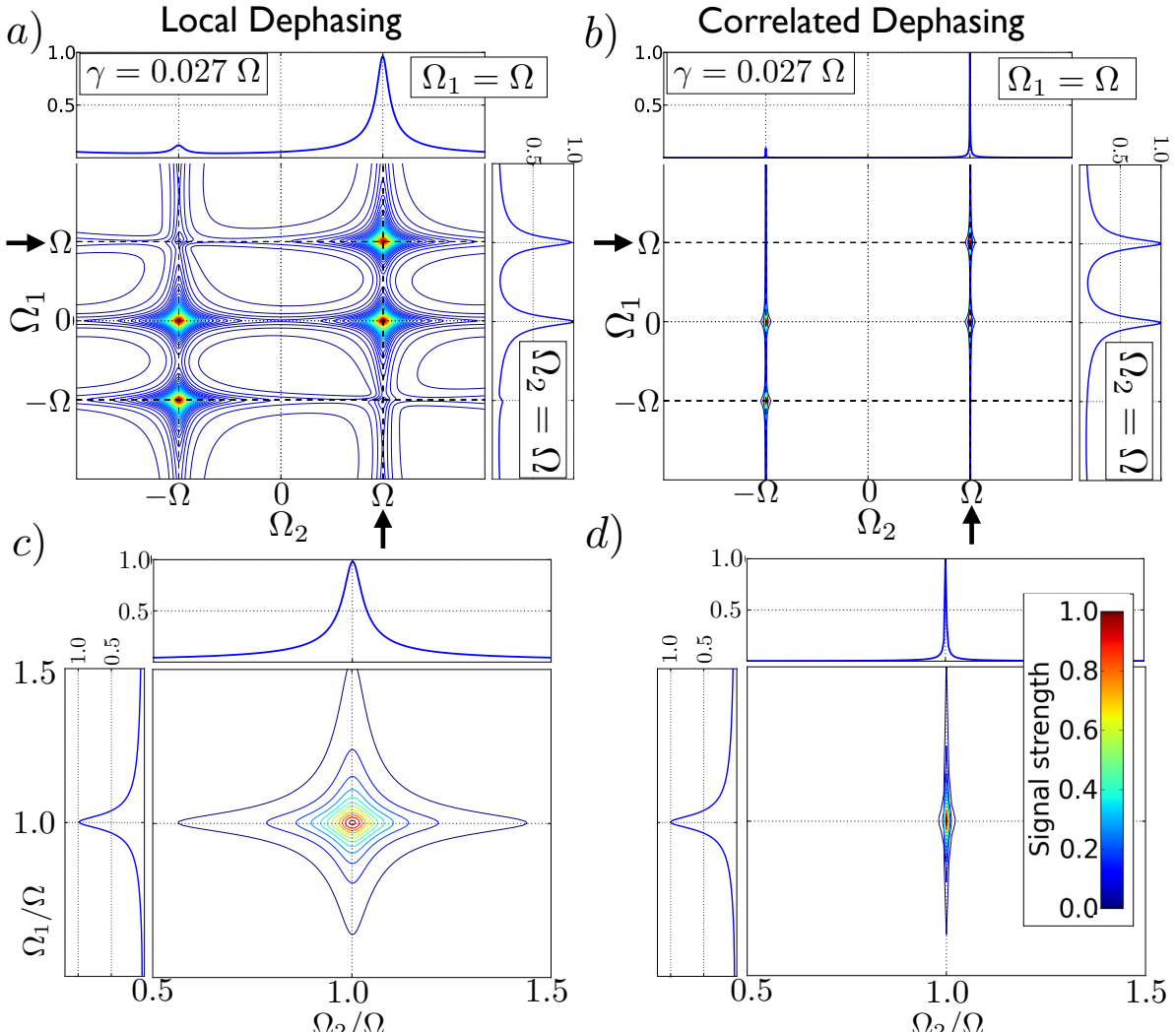

d)

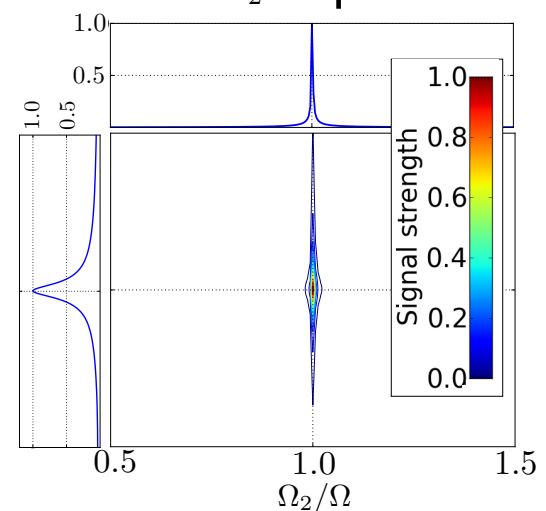

e)

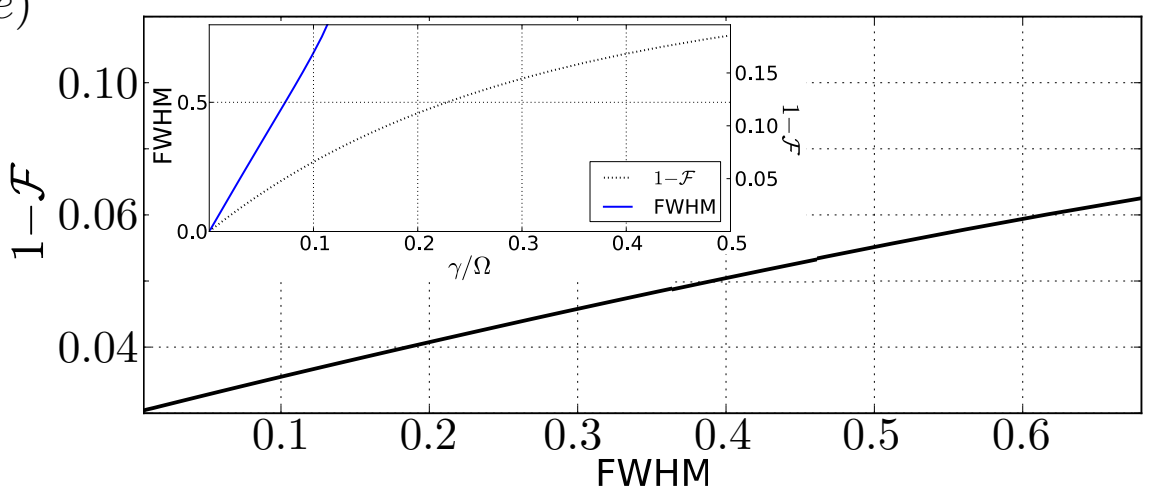

FiguRE 6.9.: The single quantum coherence signal (6.85), created by the Mølmer-Sørensen interaction (6.93). a)-b) Different noise processes can be distinguished by the associated two-dimensional lineshapes. c)-d) Close-up on the peak at $(\Omega, \Omega)$ (black arrows). e) The width of the resonance along $\Omega_{1}$ further relates to the fidelity of the corresponding entangling gate. The color code shows the absolute value of relative peak heights, on a renormalized scale. Subfigures c)-e) adapted from (Gessner et al., 2014c). 
which is produced under the influence of the respective noise processes.

Hence, the present example demonstrated that the application of the single quantum coherence signal (6.85) can be used to identify and quantify relevant decoherence processes, which is a crucial prerequisite for coherent control of the asymptotic state and reliable quantum computations.

\subsubsection{Distribution of couplings and excitations during a quantum phase transition}

The single quantum coherence signal discloses the excitation spectrum of single excited states, and, if combined with single-site addressability, the tunneling rates in a local site basis. When a system undergoes a quantum phase transition, based on our intuition from the analysis in chapter 5, we expect both these quantities to spread over broadly distributed energy ranges and displaying strong couplings which lead to long-range coherences. This should become visible in the single quantum coherence signal, even at relatively small system sizes.

The qubits in an ion chain can be brought to long-range Ising-type interaction of the type $H_{\mathrm{sc}}$ by application of suitable laser fields (Porras and Cirac, 2004b); see sections 2.2.1.7 and 6.4.1; as well as chapter 5. To analyze the induced dynamics, we employ the single quantum coherence signal based on the same excitation and readout schemes of the previous section. Thus, we excite the system with pulses of the form (6.95) to probe elementary excitations and populations in the eigenbasis of $\sigma_{z}$. We assume the initial state to be the Hamiltonian's ground state. Since this state is not generally given by $|0 \ldots 0\rangle$, it does not necessarily contain zero of these elementary excitations as the initial states in the preceding sections did. Hence, we can no longer restrict to only the left-most diagram in figure 6.6 , but rather have to take all four diagrams into account to describe the single quantum coherence signal. This is caused by the possibility to de-excite the ground state in the $\sigma_{z}$-basis and a non-vanishing expectation value of the readout observable $\sigma_{z}$ for the initial state. We must also pay special attention to the relative sign of the individual diagrams, which depends on the signs of the coefficients in the transition operator (6.95), see also figure 6.5.

Figure $6.10 \mathrm{a})$-c) shows the single quantum coherence signal, $\left|S_{11 ; 1}^{(\mathrm{SQC})}\left(\Omega_{1}, \Omega_{2}\right)\right|^{2}$, for a string of $N=5$ spins, where all excitation and readout pulses were applied to the leftmost spin. The spin chain Hamiltonian (6.63) was probed for a ferromagnetic system $\left(J_{0}>0\right)$ with intermediate interaction range $\alpha=1$, at different values of the external field. The spectrum, normalized to values between 0 and 1 , can be seen in figure 6.10 d), where the red dotted lines indicate those values of $B / J_{0}$ which correspond to the data in the subfigures a)-c).

In figure $6.10 \mathrm{a}$ ), the external field $B / J_{0}=0.1$ is rather small compared to the internal ferromagnetic spin-spin coupling. The two-dimensional spectrum shows that the local pulses mostly populate the group of first excited states which are separated from the ground state by a considerably large energy gap. Few populations of the second and third excited state manifolds can be observed, each one being strongly degenerate and energetically well separated from their neighbors. We can also observe a tiny spreading of the resonances along the $\Omega_{2}$ axis within the first excited state 

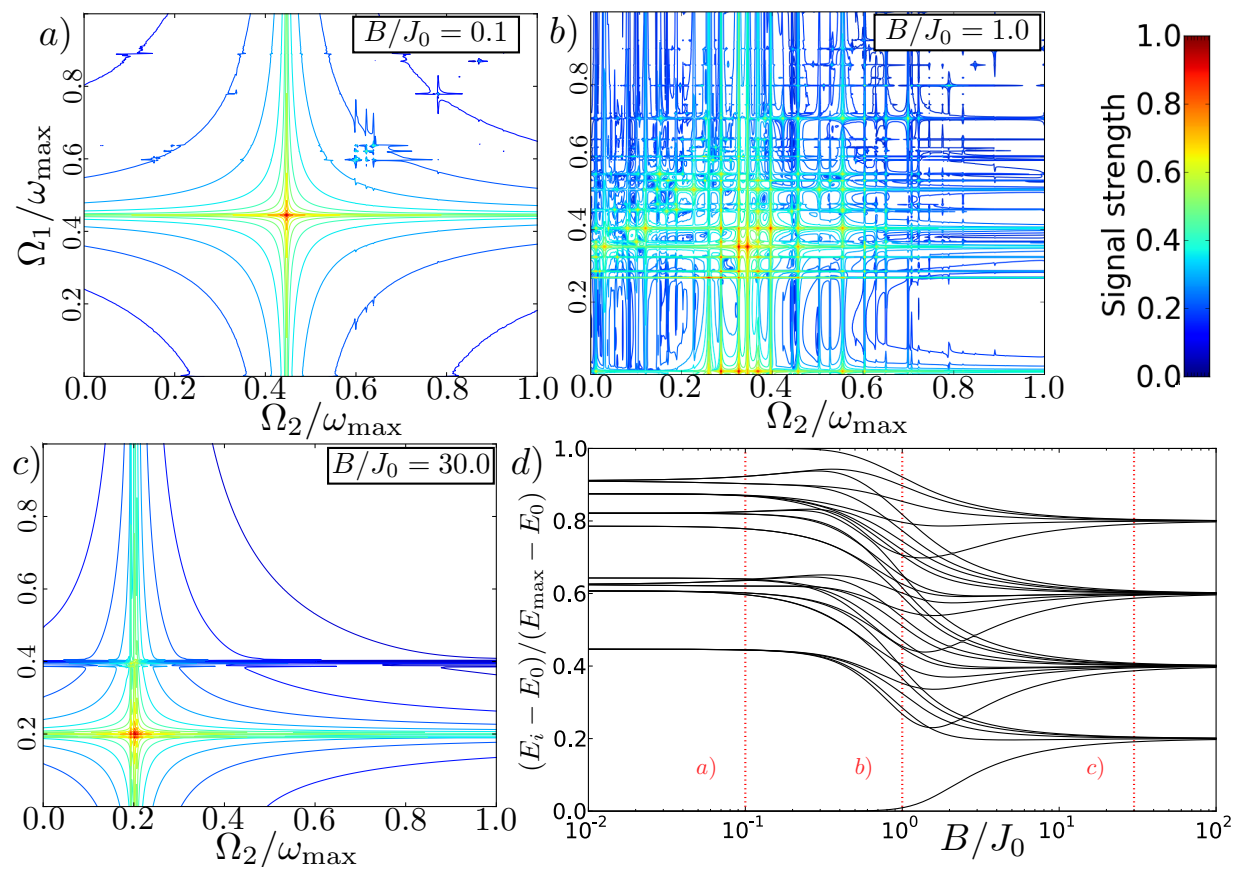

FIGURE 6.10.: a)-c) The positive-frequency part of the single quantum coherence signal, as introduced in equation (6.85), of the spin-chain model (6.63), with intermediate ferromagnetic interaction range $\alpha=1$, and $J_{0}>0$. The plot shows the rescaled absolute value $\operatorname{arcsinh}\left|S_{11 ; 1}^{(\mathrm{SQC})}\left(\Omega_{1}, \Omega_{2}\right)\right|^{2}$ to highlight smaller features on a renormalized color scale. d) The corresponding normalized spectrum can be used to interpret resonances on the $\Omega_{1}$ axis in the two-dimensional spectra. Adapted from (Schlawin et al., 2014).

manifold, indicating the hopping of spin excitations with more than one frequency.

This picture is dramatically altered when we tune $B / J_{0}=1$ into the intermediate range, as shown in figure $6.10 \mathrm{~b}$ ). The local spin excitations populate the entire energy spectrum, and we observe long-range couplings and a broad range of contributing frequencies, which generate intricate, possibly chaotic dynamics; see chapter 5 .

Finally, for very large external fields, $B / J_{0}=30$, the ferromagnetic interaction has only negligible influence on the system, which is now governed by a paramagnetic structure of equidistant energy bands. The two-dimensional spectrum, figure 6.10 c), shows that only the first two energy bands are populated, whereas the strongest contribution stems from the first excited states. Both peaks are found along the same value of $\Omega_{2}$, indicating that the coherent tunneling of spin excitations within the sites is always determined by the energy difference between neighboring bands, which, according to equation (5.64), is given by $\left(E_{k+1}-E_{k}\right) /\left(E_{\max }-E_{0}\right)=1 / N=0.2$.

The finite-size precursors of the quantum phase transition thus produce a distinct signature in the two-dimensional single quantum coherence signals. Furthermore, in 
our approach, we assumed excitation and readout of only one single spin of the entire chain. Since this spin is subject to (long-range) interactions with the rest of the chain, we were able to extract spectral information, and coherent coupling terms from local measurements.

\subsubsection{Steady-state currents}

Continuous Doppler cooling of ions with focussed lasers can effectively generate local heat baths in a chain of trapped ions. By adjusting the laser parameters, different local temperatures can be achieved, leading to steady-state heat currents and transport of phonons across the chain (Bermudez et al., 2013; Ramm et al., 2014); see also (Asadian et al., 2013). To study the role of coherent and incoherent contributions to such steady-state currents, we analyze the corresponding signatures in the single quantum coherence signal.

To this end, we expose both ends of a chain of ions to thermal heat baths of different temperatures $T_{1}$ and $T_{2}$, see figure $6.11 \mathrm{a}$ ). The excitation pulses for the single quantum coherence signal are applied at the ion in the heat bath (red arrows), while the readout (blue arrow) is carried out in the center of the chain. We simulate the corresponding signal for both cases - once with both excitation pulses being applied to the left-most ion, which is submitted to the colder temperature bath, and once with both excitation pulses on the right-most, higher-temperature ion. Readout is always performed at the center ion. To simulate the corresponding dynamics, we employ the master equation (Breuer and Petruccione, 2002)

$$
\begin{aligned}
\mathcal{L} \rho(t)=-\frac{i}{\hbar}\left[H_{\mathrm{ph}}, \rho\right] & +\gamma \sum_{i \in\{1, N\}}\left(\bar{n}_{i}+1\right)\left(a_{i} \rho(t) a_{i}^{\dagger}-\frac{1}{2}\left\{a_{i}^{\dagger} a_{i}, \rho(t)\right\}\right) \\
& +\gamma \sum_{i \in\{1, N\}} \bar{n}_{i}\left(a_{i}^{\dagger} \rho(t) a_{i}-\frac{1}{2}\left\{a_{i} a_{i}^{\dagger}, \rho(t)\right\}\right),
\end{aligned}
$$

where $\gamma$ represents the coupling strength to the heat baths, whose temperatures are characterized by the average occupation numbers $\bar{n}_{1}$, and $\bar{n}_{N}$, respectively. The phonon Hamiltonian is described by $H_{\mathrm{ph}}$ as defined in equation (6.66), with a small anharmonic correction term $U=-0.025 \nu_{x}$, which only makes a relevant contribution if the heat bath populates excited states beyond the first excited state manifold. To provide an intuitive interpretation of the signals, let us discuss the simplest non-trivial case of $N=3$, with parameters $\gamma=0.01 \nu_{x}, \bar{n}_{1}=0$ and $\bar{n}_{3}=0.5$. Again, since the initial state is not the absolute ground state, proper simulation of the single quantum coherence signals requires that all four diagrams of figure 6.6 are taken into account.

The resulting single quantum coherence signals $S_{11 ; 2}^{(\mathrm{SQC})}\left(\Omega_{1}, \Omega_{2}\right)$ and $S_{33 ; 2}^{(\mathrm{SQC})}\left(\Omega_{1}, \Omega_{2}\right)$ are shown in figure $6.11 \mathrm{~b}$ ), where the initial state is the steady-state of the dynamics (6.98). In the absence of a temperature gradient, we expect the signals to be exactly the same, independently of the position where the excitations are injected. The figure, therefore, also shows the difference between the two signals

$$
\Delta S^{(\mathrm{SQC})}\left(\Omega_{1}, \Omega_{2}\right)=S_{11 ; 2}^{(\mathrm{SQC})}\left(\Omega_{1}, \Omega_{2}\right)-S_{33 ; 2}^{(\mathrm{SQC})}\left(\Omega_{1}, \Omega_{2}\right) .
$$


a)

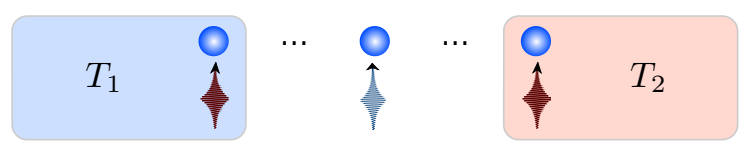

b)

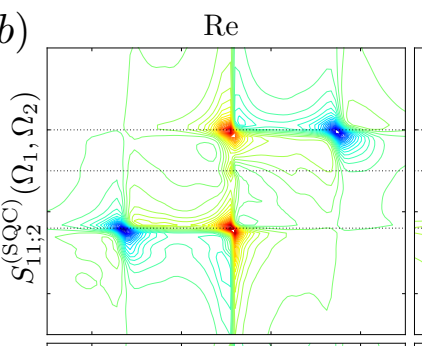

$\operatorname{Im}$
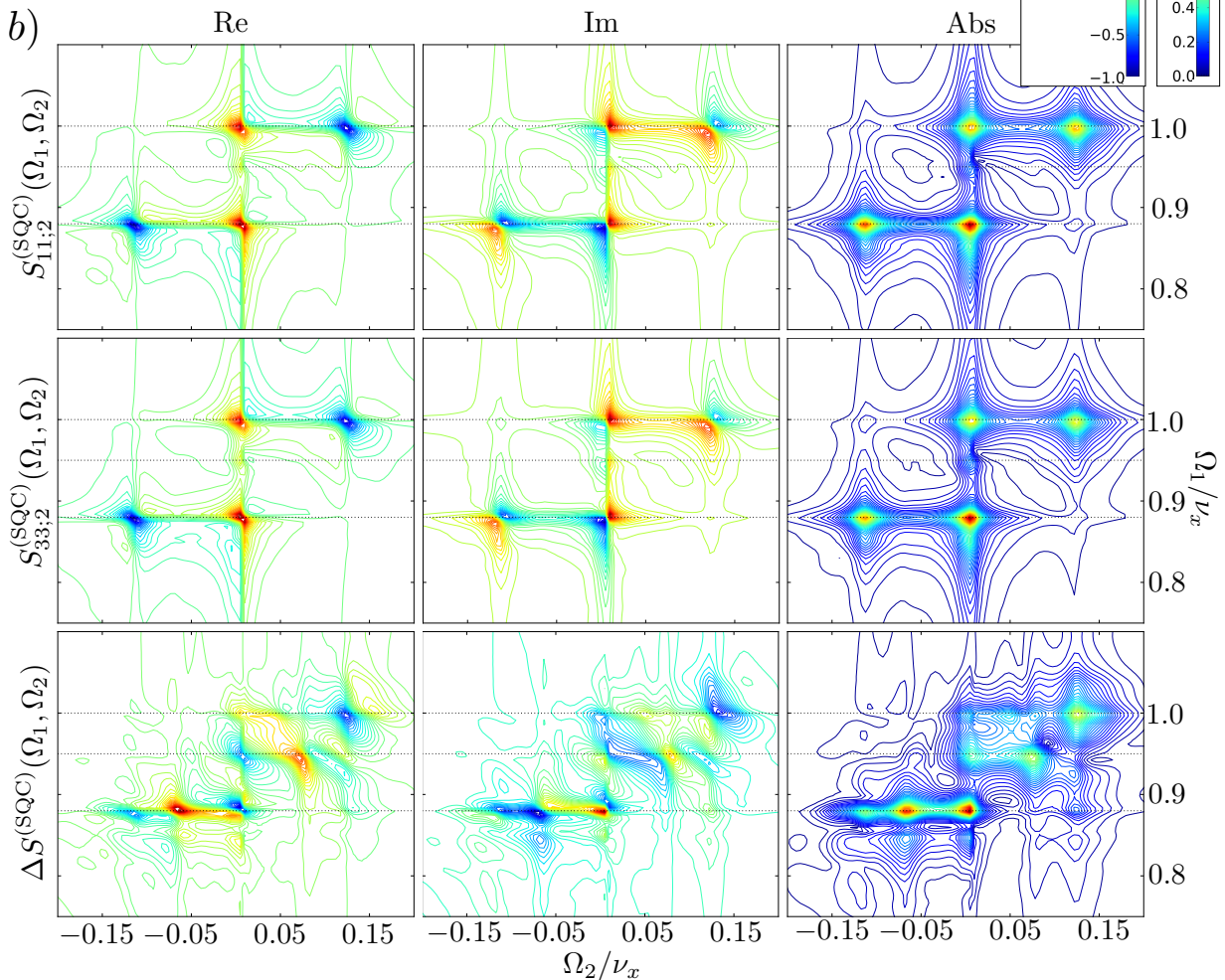

FIGURE 6.11.: Single quantum coherence signals (6.85) and their difference under the influence of a heat bath. The simulations are carried out according to equation (6.98) for $N=3$ spins, with $\bar{n}_{1}=0, \bar{n}_{3}=0.5, \gamma=0.01 \nu_{x}$. The signals are obtained by letting the system evolve into the steady state, and then applying two phasecoherent pulses on the same end of the chain before reading out at the center. The dashed lines indicate the frequencies of the eigenmodes $\omega_{1}<\omega_{2}<\omega_{3}=\nu_{x}$. Figure adapted from (Schlawin et al., 2014).

Within the single excitation subspace, the Hamiltonian (6.66) can be easily expressed in terms of the couplings $t_{i j}$ between the local sites,

$$
H_{(1)}=\hbar\left(\begin{array}{ccc}
\nu_{x}-t_{12}-t_{13} & t_{12} & t_{13} \\
t_{12} & \nu_{x}-2 t_{12} & t_{12} \\
t_{13} & t_{12} & \nu_{x}-t_{12}-t_{13}
\end{array}\right),
$$

and diagonalization yields the single excited state frequencies $\omega_{1}=\nu_{x}-3 t_{12}, \omega_{2}=$ $\nu_{x}-t_{12}-2 t_{13}$, and $\omega_{3}=\nu_{x}$. These identify the resonances along the $\Omega_{1}$ axis in the 
signals in figure $6.11 \mathrm{~b}$ ), whereas the breathing mode $\omega_{2}$ hardly contributed due to the local readout at the center. The energy differences $\omega_{i j}=\omega_{i}-\omega_{j}$ can be read off the $\Omega_{2}$ axis and are given by combinations of the coupling terms $t_{i j}$, which can be inferred from the two-dimensional spectra (Schlawin et al., 2014). Moreover, strong contributions at $\Omega_{2}=0$ indicate the presence of incoherent processes, which here are caused by the coupling to the temperature baths. From the real and imaginary parts of the signal, we can identify the characteristic shape of absorptive and dispersive peaks, respectively, which is typical for complex-valued two-dimensional signals (Hamm and Zanni, 2011).

The difference signal is entirely attributed to the broken mirror symmetry with respect to the center of the chain, induced by the nonzero temperature gradient. The plots in figure $6.11 \mathrm{~b}$ ) (bottom row) show that a significant contribution to the asymmetry is actually created by coherent transfer processes which are triggered by a thermally-induced imbalance of phonon populations across the system. Nevertheless, we also identify incoherent peaks at $\Omega_{2}=0$, indicating energy dissipation and heat flow. Also the breathing mode at $\Omega_{2}=\omega_{2}$ becomes visible in the difference, even though it plays a minor role in each of the individual signals. Thus, the incoherently created populations of this mode are significantly different, depending on the position of the excitation pulses. Finally, we can observe tiny shifts in the position of the two-dimensional resonance, which indicate effective corrections to the tunneling rates along certain directions. In fact the coupling rates are slightly suppressed against the direction of the heat current and enhanced along the temperature gradient. The shifted resonances lead to peaks in the two-dimensional system, which are especially pronounced when they involve the breathing mode (Schlawin et al., 2014).

\subsubsection{Four-pulse sequence: Double quantum coherence}

The second-order two-pulse sequences of the previous chapter have provided valuable insight into the dynamics and couplings within the first excited state manifold. Employing transition operators which create no more than one excitation per interaction, higher parts of the excitation spectrum can only be resolved with more pulses. After having discussed various applications of the single quantum coherence signal, we now turn to higher-order signals. In particular, we start by defining the fluorescence-based analog of the double quantum coherence signal, which has been employed to reveal electronic correlations in molecules (Kim et al., 2009) and collective resonances in atomic vapor (Dai et al., 2012).

In a fluorescence setting, the double quantum coherence signal is given by a fourpulse sequence with the characteristic phase signature $\phi_{1}+\phi_{2}-\phi_{3}-\phi_{4}$. Given the initial state contains no excitations, and the observable $O_{j}$ only yields a nonzero result when populations beyond the ground state are present, the only relevant diagrams are those depicted in figure 6.12 a). The first interaction creates a superposition of single excited states and ground state, which is analogous to what we observed in the single quantum coherence signal. After the first two interactions, the system evolves through a coherent superposition of double-excited states and the ground state, which, during the coherent time evolution $t_{2}$, reveals the spectrum of double excited states. 
a)

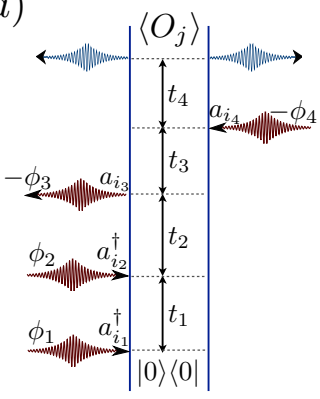

$D Q C_{1}$

c)

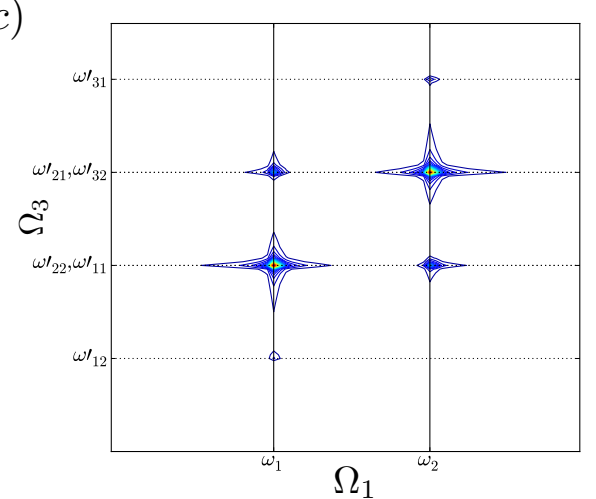

$D Q C_{2}$ b)
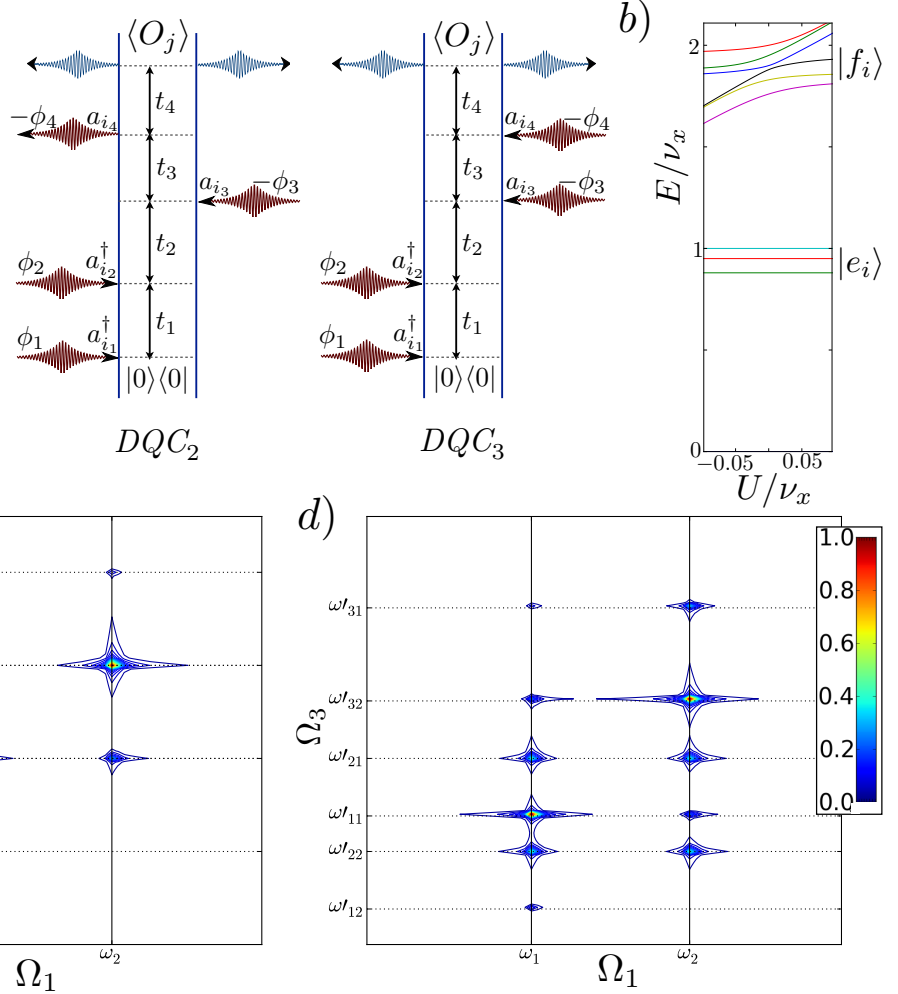

FIGURE 6.12.: a) Three relevant diagrams describe the double quantum coherence signal (see text). b) This can be used to reveal anharmonic corrections which influence higher-excited state manifolds, which can be seen in this spectrum for $N=3$ phonons; see equation (6.66). The renormalized two-dimensional spectra show $\left|S_{1111 ; 1}^{(\mathrm{DQC})}\left(\Omega_{1}, t_{2}=0, \Omega_{3}, t_{4}=0\right)\right|$ for $N=2, U=0$ (c) and for $U=-0.025 \nu_{x}$ (d). Figure adapted from (Gessner et al., 2014c).

During $t_{3}$, some of the diagrams contain contributions of coherences between single excited states and double excited states $\left(D Q C_{2}\right.$ and $\left.D Q C_{3}\right)$. Comparing the resulting resonances can reveal the presence of nonlinear couplings which influence the energy of higher excited states. The effect of the correction term $U$ is only visible in the higher excited states, as is illustrated in figure $6.12 \mathrm{~b}$ ), which shows the first two excited state manifolds of the phonons in a three-ion chain as a function of $U$, according to the Hamiltonian given in equation (6.66).

\subsubsection{Detection of anharmonicities and excitation pathways}

The time intervals $t_{2}$ and $t_{4}$ can be scanned to observe population decay, which we will show in the next section. For now, we set $t_{2}=t_{4}=0$ and scan the time intervals $t_{1}$ and 
$t_{3}$. Figure 6.12 displays the double quantum coherence signal $S_{1111 ; 1}^{(\mathrm{DQC})}\left(\Omega_{1}, \Omega_{3}\right)$, where all excitation pulses are applied to the same ion. For excitation and readout, we follow the same recipe which was applied in section 6.5.1.1. We denote the eigenfrequencies of double excited states by $\omega_{f_{i}}$, that is $H_{\mathrm{ph}}\left|f_{i}\right\rangle=\hbar \omega_{f_{i}}\left|f_{i}\right\rangle$. The ordering is chosen such that $\omega_{f_{1}} \leq \omega_{f_{2}} \leq \ldots$ Along a Fourier-transformed $t_{3}$ interval, which yields the $\Omega_{3}$ axis in a two-dimensional spectrum, the diagrams $D Q C_{2}$ and $D Q C_{3}$ thus reveal the frequency differences $\omega_{i j}^{\prime}=\omega_{f_{i}}-\omega_{j}$, whereas from $D Q C_{1}$ we recover the single excited state frequencies $\omega_{i}$. If the trap potential is perfectly harmonic, $U=0$, the energies of double excited states are given by the sum of single-excited state frequencies, $\omega_{f_{i}}=\omega_{j}+\omega_{k}$, which leads to many degeneracies, such as $\omega_{11}^{\prime}=\omega_{22}^{\prime}$, which can be seen in figure $6.12 \mathrm{c}$ ). Moreover, we can observe the resonance $\omega_{f_{i}}=2 \omega_{i}$ only if the single-excited state $\omega_{i}$ was already populated during $t_{1}$. Only then the second pulse can promote the system into the double excited state, and, consequently, such resonances are always found along the $\Omega_{1}=\omega_{i}$ axis (Gessner et al., 2014c). This demonstrates how the two-dimensional spectra express connections between double and single excited states by revealing their excitation pathways in a two-pulse scheme. The picture changes when we introduce anharmonic corrections, $U=-0.025 \nu_{x}$, as in figure $6.12 \mathrm{~d}$ ): The degeneracies of double-excited states are lifted and new excitation pathways are opened up via the induced coupling between double-excited states. This is expressed by the emergence of a new peak at $\left(\omega_{1}, \omega_{31}^{\prime}\right)$ (Gessner et al., 2014c).

\subsubsection{Spatially resolved eigenmode analysis}

Let us consider the double quantum coherence pulse sequence, but now we set $t_{1}=t_{3}=$ 0 , while scanning $t_{2}$ and $t_{4}$ to reveal the double excited state frequencies and the local tunneling rates. In a three-ion chain, we first apply all excitation and readout pulses to the same ion, at the end of the string. This produces a large ensemble of excited state frequencies, since the outer ions contribute to the motion of all eigenmodes. The corresponding spectrum is shown in figure 6.13 a). If, additionally, an anharmonic correction term contributes, the remaining degeneracies within these resonances are also lifted, leading to small splitting of the peaks into doublets, see figure $6.13 \mathrm{c}$ ). Along $\Omega_{4}$ the frequency differences can be observed, which reveal the coherent phonon couplings between the individual local sites. The picture is significantly simplified when the readout is shifted towards the center ion, which does not participate in any motion which involves the breathing mode. Without anharmonicities, $U=0$, only few degenerate energy levels and coupling terms contribute to the double quantum coherence signal in figure $6.13 \mathrm{~b}$ ). These indicate the frequencies of modes that involve the center ion. Anharmonic corrections, induced by $U=-0.01 \nu_{x}$, lead to splitting of the double-excited state frequencies, and consequently, also produce additional local phonon tunneling frequencies, and open up additional excitation pathways - see, for instance, the emergence of a peak at $\left(\Omega_{2}, \Omega_{4}\right) \approx\left(1.75 \nu_{x},-0.1 \nu_{x}\right)$ in figure $\left.6.13 \mathrm{~d}\right)$. 


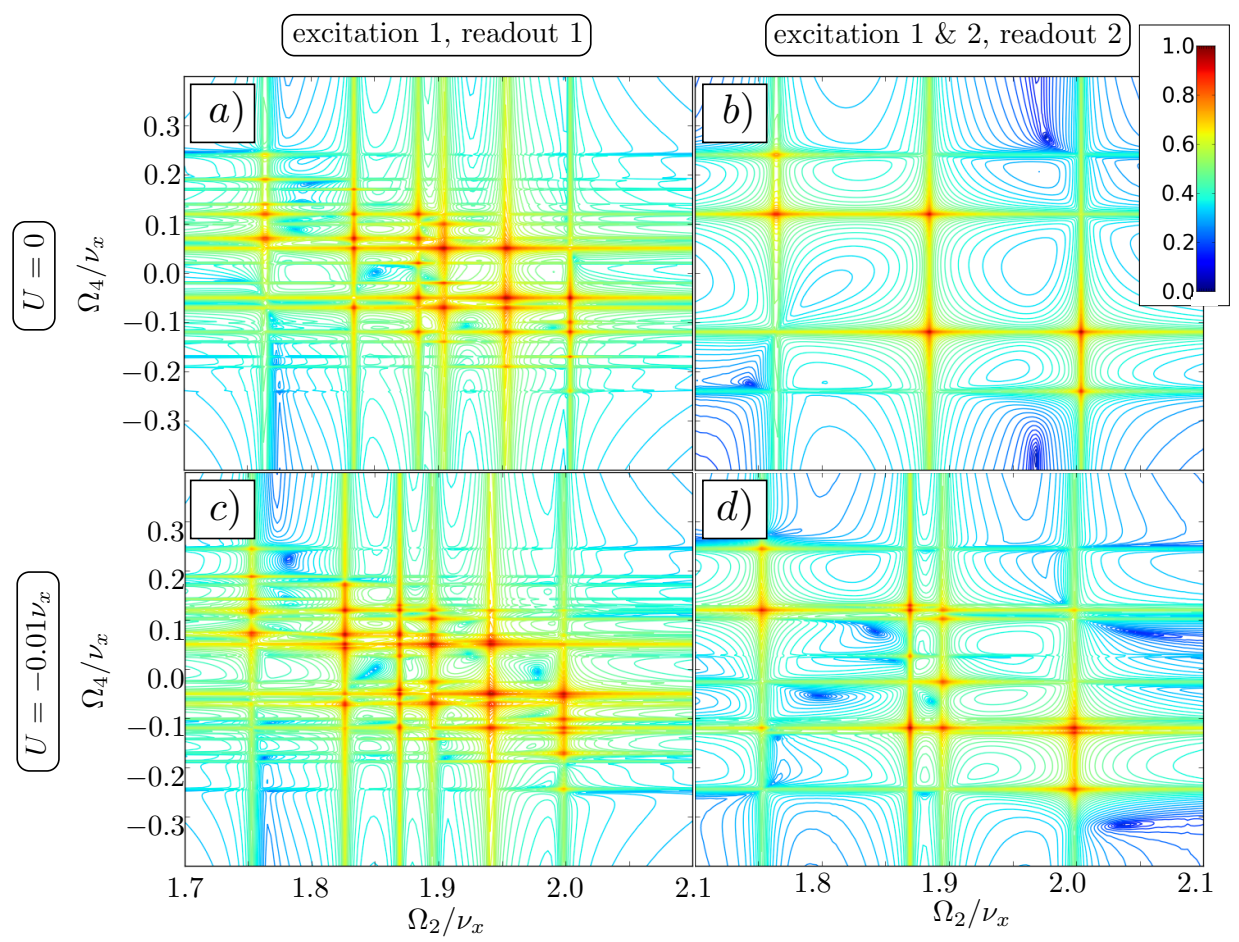

FIGURE 6.13.: Double quantum coherence signal (see text) for the phonons in a chain of three ions, described by (6.66), with and without anharmonic corrections. Signals a) and c) show $\left|S_{111 ; 1}^{(\mathrm{DQC})}\left(t_{1}=0, \Omega_{2}, t_{3}=0, \Omega_{4}\right)\right|$, while, in b) and d), local excitation and readout displays only those frequencies which contribute at the trap center in the signal $\left|S_{1122 ; 2}^{(\mathrm{DQC})}\left(t_{1}=0, \Omega_{2}, t_{3}=0, \Omega_{4}\right)\right|$. Figure adapted from (Schlawin et al., 2014).

\subsubsection{Four-pulse sequence: Photon echo}

In this final subsection, we introduce the fluorescence analog of the photon echo signal. This four-pulse sequence is often employed for studying coherent and incoherent couplings in complex environments, for instance in molecular aggregates (Brixner et al., 2005; Engel et al., 2007). It is identified via the phase signature $-\phi_{1}+\phi_{2}+\phi_{3}-\phi_{4}$ and, under the usual assumptions, the four diagrams depicted in figure 6.14 must be considered. They are labelled ground state bleaching $(G S B)$, excited state emission $(E S E)$, and excited state absorption (ESA) for historical reasons (Mukamel, 1995), whereas, in the present fluorescence scenario, there are two pathways that contribute to the $E S A$ signal.

To follow the population decay of phonons, we monitor the photon echo signal as a function of the $t_{2}$ time, while the time intervals $t_{1}$ and $t_{3}$ are Fourier transformed to reveal the contributing eigenmodes. We subject each of the ions in the chain to a 


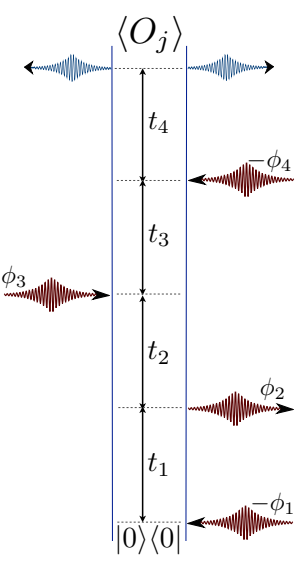

$G S B$

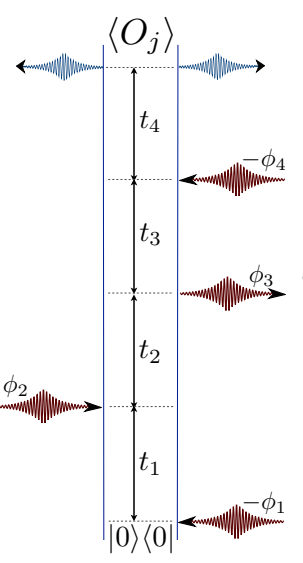

ESE

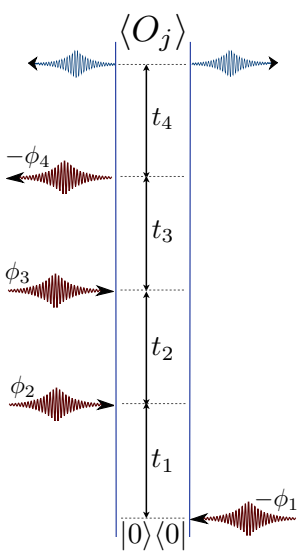

ESAa

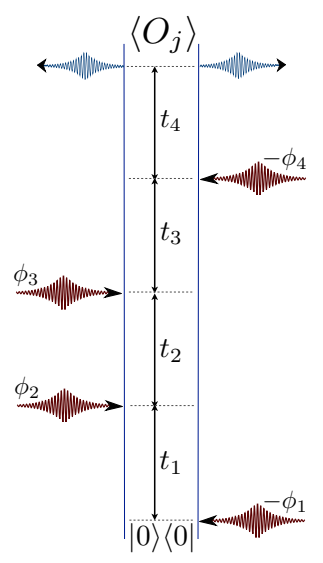

$E S A b$

FiguRE 6.14.: Four diagrams contribute to the photon echo signal (see text), which is defined via its characteristic phase signature $-\phi_{1}+\phi_{2}+\phi_{3}-\phi_{4}$. Figure adapted from (Schlawin et al., 2014).

thermal bath of the same temperature, such that the total dynamics is described by

$$
\begin{aligned}
\mathcal{L} \rho(t)=-\frac{i}{\hbar}\left[H_{\mathrm{ph}}, \rho\right] & +\gamma(\bar{n}+1) \sum_{i=1}^{N}\left(a_{i} \rho(t) a_{i}^{\dagger}-\frac{1}{2}\left\{a_{i}^{\dagger} a_{i}, \rho(t)\right\}\right) \\
& +\gamma \bar{n} \sum_{i=1}^{N}\left(a_{i}^{\dagger} \rho(t) a_{i}-\frac{1}{2}\left\{a_{i} a_{i}^{\dagger}, \rho(t)\right\}\right) .
\end{aligned}
$$

For the simulations plotted in figure 6.15, we considered the parameters $N=3, U=$ $-0.03 \nu_{x}, \bar{n}=0.1$ and $\gamma=0.0015 \nu_{x}$. The photon echo signal is obtained by applying all pulses, as well as the readout, to the center ion. During $t_{2}$, all contributions can decay to the ground state due to environmental couplings, except for the $G S B$ signal, which already describes the contribution of the ground state during $t_{2}$. The coupling to the thermal bath can in principle also lead to excitations of higher-energy states - but this process is much less likely to happen due to the small value of $\bar{n}$. Remember that, in order to yield a nonzero signal, the pathway must lead to an excited-state population at the end of the sequence. When the population decays at intermediate times, this is no longer possible for the ESE and ESAa diagrams. Thus, in the presence of population decay, the relative weights of the individual diagrams to the total signal shift as a function of $t_{2}$.

Let us start by analyzing the signal at $t_{2}=0$, which is shown in figure $6.15 \mathrm{a}$ ). Along $\Omega_{1}$, we observe the contributing single excited state frequencies $\omega_{i}$. These are also seen along $\Omega_{3}$ from the contribution of the $G S B$ and $E S E$ diagrams, whereas the two ESA diagrams contribute the frequency differences $\omega_{i j}$ between double-excited and singleexcited states. These overlap with the frequencies $\omega_{i}$ when $U=0$; however, the two 

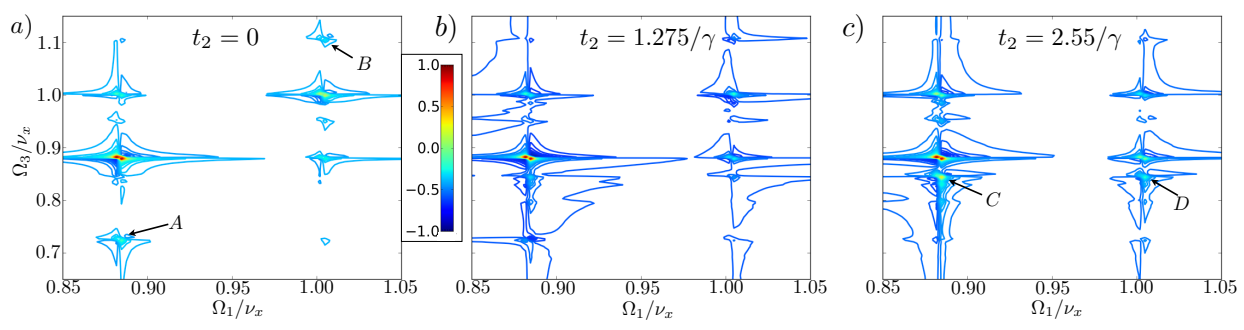

FIGURE 6.15.: Real part of the photon echo signal $S_{2222 ; 2}^{(\mathrm{PE})}\left(\Omega_{1}, t_{2}, \Omega_{3}, t_{4}=0\right)$, as introduced in the text, for a phonon chain of $N=3$ ions, coupled to local thermal baths with $\bar{n}=0.1, \gamma=0.0015 \nu_{x}, U=-0.03 \nu_{x}$ for different times $t_{2}$; see equation (6.101). The off-diagonal resonances $A$ and $B$ indicate the presence of anharmonic couplings between double-excited states. The relative weights of individual diagram contributions shifts as $t_{2}$ increases, due to incoherent population decay into the environment. As a consequence, the off-diagonal peaks diminish, while other resonances $(C$ and $D)$ emerge. Figure adapted from (Schlawin et al., 2014).

anharmonic peaks $A$ and $B$ (which cannot be found along $\Omega_{1}$ ) reveal the presence of anharmonic coupling terms in the Hamiltonian. As $t_{2}$ increases, these anharmonic peaks lose intensity, since they are mostly generated by the ESAa diagram, which, as explained above, is suppressed by population decay. Instead, those peaks which can be traced back to the $E S A b$ diagram will increase in intensity. This explains the emergence of two peaks $C$ and $D$ in figure $6.15 \mathrm{c}$ ). Note also the broadening of resonances due to the incoherent coupling to an environment.

To verify the above intuition about the relative contribution of individual diagrams, we can take advantage of the rather flexible excitation mechanisms available for the phonons in an ion trap. As was discussed in section 6.4.4.2, by applying strong resonant pulses on sideband transitions, we can generate selective excitations or de-excitations, which allows for experimental distinction between the individual diagrams that comprise the photon echo signal. In bulk systems, typical interactions include both possibilities simultaneously and must therefore be described in form of transition operators as in equation (6.83). Consequently, all diagrams interfere and cannot be distinguished. This is different now, if we make use of selective (de-)excitations in controllable quantum systems. For example, the $G S B$ diagram is distinguished from the other three diagrams by containing a de-excitation when interacting with the second pulse, see figure 6.14, while in all other pathways, the second pulse excites the system. Appropriate sequences of pulses leading to the transitions $V_{+}$and $V_{-}$, as defined in equations (6.76) and (6.76), respectively, allow to distinguish all four diagrams which build up the total photon echo signal (Schlawin et al., 2014). Their individual contributions are shown in figure 6.16. Indeed, we find that, as expected, the $G S B$ and $E S E$ pathways only contain contributions of single-excited states, whereas the cross-peaks $(A$ and $B)$ are most pronounced in the $G S B$ pathway. The anharmonic resonances (peaks $C$ and $D$ ) only occur in the two ESA pathways. Note also that the opposing signs of the two 
$E S A$ pathways almost cancel their contributions to the total signal at $t_{2}=0$. Since the $E S A a$ is suppressed by population decay when $t_{2}$ increases, a strong signature of $E S A b$ becomes visible in figure $6.15 \mathrm{c}$ ).

\subsection{Discussion}

In this chapter, we provided the theoretical foundation of phase-coherent multi-pulse spectroscopy of controllable quantum systems. This provides an elementary set of tools with which arbitrary nonlinear spectroscopic measurement schemes can be designed. A combination of specifically designed excitation pulses, followed by readout, is able to extract multi-time correlation functions, which in turn reveal the energy spectrum, internal couplings, and dissipative mechanisms induced by external environments. It is important to realize that the methods presented here do not provide a prescription for a universal measurement procedure, but instead should be considered a general toolbox, whose constituents must be combined in an appropriate way to extract the relevant information from a complex quantum system, depending on the question to be answered.

The importance of developing scalable methods for the analysis of coherent quantum dynamics, and relevant noise processes, is underlined by recent experimental achievements towards quantum simulations of medium-size systems (Häffner et al., 2008; Schneider et al., 2012; Blatt and Roos, 2012), which soon are expected to become intractable for classical computers. Artificial quantum systems further enable flexible modifications of the excitation and readout observables, which may allow us to design novel four-pulse measurement schemes, beyond the reach of conventional optical nonlinear spectroscopy. The number of pulses necessary to obtain a certain $n$-time correlation function is independent of the system size, and the presented schemes are therefore expected to be applicable to large-scale systems.

When the number of energy levels and the spectral densities increase, the resolution must be increased accordingly by adjusting the number of measured time steps, if the full information about the system's specific details is still of interest. In this limit, however, we rather expect that the center position, shape, and width of certain energy bands will be more relevant than the individual position of each quantum mechanical energy level, and, therefore, microscopic resolution would no longer be required. 


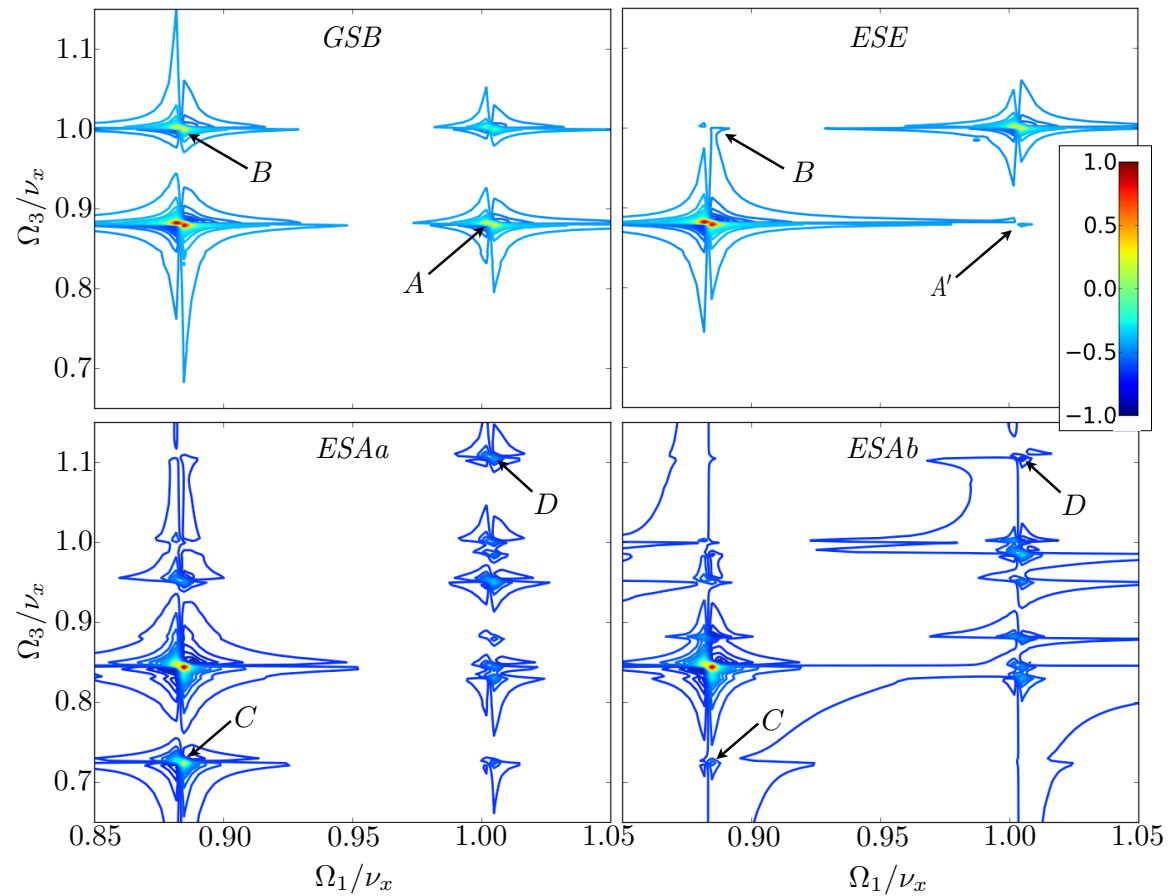

FIGURE 6.16.: Real part of the photon echo signal $S_{2222 ; 2}^{(\mathrm{PE})}\left(\Omega_{1}, t_{2}=0, \Omega_{3}, t_{4}=0\right)$, dissected into the contribution of individual diagrams (see figure 6.14), for a phonon chain of $N=3$ ions, coupled to local thermal baths with $\bar{n}=0.1, \gamma=0.0015 \nu_{x}$, $U=-0.03 \nu_{x}$; see equation (6.101). The flexibility of excitation methods for controlled quantum systems allows to monitor these diagrams individually, which is not possible in bulk material. The $G S B$ and $E S E$ contain only contributions of single excited states, with pronounced cross-peaks $A$ and $B$ in $G S B$. The diagrams $E S A a$ and $E S A b$ are suitable for studying higher excited states and reveal the anharmonic corrections via the additional peaks at $C$ and $D$. Figure adapted from (Schlawin et al., 2014). 


\section{OPEN QUANTUM SYSTEMS OF IDENTICAL PARTICLES}

We discuss the description of the reduced dynamics of a subset of interacting bosonic particles. Partially tracing over the von Neumann equation of an $N$-particle system produces a hierarchical expansion for the subdynamics of $M$ particles. In the special case of a contact interaction potential, truncating this hierarchy to first order with a pure product state assumption reproduces the mean-field dynamics as generated by the Gross-Pitaevskii equation. To describe incoherent effects beyond mean-field dynamics, we discuss possible extensions towards a second-order perturbation theory, analogous to the standard treatment of distinguishable system and environment.

\subsection{Introduction: Identical particles}

Throughout this thesis, we have discussed a variety of theoretical and experimental methods to probe the dynamics of interacting many-body systems, often by restricting to the local control of only a small subsystem. The theoretical description of the dynamics of such subsystems is in most cases provided by the theory of open quantum systems (Breuer and Petruccione, 2002), which was also briefly discussed in section 2.5.3. The relevant master equations are usually derived in a first quantized description with well separated Hilbert spaces for system and environment. This is, however, not always appropriate. Consider for example an ensemble of interacting bosonic particles, such as cold atoms forming a Bose-Einstein condensate. To predict the expectation values of single-particle observables, such as the average momentum which can be easily measured (Morsch et al., 2001; Meinert et al., 2014) -, there is no need to know the full many-body density operator. Instead, the reduced single-particle density operator provides all the necessary information. To describe the dynamics of single-particle observables, an effective description of the single-particle dynamics is required. Here, the indistinguishability of the individual particles prevents us from 
using standard derivations of master equations. Instead, the concept of reduced density operators and the partial trace operation must be adjusted to derive the relevant properties of an $M$-particle subspace from a larger $N$-particle space of identical particles. Thus, the rather simple experimental access to single-particle observables stands in severe contrast to the difficulty to theoretically describe the reduced distributions of indistinguishable, interacting particles.

We first review how the reduced density operator of the $M$-particle subspace is defined, and how a partial trace operation connects it to the full $N$-particle density operator. To this end, we introduce a general formalism in terms of symmetrized basis states.

\subsubsection{Symmetrized states}

A system of identical quantum mechanical particles is described by states in the symmetrized or anti-symmetrized subspaces of the total $N$-particle Hilbert space, depending on whether the particles are bosons or fermions (Cohen-Tannoudji et al., 1977b). Throughout this section, we will only be concerned with the dynamics of bosonic particles, which are described by the symmetric subspaces, but the following construction can be readily extended to obtain a basis of anti-symmetrized states to describe fermionic systems (Nolting, 2009). We construct a basis of the full symmetric $N$-particle space from the eigenbasis $\left\{\left|\varphi_{i}\right\rangle\right\}$ of a single-particle observable. The associated bosonic creation operator $a_{i}^{\dagger}$ creates one particle in the state $\left|\varphi_{i}\right\rangle$. Thus, if $|0\rangle$ is the vacuum state of no particles, we have

$$
a_{i}^{\dagger}|0\rangle=\left|\varphi_{i}\right\rangle .
$$

An $N$-particle state can be constructed by application of $N$ creation operators to the vacuum,

$$
\left|\varphi_{i_{1}} \ldots \varphi_{i_{N}}\right\rangle=\frac{1}{\sqrt{N !}} a_{i_{1}}^{\dagger} \cdots a_{i_{N}}^{\dagger}|0\rangle,
$$

and these states already satisfy the required symmetry property with respect to permutations of their indices, ${ }^{1}$ since the $a_{i}^{\dagger}$ satisfy the canonical commutation relations,

$$
\left[a_{i}, a_{j}^{\dagger}\right]=\delta_{i j}, \quad\left[a_{i}^{\dagger}, a_{j}^{\dagger}\right]=0, \quad\left[a_{i}, a_{j}\right]=0, \quad \forall i, j .
$$

As we will confirm explicitly later, the factor $1 / \sqrt{N !}$ ensures that the symmetrized states (7.2) are properly normalized. Note that usual formulations of second quantization would typically label the state $\left|\varphi_{i_{1}} \ldots \varphi_{i_{N}}\right\rangle$ in terms of the occurrence of each of the single-particle states, in the commonly employed number state representation (Nolting, 2009). Here, we choose to represent our observables and states in the above

\footnotetext{
${ }^{1}$ We emphasize that the symmetrized states must not be mistaken for the tensor product of the single-particle states, $\left|\varphi_{i_{1}} \ldots \varphi_{i_{N}}\right\rangle \neq\left|\varphi_{i_{1}}\right\rangle \otimes \cdots \otimes\left|\varphi_{i_{N}}\right\rangle$, as will become obvious from the equivalent definition (7.7). In the remainder of this chapter, we reserve the expression $\left|\varphi_{i_{1}} \ldots \varphi_{i_{N}}\right\rangle$ for the symmetrized tensor product of single-particle states, and indicate the standard tensor product explicitly by writing out all the $\otimes$-signs.
} 
form. One motivation is given by the particularly simple form of the completeness relation in the $N$-particle space

$$
\sum_{i_{1} \ldots i_{N}}\left|\varphi_{i_{1}} \ldots \varphi_{i_{N}}\right\rangle\left\langle\varphi_{i_{1}} \ldots \varphi_{i_{N}}\right|=\mathbb{I}^{(N)},
$$

where henceforth the superscript $(N)$ denotes operators on the $N$-particle space. The action of creation and annihilation operators $a_{k}^{\dagger}$ and $a_{k}$ on the symmetrized $N$-particle states is described by

$$
a_{k}^{\dagger}\left|\varphi_{i_{1}} \ldots \varphi_{i_{N}}\right\rangle=\sqrt{N+1}\left|\varphi_{k} \varphi_{i_{1}} \ldots \varphi_{i_{N}}\right\rangle
$$

which generalizes equation (7.1), and

$$
a_{k}\left|\varphi_{i_{1}} \ldots \varphi_{i_{N}}\right\rangle=\frac{1}{\sqrt{N}} \sum_{j=1}^{N} \delta_{k i_{j}}\left|\varphi_{i_{1}} \ldots \varphi_{i_{j-1}} \varphi_{i_{j+1}} \ldots \varphi_{i_{N}}\right\rangle .
$$

The symmetrized states can also be directly constructed from artificially symmetrizing a tensor product of single-particle states (Nolting, 2009),

$$
\left|\varphi_{i_{1}} \ldots \varphi_{i_{N}}\right\rangle=\frac{1}{N !} \sum_{\sigma \in S_{N}}\left|\varphi_{i_{\sigma(1)}}\right\rangle \otimes \cdots \otimes\left|\varphi_{i_{\sigma(N)}}\right\rangle,
$$

in analogy to the symmetrization operation (4.32) which was considered in section 4.4. Starting from the above formulation, one can, in fact, construct the bosonic creation operators $a_{i}^{\dagger}$, and show that these then satisfy the canonical commutation relations (7.3) (Nolting, 2009). Moreover, the scalar product of two symmetrized states follows as

$$
\left\langle\varphi_{i_{1}} \ldots \varphi_{i_{N}} \mid \varphi_{j_{1}} \ldots \varphi_{j_{N}}\right\rangle=\frac{1}{N !} \sum_{\sigma \in \mathcal{S}_{N}} \delta_{i_{1} j_{\sigma(1)}} \cdots \delta_{i_{N} j_{\sigma(N)}} .
$$

We now employ this representation in terms of symmetrized states to describe operators within the single-particle and two-particle subspaces. This way we gain some intuition for the symmetrized states formalism, and, in particular, for the caveats which are involved when dealing with many-body systems. In section 7.2 we develop an abstract set of rules which will establish our toolbox for the handling of symmetrized states, and then apply it in section 7.3 to generalize and formally define the notions introduced in the present chapter.

\subsubsection{The single particle subspace}

Any single-particle operator $A^{(1)}$ can be expressed in the symmetrized basis, which for $N=1$ trivially reduces to the single-particle basis from which it was constructed. We write

$$
A^{(1)}=\sum_{i j}\left|\varphi_{i}\right\rangle\left\langle\varphi_{i}\left|A^{(1)}\right| \varphi_{j}\right\rangle\left\langle\varphi_{j}\left|=\sum_{i j} A_{i j}^{(1)}\right| \varphi_{i}\right\rangle\left\langle\varphi_{j}\left|=\sum_{i j} A_{i j}^{(1)} a_{i}^{\dagger}\right| 0\right\rangle\langle 0| a_{j},
$$


with

$$
A_{i j}^{(1)}=\left\langle\varphi_{i}\left|A^{(1)}\right| \varphi_{j}\right\rangle=\operatorname{Tr}^{(1)}\left\{a_{j}^{\dagger}|0\rangle\langle 0| a_{i} A^{(1)}\right\},
$$

where the trace over the single-particle space is defined $\operatorname{as~}^{(1)} X=\sum_{i}\left\langle\varphi_{i}|X| \varphi_{i}\right\rangle$. The projector $|0\rangle\langle 0|$ on the vacuum state enters this expression via equation (7.1). In the formulation (7.9), the single-particle operator $A^{(1)}$ is restricted to the single-particle subspace, that is, the vacuum projector causes the expectation value of this operator to vanish, as soon as it is determined for a two- or more-particle state. This is caused by the emergence of expressions of the form $\left\langle 0\left|a_{j}\right| \varphi_{i_{1}} \varphi_{i_{2}}\right\rangle$, which always yield zero, since, after removing one particle from the two-particle state $\left|\varphi_{i_{1}} \varphi_{i_{2}}\right\rangle$, the resulting singleparticle state has zero overlap with the vacuum state. To determine the two-particle expectation value of a single-particle operator, we leave out the vacuum projector in the above formulation to obtain the commonly used form of a single-particle operator

$$
A^{(1)}=\sum_{i j} A_{i j}^{(1)} a_{i}^{\dagger} a_{j}
$$

The step from equation (7.9) to (7.11) can be interpreted as extending the singleparticle operator to an $\mathrm{N}$-particle operator, similarly to the tensor product with the identify operator on the remaining subspace in a scenario of distinguishable particles. To present a heuristic argument, the two-particle extension of $A^{(1)}$ can be written in an artificially symmetrized tensor product form as $A^{(2)}=\left(A^{(1)} \otimes \mathbb{I}^{(1)}+\mathbb{I}^{(1)} \otimes A^{(1)}\right) / 2$. This yields, in terms of creation and annihilation operators,

$$
\begin{aligned}
A^{(2)} & =\sum_{i j k l}\left|\varphi_{i} \varphi_{j}\right\rangle\left\langle\varphi_{i} \varphi_{j}\left|A^{(2)}\right| \varphi_{k} \varphi_{l}\right\rangle\left\langle\varphi_{k} \varphi_{l}\left|=\frac{1}{2} \sum_{i j k l} A_{i j ; k l}^{(2)} a_{i}^{\dagger} a_{j}^{\dagger}\right| 0\right\rangle\langle 0| a_{k} a_{l} \\
& =\sum_{i j k} A_{i j}^{(1)} a_{i}^{\dagger} a_{k}^{\dagger}|0\rangle\langle 0| a_{j} a_{k},
\end{aligned}
$$

where in the last step we have used equation (7.7) to obtain $A_{i j ; k l}^{(2)}=\left\langle\varphi_{i} \varphi_{j}\left|A^{(2)}\right| \varphi_{k} \varphi_{l}\right\rangle=$ $\left(A_{i k}^{(1)} \delta_{j l}+A_{j l}^{(1)} \delta_{i k}\right) / 2$. Moreover, we have introduced a semicolon to separate two permutationally invariant sets of indices. We will employ this notation throughout this chapter. For two-particle states $\left|\varphi_{n} \varphi_{m}\right\rangle$, the operator $\sum_{k} a_{i}^{\dagger} a_{k}^{\dagger}|0\rangle\langle 0| a_{j} a_{k}$ is indeed equivalent to $a_{i}^{\dagger} a_{j}$ :

$$
\begin{aligned}
\sum_{k} a_{i}^{\dagger} a_{k}^{\dagger}|0\rangle\left\langle 0\left|a_{j} a_{k}\right| \varphi_{n} \varphi_{m}\right\rangle & =\sum_{k}\left(\delta_{k n} \delta_{j m}+\delta_{k m} \delta_{j n}\right)\left|\varphi_{k} \varphi_{i}\right\rangle=\delta_{j m}\left|\varphi_{n} \varphi_{i}\right\rangle+\delta_{j n}\left|\varphi_{m} \varphi_{i}\right\rangle \\
& =a_{i}^{\dagger} a_{j}\left|\varphi_{n} \varphi_{m}\right\rangle \quad \forall n, m, i, j .
\end{aligned}
$$

Hence, we will generally omit the vacuum projectors in the decomposition of $M$-particle operators, to be able to determine their expectation values in spaces of larger particle numbers. 
A somewhat distinguished single-particle operator is the statistical operator $\rho^{(1)}$. The expectation value of the observable $A^{(1)}$ is given by

$$
\left\langle A^{(1)}\right\rangle=\operatorname{Tr}^{(1)}\left\{A^{(1)} \rho^{(1)}\right\} \stackrel{(7.11)}{=} \operatorname{Tr}^{(1)}\left\{\sum_{i j k l} A_{i j}^{(1)} \rho_{k l}^{(1)} a_{i}^{\dagger} a_{j} a_{k}^{\dagger} a_{l}\right\} \stackrel{(7.15)}{=} \sum_{i j} A_{i j}^{(1)} \rho_{j i}^{(1)} .
$$

Here we have used

$$
\begin{aligned}
\operatorname{Tr}^{(1)}\left\{a_{i}^{\dagger} a_{j} a_{k}^{\dagger} a_{l}\right\} & =\sum_{n}\left\langle\varphi_{n}\left|a_{i}^{\dagger} a_{j} a_{k}^{\dagger} a_{l}\right| \varphi_{n}\right\rangle=\sum_{n} \delta_{l n}\left\langle\varphi_{n}\left|a_{i}^{\dagger} a_{j}\right| \varphi_{k}\right\rangle \\
& =\sum_{n} \delta_{l n} \delta_{j k} \delta_{n i}=\delta_{l i} \delta_{j k},
\end{aligned}
$$

which in turn follows from equations (7.5) and (7.6). The trace of an $N$-particle operator can be computed in any $M$-particle basis. It is important to know in which basis the trace is computed. We denote the trace in the $M$-particle basis by $\operatorname{Tr}^{(M)}$. If we determine the trace (7.15) in a two-particle basis, we obtain

$$
\begin{aligned}
\operatorname{Tr}^{(2)}\left\{a_{i}^{\dagger} a_{j} a_{k}^{\dagger} a_{l}\right\} & =\sum_{m n}\left\langle\varphi_{m} \varphi_{n}\left|a_{i}^{\dagger} a_{j} a_{k}^{\dagger} a_{l}\right| \varphi_{n} \varphi_{m}\right\rangle \\
& =\delta_{i l} \delta_{j k}(2+d)+\delta_{i j} \delta_{l k},
\end{aligned}
$$

where $d$ is the dimension of the single-particle Hilbert space. By making use of the commutation relation $\left[a_{i}, a_{j}^{\dagger}\right]=\delta_{i j}$, the two-particle trace of the two-particle operator $a_{i}^{\dagger} a_{j}^{\dagger} a_{k} a_{l}$ can also be expressed in terms of the trace of $a_{i}^{\dagger} a_{j} a_{k}^{\dagger} a_{l}$. Here it is important to keep track of the space over which the trace is carried out:

$$
\begin{aligned}
\operatorname{Tr}^{(2)}\left\{a_{i}^{\dagger} a_{j}^{\dagger} a_{k} a_{l}\right\} & =\underbrace{\operatorname{Tr}^{(2)}\left\{a_{i}^{\dagger} a_{k} a_{j}^{\dagger} a_{l}\right\}}_{\delta_{i l} \delta_{j k}(2+d)+\delta_{i k} \delta_{l j}}-\delta_{j k} \underbrace{\operatorname{Tr}^{(2)}\left\{a_{i}^{\dagger} a_{l}\right\}}_{(1+d) \delta_{i l}} \\
& =\delta_{i l} \delta_{j k}+\delta_{i k} \delta_{l j} .
\end{aligned}
$$

\subsection{3 $N$-particle operators}

The above treatment of single-particle operators is readily generalized to $N$-particle operators and states. Here we have to pay attention to the normalization factors in equations (7.2) and (7.8), which lead to

$$
A^{(N)}=\frac{1}{N !} \sum_{\substack{i_{1} \ldots i_{N} \\ j_{1} \ldots j_{N}}} A_{i_{1} \ldots i_{N} ; j_{1} \ldots j_{N}}^{(N)} a_{i_{1}}^{\dagger} \ldots a_{i_{N}}^{\dagger} a_{j_{1}} \ldots a_{j_{N}}
$$

and

$$
\begin{aligned}
A_{i_{1} \ldots i_{N} ; j_{1} \ldots j_{N}}^{(N)} & =\left\langle\varphi_{i_{1}} \ldots \varphi_{i_{N}}\left|A^{(N)}\right| \varphi_{j_{1}} \ldots \varphi_{j_{N}}\right\rangle \\
& =\frac{1}{N !} \operatorname{Tr}^{(N)}\left\{a_{i_{1}} \ldots a_{i_{N}} A^{(N)} a_{j_{1}}^{\dagger} \ldots a_{j_{N}}^{\dagger}\right\} .
\end{aligned}
$$




\subsubsection{The single-particle density operator and partial trace}

Let us now consider the expectation value of a single-particle observable in an $N$ particle system:

$$
\operatorname{Tr}^{(N)}\left\{A^{(1)} \rho^{(N)}\right\}=\sum_{i j} A_{i j}^{(1)} \operatorname{Tr}^{(N)}\left\{a_{i}^{\dagger} a_{j} \rho^{(N)}\right\} .
$$

We find that to determine this expectation value, we need to know the elements $\operatorname{Tr}^{(N)}\left\{a_{i}^{\dagger} a_{j} \rho^{(N)}\right\}$, which we define as the elements of the single-particle density operator (Löwdin, 1955; Ter Haar, 1960; Ter Haar, 1961; Yang, 1962),

$$
\rho^{(1)}=\frac{1}{N} \sum_{i j} \operatorname{Tr}^{(N)}\left\{a_{i} \rho^{(N)} a_{j}^{\dagger}\right\} a_{i}^{\dagger} a_{j} .
$$

With this, the expectation value can be expressed as

$$
\operatorname{Tr}^{(N)}\left\{A^{(1)} \rho^{(N)}\right\}=N \sum_{i j} A_{i j}^{(1)} \rho_{j i}^{(1)}=N \operatorname{Tr}^{(1)}\left\{A^{(1)} \rho^{(1)}\right\} .
$$

Thus, the expectation value of the single-particle operator for a system of $N$ particles is given by $N$ times the single-particle expectation value. This also defines the partial trace operation which maps an $N$-particle state to a reduced operator of the singleparticle subspace

$$
\operatorname{Tr}_{N-1}^{(N)}\left\{X^{(N)}\right\}:=\frac{1}{N} \sum_{i j} \operatorname{Tr}^{(N)}\left\{a_{i} X^{(N)} a_{j}^{\dagger}\right\} a_{i}^{\dagger} a_{j}
$$

With this, we can write

$$
\rho^{(1)}=\operatorname{Tr}_{N-1}^{(N)}\left\{\rho^{(N)}\right\}
$$

\subsection{General formalism for symmetrized states}

In this section, we extend and generalize the concepts from the previous introductory section. To be able to handle symmetrized states efficiently, we start by deriving a series of lemmata. It is convenient to first introduce

Definition 1 (Permutation-invariant Kronecker delta).

$$
\delta_{i_{1} \ldots i_{N} ; j_{1} \ldots j_{N}}:=\frac{1}{N !} \sum_{\sigma \in \mathcal{S}_{N}} \prod_{k=1}^{N} \delta_{i_{k}, j_{\sigma(k)}} .
$$

Obviously $\delta_{i_{1} \ldots i_{N} ; j_{1} \ldots j_{N}}=\delta_{j_{1} \ldots j_{N} ; i_{1} \ldots i_{N}}$. If one of the sets $\left\{i_{1} \ldots i_{N}\right\}$ and $\left\{j_{1} \ldots j_{N}\right\}$ is invariant under all permutations this reduces to the simple product

$$
\delta_{i_{1} \ldots i_{N} ; j_{1} \ldots j_{N}}=\prod_{k=1}^{N} \delta_{i_{k}, j_{k}} .
$$


Lemma 1. Let $f\left(i_{1} \ldots i_{N}\right)$ be a function which is invariant under permutations within the set of indices $i_{1} \ldots i_{N}$,

$$
f\left(i_{1} \ldots i_{N}\right)=f\left(i_{\sigma(1)} \ldots i_{\sigma(N)}\right),
$$

for all $\sigma \in \mathcal{S}_{N}$. Then the following holds

$$
\sum_{i_{1} \ldots i_{N}} f\left(i_{1} \ldots i_{N}\right) \delta_{i_{1} \ldots i_{N} ; j_{1} \ldots j_{N}}=f\left(j_{1} \ldots j_{N}\right) .
$$

Proof.

$$
\begin{aligned}
\sum_{i_{1} \ldots i_{N}} f\left(i_{1} \ldots i_{N}\right) \delta_{i_{1} \ldots i_{N} ; j_{1} \ldots j_{N}} & =\frac{1}{N !} \sum_{\sigma \in \mathcal{S}_{N}} \sum_{i_{1} \ldots i_{N}} f\left(i_{1} \ldots i_{N}\right) \prod_{l=1}^{N} \delta_{i_{l}, j_{\sigma(l)}} \\
& =\frac{1}{N !} \sum_{\sigma \in \mathcal{S}_{N}} f\left(j_{\sigma(1)} \ldots j_{\sigma(N)}\right) \\
& =f\left(j_{1} \ldots j_{N}\right) .
\end{aligned}
$$

Since the permutation-invariant Kronecker delta itself fulfills property (7.27), this implies

$$
\sum_{k_{1} \ldots k_{N}} \delta_{i_{1} \ldots i_{N} ; k_{1} \ldots k_{N}} \delta_{k_{1} \ldots k_{N} ; j_{1} \ldots j_{N}}=\delta_{i_{1} \ldots i_{N} ; j_{1} \ldots j_{N}}
$$

\section{Lemma 2.}

$$
a_{i_{1}} \ldots a_{i_{N}}\left|\varphi_{j_{1}} \ldots \varphi_{j_{N}}\right\rangle=\delta_{i_{1} \ldots i_{N} ; j_{1} \ldots j_{N}} \sqrt{N !}|0\rangle .
$$

Proof. Subsequent application of equation (7.6) leads to

$$
\begin{aligned}
a_{i_{1}} \ldots a_{i_{N}}\left|\varphi_{j_{1}} \ldots \varphi_{j_{N}}\right\rangle & =a_{i_{N}} \ldots a_{i_{1}}\left|\varphi_{j_{1}} \ldots \varphi_{j_{N}}\right\rangle \\
& =\frac{1}{\sqrt{N !}} \sum_{\alpha_{1} \in I} \delta_{i_{1}, j_{\alpha_{1}}} \sum_{\alpha_{2} \in I \backslash\left\{\alpha_{1}\right\}} \delta_{i_{2}, j_{\alpha_{2}}} \ldots \sum_{\alpha_{N} \in I \backslash\left\{\alpha_{1} \ldots \alpha_{N-1}\right\}} \delta_{i_{N}, j_{\alpha_{N}}}|0\rangle \\
& =\frac{1}{\sqrt{N !}} \sum_{\alpha_{1} \in I} \sum_{\alpha_{2} \in I \backslash\left\{\alpha_{1}\right\}} \ldots \sum_{\alpha_{N} \in I \backslash\left\{\alpha_{1} \ldots \alpha_{N-1}\right\}} \prod_{l \in I} \delta_{i_{l}, j_{\alpha_{l}}}|0\rangle \\
& =\frac{1}{\sqrt{N !}} \sum_{\sigma \in \mathcal{S}_{N}} \prod_{\alpha \in I} \delta_{i_{\alpha}, j_{\sigma(\alpha)}}|0\rangle \\
& =\delta_{i_{1} \ldots i_{N} ; j_{1} \ldots j_{N}} \sqrt{N !}|0\rangle
\end{aligned}
$$

with $I=\{1, \ldots, N\}$. 
This is compatible with the reformulation of equation (7.8)

$$
\begin{aligned}
\left\langle\varphi_{i_{1}} \ldots \varphi_{i_{N}} \mid \varphi_{j_{1}} \ldots \varphi_{j_{N}}\right\rangle & =\frac{1}{\sqrt{N !}}\left\langle 0\left|a_{i_{1}} \ldots a_{i_{N}}\right| \varphi_{j_{1}} \ldots \varphi_{j_{N}}\right\rangle \\
& =\frac{1}{N !} \sum_{\sigma \in \mathcal{S}_{N}} \prod_{k=1}^{N} \delta_{i_{k} j_{\sigma(k)}} \\
& =\delta_{i_{1} \ldots i_{N} ; j_{1} \ldots j_{N}} .
\end{aligned}
$$

For $M \leq N$, this can be generalized to

\section{Lemma 3.}

$$
\begin{aligned}
& a_{i_{1}} \ldots a_{i_{M}}\left|\varphi_{j_{1}} \ldots \varphi_{j_{N}}\right\rangle \\
& =\sqrt{\frac{(N-M) !}{N !}} M ! \sum_{1 \leq \alpha_{1}<\cdots<\alpha_{M} \leq N} \delta_{i_{1} \ldots i_{M} ; j_{\alpha_{1}} \ldots j_{\alpha_{M}}}\left|\varphi_{\left\{j_{1} \ldots j_{N}\right\} \backslash\left\{j_{\alpha_{1}} \ldots j_{\alpha_{M}}\right\}}\right\rangle,
\end{aligned}
$$

where we have introduced the shorthand notation $\left|\varphi_{\left\{j_{1} \ldots j_{N}\right\}}\right\rangle=\left|\varphi_{j_{1}} \ldots \varphi_{j_{N}}\right\rangle$, which contains the vacuum state as $\left|\varphi_{\{\}}\right\rangle=|0\rangle$.

Proof. We make use of the symmetry of this expression under permutations within the set $\left\{i_{1} \ldots i_{M}\right\}$ :

$$
\begin{aligned}
& a_{i_{1}} \ldots a_{i_{M}}\left|\varphi_{j_{1}} \ldots \varphi_{j_{N}}\right\rangle \\
& =\frac{1}{\sqrt{N !}} a_{i_{M}} \ldots a_{i_{1}} a_{j_{1}}^{\dagger} \ldots a_{j_{N}}^{\dagger}|0\rangle \\
& =\frac{1}{\sqrt{N !}} \sum_{\alpha_{1} \in I} \ldots \sum_{\alpha_{M} \in I \backslash\left\{\alpha_{1} \ldots \alpha_{M-1}\right\}} \delta_{i_{1} j_{\alpha_{1}}} \ldots \delta_{i_{M} j_{\alpha_{M}}} \prod_{l \in I \backslash\left\{\alpha_{1} \ldots \alpha_{M}\right\}} a_{j_{l}}^{\dagger}|0\rangle \\
& =\frac{M !}{\sqrt{N !}} \sum_{1 \leq \alpha_{1}<\cdots<\alpha_{M} \leq N} \delta_{i_{1} \ldots i_{M} ; j_{\alpha_{1}} \ldots j_{\alpha_{M}}} \prod_{l \in I \backslash\left\{\alpha_{1} \ldots \alpha_{M}\right\}} a_{j_{l}}^{\dagger}|0\rangle \\
& =\sqrt{\frac{(N-M) !}{N !}} M ! \sum_{1 \leq \alpha_{1}<\cdots<\alpha_{M} \leq N} \delta_{i_{1} \ldots i_{M} ; j_{\alpha_{1}} \ldots j_{\alpha_{M}}}\left|\varphi_{\left\{j_{1} \ldots j_{N}\right\} \backslash\left\{j_{\alpha_{1}} \ldots j_{\alpha_{M}}\right\}}\right\rangle .
\end{aligned}
$$

The special case for $M=1$ was already noted in equation (7.6). Similarly, we can generalize equation (7.5) to

$$
a_{i_{1}}^{\dagger} \ldots a_{i_{M}}^{\dagger}\left|\varphi_{j_{1}} \ldots \varphi_{j_{N}}\right\rangle=\sqrt{\frac{(N+M) !}{N !}}\left|\varphi_{i_{1}} \ldots \varphi_{i_{M}} \varphi_{j_{1}} \ldots \varphi_{j_{N}}\right\rangle
$$

which leads us to 


\section{Lemma 4.}

$$
\sum_{i_{1} \ldots i_{M}} a_{i_{1}}^{\dagger} \ldots a_{i_{M}}^{\dagger} a_{i_{1}} \ldots a_{i_{M}}\left|\varphi_{j_{1}} \ldots \varphi_{j_{N}}\right\rangle=\frac{N !}{(N-M) !}\left|\varphi_{j_{1}} \ldots \varphi_{j_{N}}\right\rangle .
$$

Proof.

$$
\begin{aligned}
& \sum_{i_{1} \ldots i_{M}} a_{i_{1}}^{\dagger} \ldots a_{i_{M}}^{\dagger} a_{i_{1}} \ldots a_{i_{M}}\left|\varphi_{j_{1}} \ldots \varphi_{j_{N}}\right\rangle \\
= & \sqrt{\frac{(N-M) !}{N !}} M ! \sum_{1 \leq \alpha_{1}<\cdots<\alpha_{M} \leq N} \sum_{i_{1} \ldots i_{M}} \delta_{i_{1} \ldots i_{M} ; j_{\alpha_{1}} \ldots j_{\alpha_{M}}} a_{i_{1}}^{\dagger} \ldots a_{i_{M}}^{\dagger}\left|\varphi_{\left\{j_{1} \ldots j_{N}\right\} \backslash\left\{j_{\alpha_{1}} \ldots j_{\alpha_{M}}\right\}}\right\rangle \\
= & \sqrt{\frac{(N-M) !}{N !}} M ! \sum_{1 \leq \alpha_{1}<\cdots<\alpha_{M} \leq N} a_{j_{\alpha_{1}}}^{\dagger} \ldots a_{j_{\alpha_{M}}}^{\dagger}\left|\varphi_{\left\{j_{1} \ldots j_{N}\right\} \backslash\left\{j_{\alpha_{1}} \ldots j_{\alpha_{M}}\right\}}\right\rangle \\
= & \sqrt{\frac{(N-M) !}{N !}} \sqrt{\frac{N !}{(N-M) !}} M ! \sum_{1 \leq \alpha_{1}<\cdots<\alpha_{M} \leq N}\left|\varphi_{j_{1}} \ldots \varphi_{j_{N}}\right\rangle \\
= & M !\left(\begin{array}{l}
N \\
M
\end{array}\right)\left|\varphi_{j_{1}} \ldots \varphi_{j_{N}}\right\rangle \\
= & \frac{N !}{(N-M) !}\left|\varphi_{j_{1}} \ldots \varphi_{j_{N}}\right\rangle .
\end{aligned}
$$

Another immediate conclusion from Lemma 3 is

\section{Lemma 5.}

$$
\begin{aligned}
& \left\langle\varphi_{i_{1}} \ldots \varphi_{i_{N}}\left|a_{k_{1}}^{\dagger} \ldots a_{k_{M}}^{\dagger} a_{l_{1}} \ldots a_{l_{M}}\right| \varphi_{j_{1}} \ldots \varphi_{j_{N}}\right\rangle \\
& =\frac{(N-M) !}{N !} M !^{2} \sum_{\substack{1 \leq \alpha_{1}<\cdots<\alpha_{M} \leq N \\
1 \leq \beta_{1}<\cdots<\beta_{M} \leq N}} \delta_{k_{1} \ldots k_{M} ; i_{\alpha_{1}} \ldots i_{\alpha_{M}}} \delta_{l_{1} \ldots l_{M} ; j_{\beta_{1}} \ldots j_{\beta_{M}}} \\
& \times \delta_{\left\{i_{1} \ldots i_{N}\right\} \backslash\left\{i_{\alpha_{1}} \ldots i_{\alpha_{M}}\right\} ;\left\{j_{1} \ldots j_{N}\right\} \backslash\left\{j_{\beta_{1}} \ldots j_{\beta_{M}}\right\}} .
\end{aligned}
$$

Lemma 6.

$$
\operatorname{Tr}^{(N)}\left\{a_{i_{1}}^{\dagger} \ldots a_{i_{N}}^{\dagger} a_{j_{1}} \ldots a_{j_{N}}\right\}=N ! \delta_{i_{1} \ldots i_{N} ; j_{1} \ldots j_{N}} .
$$

Proof.

$$
\begin{aligned}
\operatorname{Tr}^{(N)}\left\{a_{i_{1}}^{\dagger} \ldots a_{i_{N}}^{\dagger} a_{j_{1}} \ldots a_{j_{N}}\right\} & =\sum_{k_{1} \ldots k_{N}}\left\langle\varphi_{k_{1}} \ldots \varphi_{k_{N}}\left|a_{i_{1}}^{\dagger} \ldots a_{i_{N}}^{\dagger} a_{j_{1}} \ldots a_{j_{N}}\right| \varphi_{k_{1}} \ldots \varphi_{k_{N}}\right\rangle \\
& =N ! \sum_{k_{1} \ldots k_{N}} \delta_{k_{1} \ldots k_{N} ; i_{1} \ldots i_{N}} \delta_{j_{1} \ldots j_{N} ; k_{1} \ldots k_{N}} \\
& =N ! \delta_{i_{1} \ldots i_{N} ; j_{1} \ldots j_{N}} .
\end{aligned}
$$


Lemma 7. Let $X$ be an arbitrary operator.

$$
\operatorname{Tr}^{(N)}\left\{a_{i_{1}} \ldots a_{i_{N}} a_{j_{1}}^{\dagger} \ldots a_{j_{N}}^{\dagger} X\right\}=N ! \delta_{i_{1} \ldots i_{N} ; j_{1} \ldots j_{N}}\langle 0|X| 0\rangle .
$$

Proof. We use Lemmata 1 and 2:

$$
\begin{aligned}
& \sum_{\alpha_{1} \ldots \alpha_{N}}\left\langle\varphi_{\alpha_{1}} \ldots \varphi_{\alpha_{N}}\left|a_{j_{1}}^{\dagger} \ldots a_{j_{N}}^{\dagger} X a_{i_{1}} \ldots a_{i_{N}}\right| \varphi_{\alpha_{1}} \ldots \varphi_{\alpha_{N}}\right\rangle \\
& =N ! \sum_{\alpha_{1} \ldots \alpha_{N}} \delta_{\alpha_{1} \ldots \alpha_{N} ; j_{1} \ldots j_{N}} \delta_{i_{1} \ldots i_{N} ; \alpha_{1} \ldots \alpha_{N}}\langle 0|X| 0\rangle \\
& =N ! \delta_{i_{1} \ldots i_{N} ; j_{1} \ldots j_{N}}\langle 0|X| 0\rangle .
\end{aligned}
$$

Lemma 8. Let $X$ be an arbitrary operator.

$$
\operatorname{Tr}^{(N)}\left\{a_{j_{1}}^{\dagger} \ldots a_{j_{N}}^{\dagger} a_{i_{1}} \ldots a_{i_{N}} X\right\}=N !\left\langle\varphi_{i_{1}} \ldots \varphi_{i_{N}}|X| \varphi_{j_{1}} \ldots \varphi_{j_{N}}\right\rangle
$$

Proof.

$$
\begin{aligned}
& \sum_{\alpha_{1} \ldots \alpha_{N}}\left\langle\varphi_{\alpha_{1}} \ldots \varphi_{\alpha_{N}}\left|X a_{j_{1}}^{\dagger} \ldots a_{j_{N}}^{\dagger} a_{i_{1}} \ldots a_{i_{N}}\right| \varphi_{\alpha_{1}} \ldots \varphi_{\alpha_{N}}\right\rangle \\
= & N ! \sum_{\alpha_{1} \ldots \alpha_{N}}\left\langle\varphi_{\alpha_{1}} \ldots \varphi_{\alpha_{N}}|X| \varphi_{j_{1}} \ldots \varphi_{j_{N}}\right\rangle \delta_{i_{1} \ldots i_{N} ; \alpha_{1} \ldots \alpha_{N}} \\
= & N !\left\langle\varphi_{i_{1}} \ldots \varphi_{i_{N}}|X| \varphi_{j_{1}} \ldots \varphi_{j_{N}}\right\rangle .
\end{aligned}
$$

\subsection{Density matrices and expectation values}

Based on the tools developed in the previous section, we establish some elementary facts about $N$-particle operators, which we use to formally introduce full $N$-particle and reduced $M$-particle density operators.

\subsection{1 $N$-particle density operator}

We introduce the $N$-particle density matrix $\rho^{(N)}$ as an $N$-particle operator, which was already introduced in section 7.1.3. The expectation value of an $N$-particle operator 
$A^{(N)}$ is given in matrix elements as

$$
\begin{aligned}
& \operatorname{Tr}^{(N)}\left\{\rho^{(N)} A^{(N)}\right\}= \frac{1}{N ! 2} \sum_{\substack{i_{1} \ldots i_{N} \\
j_{1} \ldots j_{N}}} \sum_{\substack{n_{1} \ldots n_{N} \\
m_{1} \ldots m_{N}}} \rho_{i_{1} \ldots i_{N} ; j_{1} \ldots j_{N}}^{(N)} A_{n_{1} \ldots n_{N} ; m_{1} \ldots m_{N}}^{(N)} \\
& \quad \times \operatorname{Tr}^{(N)}\left\{a_{i_{1}}^{\dagger} \ldots a_{i_{N}}^{\dagger} a_{j_{1}} \ldots a_{j_{N}} a_{n_{1}}^{\dagger} \ldots a_{n_{N}}^{\dagger} a_{m_{1}} \ldots a_{m_{N}}\right\} \\
&= \frac{1}{N !} \sum_{\substack{i_{1} \ldots i_{N} \\
j_{1} \ldots j_{N}}} \sum_{\substack{n_{1} \ldots n_{N} \\
m_{1} \ldots m_{N}}} \rho_{i_{1} \ldots i_{N} ; j_{1} \ldots j_{N}}^{(N)} A_{n_{1} \ldots n_{N} ; m_{1} \ldots m_{N}}^{(N)} \\
&=\frac{1}{N !} \sum_{i_{1} \ldots i_{N}} \sum_{n_{1} \ldots n_{N}} \rho_{i_{1} \ldots i_{N} ; n_{1} \ldots n_{N}}^{(N)} A_{n_{1} \ldots n_{N} ; i_{1} \ldots i_{N}}^{(N)},
\end{aligned}
$$

where we have used equation (7.33) and Lemma 7. This generalizes equation (7.14) to a multi-particle scenario.

The normalization condition $\operatorname{Tr}^{(N)}\left\{\rho^{(N)}\right\}=1$ for

$$
\rho^{(N)}=\frac{1}{N !} \sum_{\substack{i_{1} \ldots i_{N} \\ j_{1} \ldots j_{N}}} \rho_{i_{1} \ldots i_{N} ; j_{1} \ldots j_{N}}^{(N)} a_{i_{1}}^{\dagger} \ldots a_{i_{N}}^{\dagger} a_{j_{1}} \ldots a_{j_{N}}
$$

is fulfilled, according to Lemma 6 , if

$$
\sum_{i_{1} \ldots i_{N}} \rho_{i_{1} \ldots i_{N} ; i_{1} \ldots i_{N}}^{(N)}=1 .
$$

\subsubsection{Matrix elements and traces in a larger Hilbert space}

When describing subsystems of $M \leq N$ particles, we often have to evaluate the matrix elements and traces of $M$-particle operators in a larger $N$-particle Hilbert space.

We first determine the matrix elements

$$
\left\langle\varphi_{i_{1}} \ldots \varphi_{i_{N}}\left|A^{(M)}\right| \varphi_{j_{1}} \ldots \varphi_{j_{N}}\right\rangle .
$$

Using equation (7.19) and, subsequently, Lemma 3 we get

$$
\begin{aligned}
& \left\langle\varphi_{i_{1}} \ldots \varphi_{i_{N}}\left|A^{(M)}\right| \varphi_{j_{1}} \ldots \varphi_{j_{N}}\right\rangle \\
= & \frac{1}{M !} \sum_{\substack{k_{1} \ldots k_{M} \\
l_{1} \ldots l_{M}}} A_{k_{1} \ldots k_{M} ; l_{1} \ldots l_{M}}^{(M)}\left\langle\varphi_{i_{1}} \ldots \varphi_{i_{N}}\left|a_{k_{1}}^{\dagger} \ldots a_{k_{M}}^{\dagger} a_{l_{1}} \ldots a_{l_{M}}\right| \varphi_{j_{1}} \ldots \varphi_{j_{N}}\right\rangle \\
= & \left(\begin{array}{c}
N \\
M
\end{array}\right)^{-1} \sum_{\substack{1 \leq \alpha_{1}<\cdots<\alpha_{M} \leq N \\
1 \leq \beta_{1}<\cdots<\beta_{M} \leq N}} A_{i_{\alpha_{1}} \ldots i_{\alpha_{M}} ; j_{\beta_{1}} \ldots j_{\beta_{M}}}^{(M)} \delta_{\left\{i_{1} \ldots i_{N}\right\} \backslash\left\{i_{\alpha_{1}} \ldots i_{\alpha_{M}}\right\} ;\left\{j_{1} \ldots j_{N}\right\} \backslash\left\{j_{\beta_{1}} \ldots j_{\beta_{M}}\right\}} .
\end{aligned}
$$

The tupels $\left\{\alpha_{1} \ldots \alpha_{M}\right\}$ and $\left\{\beta_{1} \ldots \beta_{M}\right\}$ are taken away from the sets $\left\{i_{1} \ldots i_{N}\right\}$ and $\left\{j_{1} \ldots j_{N}\right\}$ respectively, which results in the sets of indices $\left\{i_{1} \ldots i_{N}\right\} \backslash\left\{i_{\alpha_{1}} \ldots i_{\alpha_{M}}\right\}$ 
and $\left\{j_{1} \ldots j_{N}\right\} \backslash\left\{j_{\beta_{1}} \ldots j_{\beta_{M}}\right\}$. These appear as arguments of the permutation-invariant delta function (7.25). There exist $\left(\begin{array}{c}N \\ M\end{array}\right)$ such tuples $\left\{\alpha_{1} \ldots \alpha_{M}\right\}$ and $\left\{\beta_{1} \ldots \beta_{M}\right\}$, respectively.

For the $N$-particle trace of $M$-particle operators we get, using Lemma 3:

$$
\begin{aligned}
& \operatorname{Tr}^{(N)}\left\{a_{n_{1}}^{\dagger} \ldots a_{n_{M}}^{\dagger} a_{m_{1}} \ldots a_{m_{M}} X\right\} \\
&= \sum_{i_{1} \ldots i_{N}}\left\langle\varphi_{i_{1}} \ldots \varphi_{i_{N}}\left|a_{n_{1}}^{\dagger} \ldots a_{n_{M}}^{\dagger} a_{m_{1}} \ldots a_{m_{M}} X\right| \varphi_{i_{1}} \ldots \varphi_{i_{N}}\right\rangle \\
&= M ! \sum_{i_{1} \ldots i_{N}} \sum_{1 \leq \alpha_{1}<\cdots<\alpha_{M} \leq N} \delta_{i_{\alpha_{1}} \ldots i_{\alpha_{M}} ; n_{1} \ldots n_{M}} \\
& \quad \times\left\langle\varphi_{\left\{i_{1} \ldots i_{N}\right\} \backslash\left\{i_{\alpha_{1}} \ldots i_{\alpha_{M}}\right\} \cup\left\{m_{1} \ldots m_{M}\right\}}|X| \varphi_{i_{1}} \ldots \varphi_{i_{N}}\right\rangle \\
&=\left(\begin{array}{c}
N \\
M
\end{array}\right) M ! \sum_{i_{M+1} \ldots i_{N}}\left\langle\varphi_{m_{1}} \ldots \varphi_{m_{M}} \varphi_{i_{M+1}} \ldots \varphi_{i_{N}}|X| \varphi_{i_{1}} \ldots \varphi_{n_{M}} \varphi_{i_{M+1}} \ldots \varphi_{i_{N}}\right\rangle \\
&= \frac{N !}{(N-M) !} \sum_{i_{M+1} \ldots i_{N}}\left\langle\varphi_{m_{1}} \ldots \varphi_{m_{M}} \varphi_{i_{M+1}} \ldots \varphi_{i_{N}}|X| \varphi_{n_{1}} \ldots \varphi_{n_{M}} \varphi_{i_{M+1}} \ldots \varphi_{i_{N}}\right\rangle
\end{aligned}
$$

where in the fourth step we renamed the indices $i_{1} \ldots i_{N}$ which leads to $\left(\begin{array}{l}N \\ M\end{array}\right)$ equal terms. In the second step, the set $\left\{i_{1} \ldots i_{N}\right\} \backslash\left\{i_{\alpha_{1}} \ldots i_{\alpha_{M}}\right\} \cup\left\{m_{1} \ldots m_{M}\right\}$ appeared, which is obtained from the set $\left\{i_{1} \ldots i_{N}\right\}$ by replacing the elements $\left\{i_{\alpha_{1}} \ldots i_{\alpha_{M}}\right\}$ with $\left\{m_{1} \ldots m_{M}\right\}$.

\subsection{3 $M$-particle reduced density operator}

To introduce the reduced density operator, we consider the expectation value of an $M$-particle operator in an $N$-particle system:

$$
\begin{aligned}
\operatorname{Tr}^{(N)}\left\{A^{(M)} \rho^{(N)}\right\} & =\frac{1}{M !} \sum_{\substack{n_{1} \ldots n_{M} \\
m_{1} \ldots m_{M}}} A_{n_{1} \ldots n_{M} ; m_{1} \ldots m_{M}}^{(M)} \operatorname{Tr}^{(N)}\left\{a_{n_{1}}^{\dagger} \ldots a_{n_{M}}^{\dagger} a_{m_{1}} \ldots a_{m_{M}} \rho^{(N)}\right\} \\
& =\left(\begin{array}{c}
N \\
M
\end{array}\right) \sum_{\substack{n_{1} \ldots n_{M} \\
m_{1} \ldots m_{M}}} A_{n_{1} \ldots n_{M} ; m_{1} \ldots m_{M}}^{(M)} \rho_{m_{1} \ldots m_{M} ; n_{1} \ldots n_{M}}^{(M)} \\
& =\frac{N !}{(N-M) !} \operatorname{Tr}^{(M)}\left\{A^{(M)} \rho^{(M)}\right\}
\end{aligned}
$$

where we used equation (7.46). We have introduced the definition of the $M$-particle reduced density operator of an $N$-particle system (Löwdin, 1955; Ter Haar, 1961; Yang, 1962; Bogoliubov, 1967a),

$$
\begin{aligned}
\rho_{i_{1} \ldots i_{M} ; j_{1} \ldots j_{M}}^{(M)} & =\frac{(N-M) !}{N !} \operatorname{Tr}^{(N)}\left\{a_{j_{1}}^{\dagger} \ldots a_{j_{M}}^{\dagger} a_{i_{1}} \ldots a_{i_{M}} \rho^{(N)}\right\} \\
& =\sum_{n_{M+1} \ldots n_{N}}\left\langle\varphi_{i_{1}} \ldots \varphi_{i_{M}} \varphi_{n_{M+1}} \ldots \varphi_{n_{N}}\left|\rho^{(N)}\right| \varphi_{j_{1}} \ldots \varphi_{j_{M}} \varphi_{n_{M+1}} \ldots \varphi_{n_{M}}\right\rangle,
\end{aligned}
$$


where in the last step we used equation (7.51). By convention, we chose the normalization constant such that all reduced density matrices are normalized to one (Ter Haar, 1960). This leads to a definition of the corresponding partial trace operation as

$$
\begin{aligned}
\rho^{(M)} & =\operatorname{Tr}_{N-M}^{(N)}\left\{\rho^{(N)}\right\} \\
& :=\frac{(N-M) !}{N ! M !} \sum_{\substack{n_{1} \ldots n_{M} \\
m_{1} \ldots m_{M}}} \operatorname{Tr}^{(N)}\left\{a_{n_{1}}^{\dagger} \ldots a_{n_{M}}^{\dagger} a_{m_{1}} \ldots a_{m_{M}} \rho^{(N)}\right\} a_{m_{1}}^{\dagger} \ldots a_{m_{M}}^{\dagger} a_{n_{1}} \ldots a_{n_{M}} .
\end{aligned}
$$

The normalization of $\rho^{(M)}$ is then given by application of Lemma 4,

$$
\begin{aligned}
\operatorname{Tr}^{(M)}\left\{\rho^{(M)}\right\}= & \frac{(N-M) !}{N !} \sum_{\substack{i_{1} \ldots i_{N} \\
j_{1} \ldots j_{N} \\
n_{1} \ldots n_{M}}} \rho_{i_{1} \ldots i_{N} ; j_{1} \ldots j_{N}}^{(N)} \\
& \quad \times\left\langle\varphi_{j_{1}} \ldots \varphi_{j_{N}}\left|a_{n_{1}}^{\dagger} \ldots a_{n_{M}}^{\dagger} a_{n_{1}} \ldots a_{n_{M}}\right| \varphi_{i_{1}} \ldots \varphi_{i_{N}}\right\rangle \\
= & \frac{(N-M) !}{N !} \frac{N !}{(N-M) !} \underbrace{\sum_{i_{1} \ldots i_{N}} \rho_{i_{1} \ldots i_{N} ; i_{1} \ldots i_{N}}^{(N)}}_{1}=1 .
\end{aligned}
$$

Alternatively, we may also project an $N$-particle operator onto the subspace spanned by $M$-particle states. For the density matrix, this yields

$$
\begin{aligned}
& \mathbb{I}^{(M)} \rho^{(N)} \mathbb{I}^{(M)}=\sum_{\substack{n_{1} \ldots n_{M} \\
m_{1} \ldots m_{M}}}\left|\varphi_{n_{1}} \ldots \varphi_{n_{M}}\right\rangle\left\langle\varphi_{n_{1}} \ldots \varphi_{n_{M}}\left|\rho^{(N)}\right| \varphi_{m_{1}} \ldots \varphi_{m_{M}}\right\rangle\left\langle\varphi_{m_{1}} \ldots \varphi_{m_{M}}\right| \\
& =\sum_{\substack{n_{1} \ldots n_{M} \\
m_{1} \ldots m_{M}}} \sum_{\substack{l_{1} \ldots l_{M} \\
k_{1} \ldots k_{N}}}\left|\varphi_{n_{1}} \ldots \varphi_{n_{M}}\right\rangle\left\langle\varphi_{n_{1}} \ldots \varphi_{n_{M}} \mid \varphi_{k_{1}} \ldots \varphi_{k_{N}}\right\rangle \\
& \times\left\langle\varphi_{k_{1}} \ldots \varphi_{k_{N}}\left|\rho^{(N)}\right| \varphi_{l_{1}} \ldots \varphi_{l_{N}}\right\rangle\left\langle\varphi_{l_{1}} \ldots \varphi_{l_{N}} \mid \varphi_{m_{1}} \ldots \varphi_{m_{M}}\right\rangle\left\langle\varphi_{m_{1}} \ldots \varphi_{m_{M}}\right| \\
& =\frac{1}{M !} \sum_{\substack{n_{1} \ldots n_{M} \\
m_{1} \ldots m_{M}}} \sum_{\substack{l_{1} \ldots l_{M} \\
k_{1} \ldots k_{N}}}\left\langle\varphi_{l_{1}} \ldots \varphi_{l_{N}} \mid \varphi_{m_{1}} \ldots \varphi_{m_{M}}\right\rangle\left\langle\varphi_{n_{1}} \ldots \varphi_{n_{M}} \mid \varphi_{k_{1}} \ldots \varphi_{k_{N}}\right\rangle \\
& \times\left\langle\varphi_{k_{1}} \ldots \varphi_{k_{N}}\left|\rho^{(N)}\right| \varphi_{l_{1}} \ldots \varphi_{l_{N}}\right\rangle a_{n_{1}}^{\dagger} \ldots a_{n_{M}}^{\dagger} a_{m_{1}} \ldots a_{m_{M}} \\
& =\frac{1}{M ! 2} \sum_{\substack{n_{1} \ldots n_{M} \\
m_{1} \ldots m_{M}}} \operatorname{Tr}^{(N)}\left\{a_{m_{1}}^{\dagger} \ldots a_{m_{M}}^{\dagger} a_{n_{1}} \ldots a_{n_{M}} \rho^{(N)}\right\} \\
& \times a_{n_{1}}^{\dagger} \ldots a_{n_{M}}^{\dagger} a_{m_{1}} \ldots a_{m_{M}} \\
& =\frac{N !}{(N-M) ! M !^{2}} \sum_{\substack{n_{1} \ldots n_{M} \\
m_{1} \ldots m_{M}}} \rho_{n_{1} \ldots n_{M} ; m_{1} \ldots m_{M}}^{(M)} a_{n_{1}}^{\dagger} \ldots a_{n_{M}}^{\dagger} a_{m_{1}} \ldots a_{m_{M}} \\
& =\left(\begin{array}{l}
N \\
M
\end{array}\right) \rho^{(M)}
\end{aligned}
$$

which leads to an equivalent definition of reduced $M$-particle density operators. 
We apply this to the special case $M=1$ to evaluate the elements of the singleparticle density matrix explicitly as a function of the full $\mathrm{N}$-particle density operator for arbitrary $N$. Using equation (7.53), we obtain

$$
\begin{aligned}
\rho^{(1)} & =\operatorname{Tr}_{N-1}^{(N)}\left\{\rho^{(N)}\right\}=\frac{1}{N} \sum_{n m} \operatorname{Tr}^{(N)}\left\{a_{m} \rho^{(N)} a_{n}^{\dagger}\right\} a_{m}^{\dagger} a_{n} \\
& =\frac{1}{N \cdot N !} \sum_{m n} \sum_{\substack{i_{1} \ldots i_{N} \\
j_{1} \ldots j_{N}}} \rho_{i_{1} \ldots i_{N} ; j_{1} \ldots j_{N}}^{(N)} \operatorname{Tr}^{(N)}\left\{a_{n}^{\dagger} a_{m} a_{i_{1}}^{\dagger} \ldots a_{i_{N}}^{\dagger} a_{j_{1}} \ldots a_{j_{N}}\right\} a_{m}^{\dagger} a_{n} \\
& =\sum_{m n} \sum_{i_{2} \ldots i_{N}} \rho_{m i_{2} \ldots i_{N} ; n i_{2} \ldots i_{N}}^{(N)} a_{m}^{\dagger} a_{n} .
\end{aligned}
$$

Here, placing the indices $n$ and $m$ at the first position is an arbitrary choice, since the entire set of indices $m, i_{2}, \ldots, i_{N}$ and $n, i_{2}, \ldots, i_{N}$ are permutationally invariant.

\subsubsection{Many-body Hamiltonian}

The Hamiltonian is another special case of an $M$-particle operator. In most models, one only considers pairwise particle-particle interactions, which also reflects the pairwise nature of fundamental forces, but effective three- or more-body interactions are sometimes useful to describe collisions or chemical reactions in dense media (Mahan, 2000; Pitaevskii and Stringari, 2003). Here we restrict to second-quantized Hamiltonians containing a single-particle energy term and a two-particle interaction,

$$
H=H^{(1)}+H^{(2)}=\sum_{i j} H_{i j}^{(1)} a_{i}^{\dagger} a_{j}+\frac{1}{2} \sum_{i j k l} H_{i j ; k l}^{(2)} a_{i}^{\dagger} a_{j}^{\dagger} a_{k} a_{l} .
$$

For such Hamiltonians, the energy expectation value is completely determined on the basis of the reduced two-particle density operator $\rho^{(2)}$, which becomes apparent from equation (7.52), with $M=2$. Special cases of systems which are described by Hamiltonians of this form have been discussed in this thesis in the context of the quantized motion of trapped ions, which resembles the Bose-Hubbard Hamiltonian (2.17), see section 6.4.3.

\subsubsection{Bosonic product states}

An important question for the description of a composite many-body system in terms of its individual constituents is: Under which conditions does the single-particle quantum state uniquely determine the full many-particle quantum state? This relates to the more general question: Which $M$-particle states can be obtained by performing the partial trace over $N-M$ particles of an $N$-particle density operator, which is particularly hard to answer for fermionic systems (Coleman, 1963; Kummer, 1967; Kummer, 1970; Radzki, 2010). The complexity of this problem is entirely caused by quantum statistics, which requires the (anti-)symmetrization of many-body states. 
For example, when dealing with distinguishable particles, we can readily express any uncorrelated $N$-particle state by the tensor product of $N$ single-particle density operators. In the simple case of $N=2$, this yields a two-particle product operator

$$
\rho_{P}^{(2)}=\rho^{(1)} \otimes \rho^{(1)}
$$

Using the symmetrized basis, for instance in the form of equation (7.7), we can use this form of $\rho_{P}^{(2)}$ to compute the matrix elements of the two-particle density matrix,

$$
\left\langle\varphi_{i} \varphi_{j}\left|\rho^{(1)} \otimes \rho^{(1)}\right| \varphi_{k} \varphi_{l}\right\rangle=\frac{1}{2}\left(\rho_{i k}^{(1)} \rho_{j l}^{(1)}+\rho_{i l}^{(1)} \rho_{j k}^{(1)}\right)
$$

expressed in terms of the elements $\rho_{i j}=\left\langle\varphi_{i}|\rho| \varphi_{j}\right\rangle$ of the single-particle density matrix. By containing the sum over all permutations (in this case of only two elements) this expression respects the required symmetry constraints and represents the correct version of a bosonic product state; see, for example (Radzki, 2010). Alternatively, we may express this operator with a second-quantized expression

$$
\rho_{P}^{(2)}=\frac{1}{2}: \rho^{(1)} \rho^{(1)}:=\frac{1}{2} \sum_{i j k l} \rho_{i j}^{(1)} \rho_{k l}^{(1)} a_{i}^{\dagger} a_{k}^{\dagger} a_{j} a_{l}
$$

where the double dots denote normal ordering. We have achieved to express a twoparticle state in terms of single-particle states. The arising complexity in the presence of identical particles becomes apparent when we try to return to the single-particle state by tracing over one of two particles: When performing the partial trace operation, as in equation (7.54), we do not recover the single-particle state $\rho^{(1)}$. This is easily seen for the two-particle case:

$$
\begin{aligned}
\left\langle n\left|\operatorname{Tr}_{1}^{(2)}\left\{\rho_{P}^{(2)}\right\}\right| m\right\rangle & =\frac{1}{2} \sum_{j}\left(\rho_{n m}^{(1)} \rho_{j j}^{(1)}+\rho_{n j}^{(1)} \rho_{j m}^{(1)}\right) \\
& =\frac{1}{2}\left(\rho_{n m}^{(1)} \operatorname{Tr}^{(1)} \rho^{(1)}+\left\langle n\left|\left(\rho^{(1)}\right)^{2}\right| m\right\rangle\right)
\end{aligned}
$$

or

$$
\operatorname{Tr}_{1}^{(2)}\left\{\rho_{P}^{(2)}\right\}=\frac{1}{2}\left(\rho^{(1)} \operatorname{Tr}^{(1)} \rho^{(1)}+\left(\rho^{(1)}\right)^{2}\right)
$$

which in general does not correspond to the single-particle density matrix $\rho^{(1)}$. This can be generalized for arbitrary $N$ and $M$ in terms of a recurrence formula, see Theorem 3.1 in (Radzki, 2010). Assuming proper normalization of the single-particle density matrix, $\operatorname{Tr}^{(1)} \rho^{(1)}=1$, we recover the single-particle density operator $\rho^{(1)}$ when $\left(\rho^{(1)}\right)^{2}=\rho^{(1)}$, that is, when $\rho^{(1)}$ is a projector describing a pure state $|\Phi\rangle$. In this case, we have $\rho_{i j}^{(1)}=\left\langle\varphi_{i} \mid \Phi\right\rangle\left\langle\Phi \mid \varphi_{j}\right\rangle$ and

$$
\left\langle\varphi_{i} \varphi_{j}\left|\rho_{P}^{(2)}\right| \varphi_{k} \varphi_{l}\right\rangle=\left\langle\varphi_{i} \mid \Phi\right\rangle\left\langle\varphi_{i} \mid \Phi\right\rangle\left\langle\Phi \mid \varphi_{k}\right\rangle\left\langle\Phi \mid \varphi_{l}\right\rangle
$$


Introducing $a_{\Phi}^{\dagger}|0\rangle=|\Phi\rangle$, we obtain $\rho^{(1)}=a_{\Phi}^{\dagger} a_{\Phi}$, which we can use to define a general bosonic pure product state as

$$
\rho_{P}^{(N)}=\frac{1}{N !}:\left(\rho^{(1)}\right)^{N}:=\frac{1}{N !} \underbrace{a_{\Phi}^{\dagger} \ldots a_{\Phi}^{\dagger}}_{\mathrm{N} \text { times }} \underbrace{a_{\Phi} \ldots a_{\Phi}}_{\mathrm{N} \text { times }} .
$$

Its matrix elements in a basis of symmetrized states read

$$
\left\langle\varphi_{i_{1}} \ldots \varphi_{i_{N}}\left|\rho_{P}^{(N)}\right| \varphi_{j_{1}} \ldots \varphi_{j_{N}}\right\rangle=c_{i_{1}} \cdots c_{i_{N}} c_{j_{1}}^{*} \cdots c_{j_{N}}^{*},
$$

with $c_{i}=\left\langle\varphi_{i} \mid \Phi\right\rangle$. The many-body system is described by such a pure product state when a particular single-particle state is macroscopically occupied, that is, when the system of bosons describes a perfect Bose-Einstein condensate. This, again, can be interpreted as a mean-field ansatz, where all individual particles are described by the same quantum state. As we will discuss in more detail in section 7.4.2, the macroscopic occupation of the single-particle state (for large $N$ ) further allows to replace the associated field operator (to be introduced in the next section) by a complex number, leading to an equation of motion for an effective classical field; recall also the discussions in sections 2.5.1 and 5.4.1.

\subsubsection{Field operator representation}

So far in this chapter, we have formulated all operators and quantum states in terms of symmetrized states. A common description of second-quantized many-body systems is based on field operators, which we introduce and put into context with symmetrized states in this section.

All of the preceding calculations were carried out in an abstract representation of an arbitrary complete basis, spanned by the eigenstates $\left\{\left|\varphi_{i}\right\rangle\right\}$ of some single-particle observable. We have not specified whether the spectrum of this observable is discrete or continuous. While in our notation we employed summations, which indicate a discrete spectrum, we could have replaced these sums by integrals to describe a continuous spectrum. One example of a single-particle operator with a continuous spectrum is given by the position operator $\hat{\mathbf{x}}$, with the eigenvalue equation

$$
\hat{\mathbf{x}}|\mathbf{x}\rangle=\mathbf{x}|\mathbf{x}\rangle .
$$

This continuous basis of states can be related to the possibly discrete set of basis states $\left|\varphi_{i}\right\rangle$ via the completeness relation,

$$
|\mathbf{x}\rangle=\sum_{i}\left|\varphi_{i}\right\rangle\left\langle\varphi_{i} \mid \mathbf{x}\right\rangle=\sum_{i} \varphi_{i}^{*}(\mathbf{x})\left|\varphi_{i}\right\rangle .
$$

We thus introduce the field operator

$$
\hat{\Psi}^{\dagger}(\mathbf{x})=\sum_{i} \varphi_{i}^{*}(\mathbf{x}) a_{i}^{\dagger},
$$


which creates a particle at position $\mathbf{x}$, that is, $\hat{\Psi}^{\dagger}(\mathbf{x})|0\rangle=|\mathbf{x}\rangle$. Conversely we obtain for the corresponding destruction operator

$$
\hat{\Psi}(\mathbf{x})=\sum_{i} \varphi_{i}(\mathbf{x}) a_{i} .
$$

These operators satisfy the canonical commutation relations for bosons,

$$
\left[\hat{\Psi}(\mathbf{x}), \hat{\Psi}^{\dagger}\left(\mathbf{x}^{\prime}\right)\right]=\delta\left(\mathbf{x}-\mathbf{x}^{\prime}\right), \quad\left[\hat{\Psi}(\mathbf{x}), \hat{\Psi}\left(\mathbf{x}^{\prime}\right)\right]=0 .
$$

Furthermore, completeness of the position basis yields

$$
\left|\varphi_{i}\right\rangle=\int d \mathbf{x}|\mathbf{x}\rangle\left\langle\mathbf{x} \mid \varphi_{i}\right\rangle=\int d \mathbf{x} \varphi_{i}(\mathbf{x})|\mathbf{x}\rangle,
$$

which leads to

$$
a_{i}^{\dagger}=\int d \mathbf{x} \varphi_{i}(\mathbf{x}) \hat{\Psi}^{\dagger}(\mathbf{x}), \quad \text { and } \quad a_{i}=\int d \mathbf{x} \varphi_{i}^{*}(\mathbf{x}) \hat{\Psi}(\mathbf{x}) .
$$

We can use this to represent the matrix elements of an arbitrary $N$-particle operator, starting from equation (7.19):

$$
\begin{aligned}
A_{i_{1} \ldots i_{N} ; j_{1} \ldots j_{N}}^{(N)}= & \left\langle\varphi_{i_{1}} \ldots \varphi_{i_{N}}\left|A^{(N)}\right| \varphi_{j_{1}} \ldots \varphi_{j_{N}}\right\rangle \\
= & \int d \mathbf{x}_{1} \ldots \int d \mathbf{x}_{N} \int d \mathbf{x}_{1}^{\prime} \ldots \int d \mathbf{x}_{N}^{\prime}\left\langle\varphi_{i_{1}} \ldots \varphi_{i_{N}} \mid \mathbf{x}_{1} \ldots \mathbf{x}_{N}\right\rangle \\
& \times\left\langle\mathbf{x}_{1} \ldots \mathbf{x}_{N}\left|A^{(N)}\right| \mathbf{x}_{1}^{\prime} \ldots \mathbf{x}_{N}^{\prime}\right\rangle\left\langle\mathbf{x}_{1}^{\prime} \ldots \mathbf{x}_{N}^{\prime} \mid \varphi_{j_{1}} \ldots \varphi_{j_{N}}\right\rangle \\
= & \int d \mathbf{x}_{1} \ldots \int d \mathbf{x}_{N} \int d \mathbf{x}_{1}^{\prime} \ldots \int d \mathbf{x}_{N}^{\prime} \varphi_{i_{1}}^{*}\left(\mathbf{x}_{1}\right) \ldots \varphi_{i_{N}}^{*}\left(\mathbf{x}_{N}\right) \\
& \times A^{(N)}\left(\mathbf{x}_{1} \ldots \mathbf{x}_{N} ; \mathbf{x}_{1}^{\prime} \ldots \mathbf{x}_{N}^{\prime}\right) \varphi_{j_{1}}\left(\mathbf{x}_{1}^{\prime}\right) \ldots \varphi_{j_{N}}\left(\mathbf{x}_{N}^{\prime}\right),
\end{aligned}
$$

with

$$
A^{(N)}\left(\mathbf{x}_{1} \ldots \mathbf{x}_{N} ; \mathbf{x}_{1}^{\prime} \ldots \mathbf{x}_{N}^{\prime}\right)=\left\langle\mathbf{x}_{1} \ldots \mathbf{x}_{N}\left|A^{(N)}\right| \mathbf{x}_{1}^{\prime} \ldots \mathbf{x}_{N}^{\prime}\right\rangle .
$$

We have made use of the symmetry of the sets $\varphi_{1}, \ldots, \varphi_{N}$ and $\mathbf{x}_{1}, \ldots, \mathbf{x}_{N}$, respectively. Using equations (7.18) and (7.73), any $N$-particle operator can be represented through field operators,

$$
\begin{aligned}
A^{(N)}= & \frac{1}{N !} \sum_{\substack{i_{1} \ldots i_{N} \\
j_{1} \ldots j_{N}}} A_{i_{1} \ldots i_{N} ; j_{1} \ldots j_{N}}^{(N)} a_{i_{1}}^{\dagger} \ldots a_{i_{N}}^{\dagger} a_{j_{1}} \ldots a_{j_{N}} \\
= & \frac{1}{N !} \int d \mathbf{x}_{1} \ldots \int d \mathbf{x}_{N} \int d \mathbf{x}_{1}^{\prime} \ldots \int d \mathbf{x}_{N}^{\prime} \\
& \times A^{(N)}\left(\mathbf{x}_{1} \ldots \mathbf{x}_{N} ; \mathbf{x}_{1}^{\prime} \ldots \mathbf{x}_{N}^{\prime}\right) \hat{\Psi}^{\dagger}\left(\mathbf{x}_{1}\right) \ldots \hat{\Psi}^{\dagger}\left(\mathbf{x}_{N}\right) \hat{\Psi}\left(\mathbf{x}_{1}^{\prime}\right) \ldots \hat{\Psi}\left(\mathbf{x}_{N}^{\prime}\right) .
\end{aligned}
$$


In the position representation one can always represent Hamilton operators with local potentials in a pseudo-diagonal form as (Cohen-Tannoudji et al., 1977a)

$$
\begin{aligned}
\left\langle\mathbf{x}\left|H^{(1)}\right| \mathbf{x}^{\prime}\right\rangle & =\left\langle\mathbf{x} \mid \mathbf{x}^{\prime}\right\rangle\left(-\frac{\hbar^{2}}{2 m} \nabla_{\mathbf{x}}^{2}+V_{0}(\mathbf{x})\right)=\delta\left(\mathbf{x}-\mathbf{x}^{\prime}\right) \mathcal{H}_{0}(\mathbf{x}), \\
\left\langle\mathbf{x}_{1} \mathbf{x}_{2}\left|H^{(2)}\right| \mathbf{x}_{1}^{\prime} \mathbf{x}_{2}^{\prime}\right\rangle & =\left\langle\mathbf{x}_{1} \mathbf{x}_{2} \mid \mathbf{x}_{1}^{\prime} \mathbf{x}_{2}^{\prime}\right\rangle V\left(\mathbf{x}_{1}, \mathbf{x}_{2}\right) .
\end{aligned}
$$

This allow us to substitute

$$
\begin{aligned}
\left\langle\mathbf{x}\left|H^{(1)}\right| \varphi_{i}\right\rangle & =\mathcal{H}_{0}(\mathbf{x}) \varphi_{i}(\mathbf{x}), \\
\left\langle\mathbf{x}_{1} \mathbf{x}_{2}\left|H^{(2)}\right| \varphi_{i} \varphi_{j}\right\rangle & =V\left(\mathbf{x}_{1}, \mathbf{x}_{2}\right)\left\langle\mathbf{x}_{1} \mathbf{x}_{2} \mid \varphi_{i} \varphi_{j}\right\rangle
\end{aligned}
$$

which leads to the common form of the Hamilton operator in field operator representation:

$$
\begin{aligned}
H=H^{(1)}+H^{(2)}= & \int d \mathbf{x} \hat{\Psi}^{\dagger}(\mathbf{x})\left(-\frac{\hbar^{2}}{2 m} \nabla_{\mathbf{x}}^{2}+V_{0}(\mathbf{x})\right) \hat{\Psi}(\mathbf{x}) \\
& +\frac{1}{2} \int d \mathbf{x}_{1} \int d \mathbf{x}_{2} \hat{\Psi}^{\dagger}\left(\mathbf{x}_{1}\right) \hat{\Psi}^{\dagger}\left(\mathbf{x}_{2}\right) V\left(\mathbf{x}_{1}, \mathbf{x}_{2}\right) \hat{\Psi}\left(\mathbf{x}_{1}\right) \hat{\Psi}\left(\mathbf{x}_{2}\right) .
\end{aligned}
$$

If the single-particle operator

$$
\rho^{(1)}=\int d \mathbf{x}_{1} \int d \mathbf{x}_{2} \hat{\Psi}^{\dagger}\left(\mathbf{x}_{1}\right) \rho^{(1)}\left(\mathbf{x}_{1} ; \mathbf{x}_{2}\right) \hat{\Psi}\left(\mathbf{x}_{2}\right)
$$

describes a pure state $|\Phi(t)\rangle$, we obtain for its matrix elements

$$
\rho^{(1)}\left(\mathbf{x}_{1} ; \mathbf{x}_{2}\right)=\left\langle\mathbf{x}_{1} \mid \Phi\right\rangle\left\langle\Phi \mid \mathbf{x}_{2}\right\rangle=\Phi\left(\mathbf{x}_{1}\right) \Phi^{*}\left(\mathbf{x}_{2}\right) .
$$

The elements of the corresponding pure product state of $N$ particles read

$$
\rho^{(N)}\left(\mathbf{x}_{1} \ldots \mathbf{x}_{N} ; \mathbf{x}_{1}^{\prime} \ldots \mathbf{x}_{N}^{\prime}\right)=\Phi\left(\mathbf{x}_{1}\right) \ldots \Phi\left(\mathbf{x}_{N}\right) \Phi^{*}\left(\mathbf{x}_{1}^{\prime}\right) \ldots \Phi^{*}\left(\mathbf{x}_{N}^{\prime}\right) .
$$

\subsection{Coherent dynamics of a subgroup of interacting bosons}

Describing the dynamics of interacting many-body systems is generally a very complicated task. Under specific assumptions, effective equations of motion can be derived, which are considerably less complex than the full many-body problem. One such example is given by the mean-field evolution of a weakly interacting system of ultracold bosons, as described by the Gross-Pitaevskii equation. In this section, we first review the standard derivation of the Gross-Pitaevskii equation. Then, we apply the formalism which was developed in the previous section, to derive a hierarchical equation of motion for the reduced $M$-particle density operator, related to the quantum version of the BBGKY-hierarchy. We will then see that a first-order truncation of this hierarchical set of equations reproduces the dynamics described by the Gross-Pitaevskii equation, in the special case of weak interactions. 


\subsubsection{Time evolution of many-body quantum systems}

We describe the dynamics of the full $N$-particle quantum state $\rho^{(N)}(t)$ in the Schrödinger picture (Bogoliubov, 1967a)

$$
\frac{\partial}{\partial t} \rho^{(N)}(t)=-\frac{i}{\hbar}\left[H, \rho^{(N)}(t)\right]
$$

where $H=H^{(1)}+H^{(2)}$, as defined in equation (7.58), contains a single-particle part $H^{(1)}$ and a two-particle interaction term $H^{(2)}$.

It is important to realize that the equation of motion (7.83) does not fully restrict to the contribution of $\mathrm{N}$-particle states: The right-hand side contains contributions of $N+1$ and $N+2$-particle operators, ${ }^{2}$ which are not accounted for by imposing, on the left-hand side of the equation, that the density operator contains exactly $N$ particles. By writing equation (7.83), we only consider the contribution of the $\mathrm{N}$ particle density matrix $\left(\rho^{(N)}\right.$-term appearing on the right-hand side) for the evolution of the $N$-particle density matrix (left-hand side). This is an approximation, since we make this assumption not for the solution $\rho^{(N)}(t)$ of the above equation, but for the equation itself. For the solution itself, this would not be an approximation, since any Hamiltonian of the form (7.58) commutes with the particle number operator (see Lemma 4 with $M=1$ for its action on an $N$-particle state)

$$
N^{(1)}=\sum_{i} a_{i}^{\dagger} a_{i},
$$

and, hence, an initial $N$-particle state $\rho^{(N)}$ will be mapped to another $N$-particle state. A more general description of the many-body dynamics could be obtained by replacing $\rho^{(N)}(t)$ with $\rho(t)=\sum_{i=1}^{N} \rho^{(i)}(t)$ on both sides of equation (7.83). In the context of the present dissertation we restrict to the above assumption of a closed evolution within the $N$-particle subspace.

Instead of working in the Schrödinger picture, we can transform to the interaction picture with $U_{1}(t)=\exp \left(-i H^{(1)} t / \hbar\right)$. We get

$$
\frac{\partial}{\partial t} \rho_{I}^{(N)}(t)=-\frac{i}{\hbar}\left[H_{I}^{(2)}(t), \rho_{I}^{(N)}(t)\right]
$$

with $\rho_{I}^{(N)}(t)=U_{1}^{\dagger}(t) \rho^{(N)}(t) U_{1}(t)$ and $H_{I}^{(2)}(t)=U_{1}^{\dagger}(t) H^{(2)} U_{1}(t)$. Typically, the singleparticle part can be solved, if not analytically, it at least allows for a numerical diagonalization, while the two-particle interaction term defies efficient numerical approaches, since it requires consideration of all particles, which renders it a many-body problem. We can, for instance, choose a representation such that $a_{i}^{\dagger}$ creates an eigenstate of $H^{(1)}$, that is, $H_{i j}^{(1)}=\epsilon_{i} \delta_{i j}$. This special choice makes the determination of the interaction picture Hamitonian particularly simple:

$$
H_{I}^{(2)}(t)=\frac{1}{2} \sum_{i j k l} e^{i\left(\epsilon_{i}+\epsilon_{j}-\epsilon_{k}-\epsilon_{l}\right) t / \hbar} H_{i j ; k l}^{(2)} a_{i}^{\dagger} a_{j}^{\dagger} a_{k} a_{l} .
$$

\footnotetext{
${ }^{2}$ In the commutator, the highest-order terms (here, $N+2$ ), drop out. This is caused by the commutativity of the annihilation operators $a_{i}$ among themselves; see equation (7.3).
} 
Finally, a commonly employed method to describe the dynamics of many-body systems which avoids the problems that emerge by restricting to $N$-particle subspaces, as discussed above, is achieved by solving for the dynamics of individual annihilation operators in the Heisenberg picture. From equations (7.18) and (7.76), we see that any $N$-particle operator can be expressed in terms of the bosonic operators $a_{i}$ or $\hat{\Psi}(\mathbf{x})$. The Heisenberg-picture field operator is given by

$$
\hat{\Psi}(t, \mathbf{x})=e^{i H t / \hbar} \hat{\Psi}(\mathbf{x}) e^{-i H t / \hbar} .
$$

We can use it to express the Hamilton operator (7.79) as

$$
\begin{aligned}
H= & e^{i H t / \hbar} H e^{-i H t / \hbar} \\
= & \int d \mathbf{x} \hat{\Psi}^{\dagger}(t, \mathbf{x})\left(-\frac{\hbar^{2}}{2 m} \nabla_{\mathbf{x}}^{2}+V_{0}(\mathbf{x})\right) \hat{\Psi}(t, \mathbf{x}) \\
& +\frac{1}{2} \int d \mathbf{x}_{1} \int d \mathbf{x}_{2} \hat{\Psi}^{\dagger}\left(t, \mathbf{x}_{1}\right) \hat{\Psi}^{\dagger}\left(t, \mathbf{x}_{2}\right) V\left(\mathbf{x}_{1}, \mathbf{x}_{2}\right) \hat{\Psi}\left(t, \mathbf{x}_{1}\right) \hat{\Psi}\left(t, \mathbf{x}_{2}\right) .
\end{aligned}
$$

The time evolution of the field operator is given by the Heisenberg equation

$$
\begin{aligned}
i \hbar \frac{\partial}{\partial t} \hat{\Psi}(t, \mathbf{x})= & {[\hat{\Psi}(t, \mathbf{x}), H] } \\
= & \int d \mathbf{x}^{\prime}\left(-\frac{\hbar^{2}}{2 m} \nabla_{\mathbf{x}^{\prime}}^{2}+V_{0}\left(\mathbf{x}^{\prime}\right)\right)\left[\hat{\Psi}(t, \mathbf{x}), \hat{\Psi}^{\dagger}\left(t, \mathbf{x}^{\prime}\right) \hat{\Psi}\left(t, \mathbf{x}^{\prime}\right)\right] \\
& +\frac{1}{2} \int d \mathbf{x}_{1} \int d \mathbf{x}_{2} V\left(\mathbf{x}_{1}, \mathbf{x}_{2}\right)\left[\hat{\Psi}(t, \mathbf{x}), \hat{\Psi}^{\dagger}\left(t, \mathbf{x}_{1}\right) \hat{\Psi}^{\dagger}\left(t, \mathbf{x}_{2}\right) \hat{\Psi}\left(t, \mathbf{x}_{1}\right) \hat{\Psi}\left(t, \mathbf{x}_{2}\right)\right]
\end{aligned}
$$

Using the canonical commutation relations, equation (7.71), we obtain

$$
\left[\hat{\Psi}(t, \mathbf{x}), \hat{\Psi}^{\dagger}\left(t, \mathbf{x}^{\prime}\right) \hat{\Psi}\left(t, \mathbf{x}^{\prime}\right)\right]=\delta\left(\mathbf{x}-\mathbf{x}^{\prime}\right) \hat{\Psi}(t, \mathbf{x})
$$

and

$$
\begin{aligned}
{\left[\hat{\Psi}(t, \mathbf{x}), \hat{\Psi}^{\dagger}\left(t, \mathbf{x}_{1}\right) \hat{\Psi}^{\dagger}\left(t, \mathbf{x}_{2}\right) \hat{\Psi}\left(t, \mathbf{x}_{1}\right) \hat{\Psi}\left(t, \mathbf{x}_{2}\right)\right]=} & \delta\left(\mathbf{x}-\mathbf{x}_{1}\right) \hat{\Psi}^{\dagger}\left(t, \mathbf{x}_{2}\right) \hat{\Psi}\left(t, \mathbf{x}_{2}\right) \hat{\Psi}\left(t, \mathbf{x}_{1}\right) \\
& +\delta\left(\mathbf{x}-\mathbf{x}_{2}\right) \hat{\Psi}^{\dagger}\left(t, \mathbf{x}_{1}\right) \hat{\Psi}\left(t, \mathbf{x}_{1}\right) \hat{\Psi}\left(t, \mathbf{x}_{2}\right)
\end{aligned}
$$

Since the interaction potential of identical particles does not distinguish between the particles, that is, $V\left(\mathbf{x}_{1}, \mathbf{x}_{2}\right)=V\left(\mathbf{x}_{2}, \mathbf{x}_{1}\right)$, this leads to the exact equation

$$
\begin{aligned}
i \hbar \frac{\partial}{\partial t} \hat{\Psi}(t, \mathbf{x})= & \int d \mathbf{x}^{\prime}\left(-\frac{\hbar^{2}}{2 m} \nabla_{\mathbf{x}^{\prime}}^{2}+V_{0}\left(\mathbf{x}^{\prime}\right)\right) \delta\left(\mathbf{x}-\mathbf{x}^{\prime}\right) \hat{\Psi}(t, \mathbf{x}) \\
& +\int d \mathbf{x}_{1} \int d \mathbf{x}_{2} V\left(\mathbf{x}_{1}, \mathbf{x}_{2}\right) \delta\left(\mathbf{x}-\mathbf{x}_{1}\right) \hat{\Psi}^{\dagger}\left(t, \mathbf{x}_{2}\right) \hat{\Psi}\left(t, \mathbf{x}_{2}\right) \hat{\Psi}\left(t, \mathbf{x}_{1}\right) \\
= & \left(-\frac{\hbar^{2}}{2 m} \nabla_{\mathbf{x}}^{2}+V_{0}(\mathbf{x})+\int d \mathbf{x}^{\prime} V\left(\mathbf{x}, \mathbf{x}^{\prime}\right) \hat{\Psi}^{\dagger}\left(t, \mathbf{x}^{\prime}\right) \hat{\Psi}\left(t, \mathbf{x}^{\prime}\right)\right) \hat{\Psi}(t, \mathbf{x})
\end{aligned}
$$




\subsubsection{The Gross-Pitaevskii equation}

To describe a dilute Bose-Einstein condensate, we start from equation (7.92) and employ the Bogoliubov approximation (Bogoliubov, 1947). This means that we describe the macroscopic occupation of a single-particle quantum state with a classical field $\Psi_{0}(t, \mathbf{x})$, and consider quantum corrections $\delta \hat{\Psi}(t, \mathbf{x})$ to it as weak

$$
\hat{\Psi}(t, \mathbf{x})=\Psi_{0}(t, \mathbf{x})+\delta \hat{\Psi}(t, \mathbf{x}) .
$$

More precisely, the classical field, $\Psi_{0}(t, \mathbf{x})$, is given by

$$
\Psi_{0}(t, \mathbf{x})=\sqrt{N_{0}} \varphi_{0}(t, \mathbf{x})
$$

where $\varphi_{0}(t, \mathbf{x})$ denotes the single-particle wavefunction of the $N_{0}$-fold $\left(N_{0} \approx N \gg 1\right)$ occupied state. ${ }^{3}$ The prefactor is required to satisfy the normalization condition

$$
N_{0}=\int d \mathbf{x} \operatorname{Tr}^{(N)}\left\{\hat{\Psi}^{\dagger}(t, \mathbf{x}) \hat{\Psi}(t, \mathbf{x}) \rho^{(N)}\right\}
$$

which corresponds to the quantum mechanical expectation value of the number operator. We now consider a weakly interacting, dilute Bose-Einstein condensate, which means we employ the following approximations: We

- ignore the quantum corrections $\delta \hat{\Psi}$,

- assume that the interaction be of short range and replace

$$
V\left(\mathbf{x}_{1}, \mathbf{x}_{2}\right)=g \delta\left(\mathbf{x}_{1}-\mathbf{x}_{2}\right) .
$$

The interaction coupling constant can be expressed in terms of the s-wave scattering length $a$ as $^{4}$

$$
g=\frac{4 \pi \hbar^{2} a}{m}
$$

Under these assumptions, the dynamics implied by (7.92) for the classical field $\Psi_{0}(t, \mathbf{x})$ takes a form known as the Gross-Pitaevskii equation (Gross, 1961; Pitaevskii, 1961; Pitaevskii and Stringari, 2003)

$$
i \hbar \frac{\partial}{\partial t} \Psi_{0}(t, \mathbf{x})=\left(-\frac{\hbar^{2}}{2 m} \nabla_{\mathbf{x}}^{2}+V_{0}(\mathbf{x})+g\left|\Psi_{0}(t, \mathbf{x})\right|^{2}\right) \Psi_{0}(t, \mathbf{x}) .
$$

\footnotetext{
${ }^{3}$ This macroscopically occupied single-particle state should not be called the ground state since the ground state of the full many-body Hamiltonian is not a single-particle state (Pitaevskii and Stringari, 2003).

${ }^{4}$ The $s$-wave scattering length $a$ determines the low-energy limit of the $s$-wave scattering amplitude (zero angular momentum contribution of the partial wave expansion of the scattering amplitude) in a central potential, and, in the same limit, determines the total cross section $\sigma_{\text {tot }}$ as $\sigma_{\text {tot }}=4 \pi a^{2}$ (Reed and Simon, 1979).
} 
Using equation (7.94) we obtain the dynamics of the single-particle wavefunction

$$
i \hbar \frac{\partial}{\partial t} \varphi_{0}(t, \mathbf{x})=\left(-\frac{\hbar^{2}}{2 m} \nabla_{\mathbf{x}}^{2}+V_{0}(\mathbf{x})+g N_{0}\left|\varphi_{0}(t, \mathbf{x})\right|^{2}\right) \varphi_{0}(t, \mathbf{x}) .
$$

If we assume the total system of $N$ particles to be described by a pure product state at all times $t$, the corresponding mean-field $N$-particle state is given by

$$
\rho^{(N)}\left(t, \mathbf{x}_{1} \ldots \mathbf{x}_{N} ; \mathbf{x}_{1}^{\prime} \ldots \mathbf{x}_{N}^{\prime}\right)=\varphi_{0}\left(t, \mathbf{x}_{1}\right) \ldots \varphi_{0}\left(t, \mathbf{x}_{N}\right) \varphi_{0}^{*}\left(t, \mathbf{x}_{1}^{\prime}\right) \ldots \varphi_{0}^{*}\left(t, \mathbf{x}_{N}^{\prime}\right) .
$$

In the context of a Bose-Einstein condensate, the pure product state assumption is well justified for the condensate part, which is characterized by macroscopic occupation of a single-particle state (Pitaevskii and Stringari, 2003), when external noise sources can be neglected.

\subsubsection{Hierarchical expansion of the reduced bosonic dynamics}

In this section, we finally employ the formalism of symmetrized states, which was introduced in the previous two sections, to derive the equations of motion for an $M$ particle subsystem of $N$ interacting bosons. To describe the dynamics of the reduced $M$-particle density operator, we perform the partial trace operation, as defined in equation (7.54), on both sides of the von Neumann equation (7.83). On the left-hand side this immediately yields the reduced $M$-particle density operator. We obtain

$$
\begin{aligned}
i \hbar \frac{\partial}{\partial t} \rho^{(M)}(t) & =\operatorname{Tr}_{N-M}^{(N)}\left\{i \hbar \frac{\partial}{\partial t} \rho^{(N)}(t)\right\} \\
& =\operatorname{Tr}_{N-M}^{(N)}\left\{\left[H^{(1)}+H^{(2)}, \rho^{(N)}(t)\right]\right\}
\end{aligned}
$$

Again, note that, by construction, we project both sides of the above equation to the $M$-particle subspace, even though there are nonzero contributions of the right-hand side to subspaces of more than $M$ particles. To evaluate the above expression, we split the right-hand side into two terms and begin with the single-particle term. Using equation (7.54) and Lemma 3, we obtain

$$
\begin{aligned}
& \operatorname{Tr}_{N-M}^{(N)}\left\{\left[H^{(1)}, \rho^{(N)}(t)\right]\right\} \\
= & \frac{(N-M) !}{N ! M !} \\
\times \sum_{\substack{n_{1} \ldots n_{M} \\
m_{1} \ldots m_{M} \\
k_{1} \ldots k_{N} \\
l_{1} \ldots l_{N}}} & {\left[\left\langle\varphi_{l_{1}} \ldots \varphi_{l_{N}}\left|a_{n_{1}}^{\dagger} \ldots a_{n_{M}}^{\dagger} a_{m_{1}} \ldots a_{m_{M}} H^{(1)}\right| \varphi_{k_{1}} \ldots \varphi_{k_{N}}\right\rangle \rho_{k_{1} \ldots k_{N} ; l_{1} \ldots l_{N}}^{(N)}(t)\right.} \\
& \left.-\left\langle\varphi_{l_{1}} \ldots \varphi_{l_{N}}\left|H^{(1)} a_{n_{1}}^{\dagger} \ldots a_{n_{M}}^{\dagger} a_{m_{1}} \ldots a_{m_{M}}\right| \varphi_{k_{1}} \ldots \varphi_{k_{N}}\right\rangle \rho_{k_{1} \ldots k_{N} ; l_{1} \ldots l_{N}}^{(N)}(t)\right] \\
& \times a_{m_{1}}^{\dagger} \ldots a_{m_{M}}^{\dagger} a_{n_{1}} \ldots a_{n_{M}} .
\end{aligned}
$$




$$
\begin{aligned}
= & \frac{(N-M) !}{N ! M !}\left(\begin{array}{c}
N \\
M
\end{array}\right) M ! \sum_{\substack{n_{1} \ldots n_{M} \\
m_{1} \ldots m_{M}}} \\
\times & {\left[\sum_{\substack{k_{1} \ldots k_{N} \\
l_{M+1} \ldots l_{N}}}\left\langle\varphi_{m_{1}} \ldots \varphi_{m_{M}} \varphi_{l_{M+1}} \ldots \varphi_{l_{N}}\left|H^{(1)}\right| \varphi_{k_{1}} \ldots \varphi_{k_{N}}\right\rangle \rho_{k_{1} \ldots k_{N} ; n_{1} \ldots n_{M} l_{M+1} \ldots l_{N}}^{(N)}(t)\right.} \\
& \left.-\sum_{k_{1} \ldots k_{M+1}}\left\langle\varphi_{l_{1}} \ldots \varphi_{l_{N}}\left|H^{(1)}\right| \varphi_{n_{1}} \ldots \varphi_{n_{M}} \varphi_{k_{M+1}} \ldots \varphi_{k_{N}}\right\rangle \rho_{m_{1} \ldots m_{M} k_{M+1} \ldots k_{N} ; l_{1} \ldots l_{N}}^{(N)}(t)\right] \\
& \times a_{m_{1} \ldots a_{m_{M}}^{\dagger} \ldots a_{m_{M}}^{\dagger} a_{n_{1}} \ldots a_{n_{M}} .}
\end{aligned}
$$

At this point, we need to determine the $N$-particle matrix elements of a single-particle operator. This was done for a general $M$-particle operator in equation (7.50). Here, we have to distinguish two cases, depending on the set of indices with which $H^{(1)}$ contracts. Obviously, there are $M$ possibilities for $1<\alpha<N$ to be found within the subset $1, \ldots, M$, in which case one index of $H^{(1)}$ is directly contracted with either the creation or the annihilation operators $a_{m_{1}}^{\dagger}, \ldots, a_{m_{M}}^{\dagger}$ and $a_{n_{1}}, \ldots, a_{n_{M}}$. In the remaining $N-M$ cases, both indices of $H^{(1)}$ are contracted with $\rho^{(N)}$. We obtain

$$
\begin{aligned}
& \operatorname{Tr}_{N-M}^{(N)}\left\{\left[H^{(1)}, \rho^{(N)}(t)\right]\right\} \\
= & \frac{(N-M) !}{N ! M !}\left(\begin{array}{c}
N \\
M
\end{array}\right) M ! \sum_{n_{n_{1} \ldots n_{M}}} \\
& \times\left[M\left(\sum_{k_{1}} H_{m_{1} k_{1}}^{(1)} \rho_{k_{1} m_{2} \ldots m_{M} ; n_{1} \ldots n_{M}}^{(M)}(t)-\sum_{l_{1}} H_{l_{1} n_{1}}^{(1)} \rho_{m_{1} \ldots m_{M} ; n_{2} \ldots n_{M} l_{1}}^{(M)}(t)\right)\right. \\
& +(N-M)\left(\sum_{l_{M+1} k_{1}} H_{l_{M+1} k_{1}}^{(1)} \rho_{k_{1} m_{1} \ldots m_{M} ; n_{1} \ldots n_{M} l_{M+1}}^{(M+1)}(t)\right. \\
& \left.\left.\quad-\sum_{l_{1} k_{M+1}} H_{l_{1} k_{M+1}}^{(1)} \rho_{m_{1} \ldots m_{M} k_{M+1} ; l_{1} n_{1} \ldots n_{M}}^{(M+1)}(t)\right)\right] \\
& \times a_{m_{1}}^{\dagger} \ldots a_{m_{M}}^{\dagger} a_{n_{1}} \ldots a_{n_{M}}
\end{aligned}
$$

Using the invariance under permutations of the indices of $\rho^{(M+1)}$, we find, after appropriate relabelling of the indices, that the terms containing $\rho^{(M+1)}$ cancel, and we 
obtain

$$
\begin{aligned}
& \operatorname{Tr}_{N-M}^{(N)}\left\{\left[H^{(1)}, \rho^{(N)}(t)\right]\right\} \\
= & \frac{M}{M !} \sum_{\substack{n_{1} \ldots n_{M} \\
m_{1} \ldots m_{M}}}\left[H_{m_{1} k_{1}}^{(1)} \rho_{k_{1} m_{2} \ldots m_{M} ; n_{1} \ldots n_{M}}^{(M)}(t)-H_{k_{1} n_{1}}^{(1)} \rho_{m_{1} \ldots m_{M} ; n_{2} \ldots n_{M} k_{1}}^{(M)}(t)\right] \\
& \times a_{m_{1}}^{\dagger} \ldots a_{m_{M}}^{\dagger} a_{n_{1}} \ldots a_{n_{M}} \\
= & {\left[H^{(1)}, \rho^{(M)}(t)\right], }
\end{aligned}
$$

where the factor $M$, accounts for the fact that we express elements of the single-particle operator $H^{(1)}$ in an $M$-particle basis. To see this, let us consider the general case of an arbitrary $S$-particle operator $J^{(S)}$ in the following expressions:

$$
\begin{aligned}
& \left\langle\varphi_{n_{1}} \ldots \varphi_{n_{M}}\left|J^{(S)} \rho^{(M)}\right| \varphi_{m_{1}} \ldots \varphi_{m_{M}}\right\rangle \\
= & \left(\begin{array}{c}
M \\
S
\end{array}\right) \sum_{i_{1} \ldots i_{S}} J_{n_{1} \ldots n_{S} ; i_{1} \ldots i_{S}}^{(S)} \rho_{i_{1} \ldots i_{S} n_{S+1} \ldots n_{M} ; m_{1} \ldots m_{M}}^{(M)},
\end{aligned}
$$

and

$$
\begin{aligned}
& \left\langle\varphi_{n_{1}} \ldots \varphi_{n_{M}}\left|\rho^{(M)} J^{(S)}\right| \varphi_{m_{1}} \ldots \varphi_{m_{M}}\right\rangle \\
= & \left(\begin{array}{c}
M \\
S
\end{array}\right) \sum_{i_{1} \ldots i_{S}} \rho_{n_{1} \ldots n_{M} ; i_{1} \ldots i_{S} m_{S+1} \ldots m_{M}}^{(M)} J_{i_{1} \ldots i_{S} ; m_{1} \ldots m_{S}}^{(S)} .
\end{aligned}
$$

Indeed, in the special case $S=1$, we obtain the prefactor $M$ which was observed in (7.104).

As expected, when neglecting the interactions $H^{(2)}$ by restricting to the singleparticle Hamiltonian $H^{(1)}$, the only remaining term, equation (7.104), describes the free evolution of $M \leq N$ particles. Now we turn to the two-particle interaction term, 
which for $M \geq 2$ yields, again using equation (7.54), and subsequently (7.50),

$$
\begin{aligned}
& \operatorname{Tr}_{N-M}^{(N)}\left\{\left[H^{(2)}, \rho^{(N)}(t)\right]\right\} \\
& =\frac{1}{M !} \sum_{\substack{n_{1} \ldots n_{M} \\
m_{1} \ldots m_{M} \\
k_{1} \ldots k_{N}}}\left[\sum_{n_{M+1} \ldots n_{N}}\left\langle\varphi_{n_{1}} \ldots \varphi_{n_{N}}\left|H^{(2)}\right| \varphi_{k_{1}} \ldots \varphi_{k_{N}}\right\rangle \rho_{k_{1} \ldots k_{N} ; m_{1} \ldots m_{M} n_{M+1} \ldots n_{N}}^{(N)}(t)\right. \\
& \left.-\sum_{m_{M+1} \ldots m_{N}} \rho_{n_{1} \ldots n_{M} m_{M+1} \ldots m_{N} ; k_{1} \ldots k_{N}}^{(N)}(t)\left\langle\varphi_{k_{1}} \ldots \varphi_{k_{N}}\left|H^{(2)}\right| \varphi_{m_{1}} \ldots \varphi_{m_{N}}\right\rangle\right] \\
& \times a_{n_{1}}^{\dagger} \ldots a_{n_{M}}^{\dagger} a_{m_{1}} \ldots a_{m_{M}} \\
& =\frac{1}{M !} \sum_{\substack{n_{1} \ldots n_{M} \\
m_{1} \ldots m_{M} \\
k_{1} \ldots k_{N}}} \\
& {\left[\sum_{n_{M+1} \ldots n_{N}} \sum_{1 \leq \alpha_{1}<\alpha_{2} \leq N} H_{n_{\alpha_{1}} n_{\alpha_{2}} ; k_{1} k_{2}}^{(2)} \rho_{k_{1} k_{2}\left\{n_{1} \ldots n_{N}\right\} \backslash\left\{n_{\alpha_{1}} n_{\alpha_{2}}\right\} ; m_{1} \ldots m_{M} n_{M+1} \ldots n_{N}}^{(N)}(t)\right.} \\
& \left.-\sum_{m_{M+1} \ldots m_{N}} \sum_{1 \leq \beta_{1}<\beta_{2} \leq N} \rho_{n_{1} \ldots n_{M} m_{M+1} \ldots m_{N} ; k_{1} k_{2}\left\{m_{1} \ldots m_{N}\right\} \backslash\left\{m_{\beta_{1}} m_{\beta_{2}}\right\}}^{(N)}(t) H_{k_{1} k_{2} ; m_{\beta_{1}} m_{\beta_{2}}}^{(2)}\right] \\
& \times a_{n_{1}}^{\dagger} \ldots a_{n_{M}}^{\dagger} a_{m_{1}} \ldots a_{m_{M}} .
\end{aligned}
$$

Again, we need to sort out which of the indices are contracted. In order to distinguish the possibilities for the different choices of $\alpha_{1}, \alpha_{2}$, as well as of $\beta_{1}$ and $\beta_{2}$, we use the following

Lemma 9. The following possibilities exist for $1 \leq \alpha_{1}<\alpha_{2} \leq r$ and $s \leq r$ :

1. Both $\alpha_{1}$ and $\alpha_{2} \leq s$. There are $\left(\begin{array}{l}s \\ 2\end{array}\right)$ possibilities to achieve this.

2. $\alpha_{1} \leq s$ and $\alpha_{2}>s$. There are $s(r-s)$ possibilities to achieve this.

3. Both $\alpha_{1}$ and $\alpha_{2}>$ s. There are $\left(\begin{array}{c}r-s \\ 2\end{array}\right)$ possibilities to achieve this. 
Using this for $r=N$ and $s=M$, we obtain

$$
\begin{aligned}
& \operatorname{Tr}_{N-M}^{(N)}\left\{\left[H^{(2)}, \rho^{(N)}(t)\right]\right\} \\
& =\frac{1}{M !} \sum_{\substack{n_{1} \ldots n_{M} \\
m_{1} \ldots m_{M} \\
k_{1} k_{2}}}\left[\left(\begin{array}{c}
M \\
2
\end{array}\right) H_{n_{1} n_{2} ; k_{1} k_{2}}^{(2)} \rho_{k_{1} k_{2} n_{3} \ldots n_{N} ; m_{1} \ldots m_{M}}^{(M)}(t)\right. \\
& +M(N-M) \sum_{n_{M+1}} H_{n_{1} n_{M+1} ; k_{1} k_{2}}^{(2)} \rho_{k_{1} k_{2} n_{2} \ldots n_{M} ; m_{1} \ldots m_{M} n_{M+1}}^{(M+1)}(t) \\
& +\left(\begin{array}{c}
N-M \\
2
\end{array}\right) \sum_{n_{M+1} n_{M+2}} H_{n_{M+1} n_{M+2} ; k_{1} k_{2}}^{(2)} \rho_{k_{1} k_{2} n_{1} \ldots n_{M} ; m_{1} \ldots m_{M} n_{M+1} n_{M+2}}^{(M+2)}(t) \\
& \text { - }\left(\begin{array}{c}
M \\
2
\end{array}\right) \rho_{n_{1} \ldots n_{M} ; k_{1} k_{2} m_{3} \ldots m_{M}}^{(M)}(t) H_{k_{1} k_{2} ; m_{1} m_{2}}^{(2)} \\
& -M(N-M) \sum_{m_{M+1}} \rho_{n_{1} \ldots n_{M} m_{M+1} ; k_{1} k_{2} m_{2} \ldots m_{M}}^{(M+1)}(t) H_{k_{1} k_{2} ; m_{1} m_{M+1}}^{(2)} \\
& \left.-\left(\begin{array}{c}
N-M \\
2
\end{array}\right) \sum_{m_{M+1} m_{M+2}} \rho_{n_{1} \ldots n_{M} m_{M+1} m_{M+2} ; k_{1} k_{2} m_{1} \ldots m_{M}}^{(M+2)}(t) H_{k_{1} k_{2} ; m_{M+1} m_{M+2}}^{(2)}\right] \\
& \times a_{n_{1}}^{\dagger} \ldots a_{n_{M}}^{\dagger} a_{m_{1}} \ldots a_{m_{M}} \\
& =\left[H^{(2)}, \rho^{(M)}(t)\right] \\
& +\frac{M(N-M)}{M !} \sum_{\substack{n_{1}, \ldots, n_{M} \\
m_{1}, \ldots, m_{M} \\
k_{1}, k_{2}}}\left[\sum_{n_{M+1}} H_{n_{1} n_{M+1} ; k_{1} k_{2}}^{(2)} \rho_{k_{1} k_{2} n_{2} \ldots n_{M} ; m_{1} \ldots m_{M} n_{M+1}}^{(M+1)}(t)\right. \\
& \left.-\sum_{m_{M+1}} \rho_{n_{1} \ldots n_{M} m_{M+1} ; k_{1} k_{2} m_{2} \ldots m_{M}}^{(M+1)}(t) H_{k_{1} k_{2} ; m_{1} m_{M+1}}^{(2)}\right] a_{n_{1}}^{\dagger} \ldots a_{n_{M}}^{\dagger} a_{m_{1}} \ldots a_{m_{M}} .
\end{aligned}
$$

The $M$-particle terms can be identified with the commutator, whereas the prefactor is caused, according to equations (7.105) and (7.106), by representing the twoparticle Hamiltonian in the $M$-particle basis. We stress again that the equality sign in equation (7.108) holds as long as the right-hand side is restricted to $M$-particle contributions, for instance by projection onto $\mathbb{I}^{(M)}$; recall the discussion following equation (7.83).

We abbreviate the second term (the last two lines in the expression above) by introducing the symbol $\mathfrak{L}^{(M)}\left(H^{(2)}, \rho^{(M+1)}(t)\right)$, leading to

$$
\operatorname{Tr}_{N-M}^{(N)}\left\{\left[H^{(2)}, \rho^{(N)}(t)\right]\right\}=\left[H^{(2)}, \rho^{(M)}(t)\right]+\frac{M(N-M)}{M !} \mathfrak{L}^{(M)}\left(H^{(2)}, \rho^{(M+1)}(t)\right) .
$$

The right-hand side contains, beyond the commutator involving the $M$-particle density matrix, also contributions of the $(M+1)$-particle density matrix. Including also the 
single-particle Hamiltonian, equation (7.104), we obtain for $M=1$

$$
\begin{aligned}
i \hbar \frac{\partial}{\partial t} \rho^{(1)}(t)= & {\left[H^{(1)}, \rho^{(1)}(t)\right] } \\
& +(N-1) \sum_{\substack{n m \\
k_{1} k_{2} l}}\left[H_{n l ; k_{1} k_{2}}^{(2)} \rho_{k_{1} k_{2} ; m l}^{(2)}(t)-\rho_{n l ; k_{1} k_{2}}^{(2)}(t) H_{k_{1} k_{2} ; m l}^{(2)}\right] a_{n}^{\dagger} a_{m} \\
= & {\left[H^{(1)}, \rho^{(1)}(t)\right]+(N-1) \operatorname{Tr}_{1}^{(2)}\left\{\left[H^{(2)}, \rho^{(2)}(t)\right]\right\} }
\end{aligned}
$$

For general $M \geq 2$, however, the $(M+1)$-particle contribution, $\mathfrak{L}^{(M)}\left(H^{(2)}, \rho^{(M+1)}(t)\right)$ in equation (7.109), does not reduce to tracing over one more particle, but will also yield contributions to the commutator. We see this easily by inserting $N=M+1$, with $M \geq 2$ into equation (7.109),

$$
\operatorname{Tr}_{1}^{(M+1)}\left\{\left[H^{(2)}, \rho^{(M+1)}(t)\right]\right\}=\left[H^{(2)}, \rho^{(M)}(t)\right]+\frac{M}{M !} \mathfrak{L}^{(M)}\left(H^{(2)}, \rho^{(M+1)}(t)\right) .
$$

This allows us to replace the term

$$
\mathfrak{L}^{(M)}\left(H^{(2)}, \rho^{(M+1)}(t)\right)=\frac{M !}{M}\left(\operatorname{Tr}_{1}^{(M+1)}\left\{\left[H^{(2)}, \rho^{(M+1)}(t)\right]\right\}-\left[H^{(2)}, \rho^{(M)}(t)\right]\right)
$$

in equation (7.109) for arbitrary $N$, leading to

$$
\begin{aligned}
& \operatorname{Tr}_{N-M}^{(N)}\left\{\left[H^{(2)}, \rho^{(N)}(t)\right]\right\} \\
= & {\left[H^{(2)}, \rho^{(M)}(t)\right]+(N-M)\left(\operatorname{Tr}_{1}^{(M+1)}\left\{\left[H^{(2)}, \rho^{(M+1)}(t)\right]\right\}-\left[H^{(2)}, \rho^{(M)}(t)\right]\right) } \\
= & {\left[(1-(N-M)) H^{(2)}, \rho^{(M)}(t)\right]+(N-M) \operatorname{Tr}_{1}^{(M+1)}\left\{\left[H^{(2)}, \rho^{(M+1)}(t)\right]\right\} . }
\end{aligned}
$$

Together with the single-particle term (7.104), this transforms (7.101) into a hierarchical expansion of the reduced dynamics,

$$
\begin{aligned}
i \hbar \frac{\partial}{\partial t} \rho^{(M)}(t)= & {\left[H^{(1)}+(1-(N-M)) H^{(2)}, \rho^{(M)}(t)\right] } \\
& +(N-M) \operatorname{Tr}_{1}^{(M+1)}\left\{\left[H^{(2)}, \rho^{(M+1)}(t)\right]\right\},
\end{aligned}
$$

which depends on $\rho^{(M)}$ and $\rho^{(M+1)}$. The dynamics of the latter, in turn, depends on $\rho^{(M+2)}$ and so on. A hierarchal expansion of this type was first derived to describe the statistical physics of classical systems (Yvon, 1935; Bogoliubov, 1946; Born and Green, 1946; Kirkwood, 1946) and is known as the BBGKY hierarchy. It was later generalized to quantum systems (Bogoliubov and Gurov, 1947); see also (Bogoliubov, 1967a; Röpke, 2013). To obtain a closed expression for the dynamics of $\rho^{(M)}(t)$, the higher-order terms have to be expressed in terms of $\rho^{(M)}(t)$ which involves some type of approximation that truncates this hierarchy. Different methods to achieve this are 
discussed in (Bogoliubov, 1967a; Bogoliubov, 1967b; Röpke, 2013). To this end, we will later employ the pure product state assumption, which was introduced in section 7.3.5.

Note also that, whenever single-particle dynamics is considered $(M=1)$, the twoparticle Hamiltonian part drops out, since, as discussed before, in this case both sides of the equation refer only to single-particle operators. Moreover, if no particle is traced out, that is when $N=M$, the evolution (7.114) reduces to the unperturbed von Neumann equation (7.83) for the $M$-particle density operator.

\subsubsection{Coherent mean-field evolution}

Making the pure product state assumption (7.65), we can truncate the hierarchical family of dynamical equations $(7.114)$, since it allows us to rewrite all $\rho^{(M+1)}$-terms as a function of $\rho^{(M)}$-terms and $\rho^{(1)}$-terms, as follows,

$$
\rho_{k_{1} k_{2} n_{2} \ldots n_{M} ; m_{1} \ldots m_{M} n_{M+1}}^{(M+1)}(t)=\rho_{k_{1} n_{M+1}}^{(1)}(t) \rho_{k_{2} n_{2} \ldots n_{M} ; m_{1} \ldots m_{M}}^{(M)}(t) .
$$

Making this replacement allows us to write the right-hand side of the hierarchy (7.114) in terms of $\rho^{(M)}$, which can be seen explicitly, for example, by employing the above decomposition in the last two lines of equation (7.108). In fact, all of the indices can be split up, and since the states are pure, also 'ket' and 'bra' side can be treated separately - see equation (7.66). We retain the density matrix notation, keeping in mind that the state is pure. Under the pure product state assumption we can write the reduced dynamics as

$$
i \hbar \frac{\partial}{\partial t} \rho^{(M)}(t)=\left[H^{(1)}+H^{(2)}+(N-M) C^{(1)}(t), \rho^{(M)}(t)\right],
$$

where $C^{(1)}(t)$ is defined as

$$
C^{(1)}(t)=\sum_{i j m n} H_{n j ; i m}^{(2)} \rho_{i j}^{(1)}(t) a_{n}^{\dagger} a_{m} .
$$

This equation still has the form of the von Neumann equation, describing coherent dynamics where the mean-field potential term $C^{(1)}(t)$ is added to the unperturbed Hamiltonian. However, since $C^{(1)}(t)$ itself depends on $\rho^{(1)}(t)$, this is a nonlinear equation.

For $M=1$, we insert a pure state,

$$
\rho^{(1)}(t)=|\Phi(t)\rangle\langle\Phi(t)|,
$$

into equation (7.116). In this case the equation is equivalent to a nonlinear Schrödinger equation

$$
i \hbar \frac{\partial}{\partial t}|\Phi(t)\rangle=H_{\mathrm{eff}}^{(1)}|\Phi(t)\rangle,
$$

with the effective Hamiltonian,

$$
H_{\text {eff }}^{(1)}=H^{(1)}+(N-1) C^{(1)}(t),
$$


where, again, $C^{(1)}(t)$ is a nonlinear term containing the contribution of $|\Phi(t)\rangle$.

For completeness, we note that a similar calculation in the interaction picture yields

$$
\begin{aligned}
i \hbar \frac{\partial}{\partial t} \rho_{I}^{(M)}(t) & =\operatorname{Tr}_{N-M}^{(N)}\left\{\left[H_{I}^{(2)}(t), \rho_{I}^{(N)}(t)\right]\right\} \\
& =\left[H_{I}^{(2)}(t)+(N-M) C^{(1)}(t, t), \rho_{I}^{(M)}(t)\right],
\end{aligned}
$$

where

$$
C^{(1)}\left(t_{1}, t_{2}\right)=\sum_{i j n m} H_{n j ; i m}^{(2)}\left(t_{1}\right) \rho_{i j}^{(1)}\left(t_{2}\right) a_{n}^{\dagger} a_{m}
$$

is the corresponding mean-field term (7.117) in the interaction-picture, expressed as a function of the matrix elements of $\rho_{I}^{(1)}(t)$ and $H_{I}^{(2)}(t)$. In general, when dealing with higher-order expansions of the interaction potential, we may encounter situations where $t_{1} \neq t_{2}$. Such situations will be discussed in section 7.5.

The dynamical equation (7.114) describes the dynamics of bosonic subsystems, without assumptions, and generates a BBGKY-type hierarchy. The formulation in terms of arbitrary second-quantized Hamilton operators allows for direct application to specific systems. The fact that the dynamics of the reduced $M$-particle system depends on the quantum state of $M+1$ particles is caused by the two-particle interaction term $H^{(2)}$ : To determine the evolution of the single-particle density matrix in the presence of pairwise interactions, we need to know the density matrix describing two particles, whose evolution, in turn, requires knowledge of the three-particle density matrix, and so on.

The truncated, pure-state equation (7.119) describes the coherent mean-field evolution for arbitrary potentials. The mean-field product state approximation allows us to express the many-body quantum state as a function of the single-particle quantum state. This single-particle state then also alters the effective potential in which each particle evolves, and thereby accounts for the effect of particle-particle interactions. In the form (7.119), we have not specified the Hamiltonian yet, thus, this equation is more general than the well-known Gross-Pitaevskii equation, which describes the evolution of a dilute Bose-Einstein condensate. In the next section we derive the GrossPitaevskii equation as a special case of the mean-field equation (7.119) for a contact interaction potential.

\subsubsection{From the hierarchical expansion to Gross-Pitaevskii}

We now employ the truncated pure-state equation (7.116), to describe a weakly interacting Bose-Einstein condensate. We assume short-range interactions $V\left(\mathbf{x}_{1}, \mathbf{x}_{2}\right)=$ $g \delta\left(\mathbf{x}_{2}-\mathbf{x}_{1}\right)$, which in field operator representation (7.79) yields the interaction Hamiltonian,

$$
H^{(2)}=\frac{g}{2} \int d \mathbf{x} \hat{\Psi}^{\dagger}(\mathbf{x}) \hat{\Psi}^{\dagger}(\mathbf{x}) \hat{\Psi}(\mathbf{x}) \hat{\Psi}(\mathbf{x}) .
$$


Hence, the effective mean-field term (7.117), reads

$$
\begin{aligned}
& C^{(1)}(t) \\
= & g \sum_{i j n m} \int d \mathbf{x} \int d \mathbf{x}_{1} \int d \mathbf{x}_{1}^{\prime} \varphi_{n}^{*}(\mathbf{x}) \varphi_{j}^{*}(\mathbf{x}) \varphi_{i}(\mathbf{x}) \varphi_{m}(\mathbf{x}) \varphi_{i}^{*}\left(\mathbf{x}_{1}\right) \rho^{(1)}\left(t ; \mathbf{x}_{1} ; \mathbf{x}_{1}^{\prime}\right) \varphi_{j}\left(\mathbf{x}_{1}^{\prime}\right) a_{n}^{\dagger} a_{m} \\
= & g \int d \mathbf{x} \hat{\Psi}^{\dagger}(\mathbf{x}) \hat{\Psi}(\mathbf{x}) \rho^{(1)}(t ; \mathbf{x} ; \mathbf{x}),
\end{aligned}
$$

where we have used (7.69), as well as the completeness relation (7.4) for $N=1$, which leads to

$$
\sum_{i} \varphi_{i}\left(\mathbf{x}_{1}\right) \varphi_{i}^{*}\left(\mathbf{x}_{2}\right)=\sum_{i}\left\langle\mathbf{x}_{1} \mid \varphi_{i}\right\rangle\left\langle\varphi_{i} \mid \mathbf{x}_{2}\right\rangle=\left\langle\mathbf{x}_{1} \mid \mathbf{x}_{2}\right\rangle=\delta\left(\mathbf{x}_{1}-\mathbf{x}_{2}\right) .
$$

Since equation (7.124) holds for pure states $|\Phi(t)\rangle$ we insert a pure single-particle density matrix (7.118) which yields

$$
C^{(1)}(t)=g \int d \mathbf{x} \hat{\Psi}^{\dagger}(\mathbf{x}) \hat{\Psi}(\mathbf{x})|\Phi(t, \mathbf{x})|^{2} .
$$

Then, the nonlinear Schrödinger equation (7.119) predicts the following time evolution of the single-particle wavefunction

$$
\begin{aligned}
i \hbar \frac{\partial}{\partial t} \Phi(t, \mathbf{x}) & =\left\langle\mathbf{x}\left|H_{\mathrm{eff}}^{(1)}\right| \Phi(t)\right\rangle \\
& =\left[-\frac{\hbar^{2}}{2 m} \nabla_{\mathbf{x}}^{2}+V_{0}(\mathbf{x})+g(N-1)|\Phi(t, \mathbf{x})|^{2}\right] \Phi(t, \mathbf{x}) .
\end{aligned}
$$

This coincides with the single-particle dynamics obtained via the Gross-Pitaevskii equation (7.99), when $\Phi(t, \mathbf{x})=\varphi_{0}(t, \mathbf{x})$ describes the macroscopically occupied singleparticle state with $N \approx N_{0} \gg 1$.

\subsection{Second-order master equation for identical particles: Incoherent effects}

In the previous section, we have performed the partial trace operation (7.54) on the many-body von Neumann equation (7.83), which lead us to a description of the subdynamics in form of a hierarchical commutator structure (7.114). The commutator structure of the von Neumann equation is maintained through all steps of the derivation, which effectively yields a description of the reduced dynamics according to a von Neumann type evolution. Such an equation is equivalent to the Schrödinger equation, which cannot describe incoherent, dissipative effects on the subdynamics. For large interacting systems, such effects are, however, expected to become visible for singleparticle observables. An example is given by the Bloch oscillations which describe the evolution of the average single-particle momentum in a periodic potential. The 
dynamics of this single-particle observable is strongly affected by particle-particle interactions, which induces decoherence of Bloch oscillations (Buchleitner and Kolovsky, 2003; Meinert et al., 2014), which are not expected to be accountable by any coherent mean-field theory.

To extend our treatment to a description of incoherent effects, we take inspiration from the microscopic derivation of weak-coupling Lindblad-type master equations, which is based on a second-order perturbative expansion in terms of the interaction Hamiltonian (Breuer and Petruccione, 2002). Regarding identical particles, the particle-particle interaction takes on the role of the interaction between system and an external reservoir. The second-order expansion leads to a characteristic doublecommutator structure, which already foreshadows the resulting Lindblad structure of the effective master equation. In conventional system-environment scenarios, tracing over the environmental degrees of freedom then leads to the Lindblad-type master equations, which, in fact, describe incoherent effects and allow for a clear interpretation of the contributing dissipative, and dephasing processes. In the case of indistinguishable particles, we, again, need to replace the tensorial partial trace operation (2.20) with the appropriate second-quantized partial trace operation (7.54).

\subsubsection{Single-particle subdynamics of a two-particle bosonic system}

We first consider the simplest case of two interacting identical particles. Before tracing over the two-particle subspace to obtain an effective description of the single-particle dynamics, we expand the full dynamics to second order employing a perturbative series in terms of the interaction Hamiltonian $H^{(2)}$. This is most conveniently realized in the interaction picture. Integrating equation (7.85) yields for $N=2$,

$$
\rho_{I}^{(2)}(t)=\rho_{I}^{(2)}(0)-\frac{i}{\hbar} \int_{0}^{t} d s\left[H_{I}^{(2)}(s), \rho_{I}^{(2)}(s)\right] .
$$

Inserting this back into equation (7.85), we obtain a second-order expression in $H_{I}^{(2)}(t)$ for the dynamics of $\rho_{I}^{(2)}(t)$, which, so far, is still exact. The dynamics of the singleparticle density matrix is given by partially tracing over the two-particle space, according to equation (7.23),

$$
\frac{\partial}{\partial t} \rho_{I}^{(1)}(t)=\operatorname{Tr}_{1}^{(2)}\left\{\frac{\partial}{\partial t} \rho_{I}^{(2)}(t)\right\}=\frac{1}{2} \sum_{i j} \operatorname{Tr}^{(2)}\left\{a_{i} \frac{\partial}{\partial t} \rho_{I}^{(2)}(t) a_{j}^{\dagger}\right\} a_{i}^{\dagger} a_{j}
$$

For the second-order expression, obtained with (7.128) in (7.85), this yields

$$
\frac{\partial}{\partial t} \rho_{I}^{(1)}(t)=\left(-\frac{i}{\hbar}\right)^{2} \int_{0}^{t} d s \operatorname{Tr}_{1}^{(2)}\left[H_{I}^{(2)}(t),\left[H_{I}^{(2)}(s), \rho_{I}^{(2)}(s)\right]\right]
$$

assuming that $\operatorname{Tr}_{1}^{(2)}\left[H_{I}^{(2)}(t), \rho_{I}^{(2)}(0)\right]=0$. The Markov approximation now consists in replacing $\rho_{I}^{(2)}(s)$ by $\rho_{I}^{(2)}(t)$, such that the future evolution does not depend on the state at previous times (Breuer and Petruccione, 2002). This means we truncate 
the perturbation expansion after the second order. Hence, we obtain a Redfield-type equation (Redfield, 1957)

$$
\begin{aligned}
\frac{\partial}{\partial t} \rho_{I}^{(1)}(t) & =-\frac{1}{\hbar^{2}} \int_{0}^{t} d s \operatorname{Tr}_{1}^{(2)}\left[H_{I}^{(2)}(t),\left[H_{I}^{(2)}(s), \rho_{I}^{(2)}(t)\right]\right] \\
& =\frac{1}{\hbar^{2}} \int_{0}^{t} d s \operatorname{Tr}_{1}^{(2)}\left\{H_{I}^{(2)}(s) \rho_{I}^{(2)}(t) H_{I}^{(2)}(t)-H_{I}^{(2)}(t) H_{I}^{(2)}(s) \rho_{I}^{(2)}(t)\right\}+\text { H.c. }
\end{aligned}
$$

Note, however, that, even for a conventional system-environment approach, this approximation alone usually does not produce a Markovian master equation (Breuer and Petruccione, 2002), and for this reason is often combined with a secular or rotatingwave approximation (Cohen-Tannoudji et al., 1992; Breuer and Petruccione, 2002; Clos and Breuer, 2012).

The right-hand side of this equation still depends on the full two-particle state. To derive a closed expression for the single-particle density matrix, we employ the pure product state assumption, see equation (7.64), which can be interpreted as the analog of the Born approximation of standard open systems. In both cases, we neglect correlations between the two subsystems, and replace the full quantum state with a uncorrelated product state. Here, however, we cannot replace the state of one of the two particles with a time-invariant state to employ a coarse-grained timescale which does not resolve excitations of the 'bath' since the concepts of 'system' and 'bath' are not applicable to a situation where both constituents cannot be distinguished. In the pure product state assumption both particles are described by the same single particle state at all times. As was discussed in section 7.3.5, the symmetry requirements of many-body systems prevents us to express the total state as a product of mixed states, which is unfortunate, since the restriction to pure states, in principle, does not allow for a description of incoherent effects. We now derive the general form of the resulting truncated master equation under the pure product state assumption. Future studies might then be able to tell under which conditions the resulting equations can be applied to describe the dynamics of mixed states.

We thus replace the elements of the interaction-picture two-particle density matrix as $\rho_{i j ; k l}^{(2)}(t)=\rho_{i k}^{(1)}(t) \rho_{j l}^{(1)}(t)$, where, in principle, we could further decompose the elements of the pure-state density matrices according to equation (7.66). The first term of the integral (7.131) can then be rewritten as

$$
\begin{aligned}
& \int_{0}^{t} d s \operatorname{Tr}_{1}^{(2)}\left\{H_{I}^{(2)}(s) \rho_{I}^{(2)}(t) H_{I}^{(2)}(t)\right\} \\
= & \int_{0}^{t} d s \frac{1}{2} \sum_{i j k l} \rho_{i k}^{(1)}(t) \rho_{j l}^{(1)}(t) \operatorname{Tr}_{1}^{(2)}\left\{H_{I}^{(2)}(s) a_{i}^{\dagger} a_{j}^{\dagger} a_{k} a_{l} H_{I}^{(2)}(t)\right\} \\
= & \int_{0}^{t} d s \frac{1}{4} \sum_{i j k l m n} \rho_{i k}^{(1)}(t) \rho_{j l}^{(1)}(t) \operatorname{Tr}^{(2)}\left\{a_{m}^{\dagger} a_{n} H_{I}^{(2)}(s) a_{i}^{\dagger} a_{j}^{\dagger} a_{k} a_{l} H_{I}^{(2)}(t)\right\} a_{n}^{\dagger} a_{m},
\end{aligned}
$$

where in the final step we have used the definition of the second-quantized partial trace operation (7.54). We reformulate the term containing the two-particle trace, using the 
invariance under cyclic permutations, and equation (7.19),

$$
\begin{aligned}
\frac{1}{2} \operatorname{Tr}^{(2)}\left\{a_{m}^{\dagger} a_{n} H_{I}^{(2)}(s) a_{i}^{\dagger} a_{j}^{\dagger} a_{k} a_{l} H_{I}^{(2)}(t)\right\} & =\frac{1}{2} \operatorname{Tr}^{(2)}\left\{a_{k} a_{l} H_{I}^{(2)}(t) a_{m}^{\dagger} a_{n} H_{I}^{(2)}(s) a_{i}^{\dagger} a_{j}^{\dagger}\right\} \\
& =\left\langle\varphi_{k} \varphi_{l}\left|H_{I}^{(2)}(t) a_{m}^{\dagger} a_{n} H_{I}^{(2)}(s)\right| \varphi_{i} \varphi_{j}\right\rangle .
\end{aligned}
$$

To simplify this expression, we derive an expression for the matrix element of the two-particle operator $\left\langle\varphi_{i} \varphi_{j}\left|X^{(2)} a_{m}^{\dagger} a_{n} Y^{(2)}\right| \varphi_{k} \varphi_{l}\right\rangle$ in terms of the matrix elements of $X$ and $Y$. Using the two-particle representation of the operators $X^{(2)}$ and $Y^{(2)}$ given in equation (7.18), we obtain

$$
\begin{aligned}
& \left\langle\varphi_{i} \varphi_{j}\left|X^{(2)} a_{m}^{\dagger} a_{n} Y^{(2)}\right| \varphi_{k} \varphi_{l}\right\rangle \\
= & \frac{1}{2 \cdot 2^{2}} \sum_{\substack{p, q, r, s \\
\alpha, \beta, \gamma, \delta}} X_{p q ; r s}^{(2)} Y_{\alpha \beta ; \gamma \delta}^{(2)} \operatorname{Tr}^{(2)}\left\{a_{i} a_{j} a_{p}^{\dagger} a_{q}^{\dagger} a_{r} a_{s} a_{m}^{\dagger} a_{n} a_{\alpha}^{\dagger} a_{\beta}^{\dagger} a_{\gamma} a_{\delta} a_{k}^{\dagger} a_{l}^{\dagger}\right\} \\
= & \sum_{r, s, \alpha, \beta} X_{i j ; r s}^{(2)} Y_{\alpha \beta ; k l}^{(2)}\left\langle\varphi_{r} \varphi_{s}\left|a_{m}^{\dagger} a_{n}\right| \varphi_{\alpha} \varphi_{\beta}\right\rangle,
\end{aligned}
$$

where we have used Lemmata 1, 2 and $\%$. This can be further simplified to

$$
\begin{aligned}
\left\langle\varphi_{i} \varphi_{j}\left|X^{(2)} a_{m}^{\dagger} a_{n} Y^{(2)}\right| \varphi_{k} \varphi_{l}\right\rangle & =\sum_{r, s, \alpha, \beta} X_{i j ; r s}^{(2)} Y_{\alpha \beta ; k l}^{(2)} \underbrace{\left\langle\varphi_{s}\left|a_{m}^{\dagger} a_{n}\right| \varphi_{\alpha} \varphi_{\beta}\right\rangle}_{\delta_{n \alpha} \delta_{r s ; m \beta}+\varphi_{n \beta} \delta_{r s ; m \alpha}} \\
& =\left(\sum_{\beta} X_{i j ; m \beta}^{(2)} Y_{n \beta ; k l}^{(2)}+\sum_{\alpha} X_{i j ; m \alpha}^{(2)} Y_{\alpha n ; k l}^{(2)}\right) \\
& =2 \sum_{\beta} X_{i j ; m \beta}^{(2)} Y_{n \beta ; k l}^{(2)} .
\end{aligned}
$$

Inserting this into equation (7.132), with $X^{(2)}=H_{I}^{(2)}(t)$ and $Y^{(2)}=H_{I}^{(2)}(s)$, yields

$$
\begin{aligned}
& \int_{0}^{t} d s \operatorname{Tr}_{1}^{(2)}\left\{H_{I}^{(2)}(s) \rho_{I}^{(2)}(t) H_{I}^{(2)}(t)\right\} \\
= & \int_{0}^{t} d s \sum_{i j k l m n p} \rho_{i k}^{(1)}(t) \rho_{j l}^{(1)}(t) H_{k l ; m p}^{(2)}(t) H_{p n ; i j}^{(2)}(s) a_{n}^{\dagger} a_{m} \\
= & \sum_{\alpha \beta} A_{\alpha \beta}^{(1)}(t) \rho_{I}^{(1)}(t) B_{\beta \alpha}^{(1)}(t),
\end{aligned}
$$

with single-particle operators $A_{\alpha \beta}^{(1)}(t)$ and $B_{\beta \alpha}^{(1)}(t)$ whose elements are defined as

$$
\left(A_{\alpha \beta}^{(1)}(s, t)\right)_{i j}:=\sum_{k} H_{i \alpha ; j k}^{(2)}(s) \rho_{k \beta}^{(1)}(t)
$$

and

$$
\left(B_{\beta \alpha}^{(1)}(t)\right)_{i j}=H_{i \beta ; j \alpha}^{(2)}(t)
$$


The operators $B_{\alpha \beta}^{(1)}$ exhibit the property

$$
\left(B_{\alpha \beta}^{(1) \dagger}\right)_{i j}=\left(B_{\alpha \beta}^{(1)}\right)_{j i}^{*}=H_{j \alpha ; i \beta}^{(2) *}=H_{i \beta ; j \alpha}^{(2)}=\left(B_{\beta \alpha}^{(1)}\right)_{i j},
$$

and, hence,

$$
B_{\alpha \beta}^{(1) \dagger}=B_{\beta \alpha}^{(1)} .
$$

The above derivation can be performed analogously for the second term of the integral (7.131), leading to

$$
\int_{0}^{t} d s \operatorname{Tr}_{1}^{(2)}\left\{H_{I}^{(2)}(t) H_{I}^{(2)}(s) \rho_{I}^{(2)}(t)\right\}=\int_{0}^{t} d s \sum_{\alpha \beta} B_{\beta \alpha}^{(1)}(t) A_{\alpha \beta}^{(1)}(s, t) \rho_{I}^{(1)}(t) .
$$

Inserting (7.136) and (7.141) into equation (7.131) leads to

$$
\begin{aligned}
\frac{\partial}{\partial t} \rho_{I}^{(1)}(t)= & \frac{1}{\hbar^{2}} \int_{0}^{t} d s \sum_{\alpha \beta}\left(A_{\alpha \beta}^{(1)}(s, t) \rho_{I}^{(1)}(t) B_{\beta \alpha}^{(1)}(t)-B_{\beta \alpha}^{(1)}(t) A_{\alpha \beta}^{(1)}(s, t) \rho_{I}^{(1)}(t)\right)+\text { H.c. } \\
= & \frac{1}{\hbar^{2}} \int_{0}^{t} d s \sum_{\alpha \beta i j k l}\left(A_{\alpha \beta}^{(1)}(s, t)\right)_{i j}\left(B_{\beta \alpha}^{(1)}(t)\right)_{k l} \\
& \quad \times\left(a_{i}^{\dagger} a_{j} \rho_{I}^{(1)}(t) a_{k}^{\dagger} a_{l}-a_{k}^{\dagger} a_{l} a_{i}^{\dagger} a_{j} \rho_{I}^{(1)}(t)\right)+\text { H.c. } \\
= & \frac{1}{\hbar^{2}} \sum_{i j k l} \Gamma_{i j k l}(t)\left(a_{i}^{\dagger} a_{j} \rho_{I}^{(1)}(t) a_{k}^{\dagger} a_{l}-a_{k}^{\dagger} a_{l} a_{i}^{\dagger} a_{j} \rho_{I}^{(1)}(t)\right)+\text { H.c., }
\end{aligned}
$$

where we have defined

$$
\begin{aligned}
\Gamma_{i j k l}(t) & =\int_{0}^{t} d s \sum_{\alpha \beta}\left(A_{\alpha \beta}^{(1)}(s, t)\right)_{i j}\left(B_{\beta \alpha}^{(1)}(t)\right)_{k l} \\
& =\int_{0}^{t} d s \sum_{\alpha \beta m} H_{i \alpha ; j m}^{(2)}(s) \rho_{m \beta}^{(1)}(t) H_{k \beta ; l \alpha}^{(2)}(t) \\
& =\int_{0}^{t} d s \sum_{\alpha \beta m} H_{i \alpha ; j m}^{(2)}(t-s) \rho_{m \beta}^{(1)}(t) H_{k \beta ; l \alpha}^{(2)}(t) \\
& =\int_{0}^{t} d s \sum_{\alpha \beta m}\left(B_{i j}^{(1)}(t-s)\right)_{\alpha m} \rho_{m \beta}^{(1)}(t)\left(B_{k l}^{(1)}(t)\right)_{\beta \alpha} \\
& =\int_{0}^{t} d s \operatorname{Tr}^{(1)}\left\{B_{i j}^{(1)}(t-s) \rho_{I}^{(1)}(t) B_{k l}^{(1)}(t)\right\} .
\end{aligned}
$$

Note that changing the time argument in the third line above is valid since

$$
\int_{0}^{t} d s f(s)=-\int_{t}^{0} d s f(t-s)=\int_{0}^{t} d s f(t-s) .
$$


Introducing (Breuer and Petruccione, 2002)

$$
\Gamma_{i j k l}(t)=\frac{1}{2} \gamma_{i j k l}(t)+i S_{i j k l}(t)
$$

allows us to substitute

$$
\begin{aligned}
\gamma_{i j k l}(t) & =\Gamma_{i j k l}(t)+\Gamma_{l k j i}^{*}(t), \\
S_{i j k l}(t) & =\frac{1}{2 i}\left(\Gamma_{i j k l}(t)-\Gamma_{l k j i}^{*}(t)\right) .
\end{aligned}
$$

We finally obtain the interaction-picture master equation

$$
\begin{aligned}
\frac{\partial}{\partial t} \rho_{I}^{(1)}(t)= & -\frac{i}{\hbar}\left[H_{L S}^{(1)}(t), \rho_{I}^{(1)}(t)\right] \\
& +\frac{1}{\hbar^{2}} \sum_{i j k l} \gamma_{i j k l}(t)\left(a_{i}^{\dagger} a_{j} \rho_{I}^{(1)}(t) a_{k}^{\dagger} a_{l}-\frac{1}{2}\left\{a_{k}^{\dagger} a_{l} a_{i}^{\dagger} a_{j}, \rho_{I}^{(1)}(t)\right\}\right),
\end{aligned}
$$

with the Lamb-Shift Hamiltonian

$$
H_{L S}^{(1)}(t)=\frac{1}{\hbar} \sum_{i j k l} S_{i j k l}(t) a_{k}^{\dagger} a_{l} a_{i}^{\dagger} a_{j} .
$$

This equation has the characteristic Lindblad structure of a time-local master equation (2.26) and, thus, is able to describe dissipative effects. Our approach was predestined to end up in a Lindblad-type structure, since we started our microscopic derivation by a second-order expansion of the von Neumann equation, which already yields a double-commutator structure (7.131). As we have already seen in the case of coherent mean-field evolutions, the partial trace operation preserves this structure to the level of the reduced master equation. The $\gamma_{i j k l}(t)$ can be interpreted as the time-dependent decay rates of the different decay channels, which are represented by the specific form of the contributing operators. These decay channels do not describe particle loss or gain, since, by construction, we derived an effective description of the single-particle reduced density matrix, which always accounts for exactly one particle. Before specifying the Hamiltonian $H$ of the system, and, in particular, the interaction term $H^{(2)}$, the role and physical interpretation of the decay channels is not determined. The functions $\gamma_{i j k l}(t)$ and $S_{i j k l}(t)$ are single-particle autocorrelation functions, which in turn depend on the state $\rho_{I}^{(1)}(t)$ - the argument of the equation of motion (7.148). The autocorrelation functions further contain the interaction-picture representation of the two-particle Hamiltonian $H^{(2)}$, as can be seen from equation (7.143). The role of these functions is closely related to the bath-autocorrelation functions which appear in the treatment of standard open systems (Breuer and Petruccione, 2002). In our present case, we observe a formal correspondence, with the crucial difference that the state of the 'bath' coincides - at all times - with the state of the system. Eventually, we obtain a nonlinear equation of motion, which is expected, given the mean-field ansatz that is implicit in the pure product state assumption. 
To establish direct comparison to the previous mean-field equations, we have to transform (7.148) back into the Schrödinger picture, which here is complicated by the fact that the argument of the time-correlation functions $\Gamma_{i j k l}(t)$, defined in equation (7.143), is also given in the interaction picture. Thus, the back-transformation involves more than just adding the commutator term with the single-particle Hamiltonian. Note also that a commonly employed expansion in terms of eigen-operators of the superoperator $\mathfrak{H}\left(X^{(1)}\right)=\left[H^{(1)}, X^{(1)}\right]$, where $H^{(1)}$ is the single-particle Hamiltonian, does not yield the straight-forward result of a Fourier transformed time-autocorrelation function, since the interaction-picture transformation of $H^{(2)}$ involves the transformation of a two-particle operator, while the back-transformation, which involves $B^{(1)}$, only affects the single-particle contributions, and therefore does not end up in the original Schrödinger-picture expression. Further details on the transformation properties will be discussed in appendix $\mathrm{C}$.

\subsubsection{General ansatz: M-particle subdynamics of an N-particle bosonic system}

Now we generalize the previous approach to describe the perturbative second-order evolution of arbitrary $M$-particle subsystems of an interacting $N$-particle bosonic system. As in the previous section, our initial point is the second-order expansion in the interaction picture,

$$
\begin{aligned}
& \frac{\partial}{\partial t} \rho_{I}^{(M)}(t) \\
= & \operatorname{Tr}_{N-M}^{(N)}\left\{-\frac{i}{\hbar}\left[H_{I}^{(2)}(t), \rho_{I}^{(N)}(0)\right]\right\} \\
& -\frac{1}{\hbar^{2}} \int_{0}^{t} d s \operatorname{Tr}_{N-M}^{(N)}\left[H_{I}^{(2)}(t),\left[H_{I}^{(2)}(s), \rho_{I}^{(N)}(s)\right]\right] \\
= & \operatorname{Tr}_{N-M}^{(N)}\left\{-\frac{i}{\hbar}\left[H_{I}^{(2)}(t), \rho_{I}^{(N)}(0)\right]\right\} \\
& +\frac{1}{\hbar^{2}} \int_{0}^{t} d s\left(\operatorname{Tr}_{N-M}^{(N)}\left\{H_{I}^{(2)}(s) \rho_{I}^{(N)}(s) H_{I}^{(2)}(t)-H_{I}^{(2)}(t) H_{I}^{(2)}(s) \rho_{I}^{(N)}(s)\right\}+\text { H.c. }\right),
\end{aligned}
$$

which describes a direct generalization of equation (7.131). Before replacing the time argument of $\rho_{I}^{(N)}(s)$ in the integral with $t$, this is an exact expression. The term containing the initial condition, $\operatorname{Tr}_{N-M}^{(N)}\left\{\left[H_{I}^{(2)}(t), \rho_{I}^{(N)}(0)\right]\right\}$, can be evaluated as a function of the matrix elements using equation (7.108). We further need to determine the two terms that occur in the double commutator. The symmetrization requirements of bosonic operators and states render this an elaborate task. We therefore shift the intermediate steps of the following derivation to the appendix A.

As detailed in the appendix, equation (7.150) is equivalent to the following expan- 
sion:

$$
\begin{aligned}
\frac{\partial}{\partial t} \rho^{(M)}(t)=- & \frac{i}{\hbar}\left[H_{I}^{(2)}(t), \rho_{I}^{(M)}(0)\right]-\frac{1}{\hbar^{2}} \int_{0}^{t} d s\left[H_{I}^{(2)}(t),\left[H_{I}^{(2)}(s), \rho_{I}^{(M)}(s)\right]\right] \\
& +(N-M) \mathcal{D}_{1}\left(t, \rho_{I}^{(M+1)}\right) \\
& +(N-M)(N-M-1) \mathcal{D}_{2}\left(t, \rho_{I}^{(M+2)}\right) \\
& +(N-M)(N-M-1)(N-M-2) \mathcal{D}_{3}\left(t, \rho_{I}^{(M+3)}\right)
\end{aligned}
$$

The coherent part of the evolution reproduces exactly the dynamics of the $M$-particle density matrix according to the interaction-picture von Neumann equation (7.85). For $N=M$ only this term remains, which was expected, since, so far, we have not realized any approximation, and when no part of the Hilbert space is traced out, the evolution must be fully coherent, similarly to equation (7.114). The decoherent parts of the evolution are now encoded in three different dissipator terms, which we express in terms of their matrix elements. The dissipator $\mathcal{D}_{1}\left(t, \rho_{I}^{(M+1)}\right)$ involves the state of one additional particle, and contains the initial term. We have

$$
\begin{aligned}
& \mathcal{D}_{1}\left(t, \rho_{I}^{(M+1)}\right) \\
=- & \frac{i}{\hbar} \int_{0}^{t} d s \sum_{\substack{n_{1} \ldots n_{M} \\
m_{1} \ldots m_{M}}} \sum_{i_{1} i_{2}} \sum_{k_{1}} M \frac{1}{M !} a_{n_{1}}^{\dagger} \ldots a_{n_{M}}^{\dagger} a_{m_{1}} \ldots a_{m_{M}} \\
& \times\left(H_{n_{1} k_{1} ; i_{1} i_{2}}^{(2)}(t) \rho_{i_{1} i_{2} n_{2} \ldots n_{M} ; m_{1} \ldots m_{M} k_{1}}^{(M+1)}(0)-\rho_{n_{1} \ldots n_{M} k_{1} ; i_{1} i_{2} m_{2} \ldots m_{M}}^{(M+1)}(0) H_{i_{1} i_{2} ; m_{1} k_{1}}^{(2)}(t)\right)
\end{aligned}
$$$$
+\frac{1}{\hbar^{2}} \int_{0}^{t} d s \sum_{\substack{n_{1} \ldots n_{M} \\ m_{1} \ldots m_{M}}} \sum_{i_{1} i_{2}} \sum_{j_{1} j_{2}} M \frac{1}{M !} a_{n_{1}}^{\dagger} \ldots a_{n_{M}}^{\dagger} a_{m_{1}} \ldots a_{m_{M}}
$$$$
\times\left[\frac{1}{2}(M-1) M \rho_{i_{1} i_{2} n_{2} \ldots n_{M} ; j_{1} j_{2} m_{3} \ldots m_{M} k_{1}}^{(M+1)}(s) H_{n_{1} k_{1} ; i_{1} i_{2}}^{(2)}(s) H_{j_{1} j_{2} ; m_{1} m_{2}}^{(2)}(t)\right.
$$$$
+\frac{1}{2}(M-1) M \rho_{i_{1} i_{2} n_{3} \ldots n_{M} k_{1} ; j_{1} j_{2} m_{2} \ldots m_{M}}^{(M+1)}(s) H_{n_{1} n_{2} ; i_{1} i_{2}}^{(2)}(s) H_{j_{1} j_{2} ; m_{1} k_{1}}^{(2)}(t)
$$$$
+M \rho_{i_{1} i_{2} n_{2} \ldots n_{M} ; j_{1} j_{2} m_{2} \ldots m_{M}}^{(M+1)}(s) H_{n_{1} k_{1} ; i_{1} i_{2}}^{(2)}(s) H_{j_{1} j_{2} ; m_{1} k_{1}}^{(2)}(t)
$$$$
-\rho_{i_{1} i_{2} n_{2} \ldots n_{M} ; m_{1} \ldots m_{M} k_{1}}^{(M+1)}(s) H_{n_{1} k_{1} ; j_{1} j_{2}}^{(2)}(t) H_{j_{1} j_{2} ; i_{1} i_{2}}^{(2)}(s)
$$$$
-(M-1) \rho_{i_{1} i_{2} j_{2} n_{3} \ldots n_{M} ; m_{1} \ldots m_{M} k_{1}}^{(M+1)}(s) H_{n_{1} n_{2} ; j_{1} j_{2}}^{(2)}(t) H_{j_{1} k_{1} ; i_{1} i_{2}}^{(2)}(s)
$$$$
-\frac{1}{2}(M-1)(M-2) \rho_{i_{1} i_{2} j_{1} j_{2} n_{4} \ldots n_{M} ; m_{1} \ldots m_{M} k_{1}}^{(M+1)}(s) H_{n_{1} n_{2} ; j_{1} j_{2}}^{(2)}(t) H_{n_{3} k_{1} ; i_{1} i_{2}}^{(2)}(s)
$$$$
-2(M-1) \rho_{i_{1} i_{2} j_{3} n_{3} \ldots n_{M} ; m_{1} \ldots m_{M} k_{1}}^{(M+1)}(s) H_{n_{1} k_{1} ; j_{1} j_{3}}^{(2)}(t) H_{j_{1} n_{2} ; i_{1} i_{2}}^{(2)}(s)
$$$$
\left.-\frac{1}{2}(M-1)(M-2) \rho_{i_{1} i_{2} j_{1} j_{2} n_{4} \ldots n_{M} ; m_{1} \ldots m_{M} k_{1}}^{(M+1)}(s) H_{n_{1} k_{1} ; j_{1} j_{2}}^{(2)}(t) H_{n_{2} n_{3} ; i_{1} i_{2}}^{(2)}(s)\right]+ \text { H.c. }
$$ 
The $(M+2)$-particle contributions are contained in $\mathcal{D}_{2}\left(t, \rho_{I}^{(M+2)}\right)$, which reads

$$
\begin{aligned}
\mathcal{D}_{2}( & \left.t, \rho_{I}^{(M+2)}\right) \\
=\frac{1}{\hbar^{2}} & \int_{0}^{t} d s \sum_{\substack{n_{1} \ldots n_{M} \\
m_{1} \ldots m_{M}}} \sum_{i_{1} i_{1} j_{2}} \sum_{j_{1} k_{2}} M \frac{1}{M !} a_{n_{1}}^{\dagger} \ldots a_{n_{M}}^{\dagger} a_{m_{1}} \ldots a_{m_{M}} \\
\times & {\left[\frac{1}{4}(M-1) \rho_{i_{1} i_{2} n_{1} \ldots n_{M} ; j_{1} j_{2} m_{3} \ldots m_{M} k_{1} k_{2}}^{(M+2)}(s) H_{k_{1} k_{2} ; i_{1} i_{2}}^{(2)}(s) H_{j_{1} j_{2} ; m_{1} m_{2}}^{(2)}(t)\right.} \\
& +M \rho_{i_{1} i_{2} n_{2} \ldots n_{M} k_{2} ; j_{1} j_{2} m_{2} \ldots m_{M} k_{1}}^{(M+2)}(s) H_{n_{1} k_{1} ; i_{1} i_{2}}^{(2)}(s) H_{j_{1} j_{2} ; m_{1} k_{2}}^{(2)}(t) \\
& +\rho_{i_{1} i_{2} n_{1} \ldots n_{M} ; j_{1} j_{2} m_{2} \ldots m_{M} k_{2}}^{(M+2)}(s) H_{k_{1} k_{2} ; i_{1} i_{2}}^{(2)}(s) H_{j_{1} j_{2} ; m_{1} k_{1}}^{(2)}(t) \\
& -\frac{1}{4}(M-1) \rho_{i_{1} i_{2} j_{1} j_{2} n_{3} \ldots n_{M} ; m_{1} \ldots m_{M} k_{1} k_{2}}^{(M+2)}(s) H_{n_{1} n_{2} ; j_{1} j_{2}}^{(2)}(t) H_{k_{1} k_{2} ; i_{1} i_{2}}^{(2)}(s) \\
& -2 \rho_{i_{1} i_{2} j_{2} n_{2} \ldots n_{M} ; m_{1} \ldots m_{M} k_{1} k_{2}}^{(M+2)}(s) H_{n_{1} k_{1} ; j_{1} j_{2}}^{(2)}(t) H_{j_{1} k_{2} ; i_{1} i_{2}}^{(2)}(s) \\
& \left.-(M-1) \rho_{i_{1} i_{2} j_{1} j_{2} n_{3} \ldots n_{M} ; m_{1} \ldots m_{M} k_{1} k_{2}}^{(M+2)}(s) H_{n_{1} k_{1} ; j_{1} j_{2}}^{(2)}(t) H_{n_{2} k_{2} ; i_{1} i_{2}}^{(2)}(s)\right]+ \text { H.c. }
\end{aligned}
$$

Finally, there are two terms containing $(M+3)$-particle contributions, which are condensed in

$$
\begin{aligned}
& \mathcal{D}_{3}\left(t, \rho_{I}^{(M+3)}\right) \\
&= \frac{1}{\hbar^{2}} \int_{0}^{t} d s \sum_{\substack{n_{1} \ldots n_{M} \\
m_{1} \ldots m_{M}}} \sum_{i_{1} i_{2}} \sum_{j_{1} j_{2}} M \frac{1}{M !} a_{2} k_{3} \\
& \quad \times \frac{1}{2}\left[\rho_{n_{1}}^{(M+3)} \ldots a_{n_{M} i_{2} n_{1} \ldots n_{M} k_{3} ; j_{1} j_{2} m_{2} \ldots m_{M} k_{1} k_{2}}^{\dagger}(s) H_{m_{1}} \ldots a_{m_{M}}^{(2)} H_{k_{1} k_{2} ; i_{1} i_{2}}(s) H_{j_{1} j_{2} ; m_{1} k_{3}}^{(2)}(t)\right. \\
&\left.\quad \quad-\rho_{i_{1} i_{2} j_{1} j_{2} n_{2} \ldots n_{M} ; m_{1} \ldots m_{M} k_{1} k_{2} k_{3}}^{(M+3)}(s) H_{k_{1} k_{2} ; j_{1} j_{2}}^{(2)}(t) H_{n_{1} k_{3} ; i_{1} i_{2}}^{(2)}(s)\right]+ \text { H.c. }
\end{aligned}
$$

These rather lengthy expressions determine the evolution of the $M$-particle reduced density matrix, as a function of terms with higher particle numbers. This is therefore not yet a closed 'open-system' description of the reduced subsystem dynamics. The reason for that is the lack of assumptions made, in particular about the initial state.

The expression (7.151) is valid for completely general $N$-particle density matrices, which may contain strong particle-particle correlations. Without assuming some type of statistical independence between the observable degrees of freedom and the remaining system, it is generally very hard, if not impossible, to derive a closed-form master equation. This issue manifests in standard open quantum systems via the necessity to assume factorizing initial conditions to derive a well-defined master equation (Breuer and Petruccione, 2002); see also (Gessner, 2011).

For bosonic systems, we have already realized that the only straight-forward way to achieve an unambiguous representation of the $N$-particle state in terms of singleparticle states is to perform the pure product state assumption. This comes with the 
significant drawback that pure states cannot directly account for decoherence and dissipation in a master-equation approach, unless when combined with stochastic MonteCarlo approaches (Dalibard et al., 1992; Gardiner and Zoller, 2004) which we do not consider here. Hence, at this point we are forced to resort to the pure product state assumption to truncate this hierarchy and to derive a closed expression for the subsystem dynamics. At the moment, it remains unclear whether the developed approach is applicable to mixed states in its present form, or, otherwise, if an appropriate generalization can be achieved.

\subsubsection{Operator structures within the pure product state approximation}

The dissipator terms in (7.151) depend on reduced distributions of higher particle numbers and generate a complicated hierarchy. Similarly to the case of coherent meanfield evolutions, we can easily truncate this hierarchy by making the pure product state assumption (7.65), since it allows us to rewrite, for instance,

$$
\rho_{i_{1} i_{2} n_{2} \ldots n_{M} ; j_{1} j_{2} m_{2} \ldots m_{M}}^{(M+1)}(s)=\rho_{i_{1} j_{1}}^{(1)}(s) \rho_{i_{2} n_{2} \ldots n_{M} ; j_{2} m_{2} \ldots m_{M}}^{(M)}(s)
$$

which we can use to effectively express the dependence on additional particles via single-particle states. We remark again that, under the pure product state assumption, $\rho_{I}^{(N)}$ can be arbitrarily decomposed into individual single-particle pure-state constituents. For now, we retain the density matrix expressions for ease of notation.

We will see in the following sections, that the dissipator terms that appear in the general expression (7.151) can be expressed in a rather compact form, when imposing the pure product state assumption.

\subsubsection{Terms involving $M+1$ particles}

We first consider the dissipator $\mathcal{D}_{1}\left(t, \rho_{I}^{(M+1)}\right)$. We expect that, by construction, double-commutator structures must emerge in the reduced dynamics. This was already observed explicitly for the special case of $N=2$ particles in section 7.5.1. In the rather complex expression $\mathcal{D}_{1}\left(t, \rho_{I}^{(M+1)}\right)$ these structures may be hidden, and especially hard to identify, since these commutators are expressed in a basis of $M$-particle states. The emerging pre-factors that arise [as was derived, for instance, in equation (7.50)] then depend on the particle number $S$ of the operator that is being represented in a basis of larger particle number $M>S$. In appendix B, we explicitly show how the relevant terms of double-commutators can be identified when represented in a basis of larger particle numbers.

For instance, expression (B.3), together with equation (B.2) in the special case $S=S^{\prime}=1$, is able identify structures of double commutators with single-particle operators. We also recall the single-particle operators $A_{\alpha \beta}^{(1)}\left(t_{1}, t_{2}\right)$ and $B_{\beta \alpha}^{(1)}(t)$ introduced in equations (7.137) and (7.138). Taken together, and using equation (B.1), we can 
indeed identify the term

$$
-\frac{1}{\hbar^{2}} \int_{0}^{t} d s \sum_{\alpha \beta}\left[B_{\beta \alpha}^{(1)}(t),\left[A_{\alpha \beta}^{(1)}(s, s), \rho_{I}^{(M)}(s)\right]\right]
$$

from (7.156), (7.157), and (7.160), whereas the latter is only accounted for with a factor $1 / 2$, and we also add the respective Hermitian conjugate parts to these terms.

To identify the next set of terms, we need to consider combinations of one- and twoparticle operators, which are given in equations (B.4) and (B.5). Using these results, together with the single-particle operators (7.122)

$$
C^{(1)}\left(t_{1}, t_{2}\right)=\sum_{n m} \operatorname{Tr}^{(1)}\left\{B_{n m}^{(1)}\left(t_{1}\right) \rho_{I}^{(1)}\left(t_{2}\right)\right\} a_{n}^{\dagger} a_{m}=\sum_{i j n m} H_{n j ; i m}^{(2)}\left(t_{1}\right) \rho_{i j}^{(1)}\left(t_{2}\right) a_{n}^{\dagger} a_{m},
$$

we can identify the term

$$
-\frac{1}{\hbar^{2}} \int_{0}^{t} d s\left[H_{I}^{(2)}(t),\left[C^{(1)}(s, s), \rho_{I}^{(M)}(s)\right]\right]
$$

with (7.154), (7.158) and (7.159), and

$$
-\frac{1}{\hbar^{2}} \int_{0}^{t} d s\left[C^{(1)}(t, s),\left[H_{I}^{(2)}(s), \rho_{I}^{(M)}(s)\right]\right]
$$

with (7.155), (7.161), and the remaining half of (7.160); that is, again, multiplied with a factor $1 / 2$. Note also that

$$
\begin{aligned}
C^{(1)}\left(t_{1}, t_{2}\right)^{\dagger} & =\sum_{i j n m} \operatorname{Tr}^{(1)}\left\{B_{m n}^{(1)}\left(t_{1}\right) \rho_{I}^{(1)}\left(t_{2}\right)\right\}^{*} a_{n}^{\dagger} a_{m} \\
& =\sum_{i j n m} \operatorname{Tr}^{(1)}\left\{\rho_{I}^{(1)}\left(t_{2}\right)^{\dagger} B_{m n}^{(1)}\left(t_{1}\right)^{\dagger}\right\} a_{n}^{\dagger} a_{m} \\
& =\sum_{i j n m} \operatorname{Tr}^{(1)}\left\{B_{n m}^{(1)}\left(t_{1}\right) \rho_{I}^{(1)}\left(t_{2}\right)\right\} a_{n}^{\dagger} a_{m} \\
& =C^{(1)}\left(t_{1}, t_{2}\right),
\end{aligned}
$$

where we used equation (7.139). Based on equation (7.121), the initial condition (7.152) can be written as

$$
-\frac{i}{\hbar}\left[C^{(1)}(t, 0), \rho_{I}^{(M)}(0)\right]
$$

Thus, we have successfully identified all of the terms depending on $\rho_{I}^{(M+1)}$. 


\subsubsection{Terms involving $M+2$ particles}

Now, we turn to the discussion of the terms which contribute to $\mathcal{D}_{2}\left(t, \rho_{I}^{(M+2)}\right)$. Using equation (B.2) for $S=S^{\prime}=1$ and (B.3), we can identify the term

$$
-\frac{1}{\hbar^{2}} \int_{0}^{t} d s\left[C^{(1)}(t, s),\left[C^{(1)}(s, s), \rho_{I}^{(M)}(s)\right]\right]
$$

with (7.164), (7.168) and one half of (7.167). Moreover, using equations (7.105) and (7.106), we can identify the terms

$$
-\frac{1}{\hbar^{2}} \int_{0}^{t} d s\left[\frac{1}{2} H_{I}^{(2)}(t) \rho_{I}^{(2)}(s) H_{I}^{(2)}(s), \rho_{I}^{(M)}(s)\right]+\text { H.c. }
$$

with (7.163) and (7.166). Furthermore, introducing

$$
D^{(1)}\left(t_{1}, t_{2}, t_{3}\right)=\sum_{i j} \operatorname{Tr}^{(1)}\left\{B_{i j}^{(1)}\left(t_{1}\right) \operatorname{Tr}_{1}^{(2)}\left\{H_{I}^{(2)}\left(t_{2}\right) \rho_{I}^{(2)}\left(t_{3}\right)\right\}\right\} a_{i}^{\dagger} a_{j}
$$

allows us to rewrite (7.165) and the remaining half of (7.167) as

$$
-\frac{1}{\hbar^{2}} \int_{0}^{t} d s\left[D^{(1)}(t, s, s), \rho_{I}^{(M)}(s)\right]+\text { H.c. }
$$

where we find the following property

$$
\begin{aligned}
D^{(1)}\left(t_{1}, t_{2}, t_{3}\right)^{\dagger} & =\sum_{i j} \operatorname{Tr}^{(1)}\left\{\operatorname{Tr}_{1}^{(2)}\left\{H_{I}^{(2)}\left(t_{2}\right) \rho_{I}^{(2)}\left(t_{3}\right)\right\}^{\dagger} B_{j i}^{(1)}\left(t_{1}\right)^{\dagger}\right\} a_{i}^{\dagger} a_{j} \\
& =\sum_{i j} \operatorname{Tr}^{(1)}\left\{B_{i j}^{(1)}\left(t_{1}\right) \operatorname{Tr}_{1}^{(2)}\left\{\rho_{I}^{(2)}\left(t_{3}\right) H_{I}^{(2)}\left(t_{2}\right)\right\}\right\} a_{i}^{\dagger} a_{j} .
\end{aligned}
$$

This identifies all terms depending on $\rho_{I}^{(M+2)}$.

\subsubsection{Terms involving $M+3$ particles}

Finally, the remaining terms in $(7.151)$ are those of $\mathcal{D}_{3}\left(t, \rho_{I}^{(M+3)}\right)$. The only contributions, (7.170) and (7.171), contain the term

$$
\begin{aligned}
E^{(1)}\left(t_{1}, t_{2}, t_{3}\right)= & \frac{1}{2} \sum_{\substack{i_{1} i_{2} j_{1} j_{2} \\
k_{1} k_{2} \\
n m}} H_{n k_{1} ; i_{1} i_{2}}^{(2)}\left(t_{1}\right) \rho_{i_{1} i_{2} k_{2} ; j_{1} j_{2} k_{1}}^{(3)}\left(t_{2}\right) H_{j_{1} j_{2} ; k_{2} m}^{(2)}\left(t_{3}\right) a_{n}^{\dagger} a_{m} \\
= & \frac{1}{2} \sum_{\substack{i_{1} i_{2} j_{1} j_{2} \\
k_{1} k_{2} \\
n m}} H_{n k_{1} ; i_{1} i_{2}}^{(2)}\left(t_{1}\right) \rho_{i_{1} k_{1}}^{(1)}\left(t_{2}\right) \rho_{i_{2} j_{1}}^{(1)}\left(t_{2}\right) H_{j_{1} j_{2} ; k_{2} m}^{(2)}\left(t_{3}\right) \rho_{k_{2} j_{2}}^{(1)}\left(t_{2}\right) a_{n}^{\dagger} a_{m} \\
= & \frac{1}{2} \sum_{\substack{i_{2} j_{1} \\
n m}} C_{n i_{2}}^{(1)}\left(t_{1}, t_{2}\right) \rho_{i_{2} j_{1}}^{(1)}\left(t_{2}\right) C_{j_{1} m}^{(1)}\left(t_{3}, t_{2}\right) a_{n}^{\dagger} a_{m} \\
= & \frac{1}{2} C^{(1)}\left(t_{1}, t_{2}\right) \rho_{I}^{(1)}\left(t_{2}\right) C^{(1)}\left(t_{3}, t_{2}\right)
\end{aligned}
$$


and can be written as

$$
-\frac{1}{\hbar^{2}} \int_{0}^{t} d s\left[E^{(1)}(s, s, t), \rho^{(M)}(s)\right]+\text { H.c. }
$$

To simplify these expressions further, note that, for $\rho=\rho^{\dagger}$,

$$
[L, \rho]+\text { H.c. }=L \rho-\rho L+\rho L^{\dagger}-L^{\dagger} \rho=\left[L-L^{\dagger}, \rho\right] .
$$

Furthermore, we find that for $\tilde{H}=i\left(L-L^{\dagger}\right)$,

$$
\tilde{H}^{\dagger}=\left[i\left(L-L^{\dagger}\right)\right]^{\dagger}=-i\left(L^{\dagger}-L\right)=\tilde{H},
$$

which expresses the fact that $\tilde{H}$ represents a Hermitian operator. Let us first simplify the term (7.182):

$$
\begin{aligned}
& -\frac{1}{\hbar^{2}} \int_{0}^{t} d s\left[D^{(1)}(t, s, s), \rho_{I}^{(M)}(s)\right]+\text { H.c. } \\
= & -\frac{i}{\hbar} \int_{0}^{t} d s\left[-\frac{i}{\hbar}\left(D^{(1)}(t, s, s)-D^{(1)}(t, s, s)^{\dagger}\right), \rho_{I}^{(M)}(s)\right] .
\end{aligned}
$$

Using equation (7.183) yields the effective, single-particle shift Hamiltonian

$$
\begin{aligned}
H_{D}^{(1)}(t, s, s) & =-\frac{i}{\hbar}\left(D^{(1)}(t, s, s)-D^{(1)}(t, s, s)^{\dagger}\right) \\
& =\sum_{i j} \operatorname{Tr}^{(1)}\left\{B_{i j}^{(1)}(t) \operatorname{Tr}_{1}^{(2)}\left\{-\frac{i}{\hbar}\left[H_{I}^{(2)}(s), \rho_{I}^{(2)}(s)\right]\right\}\right\} a_{i}^{\dagger} a_{j} .
\end{aligned}
$$

We can also identify Hermitian operators in each of the other two commutator terms, namely (7.180) and (7.185). We start with (7.180):

$$
\begin{aligned}
& -\frac{1}{\hbar^{2}} \int_{0}^{t} d s\left[\frac{1}{2} H_{I}^{(2)}(t) \rho_{I}^{(2)}(s) H_{I}^{(2)}(s), \rho_{I}^{(M)}(s)\right]+\text { H.c. } \\
= & -\frac{i}{\hbar} \int_{0}^{t} d s\left[-\frac{i}{\hbar} \frac{1}{2}\left(H_{I}^{(2)}(t) \rho_{I}^{(2)}(s) H_{I}^{(2)}(s)-H_{I}^{(2)}(s) \rho_{I}^{(2)}(s) H_{I}^{(2)}(t)\right), \rho_{I}^{(M)}(s)\right],
\end{aligned}
$$

which leads to the two-particle shift Hamiltonian

$$
H_{S}^{(2)}(t, s, s)=-\frac{i}{\hbar} \frac{1}{2}\left(H_{I}^{(2)}(t) \rho_{I}^{(2)}(s) H_{I}^{(2)}(s)-H_{I}^{(2)}(s) \rho_{I}^{(2)}(s) H_{I}^{(2)}(t)\right) .
$$

Finally, (7.185) leads to

$$
\begin{aligned}
& -\frac{1}{\hbar^{2}} \int_{0}^{t} d s\left[E^{(1)}(s, s, t), \rho_{I}^{(M)}(s)\right]+\text { H.c. } \\
= & -\frac{i}{\hbar} \int_{0}^{t} d s\left[-\frac{i}{\hbar}\left(E^{(1)}(s, s, t)-E^{(1)}(s, s, t)^{\dagger}\right), \rho_{I}^{(M)}(s)\right]
\end{aligned}
$$


with the effective single-particle shift Hamiltonian, which we express, using equation (7.184), as

$$
\begin{aligned}
H_{E}^{(1)}(s, s, t) & =-\frac{i}{\hbar}\left(E^{(1)}(s, s, t)-E^{(1)}(s, s, t)^{\dagger}\right) \\
& =-\frac{i}{\hbar} \frac{1}{2}\left(C^{(1)}(s, s) \rho_{I}^{(1)}(s) C^{(1)}(t, s)-C^{(1)}(t, s) \rho_{I}^{(1)}(s) C^{(1)}(s, s)\right) .
\end{aligned}
$$

\subsubsection{General bosonic master equation under the pure product state assumption}

Substituting these expressions into the total reduced time evolution (7.151) yields, after collecting all terms, the following equation, which is exact, except for the pure product state assumption:

$$
\begin{aligned}
& \frac{\partial}{\partial t} \rho_{I}^{(M)}(t) \\
= & -\frac{i}{\hbar}\left[H_{I}^{(2)}(t), \rho_{I}^{(M)}(0)\right]-\frac{1}{\hbar^{2}} \int_{0}^{t} d s\left[H_{I}^{(2)}(t),\left[H_{I}^{(2)}(s), \rho_{I}^{(M)}(s)\right]\right] \\
& -(N-M) \frac{i}{\hbar}\left[C^{(1)}(t, 0), \rho_{I}^{(M)}(0)\right] \\
& -(N-M) \frac{1}{\hbar^{2}} \int_{0}^{t} d s \sum_{\alpha \beta}\left[B_{\beta \alpha}^{(1)}(t),\left[A_{\alpha \beta}^{(1)}(s, s), \rho_{I}^{(M)}(s)\right]\right] \\
& -(N-M) \frac{1}{\hbar^{2}} \int_{0}^{t} d s\left[H_{I}^{(2)}(t),\left[C^{(1)}(s, s), \rho_{I}^{(M)}(s)\right]\right] \\
& -(N-M) \frac{1}{\hbar^{2}} \int_{0}^{t} d s\left[C^{(1)}(t, s),\left[H_{I}^{(2)}(s), \rho_{I}^{(M)}(s)\right]\right] \\
& -(N-M)(N-M-1) \frac{1}{\hbar^{2}} \int_{0}^{t} d s\left[C^{(1)}(t, s),\left[C^{(1)}(s, s), \rho_{I}^{(M)}(s)\right]\right] \\
& -(N-M)(N-M-1) \frac{i}{\hbar} \int_{0}^{t} d s\left[H_{D}^{(1)}(t, s, s), \rho_{I}^{(M)}(s)\right] \\
& -(N-M)(N-M-1) \frac{i}{\hbar} \int_{0}^{t} d s\left[H_{S}^{(2)}(t, s, s), \rho_{I}^{(M)}(s)\right] \\
& -(N-M)(N-M-1)(N-M-2) \frac{i}{\hbar} \int_{0}^{t} d s\left[H_{E}^{(1)}(s, s, t), \rho_{I}^{(M)}(s)\right] .
\end{aligned}
$$

Each of these terms allows for an abstract interpretation of their formal role in the dynamical equation. As was already discussed, (7.192) contributes the coherent part to the reduced dynamics, with the unshifted Hamilton operator $H_{I}^{(2)}$. Consequently, this is the only term which remains when $N=M$, that is, when nothing is traced out. The next term (7.193) represents the initial condition.

The following three terms (7.194), (7.195), and (7.196) all contain decoherence operators, where, in total, one single contribution of $\rho_{I}^{(1)}$ is contained-recall from their respective definitions (7.137) and (7.174) that both $A_{\alpha \beta}^{(1)}$ and $C^{(1)}$ contain one single 
contribution of $\rho^{(1)}$, while, for example $B_{\beta \alpha}$, defined in (7.138), and $H_{I}^{(2)}$ do not depend on $\rho^{(1)}$ at all. This implies that there must be at least one additional particle in the system, which is traced out when considering only the subsystem evolution. All three terms appear in a double-commutator structure, which can be mapped to a Lindblad-type form, as we have seen in equation (7.142). In particular (7.194) only contains single-particle decoherence operators, namely $A_{\alpha \beta}^{(1)}$ and $B_{\beta \alpha}^{(1)}$, and therefore contributes even if $M=1$. In contrast, (7.195) and (7.196) each contain a two-particle decoherence operator, $H_{I}^{(2)}$, and, thus, only appear when $M>1$.

The next set of terms (7.197), (7.198), and (7.199) depend on the quantum state of two additional particles. One contribution of $\rho_{I}^{(1)}$ is contained in each of the two occurrences of $C^{(1)}$, and the effective Hamiltonians $H_{D}^{(1)}$ and $H_{S}^{(2)}$, defined in (7.189) and (7.190), depend on $\rho_{I}^{(2)}$. Therefore these terms can only contribute when one traces over at least two particles. Consequently, the common prefactor vanishes if $N=M+1$. Here, only the first term, (7.197), appears as a double-commutator, while the other two terms are commutators which lead to environment-induced shifts of the local Hamiltonian, similar to the Lamb-shift in a standard system-environment scenario (Breuer and Petruccione, 2002). This is also the case for the very last term (7.200), which is the only term containing a three-particle state, as becomes apparent from (7.191), and therefore vanishes if $M \geq N-2$.

Regrouping terms in the full master equation (7.192)-(7.200) can further simplify the general expression for the bosonic pure product state time evolution:

$$
\begin{aligned}
& \frac{\partial}{\partial t} \rho_{I}^{(M)}(t) \\
= & -\frac{i}{\hbar}\left[H_{I}^{(2)}(t)+(N-M) C^{(1)}(t, 0), \rho_{I}^{(M)}(0)\right] \\
& -\frac{1}{\hbar^{2}} \int_{0}^{t} d s\left[H_{I}^{(2)}(t),\left[H_{I}^{(2)}(s)+(N-M) C^{(1)}(s, s), \rho_{I}^{(M)}(s)\right]\right] \\
& -\frac{1}{\hbar^{2}} \int_{0}^{t} d s\left[(N-M) C^{(1)}(t, s),\left[H_{I}^{(2)}(s)+(N-M-1) C^{(1)}(s, s), \rho_{I}^{(M)}(s)\right]\right] \\
& -(N-M) \frac{1}{\hbar^{2}} \int_{0}^{t} d s \sum_{\alpha \beta}\left[B_{\beta \alpha}^{(1)}(t),\left[A_{\alpha \beta}^{(1)}(s, s), \rho_{I}^{(M)}(s)\right]\right] \\
& -(N-M)(N-M-1) \frac{i}{\hbar} \int_{0}^{t} d s\left[H_{D}^{(1)}(t, s, s), \rho_{I}^{(M)}(s)\right] \\
& -(N-M)(N-M-1) \frac{i}{\hbar} \int_{0}^{t} d s\left[H_{S}^{(2)}(t, s, s), \rho_{I}^{(M)}(s)\right] \\
& -(N-M)(N-M-1)(N-M-2) \frac{i}{\hbar} \int_{0}^{t} d s\left[H_{E}^{(1)}(s, s, t), \rho_{I}^{(M)}(s)\right] .
\end{aligned}
$$




\subsubsection{Single-particle dynamics}

The general expression (7.192)-(7.200) simplifies significantly when we consider special choices of $N$ and $M$. Let us first consider the general single-particle dynamics, $M=1$. In this case, there can be no two-particle operators on the right-hand side of the dynamical equation, since, as we already discussed, the equality signs are valid only after projection onto the respective subspace of fixed particle number. We obtain

$$
\begin{aligned}
\frac{\partial}{\partial t} \rho_{I}^{(1)}(t)= & -(N-1) \frac{i}{\hbar}\left[C^{(1)}(t, 0), \rho_{I}^{(1)}(0)\right] \\
& -(N-1) \frac{1}{\hbar^{2}} \int_{0}^{t} d s \sum_{\alpha \beta}\left[B_{\beta \alpha}^{(1)}(t),\left[A_{\alpha \beta}^{(1)}(s, s), \rho_{I}^{(1)}(s)\right]\right] \\
& -(N-1)(N-2) \frac{1}{\hbar^{2}} \int_{0}^{t} d s\left[C^{(1)}(t, s),\left[C^{(1)}(s, s), \rho_{I}^{(1)}(s)\right]\right] \\
& -(N-1)(N-2) \frac{i}{\hbar} \int_{0}^{t} d s\left[H_{D}^{(1)}(t, s, s), \rho_{I}^{(1)}(s)\right] \\
& -(N-1)(N-2)(N-3) \frac{i}{\hbar} \int_{0}^{t} d s\left[H_{E}^{(1)}(s, s, t), \rho_{I}^{(1)}(s)\right] .
\end{aligned}
$$

If, in addition, we have only two particles in the total system, that is $N=2$, we recover the result derived in equation (7.142),

$$
\frac{\partial}{\partial t} \rho_{I}^{(1)}(t)=-\frac{i}{\hbar}\left[C^{(1)}(t, 0), \rho_{I}^{(1)}(0)\right]-\frac{1}{\hbar^{2}} \int_{0}^{t} d s \sum_{\alpha \beta}\left[B_{\beta \alpha}^{(1)}(t),\left[A_{\alpha \beta}^{(1)}(s, s), \rho_{I}^{(1)}(s)\right]\right]
$$

Here, however, we retain the term depending on the initial condition, which in section 7.5.1 was assumed to be zero.

\subsubsection{Nonlinear Redfield-type equation}

Replacing $\rho_{I}^{(M)}(s)$ with $\rho_{I}^{(M)}(t)$ in the integrands on the right-hand side of $\partial_{t} \rho_{I}^{(M)}(t)$, as given in equation (7.201), yields a second-order local-in-time master equation, similarly to the Redfield equation. However, here we deal with a nonlinear master equation, since some of the operators depend on the state at time $t$ as well. Hence, those time arguments of the operators $A^{(1)}, C^{(1)}, E^{(1)}, D^{(1)}$ that are implicitly time arguments of $\rho_{I}$, must also be adjusted from $s$ to $t$. Consider the example of $C^{(1)}\left(t_{1}, t_{2}\right)$ : Recalling its definition (7.174), we realize that $t_{1}$ describes the time argument of the interactionpicture Hamiltonian $H^{(2)}\left(t_{1}\right)$ while $t_{2}$ determines the evolution of the density matrix $\rho^{(1)}\left(t_{2}\right)$. In the third line of equation (7.202), we thus truncate the expansion via the replacement $C^{(1)}(s, s) \rightarrow C^{(1)}(s, t)$. The same rule applies for the shift Hamiltonians that are constructed as functions of these operators. Additionally, we may substitute 
the integrand $s$ by $t-s$, as in section 7.5.1. This yields

$$
\begin{aligned}
& \frac{\partial}{\partial t} \rho_{I}^{(M)}(t) \\
= & -\frac{i}{\hbar}\left[H^{(2)}(t)+(N-M) C^{(1)}(t, 0), \rho_{I}^{(M)}(0)\right] \\
& -\frac{1}{\hbar^{2}} \int_{0}^{t} d s\left[H_{I}^{(2)}(t),\left[H_{I}^{(2)}(t-s)+(N-M) C^{(1)}(t-s, t), \rho_{I}^{(M)}(t)\right]\right] \\
& -\frac{1}{\hbar^{2}} \int_{0}^{t} d s\left[(N-M) C^{(1)}(t, t),\left[H_{I}^{(2)}(t-s)+(N-M-1) C^{(1)}(t-s, t), \rho_{I}^{(M)}(t)\right]\right] \\
& -(N-M) \frac{1}{\hbar^{2}} \int_{0}^{t} d s \sum_{\alpha \beta}\left[B_{\beta \alpha}^{(1)}(t),\left[A_{\alpha \beta}^{(1)}(t-s, t), \rho_{I}^{(M)}(t)\right]\right] \\
& -(N-M)(N-M-1) \frac{i}{\hbar} \int_{0}^{t} d s\left[H_{D}^{(1)}(t, t-s, t), \rho_{I}^{(M)}(t)\right] \\
& -(N-M)(N-M-1) \frac{i}{\hbar} \int_{0}^{t} d s\left[H_{S}^{(2)}(t, t, t-s), \rho_{I}^{(M)}(t)\right] \\
& -(N-M)(N-M-1)(N-M-2) \frac{i}{\hbar} \int_{0}^{t} d s\left[H_{E}^{(1)}(t-s, t, t), \rho_{I}^{(M)}(t)\right] .
\end{aligned}
$$

Another approximate step towards a tractable master equation would be shifting the integration limit to $\infty$. It is, however, questionable how such an approximation can be justified in a system of identical particles, where the timescales of 'system' and 'environment' are not intrinsically separated. Moreover, we find that, for arbitrarily large systems, the system size only affects the couplings and not the time-autocorrelation functions itself. These are still determined on the basis of the quantum state of few additional particles, with numbers ranging between one and three. Due to the mean-field ansatz, these particles' state is at the same time also the argument of the nonlinear kinetic equation.

\subsubsection{Mean-field approximation}

Instead of employing the above approximations, we recall equation (7.201), which so far only contained the product state assumption. Before truncating the second-order expansion, we focus on the first set of terms: the double commutators in the first three lines. We can find a striking structure, resembling a second-order expansion of a fully coherent evolution, which allows us to identify a well-known mean-field term. To formally establish this, on top of the pure product state assumption, we need to establish one more mean-field approximation, which consists in

- $N-M \gg 1$, that is we assume the total sample to be large compared to the observable subspace, which allows us to replace $N-M-1 \approx N-M$, and

- $C^{(1)}(t, s) \rightarrow C^{(1)}(t, t)$, and $C^{(1)}(t, 0) \rightarrow C^{(1)}(t, t)$, where the term $C^{(1)}$ is defined in the interaction picture in equation (7.174). We thus assume that the 
time dependence of the single-particle state which occurs in the terms $C^{(1)}$, equation (7.174), is synchronized with the Hamiltonian part, which effectively assumes that it becomes part of a time-dependent effective mean-field potential.

To see this, we employ these approximations to rewrite the first terms as follows,

$$
\begin{aligned}
& -\frac{i}{\hbar}\left[H_{I}^{(2)}(t)+(N-M) C^{(1)}(t, t), \rho_{I}^{(M)}(0)\right] \\
& -\frac{1}{\hbar^{2}} \int_{0}^{t} d s\left[H_{I}^{(2)}(t),\left[H_{I}^{(2)}(s)+(N-M) C^{(1)}(s, s), \rho_{I}^{(M)}(s)\right]\right] \\
& -\frac{1}{\hbar^{2}} \int_{0}^{t} d s\left[(N-M) C^{(1)}(t, t),\left[H_{I}^{(2)}(s)+(N-M) C^{(1)}(s, s), \rho_{I}^{(M)}(s)\right]\right] \\
= & -\frac{i}{\hbar}\left[H_{I}^{(2)}(t)+(N-M) C^{(1)}(t, t),\right. \\
& \left.\quad \rho_{I}^{(M)}(0)-\frac{i}{\hbar} \int_{0}^{t} d s\left[H_{I}^{(2)}(s)+(N-M) C^{(1)}(s, s), \rho_{I}^{(M)}(s)\right]\right] \\
\approx & -\frac{i}{\hbar}\left[H_{I}^{(2)}(t)+(N-M) C^{(1)}(t, t), \rho_{I}^{(M)}(t)\right] .
\end{aligned}
$$

In the last step, we have neglected contributions of the other elements of the complete master equation to carry out the integration explicitly.

\subsubsection{Dissipative mean-field master equation for dilute gases}

Let us finally, after implementing the above mean-field approximation, carry out one more approximation, by assuming that the particle density $\varrho=N / V$, where $V$ denotes the volume to which the particles are confined, ${ }^{5}$ is sufficiently low, such that we can neglect terms of the order of $\varrho^{2}$.

Assuming $N \gg M$, the resulting factors $\propto N^{S}$ preceding the commutator terms always occur in combination with $\rho^{(S)}$; recall, for instance the discussion in section 7.5.4. The $S$-particle density matrix $\rho^{(S)}$, in turn, can be considered a quantity of the order of $1 / V^{S}$ (Bogoliubov, 1967a). Hence, these factors will turn into powers of the particle density $\varrho$, and for dilute gases, their contribution can be considered as small when $S>1$. This allows us to ignore those contributions that involve the effective potential of two or more additional particles, as represented by the effective Hamiltonians in last three lines of equation (7.201). After using (7.205) in equation (7.201), followed by a truncation of the expansion as in section 7.5.6 by appropriate substitution of the time arguments, we finally obtain the dissipative mean-field master-equation for dilute gases

$$
\begin{aligned}
\frac{\partial}{\partial t} \rho_{I}^{(M)}(t)= & -\frac{i}{\hbar}\left[H_{I}^{(2)}(t)+(N-M) C^{(1)}(t, t), \rho_{I}^{(M)}(t)\right] \\
& -(N-M) \frac{1}{\hbar^{2}} \int_{0}^{t} d s \sum_{\alpha \beta}\left[B_{\beta \alpha}^{(1)}(t),\left[A_{\alpha \beta}^{(1)}(t-s, t), \rho_{I}^{(M)}(t)\right]\right]
\end{aligned}
$$

\footnotetext{
${ }^{5}$ Not to be confused with the interaction potential $V\left(\mathbf{x}_{1}, \mathbf{x}_{2}\right)$, which always carries the particles' coordinates as argument.
} 
In this form, the first line is given by a commutator term containing the nonlinear, effective mean-field potential, which we already encountered when deriving the hierarchical commutator expansion for $\rho^{(M)}$. In fact, this commutator term exactly coincides with the mean-field truncated hierarchy expression (7.121) in the interaction picture. As was shown in section 7.4.4, in the case of a weakly interacting Bose-Einstein condensate, this mean-field term is responsible for the characteristic nonlinear term in the effective single-particle potential. The incoherent correction, the second line, is given by the term which was previously studied in the two-particle case; see equation (7.142). We already encountered this contribution in the special case studied in section 7.5.1, since it describes the time-autocorrelation functions of only one additional particle. The second term in equation (7.206), thus, contains the dissipative, second-order correction to the first-order mean-field evolution, which was shown to reproduce the Gross-Pitaevskii equation (7.127) in the special case of a contact interaction potential in section 7.4.4.

We, however, need to stress that the approximations employed in this last section are rather hand-wavy than mathematically rigorous. In fact, the three approximations (mean-field, low-density, and truncation by replacement of time arguments) do not commute with each other. Moreover, since the pure product state assumption essentially decomposes the $M$-particle state into single-particle states, it is unclear, whether the above expression, in its present form, can lead to physical insight into the $M$-particle dynamics $(M>1)$ beyond what is already contained in the singleparticle dynamics. One may further be interested to transform the equation back into the Schrödinger picture, for example, to facilitate direct comparison with the truncated mean-field hierarchy equation (7.116). The necessary transformation rules are summarized in appendix $\mathrm{C}$.

\subsection{Discussion}

In this chapter, we have developed a general formalism to deal with quantum manyparticle systems, by employing a basis of symmetrized states to account for the particles' quantum statistics. This was applied to establish analogs of the reduced density matrix and of the partial trace operation for systems of identical bosonic particles, where the reduced observables of an $N$-particle system are operators which depend on a smaller number of $M$ particles. Next, we carried out the partial trace over the von Neumann equation to determine the time evolution of the reduced $M$-particle density operator in the presence of particle-particle interactions. This led to a hierarchical expansion, in terms of the dynamics of the $(M+1)$-particle density matrix. This hierarchy can be truncated by the pure product state assumption, which implies that all $N$ particles are characterized - at all times - by the same pure single-particle state. Then, the resulting coherent mean-field evolution is described by a nonlinear effective potential, and application to the case of a weakly interacting Bose-Einstein condensate reproduces the Gross-Pitaevskii equation.

The coherent mean-field evolution has the drawback that it is incapable of describing incoherent, dissipative effects, which manifest on the level of observables on a subset of 
particles, in the presence of particle-particle interactions. To overcome this limitation, we expanded the von Neumann equation to second order in the interaction term before performing the partial trace operation, analogous to the microscopic treatment of standard open systems which couple to an environment. Yet, the indistinguishability of bosons complicates the description of the entire many-particle system on the basis of the reduced state of one or few particles. This forces us to treat the incoherent master equation within a pure-state mean-field approach to obtain a closed description of the time evolution. We obtain an incoherent mean-field master equation, which contains dissipation operators in the general Lindblad shape of a time-local master equation, on top of a coherent part which is familiar from the previous (first-order) ansatz, and amended by further shift Hamiltonians, which, in turn, involve more than one additional particle in the environment.

While the first-order results which generate the hierarchical expansion have a rather clear physical interpretation, and reduce to well-known results in certain special cases, the second-order expansion, outlined in section 7.5, must be considered as work in progress. At the present stage of this work, the exact interpretation of the decay terms, besides the intuition given by their Lindblad structure, and of the effective nonlinear Hamiltonians, remains unclear. Being forced to employ the pure product state assumption also limits the applicability of the result to the description of decoherence: Despite achieving an effective description in from of a Lindblad-type master equation, we are formally limited to the application to pure states, and, at this point, it remains unclear how a generalization to mixed states may be achieved or motivated. Possible solutions could be stochastic Monte-Carlo methods, which are able to describe decoherence using pure states. Another more sophisticated approach to the microscopic derivation of a master equation could be the adaption of projection-operator techniques to the identical-particle scenario (Breuer and Petruccione, 2002). Further work is also required for the development of reasonable, well-controlled approximations, as well as for numerical studies of example systems. The application to Bloch-oscillations in a tilted Bose-Hubbard model, with an interaction-induced, incoherent decay, would represent a key example for which the present formalism could be beneficial. Studies of a wide range of examples are expected to be possible since the results of the present section were derived for the most general many-body Hamiltonian of a single bosonic species with pairwise interactions.

We expect that a significant generalization of the present ansatz can be achieved by abandoning the restriction to any $M$-particle subspace, and, instead, allow for contributions of reduced density matrices of different particle numbers. This could be achieved via an ansatz of the form $\rho=\sum_{i=1}^{N} \rho^{(i)}$. Such a generalized description is not only formally more appealing, it is also expected to better exploit the full potential of the formalism of second quantization, by including processes that map between subspaces of different particle numbers. This would further allow for the microscopic description of incoherent particle loss or gain (Hunn et al., 2013), as generated, for example, by tunneling processes. Alternatively, by working directly in the Heisenberg picture, it may be possible to describe the evolution of an $M$-particle observable, without necessarily distinguishing between different $N$-particle density matrices. 



\section{Summary AND CONCLUSIONS}

In this dissertation we developed theoretical methods for probing composite quantum systems with feasible experimental overheads. In some cases, these were complemented by experimental realizations. Moreover, we have derived descriptions of the dynamics and spectral features of many-particle open quantum systems, with applications ranging from trapped-ion quantum registers to many-body systems of interacting identical quantum particles.

In the first part of this dissertation, we developed a method which allows to detect and to quantify bipartite discord-type correlations with local operations on a single subsystem, as well as demonstrated experimental realizations thereof with ions and single photons. Our strategy consisted in restricting to the control of a smaller, lowdimensional subsystem, which is experimentally feasible, and promises to represent a scalable approach to the characterization of correlations in the light of the exponential increase of the Hilbert space dimension with the number of interacting particles. These correlations are relevant in certain quantum information protocols. To detect them we make use of the fact that they also significantly influence the local dynamics of the accessible subsystem. This allows us, for example, to observe the single-spin signatures of the ground-state quantum correlations in one-dimensional spin-chain models with a transverse field.

To fully understand the local dynamics, it is often necessary to consider the entire excitation spectrum of the total system, which for large, complex systems may be hard to handle theoretically. In the case of the family of spin-chain models, we developed a semiclassical, multi-configurational mean-field approach to approximate the quantum spectrum, which in certain limiting cases provided exact results. The resulting semiclassical energy landscapes further provided an intuitive picture about the nature of the quantum phase transition between different magnetic phases, which applied to the entire excitation spectrum, instead of only the ground state. An interesting open question in this context is to which extent similar descriptions apply to other quantum phase transitions, and whether this observation, which was made for a large class of 
spin-chain models, represents a universal feature. We suspect that in general, quantum phase transitions do not only represent dramatic changes of the ground-state properties, but are also generically accompanied by changes of the statistical properties of the entire excitation spectrum.

To probe spectral and dynamical features of quantum optical systems, we considered sequences of phase coherent pulses to realize nonlinear spectroscopic measurement schemes. Single-site addressability provides a unique feature of artificial quantum systems, which can extend the possibilities of conventional optical nonlinear spectroscopy. This feature is, however, not a necessary prerequisite to benefit from multidimensional spectroscopy in a quantum optical scenario. In this dissertation, we have established a framework which allows to design nonlinear measurement protocols of controllable quantum systems, and we have discussed elementary steps for an experimental realization with systems of trapped ions. Theoretical studies of examples involving both the electronic and motional degree of freedom of a chain of ions demonstrated that energy levels, internal couplings and environmental processes can be identified and distinguished efficiently with these methods. In the future, the developed set of tools may be harnessed for the clever design of novel probing methods of controllable quantum systems beyond the possibilities of today's standard tools, which are mostly based on two-pulse Ramsey sequences. This is important for the scalable and reliable experimental characterization of quantum simulators which have escaped the realm of numerical simulations on classical computers.

An understanding of decoherence processes and environmental influences on the controllable quantum system is particularly important to ensure coherent quantum evolutions by avoiding or correcting the errors induced by detrimental noise sources. For ion-trap quantum computations, fluctuating, spatially homogeneous control fields produce one of the dominant error sources. We provided an analytical description of the resulting dynamics on a multipartite system for arbitrary frequency distributions, and used this to study the impact of the noise on the atomic correlations. This process was shown to be able to generate strongly correlated quantum states of nonzero discord. These are beyond the reach of local operations, which was specified by introducing the correlation rank as a complementary measure of total correlations. Moreover, we were able to identify sets of states with robust, and even time-invariant entanglement properties, which, in the multipartite case, so far, are not fully characterized and understood.

In the case of identical quantum particles, one can no longer put a label on the constituents and perform experiments on only a specific subset of them. Instead, many experiments effectively measure single-particle observables, reflecting the average of all the indistinguishable particles in an ensemble. Such observables are fully determined by suitably defined reduced quantum states. We have also discussed the time evolution of these few-particle distributions in the presence of interactions. The dynamics is governed by a hierarchical expansion which can be truncated employing a meanfield ansatz. In this case, this leads to a nonlinear coherent evolution in an effective potential, such as the Gross-Pitaevskii equation in the case of a weakly-interacting Bose-Einstein condensate. We further discussed how the provided ansatz may be able to describe incoherent effects by means of an evolution which displays the charac- 
teristic Lindblad structure of time-local master equations, which was microscopically derived. Appropriate extensions thereof may be able to provide an intuitive effective description of interaction-induced decoherence phenomena, which were observed, for example, in experiments with cold atoms in tilted optical lattices.

In conclusion, the characterization of increasingly complex quantum optical systems constitutes an important challenge for both theory and experiment. Based on the results of chapter 3 , the bipartite correlation properties of the underlying quantum states are, under rather general circumstances, accessible, if only one of two parties can be measured, which allows to reduce the size of the controllable subsystem to a manageable level. There remain few intriguing, unexplained effects, regarding the correlation properties of quantum states, such as time-invariant multipartite entanglement under collective dephasing - described in chapter 4. Otherwise, the correlations of quantum states have been studied extensively for quantum information purposes, and, at least on a fundamental level, they are rather well understood, despite the lack of analytic expressions for correlation quantifiers.

To study and characterize complex dynamics, higher-order time- or frequency autocorrelation functions usually define appropriate observables. These can be measured by phase-coherent multi-pulse sequences, as detailed in chapter 6 . Such pulse schemes are experimentally within reach for state-of-the-art quantum optical systems, and efficiently tailored, appropriate measurement protocols are expected to hold great potential for future applications to study more and more complex quantum systems of trapped ions or cold neutral atoms. Multidimensional spectra are particularly sensitive to transport properties and particle interactions, and they often carry a clear signature of the system's microscopic order (Schlawin et al., 2014; Gessner et al., 2015). Nonlinear spectroscopy may therefore be useful for the characterization of hitherto elusive quantum phases which are characterized by the interplay of interactions and the absence of transport due to disordered potentials (Basko et al., 2006).

These experimentally motivated, dynamical approaches to quantum systems need to be complemented by progress on the theoretical understanding of interacting quantum many-body systems. The multi-configurational mean-field approach, introduced in chapter 5, leads to good agreement with the exact quantum spectrum of quantum spin chains, especially in the regime of long-range interactions, and far away from the critical point (where it becomes exact). In the short-range limit, however, its applicability is limited. Spin models with short-range interactions, on the other hand, often allow for exact analytical, or efficient numerical treatments, and thus are usually already rather well understood. It is presently unclear, whether this ansatz can be modified to deliver reliable predictions for the quantum phase transition in the notoriously complicated regime of intermediate-range interactions. The application to higher-dimensional lattice geometries is expected to deliver interesting results, since the number of neighbors is increased, which generally improves the performance of mean-field approaches, while these models often defy efficient numerical and analytical descriptions. The semiclassical picture implicit to the presented mean-field ansatz is reminiscent of commonly employed analogies between phase transitions and double-well potentials - except, here, it involves the entire excitation spectrum. The connection to level clusterings within the excited states (excited-state quantum phase 
transitions) seems apparent, but also requires further analysis, especially in the limit of large particle numbers.

While the above-mentioned, static mean-field ansatz can, for certain parameter ranges, make analytical predictions about the system's spectral features, its capabilities to predict the dynamics are yet to be explored. Describing dynamical manybody systems becomes even harder, when we explicitly include the particles' quantum statistics. The dynamical mean-field approach, pursued for the dynamics of interacting bosons in chapter 7 opens up a promising route towards the derivation of the effective description of the quantum dynamics of interacting many-body systems, which has been and remains a long-standing challenge of the fields of quantum optics and solid state physics. 


\section{A Derivation of THE BOSONIC MASTER EQUATION (7.151)}

In the following we present the derivation of the second-order expression (7.151), where we also elucidate the technical details of the relevant intermediate steps. The starting point of this derivation is the expansion (7.150).

\section{A.1 First term}

To simplify the first term under the integral in equation (7.150), we first use equation (7.51) and then equation (7.50):

$$
\begin{aligned}
& \operatorname{Tr}_{N-M}^{(N)}\left\{H_{I}^{(2)}(s) \rho_{I}^{(N)}(s) H_{I}^{(2)}(t)\right\} \\
& =\frac{1}{M !} \sum_{\substack{n_{1} \ldots n_{N} \\
m_{1} \ldots m_{N}}}\left\langle\varphi_{n_{1}} \ldots \varphi_{n_{N}}\left|H_{I}^{(2)}(s) \rho_{I}^{(N)}(s) H_{I}^{(2)}(t)\right| \varphi_{m_{1}} \ldots \varphi_{m_{N}}\right\rangle \\
& \times \delta_{m_{M+1} \ldots m_{N} ; n_{M+1} \ldots n_{N}} a_{n_{1}}^{\dagger} \ldots a_{n_{M}}^{\dagger} a_{m_{1}} \ldots a_{m_{M}} \\
& =\frac{1}{M !} \sum_{\substack{n_{1} \ldots n_{N} \\
m_{1} \ldots m_{N}}} \sum_{\substack{i_{1} \ldots i_{N} \\
j_{1} \ldots j_{N}}} \rho_{i_{1} \ldots i_{N} ; j_{1} \ldots j_{N}}^{(N)}(s)\left\langle\varphi_{n_{1}} \ldots \varphi_{n_{N}}\left|H_{I}^{(2)}(s)\right| \varphi_{i_{1}} \ldots \varphi_{i_{N}}\right\rangle \\
& \times\left\langle\varphi_{j_{1}} \ldots \varphi_{j_{N}}\left|H_{I}^{(2)}(t)\right| \varphi_{m_{1}} \ldots \varphi_{m_{N}}\right\rangle \delta_{m_{M+1} \ldots m_{N} ; n_{M+1} \ldots n_{N}} a_{n_{1}}^{\dagger} \ldots a_{n_{M}}^{\dagger} a_{m_{1}} \ldots a_{m_{M}} \\
& =\frac{1}{M !} \sum_{\substack{n_{1} \ldots n_{N} \\
m_{1} \ldots m_{N}}} \sum_{\substack{i_{1} \ldots i_{N} \\
j_{1} \ldots j_{N}}} \sum_{\substack{1 \leq \alpha_{1}<\alpha_{2} \leq N \\
1 \leq \beta_{1}<\beta_{2} \leq N}} \rho_{i_{1} \ldots i_{N} ; j_{1} \ldots j_{N}}^{(N)}(s) H_{n_{\alpha_{1}} n_{\alpha_{2} ; i_{1} i_{2}}}^{(2)}(s) H_{j_{1} j_{2} ; m_{\beta_{1}} m_{\beta_{2}}}^{(2)}(t) \\
& \times \delta_{m_{M+1} \ldots m_{N} ; n_{M+1} \ldots n_{N}} \delta_{\left\{i_{3} \ldots i_{N}\right\} ;\left\{n_{1} \ldots n_{N}\right\} \backslash\left\{n_{\alpha_{1}}, n_{\alpha_{2}}\right\}} \delta_{\left\{j_{3} \ldots j_{N}\right\} ;\left\{m_{1} \ldots m_{N}\right\} \backslash\left\{m_{\beta_{1}}, m_{\beta_{2}}\right\}} \\
& \times a_{n_{1}}^{\dagger} \ldots a_{n_{M}}^{\dagger} a_{m_{1}} \ldots a_{m_{M}}
\end{aligned}
$$




$$
\begin{aligned}
= & \frac{1}{M !} \sum_{\substack{n_{1} \ldots n_{N} \\
m_{1} \ldots m_{N}}} \sum_{i_{1} i_{2} j_{1} j_{2}} \sum_{\substack{1 \leq \alpha_{1}<\alpha_{2} \leq N \\
1 \leq \beta_{1}<\beta_{2} \leq N}} \rho_{i_{1} i_{2}\left\{n_{1} \ldots n_{N}\right\} \backslash\left\{n_{\alpha_{1}}, n_{\alpha_{2}}\right\} ; j_{1} j_{2}\left\{m_{1} \ldots m_{N}\right\} \backslash\left\{m_{\beta_{1}}, m_{\beta_{2}}\right\}}^{(N)}(s) \\
& \times H_{n_{\alpha_{1}} n_{\alpha_{2} ; i_{1} i_{2}}}^{(2)}(s) H_{j_{1} j_{2} ; m_{\beta_{1}} m_{\beta_{2}}}^{(2)}(t) \delta_{m_{M+1} \ldots m_{N} ; n_{M+1} \ldots n_{N}} a_{n_{1}}^{\dagger} \ldots a_{n_{M}}^{\dagger} a_{m_{1}} \ldots a_{m_{M}}
\end{aligned}
$$

If we pay close attention to the set $\left\{\alpha_{1}, \alpha_{2}\right\}$, we realize that there are only three classes of pairs with different results. These depend on whether $\alpha_{1}, \alpha_{2}$ are picked from the set $1, \ldots, M$ or from $M+1, \ldots, N$. The same holds for the set $\left\{\beta_{1}, \beta_{2}\right\}$. We use Lemma 9 to write out all terms, grouping the three contributions generated by the sums over $\alpha_{1}$ and $\alpha_{2}$, according to the respective values of $\beta_{1}$ and $\beta_{2}$.

$$
\operatorname{Tr}_{N-M}^{(N)}\left\{H^{(2)}(s) \rho^{(N)}(s) H^{(2)}(t)\right\}=\frac{1}{M !} \sum_{\substack{n_{1} \ldots n_{M} \\ m_{1} \ldots m_{M}}} \sum_{\substack{i_{1} i_{2} \\ j_{1} j_{2}}}[
$$

- Terms with $\beta_{1}, \beta_{2} \leq M$ :

$$
\begin{gathered}
\left(\begin{array}{c}
M \\
2
\end{array}\right)^{2} \rho_{i_{1} i_{2} n_{3} \ldots n_{M} ; j_{1} j_{2} m_{3} \ldots m_{M}}^{(M)}(s) H_{n_{1} n_{2} ; i_{1} i_{2}}^{(2)}(s) H_{j_{1} j_{2} ; m_{1} m_{2}}^{(2)}(t) \\
+\sum_{n_{M+1}}\left(\begin{array}{c}
M \\
2
\end{array}\right) M(N-M) \rho_{i_{1} i_{2} n_{2} \ldots n_{M} ; j_{1} j_{2} m_{3} \ldots m_{M} n_{M+1}}^{(M+1)}(s) \\
\times H_{n_{1} n_{M+1} ; i_{1} i_{2}}^{(2)}(s) H_{j_{1} j_{2} ; m_{1} m_{2}}^{(2)}(t) \\
+\sum_{\substack{n_{M+2} \\
n_{M+2}}}\left(\begin{array}{c}
M \\
2
\end{array}\right)\left(\begin{array}{c}
N-M \\
2
\end{array}\right) \rho_{i_{1} i_{2} n_{1} \ldots n_{M} ; j_{1} j_{2} m_{3} \ldots m_{M} n_{M+1} n_{M+2}}^{(M++2)}(s) \\
\times H_{n_{M+1} n_{M+2} ; i_{1} i_{2}}^{(2)}(s) H_{j_{1} j_{2} ; m_{1} m_{2}}^{(2)}(t)
\end{gathered}
$$

- Terms with $\beta_{1} \leq M, \beta_{2}>M$ :

$$
\begin{aligned}
& +\sum_{m_{M+1}}\left(\begin{array}{c}
M \\
2
\end{array}\right) M(N-M) \rho_{i_{1} i_{2} n_{3} \ldots n_{M} m_{M+1} ; j_{1} j_{2} m_{2} \ldots m_{M}}^{(M+1)}(s) \\
& \quad \times H_{n_{1} n_{2} ; i_{1} i_{2}}^{(2)}(s) H_{j_{1} j_{2} ; m_{1} m_{M+1}}^{(2)}(t) \\
& +\sum_{\substack{n_{M+1 \ldots} \ldots n_{N} \\
m_{M+1} \ldots m_{N}}} M^{2}(N-M)^{2} \rho_{i_{1} i_{2} n_{2} \ldots n_{M}}^{(N)} n_{M+2} \ldots n_{N} ; j_{1} j_{2} m_{2} \ldots m_{M} m_{M+2} \ldots m_{N} \\
& \quad \times H_{n_{1} n_{M+1} ; i_{1} i_{2}}^{(2)}(s) H_{j_{1} j_{2} ; m_{1} m_{M+1}}^{(2)}(t) \delta_{m_{M+1} \ldots m_{N} ; n_{M+1} \ldots n_{N}} \\
& +\sum_{\substack{n_{M+1} \ldots n_{N} \\
m_{M+1} \ldots m_{N}}} M(N-M)\left(\begin{array}{c}
N-M \\
2
\end{array}\right) \rho_{i_{1} i_{2} n_{1} \ldots n_{M} n_{M+3} \ldots n_{N} ; j_{1} j_{2} m_{2} \ldots m_{M} m_{M+2} \ldots m_{N}}^{(N)}(s) \\
& \quad \times H_{n_{M+1} n_{M+2} ; i_{1} i_{2}}^{(2)}(s) H_{j_{1} j_{2} ; m_{1} m_{M+1}}^{(2)}(t) \delta_{m_{M+1} \ldots m_{N} ; n_{M+1} \ldots n_{N}}
\end{aligned}
$$


- Terms with $\beta_{1}, \beta_{2}>M$ :

$$
\begin{aligned}
&+\sum_{m_{M+1}}\left(\begin{array}{c}
M \\
m_{M+2}
\end{array}\right)\left(\begin{array}{c}
N-M \\
2
\end{array}\right) \rho_{i_{1} i_{2} n_{3} \ldots n_{M} m_{M+1} m_{M+2} ; j_{1} j_{2} m_{1} \ldots m_{M}}^{(M+2)}(s) \\
& \times H_{n_{1} n_{2} ; i_{1} i_{2}}^{(2)}(s) H_{j_{1} j_{2} ; m_{M+1} m_{M+2}}^{(2)}(t) \\
&+\sum_{\substack{n_{M+1} \ldots n_{N} \\
m_{M+1} \ldots m_{N}}} M(N-M)\left(\begin{array}{c}
N-M \\
2
\end{array}\right) \rho_{i_{1} i_{2} n_{2} \ldots n_{M} n_{M+2} \ldots n_{N} ; j_{1} j_{2} m_{1} \ldots m_{M} m_{M+3} \ldots, m_{N}}^{(N)}(s) \\
& \quad \times H_{n_{1} n_{M+1} ; i_{1} i_{2}}^{(2)}(s) H_{j_{1} j_{2} ; m_{M+1} m_{M+2}}^{(2)}(t) \delta_{m_{M+1} \ldots m_{N} ; n_{M+1} \ldots n_{N}} \\
&+\sum_{n_{M+1} \ldots n_{N}}\left(\begin{array}{c}
N-M \\
m_{M+1} \ldots m_{N}
\end{array}\right) \rho_{i_{1} i_{2} n_{1} \ldots n_{M} n_{M+3} \ldots n_{N} ; j_{1} j_{2} m_{1} \ldots m_{M} m_{M+3} \ldots m_{N}}^{(N)}(s) \\
&\left.\quad \times H_{n_{M+1} n_{M+2} ; i_{1} i_{2}}^{(2)}(s) H_{j_{1} j_{2} ; m_{M+1} m_{M+2}}^{(2)}(t) \delta_{m_{M+1} \ldots m_{N} ; n_{M+1} \ldots n_{N}}\right] \\
& \quad \times a_{n_{1}}^{\dagger} \ldots a_{n_{M}}^{\dagger} a_{m_{1}} \ldots a_{m_{M}},
\end{aligned}
$$

where we have used equation (7.53) to introduce the elements of the reduced density matrices whenever it was immediately possible. There remain four terms which cannot be identified before decomposing the symmetric $\delta$-functions into groups of permutations. In order to sort out the different permutations we need the following

Lemma 10. The following statements hold for permutations of $(1, \ldots, r)$

a) There are $s(r-1)$ ! permutations of $(1, \ldots, r)$ which map the first element onto one of the first s spots and $(r-s)(r-1)$ ! which do not.

b) There are $s(s-1)(r-2)$ ! permutations of $(1, \ldots, r)$ which map the first two elements onto the first s spots.

c) There are $2 s(r-s)(r-2)$ ! permutations of $(1, \ldots, r)$ which map either the first or the second element onto one of the first s spots.

d) There are $(r-s)(r-s-1)(r-2)$ ! permutations of $(1, \ldots, r)$ which map neither one of the first two elements onto the first $s$ spots.

First, we consider the second term in equation (A.4). The expression is invariant under permutation of the subsets $n_{M+2} \ldots n_{N}$ and $m_{M+2} \ldots m_{N}$. We have to treat the permutations within the $\delta$-function which map $n_{M+1}$ onto $m_{M+1}$ separately, since, in this case, the two Hamiltonians are contracted with each other, while in all other cases, they contract with $\rho^{(N)}$. According to Lemma 10 a) with $r=N-M$ and $s=1$, 
we obtain

$$
\begin{aligned}
& \sum_{\substack{n_{M+1} \ldots n_{N} \\
m_{M+1} \ldots m_{N}}} M^{2}(N-M)^{2} \rho_{i_{1} i_{2} n_{2} \ldots n_{M} n_{M+2} \ldots n_{N} ; j_{1} j_{2} m_{2} \ldots m_{M} m_{M+2} \ldots m_{N}}^{(N)}(s) \\
& \times H_{n_{1} n_{M+1} ; i_{1} i_{2}}^{(2)}(s) H_{j_{1} j_{2} ; m_{1} m_{M+1}}^{(2)}(t) \delta_{m_{M+1} \ldots m_{N} ; n_{M+1} \ldots n_{N}} \\
& =\sum_{n_{M+1}} \underbrace{M^{2}(N-M)^{2} \frac{(N-M-1) !}{(N-M) !}}_{M^{2}(N-M)} \rho_{i_{1} i_{2} n_{2} \ldots n_{M} ; j_{1} j_{2} m_{2} \ldots m_{M}}^{(M+1)}(s) \\
& \times H_{n_{1} n_{M+1} ; i_{1} i_{2}}^{(2)}(s) H_{j_{1} j_{2} ; m_{1} n_{M+1}}^{(2)}(t) \\
& +\sum_{n_{M+1}} \underbrace{M^{2}(N-M)^{2} \frac{(N-M-1)(N-M-1) !}{(N-M) !}}_{n^{2}(N-M)(N-M-1)} \rho_{i_{1} i_{2} n_{2} \ldots n_{M} n_{M+2} ; j_{1} j_{2} m_{2} \ldots m_{M} n_{M+1}}^{(M+2)}(s) \\
& \times H_{n_{1} n_{M+1} ; i_{1} i_{2}}^{(2)}(s) H_{j_{1} j_{2} ; m_{1} n_{M+2}}^{(2)}(t) .
\end{aligned}
$$

Next we deal with the third term in (A.4). Here, we differentiate between the cases where $m_{M+1}$ is mapped onto one of the states $n_{M+1}, n_{M+2}$ or not. We use Lemma 10 a) with $r=N-M$ and $s=2$ to obtain

$$
\begin{aligned}
\sum_{\substack{n_{M+1} \ldots n_{N} \\
m_{M+1} \ldots m_{N}}} M(N-M)\left(\begin{array}{c}
N-M \\
2
\end{array}\right) \rho_{i_{1} i_{2} n_{1} \ldots n_{M} n_{M+3} \ldots n_{N} ; j_{1} j_{2} m_{2} \ldots m_{M} m_{M+2} \ldots m_{N}}^{(N)}(s) \\
\times H_{n_{M+1} n_{M+2} ; i_{1} i_{2}}^{(2)}(s) H_{j_{1} j_{2} ; m_{1} m_{M+1}}^{(2)}(t) \delta_{m_{M+1} \ldots m_{N} ; n_{M+1} \ldots n_{N}} \\
=\sum_{n_{M+1}} \underbrace{M(N-M)\left(\begin{array}{c}
N-M \\
2
\end{array}\right) \frac{2(N-M-1) !}{(N-M) !} \rho_{i_{1} i_{2} n_{1} \ldots n_{M} ; j_{1} j_{2} m_{2} \ldots m_{M} n_{M+2}}^{(M+2)}(s)}_{M(N-M)(N-M-1)} \\
+\sum_{n_{M+1}} \underbrace{M(N-M)\left(\begin{array}{c}
N-M \\
2
\end{array}\right) \frac{(N-M-2)(N-M-1) !}{(N-M) !}}_{n_{M+2}} \\
\quad \times \rho_{n_{M+1} n_{M+2} ; i_{1} i_{2}(s) H_{j_{1} j_{2} ; m_{1} n_{M+1}}^{(2)}(t)}^{M(N-M)(N-M)}
\end{aligned}
$$


Now we simplify the second term in (A.5). We have to distinguish the same possibilities as for the previous term. We obtain

$$
\begin{aligned}
& \sum_{\substack{n_{M+1} \ldots n_{N} \\
m_{M+1} \ldots m_{N}}} M(N-M)\left(\begin{array}{c}
N-M \\
2
\end{array}\right) \rho_{i_{1} i_{2} n_{2} \ldots n_{M} n_{M+2} \ldots n_{N} ; j_{1} j_{2} m_{1} \ldots m_{M} m_{M+3} \ldots, m_{N}}^{(N)}(s) \\
& \times H_{n_{1} n_{M+1} ; i_{1} i_{2}}^{(2)}(s) H_{j_{1} j_{2} ; m_{M+1} m_{M+2}}^{(2)}(t) \delta_{m_{M+1} \ldots m_{N} ; n_{M+1} \ldots n_{N}} \\
& =\sum_{n_{M+1}} \underbrace{M(N-M)\left(\begin{array}{c}
N-M \\
n_{M+2}
\end{array}\right) \frac{2(N-M-1) !}{(N-M) !}}_{M(N-M)(N-M-1)} \rho_{i_{1} i_{2} n_{2} \ldots n_{M} n_{M+2} ; j_{1} j_{2} m_{1} \ldots m_{M}}^{(M+2)}(s) \\
& \times H_{n_{1} n_{M+1} ; i_{1} i_{2}}^{(2)}(s) H_{j_{1} j_{2} ; n_{M+1} n_{M+2}}^{(2)}(t) \\
& +\sum_{\substack{n_{M+1} \\
n_{M+2} \\
n_{M+3}}}^{M(N-M)\left(\begin{array}{c}
N-M \\
2
\end{array}\right) \frac{(N-M-2)(N-M-1) !}{(N-M) !}} \\
& \times \rho_{i_{1} i_{2} n_{2} \ldots n_{M} n_{M+2} n_{M+3} ; j_{1} j_{2} m_{1} \ldots m_{M} n_{M+1}}^{(M+3)}(s) H_{n_{1} n_{M+1} ; i_{1} i_{2}}^{(2)}(s) H_{j_{1} j_{2} ; n_{M+2} n_{M+3}}^{(2)}(t) .
\end{aligned}
$$

Finally, for the last term of (A.5), the three classes of permutations which lead to different results depend on the number of elements out of $n_{M+1}$ and $n_{M+2}$ that are mapped onto $m_{M+1}$ and $m_{M+2}$. This can be any number between zero and two. Using Lemma 10 b), c) and $d$ ), with $r=N-M$ and $s=2$, we get:

$$
\begin{aligned}
& \sum_{\substack{n_{M+1} \ldots n_{N} \\
m_{M+1} \ldots m_{N}}}\left(\begin{array}{c}
N-M \\
2
\end{array}\right)^{2} \rho_{i_{1} i_{2} n_{1} \ldots n_{M} n_{M+3} \ldots n_{N} ; j_{1} j_{2} m_{1} \ldots m_{M} m_{M+3} \ldots m_{N}}(s) \\
& \times H_{n_{M+1} n_{M+2} ; i_{1} i_{2}}^{(2)}(s) H_{j_{1} j_{2} ; m_{M+1} m_{M+2}}^{(2)}(t) \delta_{m_{M+1} \ldots m_{N} ; n_{M+1} \ldots n_{N}} \\
& =\sum_{n_{M+1}} \underbrace{\left(\begin{array}{c}
N-M \\
2
\end{array}\right)^{2} \frac{2(N-M-2) !}{(N-M) !}}_{\frac{1}{2}(N-M)(N-M-1)} \rho_{i_{1} i_{2} n_{1} \ldots n_{M} ; j_{1} j_{2} m_{1} \ldots m_{M}}^{(M+2)}(s) \\
& \times H_{n_{M+1} n_{M+2} ; i_{1} i_{2}}^{(2)}(s) H_{j_{1} j_{2} ; n_{M+1} n_{M+2}}^{(2)}(t) \\
& +\sum_{\substack{n_{M+1}+2 \\
n_{M}+2}} \underbrace{\left(\begin{array}{c}
N-M \\
2
\end{array}\right)^{2} \frac{4(N-M-2)(N-M-2) !}{(N-M) !}}_{(N-M)(N-M-1)(N-M-2)} \rho_{i_{1} i_{2} n_{1} \ldots n_{M} n_{M+3} ; j_{1} j_{2} m_{1} \ldots m_{M} n_{M+2}}^{(M+3)}(s) \\
& \times H_{n_{M+1} n_{M+2} ; i_{1} i_{2}}^{(2)}(s) H_{j_{1} j_{2} ; n_{M+1} n_{M+3}}^{(2)}(t) \\
& +\sum_{n_{M+1}} \underbrace{\left(\begin{array}{c}
N-M \\
2
\end{array}\right)^{2} \frac{(N-M-2)(N-M-3)(N-M-2) !}{(N-M) !}}_{\frac{1}{4}(N-M)(N-M-1)(N-M-2)(N-M-3)} \\
& \times \rho_{i_{1} i_{2} n_{1} \ldots n_{M} n_{M+3} n_{M+4} ; j_{1} j_{2} m_{1} \ldots m_{M} n_{M+1} n_{M+2}}^{(M+4)}(s) H_{n_{M+1} n_{M+2} ; i_{1} i_{2}}^{(2)}(s) H_{j_{1} j_{2} ; n_{M+3} n_{M+4}}^{(2)}(t) .
\end{aligned}
$$




\section{A.2 Second term}

For the second term under the integral in equation (7.150) we obtain, employing the same techniques as before,

$$
\begin{aligned}
& \operatorname{Tr}_{N-M}^{(N)}\left\{H^{(2)}(t) H^{(2)}(s) \rho^{(N)}(s)\right\} \\
& =\frac{1}{M !} \sum_{\substack{n_{1} \ldots n_{N} \\
m_{1} \ldots m_{N}}}\left\langle\varphi_{n_{1}} \ldots \varphi_{n_{N}}\left|H^{(2)}(t) H^{(2)}(s) \rho^{(N)}(s)\right| \varphi_{m_{1}} \ldots \varphi_{m_{N}}\right\rangle \\
& \times \delta_{m_{M+1} \ldots m_{N} ; n_{M+1} \ldots n_{N}} a_{n_{1}}^{\dagger} \ldots a_{n_{M}}^{\dagger} a_{m_{1}} \ldots a_{m_{M}} \\
& =\frac{1}{M !} \sum_{\substack{n_{1} \ldots n_{N} \\
m_{1} \ldots m_{N}}} \sum_{\substack{i_{1} \ldots i_{N} \\
j_{1} \ldots j_{N}}} \rho_{i_{1} \ldots i_{N} ; j_{1} \ldots j_{N}}^{(N)}(s)\left\langle\varphi_{n_{1}} \ldots \varphi_{n_{N}}\left|H^{(2)}(t) H^{(2)}(s)\right| \varphi_{i_{1}} \ldots \varphi_{i_{N}}\right\rangle \\
& \times\left\langle\varphi_{j_{1}} \ldots \varphi_{j_{N}} \mid \varphi_{m_{1}} \ldots \varphi_{m_{N}}\right\rangle \delta_{m_{M+1} \ldots m_{N} ; n_{M+1} \ldots n_{N}} a_{n_{1}}^{\dagger} \ldots a_{n_{M}}^{\dagger} a_{m_{1}} \ldots a_{m_{M}} \\
& =\frac{1}{M !} \sum_{\substack{n_{1} \ldots n_{N} \\
m_{1} \ldots m_{N}}} \sum_{\substack{i_{1} \ldots i_{N} \\
j_{1} \ldots j_{N}}} \rho_{i_{1} \ldots i_{N} ; m_{1} \ldots m_{N}}^{(N)}(s)\left\langle\varphi_{n_{1}} \ldots \varphi_{n_{N}}\left|H^{(2)}(t)\right| \varphi_{j_{1}} \ldots \varphi_{j_{N}}\right\rangle \\
& \times\left\langle\varphi_{j_{1}} \ldots \varphi_{j_{N}}\left|H^{(2)}(s)\right| \varphi_{i_{1}} \ldots \varphi_{i_{N}}\right\rangle \delta_{m_{M+1} \ldots m_{N} ; n_{M+1} \ldots n_{N}} a_{n_{1}}^{\dagger} \ldots a_{n_{M}}^{\dagger} a_{m_{1}} \ldots a_{m_{M}} \\
& =\left(\begin{array}{c}
N \\
2
\end{array}\right)^{-2} \frac{1}{M !} \sum_{\substack{n_{1} \ldots n_{N} \\
m_{1} \ldots m_{N}}} \sum_{\substack{i_{1} \ldots i_{N} \\
j_{1} \ldots j_{N}}} \sum_{\begin{array}{l}
1 \leq \alpha_{1}<\alpha_{2} \leq N \\
1 \leq \beta_{1}<\beta_{2} \leq N \\
1 \leq \gamma_{1}<\gamma_{2} \leq N \\
1 \leq \delta_{1}<\delta_{2} \leq N
\end{array}} \rho_{i_{1} \ldots i_{N} ; m_{1} \ldots m_{N}}^{(N)}(s) H_{n_{\alpha_{1}} n_{\alpha_{2}} ; j_{\beta_{1}} j_{\beta_{2}}}^{(2)}(t) \\
& \times H_{j_{\gamma_{1}} j_{\gamma_{2}} ; i_{\delta_{1}} i_{\delta_{2}}}^{(2)}(s) \delta_{m_{M+1} \ldots m_{N} ; n_{M+1} \ldots n_{N}} a_{n_{1}}^{\dagger} \ldots a_{n_{M}}^{\dagger} a_{m_{1}} \ldots a_{m_{M}} \\
& \times \delta_{\left\{n_{1} \ldots n_{N}\right\} \backslash\left\{n_{\alpha_{1}} n_{\alpha_{2}}\right\} ;\left\{j_{1} \ldots j_{N}\right\} \backslash\left\{j_{\beta_{1}} j_{\beta_{2}}\right\}} \delta_{\left\{j_{1} \ldots j_{N}\right\} \backslash\left\{j_{\gamma_{1}} j_{\gamma_{2}}\right\} ;\left\{i_{1} \ldots i_{N}\right\} \backslash\left\{i_{\delta_{1}} i_{\delta_{2}}\right\}} \\
& =\frac{1}{M !} \sum_{\substack{n_{1} \ldots n_{N} \\
m_{1} \ldots m_{N}}} \sum_{\substack{i_{1} \ldots i_{N} \\
j_{1} \ldots j_{N}}} \sum_{\substack{1 \leq \alpha_{1}<\alpha_{2} \leq N \\
1 \leq \beta_{1}<\beta_{2} \leq N}} \rho_{i_{1} \ldots i_{N} ; m_{1} \ldots m_{N}}^{(N)}(s) H_{n_{\alpha_{1}} n_{\alpha_{2}} ; j_{\beta_{1}} j_{\beta_{2}}}^{(2)}(t) \\
& \times H_{j_{1} j_{2} ; i_{1} i_{2}}^{(2)}(s) \delta_{m_{M+1} \ldots m_{N} ; n_{M+1} \ldots n_{N}} a_{n_{1}}^{\dagger} \ldots a_{n_{M}}^{\dagger} a_{m_{1}} \ldots a_{m_{M}} \\
& \times \delta_{\left\{n_{1} \ldots n_{N}\right\} \backslash\left\{n_{\alpha_{1}} n_{\alpha_{2}}\right\} ;\left\{j_{1} \ldots j_{N}\right\} \backslash\left\{j_{\beta_{1}} j_{\beta_{2}}\right\}} \delta_{j_{3} \ldots j_{N} ; i_{3} \ldots i_{N}} \\
& =\frac{1}{M !} \sum_{\substack{n_{1} \ldots n_{N} \\
m_{1} \ldots m_{M}}} \sum_{\substack{i_{1} i_{2} \\
j_{1} \ldots j_{N}}} \sum_{\substack{1 \leq \alpha_{1}<\alpha_{2} \leq N \\
1 \leq \beta_{1}<\beta_{2} \leq N}} \rho_{i_{1} i_{2} j_{3} \ldots j_{N} ; m_{1} \ldots m_{M}, n_{M+1} \ldots n_{N}}^{(N)}(s) H_{n_{\alpha_{1}} n_{\alpha_{2}} ; j_{\beta_{1}} j_{\beta_{2}}}^{(2)}(t) \\
& \times H_{j_{1} j_{2} ; i_{1} i_{2}}^{(2)}(s) \delta_{\left\{n_{1} \ldots n_{N}\right\} \backslash\left\{n_{\alpha_{1}} n_{\alpha_{2}}\right\} ;\left\{j_{1} \ldots j_{N}\right\} \backslash\left\{j_{\beta_{1}} j_{\beta_{2}}\right\}} a_{n_{1}}^{\dagger} \ldots a_{n_{M}}^{\dagger} a_{m_{1}} \ldots a_{m_{M}}
\end{aligned}
$$

We again write out all contributing terms according to different classes of permutations depending on where $\alpha_{1}, \alpha_{2}$ and $\beta_{1}, \beta_{2}$ are mapped to. As before, the different classes are characterized by whether $\alpha_{1}$ and $\alpha_{2}$ are picked from within $1, \ldots, M$ or from $M+1, \ldots, N$. For $\beta_{1}$ and $\beta_{2}$ we need to distinguish between the sets 1,2 and $3, \ldots, N$. We use Lemma 9 in its general form for the cases of $\left\{\alpha_{1}, \alpha_{2}\right\}$ while for $\left\{\beta_{1}, \beta_{2}\right\}$ we employ the special case of $s=2$. 
We obtain

$$
\operatorname{Tr}_{N-M}^{(N)}\left\{H^{(2)}(t) H^{(2)}(s) \rho^{(N)}(s)\right\}=\frac{1}{M !} \sum_{\substack{n_{1} \ldots n_{M} \\ m_{1} \ldots m_{M}}} \sum_{i_{1} i_{2}}[
$$

- Terms with $\alpha_{1}, \alpha_{2} \leq M$ :

$$
\begin{aligned}
& \sum_{j_{1} j_{2}}\left(\begin{array}{c}
M \\
2
\end{array}\right) \rho_{i_{1} i_{2} n_{3} \ldots n_{M} ; m_{1} \ldots m_{M}}^{(M)}(s) H_{n_{1} n_{2} ; j_{1} j_{2}}^{(2)}(t) H_{j_{1} j_{2} ; i_{1} i_{2}}^{(2)}(s) \\
& +\sum_{\substack{n_{M+1} \ldots n_{N} \\
j_{1} \ldots j_{N}}}\left(\begin{array}{c}
M \\
2
\end{array}\right) 2(N-2) \rho_{i_{1} i_{2} j_{3} \ldots j_{N} ; m_{1} \ldots m_{M} n_{M+1} \ldots n_{N}}^{(N)}(s) \\
& \times H_{n_{1} n_{2} ; j_{1} j_{3}}^{(2)}(t) H_{j_{1} j_{2} ; i_{1} i_{2}}^{(2)}(s) \delta_{n_{3} \ldots n_{N} ; j_{2} j_{4} \ldots j_{N}} \\
& +\sum_{\substack{n_{M+1} \ldots n_{N} \\
j_{1} \ldots j_{N}}}\left(\begin{array}{c}
M \\
2
\end{array}\right)\left(\begin{array}{c}
N-2 \\
2
\end{array}\right) \rho_{i_{1} i_{2} j_{3} \ldots j_{N} ; m_{1} \ldots m_{M} n_{M+1} \ldots n_{N}}^{(N)}(s) \\
& \times H_{n_{1} n_{2} ; j_{3} j_{4}}^{(2)}(t) H_{j_{1} j_{2} ; i_{1} i_{2}}^{(2)}(s) \delta_{n_{3} \ldots n_{N} ; j_{1} j_{2} j_{5} \ldots j_{N}}
\end{aligned}
$$

- Terms with $\alpha_{1} \leq M, \alpha_{2}>M$ :

$$
\begin{aligned}
& +\sum_{\substack{n_{M+1} \\
j_{1} j_{2}}} M(N-M) \rho_{i_{1} i_{2} n_{2} \ldots n_{M} ; m_{1} \ldots m_{M} n_{M+1}}^{(M+1)}(s) H_{n_{1} n_{M+1} ; j_{1} j_{2}}^{(2)}(t) H_{j_{1} j_{2} ; i_{1} i_{2}}^{(2)}(s) \\
& +\sum_{\substack{n_{M+1} \ldots n_{N} \\
j_{1} \ldots j_{N}}} 2(N-2) M(N-M) \rho_{i_{1} i_{2} j_{3} \ldots j_{N} ; m_{1} \ldots m_{M} n_{M+1} \ldots n_{N}}^{(N)}(s) H_{n_{1} n_{M+1} ; j_{1} j_{3}}^{(2)}(t) \\
& \times H_{j_{1} j_{2} ; i_{1} i_{2}}^{(2)}(s) \delta_{n_{2} \ldots n_{M} n_{M+2} \ldots n_{N} ; j_{2} j_{4} \ldots j_{N}} \\
& +\sum_{\substack{n_{M+1 \ldots n} \ldots n_{N} \\
j_{1} \ldots j_{N}}}\left(\begin{array}{c}
N-2 \\
2
\end{array}\right) M(N-M) \rho_{i_{1} i_{2} j_{3} \ldots j_{N} ; m_{1} \ldots m_{M} n_{M+1} \ldots n_{N}}^{(N)}(s) H_{n_{1} n_{M+1} ; j_{3} j_{4}}^{(2)}(t) \\
& \times H_{j_{1} j_{2} ; i_{1} i_{2}}^{(2)}(s) \delta_{n_{2} \ldots n_{M} n_{M+2} \ldots n_{N} ; j_{1} j_{2} j_{5} \ldots j_{N}}
\end{aligned}
$$


- Terms with $\alpha_{1}, \alpha_{2}>M$ :

$$
\begin{aligned}
& +\sum_{\substack{n_{M+1} n_{M+2} \\
j_{1} j_{2}}}\left(\begin{array}{c}
N-M \\
2
\end{array}\right) \rho_{i_{1} i_{2} n_{1} \ldots n_{M} ; m_{1} \ldots m_{M} n_{M+1} n_{M+2}}^{(M+2)}(s) \\
& \times H_{n_{M+1} n_{M+2} ; j_{1} j_{2}}^{(2)}(t) H_{j_{1} j_{2} ; i_{1} i_{2}}^{(2)}(s) \\
& +\sum_{\substack{n_{M+1} \ldots n_{N} \\
j_{1} \ldots j_{N}}}\left(\begin{array}{c}
N-M \\
2
\end{array}\right) 2(N-2) \rho_{i_{1} i_{2} j_{3} \ldots j_{N} ; m_{1} \ldots m_{M} n_{M+1} \ldots n_{N}}^{(N)}(s) H_{n_{M+1} n_{M+2} ; j_{1} j_{3}}^{(2)}(t) \\
& \times H_{j_{1} j_{2} ; i_{1} i_{2}}^{(2)}(s) \delta_{n_{1} \ldots n_{M} n_{M+3} \ldots n_{N} ; j_{2} j_{4} \ldots j_{N}} \\
& +\sum_{\substack{M+1 \ldots n_{N} \\
j_{1} \ldots j_{N}}}\left(\begin{array}{c}
N-M \\
2
\end{array}\right)\left(\begin{array}{c}
N-2 \\
2
\end{array}\right) \rho_{i_{1} i_{2} j_{3} \ldots j_{N} ; m_{1} \ldots m_{M} n_{M+1} \ldots n_{N}}^{(N)}(s) \\
& \left.\times H_{n_{M+1} n_{M+2} ; j_{3} j_{4}}^{(2)}(t) H_{j_{1} j_{2} ; i_{1} i_{2}}^{(2)}(s) \delta_{n_{1} \ldots n_{M} n_{M+3} \ldots n_{N} ; j_{1} j_{2} j_{5} \ldots j_{N}}\right] \\
& \times a_{n_{1}}^{\dagger} \ldots a_{n_{M}}^{\dagger} a_{m_{1}} \ldots a_{m_{M}}
\end{aligned}
$$

Now, there are six terms which still have to be decomposed into different permutations of the symmetric $\delta$-functions. We start with the second term of (A.12). Here the two different possibilities are given by whether $j_{2}$ is mapped to an element of $n_{3}, \ldots, n_{M}$ or of $n_{M+1}, \ldots, n_{N}$. The total number of permutations is $(N-2)$ !, and according to Lemma 10 a), with $r=N-2$ and $s=M-2$, there are $(M-2)(N-3)$ ! permutations which map $j_{2}$ within $n_{3}, \ldots, n_{M}$ and $(N-M)(N-3)$ ! permutations which do not:

$$
\begin{gathered}
\sum_{\substack{n_{M+1} \ldots n_{N} \\
j_{1} \ldots j_{N}}}\left(\begin{array}{c}
M \\
2
\end{array}\right) 2(N-2) \rho_{i_{1} i_{2} j_{3} \ldots j_{N} ; m_{1} \ldots m_{M} n_{M+1} \ldots n_{N}}^{(N)}(s) \\
\quad \times H_{n_{1} n_{2} ; j_{1} j_{3}}^{(2)}(t) H_{j_{1} j_{2} ; i_{1} i_{2}}^{(2)}(s) \delta_{n_{3} \ldots n_{N} ; j_{2} j_{4} \ldots j_{N}} \\
=\sum_{j_{1} j_{3}} \underbrace{(N-1)(M-2)}_{M\left(\begin{array}{c}
M \\
2
\end{array}\right)} \rho_{i_{1} i_{2} j_{3} n_{4} \ldots n_{M} ; m_{1} \ldots m_{M}}^{(M-2)(N-3) !}(s) \\
+\sum_{n_{M+1}}^{(M)} \underbrace{\left(\begin{array}{c}
M \\
2
\end{array}\right) 2(N-2) \frac{(N-M)(N-3) !}{(N-2) !}}_{j_{1} j_{3}} \rho_{i_{1} i_{2} j_{3} n_{3} \ldots n_{M} ; m_{1} \ldots m_{M} n_{M+1}}^{(M+1)}(s) \\
\times H_{n_{1} n_{2} ; j_{1} j_{3}}^{(2)}(t) H_{j_{1} n_{3} ; i_{1} i_{2}}^{(2)}(s) \\
\times H_{n_{1} n_{2} ; j_{1} j_{3}}^{(2)}(t) H_{j_{1} n_{M+1} ; i_{1} i_{2}}^{(2)}(s) .
\end{gathered}
$$

For the third term of (A.12), using Lemma $10 \mathrm{~b}$ ) with $r=N-2$ and $s=M-2$ we find that there are $(M-2)(M-3)(N-4)$ ! permutations which map both $j_{1}$ and $j_{2}$ within $n_{3}, \ldots, n_{M}$. From Lemma $10 \mathrm{c}$ ) we find that $2(M-2)(N-M)(N-4)$ ! permutations map one of the two indices $j_{1}$ and $j_{2}$ within $n_{3}, \ldots, n_{M}$, and, finally, from Lemma 10 
d) we obtain $(N-M)(N-M-1)(N-4)$ ! permutations where neither $j_{1}$ nor $j_{2}$ are mapped onto $n_{3}, \ldots, n_{M}$. We obtain

$$
\begin{aligned}
& \sum_{\substack{n_{M+1} \ldots n_{N} \\
j_{1} \ldots j_{N}}}\left(\begin{array}{c}
M \\
2
\end{array}\right)\left(\begin{array}{c}
N-2 \\
2
\end{array}\right) \rho_{i_{1} i_{2} j_{3} \ldots j_{N} ; m_{1} \ldots m_{M} n_{M+1} \ldots n_{N}}^{(N)}(s) \\
& \times H_{n_{1} n_{2} ; j_{3} j_{4}}^{(2)}(t) H_{j_{1} j_{2} ; i_{1} i_{2}}^{(2)}(s) \delta_{n_{3} \ldots n_{N} ; j_{1} j_{2} j_{5} \ldots j_{N}} \\
& =\sum_{j_{3} j_{4}} \underbrace{\left(\begin{array}{c}
M \\
2
\end{array}\right)\left(\begin{array}{c}
N-2 \\
2
\end{array}\right) \frac{(M-2)(M-3)(N-4) !}{(N-2) !}}_{\frac{1}{4} M(M-1)(M-2)(M-3)} \rho_{i_{1} i_{2} j_{3} j_{4} n_{5} \ldots n_{M} ; m_{1} \ldots m_{M}}^{(M)}(s) \\
& \times H_{n_{1} n_{2} ; j_{3} j_{4}}^{(2)}(t) H_{n_{3} n_{4} ; i_{1} i_{2}}^{(2)}(s) \\
& +\sum_{\substack{n_{M+1} \\
j_{3} j_{4}}} \underbrace{\left(\begin{array}{c}
M \\
2
\end{array}\right)\left(\begin{array}{c}
N-2 \\
2
\end{array}\right) \frac{2(M-2)(N-M)(N-4) !}{(N-2) !}}_{\frac{1}{2} M(M-1)(M-2)(N-M)} \rho_{i_{1} i_{2} j_{3} j_{4} n_{4} \ldots n_{M} ; m_{1} \ldots m_{M} n_{M+1}}^{(M+1)}(s) \\
& \times H_{n_{1} n_{2} ; j_{3} j_{4}}^{(2)}(t) H_{n_{3} n_{M+1} ; i_{1} i_{2}}^{(2)}(s) \\
& +\sum_{\substack{n_{M+1} n_{M+2} \\
j_{3} j_{4}}} \underbrace{\left(\begin{array}{c}
M \\
2
\end{array}\right)\left(\begin{array}{c}
N-2 \\
2
\end{array}\right) \frac{(N-M)(N-M-1)(N-4) !}{(N-2) !}}_{\frac{1}{4} M(M-1)(N-M)(N-M-1)} \\
& \times \rho_{i_{1} i_{2} j_{3} j_{4} n_{3} \ldots n_{M} ; m_{1} \ldots m_{M} n_{M+1} n_{M+2}}^{(M+2)}(s) H_{n_{1} n_{2} ; j_{3} j_{4}}^{(2)}(t) H_{n_{M+1} n_{M+2} ; i_{1} i_{2}}^{(2)}(s) .
\end{aligned}
$$

For the second term of (A.13) we have $j_{2}$ either within $n_{2}, \ldots, n_{M}$ or $n_{M+2}, \ldots, n_{N}$. This leads to a total of $(N-2)$ ! permutations and, according to Lemma 10 a), with $r=N-2$ and $s=M-1$, there are $(M-1)(N-3)$ ! cases which correspond to the first situation, and $(N-M-1)(N-3)$ ! cases for the second. This yields

$$
\begin{aligned}
& \sum_{\substack{n_{M+1} \ldots n_{N} \\
j_{1} \ldots j_{N}}} 2(N-2) M(N-M) \rho_{i_{1} i_{2} j_{3} \ldots j_{N} ; m_{1} \ldots m_{M} n_{M+1} \ldots n_{N}}^{(N)}(s) H_{n_{1} n_{M+1} ; j_{1} j_{3}}^{(2)}(t) \\
& \times H_{j_{1} j_{2} ; i_{1} i_{2}}^{(2)}(s) \delta_{n_{2} \ldots n_{M} n_{M+2} \ldots n_{N} ; j_{2} j_{4} \ldots j_{N}} \\
& =\sum_{n_{M+1}} \underbrace{2(N-2) M(N-M) \frac{(M-1)(N-3) !}{(N-2) !}}_{2 M(M-1)(N-M)} \rho_{i_{1} i_{2} j_{3} n_{3} \ldots n_{M} ; m_{1} \ldots m_{M} n_{M+1}}^{(M+1)}(s) \\
& \times H_{n_{1} n_{M+1} ; j_{1} j_{3}}^{(2)}(t) H_{j_{1} n_{2} ; i_{1} i_{2}}^{(2)}(s) \\
& +\sum_{\substack{n_{M+1} \\
n_{M+2} \\
j_{1} j_{3}}} \underbrace{2(N-2) M(N-M) \frac{(N-M-1)(N-3) !}{(N-2) !}}_{2 M(N-M)(N-M-1)} \rho_{i_{1} i_{2} j_{3} n_{2} \ldots n_{M} ; m_{1} \ldots m_{M} n_{M+1} n_{M+2}}^{(M+2)}(s) \\
& \times H_{n_{1} n_{M+1} ; j_{1} j_{3}}^{(2)}(t) H_{j_{1} n_{M+2} ; i_{1} i_{2}}^{(2)}(s) .
\end{aligned}
$$

The third term of (A.13) has either none, one, or both of the indices $j_{1}$ and $j_{2}$ mapped 
onto elements of $n_{2}, \ldots, n_{M}$ or of $n_{M+2}, \ldots, n_{N}$. Again we use Lemma 10 with $r=$ $N-2$ and $s=M-1$. According to $b), c)$ and $d)$, there are $(M-1)(M-2)(N-4)$ ! possibilities for "both", $2(M-1)(N-M-1)(N-4)$ ! for "exactly one" and $(N-$ $M-1)(N-M-2)(N-4)$ ! for "none". We get

$$
\begin{aligned}
& \sum_{\substack{n_{M+1} \ldots n_{N} \\
j_{1} \ldots j_{N}}}\left(\begin{array}{c}
N-2 \\
2
\end{array}\right) M(N-M) \rho_{i_{1} i_{2} j_{3} \ldots j_{N} ; m_{1} \ldots m_{M} n_{M+1} \ldots n_{N}}^{(N)}(s) \\
& \times H_{n_{1} n_{M+1} ; j_{3} j_{4}}^{(2)}(t) H_{j_{1} j_{2} ; i_{1} i_{2}}^{(2)}(s) \delta_{n_{2} \ldots n_{M} n_{M+2} \ldots n_{N} ; j_{1} j_{2} j_{5} \ldots j_{N}} \\
& =\sum_{n_{M+1}} \underbrace{\left(\begin{array}{c}
N-2 \\
2
\end{array}\right) M(N-M) \frac{(M-1)(M-2)(N-4) !}{(N-2) !}}_{\frac{1}{2} M(M-1)(M-2)(N-M)} \\
& \times \rho_{i_{1} i_{2} j_{3} j_{4} n_{4} \ldots n_{M} ; m_{1} \ldots m_{M} n_{M+1}}^{(M+1)}(s) H_{n_{1} n_{M+1} ; j_{3} j_{4}}^{(2)}(t) H_{n_{2} n_{3} ; i_{1} i_{2}}^{(2)}(s) \\
& +\sum_{\substack{n_{M+1} \\
n_{M+2} \\
j_{3} j_{4}}} \underbrace{\left(\begin{array}{c}
N-2 \\
2
\end{array}\right) M(N-M) \frac{2(M-1)(N-M-1)(N-4) !}{(N-2) !}}_{M(M-1)(N-M)(N-M-1)} \\
& \times \rho_{i_{1} i_{2} j_{3} j_{4} n_{3} \ldots n_{M} ; m_{1} \ldots m_{M} n_{M+1} n_{M+2}}^{(M+2)}(s) H_{n_{1} n_{M+1} ; j_{3} j_{4}}^{(2)}(t) H_{n_{2} n_{M}}^{(2)} \\
& +\sum_{\substack{n_{M+1} \\
n_{M+2} \\
n_{M+3} \\
j_{3} j_{4}}} \underbrace{\left(\begin{array}{c}
N-2 \\
2
\end{array}\right) M(N-M) \frac{(N-M-1)(N-M-2)(N-4) !}{(N-2) !}}_{\frac{1}{2} M(N-M)(N-M-1)(N-M-2)} \\
& \times \rho_{i_{1} i_{2} j_{3} j_{4}}^{(M+3)} \\
& \times H_{n_{1} n_{M+1} ; j_{3} j_{4}}^{(2)}(t) H_{n_{M+2} n_{M+3} ; i_{1} i_{2}}^{(2)}(s) .
\end{aligned}
$$

In the second term of (A.14), $j_{2}$ can be picked either from the set $n_{1}, \ldots, n_{M}$ or from $n_{M+3}, \ldots, n_{N}$. According to Lemma 10 a) with $r=N-2$, there are $M(N-3)$ ! possibilities for the first case, and $(N-M-2)(N-3)$ ! for the second. We obtain

$$
\begin{aligned}
& \sum_{\substack{n_{M+1 \ldots n_{N}} \\
j_{1} \ldots j_{N}}}\left(\begin{array}{c}
N-M \\
2
\end{array}\right) 2(N-2) \rho_{i_{1} i_{2} j_{3} \ldots j_{N} ; m_{1} \ldots m_{M} n_{M+1} \ldots n_{N}}^{(N)}(s) H_{n_{M+1} n_{M+2} ; j_{1} j_{3}}^{(2)}(t) \\
& \quad \times H_{j_{1} j_{2} ; i_{1} i_{2}}^{(2)}(s) \delta_{n_{1} \ldots n_{M} n_{M+3} \ldots n_{N} ; j_{2} j_{4} \ldots j_{N}}
\end{aligned}
$$




$$
\begin{aligned}
&= \sum_{\substack{n_{M+1} \\
n_{M+2} \\
j_{1} j_{3}}} \underbrace{\left(\begin{array}{c}
N-M \\
2
\end{array}\right) 2(N-2) \frac{M(N-3) !}{(N-2) !} \rho_{i_{1} i_{2} j_{3} n_{2} \ldots n_{M} ; m_{1} \ldots m_{M} n_{M+1} n_{M+2}}^{(M+2)}(s)}_{M(N-M)(N-M-1)} \\
& \times H_{\substack{(2) \\
n_{M+1} n_{M+2} ; j_{1} j_{3}}}^{(t) H_{j_{1} n_{1} ; i_{1} i_{2}}^{(2)}(s)} \\
&+\sum_{\substack{n_{M+1} \\
n_{M+2} \\
j_{1} j_{3}}} \underbrace{\left(\begin{array}{c}
N-M \\
2
\end{array}\right) 2(N-2) \frac{(N-M-2)(N-3) !}{(N-2) !}}_{(N-M)(N-M-1)(N-M-2)} \\
& \quad \times \rho_{i_{1} i_{2} j_{3} n_{1} \ldots n_{M} ; m_{1} \ldots m_{M} n_{M+1} n_{M+2} n_{M+3}}^{(M+3)}(s) H_{n_{M+1} n_{M+2} ; j_{1} j_{3}}^{(2)}(t) H_{j_{1} n_{M+3} ; i_{1} i_{2}}^{(2)}(s) .
\end{aligned}
$$

Finally, we decompose the third term in (A.14). Using Lemma 10 b), c) and d) with $r=N-2$, we find $M(M-1)(N-4)$ ! permutations which map $j_{1}$ and $j_{2}$ onto $n_{1}, \ldots, n_{M}$, while there are $2 M(N-M-2)(N-4)$ ! permutations for which one of $j_{1}$ and $j_{2}$ are mapped within $n_{1}, \ldots, n_{M}$, and $(N-M-2)(N-M-3)(N-4)$ ! permutations where neither $j_{1}$ nor $j_{2}$ are mapped onto $n_{1}, \ldots, n_{M}$. Inserting this, we get:

$$
\begin{aligned}
& \sum_{\substack{n_{M+1} \ldots n_{N} \\
j_{1} \ldots j_{N}}}\left(\begin{array}{c}
N-M \\
2
\end{array}\right)\left(\begin{array}{c}
N-2 \\
2
\end{array}\right) \rho_{i_{1} i_{2} j_{3} \ldots j_{N} ; m_{1} \ldots m_{M} n_{M+1} \ldots n_{N}}^{(N)}(s) H_{n_{M+1} n_{M+2} ; j_{3} j_{4}}^{(2)}(t) \\
& \times H_{j_{1} j_{2} ; i_{1} i_{2}}^{(2)}(s) \delta_{n_{1} \ldots n_{M} n_{M+3} \ldots n_{N} ; j_{1} j_{2} j_{5} \ldots j_{N}} \\
& =\sum_{\substack{n_{M+1} \\
n_{M+2} \\
j_{3} j_{4}}} \underbrace{\left(\begin{array}{c}
N-M \\
2
\end{array}\right)\left(\begin{array}{c}
N-2 \\
2
\end{array}\right) \frac{M(M-1)(N-4) !}{(N-2) !}}_{\frac{1}{4} M(M-1)(N-M)(N-M-1)} \rho_{i_{1} i_{2} j_{3} j_{4} n_{3} \ldots n_{M} ; m_{1} \ldots m_{M} n_{M+1} n_{M+2}}^{(M+2)}(s) \\
& \times H_{n_{M+1} n_{M+2} ; j_{3} j_{4}}^{(2)}(t) H_{n_{1} n_{2} ; i_{1} i_{2}}^{(2)}(s) \\
& +\sum_{\substack{n_{M+1} \\
n_{M+2} \\
n_{M+3} \\
j_{3} j_{4}}} \underbrace{\left(\begin{array}{c}
N-M \\
2
\end{array}\right)\left(\begin{array}{c}
N-2 \\
2
\end{array}\right) \frac{2 M(N-M-2)(N-4) !}{(N-2) !}}_{\frac{1}{2} M(N-M)(N-M-1)(N-M-2)} \\
& \times \rho_{i_{1} i_{2} j_{3} j_{4} n_{2} \ldots n_{M} ; m_{1} \ldots m_{M} n_{M+1} n_{M+2} n_{M+3}}^{(M+3)}(s) H_{n_{M+1} n_{M+2} ; j_{3} j_{4}}^{(2)}(t) H_{n_{1} n_{M+3} ; i_{1} i_{2}}^{(2)}(s) \\
& +\sum_{\substack{n_{M+1} n_{M+2} \\
n_{M+3} n_{M+4} \\
j_{3} j_{4}}} \underbrace{\left(\begin{array}{c}
N-M \\
2
\end{array}\right)\left(\begin{array}{c}
N-2 \\
2
\end{array}\right) \frac{(N-M-2)(N-M-3)(N-4) !}{(N-2) !}}_{\frac{1}{4}(N-M)(N-M-1)(N-M-2)(N-M-3)} \\
& \times \rho_{i_{1} i_{2} j_{3} j_{4} n_{1} \ldots n_{M} ; m_{1} \ldots m_{M} n_{M+1} n_{M+2} n_{M+3} n_{M+4}}^{(M+4)}(s) \\
& \times H_{n_{M+1} n_{M+2} ; j_{3} j_{4}}^{(2)}(t) H_{n_{M+3} n_{M+4} ; i_{1} i_{2}}^{(2)}(s) .
\end{aligned}
$$




\section{A.3 Full expression}

Now, we combine all of the contributions to the first and second term under the integral in equation (7.150), as elaborated in the preceding two sections. We group the terms according to the number of particles which contribute to the reduced density matrix. Since the two groups of terms contribute with opposite signs, some of their constituents cancel. To see this, we first write out the full expression, labeling those terms which eventually will drop out

$$
\begin{aligned}
& \operatorname{Tr}_{N-M}^{(N)}\left\{H^{(2)}(s) \rho^{(N)}(s) H^{(2)}(t)\right\}-\operatorname{Tr}_{N-M}^{(N)}\left\{H^{(2)}(t) H^{(2)}(s) \rho^{(N)}(s)\right\} \\
& =\frac{1}{M !} \sum_{\substack{n_{1} \ldots n_{M} \\
m_{1} \ldots m_{M}}} \sum_{\substack{i_{1} i_{2} \\
j_{1} j_{2}}}\left[\frac{1}{4} M^{2}(M-1)^{2} \rho_{i_{1} i_{2} n_{3} \ldots n_{M} ; j_{1} j_{2} m_{3} \ldots m_{M}}^{(M)}(s) H_{n_{1} n_{2} ; i_{1} i_{2}}^{(2)}(s) H_{j_{1} j_{2} ; m_{1} m_{2}}^{(2)}(t)\right. \\
& -\frac{1}{2} M(M-1) \rho_{i_{1} i_{2} n_{3} \ldots n_{M} ; m_{1} \ldots m_{M}}^{(M)}(s) H_{n_{1} n_{2} ; j_{1} j_{2}}^{(2)}(t) H_{j_{1} j_{2} ; i_{1} i_{2}}^{(2)}(s) \\
& -M(M-1)(M-2) \rho_{i_{1} i_{2} j_{2} n_{4} \ldots n_{M} ; m_{1} \ldots m_{M}}^{(M)}(s) H_{n_{1} n_{2} ; j_{1} j_{2}}^{(2)}(t) H_{j_{1} n_{3} ; i_{1} i_{2}}^{(2)}(s) \\
& -\frac{1}{4} M(M-1)(M-2)(M-3) \rho_{i_{1} i_{2} j_{1} j_{2} n_{5} \ldots n_{M} ; m_{1} \ldots m_{M}}^{(M)}(s) H_{n_{1} n_{2} ; j_{1} j_{2}}^{(2)}(t) H_{n_{3} n_{4} ; i_{1} i_{2}}^{(2)}(s) \\
& +\sum_{k_{1}} \frac{1}{2}(M-1) M^{2}(N-M) \rho_{i_{1} i_{2} n_{2} \ldots n_{M} ; j_{1} j_{2} m_{3} \ldots m_{M} k_{1}}^{(M+1)}(s) H_{n_{1} k_{1} ; i_{1} i_{2}}^{(2)}(s) H_{j_{1} j_{2} ; m_{1} m_{2}}^{(2)}(t) \\
& +\sum_{k_{1}} \frac{1}{2}(M-1) M^{2}(N-M) \rho_{i_{1} i_{2} n_{3} \ldots n_{M} k_{1} ; j_{1} j_{2} m_{2} \ldots m_{M}}^{(M+1)}(s) H_{n_{1} n_{2} ; i_{1} i_{2}}^{(2)}(s) H_{j_{1} j_{2} ; m_{1} k_{1}}^{(2)}(t) \\
& +\sum_{k_{1}} M^{2}(N-M) \rho_{i_{1} i_{2} n_{2} \ldots n_{M} ; j_{1} j_{2} m_{2} \ldots m_{M}}^{(M+1)}(s) H_{n_{1} k_{1} ; i_{1} i_{2}}^{(2)}(s) H_{j_{1} j_{2} ; m_{1} k_{1}}^{(2)}(t) \\
& -\sum_{k_{1}} M(N-M) \rho_{i_{1} i_{2} n_{2} \ldots n_{M} ; m_{1} \ldots m_{M} k_{1}}^{(M+1)}(s) H_{n_{1} k_{1} ; j_{1} j_{2}}^{(2)}(t) H_{j_{1} j_{2} ; i_{1} i_{2}}^{(2)}(s) \\
& -\sum_{k_{1}} M(N-M)(M-1) \rho_{i_{1} i_{2} j_{2} n_{3} \ldots n_{M} ; m_{1} \ldots m_{M} k_{1}}^{(M+1)}(s) H_{n_{1} n_{2} ; j_{1} j_{2}}^{(2)}(t) H_{j_{1} k_{1} ; i_{1} i_{2}}^{(2)}(s) \\
& -\sum_{k_{1}} \frac{1}{2} M(M-1)(M-2)(N-M) \rho_{i_{1} i_{2} j_{1} j_{2} n_{4} \ldots n_{M} ; m_{1} \ldots m_{M} k_{1}}^{(M+1)}(s) \\
& \times H_{n_{1} n_{2} ; j_{1} j_{2}}^{(2)}(t) H_{n_{3} k_{1} ; i_{1} i_{2}}^{(2)}(s) \\
& -\sum_{k_{1}} 2 M(M-1)(N-M) \rho_{i_{1} i_{2} j_{3} n_{3} \ldots n_{M} ; m_{1} \ldots m_{M} k_{1}}^{(M+1)}(s) H_{n_{1} k_{1} ; j_{1} j_{3}}^{(2)}(t) H_{j_{1} n_{2} ; i_{1} i_{2}}^{(2)}(s) \\
& -\sum_{k_{1}} \frac{1}{2} M(M-1)(M-2)(N-M) \rho_{i_{1} i_{2} j_{1} j_{2} n_{4} \ldots n_{M} ; m_{1} \ldots m_{M} k_{1}}^{(M+1)}(s) \\
& \times H_{n_{1} k_{1} ; j_{1} j_{2}}^{(2)}(t) H_{n_{2} n_{3} ; i_{1} i_{2}}^{(2)}(s)
\end{aligned}
$$




$$
\begin{aligned}
& +\sum_{k_{1} k_{2}} \frac{1}{4}(M-1) M(N-M)(N-M-1) \rho_{i_{1} i_{2} n_{1} \ldots n_{M} ; j_{1} j_{2} m_{3} \ldots m_{M} k_{1} k_{2}}^{(M+2)}(s) \\
& \times H_{k_{1} k_{2} ; i_{1} i_{2}}^{(2)}(s) H_{j_{1} j_{2} ; m_{1} m_{2}}^{(2)}(t) \\
& +\sum_{k_{1} k_{2}} \frac{1}{4}(M-1) M(N-M)(N-M-1) \rho_{i_{1} i_{2} n_{3} \ldots n_{M} k_{1} k_{2} ; j_{1} j_{2} m_{1} \ldots m_{M}}^{(M+2)}(s) \\
& \times H_{n_{1} n_{2} ; i_{1} i_{2}}^{(2)}(s) H_{j_{1} j_{2} ; k_{1} k_{2}}^{(2)}(t) \\
& +\sum_{k_{1} k_{2}} M^{2}(N-M)(N-M-1) \rho_{i_{1} i_{2} n_{2} \ldots n_{M} k_{2} ; j_{1} j_{2} m_{2} \ldots m_{M} k_{1}}^{(M+2)}(s) \\
& \times H_{n_{1} k_{1} ; i_{1} i_{2}}^{(2)}(s) H_{j_{1} j_{2} ; m_{1} k_{2}}^{(2)}(t) \\
& +\sum_{k_{1} k_{2}} M(N-M)(N-M-1) \rho_{i_{1} i_{2} n_{1} \ldots n_{M} ; j_{1} j_{2} m_{2} \ldots m_{M} k_{2}}^{(M+2)}(s) H_{k_{1} k_{2} ; i_{1} i_{2}}^{(2)}(s) H_{j_{1} j_{2} ; m_{1} k_{1}}^{(2)}(t) \\
& +\sum_{k_{1} k_{2}} M(N-M)(N-M-1) \rho_{i_{1} i_{2} n_{2} \ldots n_{M} k_{2} ; j_{1} j_{2} m_{1} \ldots m_{M}}^{(M+2)}(s) H_{n_{1} k_{1} ; i_{1} i_{2}}^{(2)}(s) H_{j_{1} j_{2} ; k_{1} k_{2}}^{(2)}(t) \\
& +\sum_{k_{1} k_{2}} \frac{1}{2}(N-M)(N-M-1) \rho_{i_{1} i_{2} n_{1} \ldots n_{M} ; j_{1} j_{2} m_{1} \ldots m_{M}}^{(M+2)}(s) H_{k_{1} k_{2} ; i_{1} i_{2}}^{(2)}(s) H_{j_{1} j_{2} ; k_{1} k_{2}}^{(2)}(t) \\
& -\sum_{k_{1} k_{2}} \frac{1}{2}(N-M)(N-M-1) \rho_{i_{1} i_{2} n_{1} \ldots n_{M} ; m_{1} \ldots m_{M} k_{1} k_{2}}^{(M+2)}(s) H_{k_{1} k_{2} ; j_{1} j_{2}}^{(2)}(t) H_{j_{1} j_{2} ; i_{1} i_{2}}^{(2)}(s) \\
& -\sum_{k_{1} k_{2}} \frac{1}{4} M(M-1)(N-M)(N-M-1) \rho_{i_{1} i_{2} j_{1} j_{2} n_{3} \ldots n_{M} ; m_{1} \ldots m_{M} k_{1} k_{2}}^{(M+2)}(s) \\
& \times H_{n_{1} n_{2} ; j_{1} j_{2}}^{(2)}(t) H_{k_{1} k_{2} ; i_{1} i_{2}}^{(2)}(s) \\
& -\sum_{k_{1} k_{2}} 2 M(N-M)(N-M-1) \rho_{i_{1} i_{2} j_{2} n_{2} \ldots n_{M} ; m_{1} \ldots m_{M} k_{1} k_{2}}^{(M+2)}(s) H_{n_{1} k_{1} ; j_{1} j_{2}}^{(2)}(t) H_{j_{1} k_{2} ; i_{1} i_{2}}^{(2)}(s) \\
& -\sum_{k_{1} k_{2}} M(M-1)(N-M)(N-M-1) \rho_{i_{1} i_{2} j_{1} j_{2} n_{3} \ldots n_{M} ; m_{1} \ldots m_{M} k_{1} k_{2}}^{(M+2)}(s) \\
& \times H_{n_{1} k_{1} ; j_{1} j_{2}}^{(2)}(t) H_{n_{2} k_{2} ; i_{1} i_{2}}^{(2)}(s) \\
& -\sum_{k_{1} k_{2}} M(N-M)(N-M-1) \rho_{i_{1} i_{2} j_{2} n_{2} \ldots n_{M} ; m_{1} \ldots m_{M} k_{1} k_{2}}^{(M+2)}(s) H_{k_{1} k_{2} ; j_{1} j_{2}}^{(2)}(t) H_{j_{1} n_{1} ; i_{1} i_{2}}^{(2)}(s)
\end{aligned}
$$$$
-\sum_{k_{1} k_{2}} \frac{1}{4} M(M-1)(N-M)(N-M-1) \rho_{i_{1} i_{2} j_{1} j_{2} n_{3} \ldots n_{M} ; m_{1} \ldots m_{M} k_{1} k_{2}}^{(M+2)}(s)
$$$$
\times H_{k_{1} k_{2} ; j_{1} j_{2}}^{(2)}(t) H_{n_{1} n_{2} ; i_{1} i_{2}}^{(2)}(s)
$$ 


$$
\begin{aligned}
& +\sum_{k_{1} k_{2} k_{3}} \frac{1}{2} M(N-M)(N-M-1)(N-M-2) \rho_{i_{1} i_{2} n_{1} \ldots n_{M} k_{3} ; j_{1} j_{2} m_{2} \ldots m_{M} k_{1} k_{2}}^{(M+3)}(s) \\
& \times H_{k_{1} k_{2} ; i_{1} i_{2}}^{(2)}(s) H_{j_{1} j_{2} ; m_{1} k_{3}}^{(2)}(t) \\
& +\sum_{k_{1} k_{2} k_{3}} \frac{1}{2} M(N-M)(N-M-1)(N-M-2) \rho_{i_{1} i_{2} n_{2} \ldots n_{M} k_{2} k_{3} ; j_{1} j_{2} m_{1} \ldots m_{M} k_{1}}^{(M+3)}(s) \\
& \times H_{n_{1} k_{1} ; i_{1} i_{2}}^{(2)}(s) H_{j_{1} j_{2} ; k_{2} k_{3}}^{(2)}(t) \\
& +\sum_{k_{1} k_{2} k_{3}}(N-M)(N-M-1)(N-M-2) \rho_{i_{1} i_{2} n_{1} \ldots n_{M} k_{3} ; j_{1} j_{2} m_{1} \ldots m_{M} k_{2}}^{(M+3)}(s) \\
& \times H_{k_{1} k_{2} ; i_{1} i_{2}}^{(2)}(s) H_{j_{1} j_{2} ; k_{1} k_{3}}^{(2)}(t) \\
& -\sum_{k_{1} k_{2} k_{3}} \frac{1}{2} M(N-M)(N-M-1)(N-M-2) \rho_{i_{1} i_{2} j_{1} j_{2} n_{2} \ldots n_{M} ; m_{1} \ldots m_{M} k_{1} k_{2} k_{3}}^{(M+3)}(s) \\
& \times H_{n_{1} k_{1} ; j_{1} j_{2}}^{(2)}(t) H_{k_{2} k_{3} ; i_{1} i_{2}}^{(2)}(s) \\
& -\sum_{k_{1} k_{2} k_{3}}(N-M)(N-M-1)(N-M-2) \rho_{i_{1} i_{2} j_{2} n_{1} \ldots n_{M} ; m_{1} \ldots m_{M} k_{1} k_{2} k_{3}}^{(M+3)}(s) \\
& \times H_{k_{1} k_{2} ; j_{1} j_{2}}^{(2)}(t) H_{j_{1} k_{3} ; i_{1} i_{2}}^{(2)}(s) \\
& -\sum_{k_{1} k_{2} k_{3}} \frac{1}{2} M(N-M)(N-M-1)(N-M-2) \rho_{i_{1} i_{2} j_{1} j_{2} n_{2} \ldots n_{M} ; m_{1} \ldots m_{M} k_{1} k_{2} k_{3}}^{(M+3)}(s) \\
& \times H_{k_{1} k_{2} ; j_{1} j_{2}}^{(2)}(t) H_{n_{1} k_{3} ; i_{1} i_{2}}^{(2)}(s) \\
& +\sum_{k_{1} k_{2} k_{3} k_{4}} \frac{1}{4}(N-M)(N-M-1)(N-M-2)(N-M-3) H_{k_{1} k_{2} ; i_{1} i_{2}}^{(2)}(s) H_{j_{1} j_{2} ; k_{3} k_{4}}^{(2)}(t) \\
& \times \rho_{i_{1} i_{2} n_{1} \ldots n_{M} k_{3} k_{4} ; j_{1} j_{2} m_{1} \ldots m_{M} k_{1} k_{2}}^{(M+4)}(s) \\
& -\sum_{k_{1} k_{2} k_{3} k_{4}} \frac{1}{4}(N-M)(N-M-1)(N-M-2)(N-M-3) H_{k_{1} k_{2} ; j_{1} j_{2}}^{(2)}(t) H_{k_{3} k_{4} ; i_{1} i_{2}}^{(2)}(s) \\
& \left.\times \rho_{i_{1} i_{2} j_{1} j_{2} n_{1} \ldots n_{M} ; m_{1} \ldots m_{M} k_{1} k_{2} k_{3} k_{4}}^{(M+4)}(s)\right] a_{n_{1}}^{\dagger} \ldots a_{n_{M}}^{\dagger} a_{m_{1}} \ldots a_{m_{M}}
\end{aligned}
$$

Several terms involving $M+2$ and $M+3$ states cancel each other, while the $M+4$ contribution vanishes completely. In particular, we are able to identify the following terms (note the opposing signs):

$$
\begin{aligned}
& (A .22)+(A .27)=0 \\
& (A .23)+(A .26)=0 \\
& (A .24)+(A .25)=0 \\
& (A .28)+(A .30)=0 \\
& (A .29)+(A .31)=0 \\
& (A .32)+(A .33)=0 .
\end{aligned}
$$


Moreover, remember that, in the final expression (7.150), the two terms that were derived in sections A.1 and A.2, respectively, are added to their own Hermitian conjugate. Taking this into account, the first four terms below (A.21), that is, all terms that depend on $\rho^{(M)}$, can be combined into a simple expression involving the concatenated commutator of the two-particle interaction-picture Hamiltonian and the $M$-particle reduced density matrix. To realize this, we need to identify the representation of products of two-particle operators in a basis of $M$-particle states. The representation of these terms is derived in general in appendix B.

Identifying $S=S^{\prime}=2, K^{(2)}=H_{I}^{(2)}(t), L^{(2)}=H_{I}^{(2)}(s)$ and $R^{(M)}=\rho_{I}^{(M)}(s)$ in equation (B.2) identifies the first term below (A.21) with $H_{I}^{(2)}(s) \rho_{I}^{(M)}(s) H_{I}^{(2)}(t)$ in a representation of $M$-particle states. To identify the next three terms we need equation (B.6). For $K^{(2)}=H_{I}^{(2)}(t), L^{(2)}=H_{I}^{(2)}(s)$ and $R^{(M)}=\rho_{I}^{(M)}(s)$, equation (B.6) indeed identifies the three following terms - the negative-sign terms that depend on $\rho^{(M)}$ below (A.21) - with the $M$-particle matrix elements of $H_{I}^{(2)}(t) H_{I}^{(2)}(s) \rho_{I}^{(M)}(s)$. Hence, these two results, together with equation (B.1), show that, when adding up the Hermitian conjugate expression, the first four terms below (A.21) yield the doublecommutator that reflects the coherent part of the time evolution:

$$
\begin{aligned}
\frac{1}{M !} & \sum_{\substack{n_{1} \ldots n_{M} \\
m_{1} \ldots m_{M}}} \sum_{i_{1} i_{2}}\left[\frac{1}{j_{1} j_{2}} M^{2}(M-1)^{2} \rho_{i_{1} i_{2} n_{3} \ldots n_{M} ; j_{1} j_{2} m_{3} \ldots m_{M}}^{(M)}(s) H_{n_{1} n_{2} ; i_{1} i_{2}}^{(2)}(s) H_{j_{1} j_{2} ; m_{1} m_{2}}^{(2)}(t)\right. \\
- & \frac{1}{2} M(M-1) \rho_{i_{1} i_{2} n_{3} \ldots n_{M} ; m_{1} \ldots m_{M}}^{(M)}(s) H_{n_{1} n_{2} ; j_{1} j_{2}}^{(2)}(t) H_{j_{1} j_{2} ; i_{1} i_{2}}^{(2)}(s) \\
- & M(M-1)(M-2) \rho_{i_{1} i_{2} j_{2} n_{4} \ldots n_{M} ; m_{1} \ldots m_{M}}^{(M)}(s) H_{n_{1} n_{2} ; j_{1} j_{2}}^{(2)}(t) H_{j_{1} n_{3} ; i_{1} i_{2}}^{(2)}(s) \\
& \left.-\frac{1}{4} M(M-1)(M-2)(M-3) \rho_{i_{1} i_{2} j_{1} j_{2} n_{5} \ldots n_{M} ; m_{1} \ldots m_{M}}^{(M)}(s) H_{n_{1} n_{2} ; j_{1} j_{2}}^{(2)}(t) H_{n_{3} n_{4} ; i_{1} i_{2}}^{(2)}(s)\right] \\
& \quad \times a_{n_{1}}^{\dagger} \ldots a_{n_{M}}^{\dagger} a_{m_{1}} \ldots a_{m_{M}}+\text { H.c. } \\
= & -\left[H_{I}^{(2)}(t),\left[H_{I}^{(2)}(s), \rho_{I}^{(M)}(s)\right]\right] .
\end{aligned}
$$

To summarize the results obtained so far for the time evolution (7.150), we group all non-vanishing terms into "coherent" and "incoherent" contributions, depending on whether they appear with a prefactor $(N-M)$ or not. The terminology is motivated by the fact that the terms depending on $N-M$ vanish when $M=N$, that is, when no particle is traced over. Using equation (7.108), we can also apply this to the initial $\operatorname{Tr}_{N-M}^{(N)}\left\{\left[H_{I}^{(2)}(t), \rho_{I}^{(N)}(0)\right]\right\}$. This finally yields equation (7.151). 



\section{B Representation of Double- COMMUTATOR TERMS IN A LARGER BASIS}

The second-order expansion of the interaction term, employed throughout section 7.5, naturally leads to dissipative terms in the shape of double-commutators, similar to the characteristic Lindblad dissipator terms of the standard open-system description. These terms generally have the form

$$
\begin{aligned}
& L^{(S)} R^{(M)} K^{\left(S^{\prime}\right)}-K^{\left(S^{\prime}\right)} L^{(S)} R^{(M)}+\text { H.c. } \\
= & L^{(S)} R^{(M)} K^{\left(S^{\prime}\right)}-K^{\left(S^{\prime}\right)} L^{(S)} R^{(M)}+K^{\left(S^{\prime}\right)} R^{(M)} L^{(S)}-R^{(M)} L^{(S)} K^{\left(S^{\prime}\right)} \\
= & {\left[L^{(S)} R^{(M)}, K^{\left(S^{\prime}\right)}\right]-\left[R^{(M)} L^{(S)}, K^{\left(S^{\prime}\right)}\right] } \\
= & -\left[K^{\left(S^{\prime}\right)},\left[L^{(S)}, R^{(M)}\right]\right]
\end{aligned}
$$

with arbitrary operators $K^{\left(S^{\prime}\right)}, L^{(S)}$ and $R^{(M)}$. In the approach followed in section 7.5, we project the full equation of motion (7.85) onto the subspace spanned by $M$-particle states. The above commutator terms are hard to identify in the resulting expressions, since the contributing operators often contain the contribution of lower particle numbers than $M$. This leads to the emergence of additional combinatorial factors, as predicted by equation (7.50). In this appendix, we explicitly derive the expression of the commutator terms (B.1) in a basis of lower particle number. 
Let us begin with the first term of equation (B.1) in all generality:

$$
\begin{aligned}
& \left\langle\varphi_{n_{1}} \ldots \varphi_{n_{M}}\left|L^{(S)} R^{(M)} K^{\left(S^{\prime}\right)}\right| \varphi_{m_{1}} \ldots \varphi_{m_{M}}\right\rangle \\
= & \sum_{\substack{i_{1} \ldots i_{M} \\
j_{1} \ldots j_{M}}}\left\langle\varphi_{n_{1}} \ldots \varphi_{n_{M}}\left|L^{(S)}\right| \varphi_{i_{1}} \ldots \varphi_{i_{M}}\right\rangle\left\langle\varphi_{j_{1}} \ldots \varphi_{j_{M}}\left|K^{\left(S^{\prime}\right)}\right| \varphi_{m_{1}} \ldots \varphi_{m_{M}}\right\rangle R_{i_{1} \ldots i_{M} ; j_{1} \ldots j_{M}}^{(M)} \\
= & \left(\begin{array}{c}
M \\
S
\end{array}\right)\left(\begin{array}{c}
M \\
S^{\prime}
\end{array}\right) \sum_{\substack{i_{1} \ldots i_{S} \\
j_{1} \ldots j_{S}}} L_{n_{1} \ldots n_{S} ; i_{1} \ldots i_{S}}^{(S)} K_{j_{1} \ldots j_{S^{\prime}} ; m_{1} \ldots m_{S^{\prime}}}^{\left(S^{\prime}\right)} R_{i_{1} \ldots i_{S} n_{S+1} \ldots n_{M} ; j_{1} \ldots j_{S^{\prime}} m_{S^{\prime}+1} \ldots m_{M}}^{(M)} .
\end{aligned}
$$

The second term is much harder to treat in the most general form. Thus, we restrict to those cases that are relevant in the context of the present dissertation, that is, oneand two-particle operators with $S, S^{\prime}=1,2$. We begin by considering single-particle operators of the form:

$$
\begin{aligned}
& \left\langle\varphi_{n_{1}} \ldots \varphi_{n_{M}}\left|K^{(1)} L^{(1)} \rho^{(M)}\right| \varphi_{m_{1}} \ldots \varphi_{m_{M}}\right\rangle \\
= & \sum_{\substack{i_{1} \ldots i_{M} \\
j_{1} \ldots j}}\left\langle\varphi_{n_{1}} \ldots \varphi_{n_{M}}\left|K^{(1)}\right| \varphi_{i_{1}} \ldots \varphi_{i_{M}}\right\rangle\left\langle\varphi_{i_{1}} \ldots \varphi_{i_{M}}\left|L^{(1)}\right| \varphi_{j_{1}} \ldots \varphi_{j_{M}}\right\rangle \rho_{j_{1} \ldots j_{M} ; m_{1} \ldots m_{M}}^{(M)} \sum_{=} \sum \sum_{i_{1} \ldots i_{M}}^{(1)} K_{n_{1} i_{1}} \delta_{n_{2} \ldots n_{M} ; i_{2} \ldots i_{M}} L_{i_{\alpha} j_{1}}^{(1)} \delta_{\left\{i_{1} \ldots i_{M}\right\} \backslash i_{\alpha} ; j_{2} \ldots j_{M}} \rho_{j_{1} \ldots j_{M} ; m_{1} \ldots m_{M}}^{(M)} \\
= & M \sum_{j_{1} \ldots j_{M}} K_{n_{1} i_{1}}^{(1)} L_{i_{1} j_{1}}^{(1)} \delta_{n_{2} \ldots n_{M} ; i_{2} \ldots i_{M}} \delta_{i_{2} \ldots i_{M} ; j_{2} \ldots j_{M}} \rho_{j_{1} \ldots j_{M} ; m_{1} \ldots m_{M}}^{(M)} \\
& +M(M-1) \sum_{j_{1} \ldots j_{M}} \sum_{i_{1} \ldots i_{M}}^{(1)} K_{n_{1} i_{1}} \delta_{n_{2} \ldots n_{M} ; i_{2} \ldots i_{M}} L_{i_{2} j_{1}}^{(1)} \delta_{i_{1} i_{3} \ldots i_{M} ; j_{2} \ldots j_{M}} \rho_{j_{1} \ldots j_{M} ; m_{1} \ldots m_{M}}^{(M)} \\
= & M \sum_{i_{1} j_{1}} K_{n_{1} i_{1}}^{(1)} L_{i_{1} j_{1}}^{(1)} \rho_{j_{1} n_{2} \ldots n_{M} ; m_{1} \ldots m_{M}}^{(M)} \\
& +M(M-1) \sum_{i_{1} j_{1}} K_{n_{1} i_{1}}^{(1)} L_{n_{2} j_{1}}^{(1)} \rho_{j_{1} i_{1} n_{3} \ldots n_{M} ; m_{1} \ldots m_{M}}^{(M)} .
\end{aligned}
$$

We now turn to combinations of operators acting on one and two particles. Using 
Lemma 10 a), we find

$$
\begin{aligned}
& \left\langle\varphi_{n_{1}} \ldots \varphi_{n_{M}}\left|K^{(2)} L^{(1)} R^{(M)}\right| \varphi_{m_{1}} \ldots \varphi_{m_{M}}\right\rangle \\
& =\sum_{\substack{i_{1} \ldots i_{M} \\
j_{1} \ldots j_{M}}}\left\langle\varphi_{n_{1}} \ldots \varphi_{n_{M}}\left|K^{(2)}\right| \varphi_{i_{1}} \ldots \varphi_{i_{M}}\right\rangle\left\langle\varphi_{i_{1}} \ldots \varphi_{i_{M}}\left|L^{(1)}\right| \varphi_{j_{1}} \ldots \varphi_{j_{M}}\right\rangle R_{j_{1} \ldots j_{M} ; m_{1} \ldots m_{M}}^{(M)} \\
& =\left(\begin{array}{c}
M \\
2
\end{array}\right) \sum_{\substack{i_{1} \ldots i_{M} \\
j_{1} \ldots j_{M}}} \sum_{1 \leq \alpha \leq M} K_{n_{1} n_{2} ; i_{1} i_{2}}^{(2)} \delta_{n_{3} \ldots n_{M} ; i_{3} \ldots i_{M}} L_{i_{\alpha} j_{1}}^{(1)} \delta_{\left\{i_{1} \ldots i_{M}\right\} \backslash i_{\alpha} ; j_{2} \ldots j_{M}} R_{j_{1} \ldots j_{M} ; m_{1} \ldots m_{M}}^{(M)} \\
& =\underbrace{\left(\begin{array}{c}
M \\
2
\end{array}\right) \frac{2(M-1) !}{M !}}_{M-1} \sum_{\substack{i_{1} \ldots i_{M} \\
j_{1} \ldots j_{M}}} K_{n_{1} n_{2} ; i_{1} i_{2}}^{(2)} \delta_{n_{3} \ldots n_{M} ; i_{3} \ldots i_{M}} L_{i_{1} j_{1}}^{(1)} \delta_{i_{2} \ldots i_{M} ; j_{2} \ldots j_{M}} R_{j_{1} \ldots j_{M} ; m_{1} \ldots m_{M}}^{(M)} \\
& +\underbrace{\left(\begin{array}{c}
M \\
2
\end{array}\right) \frac{(M-2)(M-1) !}{M !}}_{\frac{1}{2}(M-1)(M-2)} \sum_{\substack{i_{1} \ldots i_{M} \\
j_{1} \ldots j_{M}}} K_{n_{1} n_{2} ; i_{1} i_{2}}^{(2)} \delta_{n_{3} \ldots n_{M} ; i_{3} \ldots i_{M}} L_{i_{3} j_{1}}^{(1)} \\
& \times \delta_{i_{1} i_{2} i_{4} \ldots i_{M} ; j_{2} \ldots j_{M}} R_{j_{1} \ldots j_{M} ; m_{1} \ldots m_{M}}^{(M)} \\
& =(M-1) \sum_{i_{1} i_{2} j_{1}} K_{n_{1} n_{2} ; i_{1} i_{2}}^{(2)} L_{i_{1} j_{1}}^{(1)} R_{j_{1} i_{2} n_{3} \ldots n_{M} ; m_{1} \ldots m_{M}}^{(M)} \\
& +\frac{1}{2}(M-1)(M-2) \sum_{i_{1} i_{2} j_{1}} K_{n_{1} n_{2} ; i_{1} i_{2}}^{(2)} L_{n_{3} j_{1}}^{(1)} R_{j_{1} i_{1} i_{2} n_{4} \ldots n_{M} ; m_{1} \ldots m_{M}}^{(M)} .
\end{aligned}
$$

Similarly,

$$
\begin{aligned}
& \left\langle\varphi_{n_{1}} \ldots \varphi_{n_{M}}\left|K^{(1)} L^{(2)} R^{(M)}\right| \varphi_{m_{1}} \ldots \varphi_{m_{M}}\right\rangle \\
= & (M-1) \sum_{i_{1} i_{2} j_{1}} K_{n_{1} i_{1}}^{(1)} L_{n_{2} i_{1} ; j_{1} j_{2}}^{(2)} R_{j_{1} j_{2} n_{3} \ldots n_{M} ; m_{1} \ldots m_{M}}^{(M)} \\
& +\frac{1}{2}(M-1)(M-2) \sum_{i_{1} i_{2} j_{1}} K_{n_{1} i_{1}}^{(1)} L_{n_{2} n_{3} ; j_{1} j_{2}}^{(2)} R_{j_{1} j_{2} i_{1} n_{4} \ldots n_{M} ; m_{1} \ldots m_{M}}^{(M)} .
\end{aligned}
$$

Finally, using equation (7.50) and Lemma 9 with $r=M$ and $s=2$, we obtain for 
two two-particle operators

$$
\begin{aligned}
& \left\langle\varphi_{n_{1}} \ldots \varphi_{n_{M}}\left|K^{(2)} L^{(2)} R^{(M)}\right| \varphi_{m_{1}} \ldots \varphi_{m_{M}}\right\rangle \\
& =\sum_{\substack{i_{1} \ldots i_{M} \\
j_{1} \ldots j_{M}}}\left\langle\varphi_{n_{1}} \ldots \varphi_{n_{M}}\left|K^{(2)}\right| \varphi_{i_{1}} \ldots \varphi_{i_{M}}\right\rangle\left\langle\varphi_{i_{1}} \ldots \varphi_{i_{M}}\left|L^{(2)}\right| \varphi_{j_{1}} \ldots \varphi_{j_{M}}\right\rangle R_{j_{1} \ldots j_{M} ; m_{1} \ldots m_{M}}^{(M)} \\
& =\left(\begin{array}{c}
M \\
2
\end{array}\right) \sum_{\substack{i_{1} \ldots i_{M} \\
j_{1} \ldots j_{M}}} \sum_{1 \leq \alpha_{1}<\alpha_{2} \leq M} K_{n_{1} n_{2} ; i_{1} i_{2}}^{(2)} L_{i_{\alpha_{1}} i_{\alpha_{2}} ; j_{1} j_{2}}^{(2)} \delta_{n_{3} \ldots n_{M} ; i_{3} \ldots i_{M}} \delta_{\left\{i_{1} \ldots i_{M}\right\} \backslash\left\{i_{\alpha_{1}}, i_{\alpha_{2}}\right\} ; j_{3} \ldots j_{M}} \\
& \times R_{j_{1} \ldots j_{M} ; m_{1} \ldots m_{M}}^{(M)} \\
& =\left(\begin{array}{c}
M \\
2
\end{array}\right) \sum_{\substack{i_{1} \ldots i_{M} \\
j_{1} j_{2}}} K_{n_{1} n_{2} ; i_{1} i_{2}}^{(2)} L_{i_{1} i_{2} ; j_{1} j_{2}}^{(2)} \delta_{n_{3} \ldots n_{M} ; i_{3} \ldots i_{M}} R_{j_{1} j_{2} i_{3} \ldots i_{M} ; m_{1} \ldots m_{M}}^{(M)} \\
& +\left(\begin{array}{c}
M \\
2
\end{array}\right) 2(M-2) \sum_{\substack{i_{1} \ldots i_{M} \\
j_{1} j_{2}}} K_{n_{1} n_{2} ; i_{1} i_{2}}^{(2)} L_{i_{1} i_{3} ; j_{1} j_{2}}^{(2)} \delta_{n_{3} \ldots n_{M} ; i_{3} \ldots i_{M}} R_{j_{1} j_{2} i_{2} i_{4} \ldots i_{M} ; m_{1} \ldots m_{M}}^{(M)} \\
& +\left(\begin{array}{c}
M \\
2
\end{array}\right)\left(\begin{array}{c}
M-2 \\
2
\end{array}\right) \sum_{\substack{i_{1} \ldots i_{M} \\
j_{1} j_{2}}} K_{n_{1} n_{2} ; i_{1} i_{2}}^{(2)} L_{i_{3} i_{4} ; j_{1} j_{2}}^{(2)} \delta_{n_{3} \ldots n_{M} ; i_{3} \ldots i_{M}} R_{j_{1} j_{2} i_{1} i_{2} i_{5} \ldots i_{M} ; m_{1} \ldots m_{M}}^{(M)} \\
& =\frac{M(M-1)}{2} \sum_{i_{1} i_{2} j_{1} j_{2}} K_{n_{1} n_{2} ; i_{1} i_{2}}^{(2)} L_{i_{1} i_{2} ; j_{1} j_{2}}^{(2)} R_{j_{1} j_{2} n_{3} \ldots n_{M} ; m_{1} \ldots m_{M}}^{(M)} \\
& +M(M-1)(M-2) \sum_{i_{1} i_{2} j_{1} j_{2}} K_{n_{1} n_{2} ; i_{1} i_{2}}^{(2)} L_{i_{1} n_{3} ; j_{1} j_{2}}^{(2)} R_{j_{1} j_{2} i_{2} n_{4} \ldots n_{M} ; m_{1} \ldots m_{M}}^{(M)} \\
& +\frac{M(M-1)(M-2)(M-3)}{4} \sum_{i_{1} i_{2} j_{1} j_{2}} K_{n_{1} n_{2} ; i_{1} i_{2}}^{(2)} L_{n_{3} n_{4} ; j_{1} j_{2}}^{(2)} R_{j_{1} j_{2} i_{1} i_{2} n_{5} \ldots n_{M} ; m_{1} \ldots m_{M}}^{(M)} .
\end{aligned}
$$




\section{TRANSFORMATION PROPERTIES OF INTERACTION-PICTURE OPERATORS}

This appendix provides the rather abstract, general transformation properties of the operators that appear throughout section 7.5.

\section{C.1 Back-transformation to the Schrödinger picture: General prop- erties}

Section 7.5 describes the interaction-picture time evolution, based on the von Neumann equation (7.85). To obtain the corresponding evolution in the Schrödinger picture, we transform back using

$$
\rho^{(M)}(t)=U_{1}(t) \rho_{I}^{(M)}(t) U_{1}^{\dagger}(t)
$$

with $U_{1}(t)=\exp \left(-i H^{(1)} t / \hbar\right)$. We obtain

$$
i \hbar \frac{\partial}{\partial t} \rho^{(M)}(t)=\left[H^{(1)}, \rho^{(M)}(t)\right]+U_{1}(t)\left[i \hbar \frac{\partial}{\partial t} \rho_{I}^{(M)}(t)\right] U_{1}^{\dagger}(t) .
$$

Furthermore, we have

$$
U_{1}(t)\left[X, \rho_{I}^{(M)}(t)\right] U_{1}^{\dagger}(t)=\left[U_{1}(t) X U_{1}^{\dagger}(t), \rho^{(M)}(t)\right],
$$

and

$$
U_{1}(t)\left[X,\left[Y, \rho_{I}^{(M)}(t)\right]\right] U_{1}^{\dagger}(t)=\left[U_{1}(t) X U_{1}^{\dagger}(t),\left[U_{1}(t) Y U_{1}^{\dagger}(t), \rho^{(M)}(t)\right]\right] .
$$


Moreover, note that changing to a rotating frame commutes with the partial trace operation:

$$
\begin{aligned}
& \operatorname{Tr}_{N-M}^{(N)}\left\{U_{1}^{\dagger}(t) X U_{1}(t)\right\} \\
= & \frac{(N-M) !}{N ! M !} \sum_{\substack{n_{1} \ldots n_{M} \\
m_{1} \ldots m_{M}}} \operatorname{Tr}^{(N)}\left\{a_{n_{1}}^{\dagger} \ldots a_{n_{M}}^{\dagger} a_{m_{1}} \ldots a_{m_{M}} U_{1}^{\dagger}(t) X U_{1}(t)\right\} \\
& \times a_{m_{1}}^{\dagger} \ldots a_{m_{M}}^{\dagger} a_{n_{1}} \ldots a_{n_{M}} \\
= & \frac{(N-M) !}{N ! M !} \sum_{\substack{n_{1} \ldots n_{M} \\
m_{1} \ldots m_{M}}} \operatorname{Tr}^{(N)}\left\{b_{n_{1}}^{\dagger}(t) \ldots b_{n_{M}}^{\dagger}(t) b_{m_{1}}(t) \ldots b_{m_{M}}(t) X\right\} \\
& \times U_{1}^{\dagger}(t) b_{m_{1}}^{\dagger}(t) \ldots b_{m_{M}}^{\dagger}(t) b_{n_{1}}(t) \ldots b_{n_{M}}(t) U_{1}(t) \\
= & U_{1}^{\dagger}(t) \operatorname{Tr}_{N-M}^{(N)}\{X\} U_{1}(t),
\end{aligned}
$$

with the rotating basis

$$
b_{i}(t)=U_{1}(t) a_{i} U_{1}^{\dagger}(t) .
$$

In the field operator representation, the two-particle Hamiltonian in the interactionpicture can be written as

$$
\begin{aligned}
H_{I}^{(2)}(t) & =U_{1}^{\dagger}(t) H^{(2)} U_{1}(t) \\
& =\frac{1}{2} \int d \mathbf{x}_{1} \int d \mathbf{x}_{2} U_{1}^{\dagger}(t) \hat{\Psi}^{\dagger}\left(\mathbf{x}_{1}\right) \hat{\Psi}^{\dagger}\left(\mathbf{x}_{2}\right) U_{1}(t) V\left(\mathbf{x}_{1}, \mathbf{x}_{2}\right) U_{1}^{\dagger}(t) \hat{\Psi}\left(\mathbf{x}_{1}\right) \hat{\Psi}\left(\mathbf{x}_{2}\right) U_{1}(t) \\
& =\frac{1}{2} \int d \mathbf{x}_{1} \int d \mathbf{x}_{2} \hat{\Psi}_{I}^{\dagger}\left(t, \mathbf{x}_{1}\right) \hat{\Psi}_{I}^{\dagger}\left(t, \mathbf{x}_{2}\right) V\left(\mathbf{x}_{1}, \mathbf{x}_{2}\right) \hat{\Psi}_{I}\left(t, \mathbf{x}_{1}\right) \hat{\Psi}_{I}\left(t, \mathbf{x}_{2}\right)
\end{aligned}
$$

where we define

$$
\hat{\Psi}_{I}(t, \mathbf{x})=U_{1}^{\dagger}(t) \hat{\Psi}(\mathbf{x}) U_{1}(t)=\sum_{i} \varphi_{i}(\mathbf{x}) U_{1}^{\dagger}(t) a_{i} U_{1}(t) .
$$

We have $\hat{\Psi}_{I}(0, \mathbf{x})=\hat{\Psi}(\mathbf{x})$. These operators also satisfy (using that the vacuum state does not evolve under the influence of $H^{(1)}$ due to the absence of particles)

$$
\hat{\Psi}_{I}^{\dagger}(t, \mathbf{x})|0\rangle=U_{1}^{\dagger}(t)|\mathbf{x}\rangle,
$$

and, thus, we define

$$
\varphi_{i}(t, \mathbf{x})=\left\langle 0\left|\hat{\Psi}_{I}(t, \mathbf{x})\right| \varphi_{i}\right\rangle=\left\langle\mathbf{x}\left|U_{1}(t)\right| \varphi_{i}\right\rangle,
$$

allowing us to write

$$
\sum_{i} \varphi_{i}^{*}(t, \mathbf{x}) a_{i}^{\dagger}|0\rangle=\sum_{i}\left|\varphi_{i}\right\rangle\left\langle\varphi_{i}\left|U_{1}^{\dagger}(t)\right| \mathbf{x}\right\rangle=U_{1}^{\dagger}(t)|\mathbf{x}\rangle=\hat{\Psi}_{I}^{\dagger}(t, \mathbf{x})|0\rangle .
$$


Using this, we obtain for the matrix elements of the interaction-picture Hamiltonian in an arbitrary basis,

$$
\begin{aligned}
H_{i j ; k l}^{(2)}(t) & =\left\langle\varphi_{i} \varphi_{j}\left|H_{I}^{(2)}(t)\right| \varphi_{k} \varphi_{l}\right\rangle \\
& =\frac{1}{2} \int d \mathbf{x}_{1} \int d \mathbf{x}_{2}\left\langle\varphi_{i} \varphi_{j}\left|\hat{\Psi}_{I}^{\dagger}\left(t, \mathbf{x}_{1}\right) \hat{\Psi}_{I}^{\dagger}\left(t, \mathbf{x}_{2}\right) V\left(\mathbf{x}_{1}, \mathbf{x}_{2}\right) \hat{\Psi}_{I}\left(t, \mathbf{x}_{1}\right) \hat{\Psi}_{I}\left(t, \mathbf{x}_{2}\right)\right| \varphi_{k} \varphi_{l}\right\rangle \\
& =\int d \mathbf{x}_{1} \int d \mathbf{x}_{2} \varphi_{i}^{*}\left(t, \mathbf{x}_{1}\right) \varphi_{j}^{*}\left(t, \mathbf{x}_{2}\right) V\left(\mathbf{x}_{1}, \mathbf{x}_{2}\right) \varphi_{k}\left(t, \mathbf{x}_{1}\right) \varphi_{l}\left(t, \mathbf{x}_{2}\right),
\end{aligned}
$$

where we used $V\left(\mathbf{x}_{1}, \mathbf{x}_{2}\right)=V\left(\mathbf{x}_{2}, \mathbf{x}_{1}\right)$.

\section{C.2 Transformation properties of specific operators}

We now use the above rules to transform each of the relevant operators in equation (7.192)-(7.200) back into the Schrödinger picture.

Obviously, $U_{1}(t) H_{I}^{(2)}(t) U_{1}^{\dagger}(t)=H^{(2)}$.

Next, we have

$$
\begin{aligned}
C^{(1)}\left(t_{1}, t_{2}\right)= & \sum_{i j n m} H_{n j ; i m}^{(2)}\left(t_{1}\right)\left\langle\varphi_{i}\left|U_{1}^{\dagger}\left(t_{2}\right) \rho^{(1)}\left(t_{2}\right) U_{1}\left(t_{2}\right)\right| \varphi_{j}\right\rangle a_{n}^{\dagger} a_{m} \\
= & \sum_{i j n m} \int d \mathbf{x}_{1} \int d \mathbf{x}_{2} \int d \mathbf{x}_{3} \int d \mathbf{x}_{3}^{\prime} \varphi_{n}^{*}\left(t_{1}, \mathbf{x}_{1}\right) \varphi_{j}^{*}\left(t_{1}, \mathbf{x}_{2}\right) V\left(\mathbf{x}_{1}, \mathbf{x}_{2}\right) \varphi_{i}\left(t_{1}, \mathbf{x}_{1}\right) \\
& \times \varphi_{m}\left(t_{1}, \mathbf{x}_{2}\right) \varphi_{i}^{*}\left(t_{2}, \mathbf{x}_{3}\right) \rho^{(1)}\left(t_{2} ; \mathbf{x}_{3} ; \mathbf{x}_{3}^{\prime}\right) \varphi_{j}\left(t_{2}, \mathbf{x}_{3}^{\prime}\right) a_{n}^{\dagger} a_{m} .
\end{aligned}
$$

The completeness of the eigenfunctions $\left|\varphi_{i}\right\rangle$ leads to the following relation

$$
\begin{aligned}
\sum_{i} \varphi_{i}^{*}\left(t_{1}, \mathbf{x}_{1}\right) \varphi_{i}\left(t_{2}, \mathbf{x}_{2}\right) & =\sum_{i}\left\langle\mathbf{x}_{2}\left|U_{1}\left(t_{2}\right)\right| \varphi_{i}\right\rangle\left\langle\varphi_{i}\left|U_{1}^{\dagger}\left(t_{1}\right)\right| \mathbf{x}_{1}\right\rangle \\
& =\left\langle\mathbf{x}_{2}\left|U_{1}\left(t_{2}\right) U_{1}^{\dagger}\left(t_{1}\right)\right| \mathbf{x}_{1}\right\rangle \\
& =\operatorname{Tr}^{(1)}\left\{\hat{\Psi}^{\dagger}\left(t_{1}, \mathbf{x}_{1}\right) \hat{\Psi}\left(t_{2}, \mathbf{x}_{2}\right)\right\}
\end{aligned}
$$

The functions (C.14) play a fundamental role in many areas of physics. For instance, in condensed-matter theory, such functions are called Green's functions, where one typically introduces the $S$-matrix $S\left(t_{2}, t_{1}\right)=U_{1}\left(t_{2}\right) U_{1}^{\dagger}\left(t_{1}\right)$. Since the evolution $U_{1}(t)$ only involves the single-particle Hamiltonian, equation (C.14) is a free Green's function (Mahan, 2000). We assume that the single-particle Hamiltonian is time-independent which leads to the simple expression $S\left(t_{1}, t_{2}\right)=U_{1}\left(t_{2}-t_{1}\right)$. In a semi-classical pathintegral description of quantum mechanics, these functions are called propagators. For $t_{1}=t_{2}$, we recover the expression

$$
\sum_{i} \varphi_{i}^{*}\left(t, \mathbf{x}_{1}\right) \varphi_{i}\left(t, \mathbf{x}_{2}\right)=\delta\left(\mathbf{x}_{2}-\mathbf{x}_{1}\right)
$$


For equation (C.13) this leads to

$$
\begin{aligned}
C^{(1)}\left(t_{1}, t_{2}\right)= & \sum_{n m} \int d \mathbf{x}_{1} \int d \mathbf{x}_{2} \int d \mathbf{x}_{3} \int d \mathbf{x}_{3}^{\prime} \varphi_{n}^{*}\left(t_{1}, \mathbf{x}_{1}\right) V\left(\mathbf{x}_{1}, \mathbf{x}_{2}\right) \varphi_{m}\left(t_{1}, \mathbf{x}_{2}\right) a_{n}^{\dagger} a_{m} \\
& \times\left\langle\mathbf{x}_{1}\left|U\left(t_{1}-t_{2}\right)\right| \mathbf{x}_{3}\right\rangle \rho^{(1)}\left(t_{2} ; \mathbf{x}_{3} ; \mathbf{x}_{3}^{\prime}\right)\left\langle\mathbf{x}_{3}^{\prime}\left|U_{1}\left(t_{2}-t_{1}\right)\right| \mathbf{x}_{2}\right\rangle .
\end{aligned}
$$

Furthermore, equation (C.11) allows us to write

$$
\begin{aligned}
C^{(1)}\left(t_{1}, t_{2}\right)= & \int d \mathbf{x}_{1} \int d \mathbf{x}_{2} \int d \mathbf{x}_{3} \int d \mathbf{x}_{3}^{\prime} \hat{\Psi}_{I}^{\dagger}\left(t_{1}, \mathbf{x}_{1}\right) V\left(\mathbf{x}_{1}, \mathbf{x}_{2}\right) \hat{\Psi}_{I}\left(t_{1}, \mathbf{x}_{2}\right) \\
& \times\left\langle\mathbf{x}_{1}\left|U_{1}\left(t_{1}-t_{2}\right)\right| \mathbf{x}_{3}\right\rangle \rho^{(1)}\left(t_{2} ; \mathbf{x}_{3} ; \mathbf{x}_{3}^{\prime}\right)\left\langle\mathbf{x}_{3}^{\prime}\left|U_{1}\left(t_{2}-t_{1}\right)\right| \mathbf{x}_{2}\right\rangle \\
= & \int d \mathbf{x}_{1} \int d \mathbf{x}_{2} \hat{\Psi}_{I}^{\dagger}\left(t_{1}, \mathbf{x}_{1}\right) V\left(\mathbf{x}_{1}, \mathbf{x}_{2}\right) \hat{\Psi}_{I}\left(t_{1}, \mathbf{x}_{2}\right) \\
& \times\left\langle\mathbf{x}_{1}\left|U_{1}\left(t_{1}-t_{2}\right) \rho^{(1)}\left(t_{2}\right) U_{1}^{\dagger}\left(t_{1}-t_{2}\right)\right| \mathbf{x}_{2}\right\rangle .
\end{aligned}
$$

The field operators obey the transformation law

$$
U_{1}(t) \hat{\Psi}_{I}(s, \mathbf{x}) U_{1}^{\dagger}(t)=\sum_{i} \varphi_{i}(\mathbf{x}) U_{1}(t) U_{1}^{\dagger}(s) a_{i} U_{1}(s) U_{1}^{\dagger}(t)=\hat{\Psi}_{I}(s-t, \mathbf{x}) .
$$

From which we obtain for $C^{(1)}\left(t_{1}, t_{2}\right)$ in the Schrödinger picture:

$$
\begin{aligned}
U_{1}\left(t_{3}\right) C^{(1)}\left(t_{1}, t_{2}\right) U_{1}^{\dagger}\left(t_{3}\right)= & \int d \mathbf{x}_{1} \int d \mathbf{x}_{2} \hat{\Psi}_{I}^{\dagger}\left(t_{1}-t_{3}, \mathbf{x}_{1}\right) V\left(\mathbf{x}_{1}, \mathbf{x}_{2}\right) \hat{\Psi}_{I}\left(t_{1}-t_{3}, \mathbf{x}_{2}\right) \\
& \times\left\langle\mathbf{x}_{1}\left|U_{1}\left(t_{1}-t_{2}\right) \rho^{(1)}\left(t_{2}\right) U_{1}^{\dagger}\left(t_{1}-t_{2}\right)\right| \mathbf{x}_{2}\right\rangle
\end{aligned}
$$

In particular, when $t_{3}=t_{2}=t_{1}=t$, this yields $C^{(1)}(t)$, as defined in equation (7.117). This assures that the back-transformation of the coherent mean-field part (7.121), which also coincides with the first term in the dissipative mean-field master equation (7.206), reduces to the expression which was derived in the Schrödinger picture, equation (7.116).

Next, we deal with the operators $B_{\beta \alpha}^{(1)}(t)$, introduced in equation (7.138), which, in terms of field operators, read:

$$
\begin{aligned}
B_{\beta \alpha}^{(1)}(t)= & \sum_{i j} H_{i \beta ; j \alpha}^{(2)}(t) a_{i}^{\dagger} a_{j} \\
= & \frac{1}{2} \sum_{i j} \int d \mathbf{x}_{1} \int d \mathbf{x}_{2} \varphi_{i}^{*}\left(t, \mathbf{x}_{1}\right) \varphi_{\beta}^{*}\left(t, \mathbf{x}_{2}\right) V\left(\mathbf{x}_{1}, \mathbf{x}_{2}\right) \varphi_{j}\left(t, \mathbf{x}_{1}\right) \varphi_{\alpha}\left(t, \mathbf{x}_{2}\right) a_{i}^{\dagger} a_{j} \\
& +\frac{1}{2} \sum_{i j} \int d \mathbf{x}_{1} \int d \mathbf{x}_{2} \varphi_{i}^{*}\left(t, \mathbf{x}_{2}\right) \varphi_{\beta}^{*}\left(t, \mathbf{x}_{1}\right) V\left(\mathbf{x}_{1}, \mathbf{x}_{2}\right) \varphi_{j}\left(t, \mathbf{x}_{1}\right) \varphi_{\alpha}\left(t, \mathbf{x}_{2}\right) a_{i}^{\dagger} a_{j} \\
= & \frac{1}{2} \int d \mathbf{x}_{1} \int d \mathbf{x}_{2} \hat{\Psi}_{I}^{\dagger}\left(t, \mathbf{x}_{1}\right) \varphi_{\beta}^{*}\left(t, \mathbf{x}_{2}\right) V\left(\mathbf{x}_{1}, \mathbf{x}_{2}\right) \varphi_{\alpha}\left(t, \mathbf{x}_{2}\right) \hat{\Psi}_{I}\left(t, \mathbf{x}_{1}\right) \\
& +\frac{1}{2} \int d \mathbf{x}_{1} \int d \mathbf{x}_{2} \hat{\Psi}_{I}^{\dagger}\left(t, \mathbf{x}_{2}\right) \varphi_{\beta}^{*}\left(t, \mathbf{x}_{1}\right) V\left(\mathbf{x}_{1}, \mathbf{x}_{2}\right) \varphi_{\alpha}\left(t, \mathbf{x}_{2}\right) \hat{\Psi}_{I}\left(t, \mathbf{x}_{1}\right) .
\end{aligned}
$$


Transformation to the Schrödinger picture yields

$$
\begin{aligned}
& U_{1}\left(t_{2}\right) B_{\beta \alpha}^{(1)}\left(t_{1}\right) U_{1}^{\dagger}\left(t_{2}\right) \\
= & \frac{1}{2} \int d \mathbf{x}_{1} \int d \mathbf{x}_{2} \hat{\Psi}_{I}^{\dagger}\left(t_{1}-t_{2}, \mathbf{x}_{1}\right) \varphi_{\beta}^{*}\left(t_{1}, \mathbf{x}_{2}\right) V\left(\mathbf{x}_{1}, \mathbf{x}_{2}\right) \varphi_{\alpha}\left(t_{1}, \mathbf{x}_{2}\right) \hat{\Psi}_{I}\left(t_{1}-t_{2}, \mathbf{x}_{1}\right) \\
& +\frac{1}{2} \int d \mathbf{x}_{1} \int d \mathbf{x}_{2} \hat{\Psi}_{I}^{\dagger}\left(t_{1}-t_{2}, \mathbf{x}_{2}\right) \varphi_{\beta}^{*}\left(t_{1}, \mathbf{x}_{1}\right) V\left(\mathbf{x}_{1}, \mathbf{x}_{2}\right) \varphi_{\alpha}\left(t_{1}, \mathbf{x}_{2}\right) \hat{\Psi}_{I}\left(t_{1}-t_{2}, \mathbf{x}_{1}\right) .
\end{aligned}
$$

Similarly, $A_{\alpha \beta}\left(t_{1}, t_{2}\right)$, introduced in equation (7.137), read in terms of field operators:

$$
\begin{aligned}
A_{\alpha \beta}^{(1)}\left(t_{1}, t_{2}\right)= & \sum_{i j k} H_{i \alpha ; j k}^{(2)}\left(t_{1}\right) \rho_{k \beta}^{(1)}\left(t_{2}\right) a_{i}^{\dagger} a_{j} \\
= & \sum_{i j k} \int d \mathbf{x}_{1} \int d \mathbf{x}_{2} \frac{1}{2}\left[\varphi_{i}^{*}\left(t_{1}, \mathbf{x}_{1}\right) \varphi_{\alpha}^{*}\left(t_{1}, \mathbf{x}_{2}\right)+\varphi_{i}^{*}\left(t_{1}, \mathbf{x}_{2}\right) \varphi_{\alpha}^{*}\left(t_{1}, \mathbf{x}_{1}\right)\right] \\
& \times V\left(\mathbf{x}_{1}, \mathbf{x}_{2}\right) \varphi_{j}\left(t_{1}, \mathbf{x}_{1}\right) \varphi_{k}\left(t_{1}, \mathbf{x}_{2}\right)\left\langle\varphi_{k}\left|U_{1}^{\dagger}\left(t_{2}\right) \rho^{(1)}\left(t_{2}\right) U_{1}\left(t_{2}\right)\right| \varphi_{\beta}\right\rangle a_{i}^{\dagger} a_{j} \\
= & \frac{1}{2} \int d \mathbf{x}_{1} \int d \mathbf{x}_{2} \hat{\Psi}_{I}^{\dagger}\left(t_{1}, \mathbf{x}_{1}\right) V\left(\mathbf{x}_{1}, \mathbf{x}_{2}\right) \hat{\Psi}_{I}\left(t_{1}, \mathbf{x}_{1}\right) \\
& \times\left\langle\varphi_{\alpha}\left|\hat{\Psi}_{I}^{\dagger}\left(t_{1}, \mathbf{x}_{2}\right) \hat{\Psi}_{I}\left(t_{1}, \mathbf{x}_{2}\right) U_{1}^{\dagger}\left(t_{2}\right) \rho^{(1)}\left(t_{2}\right) U_{1}\left(t_{2}\right)\right| \varphi_{\beta}\right\rangle \\
+ & \frac{1}{2} \int d \mathbf{x}_{1} \int d \mathbf{x}_{2} \hat{\Psi}_{I}^{\dagger}\left(t_{1}, \mathbf{x}_{2}\right) V\left(\mathbf{x}_{1}, \mathbf{x}_{2}\right) \hat{\Psi}_{I}\left(t_{1}, \mathbf{x}_{1}\right) \\
& \times\left\langle\varphi_{\alpha}\left|\hat{\Psi}_{I}^{\dagger}\left(t_{1}, \mathbf{x}_{1}\right) \hat{\Psi}_{I}\left(t_{1}, \mathbf{x}_{2}\right) U_{1}^{\dagger}\left(t_{2}\right) \rho^{(1)}\left(t_{2}\right) U_{1}\left(t_{2}\right)\right| \varphi_{\beta}\right\rangle,
\end{aligned}
$$

and transforms to

$$
\begin{aligned}
U_{1}\left(t_{3}\right) A_{\alpha \beta}^{(1)}\left(t_{1}, t_{2}\right) U_{1}^{\dagger}\left(t_{3}\right)= & \frac{1}{2} \int d \mathbf{x}_{1} \int d \mathbf{x}_{2} \hat{\Psi}_{I}^{\dagger}\left(t_{1}-t_{3}, \mathbf{x}_{1}\right) V\left(\mathbf{x}_{1}, \mathbf{x}_{2}\right) \hat{\Psi}_{I}\left(t_{1}-t_{3}, \mathbf{x}_{1}\right) \\
& \times\left\langle\varphi_{\alpha}\left|\hat{\Psi}_{I}^{\dagger}\left(t_{1}, \mathbf{x}_{2}\right) \hat{\Psi}_{I}\left(t_{1}, \mathbf{x}_{2}\right) U_{1}^{\dagger}\left(t_{2}\right) \rho^{(1)}\left(t_{2}\right) U_{1}\left(t_{2}\right)\right| \varphi_{\beta}\right\rangle \\
+ & \frac{1}{2} \int d \mathbf{x}_{1} \int d \mathbf{x}_{2} \hat{\Psi}_{I}^{\dagger}\left(t_{1}-t_{3}, \mathbf{x}_{2}\right) V\left(\mathbf{x}_{1}, \mathbf{x}_{2}\right) \hat{\Psi}_{I}\left(t_{1}-t_{3}, \mathbf{x}_{1}\right) \\
& \times\left\langle\varphi_{\alpha}\left|\hat{\Psi}_{I}^{\dagger}\left(t_{1}, \mathbf{x}_{1}\right) \hat{\Psi}_{I}\left(t_{1}, \mathbf{x}_{2}\right) U_{1}^{\dagger}\left(t_{2}\right) \rho^{(1)}\left(t_{2}\right) U_{1}\left(t_{2}\right)\right| \varphi_{\beta}\right\rangle .
\end{aligned}
$$

To transform the effective Hamiltonian $H_{D}^{(1)}\left(t_{1}, t_{2}, t_{3}\right)$, see equation (7.189), back to 
the Schrödinger picture, we rewrite (C.14) as

$$
\begin{aligned}
D^{(1)}\left(t_{1}, t_{2}, t_{3}\right)= & \sum_{i j k l m p q} H_{i k ; j l}^{(2)}\left(t_{1}\right) H_{m l ; p q}^{(2)}\left(t_{2}\right) \rho_{p q ; k m}^{(2)}\left(t_{3}\right) a_{i}^{\dagger} a_{j} \\
= & \frac{1}{2} \int d \mathbf{x}_{1} \ldots \int d \mathbf{x}_{4} \hat{\Psi}_{I}^{\dagger}\left(t_{1}, \mathbf{x}_{1}\right) V\left(\mathbf{x}_{1}, \mathbf{x}_{2}\right) V\left(\mathbf{x}_{3}, \mathbf{x}_{4}\right)\left\langle\mathbf{x}_{2}\left|U_{1}\left(t_{1}-t_{2}\right)\right| \mathbf{x}_{4}\right\rangle \\
& \times\left\langle\mathbf{x}_{4}\left|U_{1}\left(t_{2}-t_{3}\right) \rho^{(1)}\left(t_{3}\right) U_{1}^{\dagger}\left(t_{2}-t_{3}\right)\right| \mathbf{x}_{3}\right\rangle \\
& \times\left\langle\mathbf{x}_{3}\left|U_{1}\left(t_{2}-t_{3}\right) \rho^{(1)}\left(t_{3}\right) U_{1}^{\dagger}\left(t_{1}-t_{3}\right)\right| \mathbf{x}_{2}\right\rangle \hat{\Psi}_{I}\left(t_{1}, \mathbf{x}_{1}\right) \\
+ & \frac{1}{2} \int d \mathbf{x}_{1} \ldots \int d \mathbf{x}_{4} \hat{\Psi}_{I}^{\dagger}\left(t_{1}, \mathbf{x}_{1}\right) V\left(\mathbf{x}_{1}, \mathbf{x}_{2}\right) V\left(\mathbf{x}_{3}, \mathbf{x}_{4}\right)\left\langle\mathbf{x}_{1}\left|U_{1}\left(t_{1}-t_{2}\right)\right| \mathbf{x}_{4}\right\rangle \\
& \times\left\langle\mathbf{x}_{4}\left|U_{1}\left(t_{2}-t_{3}\right) \rho^{(1)}\left(t_{3}\right) U_{1}^{\dagger}\left(t_{2}-t_{3}\right)\right| \mathbf{x}_{3}\right\rangle \\
& \times\left\langle\mathbf{x}_{3}\left|U_{1}\left(t_{2}-t_{3}\right) \rho^{(1)}\left(t_{3}\right) U_{1}^{\dagger}\left(t_{1}-t_{3}\right)\right| \mathbf{x}_{2}\right\rangle \hat{\Psi}_{I}\left(t_{1}, \mathbf{x}_{2}\right) .
\end{aligned}
$$

Finally, as above, a transformation with $U_{1}\left(t_{4}\right) D^{(1)}\left(t_{1}, t_{2}, t_{3}\right) U_{1}^{\dagger}\left(t_{4}\right)$ shifts the time arguments of the interaction-picture field operators. This can be used to transform $H_{D}^{(1)}\left(t_{1}, t_{2}, t_{3}\right)$ on the basis of equation (7.189). Moreover, the transformation properties of the other two remaining Hamiltonians $H_{S}^{(2)}\left(t_{1}, t_{2}, t_{3}\right)$ and $H_{E}^{(1)}\left(t_{1}, t_{2}, t_{3}\right)$, defined in equations (7.190) and (7.191), respectively, are fully determined by the properties of $H_{I}^{(2)}(t)$ and $C^{(1)}\left(t_{1}, t_{2}\right)$, which we derived above.

\section{C.3 Results for the delta-shaped interaction potential}

In this section, we apply the general transformation properties, as obtained in the preceding section, to the special case of a delta-shaped contact interaction potential, $V\left(\mathbf{x}_{1}, \mathbf{x}_{2}\right)=g \delta\left(\mathbf{x}_{1}-\mathbf{x}_{2}\right)$. We further consider the time arguments that appear in equation (7.204). We obtain

$$
U_{1}(t) C^{(1)}(t, t) U_{1}^{\dagger}(t)=g \int d \mathbf{x} \hat{\Psi}^{\dagger}(\mathbf{x}) \hat{\Psi}(\mathbf{x})\left\langle\mathbf{x}\left|\rho^{(1)}(t)\right| \mathbf{x}\right\rangle=C^{(1)}(t) .
$$

Furthermore,

$$
U_{1}(t) B_{\beta \alpha}^{(1)}(t) U_{1}^{\dagger}(t)=g \int d \mathbf{x} \hat{\Psi}^{\dagger}(\mathbf{x}) \varphi_{\beta}^{*}(t, \mathbf{x}) \varphi_{\alpha}(t, \mathbf{x}) \hat{\Psi}(\mathbf{x}),
$$

and, using equations (C.11) and (7.73) in (C.23), we obtain

$$
\begin{aligned}
& U_{1}(t) A_{\alpha \beta}^{(1)}(t-s, t) U_{1}^{\dagger}(t) \\
= & g \int d \mathbf{x} \hat{\Psi}_{I}^{\dagger}(-s, \mathbf{x}) \hat{\Psi}_{I}(-s, \mathbf{x})\left\langle\varphi_{\alpha}\left|\hat{\Psi}_{I}^{\dagger}(t-s, \mathbf{x}) \hat{\Psi}_{I}(t-s, \mathbf{x}) U_{1}^{\dagger}(t) \rho^{(1)}(t) U_{1}(t)\right| \varphi_{\beta}\right\rangle \\
= & g \int d \mathbf{x} \int d \mathbf{x}_{1} \int d \mathbf{x}_{2} \hat{\Psi}^{\dagger}\left(\mathbf{x}_{1}\right) \hat{\Psi}\left(\mathbf{x}_{2}\right)\left\langle\varphi_{\alpha}\left|U_{1}^{\dagger}(t) U_{1}(s)\right| \mathbf{x}\right\rangle\left\langle\mathbf{x}\left|U_{1}^{\dagger}(s)\right| \mathbf{x}_{2}\right\rangle \\
& \times\left\langle\mathbf{x}_{1}\left|U_{1}(s)\right| \mathbf{x}\right\rangle\left\langle\mathbf{x}\left|U_{1}^{\dagger}(s) \rho^{(1)}(t) U_{1}(t)\right| \varphi_{\beta}\right\rangle .
\end{aligned}
$$


From

$$
\begin{aligned}
& U_{1}(t) D^{(1)}(t, t-s, t) U_{1}^{\dagger}(t) \\
& =g^{2} \int d \mathbf{x}_{1} \int d \mathbf{x}_{2} \hat{\Psi}^{\dagger}\left(\mathbf{x}_{1}\right)\left\langle\mathbf{x}_{1}\left|U_{1}(s)\right| \mathbf{x}_{2}\right\rangle \\
& \quad \times\left\langle\mathbf{x}_{2}\left|U_{1}(-s) \rho^{(1)}(t) U_{1}^{\dagger}(-s)\right| \mathbf{x}_{2}\right\rangle\left\langle\mathbf{x}_{2}\left|U_{1}(-s) \rho^{(1)}(t)\right| \mathbf{x}_{1}\right\rangle \hat{\Psi}\left(\mathbf{x}_{1}\right)
\end{aligned}
$$

we obtain — recall the definition of $H_{D}^{(1)}$ in equation (7.189) via $D^{(1)}$,

$$
\begin{aligned}
& U_{1}(t) H_{D}^{(1)}(t, t-s, t) U_{1}^{\dagger}(t) \\
& =g^{2} \int d \mathbf{x}_{1} \int d \mathbf{x}_{2} \hat{\Psi}^{\dagger}\left(\mathbf{x}_{1}\right)\left\langle\mathbf{x}_{2}\left|U_{1}^{\dagger}(s) \rho^{(1)}(t) U_{1}(s)\right| \mathbf{x}_{2}\right\rangle \\
& \quad \times\left\langle\mathbf{x}_{1}\left|\left(-\frac{i}{\hbar}\right)\left[U_{1}(s)\left|\mathbf{x}_{2}\right\rangle\left\langle\mathbf{x}_{2}\right| U_{1}^{\dagger}(s), \rho^{(1)}(t)\right]\right| \mathbf{x}_{1}\right\rangle \hat{\Psi}\left(\mathbf{x}_{1}\right) .
\end{aligned}
$$

Finally, we find

$$
U_{1}(t) H_{I}^{(2)}(t) \rho_{I}^{(2)}(t) H_{I}^{(2)}(t-s) U_{1}^{\dagger}(t)=H^{(2)} \rho^{(2)}(t) U_{1}(t) H_{I}^{(2)}(t-s) U_{1}^{\dagger}(t),
$$

leading to

$$
\begin{aligned}
& U_{1}(t) H_{S}^{(2)}(t, t, t-s) U_{1}^{\dagger}(t) \\
= & -\frac{i}{\hbar} \frac{1}{2}\left(H^{(2)} \rho^{(2)}(t) U_{1}(t) H_{I}^{(2)}(t-s) U_{1}^{\dagger}(t)-U_{1}(t) H_{I}^{(2)}(t-s) U_{1}^{\dagger}(t) \rho^{(2)}(t) H^{(2)}\right),
\end{aligned}
$$

and

$$
\begin{aligned}
& U_{1}(t) C^{(1)}(t-s, t) \rho_{I}^{(1)}(t) C^{(1)}(t, t) U_{1}^{\dagger}(t) \\
= & U_{1}(t) C^{(1)}(t-s, t) U_{1}^{\dagger}(t) \rho^{(1)}(t) C^{(1)}(t) .
\end{aligned}
$$

From this last result, we obtain with equation (7.191)

$$
\begin{aligned}
& H_{E}^{(1)}(t-s, t, t) \\
= & -\frac{i}{\hbar} \frac{1}{2}\left(U_{1}(t) C^{(1)}(t-s, t) U_{1}^{\dagger}(t) \rho^{(1)}(t) C^{(1)}(t)\right. \\
& \left.-C^{(1)}(t) \rho^{(1)}(t) U_{1}(t) C^{(1)}(t-s, t) U_{1}^{\dagger}(t)\right) \\
= & -\frac{i}{\hbar} \frac{g^{2}}{2} \int d \mathbf{x}_{1} \int d \mathbf{x}_{2}\left\langle\mathbf{x}_{1}\left|U_{1}^{\dagger}(s) \rho^{(1)}(t) U_{1}(s)\right| \mathbf{x}_{1}\right\rangle\left\langle\mathbf{x}_{2}\left|\rho^{(1)}(t)\right| \mathbf{x}_{2}\right\rangle \\
& \times\left(U_{1}(s)\left|\mathbf{x}_{1}\right\rangle\left\langle\mathbf{x}_{1}\left|U_{1}^{\dagger}(s) \rho^{(1)}(t)\right| \mathbf{x}_{2}\right\rangle\left\langle\mathbf{x}_{2}|-| \mathbf{x}_{2}\right\rangle\left\langle\mathbf{x}_{2}\left|\rho^{(1)}(t) U_{1}(s)\right| \mathbf{x}_{1}\right\rangle\left\langle\mathbf{x}_{1}\right| U_{1}^{\dagger}(s)\right) .
\end{aligned}
$$

Thus, we find that even for the simple case of a contact-interaction potential, this back-transformation does not immediately reduce to an easily interpretable master equation. Without writing out the full expression, we remark that, when all these 
terms are inserted back into the respective equations (7.204) or (7.206), the dependence on the single-particle time-evolution operator can be lumped into expressions of the form

$$
\Lambda_{t}(X)=\int_{0}^{t} d s \int d \mathbf{x} U_{1}(s)|\mathbf{x}\rangle\left\langle\mathbf{x}\left|U_{1}^{\dagger}(s) X U_{1}(s)\right| \mathbf{x}\right\rangle\langle\mathbf{x}| U_{1}^{\dagger}(s),
$$

which represent complete dephasing in a basis of states $U(s)|\mathbf{x}\rangle$-remember, for example, the shape and interpretation of the controlled dephasing operation (3.3). We expect that the derivation of approximate expressions for such terms will require some sort of rotating-wave approximation, which might be implemented on the basis of an expansion in terms of eigen-operators of the superoperator generated by the commutator with the single-particle Hamiltonian. The explicit derivation thereof is left open for future work.

Let us finally discuss an alternative, explicit, approximate expression for equation (C.34), for a special case. If within some form of Markovian approximation the upper time integration limit can be shifted to infinity, and if the single-particle Hamiltonian describes an ergodic system, such that the time-average corresponds to the unitary average according to the Haar measure $d \mu(U)$ on the unitary group (Gessner and Breuer, 2013b), we can explicitly evaluate the integral to obtain

$$
\Lambda(X)=\lim _{t \rightarrow \infty} \Lambda_{t}(X) \approx \int d \mu(U) \int d \mathbf{x} U|\mathbf{x}\rangle\left\langle\mathbf{x}\left|U^{\dagger} X U\right| \mathbf{x}\right\rangle\langle\mathbf{x}| U^{\dagger} .
$$

We denote the volume of the state space spanned by the position eigenstates $|\mathbf{x}\rangle$ with $V$. This means we assume the particles to move in a confined volume. Then, the integral above can be evaluated explicitly and yields (Gessner and Breuer, 2013a)

$$
\Lambda(X) \approx \frac{V-1}{V^{2}-1}(X+(\operatorname{Tr} X) \mathbb{I}) \approx \frac{1}{V}(X+(\operatorname{Tr} X) \mathbb{I}) .
$$

Note, however, that this is unlikely to represent a generally applicable approach, since most model systems do not contain generic and ergodic single-particle Hamiltonians. When inserted in equation (7.206), we obtain, making the above approximations, the nonlinear single-particle master equation in the the Schrödinger picture:

$$
\begin{aligned}
\frac{\partial}{\partial t} \rho^{(1)}(t)= & -\frac{i}{\hbar}\left[H^{(1)}+(N-1) C^{(1)}(t), \rho^{(1)}(t)\right] \\
& -(N-1) \frac{g^{2}}{V \hbar^{2}} \int d \mathbf{x}\left[\hat{\Psi}^{\dagger}(\mathbf{x}) \hat{\Psi}(\mathbf{x}),\left[\rho^{(1)}(t) \hat{\Psi}^{\dagger}(\mathbf{x}) \hat{\Psi}(\mathbf{x}), \rho^{(1)}(t)\right]\right] .
\end{aligned}
$$




\section{PublicATiOns}

The following publications are based on the present dissertation:

- M. Gessner, F. Schlawin, and A. Buchleitner Probing Polariton Dynamics in Trapped Ions with Phase-Coherent Two-Dimensional Spectroscopy

The Journal of Chemical Physics 142, 212439 (2015).

- E. Carnio, A. Buchleitner, and M. Gessner Robust Asymptotic Entanglement under Multipartite Collective Dephasing Physical Review Letters 115, 010404 (2015).

- F. Schlawin, M. Gessner, S. Mukamel, and A. Buchleitner Nonlinear spectroscopy of trapped ions Physical Review A 90, 023603 (2014).

- M. Gessner, M. Ramm, H. Häffner, A. Buchleitner, and H.-P. Breuer Observing a Quantum Phase Transition by Measuring a Single Spin Europhysics Letters 107, 40005 (2014).

- M. Gessner, F. Schlawin, H. Häffner, S. Mukamel, and A. Buchleitner Nonlinear Spectroscopy of Controllable Many-Body Quantum Systems New Journal of Physics 16, 092001 (2014).

- M. Gessner, M. Ramm, T. Pruttivarasin, A. Buchleitner, H.-P. Breuer, and H. Häffner Local Detection of Quantum Correlations with a Single Trapped Ion Nature Physics 10, 105 (2014).

- M. Gessner and H.-P. Breuer Local witness for bipartite quantum discord Physical Review A 87, 042107 (2013). 
- M. Gessner, E.-M. Laine, H.-P. Breuer, and J. Piilo

Correlations in Quantum States and the Local Creation of Quantum Discord Physical Review A 85, 052122 (2012).

This dissertation further presents the theoretical foundations for the following experimental publications:

- J.-S. Tang, Y.-T. Wang, G. Chen, Y. Zou, C.-F. Li, G.-C. Guo, Y. Yu, M.-F. Li, G.-W. Zha, H.-Q. Ni, Z.-C. Niu, M. Gessner, and H.-P. Breuer

Experimental detection of polarization-frequency quantum correlations in a photonic quantum channel by local operations arXiv:1312.3365

- B. P. Lanyon, P. Jurcevic, C. Hempel, M. Gessner, V. Vedral, R. Blatt, and C. F. Roos

Experimental Generation of Quantum Discord via Noisy Processes Physical Review Letters 111, 100504 (2013). 


\section{List OF Figures}

2.1 Design principle of a Paul trap and the generated time-dependent electric field. . . . . . . . . . . . . . . . . . . . 13

2.2 Photograph of the Paul trap used in the Berkeley experiments and fluorescence of a string of ions. . . . . . . . . . . . . . . . . . . 14

2.3 Electronic states of ${ }^{40} \mathrm{Ca}^{+}$ions and laser transitions. . . . . . . . . . . . 17

2.4 Sideband transitions and laser-induced couplings between electronic state and motion. . . . . . . . . . . . . . . . . 21

2.5 Single-site resolution and individual addressing of cold atoms in an optical lattice. . . . . . . . . . . . . . . . . . . . 27

2.6 Scheme of a typical nonlinear spectroscopy experiment, the induced excitation pathway, and the resulting signal. . . . . . . . . . . . . 35

2.7 Poissonian and Wigner-Dyson level statistics. . . . . . . . . . . . . . 39

2.8 Factorizing initial conditions and the dynamical map. . . . . . . . . . . 42

3.1 Schematic representation of the local detection protocol. . . . . . . . . . 54

3.2 Coupling of electronic qubit and harmonic motion for ${ }^{40} \mathrm{Ca}^{+}$ions. . . . 68

3.3 Local detection scheme applied to a single trapped ion while driving the first blue sideband. . . . . . . . . . . . . . . . 70

3.4 Local trace distance for different initial states. . . . . . . . . . . . . . 73

3.5 Maximum local trace distance provides a lower bound for the dephasing disturbance. . . . . . . . . . . . . . . . 74

3.6 Temperature dependence of the local signal. . . . . . . . . . . . . . 75

3.7 Time-averaged local Hilbert-Schmidt distance quantifies total HilbertSchmidt based dephasing disturbance. . . . . . . . . . . . . . . 77

3.8 Experimental setup for the photonic experiment on local detection of polarization-frequency correlations. . . . . . . . . . . . . 81

3.9 Measured local trace distance evolution and theoretical prediction. . . . 87 
3.10 Maximum local distance bounds the total distance in the photonic experiment. . . . . . . . . . . . . . . . 89

3.11 Single-spin trace distance evolution shows a distinct peak at the quantum phase transition. . . . . . . . . . . . . . . 101

3.12 Thermal discord-type correlations of low-temperature states and their single-spin signature. . . . . . . . . . . . . . . . . . . . 102

3.13 Lower part of the excitation spectrum and energy gap between ground states of the spin chain. . . . . . . . . . . . . . . . . . . 104

4.1 State tomography and singular values during local amplitude damping. . 112

4.2 Final correlation rank as a function of geometric relations. . . . . . . . . 115

4.3 State tomography and singular value during collective dephasing. . . . . 116

4.4 Hierarchies of multipartite entanglement after collective dephasing. . . . 124

5.1 Dephasing-induced excitations by local dephasing of the spin-chain ground state. . . . . . . . . . . . . . . . . . . . . 131

5.2 Local dephasing-induced excitations as a function of the eigenstate index.133

5.3 Global time-autocorrelation function of the dephased state. . . . . . . 134

5.4 Spectra of small spin systems separated into parity subspaces. . . . . . . 135

5.5 Density of states for different $B$ at $N=12$ and $\alpha=1 \ldots \ldots$. . . . 136

5.6 Statistics of nearest-neighbor energy level spacing distributions. . . . . 138

5.7 Comparison of the numerically exact quantum spectrum and the semiclassical ground state energy for short- and long-range spin chains. . . . 145

5.8 Comparison of the multidimensional saddle point optimization with the numerically exact quantum spectrum. . . . . . . . . . . . . . . 147

5.9 Breakdown of the semiclassical saddle-point optimization for long-range spin chains. . . . . . . . . . . . . . . . . . . 148

5.10 Bifurcations of the semiclassical energy landscapes reflect the quantum phase transition. . . . . . . . . . . . . . . . . . . . . . 149

5.11 Comparison of the semiclassical spectrum and the numerically exact quantum spectrum for an anti-ferromagnet with long-range interactions. 154

5.12 Exact correspondence of the semiclassical energy levels with the quantum spectrum for $B \ll J_{0}$ and $B \gg J_{0}$. . . . . . . . . . . 156

5.13 Integrated density of states at $B=0$ as a function of the spin-spin interaction range. . . . . . . . . . . . . . . . . . 159

5.14 Deviation of semiclassical and numerically exact ground state energies. . 160

5.15 Convergence of the effective spin-spin couplings towards Riemann's zeta function and Dirichlet's eta function. . . . . . . . . . . . . . . . 162

5.16 Thermodynamic limit and finite-size scaling of effective spin-spin couplings. . . . . . . . . . . . . . . . . . . . . . . 164

5.17 The ferromagnetic spectra at $B=0$ as a function of alpha are bounded by Zeta and Eta functions. . . . . . . . . . . . . . . . 165

5.18 Histogram distributions of critical fields. . . . . . . . . . . . . . 169 
6.1 Diagrammatic representation of the contributions to the total signal in a simple two-pulse measurement scheme. . . . . . . . . . . . . . 180

6.2 Elementary excitations and second-order quantum coherence signals . . 185

6.3 Diagrams describing the third-order quantum coherence signal. . . . . . 187

6.4 Experimental scheme for a two-pulse nonlinear measurement protocol with single-site addressability. . . . . . . . . . . . . . . . . . . 192

6.5 Elementary building blocks for the diagrammatic representation of nonlinear measurement protocols. . . . . . . . . . . . . . . 193

6.6 Most relevant diagrams for the single quantum coherence signal. . . . 195

6.7 Excitation schemes to create phonons in an ion crystal. . . . . . . . . 199

6.8 Single quantum coherence signal for the phonons in an ion trap. . . . . 207

6.9 Two-dimensional lineshapes of the single quantum coherence signal distinguish different noise processes of ionic qubits. . . . . . . . . . . . 212

6.10 Single quantum coherence signal probing the quantum phase transition in a long-range interacting spin chain. . . . . . . . . . . . . . . . . 214

6.11 Signatures of steady-state currents in the single quantum coherence signal. 216

6.12 Double quantum coherence signal reveals anharmonic corrections to the trap potential. . . . . . . . . . . . . . . . . 218

6.13 Double quantum coherence signal discloses local participation of ions in the motion of common eigenmodes. . . . . . . . . . . . . . 220

6.14 Diagrams which contribute to the photon echo signal. . . . . . . . . . 221

6.15 Full photon echo signal as a function of the second time interval. . . . . 222

6.16 Contributions of individual diagrams to the full photon echo signal. . . . 224 



\section{BIBLIOGRAPHY}

Aaronson, S. and Arkhipov, A. The computational complexity of linear optics. Theory of Computing 9, 143-252 (2013).

Acín, A., Gisin, N., and Masanes, L. From Bell's Theorem to Secure Quantum Key Distribution. Physical Review Letters 97, 120405 (2006).

Acosta, V.M., Bauch, E., Ledbetter, M.P., Waxman, A., Bouchard, L.S., and Budker, D. Temperature Dependence of the Nitrogen-Vacancy Magnetic Resonance in Diamond. Physical Review Letters 104, 070801 (2010).

Adesso, G., D'Ambrosio, V., Nagali, E., Piani, M., and Sciarrino, F. Experimental Entanglement Activation from Discord in a Programmable Quantum Measurement. Physical Review Letters 112, 140501 (2014).

Aeschlimann, M., Brixner, T., Fischer, A., Kramer, C., Melchior, P., Pfeiffer, W., Schneider, C., Strüber, C., Tuchscherer, P., and Voronine, D.V. Coherent TwoDimensional Nanoscopy. Science 333, 1723-1726 (2011).

Alicki, R. Comment on "Reduced Dynamics Need Not Be Completely Positive". Physical Review Letters 75, 3020 (1995).

Alicki, R. and Lendi, K. Quantum Dynamical Semigroups and Applications (Springer, Berlin, Germany, 1987).

Allen, L., Beijersbergen, M.W., Spreeuw, R.J.C., and Woerdman, J.P. Orbital angular momentum of light and the transformation of Laguerre-Gaussian laser modes. Physical Review A 45, 8185-8189 (1992).

Amico, L., Fazio, R., Osterloh, A., and Vedral, V. Entanglement in many-body systems. Reviews of Modern Physics 80, 517-576 (2008). 
Anderlini, M., Lee, P.J., Brown, B.L., Sebby-Strabley, J., Phillips, W.D., and Porto, J.V. Controlled exchange interaction between pairs of neutral atoms in an optical lattice. Nature 448, 452-456 (2007).

Anderson, M.H., Ensher, J.R., Matthews, M.R., Wieman, C.E., and Cornell, E.A. Observation of Bose-Einstein Condensation in a Dilute Atomic Vapor. Science 269, 198-201 (1995).

Anderson, P.W. Absence of Diffusion in Certain Random Lattices. Physical Review 109, 1492-1505 (1958).

Arecchi, F.T., Courtens, E., Gilmore, R., and Thomas, H. Atomic Coherent States in Quantum Optics. Physical Review A 6, 2211-2237 (1972).

Asadian, A., Manzano, D., Tiersch, M., and Briegel, H.J. Heat transport through lattices of quantum harmonic oscillators in arbitrary dimensions. Physical Review E 87, 012109 (2013).

Aspect, A., Dalibard, J., and Roger, G. Experimental Test of Bell's Inequalities Using Time- Varying Analyzers. Physical Review Letters 49, 1804-1807 (1982a).

Aspect, A., Grangier, P., and Roger, G. Experimental Realization of Einstein-PodolskyRosen-Bohm Gedankenexperiment: A New Violation of Bell's Inequalities. Physical Review Letters 49, 91-94 (1982b).

Auccaise, R., Maziero, J., Céleri, L.C., Soares-Pinto, D.O., deAzevedo, E.R., Bonagamba, T.J., Sarthour, R.S., Oliveira, I.S., and Serra, R.M. Experimentally Witnessing the Quantumness of Correlations. Physical Review Letters 107, 070501 (2011).

Bacon, D., Lidar, D.A., and Whaley, K.B. Robustness of decoherence-free subspaces for quantum computation. Physical Review A 60, 1944-1955 (1999).

Bakr, W.S., Peng, A., Tai, M.E., Ma, R., Simon, J., Gillen, J.I., Fölling, S., Pollet, L., and Greiner, M. Probing the Superfluid-to-Mott Insulator Transition at the Single-Atom Level. Science 329, 547-550 (2010).

Bakr, W.S., Gillen, J.I., Peng, A., Folling, S., and Greiner, M. A quantum gas microscope for detecting single atoms in a Hubbard-regime optical lattice. Nature 462, 74-77 (2009).

Balakrishnan, R. and Bishop, A.R. Nonlinear excitations on a quantum ferromagnetic chain. Physical Review Letters 55, 537-540 (1985).

Barnett, S.M. and Phoenix, S.J.D. Information theory, squeezing, and quantum correlations. Physical Review A 44, 535-545 (1991).

Barreiro, J.T., Müller, M., Schindler, P., Nigg, D., Monz, T., Chwalla, M., Hennrich, M., Roos, C.F., Zoller, P., and Blatt, R. An Open-System Quantum Simulator with Trapped Ions. Nature 470, 486 (2011). 
Barrett, M., Chiaverini, J., Schaetz, T., Britton, J., Itano, W., Jost, J., Knill, E., Langer, C., Leibfried, D., Ozeri, R., and Wineland, D. Deterministic Quantum Teleportation of Atomic Qubits. Nature 429, 737-739 (2004).

Barton, P.A., Donald, C.J.S., Lucas, D.M., Stevens, D.A., Steane, A.M., and Stacey, D.N. Measurement of the lifetime of the $3 d^{2} D_{5 / 2}$ state in ${ }^{40} \mathrm{Ca}^{+}$. Physical Review A 62, 032503 (2000).

Basko, D., Aleiner, I., and Altshuler, B. Metal-insulator transition in a weakly interacting many-electron system with localized single-particle states. Annals of Physics 321, 1126-1205 (2006).

Bastidas, V.M., Pérez-Fernández, P., Vogl, M., and Brandes, T. Quantum Criticality and Dynamical Instability in the Kicked-Top Model. Physical Review Letters 112, 140408 (2014).

Batrouni, G.G. and Scalettar, R.T. Quantum Phase Transitions. In C. Miniatura, L.C. Kwek, M. Ducloy, B. Grémaud, B.G. Englert, L. Cugliandolo, A. Ekert, and K.K. Phua (Editors), Ultracold Gases and Quantum Information - Lecture Notes of the Les Houches Summer School, pp. 356-394 (Oxford University Press, Oxford, UK, 2011).

Beenakker, C.W.J. Random-matrix theory of quantum transport. Reviews of Modern Physics 69, 731-808 (1997).

Bell, J.S. On the Einstein-Podolsky-Rosen Paradox. Physics 1, 195-200 (1964).

Benassi, A., Vanossi, A., and Tosatti, E. Nanofriction in cold ion traps. Nature Communications 2, 236 (2011).

Benatti, F., Floreanini, R., and Olivares, S. Non-divisibility and non-Markovianity in a Gaussian dissipative dynamics. Physics Letters A 376, 2951-2954 (2012).

Bengtsson, I. and Życzkowski, K. Geometry of Quantum States (Cambridge University Press, Cambridge, UK, 2006).

Bennett, C.H., Bernstein, H.J., Popescu, S., and Schumacher, B. Concentrating partial entanglement by local operations. Physical Review A 53, 2046-2052 (1996a).

Bennett, C.H., Brassard, G., Crépeau, C., Jozsa, R., Peres, A., and Wootters, W.K. Teleporting an Unknown Quantum State via Dual Classical and EinsteinPodolsky-Rosen Channels. Physical Review Letters 70, 1895-1899 (1993).

Bennett, C.H., DiVincenzo, D.P., Smolin, J.A., and Wootters, W.K. Mixed-state entanglement and quantum error correction. Physical Review A 54, 3824-3851 (1996b).

Bennett, C. and Brassard, G. Quantum cryptography: Public key distribution and coin tossing. In Proceedings of the IEEE International Conference on Computers, Systems and Signal Processing, Bangalore, India, p. 175 (IEEE, New York, 1984). 
Benson, O., Raithel, G., and Walther, H. Quantum jumps of the micromaser field: Dynamic behavior close to phase transition points. Physical Review Letters $\mathbf{7 2}$, 3506-3509 (1994).

Bergmann, K., Theuer, H., and Shore, B.W. Coherent population transfer among quantum states of atoms and molecules. Reviews of Modern Physics 70, 10031025 (1998).

Berkeland, D.J., Miller, J.D., Bergquist, J.C., Itano, W.M., and Wineland, D.J. LaserCooled Mercury Ion Frequency Standard. Physical Review Letters 80, 2089-2092 (1998).

Bermudez, A., Bruderer, M., and Plenio, M.B. Controlling and Measuring Quantum Transport of Heat in Trapped-Ion Crystals. Physical Review Letters 111, 040601 (2013).

Berry, M.V. and Tabor, M. Level Clustering in the Regular Spectrum. Proceedings of the Royal Society of London A 356, 375-394 (1977).

Bethe, H. Zur Theorie der Metalle. Zeitschrift für Physik 71, 205-226 (1931).

Billy, J., Josse, V., Zuo, Z., Bernard, A., Hambrecht, B., Lugan, P., Clément, D., Sanchez-Palencia, L., Bouyer, P., and Aspect, A. Direct observation of Anderson localization of matter waves in a controlled disorder. Nature 453, 891-894 (2008).

Birkl, G., Kassner, S., and Walther, H. Multiple-shell structures of laser-cooled $24 M g+$ ions in a quadrupole storage ring. Nature 357, 310-313 (1992).

Blatt, R. and Roos, C.F. Quantum simulations with trapped ions. Nature Physics 8, 277-284 (2012).

Bloch, F. Über die Quantenmechanik der Elektronen in Kristallgittern. Zeitschrift für Physik 52, 555-600 (1928).

Bloch, F. Nuclear Induction. Physical Review 70, 460-474 (1946).

Bloch, I., Dalibard, J., and Zwerger, W. Many-body physics with ultracold gases. Reviews of Modern Physics 80, 885-964 (2008).

Blockley, C.A., Walls, D.F., and Risken, H. Quantum Collapses and Revivals in a Quantized Trap. Europhysics Letters 17, 509 (1992).

Bogoliubov, N.N. Kinetic Equations. Journal of Physics USSR 10, 265-274 (1946).

Bogoliubov, N.N. On the Theory of Superfluidity. Journal of Physics USSR 11, 23-32 (1947).

Bogoliubov, N.N. Lectures on Quantum Statistics, Volume 1. Quantum Statistics (Gordon \& Breach, New York, NY, 1967a). 
Bogoliubov, N.N. Lectures on Quantum Statistics, Volume 2. Quasi-Averages (Gordon \& Breach, New York, NY, 1967b).

Bogoliubov, N.N. and Gurov, K.P. Kinetic Equations in Quantum Mechanics. Journal of Experimental and Theoretical Physics (in Russian) 17, 614-628 (1947).

Bohigas, O., Giannoni, M.J., and Schmit, C. Characterization of Chaotic Quantum Spectra and Universality of Level Fluctuation Laws. Physical Review Letters 52, $1-4$ (1984).

Born, M. and Green, H.S. A General Kinetic Theory of Liquids. I. The Molecular Distribution Functions. Proceedings of the Royal Society of London A: Mathematical, Physical and Engineering Sciences 188, 10-18 (1946).

Borrelli, M., Haikka, P., De Chiara, G., and Maniscalco, S. Non-Markovian qubit dynamics induced by Coulomb crystals. Physical Review A 88, 010101 (2013).

Boschi, D., Branca, S., De Martini, F., Hardy, L., and Popescu, S. Experimental Realization of Teleporting an Unknown Pure Quantum State via Dual Classical and Einstein-Podolsky-Rosen Channels. Physical Review Letters 80, 1121-1125 (1998).

Botet, R. and Jullien, R. Large-size critical behavior of infinitely coordinated systems. Physical Review B 28, 3955-3967 (1983).

Botet, R., Jullien, R., and Pfeuty, P. Size Scaling for Infinitely Coordinated Systems. Physical Review Letters 49, 478-481 (1982).

Bouwmeester, D., Pan, J.W., Mattle, K., Eibl, M., Weinfurter, H., and Zeilinger, A. Experimental quantum teleportation. Nature 390, 575-579 (1997).

Bouyer, P. Quantum gases and optical speckle: a new tool to simulate disordered quantum systems. Reports on Progress in Physics 73, 062401 (2010).

Brack, M. and Bhaduri, R.K. Semiclassical Physics (Addison-Wesley, Reading, MA, 1997).

Brańczyk, A.M., Turner, D.B., and Scholes, G.D. Crossing disciplines - A view on two-dimensional optical spectroscopy. Annalen der Physik 526, 31-49 (2014).

Breuer, H.P. and Petruccione, F. The Theory of Open Quantum Systems (Oxford University Press, Oxford, UK, 2002).

Breuer, H.P. Foundations and measures of quantum non-Markovianity. Journal of Physics B: Atomic, Molecular and Optical Physics 45, 154001 (2012).

Breuer, H.P., Kappler, B., and Petruccione, F. The Time-Convolutionless Projection Operator Technique in the Quantum Theory of Dissipation and Decoherence. Annals of Physics 291, 36-70 (2001). 
Breuer, H.P., Laine, E.M., and Piilo, J. Measure for the Degree of non-Markovian Behavior of Quantum Processes in Open Systems. Physical Review Letters 103, 210401 (2009).

Briegel, H.J. and Raussendorf, R. Persistent Entanglement in Arrays of Interacting Particles. Physical Review Letters 86, 910-913 (2001).

Britton, J.W., Sawyer, B.C., Keith, A.C., Wang, C.C.J., Freericks, J.K., Uys, H., Biercuk, M.J., and Bollinger, J.J. Engineered two-dimensional Ising interactions in a trapped-ion quantum simulator with hundreds of spins. Nature 484, 489-492 (2012).

Brixner, T., Stenger, J., Vaswani, H.M., Cho, M., Blankenship, R.E., and Fleming, G.R. Two-dimensional spectroscopy of electronic couplings in photosynthesis. Nature 434, 625-628 (2005).

Broome, M.A., Fedrizzi, A., Rahimi-Keshari, S., Dove, J., Aaronson, S., Ralph, T.C., and White, A.G. Photonic Boson Sampling in a Tunable Circuit. Science $\mathbf{3 3 9}$, 794-798 (2013).

Brouwer, P.W. and Beenakker, C.W.J. Diagrammatic Method of Integration over the Unitary Group, with Applications to Quantum Transport in Mesoscopic Systems. Journal of Mathematical Physics 37, 4904 (1996).

Brown, K.R., Ospelkaus, C., Colombe, Y., Wilson, A.C., Leibfried, D., and Wineland, D.J. Coupled quantized mechanical oscillators. Nature 471, 196-199 (2011).

Bruder, L. private communication (2014).

Buchleitner, A., Delande, D., and Zakrzewski, J. Non-dispersive wave packets in periodically driven quantum systems. Physics Reports 368, 409-547 (2002).

Buchleitner, A. and Kolovsky, A.R. Interaction-Induced Decoherence of Atomic Bloch Oscillations. Physical Review Letters 91, 253002 (2003).

Cahill, K.E. and Glauber, R.J. Density Operators and Quasiprobability Distributions. Physical Review 177, 1882-1902 (1969).

Caldeira, A. and Leggett, A. Quantum tunnelling in a dissipative system. Annals of Physics 149, 374-456 (1983).

Campbell, S., Mazzola, L., and Paternostro, M. Global Quamtum Correlations in the Ising Model. International Journal of Quantum Information 9, 1685-1699 (2011).

Cannas, S.A. and Tamarit, F.A. Long-range interactions and nonextensivity in ferromagnetic spin models. Physical Review B 54, R12661-R12664 (1996).

Caprio, M., Cejnar, P., and Iachello, F. Excited state quantum phase transitions in many-body systems. Annals of Physics 323, 1106-1135 (2008). 
Carnio, E.G. Multipartite correlated dephasing. Master's thesis, Albert-LudwigsUniversität Freiburg (2014).

Carnio, E.G., Buchleitner, A., and Gessner, M. Robust Asymptotic Entanglement under Multipartite Collective Dephasing. Phys. Rev. Lett. 115, 010404 (2015).

Cejnar, P., Macek, M., Heinze, S., Jolie, J., and Dobeš, J. Monodromy and excited-state quantum phase transitions in integrable systems: collective vibrations of nuclei. Journal of Physics A: Mathematical and General 39, L515 (2006).

Cejnar, P. and Stránský, P. Impact of quantum phase transitions on excited-level dynamics. Physical Review E 78, 031130 (2008).

Chu, S. Nobel Lecture: The manipulation of neutral particles. Reviews of Modern Physics 70, 685-706 (1998).

Chu, S., Bjorkholm, J.E., Ashkin, A., and Cable, A. Experimental Observation of Optically Trapped Atoms. Physical Review Letters 57, 314-317 (1986).

Chuan, T.K., Maillard, J., Modi, K., Paterek, T., Paternostro, M., and Piani, M. Quantum Discord Bounds the Amount of Distributed Entanglement. Physical Review Letters 109, 070501 (2012).

Chung, H.S., Ganim, Z., Jones, K.C., and Tokmakoff, A. Transient 2D IR spectroscopy of ubiquitin unfolding dynamics. Proceedings of the National Academy of Sciences 104, 14237-14242 (2007).

Cialdi, S., Smirne, A., Paris, M.G.A., Olivares, S., and Vacchini, B. Two-step procedure to discriminate discordant from classical correlated or factorized states. Physical Review A 90, 050301 (2014).

Ciccarello, F. and Giovannetti, V. Creating quantum correlations through local nonunitary memoryless channels. Physical Review A 85, 010102 (2012).

Cirac, J.I. and Zoller, P. Quantum Computations with Cold Trapped Ions. Physical Review Letters 74, 4091-4094 (1995).

Cirac, J.I. and Zoller, P. A scalable quantum computer with ions in an array of microtraps. Nature 404, 579-581 (2000).

Clarke, J. and Wilhelm, F.K. Superconducting quantum bits. Nature 453, 1031-1042 (2008).

Clos, G. and Breuer, H.P. Quantification of memory effects in the spin-boson model. Physical Review A 86, 012115 (2012).

Cohen-Tannoudji, C., Dupont-Roc, J., and Grynberg, G. Atom-Photon Interactions (Wiley, Weinheim, Germany, 1992). 
Cohen-Tannoudji, C., Diu, B., and Laloë, F. Quantum Mechanics, volume I (Hermann \& WILEY-VCH, Paris, France, 1977a).

Cohen-Tannoudji, C., Diu, B., and Laloë, F. Quantum Mechanics, volume II (Hermann \& WILEY-VCH, Paris, France, 1977b).

Cohen-Tannoudji, C.N. Nobel Lecture: Manipulating atoms with photons. Reviews of Modern Physics 70, 707-719 (1998).

Coleman, A. Structure of Fermion Density Matrices. Reviews of Modern Physics 35, 668-686 (1963).

Collins, B. Moments and Cumulants of Polynomial Random Variables on Unitary Groups, the Itzykson-Zuber Integral, and Free Probability. International Mathematics Research Notices 17, 953-982 (2003).

Collins, B. and Sniady, P. Integration with Respect to the Haar Measure on Unitary, Orthogonal and Symplectic Group. Communications in Mathematical Physics 264, 773-795 (2006).

Conway, J.H. and Guy, R.K. The book of numbers (Copernicus, New York, NY, 1998).

Crespi, A., Osellame, R., Ramponi, R., Brod, D.J., Galvao, E.F., Spagnolo, N., Vitelli, C., Maiorino, E., Mataloni, P., and Sciarrino, F. Integrated multimode interferometers with arbitrary designs for photonic boson sampling. Nature Photonics $\mathbf{7}$, 545-549 (2013).

Cubitt, T.S., Verstraete, F., Dür, W., and Cirac, J.I. Separable States Can Be Used To Distribute Entanglement. Physical Review Letters 91, 037902 (2003).

Cundiff, S.T. and Mukamel, S. Optical multidimensional coherent spectroscopy. Physics Today 66, 44-49 (2013).

Curie, P. Propriétés magnétiques des corps a diverses températures. Annales de Chimie et de Physique 5, 289-405 (1895).

Dai, X., Bristow, A.D., Karaiskaj, D., and Cundiff, S.T. Two-dimensional Fouriertransform spectroscopy of potassium vapor. Physical Review A 82, 052503 (2010).

Dai, X., Richter, M., Li, H., Bristow, A.D., Falvo, C., Mukamel, S., and Cundiff, S.T. Two-Dimensional Double-Quantum Spectra Reveal Collective Resonances in an Atomic Vapor. Physical Review Letters 108, 193201 (2012).

Dakić, B., Lipp, Y.O., Ma, X., Ringbauer, M., Kropatschek, S., Barz, S., Paterek, T., Vedral, V., Zeilinger, A., Brukner, C., and Walther, P. Quantum discord as resource for remote state preparation. Nature Physics 8, 666-670 (2012).

Dakić, B., Vedral, V., and Brukner, C. Necessary and Sufficient Condition for Nonzero Quantum Discord. Physical Review Letters 105, 190502 (2010). 
Dalibard, J. and Cohen-Tannoudji, C. Dressed-atom approach to atomic motion in laser light: the dipole force revisited. Journal of the Optical Society of America B 2, 1707-1720 (1985).

Dalibard, J., Castin, Y., and Mølmer, K. Wave-function approach to dissipative processes in quantum optics. Physical Review Letters 68, 580-583 (1992).

Dalichaouch, R., Armstrong, J.P., Schultz, S., Platzman, P.M., and McCall, S.L. Microwave localization by two-dimensional random scattering. Nature 354, 53-55 (1991).

Daniilidis, N., Gerber, S., Bolloten, G., Ramm, M., Ransford, A., Ulin-Avila, E., Talukdar, I., and Häffner, H. Surface noise analysis using a single-ion sensor. Physical Review B 89, 245435 (2014).

Danzl, J.G., Haller, E., Gustavsson, M., Mark, M.J., Hart, R., Bouloufa, N., Dulieu, O., Ritsch, H., and Nägerl, H.C. Quantum Gas of Deeply Bound Ground State Molecules. Science 321, 1062-1066 (2008).

Datta, A., Flammia, S.T., and Caves, C.M. Entanglement and the power of one qubit. Physical Review A 72, 042316 (2005).

Datta, A., Shaji, A., and Caves, C.M. Quantum Discord and the Power of One Qubit. Physical Review Letters 100, 050502 (2008).

Davies, E.B. Quantum Theory of Open Systems (Academic Press, London, UK, 1976).

Davis, K.B., Mewes, M.O., Andrews, M.R., van Druten, N.J., Durfee, D.S., Kurn, D.M., and Ketterle, W. Bose-Einstein Condensation in a Gas of Sodium Atoms. Physical Review Letters 75, 3969-3973 (1995).

De Chiara, G., Calarco, T., Fishman, S., and Morigi, G. Ramsey interferometry with a spin embedded in a Coulomb chain. Physical Review A 78, 043414 (2008).

de Finetti, B. La prévision: ses lois logiques, ses sources subjectives. Annales de l'institut Henri Poincaré 7, 1-68 (1937).

Delande, D. and Gay, J.C. Quantum Chaos and Statistical Properties of Energy Levels: Numerical Study of the Hydrogen Atom in a Magnetic Field. Physical Review Letters 57, 2006-2009 (1986).

Delande, D. and Buchleitner, A. Classical and Quantum Chaos in Atomic Systems. Advances In Atomic, Molecular, and Optical Physics 34, 85-123 (1994).

Deslauriers, L., Olmschenk, S., Stick, D., Hensinger, W.K., Sterk, J., and Monroe, C. Scaling and Suppression of Anomalous Heating in Ion Traps. Physical Review Letters 97, 103007 (2006).

Dicke, R.H. Coherence in Spontaneous Radiation Processes. Physical Review 93, 99-110 (1954). 
Diedrich, F., Bergquist, J.C., Itano, W.M., and Wineland, D.J. Laser Cooling to the Zero-Point Energy of Motion. Physical Review Letters 62, 403-406 (1989).

Dieks, D. Communication by EPR devices. Physics Letters A 92, 271-272 (1982).

DiVincenzo, D.P. Two-bit gates are universal for quantum computation. Physical Review A 51, 1015-1022 (1995).

DiVincenzo, D. Dogma and heresy in quantum computing. Quantum Information and Computation 1, 1 (2001).

Duan, L.M., Demler, E., and Lukin, M.D. Controlling Spin Exchange Interactions of Ultracold Atoms in Optical Lattices. Physical Review Letters 91, 090402 (2003).

Duan, L.M., Lukin, M.D., Cirac, J.I., and Zoller, P. Long-distance quantum communication with atomic ensembles and linear optics. Nature 414, 413-418 (2001).

Dür, W., Vidal, G., and Cirac, J.I. Three qubits can be entangled in two inequivalent ways. Physical Review A 62, 062314 (2000).

Dusuel, S. and Vidal, J. Finite-Size Scaling Exponents of the Lipkin-Meshkov-Glick Model. Physical Review Letters 93, 237204 (2004).

Dusuel, S. and Vidal, J. Continuous unitary transformations and finite-size scaling exponents in the Lipkin-Meshkov-Glick model. Physical Review B 71, 224420 (2005).

Dyson, F.J. Statistical Theory of the Energy Levels of Complex Systems. I. Journal of Mathematical Physics 3, 140 (1962).

Dyson, F. Existence of a phase-transition in a one-dimensional Ising ferromagnet. Communications in Mathematical Physics 12, 91-107 (1969).

Edwards, H.M. Riemann's Zeta function (Academic Press, New York, NY, 1974).

Eggeling, T. and Werner, R.F. Separability properties of tripartite states with $U \otimes U \otimes U$ symmetry. Physical Review A 63, 042111 (2001).

Ehrenfest, P. Bemerkung über die angenäherte Gültigkeit der klassischen Mechanik innerhalb der Quantenmechanik. Zeitschrift für Physik 45, 455-457 (1927).

Einstein, A., Podolsky, B., and Rosen, N. Can Quantum-Mechanical Description of Physical Reality Be Considered Complete? Physical Review 47, 777-780 (1935).

Eisaman, M.D., Fan, J., Migdall, A., and Polyakov, S.V. Invited Review Article: Single-photon sources and detectors. Review of Scientific Instruments 82, 071101 (2011).

Ekert, A. and Knight, P.L. Entangled quantum systems and the Schmidt decomposition. American Journal of Physics 63, 415-423 (1995). 
Ekert, A.K. Quantum Cryptography Based on Bell's Theorem. Physical Review Letters 67, 661-663 (1991).

Emary, C. and Brandes, T. Chaos and the quantum phase transition in the Dicke model. Physical Review E 67, 066203 (2003a).

Emary, C. and Brandes, T. Quantum Chaos Triggered by Precursors of a Quantum Phase Transition: The Dicke Model. Physical Review Letters 90, 044101 (2003b).

Engel, G.S., Calhoun, T.R., Read, E.L., Ahn, T.K., Mancal, T., Cheng, Y.C., Blankenship, R.E., and Fleming, G.R. Evidence for Wavelike Energy Transfer Through Quantum Coherence in Photosynthetic Systems. Nature 446, 782-786 (2007).

Engelhardt, G., Bastidas, V.M., Kopylov, W., and Brandes, T. Excited-state quantum phase transitions and periodic dynamics. Physical Review A 91, 013631 (2015).

Engl, T., Dujardin, J., Argüelles, A., Schlagheck, P., Richter, K., and Urbina, J.D. Coherent Backscattering in Fock Space: A Signature of Quantum Many-Body Interference in Interacting Bosonic Systems. Physical Review Letters 112, 140403 (2014a).

Engl, T., Plößl, P., Urbina, J., and Richter, K. The semiclassical propagator in fermionic Fock space. Theoretical Chemistry Accounts 133, 1563 (2014b).

Englert, B.G. On quantum theory. The European Physical Journal D 67, 238 (2013).

Ernst, R., Bodenhausen, G., and Wokaun, A. Principles of Nuclear Magnetic Resonance in One and Two Dimensions. International series of monographs on chemistry (Oxford University Press, Oxford, UK, 1987).

Eschner, J., Morigi, G., Schmidt-Kaler, F., and Blatt, R. Laser cooling of trapped ions. Journal of the Optical Society of America B 20, 1003-1015 (2003).

Evers, F. and Mirlin, A.D. Anderson transitions. Reviews of Modern Physics 80, 1355-1417 (2008).

Fannes, M., Lewis, J., and Verbeure, A. Symmetric states of composite systems. Letters in Mathematical Physics 15, 255-260 (1988).

Fannes, M., Nachtergaele, B., and Werner, R. Finitely correlated states on quantum spin chains. Communications in Mathematical Physics 144, 443-490 (1992).

Fano, U. Pairs of two-level systems. Reviews of Modern Physics 55, 855-874 (1983).

Fedrizzi, A., Zuppardo, M., Gillett, G.G., Broome, M.A., Almeida, M.P., Paternostro, M., White, A.G., and Paterek, T. Experimental Distribution of Entanglement with Separable Carriers. Physical Review Letters 111, 230504 (2013).

Feng, D.H., Gilmore, R., and Deans, S.R. Phase transitions and the geometric properties of the interacting boson model. Physical Review C 23, 1254-1258 (1981). 
Ferraro, A., Aolita, L., Cavalcanti, D., Cucchietti, F.M., and Acín, A. Almost All Quantum States Have Nonclassical Correlations. Physical Review A 81, 052318 (2010).

Feynman, R.P. Forces in Molecules. Physical Review 56, 340-343 (1939).

Feynman, R.P. Space-Time Approach to Non-Relativistic Quantum Mechanics. Reviews of Modern Physics 20, 367-387 (1948).

Feynman, R.P. Simulating Physics with Computers. International Journal of Theoretical Physics 21, 467-488 (1982).

Fischer, S. and Breuer, H.P. Coherence in a network of two-level systems coupled to a bosonic field. Physical Review A 88, 062103 (2013).

Foot, C.J. Atomic Physics (Oxford University Press, Oxford, UK, 2007).

Fourkas, J.T., Wilson, W.L., Wäckerle, G., Frost, A.E., and Fayer, M.D. Picosecond time-scale phase-related optical pulses: measurement of sodium optical coherence decay by observation of incoherent fluorescence. Journal of the Optical Society of America B 6, 1905-1910 (1989).

Friedenauer, A., Schmitz, H., Glueckert, J.T., Porras, D., and Schaetz, T. Simulating a quantum magnet with trapped ions. Nature Physics 4, 757-761 (2008).

Gaetan, A., Miroshnychenko, Y., Wilk, T., Chotia, A., Viteau, M., Comparat, D., Pillet, P., Browaeys, A., and Grangier, P. Observation of collective excitation of two individual atoms in the Rydberg blockade regime. Nature Physics 5, 115-118 (2009).

Gallagher, T.F. Rydberg Atoms (Cambridge University Press, Cambridge, UK, 1994).

Gao, T., Hong, Y., Lu, Y., and Yan, F. Efficient k-separability criteria for mixed multipartite quantum states. Europhysics Letters 104, 20007 (2013).

Gao, T., Yan, F., and van Enk, S.J. Permutationally Invariant Part of a Density Matrix and Nonseparability of $N$-Qubit States. Physical Review Letters 112, 180501 (2014).

García-Mata, I., Zhirov, O.V., and Shepelyansky, D.L. Frenkel-Kontorova model with cold trapped ions. The European Physical Journal D 41, 325-330 (2007).

Gardiner, C. and Zoller, P. Quantum Noise: A Handbook of Markovian and NonMarkovian Quantum Stochastic Methods with Applications to Quantum Optics (Springer, Berlin, Germany, 2004).

Gardiner, S.A., Cirac, J.I., and Zoller, P. Nonclassical states and measurement of general motional observables of a trapped ion. Physical Review A 55, 1683-1694 (1997). 
Georges, A., Kotliar, G., Krauth, W., and Rozenberg, M.J. Dynamical mean-field theory of strongly correlated fermion systems and the limit of infinite dimensions. Reviews of Modern Physics 68, 13-125 (1996).

Gerry, C.C. and Knight, P.L. Introductory Quantum Optics (Cambridge University Press, Cambridge, UK, 2005).

Gessner, M., Ramm, M., Pruttivarasin, T., Buchleitner, A., Breuer, H.P., and Häffner, $\mathrm{H}$. Local detection of quantum correlations with a single trapped ion. Nature Physics 10, 105-109 (2014a).

Gessner, M. Initial Correlations in Open Quantum Systems. Diplomarbeit, AlbertLudwigs-Universität Freiburg (2011).

Gessner, M. and Breuer, H.P. Detecting Nonclassical System-Environment Correlations by Local Operations. Physical Review Letters 107, 180402 (2011).

Gessner, M. and Breuer, H.P. Generic features of the dynamics of complex open quantum systems: Statistical approach based on averages over the unitary group. Physical Review E 87, 042128 (2013a).

Gessner, M. and Breuer, H.P. Local witness for bipartite quantum discord. Physical Review A 87, 042107 (2013b).

Gessner, M., Laine, E.M., Breuer, H.P., and Piilo, J. Correlations in quantum states and the local creation of quantum discord. Physical Review A 85, 052122 (2012).

Gessner, M., Ramm, M., Häffner, H., Buchleitner, A., and Breuer, H.P. Observing a quantum phase transition by measuring a single spin. Europhysics Letters 107, 40005 (2014b).

Gessner, M., Schlawin, F., and Buchleitner, A. Probing Polariton Dynamics in Trapped Ions with Phase-Coherent Two-Dimensional Spectroscopy. The Journal of Chemical Physics 142, 212439 (2015).

Gessner, M., Schlawin, F., Häffner, H., Mukamel, S., and Buchleitner, A. Nonlinear spectroscopy of controllable many-body quantum systems. New Journal of Physics 16, 092001 (2014c).

Ghirardi, G.C., Rimini, A., and Weber, T. Unified dynamics for microscopic and macroscopic systems. Physical Review D 34, 470-491 (1986).

Gilmore, R. and Feng, D. Phase transitions in nuclear matter described by pseudospin Hamiltonians. Nuclear Physics A 301, 189-204 (1978).

Giorgi, G.L. Quantum discord and remote state preparation. Physical Review A 88 , 022315 (2013). 
Girolami, D., Paternostro, M., and Adesso, G. Faithful nonclassicality indicators and extremal quantum correlations in two-qubit states. Journal of Physics A: Mathematical and Theoretical 44, 352002 (2011).

Girolami, D., Souza, A.M., Giovannetti, V., Tufarelli, T., Filgueiras, J.G., Sarthour, R.S., Soares-Pinto, D.O., Oliveira, I.S., and Adesso, G. Quantum Discord Determines the Interferometric Power of Quantum States. Physical Review Letters 112, 210401 (2014).

Girolami, D., Tufarelli, T., and Adesso, G. Characterizing Nonclassical Correlations via Local Quantum Uncertainty. Physical Review Letters 110, 240402 (2013).

Glauber, R.J. Coherent and Incoherent States of the Radiation Field. Physical Review 131, 2766-2788 (1963).

Glick, A.J., Lipkin, H.J., and Meshkov, N. Validity of many-body approximation methods for a solvable model: (III). Diagram summations. Nuclear Physics 62, 211-224 (1965).

Gnutzmann, S., Haake, F., and Kuś, M. Quantum chaos of SU 3 observables. Journal of Physics A: Mathematical and General 33, 143 (2000).

Gnutzmann, S. and Kuś, M. Coherent states and the classical limit on irreducible $\mathrm{SU}_{3}$ representations. Journal of Physics A: Mathematical and General 31, 9871 (1998).

Gorin, T. and Seligman, T.H. A random matrix approach to decoherence. Journal of Optics B: Quantum and Semiclassical Optics 4, S386 (2002).

Gorin, T., Prosen, T., Seligman, T.H., and Žnidarič, M. Dynamics of Loschmidt echoes and fidelity decay. Physics Reports 435, 33-156 (2006).

Gorini, V., Kossakowski, A., and Sudarshan, E.C.G. Completely Positive Dynamical Semigroups of N-Level Systems. Journal of Mathematical Physics 17, 821 (1976).

Gorini, V., Verri, M., and Frigerio, A. Non-Markovian Behavior in Low-Temperature Damping. Physica A: Statistical Mechanics and its Applications 161, 357-384 (1989).

Grabert, H. Projection Operator Techniques in Nonequilibrium Statistical Mechanics (Springer, Berlin, Germany, 1982).

Grabert, H., Schramm, P., and Ingold, G.L. Quantum Brownian Motion: The Functional Integral Approach. Physics Reports 168, 115-207 (1988).

Graham, R.L., Knuth, D.E., and Patashnik, O. Concrete Mathematics (AddisonWesley, Reading, MA, 1989). 
Greenberger, D.M., Horne, M.A., and Zeilinger, A. Going Beyond Bell's Theorem. In M. Kafatos (Editor), Bell's Theorem, Quantum Theory, and Conceptions of the Universe, pp. 69-72 (Kluwer, Dordrecht, Netherlands, 1989).

Greiner, M., Mandel, O., Esslinger, T., Hänsch, T.W., and Bloch, I. Quantum phase transition from a superfluid to a Mott insulator in a gas of ultracold atoms. Nature 415, 39-44 (2002).

Greiner, M., Regal, C.A., and Jin, D.S. Emergence of a molecular Bose-Einstein condensate from a Fermi gas. Nature 426, 537-540 (2003).

Grimm, R., Weidemüller, M., and Ovchinnikov, Y.B. Optical dipole traps for neutral atoms. Advances in Atomic, Molecular, and Optical Physics 42, 95-170 (2000).

Gross, D., Liu, Y.K., Flammia, S.T., Becker, S., and Eisert, J. Quantum State Tomography via Compressed Sensing. Physical Review Letters 105, 150401 (2010).

Gross, E. Structure of a quantized vortex in boson systems. Il Nuovo Cimento 20, 454-477 (1961).

Grover, L.K. Quantum Mechanics Helps in Searching for a Needle in a Haystack. Physical Review Letters 79, 325-328 (1997).

Gühne, O. and Seevinck, M. Separability criteria for genuine multiparticle entanglement. New Journal of Physics 12, 053002 (2010).

Gühne, O. and Tóth, G. Entanglement detection. Physics Reports 474, 1-75 (2009).

Guhr, T., Müller-Groeling, A., and Weidenmüller, H.A. Random-Matrix Theories in Quantum Physics: Common Concepts. Physics Reports 299, 189-425 (1998).

Günter, G., Schempp, H., Robert-de Saint-Vincent, M., Gavryusev, V., Helmrich, S., Hofmann, C.S., Whitlock, S., and Weidemüller, M. Observing the Dynamics of Dipole-Mediated Energy Transport by Interaction-Enhanced Imaging. Science $\mathbf{3 4 2}$, 954-956 (2013).

Gutzwiller, M.C. Effect of Correlation on the Ferromagnetism of Transition Metals. Physical Review Letters 10, 159-162 (1963).

Gutzwiller, M.C. Phase-Integral Approximation in Momentum Space and the Bound States of an Atom. Journal of Mathematical Physics 8, 1979-2000 (1967).

Gutzwiller, M.C. Energy Spectrum According to Classical Mechanics. Journal of Mathematical Physics 11, 1791-1806 (1970).

Gutzwiller, M.C. Periodic Orbits and Classical Quantization Conditions. Journal of Mathematical Physics 12, 343-358 (1971).

Gutzwiller, M.C. Chaos in Classical and Quantum Mechanics (Springer, New York, NY, 1990). 
Gutzwiller, M.C. The semi-classical quantization of chaotic Hamiltonian systems. In M.J. Giannoni, A. Voros, and J. Zinn-Justin (Editors), Chaos and Quantum Physics (Elsevier Science Publishers B.V., Amsterdam, Netherlands, 1991).

Haake, F. Quantum Signatures of Chaos (Springer, Berlin, Germany, 2001).

Haake, F. and Reibold, R. Strong damping and low-temperature anomalies for the harmonic oscillator. Physical Review A 32, 2462-2475 (1985).

Häffner, H., Hänsel, W., Roos, C.F., Benhelm, J., Chek-al kar, D., Chwalla, M., Korber, T., Rapol, U.D., Riebe, M., Schmidt, P.O., Becher, C., Guhne, O., Dür, W., and Blatt, R. Scalable multiparticle entanglement of trapped ions. Nature 438, 643-646 (2005a).

Häffner, H., Roos, C., and Blatt, R. Quantum computing with trapped ions. Physics Reports 469, 155-203 (2008).

Häffner, H., Schmidt-Kaler, F., Hänsel, W., Roos, C., Körber, T., Chwalla, M., Riebe, M., Benhelm, J., Rapol, U., Becher, C., and Blatt, R. Robust entanglement. Applied Physics B 81, 151-153 (2005b).

Hakim, V. and Ambegaokar, V. Quantum theory of a free particle interacting with a linearly dissipative environment. Physical Review A 32, 423-434 (1985).

Hamm, P., Lim, M., and Hochstrasser, R.M. Structure of the Amide I Band of Peptides Measured by Femtosecond Nonlinear-Infrared Spectroscopy. The Journal of Physical Chemistry B 102, 6123-6138 (1998).

Hamm, P. and Zanni, M. Concepts and Methods of $2 D$ Infrared Spectroscopy (Cambridge University Press, Cambridge, UK, 2011).

Hänsch, T.W. and Schawlow, A.L. Cooling of gases by laser radiation. Optics Communications 13, 68 (1975).

Harlander, M., Lechner, R., Brownnutt, M., Blatt, R., and Hänsel, W. Trapped-Ion Antennae for the Transmission of Quantum Information. Nature 471, 200 (2011).

Haroche, S. Nobel Lecture: Controlling photons in a box and exploring the quantum to classical boundary. Reviews of Modern Physics 85, 1083-1102 (2013).

Haroche, S. and Raimond, J.M. Exploring the Quantum: Atoms, Cavities, and Photons (Oxford Graduate Texts, Oxford, UK, 2006).

Hastings, M.B. Superadditivity of Communication Capacity Using Entangled Inputs. Nature Physics 5, 255-257 (2009).

Hayashi, M. Quantum Information (Springer, Berlin, Germany, 2006).

Hayes, D., Clark, S.M., Debnath, S., Hucul, D., Inlek, I.V., Lee, K.W., Quraishi, Q., and Monroe, C. Coherent Error Suppression in Multiqubit Entangling Gates. Physical Review Letters 109, 020503 (2012). 
Haze, S., Tateishi, Y., Noguchi, A., Toyoda, K., and Urabe, S. Observation of phonon hopping in radial vibrational modes of trapped ions. Physical Review A 85, 031401 (2012).

Hazzard, Kaden R. A., Gadway, B., Foss-Feig, M., Yan, B., Moses, S.A., Covey, J.P., Yao, N.Y., Lukin, M.D., Ye, J., Jin, D.S., and Rey, A.M. Many-Body Dynamics of Dipolar Molecules in an Optical Lattice. Physical Review Letters 113, 195302 (2014).

Heinzen, D.J. and Wineland, D.J. Quantum-limited cooling and detection of radiofrequency oscillations by laser-cooled ions. Physical Review A 42, 2977-2994 (1990).

Heisenberg, W. Über den anschaulichen Inhalt der quantentheoretischen Kinematik und Mechanik. Zeitschrift für Physik 43, 172-198 (1927).

Heisenberg, W. Zur Theorie des Ferromagnetismus. Zeitschrift für Physik 49, 619-636 (1928).

Heiss, W. Phase transitions of finite Fermi systems and quantum chaos. Physics Reports 242, 443-451 (1994).

Helstrom, C.W. Detection theory and quantum mechanics. Information and Control 10, 254-291 (1967).

Henderson, L. and Vedral, V. Classical, Quantum and Total Correlations. Journal of Physics A: Mathematical and General 34, 6899 (2001).

Hensinger, W.K., Häffner, H., Browaeys, A., Heckenberg, N.R., Helmerson, K., McKenzie, C., Milburn, G.J., Phillips, W.D., Rolston, S.L., Rubinsztein-Dunlop, H., and Upcroft, B. Dynamical tunnelling of ultracold atoms. Nature 412, 52-55 (2001).

Hertz, J.A. Quantum critical phenomena. Physical Review B 14, 1165-1184 (1976).

Hildner, R., Brinks, D., and van Hulst, N.F. Femtosecond coherence and quantum control of single molecules at room temperature. Nature Physics 7, 172-177 (2011).

Hiller, M., Kottos, T., and Geisel, T. Wave-packet dynamics in energy space of a chaotic trimeric Bose-Hubbard system. Phys. Rev. A 79, 023621 (2009).

Hite, D.A., Colombe, Y., Wilson, A.C., Brown, K.R., Warring, U., Jördens, R., Jost, J.D., McKay, K.S., Pappas, D.P., Leibfried, D., and Wineland, D.J. 100-Fold Reduction of Electric-Field Noise in an Ion Trap Cleaned with In Situ Argon-IonBeam Bombardment. Physical Review Letters 109, 103001 (2012).

Hofheinz, M., Wang, H., Ansmann, M., Bialczak, R.C., Lucero, E., Neeley, M., O'Connell, A.D., Sank, D., Wenner, J., Martinis, J.M., and Cleland, A.N. Synthesizing arbitrary quantum states in a superconducting resonator. Nature 459, 546-549 (2009). 
Holstein, T. and Primakoff, H. Field Dependence of the Intrinsic Domain Magnetization of a Ferromagnet. Physical Review 58, 1098-1113 (1940).

Hong, C.K., Ou, Z.Y., and Mandel, L. Measurement of subpicosecond time intervals between two photons by interference. Physical Review Letters 59, 2044-2046 (1987).

Horodecki, R. and Horodecki, M. Information-theoretic aspects of inseparability of mixed states. Physical Review A 54, 1838-1843 (1996).

Horodecki, R., Horodecki, P., Horodecki, M., and Horodecki, K. Quantum Entanglement. Reviews of Modern Physics 81, 865-942 (2009).

Hu, B.L., Paz, J.P., and Zhang, Y. Quantum Brownian motion in a general environment: Exact master equation with nonlocal dissipation and colored noise. Physical Review D 45, 2843-2861 (1992).

Hu, H., Strybulevych, A., Page, J.H., Skipetrov, S.E., and van Tiggelen, B.A. Localization of ultrasound in a three-dimensional elastic network. Nat Phys 4, 945-948 (2008).

$\mathrm{Hu}, \mathrm{X} ., \mathrm{Gu}, \mathrm{Y}$. , Gong, Q., and Guo, G. Necessary and sufficient condition for Markovian-dissipative-dynamics-induced quantum discord. Physical Review A 84, 022113 (2011).

Hubbard, J. Electron Correlations in Narrow Energy Bands. Royal Society of London Proceedings Series A 276, 238-257 (1963).

Huber, M., Mintert, F., Gabriel, A., and Hiesmayr, B.C. Detection of HighDimensional Genuine Multipartite Entanglement of Mixed States. Physical Review Letters 104, 210501 (2010).

Hughes, R.J., James, D.F.V., Knill, E.H., Laflamme, R., and Petschek, A.G. Decoherence Bounds on Quantum Computation with Trapped Ions. Physical Review Letters 77, 3240-3243 (1996).

Hunn, S., Zimmermann, K., Hiller, M., and Buchleitner, A. Tunneling decay of two interacting bosons in an asymmetric double-well potential: A spectral approach. Physical Review A 87, 043626 (2013).

Huxter, V.M., Oliver, T.A.A., Budker, D., and Fleming, G.R. Vibrational and electronic dynamics of nitrogen-vacancy centres in diamond revealed by twodimensional ultrafast spectroscopy. Nature Physics 9, 744-749 (2013).

Ising, E. Beitrag zur Theorie des Ferromagnetismus. Zeitschrift für Physik 31, 253-258 (1925). 
Islam, R., Edwards, E.E., Kim, K., Korenblit, S., Noh, C., Carmichael, H., Lin, G.D., Duan, L.M., Joseph Wang, C.C., Freericks, J.K., and Monroe, C. Onset of a quantum phase transition with a trapped ion quantum simulator. Nature Communications 2, 377 (2011).

Ivanov, P.A., Ivanov, S.S., Vitanov, N.V., Mering, A., Fleischhauer, M., and Singer, K. Simulation of a quantum phase transition of polaritons with trapped ions. Physical Review A 80, 060301 (2009).

Jackson, J.D. Classical Electrodynamics (Wiley, New York, NY, 1999).

Jaksch, D., Bruder, C., Cirac, J.I., Gardiner, C.W., and Zoller, P. Cold Bosonic Atoms in Optical Lattices. Physical Review Letters 81, 3108-3111 (1998).

Jalabert, R.A. and Pastawski, H.M. Environment-Independent Decoherence Rate in Classically Chaotic Systems. Physical Review Letters 86, 2490-2493 (2001).

James, D. Quantum dynamics of cold trapped ions with application to quantum computation. Applied Physics B 66, 181-190 (1998).

Jaynes, E. and Cummings, F. Comparison of quantum and semiclassical radiation theories with application to the beam maser. Proceedings of the IEEE 51, 89-109 (1963).

Jelezko, F., Gaebel, T., Popa, I., Gruber, A., and Wrachtrup, J. Observation of Coherent Oscillations in a Single Electron Spin. Physical Review Letters 92, 076401 (2004).

Jendrzejewski, F., Müller, K., Richard, J., Date, A., Plisson, T., Bouyer, P., Aspect, A., and Josse, V. Coherent Backscattering of Ultracold Atoms. Physical Review Letters 109, 195302 (2012).

Jin, J. and Church, D.A. Precision lifetimes for the $\mathrm{Ca}^{+} 4 p^{2} P$ levels: Experiment challenges theory at the 1\% level. Physical Review Letters 70, 3213-3216 (1993).

Johanning, M., Braun, A., Timoney, N., Elman, V., Neuhauser, W., and Wunderlich, C. Individual Addressing of Trapped Ions and Coupling of Motional and Spin States Using rf Radiation. Physical Review Letters 102, 073004 (2009).

Johansson, J., Nation, P., and Nori, F. QuTiP 2: A Python framework for the dynamics of open quantum systems. Computer Physics Communications 184, 1234-1240 (2013).

Jordan, P. and Wigner, E. Über das Paulische Äquivalenzverbot. Zeitschrift für Physik 47, 631-651 (1928).

Jördens, R., Strohmaier, N., Gunter, K., Moritz, H., and Esslinger, T. A Mott insulator of fermionic atoms in an optical lattice. Nature 455, 204-207 (2008). 
Jurcevic, P., Lanyon, B.P., Hauke, P., Hempel, C., Zoller, P., Blatt, R., and Roos, C.F. Quasiparticle engineering and entanglement propagation in a quantum many-body system. Nature 511, 202-205 (2014).

Kamenev, A. Many-body theory of non-equilibrium systems. In H. Bouchiat, Y. Gefen, S. Gu'eron, G. Montambaux, and J. Dalibard (Editors), Nanophysics: Coherence and Transport, volume 81 of Les Houches, pp. 177-246 (Elsevier, Amsterdam, Netherlands, 2005).

Kanamori, J. Electron Correlation and Ferromagnetism of Transition Metals. Progress of Theoretical Physics 30, 275-289 (1963).

Kielpinski, D., Monroe, C., and Wineland, D.J. Architecture for a large-scale ion-trap quantum computer. Nature 417, 709-711 (2002).

Kierig, E., Schnorrberger, U., Schietinger, A., Tomkovic, J., and Oberthaler, M.K. Single-Particle Tunneling in Strongly Driven Double-Well Potentials. Physical Review Letters 100, 190405 (2008).

Kim, J., Mukamel, S., and Scholes, G.D. Two-Dimensional Electronic DoubleQuantum Coherence Spectroscopy. Accounts of Chemical Research 42, 1375-1384 (2009).

Kim, K., Chang, M.S., Korenblit, S., Islam, R., Edwards, E.E., Freericks, J.K., Lin, G.D., Duan, L.M., and Monroe, C. Quantum simulation of frustrated Ising spins with trapped ions. Nature 465, 590-593 (2010).

Kirkwood, J.G. The Statistical Mechanical Theory of Transport Processes I. General Theory. The Journal of Chemical Physics 14, 180-201 (1946).

Klauder, J.R. Continuous-Representation Theory. II. Generalized Relation between Quantum and Classical Dynamics. Journal of Mathematical Physics 4, 10581073 (1963).

Kleinert, H. Path Integrals in Quantum Mechanics, Statistics, Polymer Physics, and Financial Markets (World Scientific, Singapore, 2009).

Kleppner, D. Inhibited Spontaneous Emission. Physical Review Letters 47, 233-236 (1981).

Knap, M., Kantian, A., Giamarchi, T., Bloch, I., Lukin, M.D., and Demler, E. Probing Real-Space and Time-Resolved Correlation Functions with Many-Body Ramsey Interferometry. Physical Review Letters 111, 147205 (2013).

Knill, E. and Laflamme, R. Power of One Bit of Quantum Information. Physical Review Letters 81, 5672-5675 (1998).

Knill, E., Laflamme, R., and Milburn, G.J. A scheme for efficient quantum computation with linear optics. Nature 409, 46-52 (2001). 
Knopp, K. Infinite Sequences and Series (Dover Publications, New York, NY, 1956).

Koffel, T., Lewenstein, M., and Tagliacozzo, L. Entanglement Entropy for the LongRange Ising Chain in a Transverse Field. Physical Review Letters 109, 267203 (2012).

Kok, P., Munro, W.J., Nemoto, K., Ralph, T.C., Dowling, J.P., and Milburn, G.J. Linear optical quantum computing with photonic qubits. Reviews of Modern Physics 79, 135-174 (2007).

Kolovsky, A.R. New Bloch Period for Interacting Cold Atoms in 1D Optical Lattices. Physical Review Letters 90, 213002 (2003).

Kolovsky, A.R. and Buchleitner, A. Floquet-Bloch operator for the Bose-Hubbard model with static field. Physical Review E 68, 056213 (2003).

Kraus, K. States, Effects and Operations: Fundamental Notions of Quantum Theory (Springer, Berlin, Germany, 1983).

Kreuter, A., Becher, C., Lancaster, G.P.T., Mundt, A.B., Russo, C., Häffner, H., Roos, C., Hänsel, W., Schmidt-Kaler, F., Blatt, R., and Safronova, M.S. Experimental and theoretical study of the $3 d^{2} D$ level lifetimes of ${ }^{40} \mathrm{Ca}^{+}$. Physical Review A 71, 032504 (2005).

Kucsko, G., Maurer, P.C., Yao, N.Y., Kubo, M., Noh, H.J., Lo, P.K., Park, H., and Lukin, M.D. Nanometre-scale thermometry in a living cell. Nature 500, 54-58 (2013).

Kummer, H. Mathematical Description of a System Consisting of Identical QuantumMechanical Particles. Journal of Mathematical Physics 11, 449-474 (1970).

Kummer, H. n-Representability Problem for Reduced Density Matrices. Journal of Mathematical Physics 8, 2063-2081 (1967).

Kurtsiefer, C., Mayer, S., Zarda, P., and Weinfurter, H. Stable Solid-State Source of Single Photons. Physical Review Letters 85, 290-293 (2000).

Kwiat, P.G., Mattle, K., Weinfurter, H., Zeilinger, A., Sergienko, A.V., and Shih, Y. New High-Intensity Source of Polarization-Entangled Photon Pairs. Physical Review Letters 75, 4337-4341 (1995).

Laine, E.M., Piilo, J., and Breuer, H.P. Witness for Initial System-Environment Correlations in Open-System Dynamics. Europhysics Letters 92, 60010 (2010).

Lambropoulos, P., Nikolopoulos, G.M., Nielsen, T.R., and Bay, S. Fundamental quantum optics in structured reservoirs. Reports on Progress in Physics 63, 455 (2000).

Landau, L. and Lifshitz, E. Statistical Physics (Pergamon Press, Oxford, UK, 1969), 2 edition. 
Lanyon, B.P., Barbieri, M., Almeida, M.P., and White, A.G. Experimental Quantum Computing without Entanglement. Physical Review Letters 101, 200501 (2008).

Lanyon, B.P., Hempel, C., Nigg, D., Müller, M., Gerritsma, R., Zähringer, F., Schindler, P., Barreiro, J.T., Rambach, M., Kirchmair, G., Hennrich, M., Zoller, P., Blatt, R., and Roos, C.F. Universal Digital Quantum Simulation with Trapped Ions. Science 334, 57-61 (2011).

Lanyon, B.P., Jurcevic, P., Hempel, C., Gessner, M., Vedral, V., Blatt, R., and Roos, C.F. Experimental Generation of Quantum Discord via Noisy Processes. Physical Review Letters 111, 100504 (2013).

Leggett, A.J., Chakravarty, S., Dorsey, A.T., Fisher, M.P.A., Garg, A., and Zwerger, W. Dynamics of the dissipative two-state system. Reviews of Modern Physics 59, 1-85 (1987).

Leibfried, D., Blatt, R., Monroe, C., and Wineland, D. Quantum dynamics of single trapped ions. Reviews of Modern Physics 75, 281-324 (2003a).

Leibfried, D., DeMarco, B., Meyer, V., Lucas, D., Barrett, M., Britton, J., Itano, W.M., Jelenkovic, B., Langer, C., Rosenband, T., and Wineland, D.J. Experimental demonstration of a robust, high-fidelity geometric two ion-qubit phase gate. Nature 422, 412-415 (2003b).

Levi, F. and Mintert, F. Hierarchies of Multipartite Entanglement. Physical Review Letters 110, 150402 (2013).

Leyvraz, F. and Heiss, W.D. Large-N Scaling Behavior of the Lipkin-Meshkov-Glick Model. Physical Review Letters 95, 050402 (2005).

Li, C.F., Tang, J.S., Li, Y.L., and Guo, G.C. Experimentally witnessing the initial correlation between an open quantum system and its environment. Physical Review A 83, 064102 (2011).

Li, N. and Luo, S. Classical States Versus Separable States. Physical Review A 78, 024303 (2008).

Li, X., Zhang, T., Borca, C.N., and Cundiff, S.T. Many-Body Interactions in Semiconductors Probed by Optical Two-Dimensional Fourier Transform Spectroscopy. Physical Review Letters 96, 057406 (2006).

Lidar, D.A., Chuang, I.L., and Whaley, K.B. Decoherence-Free Subspaces for Quantum Computation. Physical Review Letters 81, 2594-2597 (1998).

Lieb, E., Schultz, T., and Mattis, D. Two soluble models of an antiferromagnetic chain. Annals of Physics 16, 407-466 (1961).

Lindblad, G. On the Generators of Quantum Dynamical Semigroups. Communications in Mathematical Physics 48, 119-130 (1976). 
Lindblad, G. On the Existence of Quantum Subdynamics. Journal of Physics A: Mathematical and General 29, 4197 (1996).

Linden, N. and Popescu, S.Popescu, S. On Multi-Particle Entanglement. Fortschritte der Physik 46, 567-578 (1998).

Links, J., Zhou, H.Q., McKenzie, R.H., and Gould, M.D. Algebraic Bethe ansatz method for the exact calculation of energy spectra and form factors: applications to models of Bose-Einstein condensates and metallic nanograins. Journal of Physics A: Mathematical and General 36, R63 (2003).

Lipkin, H., Meshkov, N., and Glick, A. Validity of many-body approximation methods for a solvable model: (I). Exact solutions and perturbation theory. Nuclear Physics 62, 188-198 (1965).

Littich, G. Electrostatic Control and Transport of Ions on a Planar Trap for Quantum Information Processing. Master's thesis, ETH Zurich (2011).

Liu, B.H., Li, L., Huang, Y.F., Li, C.F., Guo, G.C., Laine, E.M., Breuer, H.P., and Piilo, J. Experimental control of the transition from Markovian to non-Markovian dynamics of open quantum systems. Nature Physics 7, 931-934 (2011).

Lloyd, S. Universal Quantum Simulators. Science 273, 1073-1078 (1996).

Lott, G.A., Perdomo-Ortiz, A., Utterback, J.K., Widom, J.R., Aspuru-Guzik, A., and Marcus, A.H. Conformation of self-assembled porphyrin dimers in liposome vesicles by phase-modulation 2D fluorescence spectroscopy. Proceedings of the National Academy of Sciences 108, 16521-16526 (2011).

Loudon, R. The Quantum Theory of Light (Oxford University Press, Oxford, UK, 1973).

Löwdin, P.O. Quantum Theory of Many-Particle Systems. I. Physical Interpretations by Means of Density Matrices, Natural Spin-Orbitals, and Convergence Problems in the Method of Configurational Interaction. Physical Review 97, 1474-1489 (1955).

Lukin, M.D., Fleischhauer, M., Cote, R., Duan, L.M., Jaksch, D., Cirac, J.I., and Zoller, P. Dipole Blockade and Quantum Information Processing in Mesoscopic Atomic Ensembles. Physical Review Letters 87, 037901 (2001).

Luo, S. Wigner-Yanase Skew Information and Uncertainty Relations. Physical Review Letters 91, 180403 (2003).

Luo, S. Using Measurement-Induced Disturbance to Characterize Correlations as Classical or Quantum. Physical Review A 77, 022301 (2008).

Ma, S.k. Introduction to the Renormalization Group. Reviews of Modern Physics 45, 589-614 (1973). 
Madroñero, J., Ponomarev, A., Carvalho, A.R., Wimberger, S., Viviescas, C., Kolovsky, A., Hornberger, K., Schlagheck, P., Krug, A., and Buchleitner, A. Quantum Chaos, Transport, and Control-in Quantum Optics. Advances In Atomic, Molecular, and Optical Physics 53, 33-73 (2006).

Mahan, G.D. Many-Particle Physics (Kluwer Academic / Plenum Publishers, New York, NY, 2000).

Mahmud, K.W., Perry, H., and Reinhardt, W.P. Quantum phase-space picture of BoseEinstein condensates in a double well. Physical Review A 71, 023615 (2005).

Mandel, L. and Wolf, E. Optical Coherence and Quantum Optics (Cambridge University Press, Cambridge, UK, 1995).

Matsumoto, S. General Moments of Matrix Elements from Circular Orthogonal Ensembles. Random Matrices: Theory and Applications 1, 1250005 (2012).

Mayer, K., Rodriguez, A., and Buchleitner, A. Matter-wave scattering from interacting bosons in an optical lattice. Physical Review A 90, 023629 (2014).

Maze, J.R., Stanwix, P.L., Hodges, J.S., Hong, S., Taylor, J.M., Cappellaro, P., Jiang, L., Dutt, M.V.G., Togan, E., Zibrov, A.S., Yacoby, A., Walsworth, R.L., and Lukin, M.D. Nanoscale magnetic sensing with an individual electronic spin in diamond. Nature 455, 644-647 (2008).

Meekhof, D.M., Monroe, C., King, B.E., Itano, W.M., and Wineland, D.J. Generation of Nonclassical Motional States of a Trapped Atom. Physical Review Letters 76, 1796-1799 (1996).

Mehta, M.L. Random Matrices (Elsevier / Academic Press, Amsterdam, Netherlands, 1991).

Meinert, F., Mark, M.J., Kirilov, E., Lauber, K., Weinmann, P., Daley, A.J., and Nägerl, H.C. Quantum Quench in an Atomic One-Dimensional Ising Chain. Physical Review Letters 111, 053003 (2013).

Meinert, F., Mark, M.J., Kirilov, E., Lauber, K., Weinmann, P., Gröbner, M., and Nägerl, H.C. Interaction-Induced Quantum Phase Revivals and Evidence for the Transition to the Quantum Chaotic Regime in 1D Atomic Bloch Oscillations. Physical Review Letters 112, 193003 (2014).

Mello, P.A. and Seligman, T.H. On the entropy approach to statistical nuclear reactions. Nuclear Physics A 344, 489-508 (1980).

Mello, P.A. Averages on the Unitary Group and Applications to the Problem of Disordered Conductors. Journal of Physics A: Mathematical and General 23, 406-4080 (1990).

Meschede, D. and Rauschenbeutel, A. Manipulating single atoms. Advances In Atomic, Molecular, and Optical Physics 53, 75 (2006). 
Meschede, D., Walther, H., and Müller, G. One-Atom Maser. Physical Review Letters 54, 551-554 (1985).

Meshkov, N., Glick, A., and Lipkin, H. Validity of many-body approximation methods for a solvable model: (II). Linearization procedures. Nuclear Physics 62, 199-210 (1965).

Meyer, Jr., C. Generalized Inverses and Ranks of Block Matrices. SIAM Journal on Applied Mathematics 25, 597-602 (1973).

Meystre, P. A short walk through quantum optomechanics. Annalen der Physik 525, 215-233 (2013).

Michler, P., Kiraz, A., Becher, C., Schoenfeld, W.V., Petroff, P.M., Zhang, L., Hu, E., and Imamoglu, A. A Quantum Dot Single-Photon Turnstile Device. Science 290, 2282-2285 (2000).

Milnor, J. Morse Theory (Princeton University Press, Princeton, NJ, 1963).

Milota, F., Sperling, J., Nemeth, A., Mančal, T., and Kauffmann, H.F. TwoDimensional Electronic Spectroscopy of Molecular Excitons. Accounts of Chemical Research 42, 1364-1374 (2009).

Mintert, F., Carvalho, A.R., Kuś, M., and Buchleitner, A. Measures and dynamics of entangled states. Physics Reports 415, 207-259 (2005).

Mintert, F. and Wunderlich, C. Ion-Trap Quantum Logic Using Long-Wavelength Radiation. Physical Review Letters 87, 257904 (2001).

Mirlin, A.D. Statistics of energy levels and eigenfunctions in disordered systems. Physics Reports 326, 259-382 (2000).

Modi, K., Brodutch, A., Cable, H., Paterek, T., and Vedral, V. The classical-quantum boundary for correlations: Discord and related measures. Reviews of Modern Physics 84, 1655-1707 (2012).

Modugno, G. Anderson localization in Bose-Einstein condensates. Reports on Progress in Physics 73, 102401 (2010).

Monroe, C., Meekhof, D.M., King, B.E., Itano, W.M., and Wineland, D.J. Demonstration of a Fundamental Quantum Logic Gate. Physical Review Letters 75, 4714-4717 (1995).

Monroe, C., Meekhof, D.M., King, B.E., and Wineland, D.J. A "Schrödinger Cat" Superposition State of an Atom. Science 272, 1131-1136 (1996).

Monz, T., Schindler, P., Barreiro, J.T., Chwalla, M., Nigg, D., Coish, W.A., Harlander, M., Hänsel, W., Hennrich, M., and Blatt, R. 14-Qubit Entanglement: Creation and Coherence. Physical Review Letters 106, 130506 (2011). 
Moore, F.L., Robinson, J.C., Bharucha, C.F., Sundaram, B., and Raizen, M.G. Atom Optics Realization of the Quantum $\delta$-Kicked Rotor. Physical Review Letters 75, 4598-4601 (1995).

Morsch, O., Müller, J.H., Cristiani, M., Ciampini, D., and Arimondo, E. Bloch Oscillations and Mean-Field Effects of Bose-Einstein Condensates in 1D Optical Lattices. Physical Review Letters 87, 140402 (2001).

Morsch, O. and Oberthaler, M. Dynamics of Bose-Einstein condensates in optical lattices. Reviews of Modern Physics 78, 179-215 (2006).

Morse, P.M. and Feshbach, H. Methods of Theoretical Physics, Part I (McGraw-Hill, New York, NY, 1953).

Muir, T. A Treatise on the Theory of Determinants (Dover Publications, New York, NY, 1960).

Mukamel, S. Principles of Nonlinear Optical Spectroscopy (Oxford University Press, Oxford, UK, 1995).

Mukamel, S., Abramavicius, D., Yang, L., Zhuang, W., Schweigert, I.V., and Voronine, D.V. Coherent Multidimensional Optical Probes for Electron Correlations and Exciton Dynamics: From NMR to X-rays. Accounts of Chemical Research 42, 553-562 (2009).

Myatt, C.J., King, B.E., Turchette, Q.A., Sackett, C.A., Kielpinski, D., Itano, W.M., Monroe, C., and Wineland, D.J. Decoherence of quantum superpositions through coupling to engineered reservoirs. Nature 403, 269-273 (2000).

Nakano, T., Piani, M., and Adesso, G. Negativity of quantumness and its interpretations. Physical Review A 88, 012117 (2013).

Neuhauser, W., Hohenstatt, M., Toschek, P.E., and Dehmelt, H. Localized visible Ba ${ }^{+}$ mono-ion oscillator. Physical Review A 22, 1137-1140 (1980).

Ni, K.K., Ospelkaus, S., de Miranda, M.H.G., Pe'er, A., Neyenhuis, B., Zirbel, J.J., Kotochigova, S., Julienne, P.S., Jin, D.S., and Ye, J. A High Phase-Space-Density Gas of Polar Molecules. Science 322, 231-235 (2008).

Nielsen, M.A. and Chuang, I.L. Quantum Computation and Quantum Information (Cambridge University Press, New York, NY, 2000).

Nogrette, F., Labuhn, H., Ravets, S., Barredo, D., Béguin, L., Vernier, A., Lahaye, T., and Browaeys, A. Single-Atom Trapping in Holographic 2D Arrays of Microtraps with Arbitrary Geometries. Physical Review X 4, 021034 (2014).

Nolting, W. Grundkurs Theoretische Physik 7: Viel-Teilchen-Theorie (Springer, Berlin, Germany, 2009). 
Oberthaler, M.K., Godun, R.M., d'Arcy, M.B., Summy, G.S., and Burnett, K. Observation of Quantum Accelerator Modes. Physical Review Letters 83, 4447-4451 (1999).

O'Brien, J.L., Furusawa, A., and Vuckovic, J. Photonic quantum technologies. Nature Photonics 3, 687-695 (2009).

Ollivier, H. and Zurek, W.H. Quantum Discord: A Measure of the Quantumness of Correlations. Physical Review Letters 88, 017901 (2001).

Orth, P.P., Imambekov, A., and Le Hur, K. Nonperturbative stochastic method for driven spin-boson model. Physical Review B 87, 014305 (2013).

Osborne, T.J. and Nielsen, M.A. Entanglement in a simple quantum phase transition. Physical Review A 66, 032110 (2002).

Ospelkaus, C., Warring, U., Colombe, Y., Brown, K.R., Amini, J.M., Leibfried, D., and Wineland, D.J. Microwave quantum logic gates for trapped ions. Nature 476, 181-184 (2011).

Osterloh, A., Amico, L., Falci, G., and Fazio, R. Scaling of Entanglement Close to a Quantum Phase Transition. Nature 416, 608-610 (2002).

Ozawa, M. Entanglement measures and the Hilbert-Schmidt distance. Physics Letters A 268, 158-160 (2000).

Ozeri, R., Langer, C., Jost, J.D., DeMarco, B., Ben-Kish, A., Blakestad, B.R., Britton, J., Chiaverini, J., Itano, W.M., Hume, D.B., Leibfried, D., Rosenband, T., Schmidt, P.O., and Wineland, D.J. Hyperfine Coherence in the Presence of Spontaneous Photon Scattering. Physical Review Letters 95, 030403 (2005).

Ozorio de Almeida, A.M. Hamiltonian Systems: Chaos and Quantization (Cambridge University Press, Cambridge, UK, 1988).

Palma, G.M., Suominen, K.A., and Ekert, A.K. Quantum Computers and Dissipation. Proceedings of the Royal Society of London A: Mathematical, Physical and Engineering Sciences 452, 567-584 (1996).

Pan, F. and Draayer, J. Analytical solutions for the LMG model. Physics Letters B 451, 1-10 (1999).

Paris, M. and Řeháček, J. (Editors). Quantum State Estimation (Springer, 2004).

Paterson, C. Atmospheric Turbulence and Orbital Angular Momentum of Single Photons for Optical Communication. Physical Review Letters 94, 153901 (2005).

Paul, W., Osberghaus, O., and Fischer, E. Ein Ionenkäfig. Forschungsberichte des Wirtschafts- und Verkehrsministeriums Nordrhein-Westfalen 415 (1958). 
Paul, W. Electromagnetic traps for charged and neutral particles. Reviews of Modern Physics 62, 531 (1990).

Paula, F.M., de Oliveira, T.R., and Sarandy, M.S. Geometric quantum discord through the Schatten 1-norm. Physical Review A 87, 064101 (2013).

Pechukas, P. Reduced Dynamics Need Not Be Completely Positive. Physical Review Letters 73, 1060-1062 (1994).

Pechukas, P. Pechukas Replies:: Physical Review Letters 75, 3021 (1995).

Penning, F. Die Glimmentladung bei niedrigem Druck zwischen koaxialen Zylindern in einem axialen Magnetfeld. Physica 3, 873-894 (1936).

Peres, A. Separability Criterion for Density Matrices. Physical Review Letters 77, 1413-1415 (1996).

Peruzzo, A., Lobino, M., Matthews, J.C.F., Matsuda, N., Politi, A., Poulios, K., Zhou, X.Q., Lahini, Y., Ismail, N., Wörhoff, K., Bromberg, Y., Silberberg, Y., Thompson, M.G., and O'Brien, J.L. Quantum Walks of Correlated Photons. Science 329, 1500-1503 (2010).

Peuntinger, C., Chille, V., Mišta, L., Korolkova, N., Förtsch, M., Korger, J., Marquardt, C., and Leuchs, G. Distributing Entanglement with Separable States. Physical Review Letters 111, 230506 (2013).

Phillips, W.D. Nobel Lecture: Laser cooling and trapping of neutral atoms. Reviews of Modern Physics 70, 721-741 (1998).

Piani, M. Problem with geometric discord. Physical Review A 86, 034101 (2012).

Piani, M. and Adesso, G. Quantumness of correlations revealed in local measurements exceeds entanglement. Physical Review A 85, 040301 (2012).

Piani, M., Gharibian, S., Adesso, G., Calsamiglia, J., Horodecki, P., and Winter, A. All Nonclassical Correlations Can Be Activated into Distillable Entanglement. Physical Review Letters 106, 220403 (2011).

Pitaevskii, L.P. Vortex Lines in an Imperfect Bose Gas. Soviet Physics JETP-USSR 13, 451 (1961).

Pitaevskii, L.P. and Stringari, S. Bose-Einstein-Condensation (Oxford University Press, Oxford, UK, 2003).

Polkovnikov, A. Phase space representation of quantum dynamics. Annals of Physics 325, 1790-1852 (2010).

Popescu, S. Bell's inequalities versus teleportation: What is nonlocality? Physical Review Letters 72, 797-799 (1994). 
Porras, D. and Cirac, J.I. Bose-Einstein Condensation and Strong-Correlation Behavior of Phonons in Ion Traps. Physical Review Letters 93, 263602 (2004a).

Porras, D. and Cirac, J.I. Effective Quantum Spin Systems with Trapped Ions. Physical Review Letters 92, 207901 (2004b).

Poyatos, J.F., Cirac, J.I., and Zoller, P. Quantum Reservoir Engineering with Laser Cooled Trapped Ions. Physical Review Letters 77, 4728-4731 (1996).

Prosen, T. and Žnidarič, M. Is the efficiency of classical simulations of quantum dynamics related to integrability? Physical Review E 75, 015202 (2007).

Pruttivarasin, T. Spectroscopy, fundamental symmetry tests and quantum simulation with trapped ions. Ph.D. thesis, University of California, Berkeley (2014).

Pruttivarasin, T., Ramm, M., Talukdar, I., Kreuter, A., and Häffner, H. Trapped Ions in Optical Lattices for Probing Oscillator Chain Models. New Journal of Physics 13, 075012 (2011).

Purcell, E.M. Spontaneous Emission Probabilities at Radio Frequencies, (Note B10 in Proceedings of the American Physical Society). Physical Review 69, 674-702 (1946).

Puri, R.R. and Agarwal, G.S. Collapse and revival phenomena in the JaynesCummings model with cavity damping. Physical Review A 33, 3610-3613 (1986).

Ra, Y.S., Tichy, M.C., Lim, H.T., Kwon, O., Mintert, F., Buchleitner, A., and Kim, Y.H. Nonmonotonic quantum-to-classical transition in multiparticle interference. Proceedings of the National Academy of Sciences 110, 1227-1231 (2013).

Raab, E.L., Prentiss, M., Cable, A., Chu, S., and Pritchard, D.E. Trapping of Neutral Sodium Atoms with Radiation Pressure. Physical Review Letters 59, 2631-2634 (1987).

Radcliffe, J.M. Some properties of coherent spin states. Journal of Physics A: General Physics 4, 313 (1971).

Radzki, W. Contractions of Product Density Operators of Systems of Identical Fermions and Bosons. International Journal of Mathematics and Mathematical Sciences 2010, 890523 (2010).

Raghavan, S., Smerzi, A., Fantoni, S., and Shenoy, S.R. Coherent oscillations between two weakly coupled Bose-Einstein condensates: Josephson effects, $\pi$ oscillations, and macroscopic quantum self-trapping. Physical Review A 59, 620-633 (1999).

Raizen, M.G. Quantum Chaos with Cold Atoms. Advances In Atomic, Molecular, and Optical Physics 41, 43-81 (1999).

Ramm, M. Quantum Correlations and Energy Transport in Trapped Ions. Ph.D. thesis, University of California, Berkeley (2014). 
Ramm, M., Pruttivarasin, T., and Häffner, H. Energy transport in trapped ion chains. New Journal of Physics 16, 063062 (2014).

Ramm, M., Pruttivarasin, T., Kokish, M., Talukdar, I., and Häffner, H. Precision Measurement Method for Branching Fractions of Excited $P_{1 / 2}$ States Applied to ${ }^{40} \mathrm{Ca}^{+}$. Physical Review Letters 111, 023004 (2013).

Ramsey, N.F. Experiments with separated oscillatory fields and hydrogen masers. Reviews of Modern Physics 62, 541-552 (1990).

Raussendorf, R. and Briegel, H.J. A One-Way Quantum Computer. Physical Review Letters 86, 5188-5191 (2001).

Reck, M., Zeilinger, A., Bernstein, H.J., and Bertani, P. Experimental realization of any discrete unitary operator. Physical Review Letters 73, 58-61 (1994).

Redfield, A.G. On the Theory of Relaxation Processes. IBM Journal of Research and Development 1, 19-31 (1957).

Reed, M. and Simon, B. Methods of Modern Mathematical Physics III: Scattering Theory (Academic Press, San Diego, CA, 1979).

Řeháček, J., Mogilevtsev, D., and Hradil, Z. Tomography for quantum diagnostics. New Journal of Physics 10, 043022 (2008).

Renner, R. Symmetry of large physical systems implies independence of subsystems. Nat Phys 3, 645-649 (2007).

Ribeiro, P., Vidal, J., and Mosseri, R. Thermodynamical Limit of the Lipkin-MeshkovGlick Model. Physical Review Letters 99, 050402 (2007).

Ribeiro, P., Vidal, J., and Mosseri, R. Exact spectrum of the Lipkin-Meshkov-Glick model in the thermodynamic limit and finite-size corrections. Physical Review $\mathrm{E}$ 78, 021106 (2008).

Richerme, P., Gong, Z.X., Lee, A., Senko, C., Smith, J., Foss-Feig, M., Michalakis, S., Gorshkov, A.V., and Monroe, C. Non-local propagation of correlations in quantum systems with long-range interactions. Nature 511, 198-201 (2014).

Richter, K. Semiclassical Theory of Mesoscopic Quantum Systems (Springer, Berlin, Germany, 2000).

Richter, M. and Mukamel, S. Ultrafast double-quantum-coherence spectroscopy of excitons with entangled photons. Physical Review A 82, 013820 (2010).

Richter, M., Schlosser, F., Schoth, M., Burger, S., Schmidt, F., Knorr, A., and Mukamel, S. Reconstruction of the wave functions of coupled nanoscopic emitters using a coherent optical technique. Physical Review B 86, 085308 (2012). 
Riebe, M., Häffner, H., Roos, C.F., Hänsel, W., Benhelm, J., Lancaster, G.P.T., Körber, T.W., Becher, C., Schmidt-Kaler, F., James, D.F.V., and Blatt, R. Deterministic Quantum Teleportation with Atoms. Nature 429, 734 (2004).

Riemann, B. Über die Anzahl der Primzahlen unter einer gegebenen Grösse. Monatsberichte der Königlich Preußischen Akademie der Wissenschaften zu Berlin (1859).

Riseborough, P.S., Hanggi, P., and Weiss, U. Exact results for a damped quantummechanical harmonic oscillator. Physical Review A 31, 471-478 (1985).

Ritz, W. Über eine neue Methode zur Lösung gewisser Variationsprobleme der mathematischen Physik. Journal für die Reine und Angewandte Mathematik 135, 1-61 (1909).

Rivas, A., Huelga, S.F., and Plenio, M.B. Entanglement and Non-Markovianity of Quantum Evolutions. Physical Review Letters 105, 050403 (2010).

Roati, G., D'Errico, C., Fallani, L., Fattori, M., Fort, C., Zaccanti, M., Modugno, G., Modugno, M., and Inguscio, M. Anderson localization of a non-interacting Bose-Einstein condensate. Nature 453, 895-898 (2008).

Roghani, M., Helm, H., and Breuer, H.P. Entanglement Dynamics of a Strongly Driven Trapped Atom. Physical Review Letters 106, 040502 (2011).

Röpke, G. Nonequilibrium Statistical Physics (WILEY-VCH, Weinheim, Germany, 2013).

Rossi, M.A.C., Benedetti, C., and Paris, M.G.A. Engineering decoherence for twoqubit systems interacting with a classical environment. International Journal of Quantum Information 12, 1560003 (2014).

Rowe, M., Ben-Kish, A., DeMarco, B., Leibfried, D., Meyer, V., Beall, J., Britton, J., Hughes, J., Itano, W., Jelenkovic, B., Langer, C., Rosenband, T., and Wineland, D. Transport of quantum states and separation of ions in a dual RF ion trap. Quantum Information and Computation 2, 257-271 (2002).

Rudin, W. Fourier Analysis on Groups (John Wiley \& Sons, Hoboken, NJ, 1990).

Ruskai, M.B. Beyond Strong Subsadditivity? Improved Bounds on the Contraction of Generalized Relative Entropy. Reviews in Mathematical Physics 6, 1147-1161 (1994).

Sachdev, S. Quantum Phase Transitions (Cambridge University Press, Cambridge, UK, 1999), 1 edition.

Sachdev, S., Sengupta, K., and Girvin, S.M. Mott insulators in strong electric fields. Physical Review B 66, 075128 (2002).

Saffman, M., Walker, T.G., and Mølmer, K. Quantum information with Rydberg atoms. Reviews of Modern Physics 82, 2313-2363 (2010). 
Sakurai, J.J. and Napolitano, J. Modern Quantum Mechanics (Addison-Wesley, San Francisco, CA, 1994).

Sallen, G., Tribu, A., Aichele, T., Andre, R., Besombes, L., Bougerol, C., Richard, M., Tatarenko, S., Kheng, K., and Poizat, J.P. Subnanosecond spectral diffusion measurement using photon correlation. Nature Photonics 4, 696-699 (2010).

Sassetti, M. and Weiss, U. Correlation functions for dissipative two-state systems: Effects of the initial preparation. Physical Review A 41, 5383-5393 (1990).

Scherer, N.F., Carlson, R.J., Matro, A., Du, M., Ruggiero, A.J., Romero-Rochin, V., Cina, J.A., Fleming, G.R., and Rice, S.A. Fluorescence-detected wave packet interferometry: Time resolved molecular spectroscopy with sequences of femtosecond phase-locked pulses. The Journal of Chemical Physics 95, 1487-1511 (1991).

Schindler, P., Nigg, D., Monz, T., Barreiro, J.T., Martinez, E., Wang, S.X., Quint, S., Brandl, M.F., Nebendahl, V., Roos, C.F., Chwalla, M., Hennrich, M., and Blatt, R. A quantum information processor with trapped ions. New Journal of Physics 15, $123012(2013)$.

Schlawin, F. Nonlinear Quantum-Optical Spectroscopy. Dissertation, Albert-LudwigsUniversität Freiburg, Universität Freiburg (2015).

Schlawin, F., Dorfman, K.E., Fingerhut, B.P., and Mukamel, S. Manipulation of two-photon-induced fluorescence spectra of chromophore aggregates with entangled photons: A simulation study. Physical Review A 86, 023851 (2012).

Schlawin, F., Dorfman, K.E., Fingerhut, B.P., and Mukamel, S. Suppression of population transport and control of exciton distributions by entangled photons. Nature Communications 4, 1782 (2013).

Schlawin, F., Gessner, M., Mukamel, S., and Buchleitner, A. Nonlinear spectroscopy of trapped ions. Physical Review A 90, 023603 (2014).

Schleich, W. Quantum Optics in Phase Space (WILEY-VCH, Berlin, Germany, 2001).

Schliemann, J. and Mertens, F.G. Semiclassical description of Heisenberg models via spin-coherent states. Journal of Physics: Condensed Matter 10, 1091 (1998).

Schmidt, E. Zur Theorie der linearen und nichtlinearen Integralgleichungen. Mathematische Annalen 63, 433-476 (1907).

Schmidt-Kaler, F., Häffner, H., Riebe, M., Gulde, S., Lancaster, G.P.T., Deuschle, T., Becher, C., Roos, C.F., Eschner, J., and Blatt, R. Realization of the Cirac-Zoller controlled-NOT quantum gate. Nature 422, 408-411 (2003).

Schneider, C., Porras, D., and Schaetz, T. Experimental quantum simulations of manybody physics with trapped ions. Reports on Progress in Physics 75, 024401 (2012). 
Schneider, S., Herkommer, A.M., Leonhardt, U., and Schleich, W.P. Cavity field tomography via atomic beam deflection. Journal of Modern Optics 44, 2333-2342 (1997).

Schollwöck, U. The density-matrix renormalization group. Reviews of Modern Physics 77, 259-315 (2005).

Schrödinger, E. Are there quantum jumps? British Journal for the Philosophy of Science 3, 233 (1952).

Schröter, M., Ivanov, S.D., Schulze, J., Polyutov, S.P., Yan, Y., Pullerits, T., and Kühn, O. Exciton-vibrational coupling in the dynamics and spectroscopy of Frenkel excitons in molecular aggregates. Physics Reports 567, 1-78 (2015).

Schuch, N., Wolf, M.M., Verstraete, F., and Cirac, J.I. Entropy Scaling and Simulability by Matrix Product States. Physical Review Letters 100, 030504 (2008).

Schwemmer, C., Knips, L., Richart, D., Weinfurter, H., Moroder, T., Kleinmann, M., and Gühne, O. Systematic Errors in Current Quantum State Tomography Tools. Physical Review Letters 114, 080403 (2015).

Schwemmer, C., Tóth, G., Niggebaum, A., Moroder, T., Gross, D., Gühne, O., and Weinfurter, H. Experimental Comparison of Efficient Tomography Schemes for a Six-Qubit State. Physical Review Letters 113, 040503 (2014).

Scully, M.O. and Zubairy, M.S. Quantum Optics (Cambridge University Press, Cambridge, UK, 1997).

Segal, D.M. and Wunderlich, C. Cooling Techniques for Trapped Ions. In Physics with Trapped Charged Particles - Lectures from the Les Houches Winter School, pp. 43-81 (World Scientific, Singapore, Singapore, 2014).

Senko, C., Smith, J., Richerme, P., Lee, A., Campbell, W.C., and Monroe, C. Coherent imaging spectroscopy of a quantum many-body spin system. Science 345, 430-433 (2014).

Serbyn, M., Knap, M., Gopalakrishnan, S., Papić, Z., Yao, N.Y., Laumann, C.R., Abanin, D.A., Lukin, M.D., and Demler, E.A. Interferometric Probes of ManyBody Localization. Physical Review Letters 113, 147204 (2014).

Shchesnovich, V.S. and Konotop, V.V. Nonlinear tunneling of Bose-Einstein condensates in an optical lattice: Signatures of quantum collapse and revival. Physical Review A 75, 063628 (2007).

Shen, C., Zhang, Z., and Duan, L.M. Scalable Implementation of Boson Sampling with Trapped Ions. Physical Review Letters 112, 050504 (2014).

Sherson, J.F., Weitenberg, C., Endres, M., Cheneau, M., Bloch, I., and Kuhr, S. Single-atom-resolved fluorescence imaging of an atomic Mott insulator. Nature 467, 68-72 (2010). 
Shor, P. Algorithms for quantum computation: Discrete logarithms and factoring. In S. Goldwasser (Editor), Proc. 35th Annu. Symp. on the Foundations of Computer Science, pp. 124-134 (IEEE Computer Society Press, Los Alamitos, California, 1994).

Siemens, M.E., Moody, G., Li, H., Bristow, A.D., and Cundiff, S.T. Resonance lineshapes in two-dimensional Fourier transform spectroscopy. Optics Express 18, 17699-17708 (2010).

Silva, I.A., Girolami, D., Auccaise, R., Sarthour, R.S., Oliveira, I.S., Bonagamba, T.J., deAzevedo, E.R., Soares-Pinto, D.O., and Adesso, G. Measuring Bipartite Quantum Correlations of an Unknown State. Physical Review Letters 110, 140501 (2013).

Simon, J., Bakr, W.S., Ma, R., Tai, M.E., Preiss, P.M., and Greiner, M. Quantum simulation of antiferromagnetic spin chains in an optical lattice. Nature $\mathbf{4 7 2}$, 307-312 (2011).

Sinatra, A., Lobo, C., and Castin, Y. Classical-Field Method for Time Dependent Bose-Einstein Condensed Gases. Physical Review Letters 87, 210404 (2001).

Smerzi, A., Fantoni, S., Giovanazzi, S., and Shenoy, S.R. Quantum Coherent Atomic Tunneling between Two Trapped Bose-Einstein Condensates. Physical Review Letters 79, 4950-4953 (1997).

Smirne, A., Brivio, D., Cialdi, S., Vacchini, B., and Paris, M.G.A. Experimental investigation of initial system-environment correlations via trace-distance evolution. Physical Review A 84, 032112 (2011).

Smith, C.M. and Caldeira, A.O. Generalized Feynman-Vernon approach to dissipative quantum systems. Physical Review A 36, 3509-3511 (1987).

Smith, C.M. and Caldeira, A.O. Application of the generalized Feynman-Vernon approach to a simple system: The damped harmonic oscillator. Physical Review A 41, 3103-3115 (1990).

Sondhi, S.L., Girvin, S.M., Carini, J.P., and Shahar, D. Continuous quantum phase transitions. Reviews of Modern Physics 69, 315-333 (1997).

Sørensen, A., Duan, L.M., Cirac, J.I., and Zoller, P. Many-particle entanglement with Bose-Einstein condensates. Nature 409, 63-66 (2001).

Sørensen, A. and Mølmer, K. Quantum Computation with Ions in Thermal Motion. Physical Review Letters 82, 1971-1974 (1999).

Spohn, H. Kinetic equations from Hamiltonian dynamics: Markovian limits. Reviews of Modern Physics 52, 569-615 (1980). 
Spring, J.B., Metcalf, B.J., Humphreys, P.C., Kolthammer, W.S., Jin, X.M., Barbieri, M., Datta, A., Thomas-Peter, N., Langford, N.K., Kundys, D., Gates, J.C., Smith, B.J., Smith, P.G.R., and Walmsley, I.A. Boson Sampling on a Photonic Chip. Science 339, 798-801 (2013).

Štelmachovič, P. and Bužek, V. Quantum-information approach to the Ising model: Entanglement in chains of qubits. Physical Review A 70, 032313 (2004).

Stenholm, S. The semiclassical theory of laser cooling. Reviews of Modern Physics 58, 699-739 (1986).

Streltsov, A., Kampermann, H., and Bruß, D. Behavior of Quantum Correlations under Local Noise. Physical Review Letters 107, 170502 (2011a).

Streltsov, A., Kampermann, H., and Bruß, D. Linking Quantum Discord to Entanglement in a Measurement. Physical Review Letters 106, 160401 (2011b).

Streltsov, A., Kampermann, H., and Bruß, D. Quantum Cost for Sending Entanglement. Physical Review Letters 108, 250501 (2012).

Sudarshan, E.C.G. Equivalence of Semiclassical and Quantum Mechanical Descriptions of Statistical Light Beams. Physical Review Letters 10, 277-279 (1963).

Suzuki, M. Relationship between d-Dimensional Quantal Spin Systems and $(d+1)$ Dimensional Ising Systems - Equivalence, Critical Exponents and Systematic Approximants of the Partition Function and Spin Correlations-. Progress of Theoretical Physics 56, 1454-1469 (1976).

Tang, J.S., Wang, Y.T., Chen, G., Zou, Y., Li, C.F., Guo, G.C., Yu, Y., Li, M.F., Zha, G.W., Ni, H.Q., Niu, Z.C., Gessner, M., and Breuer, H.P. Experimental detection of polarization-frequency quantum correlations in a photonic quantum channel by local operations. arXiv:1311.5034 [quant-ph] (2013).

Tanimura, Y. and Mukamel, S. Two-dimensional femtosecond vibrational spectroscopy of liquids. The Journal of Chemical Physics 99, 9496-9511 (1993).

Taylor, J.M., Cappellaro, P., Childress, L., Jiang, L., Budker, D., Hemmer, P.R., Yacoby, A., Walsworth, R., and Lukin, M.D. High-sensitivity diamond magnetometer with nanoscale resolution. Nature Physics 4, 810-816 (2008).

Tekavec, P.F., Dyke, T.R., and Marcus, A.H. Wave packet interferometry and quantum state reconstruction by acousto-optic phase modulation. The Journal of Chemical Physics 125, 194303 (2006).

Tekavec, P.F., Lott, G.A., and Marcus, A.H. Fluorescence-detected two-dimensional electronic coherence spectroscopy by acousto-optic phase modulation. The Journal of Chemical Physics 127, 214307 (2007).

Tennyson, J.L., Cary, J.R., and Escande, D.F. Change of the Adiabatic Invariant due to Separatrix Crossing. Physical Review Letters 56, 2117-2120 (1986). 
Ter Haar, D. On the density matrices used in Hartree-Fock calculations. Physica 26, 1041-1044 (1960).

Ter Haar, D. Theory and applications of the density matrix. Reports on Progress in Physics 24, 304 (1961).

Tian, P., Keusters, D., Suzaki, Y., and Warren, W.S. Femtosecond Phase-Coherent Two-Dimensional Spectroscopy. Science 300, 1553-1555 (2003).

Tichy, M.C. Entanglement and interference of identical particles. Dissertation, AlbertLudwigs-Universität Freiburg, Universität Freiburg (2011).

Tichy, M.C. Interference of identical particles from entanglement to boson-sampling. Journal of Physics B: Atomic, Molecular and Optical Physics 47, 103001 (2014).

Tillmann, M., Dakic, B., Heilmann, R., Nolte, S., Szameit, A., and Walther, P. Experimental boson sampling. Nature Photonics 7, 540-544 (2013).

Titchmarsh, E.C. The theory of the Riemann zeta-function (Clarendon Press, Oxford, UK, 1986).

Tokmakoff, A. Two-Dimensional Line Shapes Derived from Coherent Third-Order Nonlinear Spectroscopy. The Journal of Physical Chemistry A 104, 4247-4255 (2000).

Toyoda, K., Matsuno, Y., Noguchi, A., Haze, S., and Urabe, S. Experimental Realization of a Quantum Phase Transition of Polaritonic Excitations. Physical Review Letters 111, 160501 (2013).

Uhlmann, A. Entropy and optimal decompositions of states relative to a maximal commutative subalgebra. Open Systems \& Information Dynamics 5, 209-228 (1998).

Upton, L., Harpham, M., Suzer, O., Richter, M., Mukamel, S., and Goodson, T. Optically Excited Entangled States in Organic Molecules Illuminate the Dark. The Journal of Physical Chemistry Letters 4, 2046-2052 (2013).

Urban, E., Johnson, T.A., Henage, T., Isenhower, L., Yavuz, D.D., Walker, T.G., and Saffman, M. Observation of Rydberg blockade between two atoms. Nature Physics 5, 110-114 (2009).

Ursin, R., Tiefenbacher, F., Schmitt-Manderbach, T., Weier, H., Scheidl, T., Lindenthal, M., Blauensteiner, B., Jennewein, T., Perdigues, J., Trojek, P., Omer, B., Furst, M., Meyenburg, M., Rarity, J., Sodnik, Z., Barbieri, C., Weinfurter, H., and Zeilinger, A. Entanglement-based quantum communication over $144 \mathrm{~km}$. Nature Physics 3, 481-486 (2007).

Uys, H., Biercuk, M.J., VanDevender, A.P., Ospelkaus, C., Meiser, D., Ozeri, R., and Bollinger, J.J. Decoherence due to Elastic Rayleigh Scattering. Physical Review Letters 105, 200401 (2010). 
Van Vleck, J.H. The Correspondence Principle in the Statistical Interpretation of Quantum Mechanics. Proceedings of the National Academy of Sciences of the United States of America 14, 178-188 (1928).

Vandersypen, L.M.K., Steffen, M., Breyta, G., Yannoni, C.S., Sherwood, M.H., and Chuang, I.L. Experimental realization of Shor's quantum factoring algorithm using nuclear magnetic resonance. Nature 414, 883-887 (2001).

Vasa, P., Ropers, C., Pomraenke, R., and Lienau, C. Ultra-fast nano-optics. Laser \& Photonics Reviews 3, 483-507 (2009).

Vedral, V., Plenio, M.B., Rippin, M.A., and Knight, P.L. Quantifying Entanglement. Physical Review Letters 78, 2275-2279 (1997).

Venzl, H., Daley, A.J., Mintert, F., and Buchleitner, A. Statistics of Schmidt coefficients and the simulability of complex quantum systems. Physical Review E 79, 056223 (2009).

Verstraete, F., Murg, V., and Cirac, J. Matrix product states, projected entangled pair states, and variational renormalization group methods for quantum spin systems. Advances in Physics 57, 143-224 (2008).

Vidal, G., Latorre, J.I., Rico, E., and Kitaev, A. Entanglement in Quantum Critical Phenomena. Physical Review Letters 90, 227902 (2003).

Vidal, G. and Werner, R.F. Computable measure of entanglement. Physical Review A 65, 032314 (2002).

Vidal, J., Mosseri, R., and Dukelsky, J. Entanglement in a first-order quantum phase transition. Physical Review A 69, 054101 (2004).

Vogel, W. and Filho, R.L.d.M. Nonlinear Jaynes-Cummings dynamics of a trapped ion. Physical Review A 52, 4214-4217 (1995).

Vojta, M. Quantum phase transitions. Reports on Progress in Physics 66, 2069 (2003).

Vollbrecht, K.G.H. and Werner, R.F. Entanglement Measures Under Symmetry. Physical Review A 64, 062307 (2001).

Vollmer, C.E., Schulze, D., Eberle, T., Händchen, V., Fiurášek, J., and Schnabel, R. Experimental Entanglement Distribution by Separable States. Physical Review Letters 111, 230505 (2013).

Volz, J., Weber, M., Schlenk, D., Rosenfeld, W., Vrana, J., Saucke, K., Kurtsiefer, C., and Weinfurter, H. Observation of Entanglement of a Single Photon with a Trapped Atom. Physical Review Letters 96, 030404 (2006).

Waki, I., Kassner, S., Birkl, G., and Walther, H. Observation of ordered structures of laser-cooled ions in a quadrupole storage ring. Physical Review Letters 68, 2007-2010 (1992). 
Wallraff, A., Schuster, D.I., Blais, A., Frunzio, L., Huang, R.S., Majer, J., Kumar, S., Girvin, S.M., and Schoelkopf, R.J. Strong coupling of a single photon to a superconducting qubit using circuit quantum electrodynamics. Nature 431, 162167 (2004).

Walter, M., Doran, B., Gross, D., and Christandl, M. Entanglement Polytopes: Multiparticle Entanglement from Single-Particle Information. Science 340, 1205-1208 (2013).

Walther, A., Ziesel, F., Ruster, T., Dawkins, S.T., Ott, K., Hettrich, M., Singer, K., Schmidt-Kaler, F., and Poschinger, U. Controlling Fast Transport of Cold Trapped Ions. Physical Review Letters 109, 080501 (2012).

Walther, H. Experiments on cavity quantum electrodynamics. Physics Reports 219, 263-281 (1992).

Walther, H., Varcoe, B.T.H., Englert, B.G., and Becker, T. Cavity quantum electrodynamics. Reports on Progress in Physics 69, 1325 (2006).

Wang, L., Huang, J.H., Dowling, J., and Zhu, S.Y. Quantum information transmission. Quantum Information Processing 12, 899-906 (2013).

Warren, W.S. The Usefulness of NMR Quantum Computing. Science 277, 1688-1690 (1997).

Weiss, P. L’hypothèse du champ moléculaire et la propriété ferromagnétique. Journal de Physique Théorique et Appliquée 6, 661-690 (1907).

Weiss, U. Quantum Dissipative Systems (World Scientific, Singapore, Singapore, 1993).

Weisskopf, V. and Wigner, E.P. Berechnung der natürlichen Linienbreite auf Grund der Diracschen Lichttheorie. Zeitschrift für Physik 63, 54-73 (1930).

Weitenberg, C., Endres, M., Sherson, J.F., Cheneau, M., Schausz, P., Fukuhara, T., Bloch, I., and Kuhr, S. Single-spin addressing in an atomic Mott insulator. Nature 471, 319-324 (2011).

Wen, X.G. Quantum Field Theory of Many-Body Systems (Oxford Graduate Texts, Oxford, UK, 2004).

Wenz, A.N., Zürn, G., Murmann, S., Brouzos, I., Lompe, T., and Jochim, S. From Few to Many: Observing the Formation of a Fermi Sea One Atom at a Time. Science 342, 457-460 (2013).

Werner, R.F. Quantum States with Einstein-Podolsky-Rosen Correlations Admitting a Hidden-Variable Model. Physical Review A 40, 4277-4281 (1989).

Weyl, H. The Theory of Groups and Quantum Mechanics (Dover Publications, New York, NY, 1950). 
White, S.R. Density matrix formulation for quantum renormalization groups. Physical Review Letters 69, 2863-2866 (1992).

Wiersma, D.S., Bartolini, P., Lagendijk, A., and Righini, R. Localization of light in a disordered medium. Nature 390, 671-673 (1997).

Wigner, E.P. and Yanase, M.M. Information Contents of Distributions. Proceedings of the National Academy of Sciences 49, 910 (1963).

Wigner, E.P. On the statistical distribution of the widths and spacings of nuclear resonance levels. Mathematical Proceedings of the Cambridge Philosophical Society 47, 790-798 (1951).

Wigner, E.P. Group Theory and its Application to the Quantum Mechanics of Atomic Spectra (Academic Press, New York, NY, 1959).

Wilson, K.G. The renormalization group: Critical phenomena and the Kondo problem. Reviews of Modern Physics 47, 773-840 (1975).

Wimberger, S., Guarneri, I., and Fishman, S. Quantum resonances and decoherence for $\delta$-kicked atoms. Nonlinearity 16, 1381 (2003).

Wineland, D.J. and Dehmelt, H. Proposed $10^{-14} \Delta \nu<\nu$ laser fluorescence spectroscopy on $\mathrm{Tl}^{+}$mono-ion oscillator. Bulletin of the American Physical Society 20, 637 (1975).

Wineland, D.J., Ekstrom, P., and Dehmelt, H. Monoelectron Oscillator. Physical Review Letters 31, 1279 (1973).

Wineland, D.J. and Itano, W.M. Laser cooling of atoms. Physical Review A 20, 1521-1540 (1979).

Wineland, D.J., Monroe, C., Itano, W.M., Leibfried, D., King, B.E., and Meekhof, D.M. Experimental issues in coherent quantum-state manipulation of trapped atomic ions. Journal of Research of the National Institute of Standards and Technology 103, 259-328 (1998).

Wineland, D.J. Nobel Lecture: Superposition, entanglement, and raising Schrödinger's cat. Reviews of Modern Physics 85, 1103-1114 (2013).

Wootters, W.K. and Zurek, W.H. A single quantum cannot be cloned. Nature 299, 802-803 (1982).

Wootters, W.K. Entanglement of Formation of an Arbitrary State of Two Qubits. Physical Review Letters 80, 2245-2248 (1998).

Wu, H., Feng, D.H., and Vallieres, M. 'Chaotic' level statistics and quantum phase transition. Journal of Physics G: Nuclear and Particle Physics 16, L149 (1990). 
Wu, L.A., Sarandy, M.S., and Lidar, D.A. Quantum Phase Transitions and Bipartite Entanglement. Physical Review Letters 93, 250404 (2004).

Würtz, P., Langen, T., Gericke, T., Koglbauer, A., and Ott, H. Experimental Demonstration of Single-Site Addressability in a Two-Dimensional Optical Lattice. Physical Review Letters 103, 080404 (2009).

Yan, B., Moses, S.A., Gadway, B., Covey, J.P., Hazzard, K.R.A., Rey, A.M., Jin, D.S., and $\mathrm{Ye}, \mathrm{J}$. Observation of dipolar spin-exchange interactions with lattice-confined polar molecules. Nature 501, 521-525 (2013).

Yang, C. Concept of Off-Diagonal Long-Range Order and the Quantum Phases of Liquid He and of Superconductors. Reviews of Modern Physics 34, 694-704 (1962).

Young, B.C., Cruz, F.C., Itano, W.M., and Bergquist, J.C. Visible Lasers with Subhertz Linewidths. Physical Review Letters 82, 3799-3802 (1999).

Yvon, J. La théorie statistique des fluides et l'équation d'état (Hermann, Paris, France, 1935).

Zeilinger, A. General properties of lossless beam splitters in interferometry. American Journal of Physics 49, 882-883 (1981).

Zener, C. A Theory of the Electrical Breakdown of Solid Dielectrics. Proceedings of the Royal Society of London. Series A, Containing Papers of a Mathematical and Physical Character 145, 523-529 (1934).

Zhang, T., Kuznetsova, I., Meier, T., Li, X., Mirin, R.P., Thomas, P., and Cundiff, S.T. Polarization-dependent optical 2D Fourier transform spectroscopy of semiconductors. Proceedings of the National Academy of Sciences 104, 14227-14232 (2007).

Zhang, W.M., Feng, D.H., and Gilmore, R. Coherent states: Theory and some applications. Reviews of Modern Physics 62, 867-927 (1990).

Zhao, M.J. private communication (2012). 